



\section{Sexually Transmitted Disease Surveillance 2018}

\section{Division of STD Prevention October 2019}

U.S. Department of Health and Human Services Centers for Disease Control and Prevention

National Center for HIV/AIDS, Viral Hepatitis, STD, and TB Prevention

Division of STD Prevention

Atlanta, Georgia, 30329-4027 


\section{This report was prepared by}

\section{Surveillance and Data Management Branch}

\section{Division of STD Prevention}

National Center for HIV/AIDS, Viral Hepatitis, STD, and TB Prevention

Centers for Disease Control and Prevention
Virginia Bowen
Jim Braxton
Darlene Davis
Elaine Flagg
Jeremy Grey
LaZetta Grier
Alesia Harvey
Sarah Kidd
Kristen Kreisel
Eloisa Llata
Kerry Mauk

\begin{abstract}
Melissa Pagaoa
Viani Picchetti

Rodney Presley

Philip Ricks

Steven Shapiro

Sancta St. Cyr

Mark Stenger

Elizabeth Torrone

Hillard Weinstock

Emily Weston

Niketta Womack
\end{abstract}

Others contributing to the production and dissemination of this publication

\section{Division of STD Prevention}

\author{
Tranita Anderson \\ Diane Ballard \\ Gail Bolan \\ Keith Davis \\ Jaeyoung Hong \\ Britney Johnson \\ Ellen Kersh \\ Neeraja Lakshmipathy \\ Jennifer Ludovic
}

\author{
Nikki Mayes \\ Mary McFarlane \\ Alejandro Perez \\ Cau Pham \\ Tiffani Phelps \\ Raul Romaguera \\ Salina Smith \\ Guoyu Tao \\ Jo Valentine
}

\section{National Center for HIV/AIDS, Viral Hepatitis, STD, and TB Prevention Daniel Johnson \\ Jacqueline Petty \\ Rachel Wingard}

Publication of this report would not have been possible without the contributions of the state and territorial health departments, STD control programs, and public health laboratories that provided surveillance data to CDC. 


\section{Copyright Information}

All material contained in this report is in the public domain and may be used and reprinted without special permission; however, citation as to source is appreciated.

\section{Suggested Citation}

Centers for Disease Control and Prevention. Sexually Transmitted Disease Surveillance 2018. Atlanta: U.S. Department of Health and Human Services; 2019. DOI: 10.15620/cdc.79370.

\section{Web Site}

The online version of this report is available at https:/www.cdc.gov/std/stats

\section{Selected STD Surveillance and Prevention References and Web Sites}

STD Surveillance Reports 1997-2017

https://www.cdc.gov/std/stats/archive.htm

STD Data in the NCHHSTP AtlasPlus

https://www.cdc.gov/nchhstp/atlas/

STD Data on Wonder

https://wonder.cdc.gov/std.html

STD Data Management \& Information Technology

https://www.cdc.gov/std/Program/data-mgmt.htm

STD Fact Sheets

https://www.cdc.gov/std/healthcomm/fact sheets.htm

STD Treatment Guidelines

https://www.cdc.gov/STD/treatment/

STD Program Evaluation Guidelines

https://www.cdc.gov/std/program/pupestd.htm

STD Program Operation Guidelines

https://www.cdc.gov/std/program/GL-2001.htm

Recommendations for Public Health Surveillance of Syphilis in the United States

https://www.cdc.gov/std/SyphSurvReco.pdf

Gonococcal Isolate Surveillance Project (GISP)

https://www.cdc.gov/std/gisp/default.htm

STD Surveillance Network (SSuN)

https://www.cdc.gov/std/ssun/default.htm

National Health and Nutrition Examination Survey (NHANES)

https://www.cdc.gov/nchs/nhanes/index.htm 
This page intentionally left blank. 
STDs have long been an underestimated opponent in the public health battle. A 1997 Institute of Medicine (IOM) report described STDs as "hidden epidemics of tremendous health and economic consequence in the United States," and stated that the "scope, impact, and consequences of STDs are under recognized by the public and healthcare professionals." Since well before this report was published, and two decades later, those facts remain unchanged.

Yet not that long ago, gonorrhea rates were at historic lows, syphilis was close to elimination, and we were able to point to advances in STD prevention, such as better chlamydia diagnostic tests and more screening, contributing to increases in detection and treatment of chlamydial infections. That progress has since unraveled. The number of reported syphilis cases is climbing after being largely on the decline since 1941, and gonorrhea rates are now increasing. This is especially concerning given that we are slowly running out of treatment options to cure Neisseria gonorrhoeae. Many young women continue to have undiagnosed chlamydial infections, putting them at risk for infertility.

Half of STDs are among young people aged 15 to 24 years. ${ }^{2}$ These infections can lead to long-term health consequences, such as infertility; they can facilitate HIV transmission; and they have stigmatized entire subgroups of Americans. Beyond the impact on an individual's health, STDs are also an economic drain on the US healthcare system, costing billions annually. ${ }^{3}$ To complicate the matter, STD public health programs are increasingly facing challenges and barriers in achieving their mission.

It is imperative that federal, state, and local programs employ strategies that maximize long-term population impact by reducing STD incidence and promoting sexual, reproductive, maternal, and infant health. The resurgence of syphilis, and particularly congenital syphilis, is not an arbitrary event, but rather a symptom of a deteriorating public health infrastructure and lack of access to health care. It is exposing hidden, fragile populations in need that are not getting the health care and preventive services they deserve. This points to our need for public health and health care action for each of the cases in this report, as they represent real people, not just numbers.

We also need to modernize surveillance to move beyond counting only those cases in persons who have access to diagnosis and treatment, to develop innovative strategies to understand the burden of disease in those who may not access care, and to improve our surveillance systems to collect the information needed to target prevention activities. Further, it will be important for us to measure and monitor the adverse health consequences of STDs, such as ocular and neurosyphilis, pelvic inflammatory disease, ectopic pregnancy, infertility, HIV, congenital syphilis, and neonatal herpes.

It is my hope that in future years, we will be reporting on progress, instead of more health inequity in our society. This is our challenge and our call to effectively respond to the information shared in this report.

Gail Bolan, M.D.

Director, Division of STD Prevention

National Center for HIV/AIDS, Viral Hepatitis, STD, and

TB Prevention

US Centers for Disease Control and Prevention

\section{References}

1. Eng TR, Butler WT, editors; Institute of Medicine (US). Summary: The hidden epidemic: Confronting sexually transmitted diseases. Washington (DC): National Academy Press; 1997.

2. Satterwhite CL, Torrone E, Meites E, et al. Sexually transmitted infections among US women and men: Prevalence and incidence estimates, 2008. Sex Transm Dis. 2013;40(3):187-193. DOI: 10.1097/OLQ.0b013e318286bb53.

3. Owusu-Edusei K Jr, Chesson HW, Gift TL, et al. The estimated direct medical cost of selected sexually transmitted infections in the United States, 2008. Sex Transm Dis. 2013;40(3):197-201. DOI: 10.1097/ OLQ.0b013e318285c6d2. 
This page intentionally left blank. 


\section{Preface}

Sexually Transmitted Disease Surveillance 2018 presents statistics and trends for STDs in the United States through 2018. This annual publication is intended as a reference document for policy makers, program managers, health planners, researchers, and others who are concerned with the public health implications of these diseases. The figures and tables in this edition supersede those in earlier publications of these data.

The surveillance information in this report is based on the following sources of data: (1) notifiable disease reporting from state and local STD programs; (2) projects and programs that monitor STDs in various settings, including the National Job Training Program, the STD Surveillance Network, and the Gonococcal Isolate Surveillance Project; and (3) national surveys and other data collection systems implemented by federal and private organizations.

Four STDs are nationally notifiable, chlamydia, gonorrhea, syphilis, and chancroid, and state and local STD control programs provide CDC with case reports for these conditions. These case reports are the data source for many of the figures and most of the statistical tables in this publication; however, it is important to note that these case reports reflect only a portion of STDs occurring in the US population. First, other common STDs, such as human papillomavirus (HPV) and herpes simplex virus (HSV) are not nationally notifiable diseases. Additionally, STDs are often asymptomatic and may not be diagnosed; therefore, case report data underestimate the number of infections that occurred.

In January 2018, a revised case definition for syphilis went into effect, including changing the stage previously termed "early latent syphilis" to "syphilis, early non-primary non-secondary". This change in terminology more accurately reflects this stage of infection, as neurologic symptoms, including ocular syphilis, can occur at this stage. Additionally, the stages of "late latent syphilis" and "late syphilis with clinical manifestations" were removed and "syphilis, unknown duration or late" was added. More information on syphilis morbidity reporting and the current case definition can be found in Appendices A and $\mathrm{C}$ of this report.

Sexually Transmitted Disease Surveillance 2018 consists of four sections: the National Profile, the Special Focus Profiles, the Tables, and the Appendix. The National Profile section contains figures that provide an overview of STD morbidity in the United States. The accompanying text identifies major findings and trends for selected STDs. The Special Focus Profiles section contains figures and text that describe STDs in selected populations that are a focus of national and state prevention efforts. The Tables section provides statistical information about STDs at county, metropolitan statistical area, regional, state, and national levels. The Appendix includes information on how to interpret the STD surveillance data used to produce this report, as well as information about Healthy People 2020 STD objectives and progress toward meeting these objectives, Congressional Budget Justification goals and progress toward meeting these goals, and STD surveillance case definitions.

Any comments and suggestions that would improve future publications are appreciated and should be sent to:

Director, Division of STD Prevention

National Center for HIV/AIDS, Viral Hepatitis, STD, and TB Prevention

Centers for Disease Control and Prevention

1600 Clifton Road NE, Mailstop US12-2

Atlanta, Georgia 30329-4027 
This page intentionally left blank. 


\section{Guide to Acronyms}

ACIP

$\mathrm{AI} / \mathrm{AN}$

ARLN

APC

AS

CDC

CI

CIA

CIN2+

CS

CSF

CSTE

CT

ED

EIA

EP

FDA

FTA-ABS

GC

GISP

GU

HCUP

HD

HEDIS

HMOs

HIV

HP2020

HPV

HSV

HSV-1

HSV-2

IHC

MHA-TP

MICs

MP

MPC

MSAs

MSM

MSMW
Advisory Committee on Immunization Practices

American Indians/Alaska Natives

Antibiotic Resistance Laboratory Network

annual percent change

American Samoa

Centers for Disease Control and Prevention

confidence interval

chemiluminescence immunoassay

cervical intraepithelial neoplasia grades 2 and 3

congenital syphilis

cerebrospinal fluid

Council of State and Territorial Epidemiologists

chlamydia

emergency department

enzyme immunoassay

ectopic pregnancy

Food and Drug Administration

fluorescent treponemal antibody absorbed

gonorrhea

Gonococcal Isolate Surveillance Project

Guam

Healthcare Cost and Utilization Project

health department

Healthcare Effectiveness Data and Information Set

health maintenance organizations

human immunodeficiency virus

Healthy People 2020

human papillomavirus

herpes simplex virus

herpes simplex virus type 1

herpes simplex virus type 2

immunohistochemistry

microhemagglutination assay for antibody to Treponema pallidum

minimum inhibitory concentrations

Northern Mariana Islands

mucopurulent cervicitis

metropolitan statistical areas

gay, bisexual, and other men who have sex with men

men who have sex with both men and women 


$\begin{array}{ll}\text { MSW } & \text { men who have sex with women only } \\ \text { NAATs } & \text { nucleic acid amplification tests } \\ \text { NCHHSTP } & \text { National Center for HIV/AIDS, Viral Hepatitis, STD, and TB Prevention } \\ \text { NCHS } & \text { National Center for Health Statistics } \\ \text { NHOPI } & \text { Native Hawaiians/Other Pacific Islanders } \\ \text { NDTI } & \text { National Disease and Therapeutic Index } \\ \text { NEDSS } & \text { National Electronic Disease Surveillance System } \\ \text { NETSS } & \text { National Electronic Telecommunications System for Surveillance } \\ \text { NGU } & \text { nongonococcal urethritis } \\ \text { NHANES } & \text { National Health and Nutrition Examination Survey } \\ \text { NJTP } & \text { National Job Training Program } \\ \text { NNDSS } & \text { National Notifiable Diseases Surveillance System } \\ \text { OMB } & \text { Office of Management and Budget } \\ \text { P\&S } & \text { primary and secondary } \\ \text { PCR } & \text { polymerase chain reaction } \\ \text { PID } & \text { pelvic inflammatory disease } \\ \text { PR } & \text { Puerto Rico } \\ \text { RPR } & \text { rapid plasma reagin } \\ \text { SSuN } & \text { STD Surveillance Network } \\ \text { STD } & \text { sexually transmitted disease } \\ \text { STI } & \text { sexually transmitted infection } \\ \text { TP-PA } & \text { T. pallidum particle agglutination } \\ \text { VDRL } & \text { Venereal Disease Research Laboratory } \\ \text { VI } & \text { Virgin Islands } \\ \text { WBC } & \text { white blood cell }\end{array}$




\section{Table of Contents}

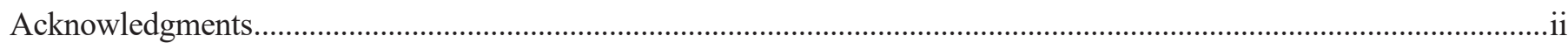

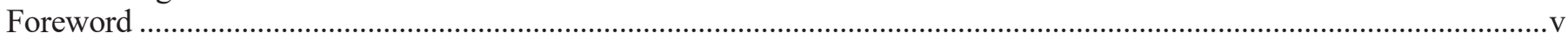

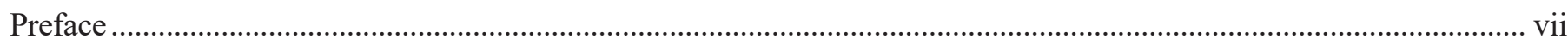

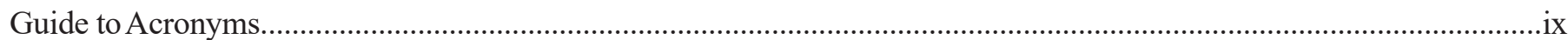

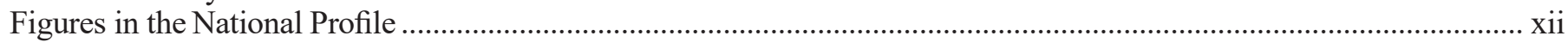

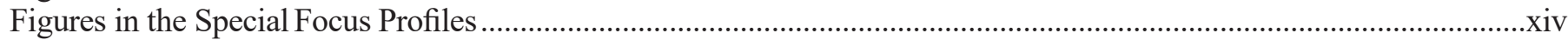

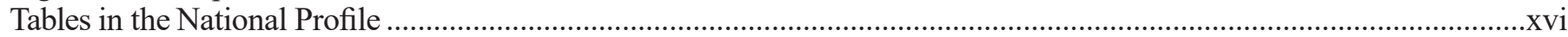

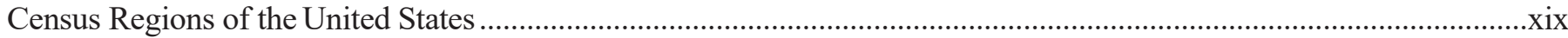

\section{National Profile}

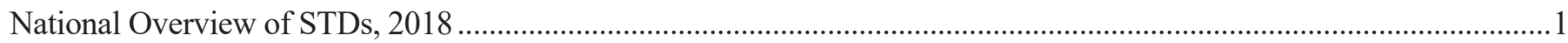

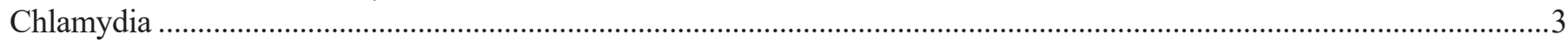

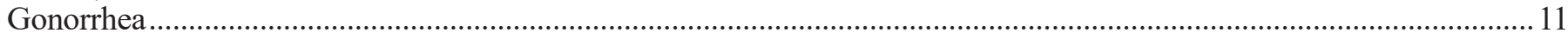

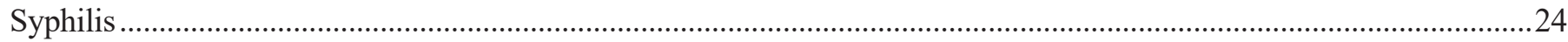

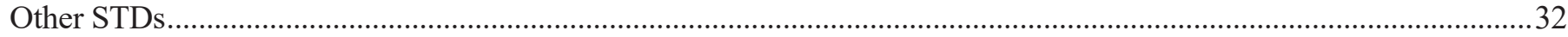

\section{Special Focus Profiles}

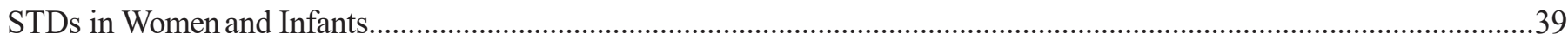

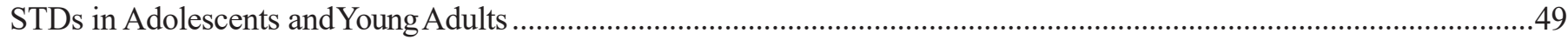

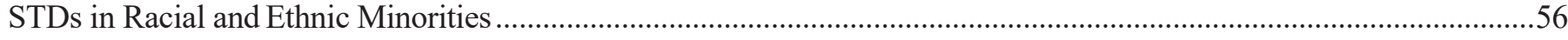

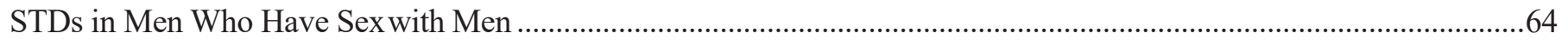

\section{Tables}

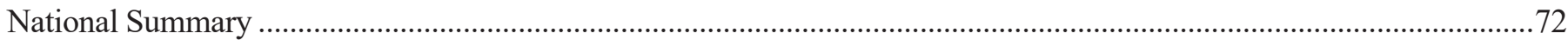

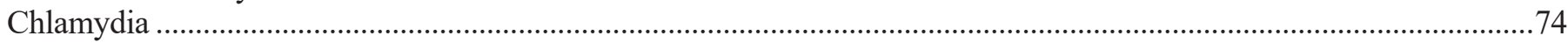

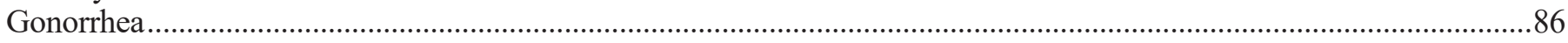

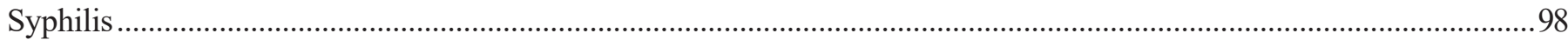

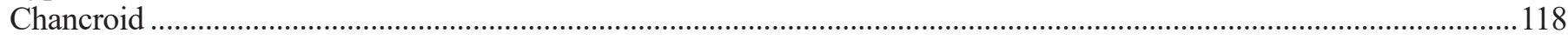

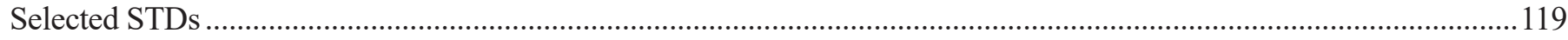

\section{Appendix}

A. Interpreting STD Surveillance Data

TableA1. Selected STDs - Percentage of Unknown, Missing, or Invalid Values for Selected Variables by State

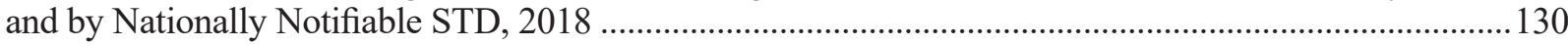

TableA2. Reported Cases of STDs by Reporting Source and Sex, United States, 2018 ........................................132

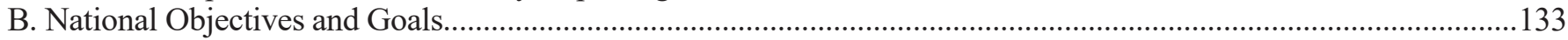

Table B1. Healthy People 2020 (HP2020) Sexually Transmitted Diseases Objectives .........................................134

Table B2. Congressional Budget Justification Sexually Transmitted Diseases Goals, Measures, and Target...............135

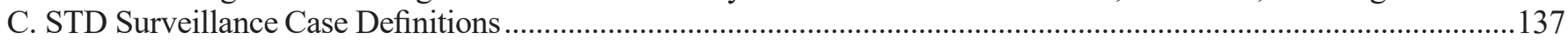

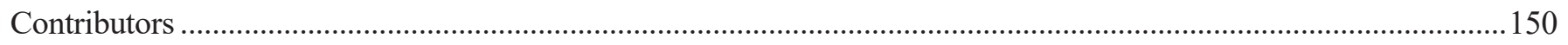




\section{Chlamydia}

Figure 1.

Figure 2.

Figure 3.

Figure 4.

Figure 5.

Figure 6.

Figure 7.

Chlamydia — Rates of Reported Cases by Sex, United States, 2000-2018 ….......................................... 4

Chlamydia — Rates of Reported Cases by Region, United States, 2009-2018_....................................... 4

Chlamydia — Rates of Reported Cases by State and Territory, United States, 2018 ..................................5

Chlamydia — Rates of Reported Cases by County, United States, 2018 .....................................................

Chlamydia — Rates of Reported Cases by Age Group and Sex, United States, 2018................................ 6

Chlamydia — Rates of Reported Cases Among Females Aged 15-44 Years by Age Group, United

States, 2009-2018......

Chlamydia — Rates of Reported Cases Among Males Aged 15-44 Years by Age Group, United States, 2009-2018.

Figure 8.

Chlamydia — Rates of Reported Cases by Race/Hispanic Ethnicity, United States, 2014-2018......

Figure 9.

Figure 10.

Chlamydia - Percentage of Reported Cases Among Females by Reporting Source, United States, 2009-2018.

Chlamydia — Percentage of Reported Cases Among Males by Reporting Source, United States,

2009-2018.

Figure 11. Chlamydia - National Estimates of Prevalence Among Persons Aged 14-39 Years by Sex,

Race/Hispanic Ethnicity, or Age Group, National Health and Nutrition Examination Survey (NHANES),

2013-2016.

Figure 12. Chlamydia - National Estimates of Prevalence Among Sexually-Active Females Aged 14-39 Years by Race/Hispanic Ethnicity and Age Group, National Health and Nutrition Examination Survey (NHANES), 2013-2016...

Figure 13.

Chlamydia - Proportion of STD Clinic Patients Testing Positive by Age Group and Sex and Sex of

Sex Partners, STD Surveillance Network (SSuN), 2018

\section{Gonorrhea}

Figure 14.

Figure 15.

Figure 16.

Figure 17.

Figure 18.

Figure 19.

Figure 20.

Gonorrhea - Rates of Reported Cases by Year, United States, 1941-2018

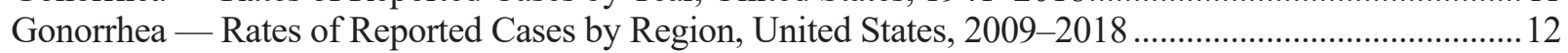

Gonorrhea — Rates of Reported Cases by State and Territory, United States, 2018 ..................................... 12

Gonorrhea — Rates of Reported Cases by County, United States, 2018 ................................................. 13

Gonorrhea — Rates of Reported Cases by Sex, United States, 2009-2018 …........................................... 13

Gonorrhea — Rates of Reported Cases by Age Group and Sex, United States, 2018 ............................... 14

Gonorrhea - Rates of Reported Cases Among Females Aged 15-44 Years by Age Group, United

States, 2009-2018

Figure 21. Gonorrhea — Rates of Reported Cases Among Males Aged 15-44 Years by Age Group, United States,

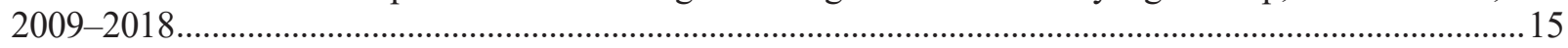

Figure 22. Gonorrhea - Rates of Reported Cases by Race/Hispanic Ethnicity, United States, 2014-2018 ................15

Figure 23. Gonorrhea - Percentage of Reported Cases Among Females by Reporting Source, United States, 2009-2018.

Figure 24. Gonorrhea - Percentage of Reported Cases Among Males by Reporting Source, United States, 2009-2018.

Figure 25. Estimated Proportion of MSM, MSW, and Women Among Gonorrhea Cases by Jurisdiction, STD Surveillance Network (SSuN), 2018.

Figure 26. Gonorrhea - Estimated Rates of Reported Gonorrhea Cases by MSM, MSW, and Women, STD Surveillance Network (SSuN), 2010-2018.

Figure 27. Gonorrhea - Proportion of STD Clinic Patients Testing Positive by Age Group and Sex and Sex of Sex Partners, STD Surveillance Network (SSuN), 2018.

Figure 28. Location of Participating Sentinel Sites and Regional Laboratories, Gonococcal Isolate Surveillance

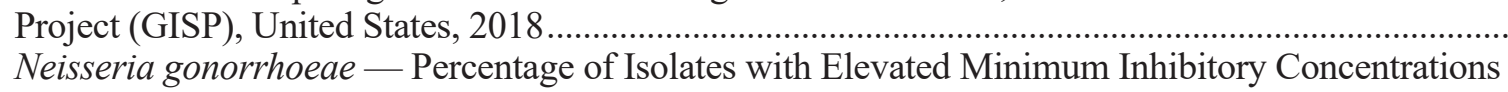

Figure 29. Neisseria gonorrhoeae - Percentage of Isolates with Elevated Minimum Inhibitory Concentrations
(MICs) to Azithromycin, Cefixime, and Ceftriaxone, Gonococcal Isolate Surveillance Project (GISP), 2009-2018.. 
Figure 30. Neisseria gonorrhoeae - Distribution of Gentamicin Minimum Inhibitory Concentrations (MICs)

by Year, Gonococcal Isolate Surveillance Project (GISP), 2015-2018 .....

Figure 31. Neisseria gonorrhoeae - Prevalence of Tetracycline, Penicillin, or Fluoroquinolone Resistance or Elevated Cefixime, Ceftriaxone, or Azithromycin Minimum Inhibitory Concentrations (MICs), by Year - Gonococcal Isolate Surveillance Project (GISP), 2000-2018...

Figure 32. Resistance or Elevated MIC Patterns of Neisseria gonorrhoeae Isolates to Antimicrobials, Gonococcal Isolate Surveillance Project (GISP), 2018.

Figure 33. Distribution of Primary Antimicrobial Drug Used to Treat Gonorrhea Among Participants, Gonococcal Isolate Surveillance Project (GISP), 1988-2018

Figure 34. Gonorrhea - Estimated Proportion of Cases Treated by Recommended Treatment Regimen and Jurisdiction, STD Surveillance Network (SSuN), $2018 .$.

\section{Syphilis} Figure 36. Figure 37.

Figure 38.

Figure 39.

Figure 40.

Figure 41.

Figure 42.

Figure 43.

Figure 44.

Figure 45 .

Figure 46.

Figure 47.

Figure 48 .

Figure 49 .
Figure 35.

Syphilis - Rates of Reported Cases by Stage of Infection, United States, 1941-2018 24 Primary and Secondary Syphilis — Rates of Reported Cases by Region, United States, 2009-2018 .........25 Primary and Secondary Syphilis — Rates of Reported Cases by State and Territory, United States, 2018.

Primary and Secondary Syphilis — Rates of Reported Cases by County, United States, 2018 ..... 25

Primary and Secondary Syphilis - Distribution of Cases by Sex and Sex of Sex Partners, United States, 2018.

Primary and Secondary Syphilis — Rates of Reported Cases by Sex and Male-to-Female Rate Ratios, United States, 1990-2018.

Primary and Secondary Syphilis — Reported Cases by Sex and Sex of Sex Partners, 36 States, 2014-2018.

Primary and Secondary Syphilis — Rates of Reported Cases by Age Group and Sex, United States, 2018.

Primary and Secondary Syphilis — Rates of Reported Cases Among Females Aged 15-44 Years by Age Group, United States, 2009-2018..

Primary and Secondary Syphilis - Rates of Reported Cases Among Males Aged 15-44 Years by Age Group, United States, 2009-2018..

Primary and Secondary Syphilis - Rates of Reported Cases by Race/Hispanic Ethnicity, United States, 2014-2018.

Primary and Secondary Syphilis - Reported Cases by Sex and Sex of Sex Partners and HIV Status, United States, 2018

Primary and Secondary Syphilis — Reported Cases by Reporting Source and Sex, United States, 2009-2018......

Primary and Secondary Syphilis - Percentage of Reported Cases by Sex and Sex of Sex Partners and Selected Reporting Sources, United States, 2018. 31

Congenital Syphilis - Reported Cases by Year of Birth and Rates of Reported Cases of Primary and Secondary Syphilis Among Females Aged 15-44 Years, United States, 2009-2018

\section{Other STDs}

Figure 50. Chancroid — Reported Cases by Year, United States, 1941-2018. .32

Figure 51.

Figure 52.

Cervical Intraepithelial Neoplasia Grades 2 and 3 - Prevalence per 1000 Person-Years Among

Female Enrollees in Private Health Plans Aged 15-39 Years, by Age Group and Year, 2007-2014 . .33

Anogenital Warts - Prevalence per 1000 Person-Years Among Enrollees in Private Health Plans Aged 15-39 Years by Sex, Age Group, and Year, 2006-2014....

Figure 53. Herpes Simplex Virus Type 2 - National Estimates of Trends in Age-Adjusted Seroprevalence Among Persons Aged 14-49 Years by Race/Hispanic Ethnicity, National Health and Nutrition Examination Survey (NHANES), 1999-2000 through 2015-2016

Figure 54. Trichomonas vaginalis and Other Vaginal Infections Among Females — Initial Visits to Physicians' Offices, United States, 1966-2016. 36 


\section{STDs in Women and Infants}

Figure A. Chlamydia - Rates of Reported Cases Among Females by State and Territory, United States, 2018

Figure B.

Chlamydia - Positivity Among Females Aged 14-39 Years by Race/Hispanic Ethnicity and Age

Group in Clinics Providing Family Planning and Reproductive Health Services, STD Surveillance

Network (SSuN), 2018.

Figure C. Gonorrhea - Rates of Reported Cases Among Females by State and Territory, United States, 2018 ........41

Figure D. Pelvic Inflammatory Disease — Initial Visits to Physicians' Offices Among Females Aged 15-44 Years,

United States, 2007-2016.

Figure E. Estimated Percentage of Acute Pelvic Inflammatory Disease Emergency Department Visits Among

Females Aged 15-44 Years by Age Group and Year, United States, 2006-2013...

Figure F.

Pelvic Inflammatory Disease - National Estimates of Lifetime Prevalence Among Sexually

Experienced Women Aged 18-44 Years by Race/Hispanic Ethnicity and Previous STD Diagnosis,

National Health and Nutrition Examination Survey (NHANES), 2013-2014.

Figure G. Ectopic Pregnancy — Ratio Among Commercially Insured Females with Live Births Aged 15-44 Years

by Age Group, 2006-2017 ..................................................................................................................... 44

Figure H. Chlamydia and Gonorrhea - Rates of Reported Cases Among Infants $<1$ Year of Age by Year and

Specimen Source, United States, 2014-2018 ………................................................................................ 44

Figure I. Congenital Syphilis — Rates of Reported Cases by State and Territory, United States, $2018 \ldots \ldots \ldots \ldots \ldots \ldots \ldots . . . .45$

Figure J. $\quad$ Primary and Secondary Syphilis — Rates of Reported Cases Among Females by State and Territory, United States, 2018

\section{STDs in Adolescents and Young Adults}

Figure K. Chlamydia — Rates of Reported Cases Among Females Aged 15-24 Years by State and Territory, United States, 2018

Figure L. Chlamydia - Rates of Reported Cases Among Males Aged 15-24 Years by State and Territory, United States, 2018 ..

Figure M. Gonorrhea - Rates of Reported Cases Among Females Aged 15-24 Years by State and Territory, United States, 2018.

Figure N. Gonorhea - Rates of Reported Cases Among Males Aged 15-24 Years by State and Territory, United States, 2018.

Figure O.

Chlamydia - Prevalence Among Females Aged 16-24 Years Entering the National Job Training

Program (NJTP) by State and Territory of Residence, United States, 2018

Figure P. Chlamydia - Prevalence Among Males Aged 16-24 Years Entering the National Job Training

Program (NJTP) by State and Territory of Residence, United States, 2018.

Figure Q. Gonorrhea - Prevalence Among Females Aged 16-24 Years Entering the National Job Training Program (NJTP) by State and Territory of Residence, United States, 2018 .............................................54

Figure R. Gonorrhea - Prevalence Among Males Aged 16-24 Years Entering the National Job Training Program (NJTP) by State and Territory of Residence, United States, 2018.

\section{STDs in Racial and Ethnic Minorities}

Figure S.

Figure T.

Figure U.

Figure V.

Figure W.

Figure X.

Figure Y.

Figure Z.
Chlamydia — Rates of Reported Cases by Race/Hispanic Ethnicity and Sex, United States, 2018. Chlamydia — Rate Ratios Among Females Aged 15-24 Years by Race/Hispanic Ethnicity and

Region, United States, 2018

Gonorrhea - Rates of Reported Cases by Race/Hispanic Ethnicity and Sex, United States, 2018

Gonorrhea - Rate Ratios by Race/Hispanic Ethnicity and Region, United States, 2018

Primary and Second

Primary and Second

United States, 2018

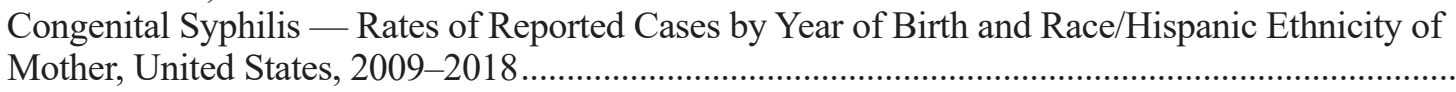

Primary and Secondary Syphilis 


\section{STDs in Men Who Have Sex with Men}

Figure AA. Primary and Secondary Syphilis - Estimated Rates of Reported Cases Among MSM by State, United States, 2018.

Figure BB. Neisseria gonorrhoeae - Percentage of Urethral Isolates Obtained from MSM Attending STD Clinics, Gonococcal Isolate Surveillance Project (GISP), 1989-2018

Figure CC. Neisseria gonorrhoeae - Percentage of Urethral Isolates with Elevated Minimum Inhibitory Concentrations (MICs) to Azithromycin and Ceftriaxone by Sex and Sex of Sex Partners, Gonococcal Isolate Surveillance Project (GISP), 2009-2018

Figure DD. Gonorrhea and Chlamydia - Proportion of MSM STD Clinic Patients Tested and Testing Positive for Urogenital Gonorrhea and Chlamydia by Jurisdiction, STD Surveillance Network (SSuN), 2018.

67

Figure EE. Gonorrhea and Chlamydia - Proportion of MSM STD Clinic Patients Tested and Testing Positive for Rectal Gonorrhea and Chlamydia by Jurisdiction, STD Surveillance Network (SSuN), 2018

Figure FF. Gonorrhea and Chlamydia - Proportion of MSM STD Clinic Patients Tested and Testing Positive for Pharyngeal Gonorrhea and Chlamydia by Jurisdiction, STD Surveillance Network (SSuN), 2018 ...........68

Figure GG. Proportion of MSM Attending STD Clinics with Primary and Secondary Syphilis, Urogenital Gonorrhea, or Urogenital Chlamydia by Known HIV Status, STD Surveillance Network (SSuN), 2018 ..69 


\section{Tables in the National Profile}

\section{National Summary}

Table 1. Sexually Transmitted Diseases - Reported Cases and Rates of Reported Cases, United States, 1941-2018.

\section{Chlamydia}

Table 2.

Chlamydia - Reported Cases and Rates of Reported Cases by State, Ranked by Rates, United States,

Table 3. 2018

Chlamydia - Reported Cases and Rates of Reported Cases by State/Territory and Region in

Alphabetical Order, United States, 2014-2018

Table 4.

Chlamydia Among Females — Reported Cases and Rates of Reported Cases by State/Territory and

Region in Alphabetical Order, United States, 2014-2018............................................................................76

Table 5. Chlamydia Among Males - Reported Cases and Rates of Reported Cases by State/Territory and Region in Alphabetical Order, United States, 2014-2018.......

Table 6. Chlamydia - Reported Cases and Rates of Reported Cases in Selected Metropolitan Statistical Areas (MSAs) in Alphabetical Order, United States, 2014-2018......

Table 7. Chlamydia Among Females - Reported Cases and Rates of Reported Cases in Selected Metropolitan Statistical Areas (MSAs) in Alphabetical Order, United States, 2014-2018 ......

Table 8 . Chlamydia Among Males — Reported Cases and Rates of Reported Cases in Selected Metropolitan Statistical Areas (MSAs) in Alphabetical Order, United States, 2014-2018 ................................................80

Table 9. Chlamydia - Reported Cases and Rates of Reported Cases in Counties and Independent Cities Ranked by Number of Reported Cases, United States, $2018 .$.

Table 10. Chlamydia - Reported Cases and Rates of Reported Cases by Age Group and Sex, United States,

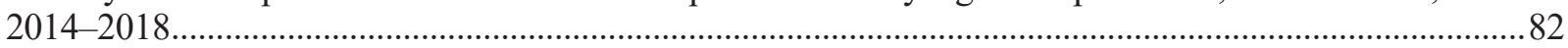

Table 11A. Chlamydia - Reported Cases by Race/Hispanic Ethnicity, Age Group, and Sex, United States, 2018 .....83

Table 11B. Chlamydia - Rates of Reported Cases by Race/Hispanic Ethnicity, Age Group, and Sex, United States, 2018

Table 12A. Chlamydia - Reported Cases and Rates of Reported Cases Among Females Aged 15-24 Years by Age, United States, 2014-2018.

Table 12B.

Chlamydia - Reported Cases and Rates of Reported Cases Among Males Aged 15-24 Years by Age,

United States, 2014-2018..

\section{Gonorrhea}

Table 13. Gonorrhea - Reported Cases and Rates of Reported Cases by State, Ranked by Rates, United States, 2018 .

Table 14. Gonorrhea - Reported Cases and Rates of Reported Cases by State/Territory and Region in Alphabetical Order, United States, 2014-2018.....

Table 15.

Gonorrhea Among Females - Reported Cases and Rates of Reported Cases by State/Territory and

Region in Alphabetical Order, United States, 2014-2018 ....................................................................... 88

Table 16.

Gonorrhea Among Males - Reported Cases and Rates of Reported Cases by State/Territory and

Region in Alphabetical Order, United States, 2014-2018.

Table 17. Gonorrhea - Reported Cases and Rates of Reported Cases in Selected Metropolitan Statistical Areas (MSAs) in Alphabetical Order, United States, 2014-2018

Table 18. Gonorrhea Among Females - Reported Cases and Rates of Reported Cases in Selected Metropolitan Statistical Areas (MSAs) in Alphabetical Order, United States, 2014-2018 ...

Table 19. Gonorrhea Among Males - Reported Cases and Rates of Reported Cases in Selected Metropolitan Statistical Areas (MSAs) in Alphabetical Order, United States, 2014-2018.....

Table 20. Gonorrhea - Reported Cases and Rates of Reported Cases in Counties and Independent Cities Ranked by Number of Reported Cases, United States, 2018 .....

Table 21. Gonorrhea 
Table 22A. Gonorrhea - Reported Cases by Race/Hispanic Ethnicity, Age Group, and Sex, United States, 2018 ......95

Table 22B. Gonorrhea - Rates of Reported Cases by Race/Hispanic Ethnicity, Age Group, and Sex, United

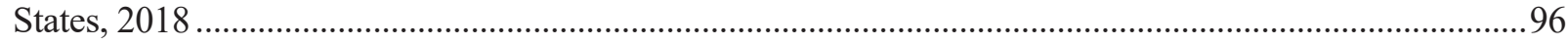

Table 23A. Gonorrhea - Reported Cases and Rates of Reported Cases Among Females Aged 15-24 Years

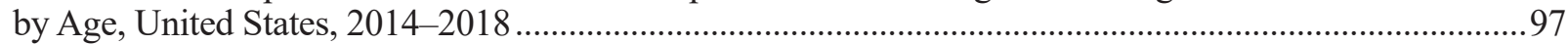

Table 23B. Gonorrhea - Reported Cases and Rates of Reported Cases Among Males Aged 15-24 Years

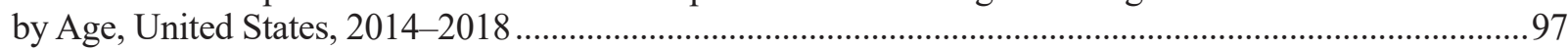

\section{Syphilis}

Table 24.

All Stages of Syphilis - Reported Cases and Rates of Reported Cases by State/Territory and Region in Alphabetical Order, United States, 2014-2018.......

Table 25. All Stages of Syphilis - Reported Cases and Rates of Reported Cases in Selected Metropolitan Statistical Areas (MSAs) in Alphabetical Order, United States, 2014-2018.

Table 26. Primary and Secondary Syphilis - Reported Cases and Rates of Reported Cases by State, Ranked by Rates, United States, 2018.

Table 27. Primary and Secondary Syphilis - Reported Cases and Rates of Reported Cases by State/Territory and Region in Alphabetical Order, United States, 2014-2018.

Table 28. Primary and Secondary Syphilis Among Females - Reported Cases and Rates of Reported Cases by State/Territory and Region in Alphabetical Order, United States, 2014-2018 .

Table 29.

Primary and Secondary Syphilis Among Males — Reported Cases and Rates of Reported Cases by State/Territory and Region in Alphabetical Order, United States, 2014-2018.

Table 30.

Primary and Secondary Syphilis - Reported Cases and Rates of Reported Cases in Selected

Metropolitan Statistical Areas (MSAs) in Alphabetical Order, United States, 2014-2018.

Table 31.

Table 32. Primary and Secondary Syphilis Among Males - Reported Cases and Rates of Reported Cases in Selected Metropolitan Statistical Areas (MSAs) in Alphabetical Order, United States, 2014-2018 .......... 105 Selected Metropolitan Statistical Areas (MSAs) in Alphabetical Order, United States, 2014-2018.......... 106

Table 33. Primary and Secondary Syphilis - Reported Cases and Rates of Reported Cases in Counties and Independent Cities Ranked by Number of Reported Cases, United States, 2018 .....

Table 34. Primary and Secondary Syphilis - Reported Cases and Rates of Reported Cases by Age Group and Sex, United States, 2014-2018.

Table 35A. Primary and Secondary Syphilis — Reported Cases by Race/Hispanic Ethnicity, Age Group, and Sex, United States, 2018.

Table 35B. Primary and Secondary Syphilis - Rates of Reported Cases by Race/Hispanic Ethnicity, Age Group, and Sex, United States, 2018. 110

Table 36. Early Non-Primary Non-Secondary Syphilis - Reported Cases and Rates of Reported Cases by State/Territory and Region in Alphabetical Order, United States, 2014-2018.

Table 37. Early Non-Primary Non-Secondary Syphilis - Reported Cases and Rates of Reported Cases in Selected Metropolitan Statistical Areas (MSAs) in Alphabetical Order, United States, 2014-2018......

Table 38. Unknown Duration or Late Syphilis - Reported Cases and Rates of Reported Cases by State/Territory and Region in Alphabetical Order, United States, 2014-2018.

Table 39. Unknown Duration or Late Syphilis - Reported Cases and Rates of Reported Cases in Selected Metropolitan Statistical Areas (MSAs) in Alphabetical Order, United States, 2014-2018.

Table 40. Congenital Syphilis - Reported Cases and Rates of Reported Cases by State, Ranked by Rates, United States, 2018.

Table 41.

Congenital Syphilis - Reported Cases and Rates of Reported Cases by Year of Birth,

State/Territory and Region in Alphabetical Order, United States, 2014-2018....

Table 42.

Congenital Syphilis - Reported Cases and Rates of Reported Cases by Year of Birth and

Race/Hispanic Ethnicity of Mother, United States, 2014-2018.

\section{Chancroid}

Table 43.

Chancroid - Reported Cases and Rates of Reported Cases by State/Territory in Alphabetical Order, United States, 2014-2018. 


\section{Selected STDs}

Table 44. Selected STDs and Complications — Initial Visits to Physicians' Offices, National Disease and Therapeutic Index (NDTI), United States, 1966-2016

\section{Appendix}

Table A1. Selected STDs — Percentage of Unknown, Missing, or Invalid Values for Selected Variables

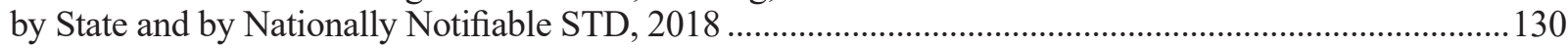

Table A2. Reported Cases of STDs by Reporting Source and Sex, United States, 2018 .......................................132

Table B1. Healthy People 2020 (HP2020) Sexually Transmitted Diseases Objectives.............................................134

Table B2. Congressional Budget Justification Sexually Transmitted Diseases Goals, Measures, and Target..............135 

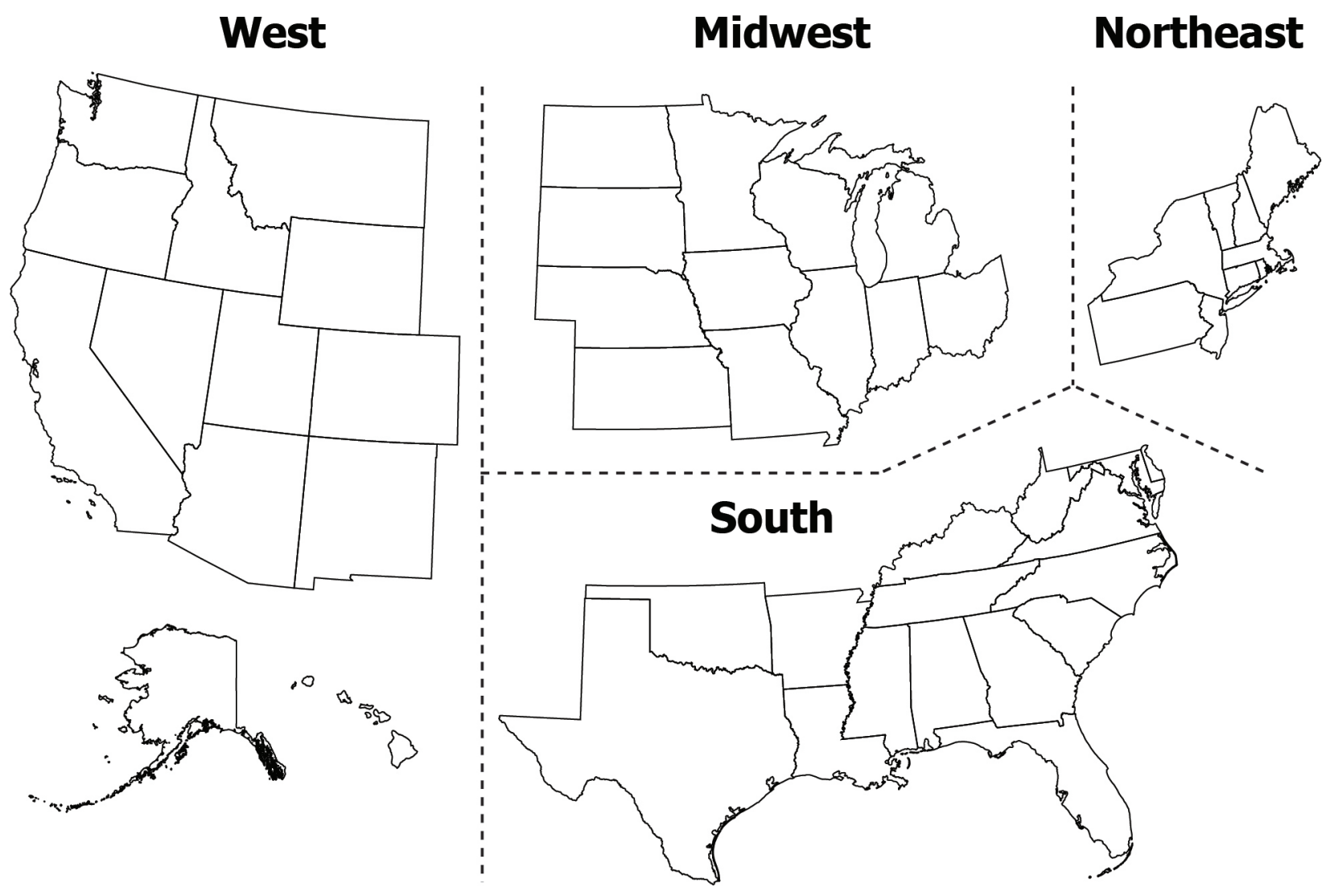

$\begin{array}{llll}\text { West } & \text { Midwest } & \text { South } & \text { Northeast } \\ \text { Alaska } & \text { Illinois } & \text { Alabama } & \text { Connecticut } \\ \text { Arizona } & \text { Indiana } & \text { Arkansas } & \text { Maine } \\ \text { California } & \text { Iowa } & \text { Delaware } & \text { Massachusetts } \\ \text { Colorado } & \text { Kansas } & \text { District of Columbia } & \text { New Hampshire } \\ \text { Hawaii } & \text { Michigan } & \text { Florida } & \text { New Jersey } \\ \text { Idaho } & \text { Minnesota } & \text { Georgia } & \text { New York } \\ \text { Montana } & \text { Missouri } & \text { Kentucky } & \text { Pennsylvania } \\ \text { Nevada } & \text { Nebraska } & \text { Louisiana } & \text { Rhode Island } \\ \text { New Mexico } & \text { North Dakota } & \text { Maryland } & \text { Vermont } \\ \text { Oregon } & \text { Ohio } & \text { Mississippi } & \\ \text { Utah } & \text { South Dakota } & \text { North Carolina } & \\ \text { Washington } & \text { Wisconsin } & \text { Oklahoma } & \\ \text { Wyoming } & & \text { South Carolina } & \\ & & \text { Tennessee } & \\ & & \text { Texas } & \end{array}$


This page intentionally left blank. 


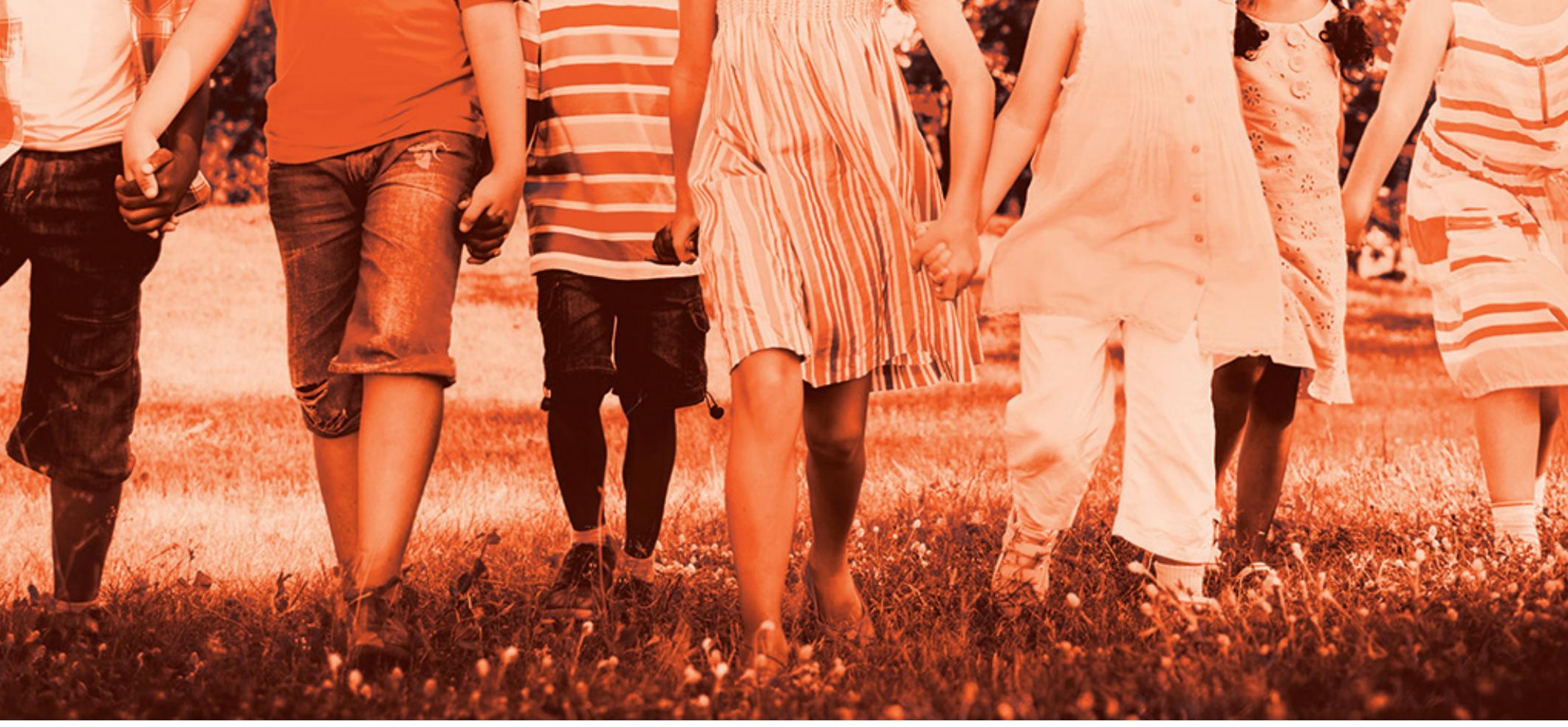

\section{National Profile}

The National Profile section contains figures that show trends and the distribution of nationally notifiable STDs (chlamydia, gonorrhea, syphilis, and chancroid) by age, sex, race/Hispanic ethnicity, and location for the United States.

\section{National Overview of STDs, 2018}

All Americans should have the opportunity to make choices that lead to health and wellness. Working together, interested, committed public and private organizations, communities, and individuals can take action to prevent STDs and their related health consequences. In addition to federal, state, and local public support for STD prevention activities, local community leaders can promote STD prevention education. Health care providers can take sexual histories as a part of routine preventive exams to assess patients' risks and identify STD testing and treatment opportunities. Parents can talk to their children about STDs and sexual health. Individuals can discuss ways to protect their health with sexual partners, including using condoms consistently and correctly. As noted in the Institute of Medicine report,
The Hidden Epidemic: Confronting Sexually Transmitted Diseases, surveillance is a key component of all our efforts to prevent and control these diseases. ${ }^{1}$ This report provides trends in STDs to inform prevention and control strategies. This overview summarizes national surveillance data for 2018 on the three notifiable diseases for which there are federally funded control programs: chlamydia, gonorrhea, and syphilis.

\section{Chlamydia}

In 2018, a total of $1,758,668$ cases of Chlamydia trachomatis infection were reported to the $\mathrm{CDC}$, making it the most common notifiable condition in the United States. This case count corresponds to a rate of 539.9 cases per 100,000 population, an increase of $2.9 \%$ compared with the rate in 2017. During 2017-2018, rates of reported chlamydia increased among both males and females, in all regions of the United States, and among all racial/Hispanic ethnicity groups.

Rates of reported chlamydia are highest among adolescent and young adults. In 2018, almost two-thirds of all reported chlamydia cases were among persons aged 15-24 years. Among females aged 15-24 years, the population targeted for chlamydia screening, the overall rate of reported cases of chlamydia was 3,693.6 cases per 100,000 females, an increase of $1.0 \%$ from 2017 and of $11.8 \%$ from 2014.

Although rates of reported cases among men are generally lower than rates among women, reflecting the larger number of women screened for this infection, rates among men 
increased 37.8\% during 2014-2018. Increases in rates among men may reflect an increased number of men, including gay, bisexual, and other men who have sex with men (MSM) being tested and diagnosed with a chlamydial infection due to increased availability of urine testing and extragenital screening, increased transmission among men, or both.

\section{Gonorrhea}

In 2018, a total of 583,405 cases of gonorrhea were reported to the $\mathrm{CDC}$, making it the second most common notifiable condition in the United States. Rates of reported gonorrhea have increased $82.6 \%$ since the historic low in 2009. During 20172018 , the overall rate of reported gonorrhea increased $5.0 \%$, and rates increased among both males and females, in all regions of the United States, and among all racial/Hispanic ethnicity groups.

Since 2013, the rate of reported gonorrhea has been higher among men compared to women. Among men, the rate of reported gonorrhea increased 6.0\% during 2017-2018 and $78.7 \%$ during $2014-2018$, while rates among women increased $3.6 \%$ during $2017-2018$ and $45.2 \%$ during 2014-2018. The higher case rate among men and the magnitude of recent increases suggests either increased transmission, increased case ascertainment (e.g., through increased extra-genital screening among MSM), or both. The concurrent increase in cases reported among women suggests parallel increases in heterosexual transmission, increased screening among women, or both.

Gonorrhea can quickly develop resistance to antibiotics used to treat infection, and in 2018, more than half of all infections were estimated to be resistant to at least one antibiotic.
Since 2009, almost all circulating strains in the United States, based on gonococcal isolates collected through sentinel surveillance, remain susceptible to ceftriaxone, the primary treatment for gonorrhea; ${ }^{2}$ only $0.2 \%$ of isolates displayed elevated ceftriaxone minimum inhibitory concentrations in 2018. Continued monitoring of susceptibility patterns to antibiotics is critical to inform treatment gonorrhea guidelines.

\section{Syphilis}

In $2018,115,045$ cases of all stages of syphilis were reported, including 35,063 cases of primary and secondary (P\&S) syphilis, the most infectious stages of the disease. Since reaching a historic low in 2000 and 2001, the rate of P\&S syphilis has increased almost every year, increasing 14.9\% during 2017-2018. Rates increased among both males and females, in all regions of the United States, and among all racial/ Hispanic ethnicity groups.

Since 2000, rates of P\&S syphilis have increased among men, primarily attributable to increases in cases among MSM. Similar to past years, in 2018, MSM accounted for the majority $(53.5 \%)$ of all reported cases of P\&S syphilis and, of these, $41.6 \%$ were known to be living with diagnosed HIV. Although rates of P\&S syphilis are lower among women, rates have increased substantially in recent years, increasing 30.4\% during 2017-2018 and $172.7 \%$ during $2014-2018$, suggesting a rapidly growing heterosexual epidemic.

The 2013 rate of congenital syphilis ( 9.2 cases per 100,000 live births) marked the first increase in congenital syphilis since 2008. Since 2013, the rate of congenital syphilis has increased each year. In 2018, 1,306 cases of congenital syphilis were reported. Although the majority of cases were reported from a few states, 41 states reported at least one case of congenital syphilis in 2018. The national rate of 33.1 cases per 100,000 live births in 2018 represents a $39.7 \%$ increase relative to 2017 and a $185.3 \%$ increase relative to 2014 . During 2017-2018 the number of syphilitic stillbirths increased (from 64 to 78 stillbirths), as did the number of congenital syphilis related infant deaths (from 13 to 16 deaths).

\section{References}

1. Eng TR, Butler WT, editors; Institute of Medicine (US). Summary: The hidden epidemic: Confronting sexually transmitted diseases. Washington (DC): National Academy Press;1997.

2. Centers for Disease Control and Prevention. Sexually transmitted diseases treatment guidelines, 2015. MMWR Recomm Rep. 2015;64(No. RR-3):1-137. 


\section{Chlamydia}

\section{Background}

Chlamydia, caused by infection with Chlamydia trachomatis, is the most common notifiable disease in the United States. It is among the most prevalent of all STDs, and since 1994, has comprised the largest proportion of all STDs reported to CDC (Table 1). Studies also demonstrate the high prevalence of chlamydial infections in the civilian, non-institutionalized US population, particularly among young women. ${ }^{1}$

Chlamydial infections in women are usually asymptomatic. ${ }^{2}$ Untreated infection can result in pelvic inflammatory disease (PID), which is a major cause of infertility, ectopic pregnancy, and chronic pelvic pain. Data from randomized controlled trials of chlamydia screening suggested that screening programs can lead to a reduction in the incidence of PID. ${ }^{3,4}$ As with other inflammatory STDs, chlamydial infection could facilitate the transmission of HIV infection. ${ }^{5}$ In addition, pregnant women infected with chlamydia can pass the infection to their infants during delivery, potentially resulting in ophthalmia neonatorum, which can lead to blindness, and pneumonia. ${ }^{6}$ Because of the large burden of disease and risks associated with infection, CDC recommends annual chlamydia screening for all sexually-active women younger than age 25 years and women $\geq 25$ years at increased risk for infection (e.g., women with new or multiple sex partners). ${ }^{7}$

The Healthcare Effectiveness Data and Information Set (HEDIS) contains a measure which assesses chlamydia screening coverage of sexually-active young women who receive medical care through commercial or Medicaid managed care organizations. Among sexuallyactive women aged 16-24 years in commercial health maintenance organization (HMO) plans, chlamydia screening increased from $23.1 \%$ in 2001 to $48.9 \%$ in 2017. Among sexually-active women aged 16-24 years covered by Medicaid, screening rates increased from $40.4 \%$ in 2001 to $57.6 \%$ in $2017 .{ }^{8}$ Although chlamydia screening has expanded over the past two decades, many women who are at risk are still not being tested - reflecting, in part, the lack of awareness among some health-care providers and the limited resources available to support these screenings.

\section{Interpreting Rates of Reported Cases of Chlamydia}

Trends in rates of reported cases of chlamydia are influenced by changes in incidence of infection, as well as changes in diagnostic, screening, and reporting practices. As chlamydial infections are usually asymptomatic, the number of infections identified and reported can increase as more people are screened even when incidence is flat or decreasing. During 2000-2011, the expanded use of more sensitive diagnostic tests (e.g., nucleic acid amplification tests [NAATs]) likely increased the number of infections identified and reported independently of increases in incidence. Also, although chlamydia has been a nationally notifiable condition since 1995, it was not until 2000 that all 50 states and the District of Columbia required reporting of chlamydia cases. National case rates prior to 2000 reflect incomplete reporting. The increased use of electronic laboratory reporting over the last decade or so also likely increased the proportion of diagnosed cases reported. Consequently, an increasing chlamydia case rate over time may reflect increases in incidence of infection, screening coverage, and use of more sensitive tests, as well as more complete reporting. Likewise, decreases in chlamydia case rates may suggest decreases in incidence of infection or screening coverage.

\section{Chlamydia - United States}

In 2018, a total of $1,758,668$

chlamydial infections were reported to CDC in 50 states and the District of Columbia (Table 1). This case count corresponds to a rate of 539.9 cases per 100,000 population. During 2000-2011, the rate of reported chlamydial infection increased from 251.4 to 453.4 cases per 100,000 population (Figure 1, Table 1). During 2011-2013, the rate of reported cases decreased to 443.5 cases per 100,000 population, followed by an increase in the rate of reported cases over each of the next five years. During 2017-2018, the rate increased 2.9\%, from 524.6 to 539.9 cases per 100,000 population (Figure 1, Table 1).

\section{Chlamydia by Region}

In 2018, rates of reported cases of chlamydia were highest in the South (565.2 cases per 100,000 population, $1.9 \%$ increase from 2017 ), followed by the West (548.5, 5.6\% increase from 2017), Midwest (524.0, 2.2\% increase from 2017), and Northeast (492.1, 2.3\% increase from 2017) (Table 3). During 2009-2018, rates of reported cases of chlamydia increased in all regions (Figure 2). During 2012-2013, rates decreased in the Northeast, Midwest, and South and remained stable in the West. Rates started increasing in 2013, and have increased in all regions over each of the last five years. During 2014-2018, the largest increase occurred in the West (436.4 to 548.5 cases per 100,000 population, $25.7 \%$ increase) (Figure 2, Table 3). 


\section{Figure 1. Chlamydia - Rates of Reported Cases by Sex, United States, 2000-2018}

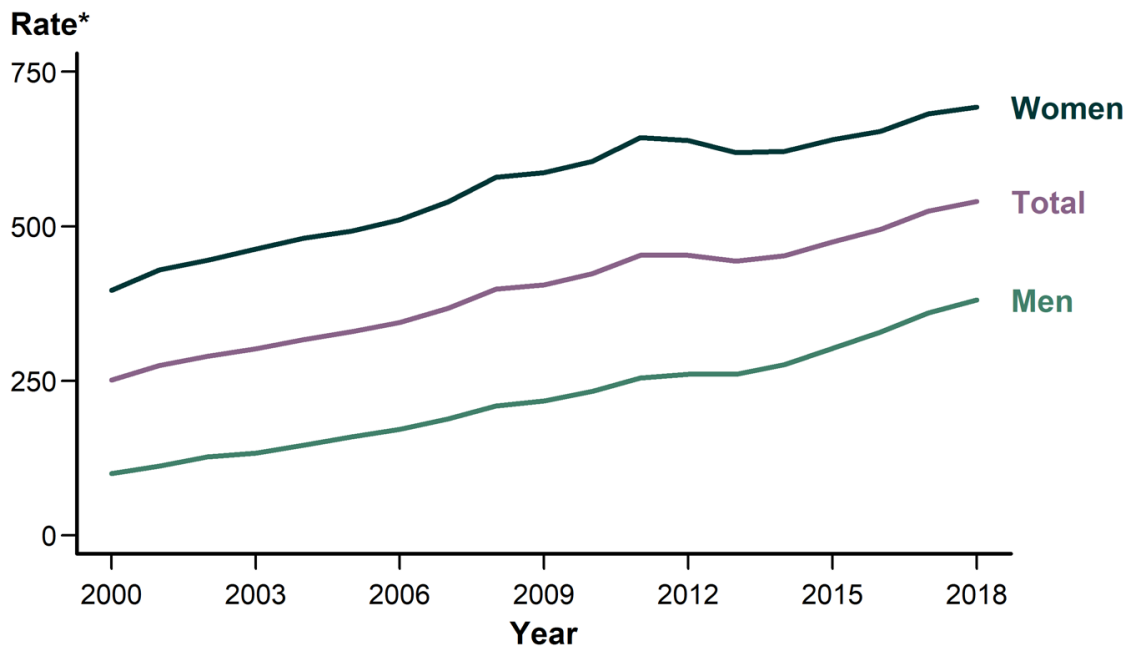

* Per 100,000

NOTE: See sections A1.3 and A1.8 in the Appendix for more information on chlamydia case reporting and interpreting trends in chlamydia case reports.

\section{Figure 2. Chlamydia - Rates of Reported Cases by Region, United States, 2009-2018}

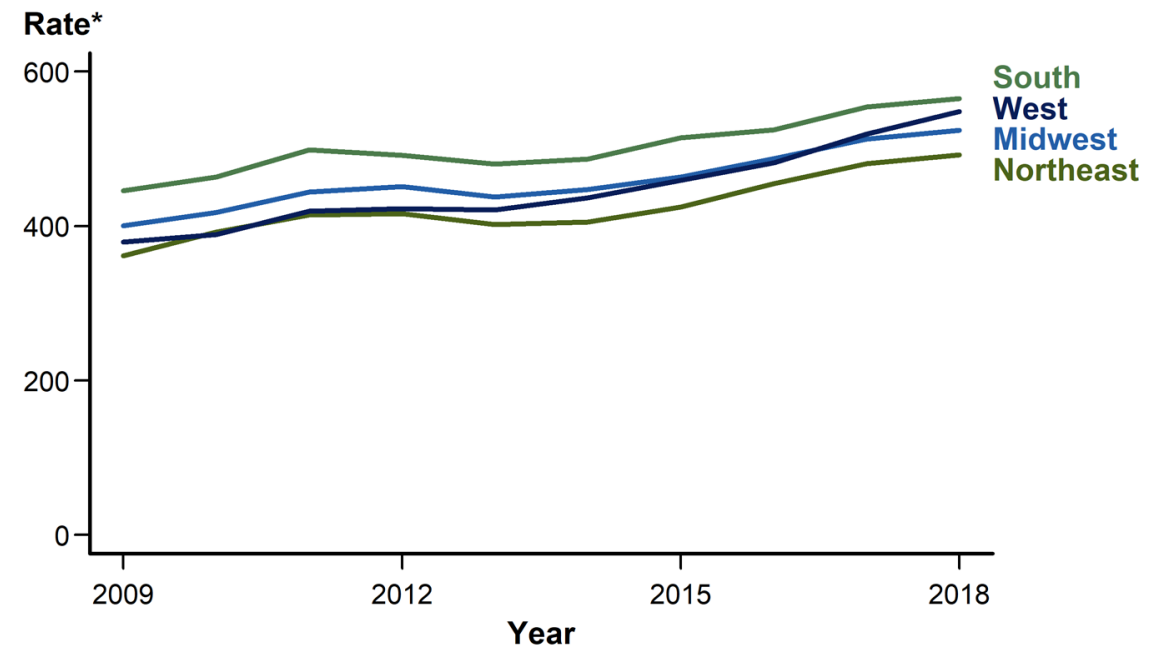

* Per 100,000

\section{Chlamydia by State}

In 2018, rates of reported cases of chlamydia by state ranged from 198.2 cases per 100,000 population in West Virginia to 832.5 cases per 100,000 population in Alaska (Figure 3 , Table 2); the rate for the District of Columbia was 1,298.9 cases per 100,000 population (Table 3 ). During 2017-2018, rates of reported chlamydia cases increased in 40 states. The rate of reported chlamydia cases in 2018 was above the US total in 21 states (Table 2).

\section{Chlamydia by Metropolitan Statistical Area}

The overall rate of reported cases of chlamydia for the 50 most populous metropolitan statistical areas (MSAs) increased 3.0\% during 2017-2018 (from 555.7 to 572.1 cases per 100,000 population) (Table 6 ). In $2018,58.5 \%$ of chlamydia cases were reported by these MSAs. During 2017-2018, the rate of reported cases of chlamydia in these MSAs increased $1.3 \%$ among females (from 697.0 to 706.0 cases per 100,000 females) and $6.0 \%$ among males (from 406.7 to 430.9 cases per 100,000 males) (Tables 7 and 8).

\section{Chlamydia by County}

In 2018, 628 (20.0\%) of 3,142 counties had rates of reported chlamydia at or higher than 560 cases per 100,000 population (Figure 4). Seventy counties and independent cities reported $44 \%$ of all chlamydia cases in 2018 (Table 9). Of the 70 counties and independent cities reporting the highest number of chlamydia cases, 47 (67.1\%) were located in the South and West (Table 9).

\section{Chlamydia by Sex}

In $2018,1,145,063$ cases of chlamydia were reported among females for a rate of 692.7 cases per 100,000 females (Table 4). After increasing each year during 2000 2011, the rate of reported chlamydia cases among females decreased during 2011-2013, followed by an increase in the rate of reported cases over each of the next five years (Figure 1, Table 4). The total rate increase during 2014-2018 among females was $11.4 \%$.

Among males, 610,447 cases of chlamydia were reported in 2018 for a rate of 380.6 cases per 100,000 males (Table 5). The rate of reported cases among males increased each year 


\section{Figure 3. Chlamydia - Rates of Reported Cases by State and Territory, United States, 2018}

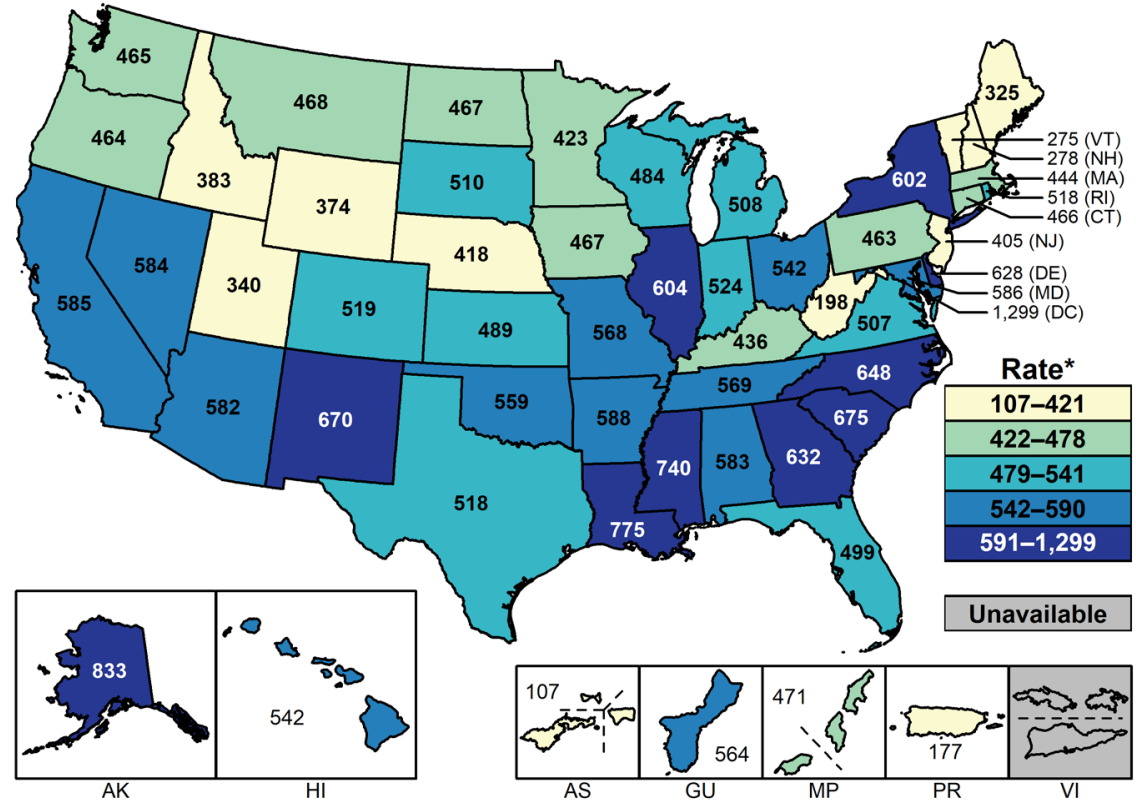

* Per 100,000 .

NOTE: See Section A1.11 in the Appendix for more information on interpreting reported rates in US territories.

\section{Figure 4. Chlamydia - Rates of Reported Cases by County, United States, 2018}

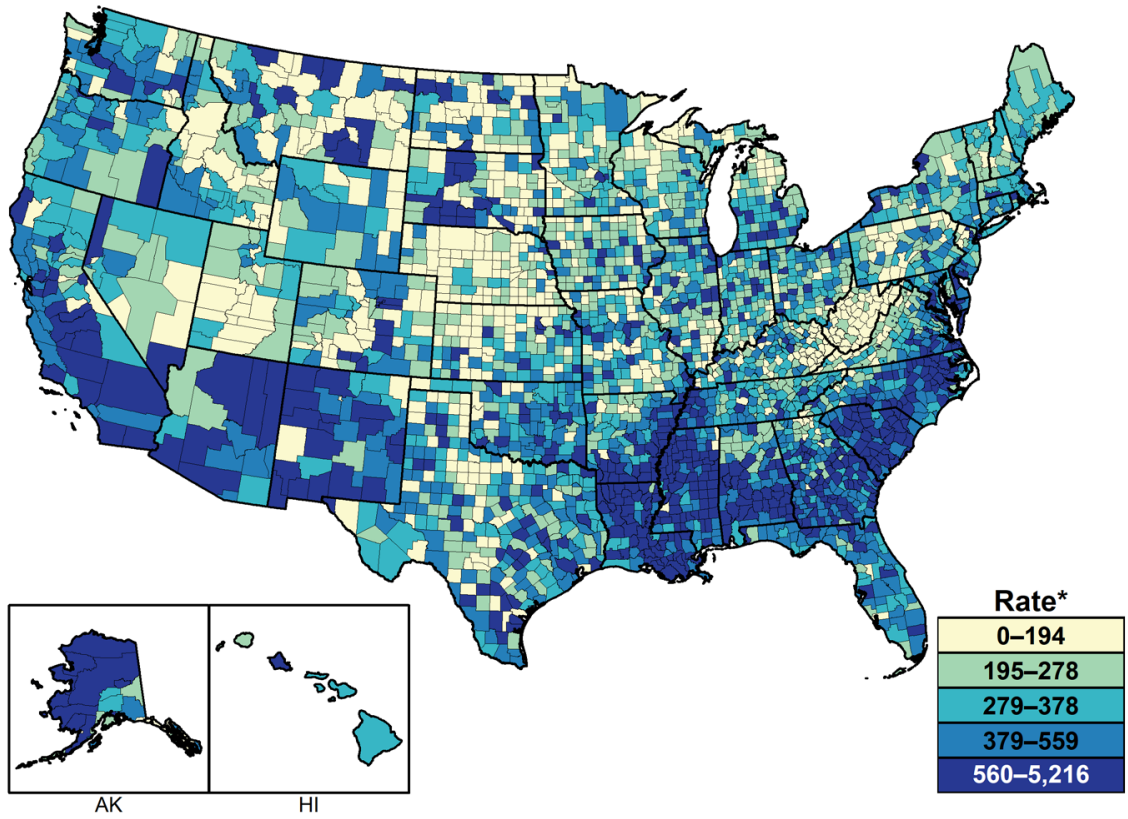

* Per 100,000 .

NOTE: See section A1.5 in the Appendix for more information on county-level rates. during 2000-2018, with the exception of 2012-2013, when rates remained stable (Figure 1). During 2017-2018 alone, the rate among males increased 5.7\%; during 2014-2018, rates of reported cases among males increased $37.8 \%$ (Tables 4 and 5). This

pronounced increase among males could be attributed to either increased transmission or improved case identification (e.g., through intensified extra-genital screening efforts) among gay, bisexual, and other men who have sex with men (MSM). This cannot be assessed, however, as most jurisdictions do not routinely report sex of sex partners or anatomic site of infection for cases of chlamydia.

Despite this considerable increase in males, the rate of reported chlamydia cases among females was still about two times the rate among males in 2018 , likely reflecting a larger number of females screened for this infection (Figure 1, Tables 4 and 5). The lower rate among males also suggests that many of the sex partners of females with chlamydia are not receiving a diagnosis of chlamydia or being reported as having chlamydia.

\section{Chlamydia by Age}

The rates of reported cases of chlamydia were highest among adolescents and young adults aged 15-24 years during 2014-2018 (Table 10). In 2018, the age-specific rate of reported cases of chlamydia among 15-19 year olds was $2,110.6$ cases per 100,000 population and the rate among 20-24 year olds was 2,899.2 cases per 100,000 population (Table $10)$.

In $2018,97.4 \%$ of all reported chlamydia cases in females were among those aged 15-44 years (Table 10). The highest age-specific rates of reported cases of chlamydia in 2018 were among those aged 15-19 years (3,306.8 cases per 100,000 females) and $20-24$ years $(4,064.6$ cases per 100,000 females) (Figure 5, Table 
10). Within these age groups, rates were highest among women aged 19 years $(5,485.8$ cases per 100,000 females) and 20 years $(5,309.8$ cases per 100,000 females) (Table 12A).

Increases have been observed in recent years in rates of reported cases of chlamydia among all age groups in females aged 15-44 years (Figure $6)$. The rate among 15-19 year old females increased $1.3 \%$ during $2017-$ 2018 , with a total increase of $12.1 \%$ during 2014-2018 (2,949.3 to 3,306.8 cases per 100,000 females) (Table $10)$. The rate among 20-24 year old females increased $0.8 \%$ during 2017-2018, with a total increase of

\section{Figure 5. Chlamydia - Rates of Reported Cases by Age Group and}

Sex, United States, 2018

\section{Male Rate*}

4,500

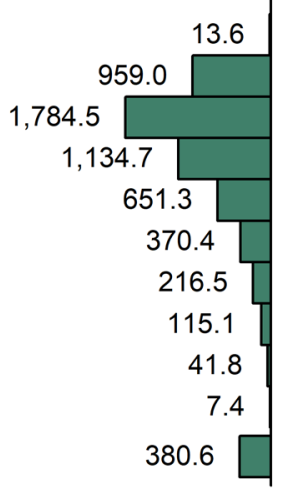

0 Age Group 0

$10-14$

$15-19$

20-24

$25-29$

30-34

35-39

$40-44$

$45-54$

$55-64$

$65+$

Total

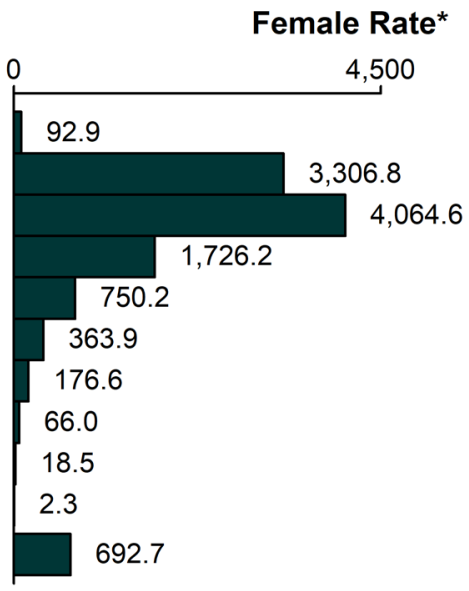

* Per 100,000 .

Figure 6. Chlamydia - Rates of Reported Cases Among Females Aged 15-44 Years by Age Group, United States, 2009-2018

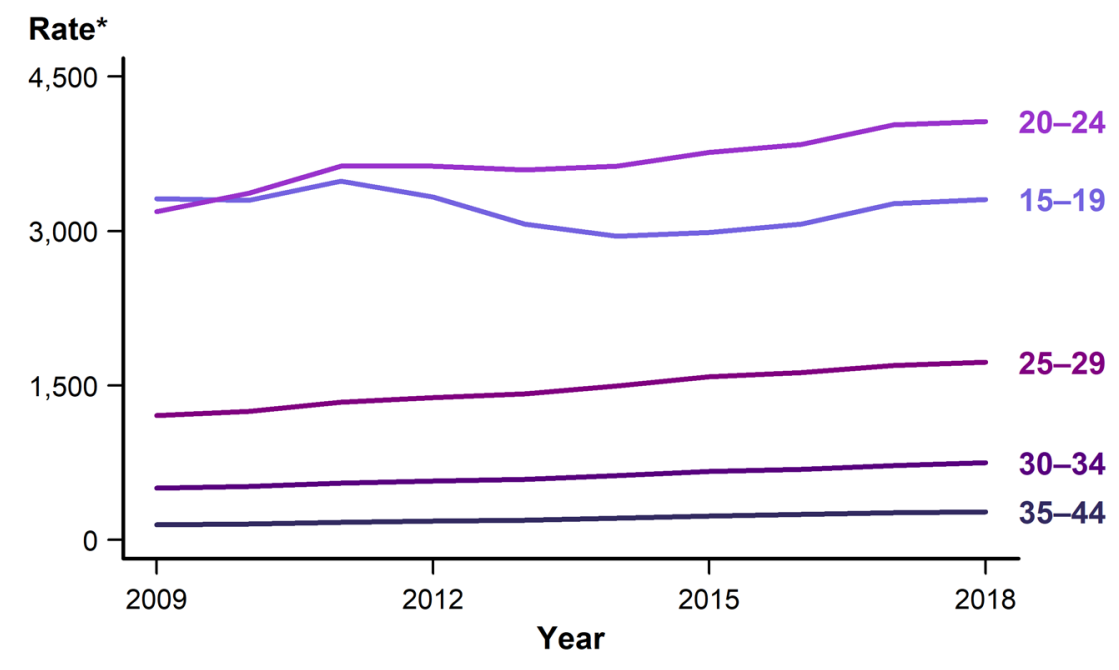

* Per 100,000
$11.9 \%$ during $2014-2018(3,632.7$ to $4,064.6$ cases per 100,000 females) (Table 10).

In $2018,94.0 \%$ of all reported chlamydia cases in males were among those aged 15-44 years (Table 10). The age-specific rates of reported cases of chlamydia among males, although substantially lower than rates among females, were highest in those aged $20-24$ years $(1,784.5$ cases per 100,000 males) (Figure 5, Table 10). Similar to trends in females, increases have been observed in rates of reported cases of chlamydia among all age groups in males aged 15-44 years, although for males these increases have been more pronounced (Figure 7). The rate among 15-19 year olds increased $3.7 \%$ during 2017-2018, with a total increase of $32.8 \%$ during 2014-2018 (from 722.4 to 959.0 cases per 100,000 males). The rate among 20-24 year old males increased 3.3\% during 2017-2018, with a total increase of $31.1 \%$ during 2014-2018 (1,361.3 to $1,784.5$ cases per 100,000 males) (Table 10).

\section{Chlamydia by Race/Hispanic Ethnicity}

In 2018, rates of reported cases of chlamydia were highest among Black, American Indian/Alaska Native (AI/ AN), and Native Hawaiian/Other Pacific Islander (NHOPI) persons (Figure S, Table 11B). Overall, the rate of reported cases of chlamydia among Blacks was 5.6 times the rate among Whites $(1,192.5$ and 212.1 cases per 100,000 population, respectively). The rate among AI/ANs (784.8 cases per 100,000 population) was 3.7 times the rate among Whites. The rate among NHOPIs (700.8 cases per 100,000 population) was 3.3 times the rate among Whites. The rate among Hispanics (392.5 cases per 100,000 population) was 1.9 times the rate among Whites. The rate among Asians (132.1 cases per 100,000 population) was 0.6 times the rate among Whites. 
During 2014-2018, rates of reported chlamydia cases increased among all $\mathrm{racial} /$ Hispanic ethnicity groups, with $\mathrm{AI} / \mathrm{ANs}$ increasing $7.0 \%$, Hispanics $8.1 \%$, Blacks $9.0 \%$, Whites $17.6 \%$, NHOPIs $20.1 \%$, Asians $29.3 \%$, and
Multirace 59.7\% (Figure 8). During 2017-2018, rates increased among some racial/Hispanic ethnicity groups (Whites: 0.3\%, AI/ANs: 1.1\%, Blacks: $2.5 \%$, Asians: $5.7 \%$, and Multirace: $16.4 \%$ ), and decreased

\section{Figure 7. Chlamydia - Rates of Reported Cases Among Males Aged 15-44 Years by Age Group, United States, 2009-2018}

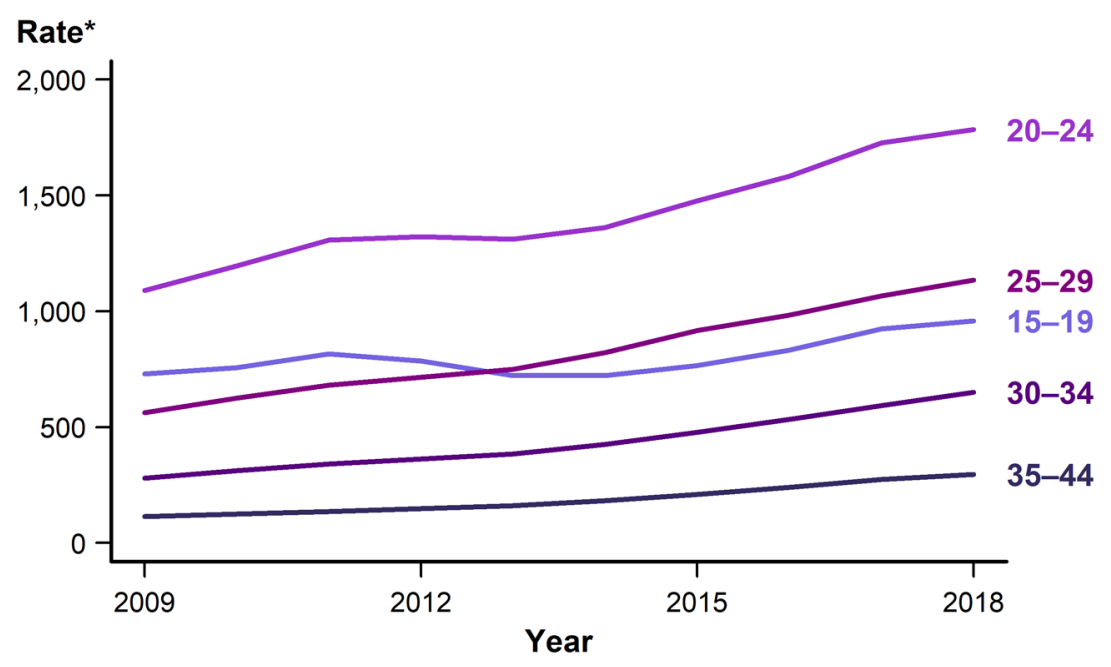

* Per 100,000 .

Figure 8. Chlamydia - Rates of Reported Cases by Race/Hispanic Ethnicity, United States, 2014-2018

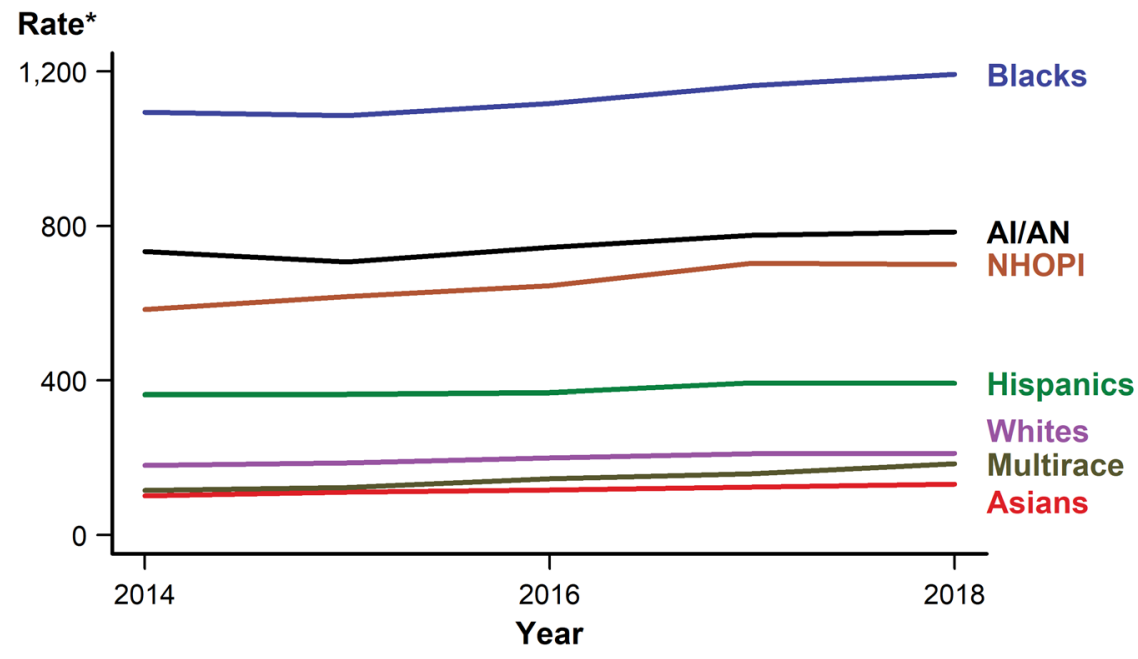

* Per 100,000.

NOTE: See Section A1.5 in the Appendix for information on reporting STD case data for race/Hispanic ethnicity.

ACRONYMS: AI/AN = American Indians/Alaska Natives; NHOPI = Native Hawaiians/Other Pacific Islanders.

among others (NHOPIs: $-0.4 \%$ and Hispanics: $-0.4 \%)$.

More information on chlamydia rates among race/Hispanic ethnicity groups can be found in the Special Focus Profiles, STDs in Racial and Ethnic Minorities.

\section{Chlamydia by Reporting Source}

In $2018,5.2 \%$ of chlamydia cases were reported from STD clinics, $80.2 \%$ were reported from venues outside of STD clinics, and 14.6\% had an unknown reporting source (Table A2). In 2018, among females, only $3.5 \%$ of chlamydia cases were reported through an STD clinic, with a large proportion of cases (30.9\%) being reported from a private physician/HMO (Figure 9 and Table A2). Over time, the proportion of male cases reported from STD clinic sites has decreased substantially, from $30.1 \%$ in 2009 to $8.6 \%$ in 2018 (Figure 10). Nearly one quarter $(24.0 \%)$ of male cases were reported from a private physician/HMO.

\section{Chlamydia Prevalence in the Population}

The National Health and Nutrition Examination Survey (NHANES; see Section A2.4 in the Appendix) is a nationally representative survey of the US civilian, non-institutionalized population that provides an important measure of chlamydia disease burden in respondents aged 14-39 years. During 2013-2016, the overall prevalence of chlamydia among persons aged 14-39 years was $1.7 \%$ (95\% Confidence Interval [CI]: 1.3-2.1) (Figure 11). Among sexually-active females aged 14-24 years, the population targeted for screening, prevalence was $4.3 \%$ (95\% CI: 2.7-5.8), with the highest prevalence among Mexican American females $(10.0 \%, 95 \%$ CI: 4.0-15.9) (Figure 12). 


\section{Figure 9. Chlamydia - Percentage of Reported Cases Among Females by Reporting Source*, United States, 2009-2018}

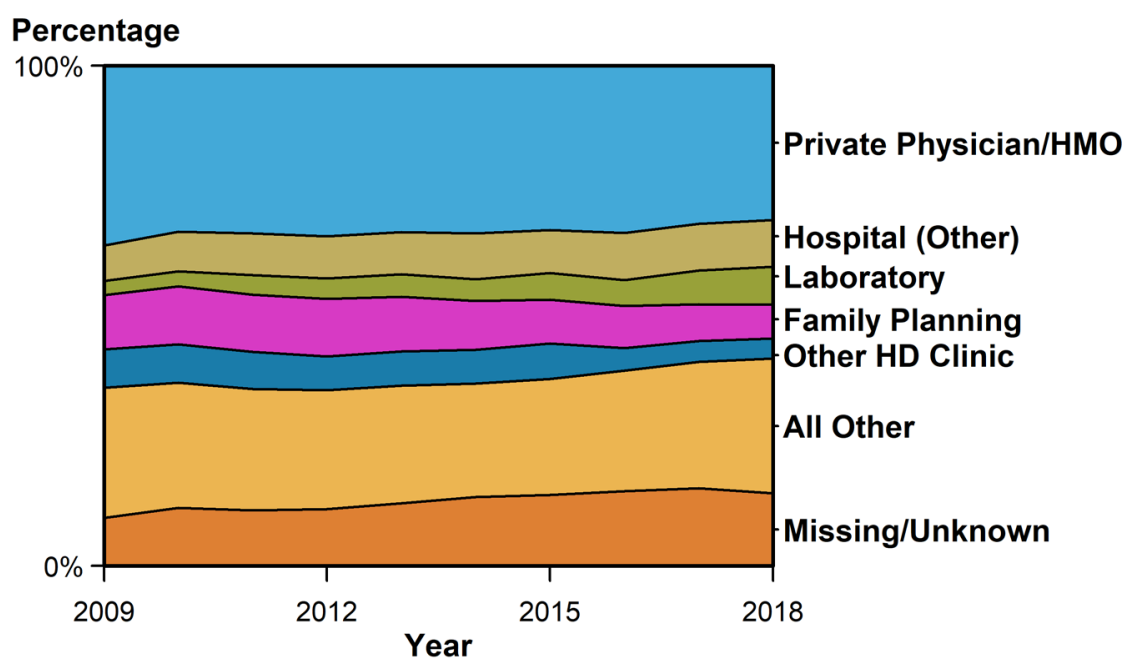

* See section A1.7 in the Appendix for information on classification of reporting sources and a full list of reporting sources.

ACRONYMS: $\mathrm{HMO}=$ Health maintenance organization; $\mathrm{HD}=$ Health department.

\section{Figure 10. Chlamydia - Percentage of Reported Cases Among Males by Reporting Source*, United States, 2009-2018}

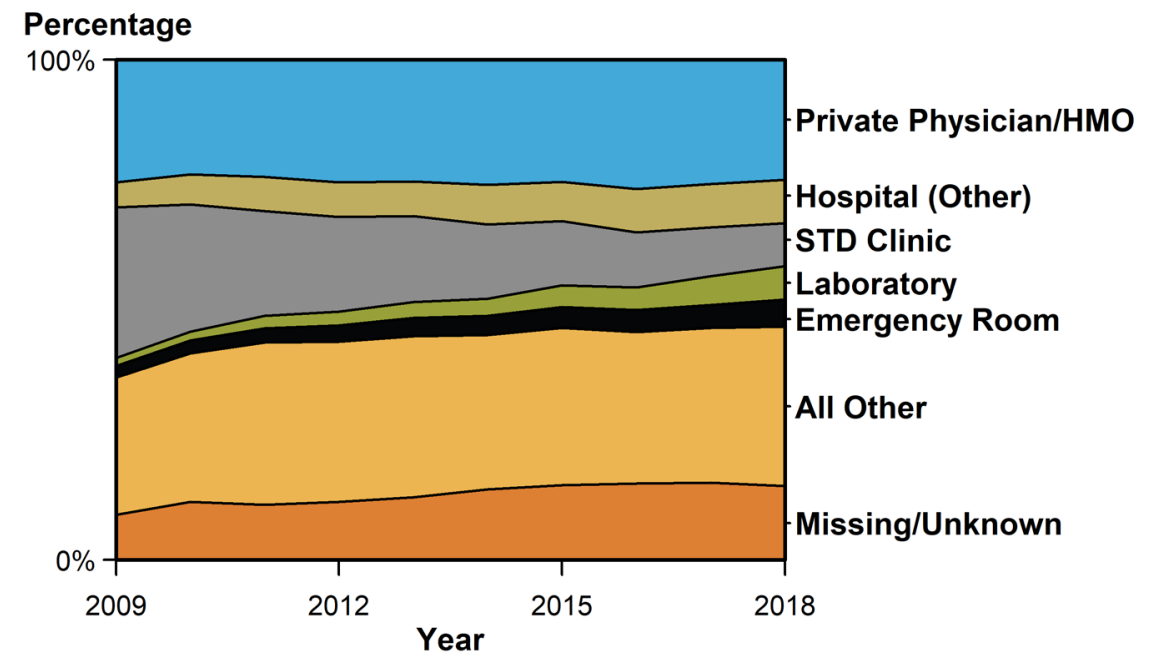

* See section A1.7 in the Appendix for information on classification of reporting sources and a full list of reporting sources.

ACRONYMS: $\mathrm{HMO}=$ Health maintenance organization .

\section{Chlamydia Positivity in Selected Populations}

The STD Surveillance Network $(\mathrm{SSuN})$ is an ongoing collaboration of state, county, and city health departments conducting sentinel and enhanced surveillance activities. These include collecting enhanced clinical and behavioral information among all patients attending selected STD clinics, among women aged
15-44 years in selected reproductive health clinics, and conducting enhanced patient and provider investigations on a representative sample of gonorrhea cases diagnosed and reported from all reporting sources in their jurisdiction (See Section A2.2 of the Appendix).

In 2018, the proportion of STD clinic patients testing positive for chlamydia varied by sex and sex of sex partners, as well as age. Women and men who have sex with women only (MSW) aged $\leq 19$ years had the highest positivity; positivty was nearly equal at $30.6 \%$ and $30.5 \%$, respectively (Figure 13). Positivity among all those tested decreased with age, though the decrease in positivity by age was not as pronounced for MSM (Figure 13). The overall positivity, represented by the average of the mean value by the contributing SSuN jurisdictions, was $16.9 \%$ for MSM, $13.8 \%$ for MSW, and $10.9 \%$ for women.

\section{Chlamydia Among Special Populations}

More information on chlamydia among females of reproductive age, adolescents and young adults, MSM, and racial and ethnic minorities can be found in the Special Focus Profiles.

\section{Chlamydia Summary}

Chlamydia continues to be the most commonly reported nationally notifiable disease, with 1,758,668 cases reported in 2018 and increasing rates of reported cases over each of the last five years. While the rate of reported chlamydia cases increased just 2.9\% during 2017-2018, the rate increased $19.4 \%$ during 2014-2018. The Southern region of the US reported the highest rate of chlamydial infection in 2018; the West reported the largest rate increase during 2017-2018. In 2018, similar to prior years, the rate of reported cases of chlamydia in females was nearly two times the rate in males. However, during 2014-2018, the rate in males 
Figure 11. Chlamydia - National Estimates of Prevalence Among Persons Aged 14-39 Years by Sex, Race/Hispanic Ethnicity, or Age Group, National Health and Nutrition Examination Survey (NHANES), 2013-2016

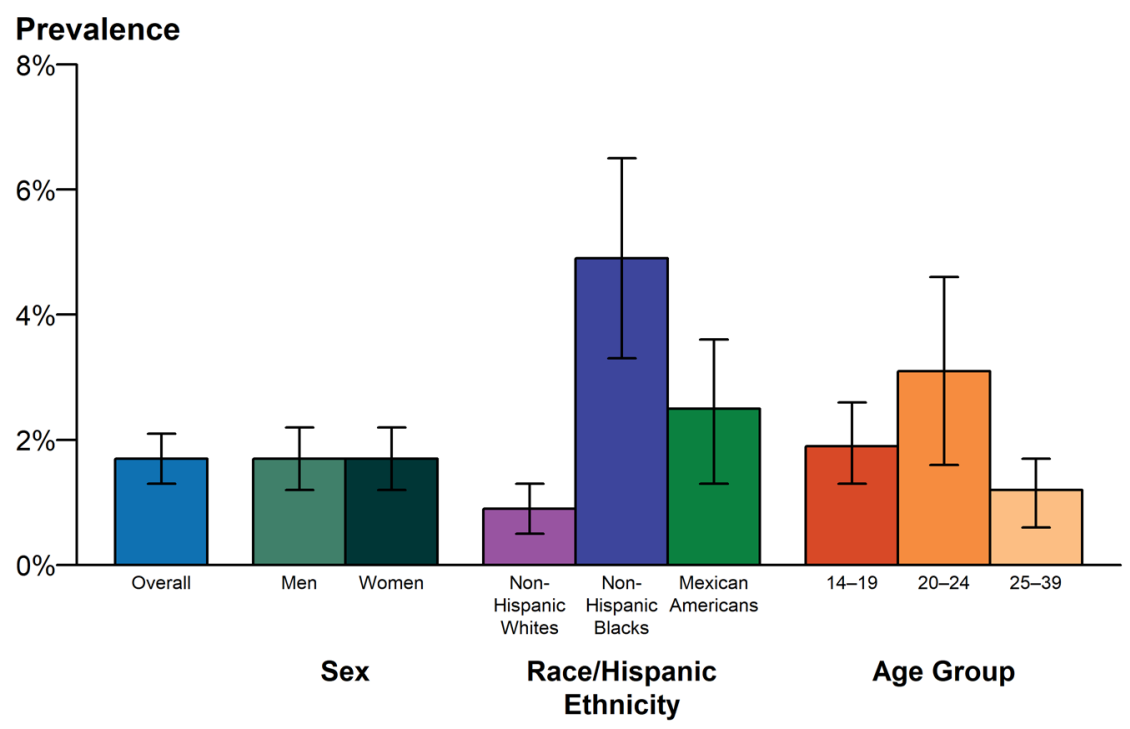

NOTE: Error bars indicate $95 \%$ confidence intervals. Overall prevalence estimates include all race/Hispanic ethnicity groups, including those not shown separately.

ADAPTED FROM: Torrone E, Papp J, Weinstock H. Prevalence of Chlamydia

trachomatis genital infection among persons aged 14-39 years - United States, 2007-2012. MMWR Morb Mortal Wkly Rep. 2014; 63(38):834-838.

Figure 12. Chlamydia - National Estimates of Prevalence Among Sexually-Active Females Aged 14-39 Years by Race/Hispanic Ethnicity and Age Group, National Health and Nutrition Examination Survey (NHANES), 2013-2016

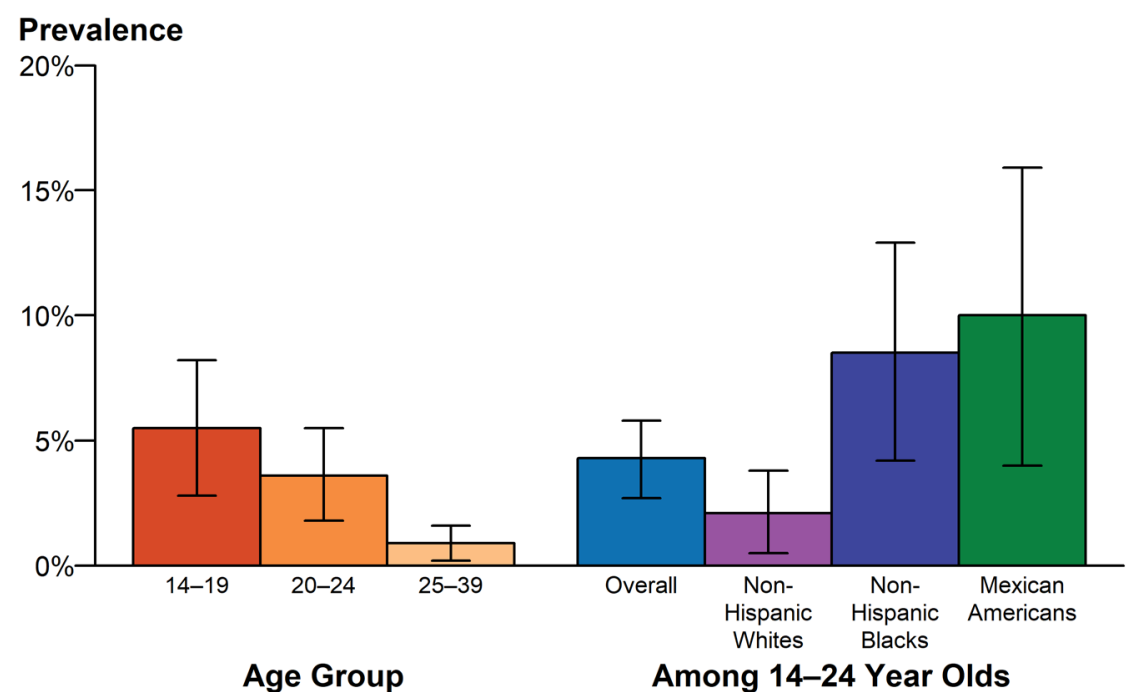

NOTE: Error bars indicate $95 \%$ confidence intervals. Overall prevalence estimates include all race/Hispanic ethnicity groups, including those not shown separately.

ADAPTED FROM: Torrone E, Papp J, Weinstock H. Prevalence of Chlamydia trachomatis genital infection among persons aged 14-39 years - United States, 2007-2012. MMWR Morb Mortal Wkly Rep. 2014; 63(38):834-838. increased $37.8 \%$, whereas the rate in females increased only $11.4 \%$. Potential reasons for this considerable increase in male cases could be due to a true increase in infections or to improved screening coverage in males, especially increased extragenital screening in MSM, or both.

The facilities reporting chlamydial infections have changed over the last 10 years with most $(80.2 \%)$ chlamydia cases in 2018 reported from venues outside of STD clinics. The proportion of males being diagnosed with chlamydia in STD clinics decreased $71.4 \%$ from $30.1 \%$ in 2009 to $8.6 \%$ in 2018 . In females, approximately one-third of chlamydia cases were reported in 2018 from a private physician/HMO, while only $3.5 \%$ were reported from STD clinics. Racial differences also persist. Reported case rates among Blacks continue to be substantially higher than among all other racial/Hispanic ethnicity groups, although rates have increased substantially among all racial/Hispanic ethnicity groups over the last five years. Ultimately, both test positivity and the number of reported cases of $C$. trachomatis infections remain high among younger age groups, and most racial/ Hispanic ethnicity groups, geographic areas, and both sexes.

\section{References}

1. Torrone E, Papp J, Weinstock H. Prevalence of Chlamydia trachomatis genital infection among persons aged 14-39 years - United States, 2007-2012. MMWR Morb Mortal Wkly Rep. 2014;63(38):834-838.

2. Stamm WE. Chlamydia trachomatis infections in the adult. In: Holmes KK, Sparling PF, Stamm WE, et al., eds. Sexually Transmitted Diseases. 4th ed. New York, NY: McGraw-Hill; 2008:575-606.

3. Scholes D, Stergachis A, Heidrich FE, et al. Prevention of pelvic inflammatory disease by screening for cervical chlamydial infection. $N$ Engl J Med. 1996;334(21):1362-1366.

4. Oakeschott P, Kerry S, Aghaizu A, et al. Randomised controlled trial of screening for Chlamydia trachomatis to prevent pelvic inflammatory disease: The POPI (prevention of pelvic infection) trial. $B M J .2010 ; 340: c 1642$. 
Figure 13. Chlamydia - Proportion of STD Clinic Patients Testing Positive* by Age Group and Sex and Sex of Sex Partners, STD Surveillance Network (SSuN), 2018

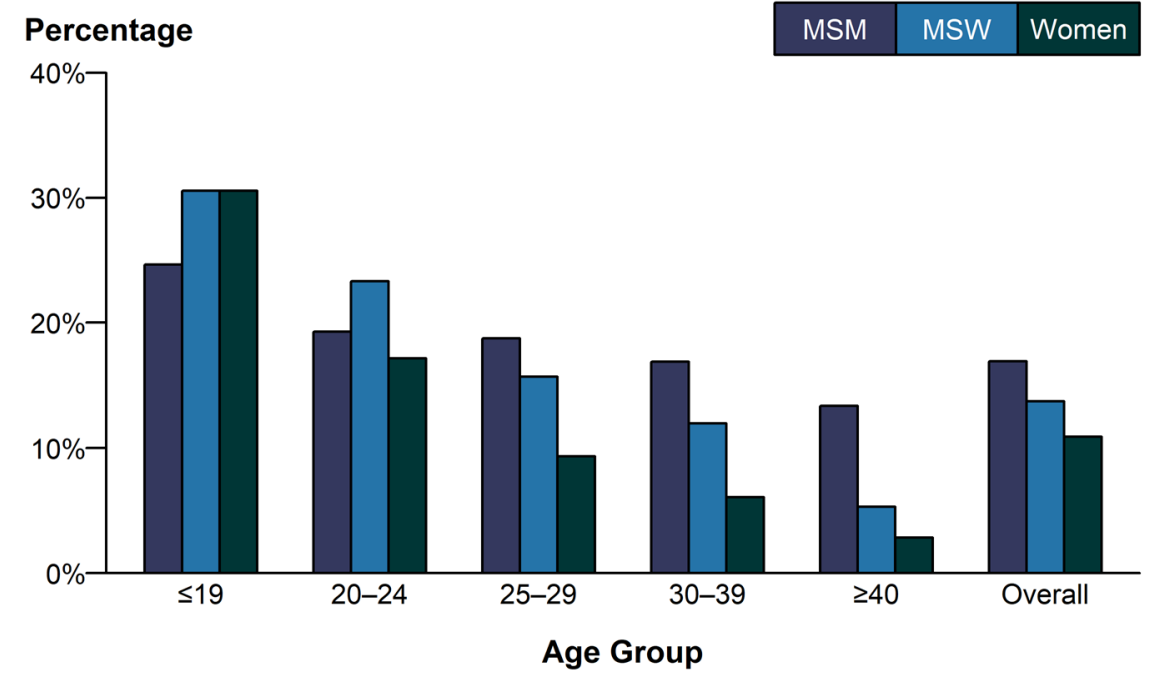

* Results are based on unique patients with known sex of sex partners $(n=83,691)$ attending SSUN STD clinics who were tested $\geq 1$ time for chlamydia in 2018 . NOTE: See Section A2.2 in the Appendix for SSUN methods.

ACRONYMS: $M S M=$ Gay, bisexual, and other men who have sex with men; MSW = Men who have sex with women only.
5. Fleming DT, Wasserheit JN. From epidemiological synergy to public health policy and practice: The contribution of other sexually transmitted diseases to sexual transmission of HIV infection. Sex Transm Infect. 1999;75(1):317.

6. Hammerschlag MR, Chandler JW, Alexander ER, et al. Longitudinal studies of chlamydial infection in the first year of life. Pediatr Infect Dis. 1982;1(6):395-401.

7. Centers for Disease Control and Prevention. Sexually transmitted diseases treatment guidelines, 2015. MMWR Recomm Rep. 2015;64(No. RR-3):1-137.

8. National Committee for Quality Assurance. The State of Healthcare Quality: Chlamydia Screening in Women (CHL). Available at: https://www.ncqa.org/hedis/measures/ chlamydia-screening-in-women/. Accessed July 24, 2019. 


\section{Background}

Gonorrhea is the second most commonly reported notifiable disease in the United States. Infections due to Neisseria gonorrhoeae, like those resulting from Chlamydia trachomatis, are a cause of pelvic inflammatory disease (PID) in the United States. PID can lead to serious outcomes in women, such as tubal infertility, ectopic pregnancy, and chronic pelvic pain. In addition, epidemiologic and biologic studies provide evidence that gonococcal infections facilitate the transmission of HIV infection. ${ }^{1}$ Together, sexual behavior and community prevalence can increase the risk of acquiring gonorrhea. Social determinants of health, such as socioeconomic status, discrimination, and access to quality health care, may contribute to the burden of gonorrhea in a community. ${ }^{2}$

$N$. gonorrhoeae has progressively developed resistance to each of the antimicrobials used for treatment of gonorrhea. Declining susceptibility to cefixime (an oral cephalosporin antibiotic) resulted in a change to the CDC treatment guidelines in 2015 to the current CDCrecommended regimen of ceftriaxone (an injectable cephalosporin) and azithromycin. ${ }^{3}$ The emerging threat of cephalosporin resistance highlights the need for continued surveillance of $N$. gonorrhoeae antimicrobial susceptibility.

The combination of persistently high gonorrhea morbidity in some populations and the threat of cephalosporin-resistant gonorrhea reinforces the need to better understand the epidemiology of gonorrhea.

\section{Interpreting Rates of Reported Cases of Gonorrhea}

Although gonorrhea case reporting is useful for monitoring disease trends, the number of gonorrhea cases reported to CDC is affected by many factors in addition to the actual occurrence of the infection within the population. Changes in the burden of gonorrhea may be masked by changes in screening practices (e.g., screening for chlamydia with tests that also detect $N$. gonorrhoeae infections or increased screening at extra-genital anatomic sites), the use of diagnostic tests with different test performance (e.g., the broader use of nucleic acid amplification tests [NAATs]), and changes in reporting practices. As with other STDs, the reporting of gonorrhea cases to CDC is incomplete. ${ }^{4}$ For these reasons, supplemental data on gonorrhea prevalence in persons screened in a variety of settings are useful in assessing the burden of disease in selected populations.

\section{Gonorrhea - United States}

In 2018, a total of 583,405 cases of gonorrhea were reported in the United States, yielding a rate of 179.1 cases per 100,000 population (Figure 14, Table 1). During 2017-2018, the rate of reported gonorrhea cases increased $5.0 \%$, and increased $82.6 \%$ since the historic low in 2009.

\section{Gonorrhea by Region}

The South had the highest rate of reported gonorrhea cases (194.4 cases per 100,000 population) among the four regions of the United States in 2018, followed by the Midwest (184.5 cases per 100,000 population), the West (179.7 cases per 100,000 population), and the Northeast (138.4 cases per 100,000 population) (Figure 15, Table 14). During 2017-2018, the gonorrhea rate increased in all four

\section{Figure 14. Gonorrhea - Rates of Reported Cases by Year, United States, 1941-2018}

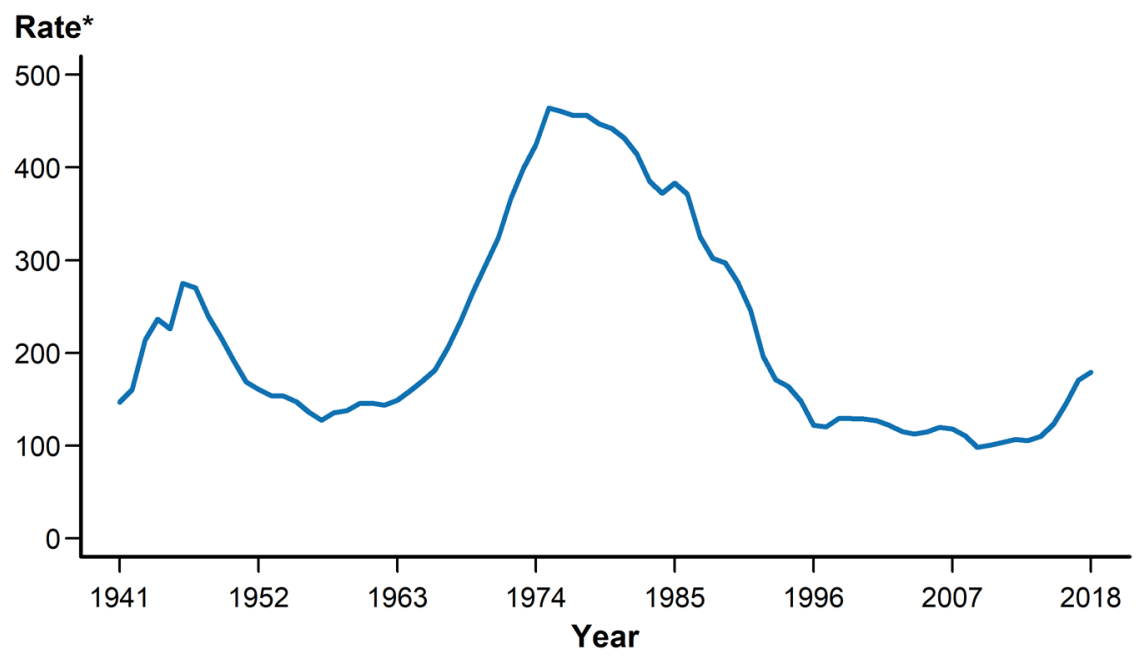

* Per 100,000.

NOTE: See section A1.3 in the Appendix for more information on gonorrhea case reporting. 


\section{Figure 15. Gonorrhea - Rates of Reported Cases by Region, United States, 2009-2018}

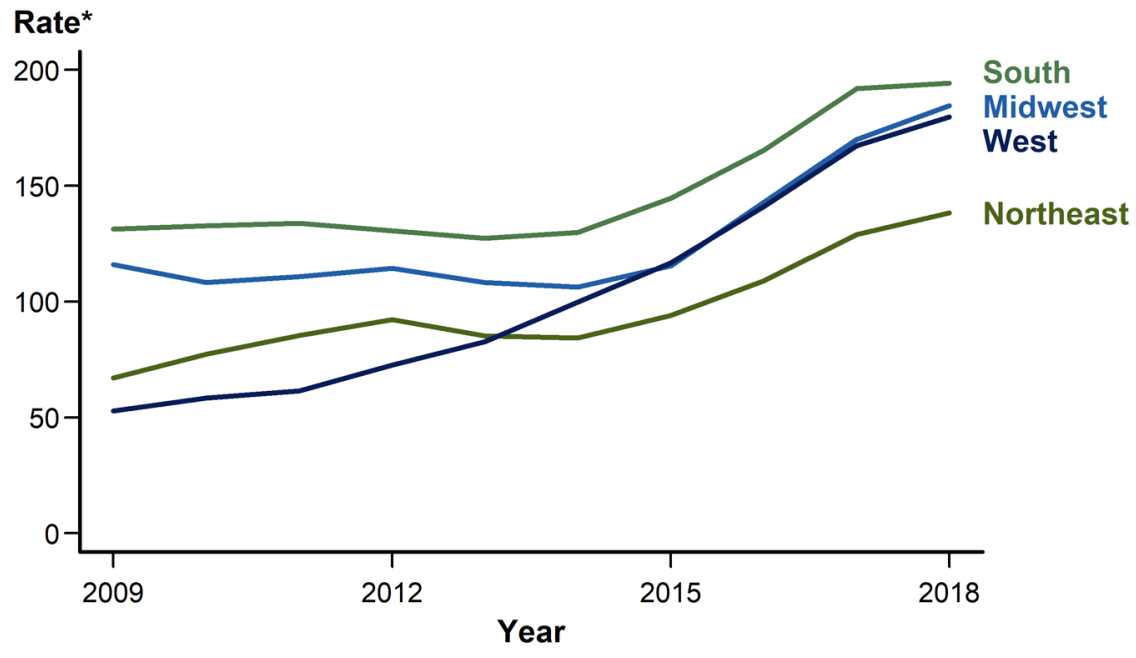

* Per 100,000

regions: $8.5 \%$ in the Midwest, $7.4 \%$ in the West, $7.3 \%$ in the Northeast, and $1.3 \%$ in the South (Figure 15, Table 14). During 2014-2018, the rate of gonorrhea in the West increased by $79.9 \%$ (99.9 to 179.7 cases per 100,000 population) and the rate in the Midwest increased by $73.7 \%$ (106.2 to 184.5 cases per 100,000 population), while the Northeast and South had lower overall increases during this time period $(64.0 \%$ and $49.7 \%$, respectively).

\section{Gonorrhea by State}

In 2018, rates of reported gonorrhea cases per 100,000 population ranged by state from 43.0 in Vermont to 326.7 in Mississippi; the gonorrhea rate in the District of Columbia was 611.0 cases per 100,000 population (Figure 16, Tables 13 and 14).

During 2017-2018, gonorrhea rates increased in 40 states $(78.4 \%)$ and decreased in 10 states and the District of Columbia (21.6\%) (Table 14).

\section{Gonorrhea by Metropolitan Statistical Area}

The overall rate of reported gonorrhea cases in the 50 most populous rate in 2017 (189.4 cases per 100,000 population) (Table 17). In 2018, $60.1 \%$ of reported gonorrhea cases were reported by these MSAs. Since 2014, the gonorrhea rate among females in the 50 most populous MSAs has been lower than the rate among males (Tables 18 and 19). In 2018 , the rate among females in these MSAs was 139.0 cases per 100,000 females, while the rate among males was 252.5 cases per 100,000 males.

\section{Gonorrhea by County}

In $2018,93.4 \%$ of all counties in the United States reported at least one case of gonorrhea; however, $49.0 \%$ of reported gonorrhea cases occurred in just 70 counties or independent cities (Figure 17). The rate ranged from 0.0 to 30.0 cases per 100,000 population in 627 counties $(20.0 \%)$, from 31.0 to 60.0 cases per 100,000 population in 625 counties $(20.0 \%)$, from 61.0 to 102.0 cases per 100,000 population in was 194.9 cases per 100,000 population in 2018, representing a $2.9 \%$ increase compared with the
Figure 16. Gonorrhea - Rates of Reported Cases by State and Territory, United States, 2018

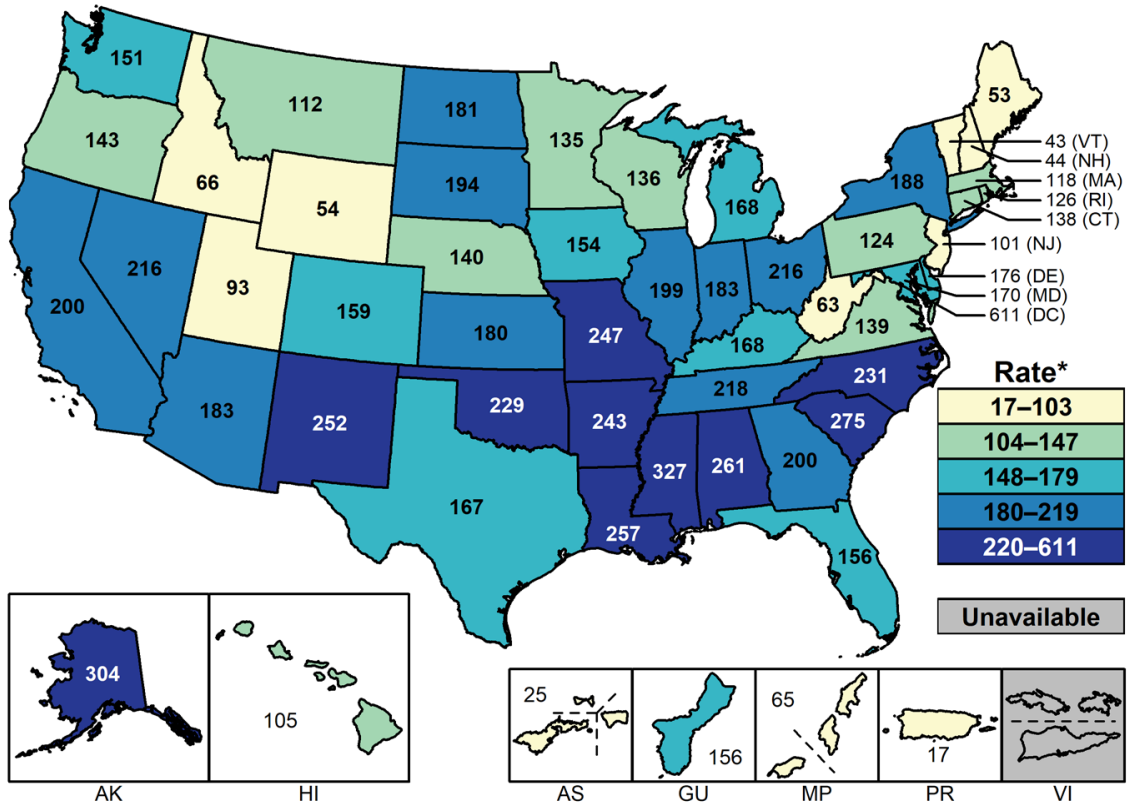

* Per 100,000.

NOTE: See Section A1.11 in the Appendix for more information on interpreting reported rates in US territories. 


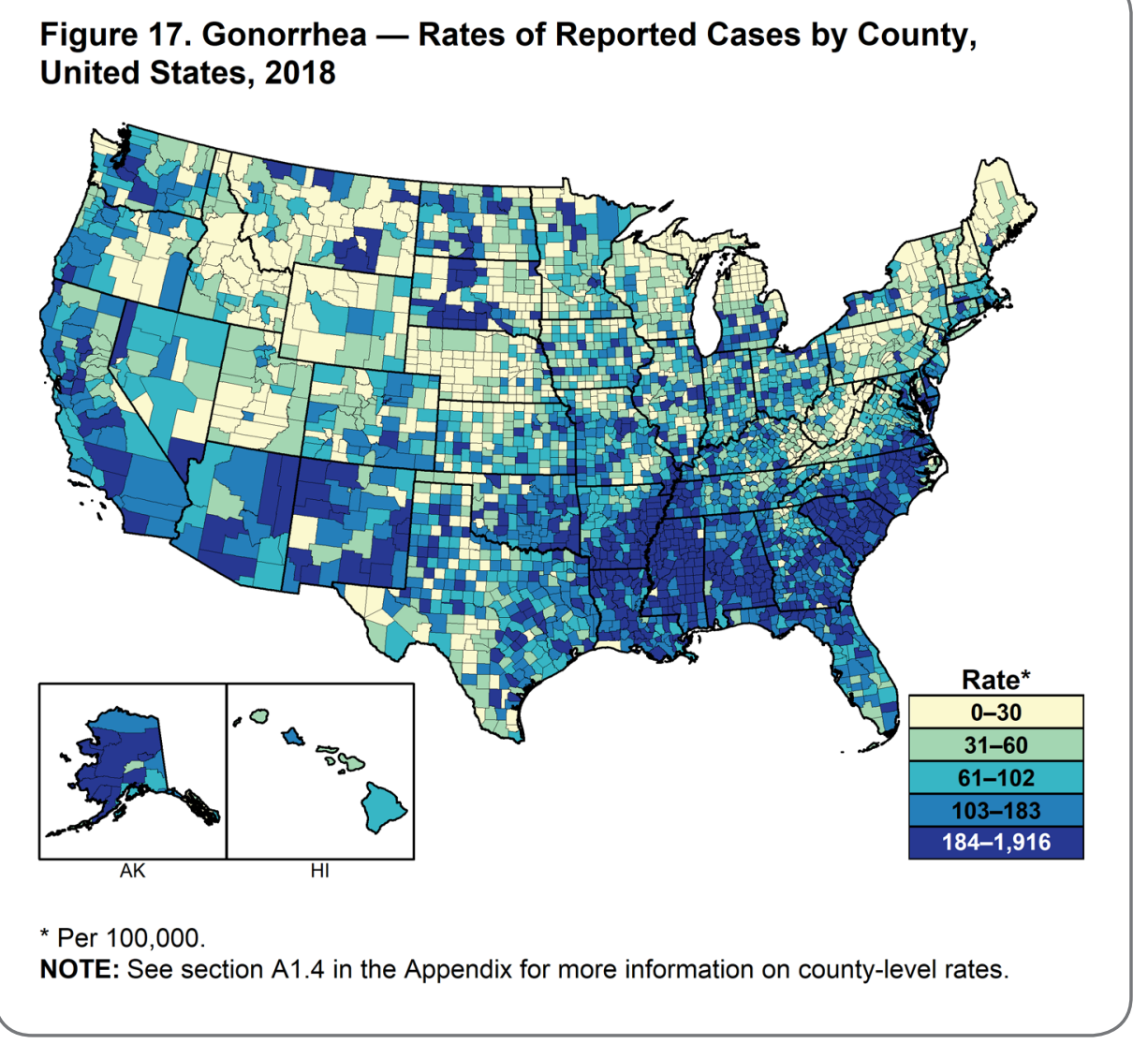

629 counties $(20.0 \%)$, from 103.0 to 183.0 cases per 100,000 population in 628 counties $(20.0 \%)$, and was 184.0 cases per 100,000 population or more in 633 counties (20.1\%). As in previous years, counties with the highest gonorrhea rates were concentrated in the South.

\section{Gonorrhea by Sex}

As was observed during 2014-2017, the rate of reported gonorrhea cases among males was higher than the rate among females in 2018 (Figure 18, Tables 15 and 16). During 2017-2018, the gonorrhea rate among males increased $6.0 \%$ (200.8 to 212.8 cases per 100,000 males) and the rate among females increased $3.6 \%$ (140.7 to 145.8 cases per 100,000 females). During 2014-2018, the rate among males increased $78.7 \%$ (119.1 to 212.8 cases per 100,000 males) and the rate among females increased $45.2 \%$ (100.4 to 145.8 cases per 100,000 females). The magnitude of the increase among males suggests either increased transmission or increased case ascertainment (e.g., through increased extra-genital screening) among gay, bisexual, and other men who have sex with men (MSM). However, most jurisdictions

do not routinely report sex of sex partners or site of infection for gonorrhea cases, so national trends in gonorrhea rates among MSM over time cannot be assessed.

\section{Gonorrhea by Region and Sex}

In most regions, the rate of gonorrhea increased among both males and females during 2017-2018 and during 2014-2018 (Tables 15 and 16). The rate of reported gonorrhea cases among females increased the most in the West (8.7\% during 2017-2018 and $71.0 \%$ during 2014-2018) and Midwest (7.1\% during 2017-2018 and $57.5 \%$ during 2014-2018) with smaller increases in the Northeast (3.1\% during 2017-2018 and 30.8\% during 2014-2018) and South (33.2\% during 2014-2018) (Table 15). The rate of reported gonorrhea cases among males increased the most in the Midwest (10.1\% during 2017-2018 and $90.8 \%$ during 2014-2018) and Northeast (9.3\% during $2017-2018$ and $86.2 \%$ during 2014-2018); however, increases were all also reported in the West $(6.6 \%$ during 2017-2018 and $85.0 \%$ during 2014-2018) and South (2.4\% during

\section{Figure 18. Gonorrhea - Rates of Reported Cases by Sex, United States, 2009-2018}

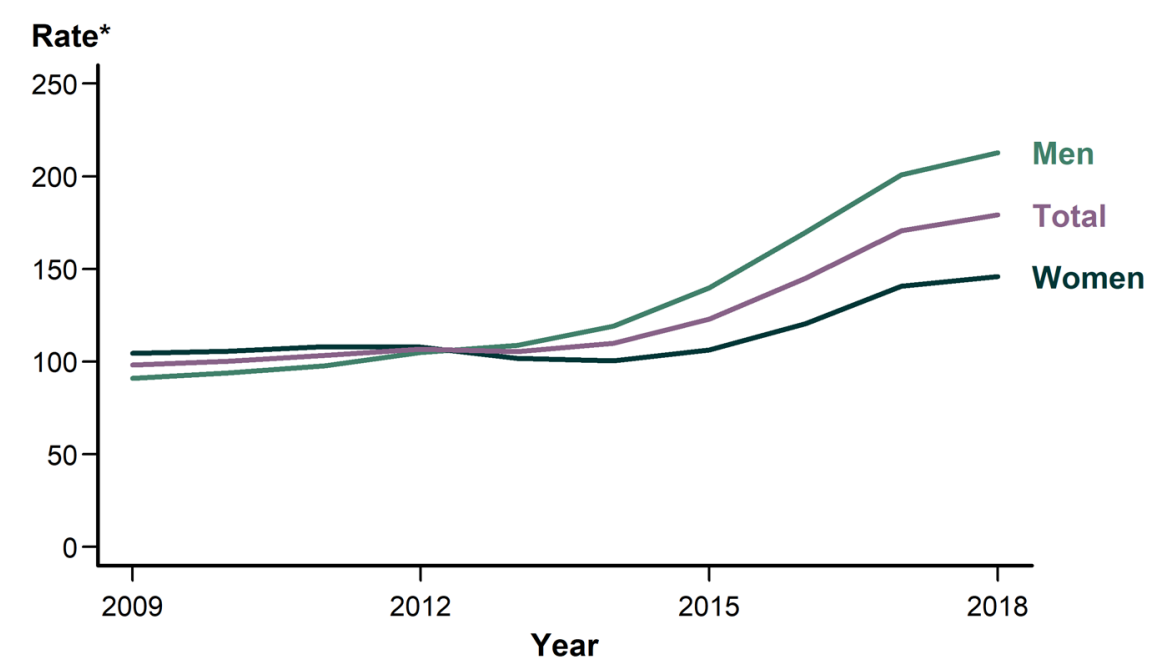

* Per 100,000. 
2017-2018 and 66.3\% during 20142018) (Table 16).

\section{Gonorrhea by Age}

In 2018, rates of reported gonorrhea cases continued to be highest among adolescents and young adults (Figure 19, Table 21). In 2018, the highest rates among females were observed among those aged $20-24$ years $(702.6$ cases per 100,000 females) and 15-19 years (548.1 cases per 100,000 females). Among males, the rate was highest among those aged 20-24 years (720.9 cases per 100,000 males) and 25-29 years (674.0 cases per 100,000 males).

In 2018, persons aged 15-44 years accounted for $91.6 \%$ of reported gonorrhea cases with known age (Table 21). Among 15-19 year olds, rates decreased $1.3 \%$ during 2017-2018. However, the gonorrhea rate increased among the following age groups during 2017-2018: 1.2\% among those aged 20-24 years, $6.2 \%$ among those aged 25-29 years, $12.4 \%$ among those aged 30-34 years, $10.5 \%$ among those aged $35-39$ years, and $11.8 \%$ among those aged 40-44 years (Table 21). Increases in rates were observed in most age groups for both males and females during 2017-2018. However, rates decreased $1.7 \%$ among females aged $15-19$ years ( 557.3 to 548.1 cases per 100,000 females) and $0.9 \%$ among males aged $15-19$ years $(323.3$ to 320.5 cases per 100,000 males) (Figures 20 and 21).

\section{Gonorrhea by Race/Hispanic Ethnicity}

In 2018, the rate of reported gonorrhea cases remained highest among Blacks (548.9 cases per 100,000 population) (Table 22B). The rate among Blacks was 7.7 times the rate among Whites (71.1 cases per 100,000 population). The gonorrhea rate among American Indians/Alaska Natives (AI/AN) (329.5 cases per

\section{Figure 19. Gonorrhea - Rates of Reported Cases by Age Group and Sex, United States, 2018}

Male Rate*

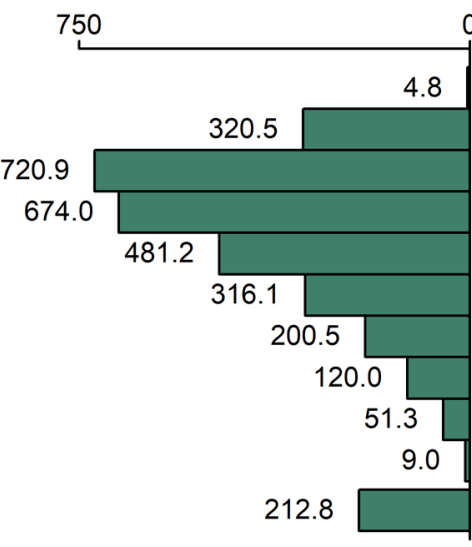

0 Age Group 0 10-14 15-19 20-24 25-29 30-34 35-39 40-44 45-54 55-64 $65+$ Total Female Rate*

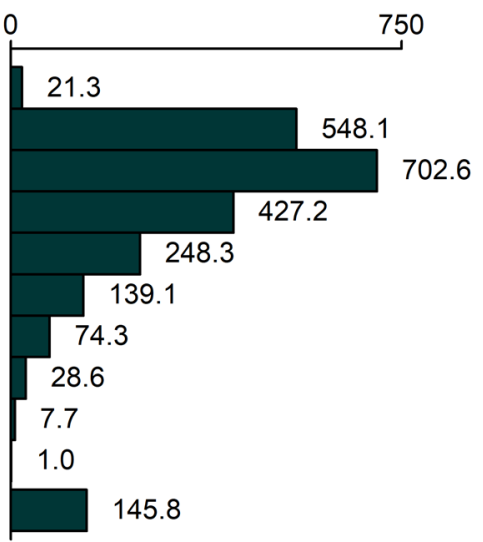

* Per 100,000.

Figure 20. Gonorrhea - Rates of Reported Cases Among Females Aged 15-44 Years by Age Group, United States, 2009-2018

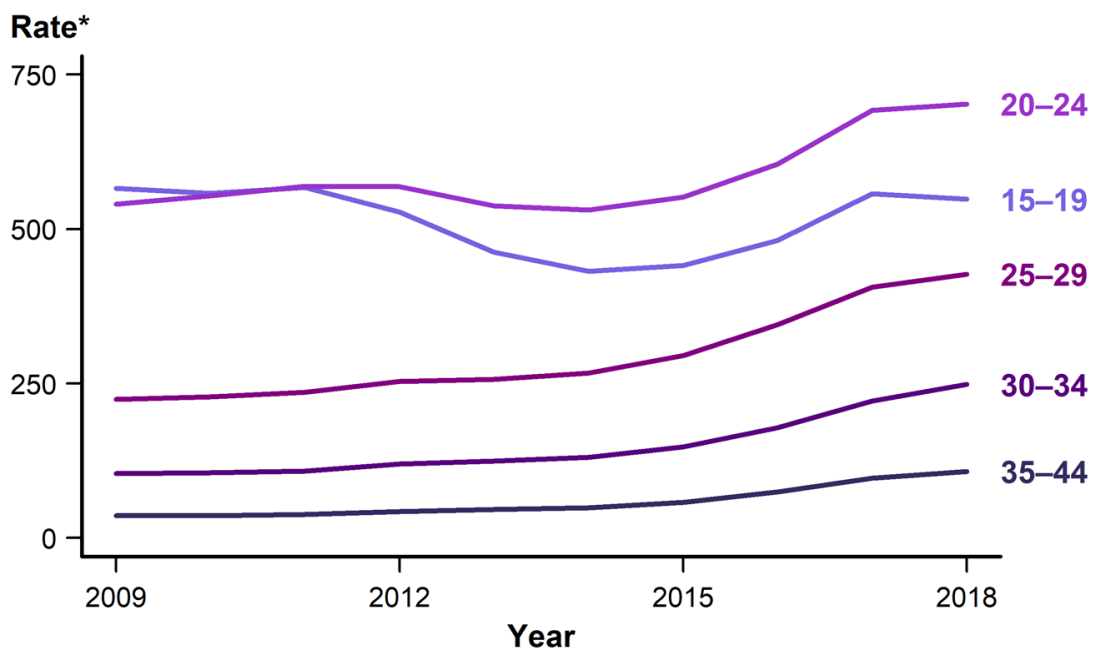

* Per 100,000.

100,000 population) was 4.6 times that of Whites, the rate among Native Hawaiians/Other Pacific Islanders (NHOPI) (181.4 cases per 100,000 population) was 2.6 times that of Whites, the rate among Hispanics (115.9 cases per 100,000 population) was 1.6 times that of Whites, the rate among Multirace persons (94.4 cases per 100,000 population) was
1.3 times that of Whites, and the rate among Asians (35.1 cases per 100,000 population) was half the rate of Whites (Table 22B).

During 2014-2018, for all five years during that period, the gonorrhea rate increased among all race/Hispanic ethnicity groups: $119.5 \%$ among Multirace persons, 99.4\% among 


\section{Figure 21. Gonorrhea - Rates of Reported Cases Among Males Aged 15-44 Years by Age Group, United States, 2009-2018}

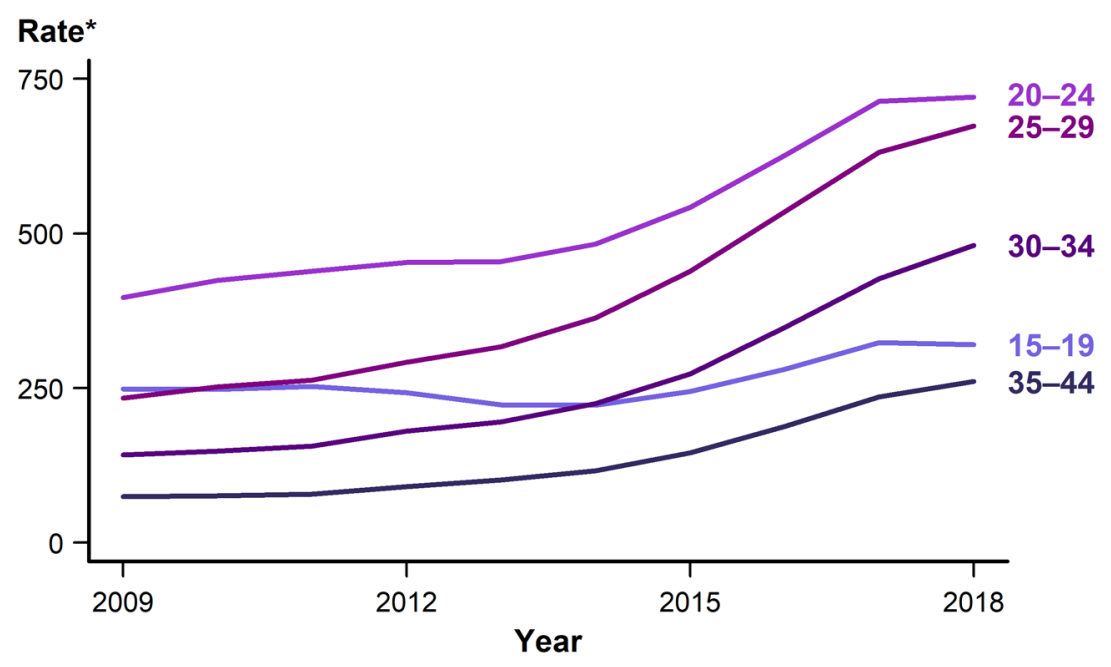

* Per 100,000 .

Asians, 90.3\% among NHOPI, 89.1\% among Whites, $84.2 \%$ among $\mathrm{AI} / \mathrm{AN}$, $66.0 \%$ among Hispanics, and $38.8 \%$ among Blacks (Figure 22).

More information on gonorrhea rates among race/Hispanic ethnicity groups can be found in the Special Focus Profiles, STDs in Racial and Ethnic Minorities.

\section{Gonorrhea by Reporting Source}

In $2018,8.4 \%$ of gonorrhea cases were reported from STD clinics, $77.7 \%$ were reported from venues outside of STD clinics, and 13.9\% had an unknown reporting source (Table A2).

During 2009-2018, the percent of gonorrhea cases reported by STD clinics declined $42.6 \%$ among females and $19.8 \%$ among males; however, the percent of gonorrhea cases with missing/unknown reporting source increased $80.4 \%$ among females and $277.3 \%$ among males (Figures 23 and 24). During 2017-2018, the percent of gonorrhea cases reported by STD clinics decreased $7.5 \%$ among females and $4.9 \%$ among males.
$(9.0 \%)$, emergency rooms $(6.0 \%)$, and STD clinics (5.8\%) (Figure 23). Among males, private physicians/ HMOs (20.7\%) were the most common reporting source, followed by other hospital clinics/facilities (12.3\%), STD clinics (10.3\%), emergency rooms $(7.1 \%)$, and laboratories (6.9\%) (Figure 24).

\section{STD Surveillance Network}

The STD Surveillance Network ( $\mathrm{SSuN})$ is an ongoing collaboration of state, county, and city health departments conducting sentinel and enhanced surveillance activities. These include collecting enhanced clinical and behavioral information among all patients attending selected STD clinics, among women aged 15-44 years in selected reproductive health clinics, and conducting enhanced patient and provider investigations on a representative sample of gonorrhea cases diagnosed and reported from all reporting sources in their jurisdiction.
In 2018, the largest proportion of cases among females were reported by private physicians/health maintenance organizations (HMOs) (24.1\%), followed by other hospital clinics/facilities (13.6\%), laboratories

\section{Figure 22. Gonorrhea - Rates of Reported Cases by Race/Hispanic Ethnicity, United States, 2014-2018}

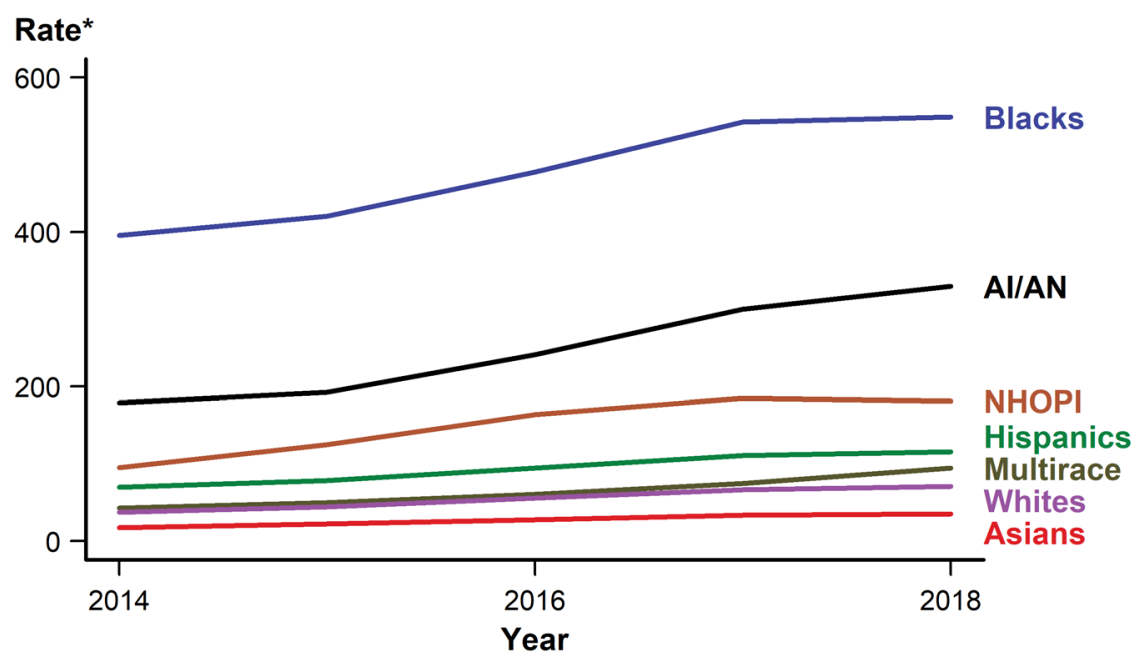

* Per 100,000.

NOTE: See Section A1.5 in the Appendix for information on reporting STD case data for race/Hispanic ethnicity.

ACRONYMS: Al/AN = American Indians/Alaska Natives; $\mathrm{NHOPI}=$ Native Hawaiians/Other Pacific Islanders. 
Figure 23. Gonorrhea - Percentage of Reported Cases Among Females by Reporting Source*, United States, 2009-2018

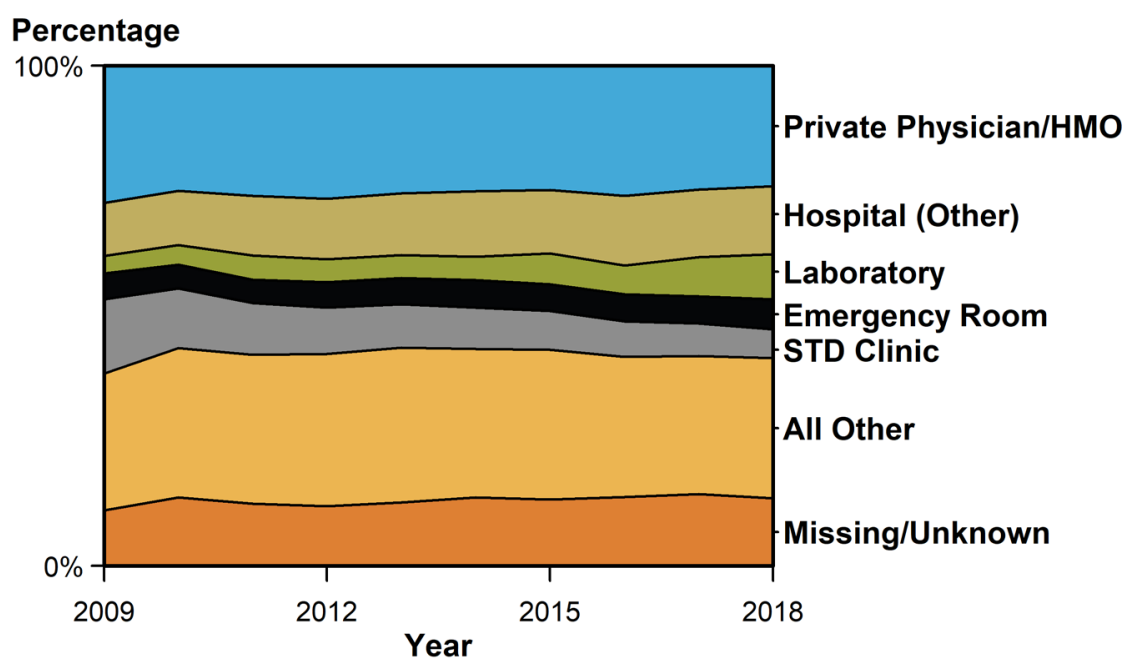

* See section A1.7 in the Appendix for information on classification of reporting sources and a full list of reporting sources.

ACRONYMS: $\mathrm{HMO}=$ Health maintenance organization .

\section{Figure 24. Gonorrhea - Percentage of Reported Cases Among Males by Reporting Source*, United States, 2009-2018}

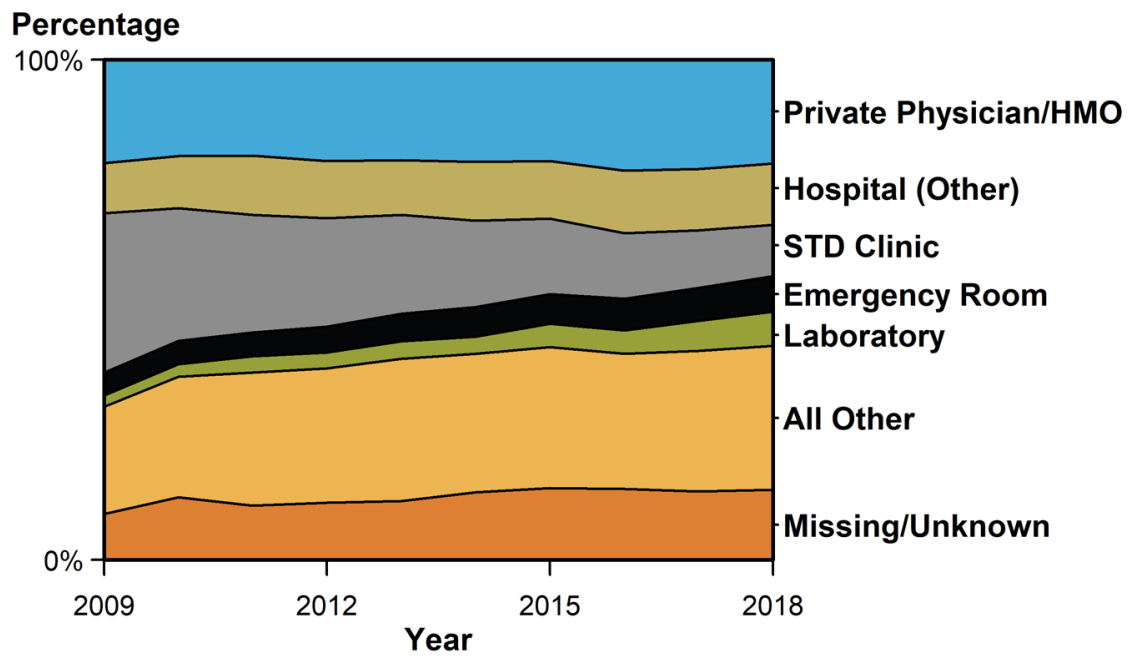

* See section A1.7 in the Appendix for information on classification of reporting sources and a full list of reporting sources.

ACRONYMS: $\mathrm{HMO}=$ Health maintenance organization .

Enhanced gonorrhea case report data for 2018 were obtained from Cycle 3 of SSuN, which includes 10 jurisdictions randomly sampling all cases reported in their jurisdictions. In 2018, SSuN collaborators interviewed
6,842 gonorrhea cases, representing $4.3 \%$ of all cases reported from participating jurisdictions. The estimated burden of disease represented by men who have sex with men (MSM; including men who have sex with both men and women), men who have sex with women only (MSW), and women varied substantially across collaborating sites based on weighted analysis (Figure 25). San Francisco had the highest proportion of cases estimated to be MSM (86.4\%), while Baltimore had the lowest proportion of MSM cases (20.1\%). In total, across all SSuN sites, $42.5 \%$ of gonorrhea cases were estimated to be among MSM, 25.1\% among MSW, and 32.4\% among women.

Among six jurisdictions participating in SSuN continuously from 2010 to 2018, estimated rates of gonorrhea among MSM, MSW, and women were calculated by extending published estimates of the MSM population and are presented in Figure 26. ${ }^{5,6}$ The estimated gonorrhea case rate among MSM increased $375.5 \%$ during 2010-2018 from $1,368.6$ cases per 100,000 MSM in 2010 to $6,508.0$ cases per 100,000 MSM in 2018. Over the same time period, case rates among MSW and women also increased by $69.3 \%$ and $95.2 \%$, respectively.

Collaborating SSuN jurisdictions also conduct sentinel surveillance on all patients seeking care in selected STD clinics. In 2018, the proportion of STD clinic patients who tested positive for gonorrhea varied by sex and sex of sex partners, as well as age group (Figure 27). The overall prevalence, represented by the average of the mean value by nine of the $10 \mathrm{SSuN}$ jurisdictions, where data was available, was $20.5 \%$ for MSM, $9.1 \%$ for MSW, and $5.4 \%$ for women. Among those attending these clinics, MSM disproportionately had higher positivity rates when compared to MSW and women in all age groups. Although overall gonorrhea positivity rates declined with increasing age for women, MSM, and MSW, a slower decline by age was observed in MSM $<40$ years of age. 
Additional information about the SSuN methodology can be found in Section A2.2 of the Appendix.

\section{Gonococcal Isolate Surveillance Project}

Antimicrobial resistance remains an important consideration in the treatment of gonorrhea. ${ }^{3,7-9}$ In 1986, the Gonococcal Isolate Surveillance Project (GISP), a national sentinel surveillance system, was established to monitor trends in antimicrobial susceptibilities of urethral $N$. gonorrhoeae strains in the United States. ${ }^{7}$ Data are collected from selected STD clinic sentinel sites and from regional laboratories (Figure 28).

Antimicrobial susceptibility is measured by the minimum inhibitory concentration (MIC), the lowest antimicrobial concentration that inhibits bacterial growth in the laboratory. Increases in MICs demonstrate that the bacteria can survive at higher antimicrobial concentrations in the laboratory. Monitoring of MIC trends is useful because increasing MICs can oftentimes be an early indicator of the emergence of antimicrobial resistance.

Information on the antimicrobial susceptibility criteria used in GISP can be found in Section A2.3 in the Appendix. More information about GISP and additional data can be found at: https://www.cdc.gov/std/ GISP.

\section{Ceftriaxone Susceptibility}

Susceptibility testing for ceftriaxone began in 1987. During 2009-2018, the percentage of GISP isolates that exhibited elevated ceftriaxone MICs, defined as $\geq 0.125 \mu \mathrm{g} / \mathrm{mL}$, fluctuated between $0.1 \%$ and $0.4 \%$ (Figure 29 ). In $2018,0.2 \%$ of isolates had elevated ceftriaxone MICs. Five isolates with decreased ceftriaxone susceptibility $(\mathrm{MIC}=0.5 \mu \mathrm{g} / \mathrm{mL})$ have been

\section{Figure 25. Estimated Proportion* of MSM, MSW, and Women Among Gonorrhea Cases by Jurisdiction, STD Surveillance Network (SSuN), 2018}

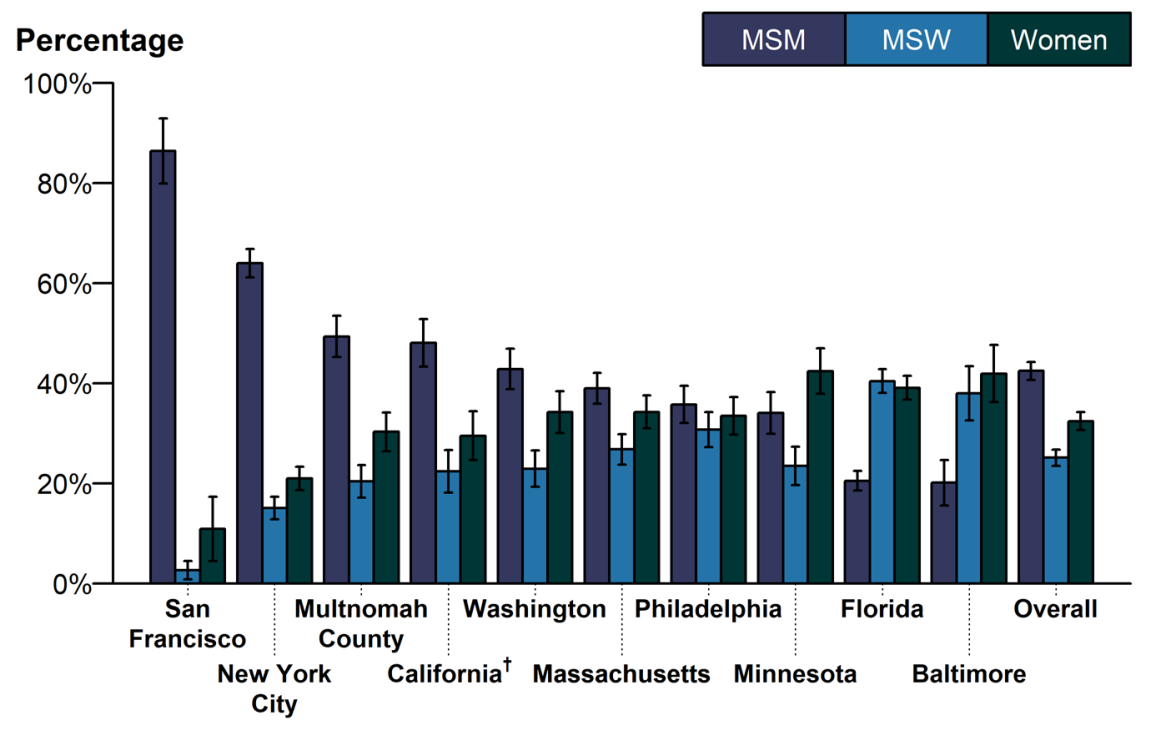

\footnotetext{
* Estimate based on weighted analysis of data obtained from interviews $(n=6,842)$ conducted among a random sample of reported gonorrhea cases during January to December 2018.

${ }^{\dagger}$ California data exclude San Francisco (shown separately). NOTE: See section A2.2 in the Appendix for SSUN methods. ACRONYMS: MSM = Gay, bisexual, and other men who have sex with men; MSW = Men who have sex with women only.
}

previously identified in GISP: one from San Diego, California (1987), two from Cincinnati, Ohio (1992 and 1993), one from Philadelphia, Pennsylvania (1997), and one from Oklahoma City, Oklahoma (2012).

\section{Cefixime Susceptibility}

Susceptibility testing for cefixime began in 1992, was discontinued in 2007, and was restarted in 2009. The percentage of isolates with elevated cefixime MICs $(\geq 0.25 \mu \mathrm{g} / \mathrm{mL})$ declined from $1.4 \%$ in 2011 to $0.3 \%$ in 2018 (Figure 29).

\section{Azithromycin Susceptibility}

Susceptibility testing for azithromycin began in 1992. Figure 29 displays the distribution of azithromycin MICs among GISP isolates collected during
2009-2018. Most isolates had MICs of $0.125-0.5 \mu \mathrm{g} / \mathrm{mL}$. During $2012-$ 2014 , the percentage of isolates with elevated azithromycin MICs ( $\geq 2 \mu \mathrm{g}$ / $\mathrm{mL}$ ) ranged from $0.3 \%$ to $2.5 \%$ with a sharp increase during 2013-2014 (from $0.6 \%$ to $2.5 \%$ ); during 2014 2018 , the percentage increased from $2.5 \%$ to $4.6 \%$.

\section{Susceptibility to Other Antimicrobials}

Susceptibility testing for gentamicin began in 2015. Between 2015 and $2017,66.7-75.3 \%$ of all tested isolates had an MIC value of $8 \mu \mathrm{g} /$ $\mathrm{mL}$ (Figure 30). Starting in 2018, the range of MIC values tested in GISP was expanded to include MICs as low as $0.25 \mu \mathrm{g} / \mathrm{mL}$ and as high as 64 $\mu \mathrm{g} / \mathrm{mL}$. In $2018,0.02 \%$ of all tested isolates had an MIC above $16 \mu \mathrm{g} / \mathrm{mL}$. 


\section{Figure 26. Gonorrhea - Estimated* Rates of Reported Gonorrhea Cases by MSM, MSW, and Women, STD Surveillance Network (SSUN) $^{\dagger}$, 2010-2018}

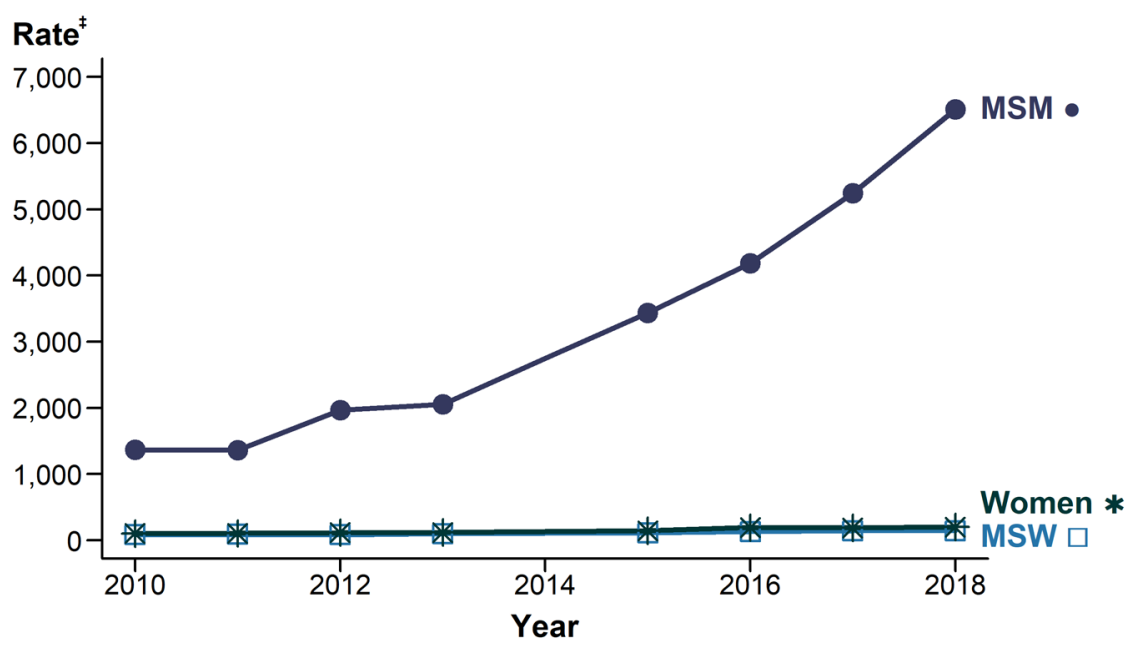

* Estimates based on interviews among a random sample of reported cases of gonorrhea $(n=21,417)$; cases weighted for analysis. Data not available for 2014; 2013-2015 trend interpolated; trends lines overlap for MSW and women in this figure.

${ }^{\dagger}$ Sites include Baltimore, Philadelphia, New York City, Washington State, San Francisco, and California (excluding San Francisco).

${ }^{\ddagger}$ Per 100,000 .

ADAPTED FROM: Stenger M, Pathela $\mathrm{P}$, Anschuetz $\mathrm{G}$, et al. Increases in the rate of Neisseria gonorrhoeae among gay, bisexual and other men who have sex with men (MSM) - findings from the STD Surveillance Network 2010-2015. Sex Transm Dis. 2017; 44(7): 393-397.

ACRONYMS: $M S M=$ Gay, bisexual, and other men who have sex with men; MSW = Men who have sex with women only.

In $2018,31.2 \%$ of isolates collected from GISP sites were resistant to ciprofloxacin, $25.6 \%$ to tetracycline, and $13.7 \%$ to penicillin (Figure 31). Although these antimicrobials are no longer recommended for treatment of gonorrhea, the resistance phenotypes remain common. The different susceptibility patterns seen in GISP in 2018 are shown in Figure 32. Panel A demonstrates the percentage of isolates with resistance or elevated MICs to various numbers of antimicrobials. Panel B further highlights the specific combinations of resistance or elevated MICs across antimicrobials (vertical bars).
The overall percentage of reduced susceptibility for each antimicrobial (horizontal bars) are the same percentages seen for 2018 in Figure 31. Of all the isolates collected in GISP in 2018, 4.5\% demonstrated resistance or elevated MICs to at least three antibiotics tested with the majority of the combinations including tetracycline, penicillin and ciprofloxacin; no isolates with elevated azithromycin MICs had elevated ceftriaxone MICs (Figure 32 ). In $2018,48.7 \%$ of all tested isolates were susceptible to all antibiotics tested.

\section{Antimicrobial Treatments Given for Gonorrhea}

The antimicrobial agents given to GISP patients for gonorrhea therapy are shown in Figure 33. The proportion of patients treated with ceftriaxone $250 \mathrm{mg}$ increased from $84.0 \%$ in 2011 to $98.1 \%$ in 2017 but decreased slightly to $96.5 \%$ in 2018 . Patients treated with gentamicin 240 $\mathrm{mg}$ increased from $0.2 \%$ in 2015 to $1.4 \%$ in 2018 and patients treated with cefixime $400 \mathrm{mg}$ decreased from $0.3 \%$ in 2015 to $0.1 \%$ in 2018 .

In 2018, based on weighted analysis of SSuN jurisdictions with documented treatment information (i.e., antimicrobials and dosages) for $\geq 80 \%$ of cases, $85.8 \%$ (95\% CI: 84.1-87.5) of reported patients with gonorrhea in SSuN jurisdictions received the recommended treatment for uncomplicated gonorrhea (Figure 34). The proportion of reported patients that received the recommended dual treatment ranged from $83.8 \%$ (95\% CI: 81.9-85.6) in Florida to 95.5\% (95\% CI: 92.9-98.1) in San Francisco, California.

\section{Gonorrhea Among Special Populations}

More information about gonorrhea in race/Hispanic ethnicity groups, females of reproductive age, adolescents, young adults, and MSM can be found in the Special Focus Profiles.

\section{Gonorrhea Summary}

The national rate of reported gonorrhea cases reached a historic low in 2009, but increased each year during 2009-2012. After a temporary decrease in 2013, the gonorrhea rate increased again during 2014-2018. This increase was largely attributable to increases among men. Enhanced 
Figure 27. Gonorrhea - Proportion of STD Clinic Patients* Testing Positive by Age Group and Sex and Sex of Sex Partners, STD Surveillance Network (SSuN), 2018

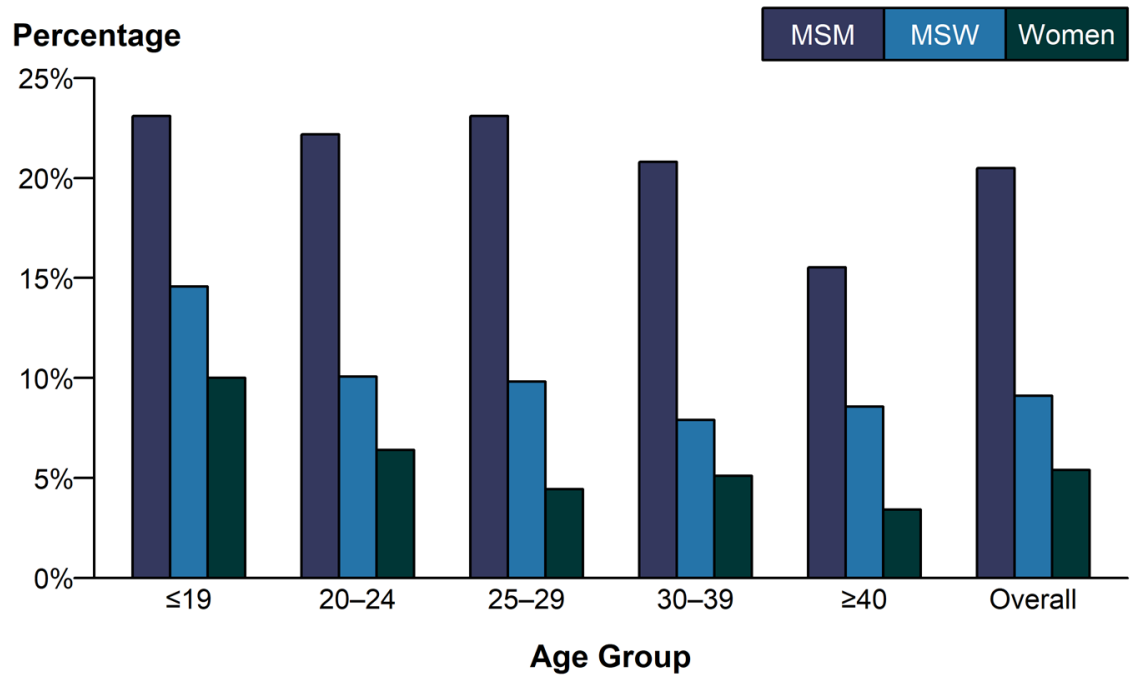

* Results are based on data obtained from unique patients with known sex of sex partners $(n=77,314)$ attending SSUN STD clinics who were tested $\geq 1$ time for gonorrhea in 2018. NOTE: See section A2.2 in the Appendix for SSuN methods.

ACRONYMS: $M S M=$ Gay, bisexual, and other men who have sex with men; $M S W=$ Men who have sex with women only.

\section{Figure 28. Location of Participating Sentinel Sites and Regional Laboratories, Gonococcal Isolate Surveillance Project (GISP), United States, 2018}

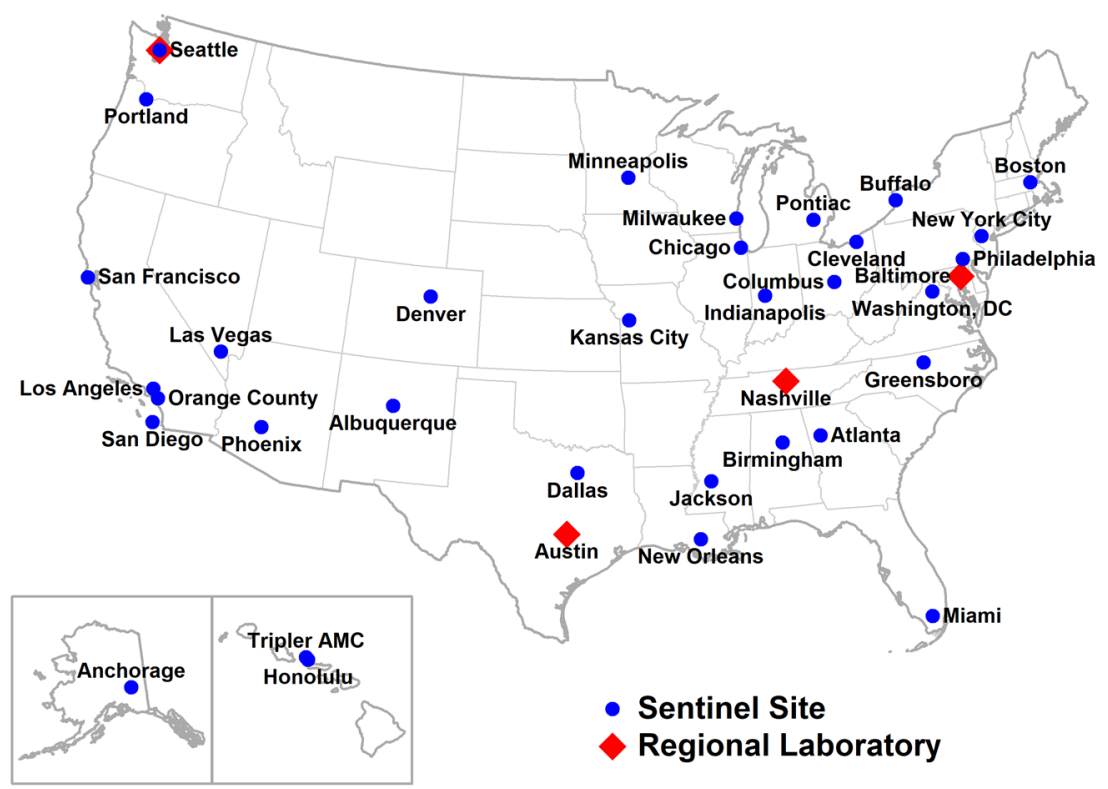

NOTE: Seattle is both a sentinel site and a regional laboratory. surveillance data suggest the largest increases are among MSM. However, high gonorrhea rates persist in certain geographic areas, among adolescents and young adults, and in some racial/ Hispanic ethnicity groups. Continued surveillance for antimicrobial resistant gonorrhea is critical to monitor for the emergence of reduced susceptibility and resistance to cephalosporins and azithromycin.

\section{References}

1. Fleming DT, Wasserheit JN. From epidemiological synergy to public health policy and practice: The contribution of other sexually transmitted diseases to sexual transmission of HIV infection. Sex Transm Infect. 1999;75(1):317.

2. Hogben M, Leichliter JS. Social determinants and sexually transmitted disease disparities. Sex Transm Dis. 2008;35(12 Suppl):S13-18.

3. Centers for Disease Control and Prevention. Sexually transmitted diseases treatment guidelines, 2015. MMWR Recomm Rep. 2015;64(No. RR-3):1-137.

4. Satterwhite CL, Torrone E, Meites E, et al. Sexually transmitted infections among US women and men: Prevalence and incidence estimates, 2008. Sex Transm Dis. 2013;40(3):187-193. DOI: 10.1097/ OLQ.0b013e318286bb53. Review.

5. Grey JA, Bernstein KT, Sullivan PS, et al. Estimating the population sizes of men who have sex with men in US states and counties using data from the American Community Survey. JMIR Public Health Surveill. 2016;2(1):e14.

6. Stenger M, Pathela P, Anschuetz G, et al Increases in the rate of Neisseria gonorrhoeae among gay, bisexual and other men who have sex with men (MSM) - findings from the STD Surveillance Network 2010-2015. Sex Transm Dis. 2017;44(7):393-397.

7. Centers for Disease Control and Prevention. Update to CDC's sexually transmitted diseases treatment guidelines, 2006: Fluoroquinolones no longer recommended for treatment of gonococcal infections. MMWR Morb Mortal Wkly Rep. 2007;56(14):332-336.

8. Centers for Disease Control and Prevention. Sexually transmitted diseases treatment guidelines, 2010. MMWR Recomm Rep. 2010;59(No. RR-12):1-110.

9. Schwarcz S, Zenilman J, Schnell D, et al. National surveillance of antimicrobial resistance in Neisseria gonorrhoeae. JAMA. 1990;264(111):1413-1417. 
Figure 29. Neisseria gonorrhoeae - Percentage of Isolates with Elevated Minimum Inhibitory Concentrations (MICs) to Azithromycin, Cefixime, and Ceftriaxone, Gonococcal Isolate Surveillance Project (GISP), 2009-2018

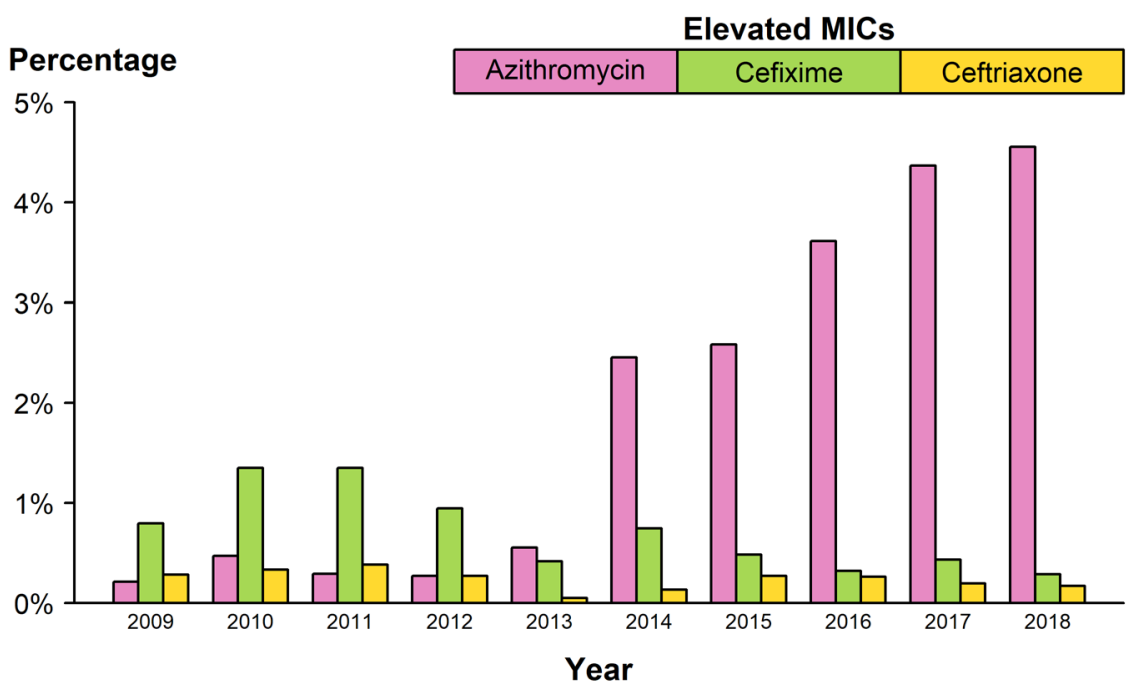

NOTE: Elevated MIC = Azithromycin: $\geq 2.0 \mu \mathrm{g} / \mathrm{mL}$; Cefixime: $\geq 0.25 \mu \mathrm{g} / \mathrm{mL}$; Ceftriaxone: $\geq 0.125 \mu \mathrm{g} / \mathrm{mL}$.

Figure 30. Neisseria gonorrhoeae - Distribution of Gentamicin Minimum Inhibitory Concentrations (MICs) by Year, Gonococcal Isolate Surveillance Project (GISP), 2015-2018*

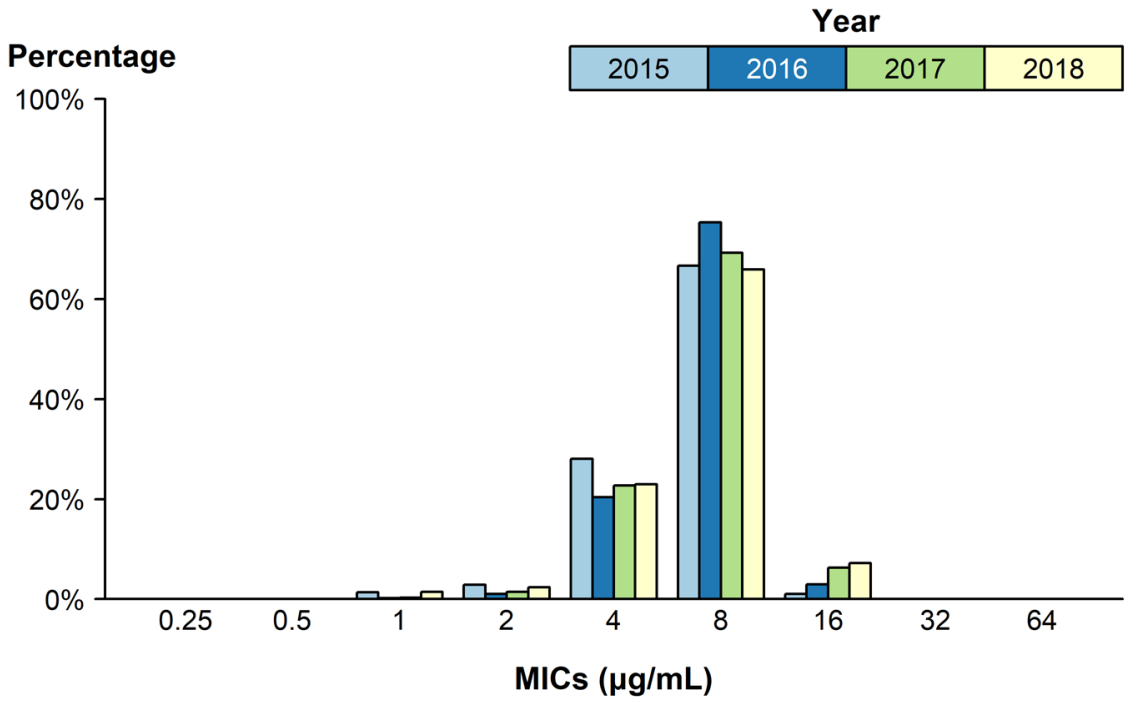

* In 2018, the antibiotic susceptibility testing range for gentamicin was expanded from MICs of $1 \mu \mathrm{g} / \mathrm{mL}-32 \mu \mathrm{g} / \mathrm{mL}$ in previous years to $0.25 \mu \mathrm{g} / \mathrm{mL}-64 \mu \mathrm{g} / \mathrm{mL}$ 
Figure 31. Neisseria gonorrhoeae - Prevalence of Tetracycline, Penicillin, or Fluoroquinolone Resistance* or Elevated Cefixime, Ceftriaxone, or Azithromycin Minimum Inhibitory Concentrations (MICs) $^{\dagger}$, by Year - Gonococcal Isolate Surveillance Project (GISP), 2000-2018

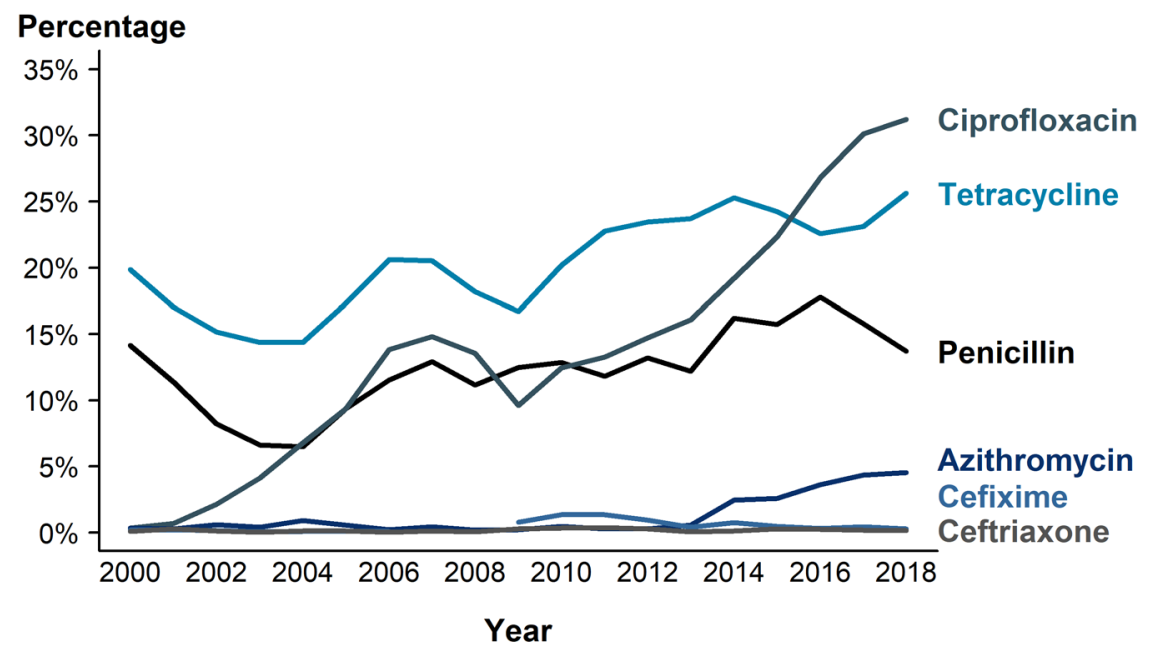

${ }^{*}$ Resistance $=$ Fluoroquinolone (ciprofloxacin): $\mathrm{MIC} \geq 1.0 \mu \mathrm{g} / \mathrm{mL}$; Penicillin: MIC $\geq 2.0$ $\mu \mathrm{g} / \mathrm{mL}$ or Beta-lactamase positive; Tetracycline: MIC $\geq 2.0 \mu \mathrm{g} / \mathrm{mL}$.

${ }^{\dagger}$ Elevated MICs = Azithromycin: MIC $\geq 1.0 \mu \mathrm{g} / \mathrm{mL}$ (2000-2004), MIC $\geq 2.0 \mu \mathrm{g} / \mathrm{mL}$ (2005-2018); Ceftriaxone: MIC $\geq 0.125 \mu \mathrm{g} / \mathrm{mL}$; Cefixime: $\mathrm{MIC} \geq 0.25 \mu \mathrm{g} / \mathrm{mL}$.

NOTE: Cefixime susceptibility was not tested in 2007 and 2008. 
Figure 32. Resistance or Elevated MIC Patterns of Neisseria gonorrhoeae Isolates to Antimicrobials, Gonococcal Isolate Surveillance Project (GISP), 2018

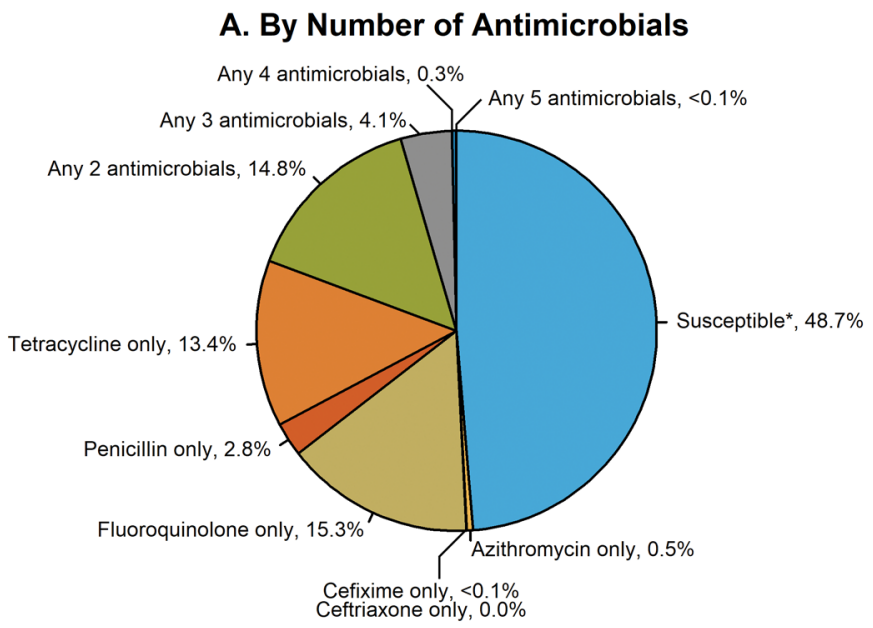

B. By Specific Antimicrobial Combinations

Percentage

by Antimicrobia

Combination

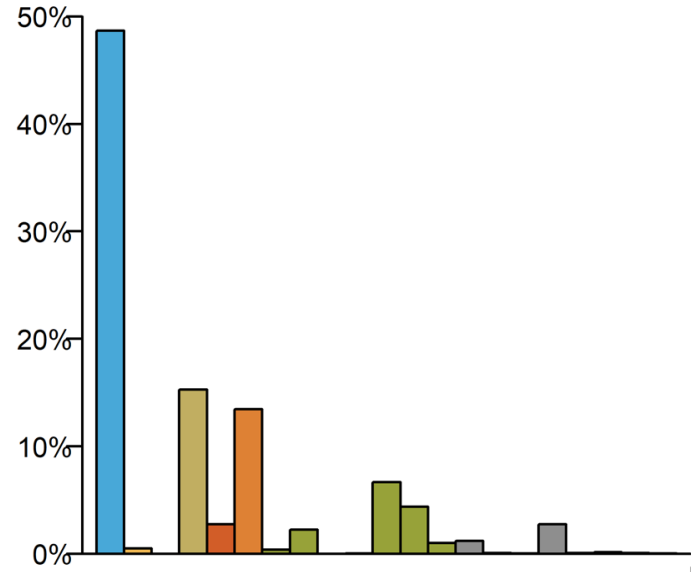

Azithromycin 000000000000000000000

Cefixime 000000000000000000000

Ceftriaxone 000000000000000000000

Fluoroquinolone 000000000000000000000

Penicillin 00000000000000000000

Tetracycline 000000000000000000000

Cumulative Percentage by Antimicrobial

* Susceptible category only includes isolates with penicillin, tetracycline, and fluoroquinolone MIC values that are considered susceptible and isolates with ceftriaxone, cefixime, and azithromycin MIC values that are not considered elevated.

NOTE: Elevated MIC = Ceftriaxone: $\mathrm{MIC} \geq 0.125 \mu \mathrm{g} / \mathrm{mL}$; Cefixime: $\mathrm{MIC} \geq 0.25 \mu \mathrm{g} / \mathrm{mL}$; Azithromycin: $\mathrm{MIC} \geq 2.0 \mu \mathrm{g} / \mathrm{mL}$. Resistance $=$ Tetracycline: $\mathrm{MIC} \geq 2.0 \mu \mathrm{g} / \mathrm{mL}$;

Fluoroquinolone: $\mathrm{MIC} \geq 1.0 \mu \mathrm{g} / \mathrm{mL}$; Penicillin: $\mathrm{MIC} \geq 2.0 \mu \mathrm{g} / \mathrm{mL}$ or Beta-lactamase positive. In Panel $\mathrm{B}$, a filled circle reflects resistance or elevated MIC to a specific antimicrobial; only antimicrobial combinations with non-zero percentages are shown. ACRONYMS: MIC $=$ Minimum Inhibitory Concentration . 
Figure 33. Distribution of Primary Antimicrobial Drug Used to Treat Gonorrhea Among Participants, Gonococcal Isolate Surveillance Project (GISP), 1988-2018

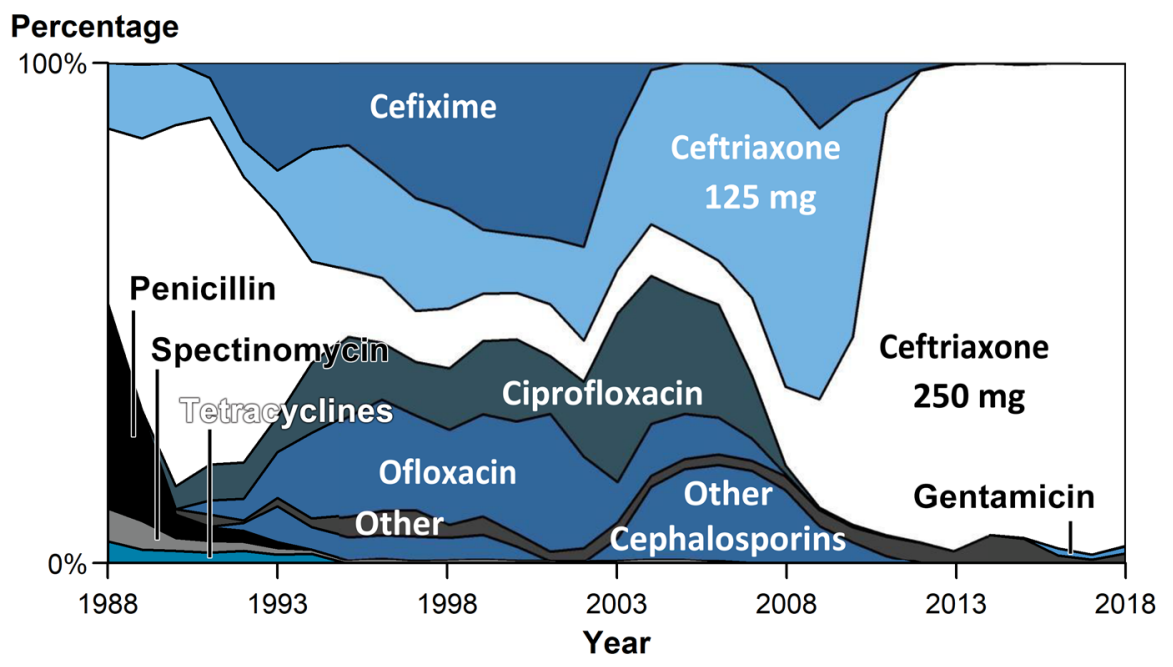

NOTE: For 2018, "Other" includes azithromycin $2 \mathrm{~g}(0.3 \%)$, no therapy documented $(0.5 \%)$, and other less frequently used drugs $(1.2 \%)$

Figure 34. Gonorrhea - Estimated Proportion of Cases Treated by Recommended Treatment Regimen* and Jurisdiction ${ }^{\dagger}$, STD Surveillance Network (SSuN), 2018

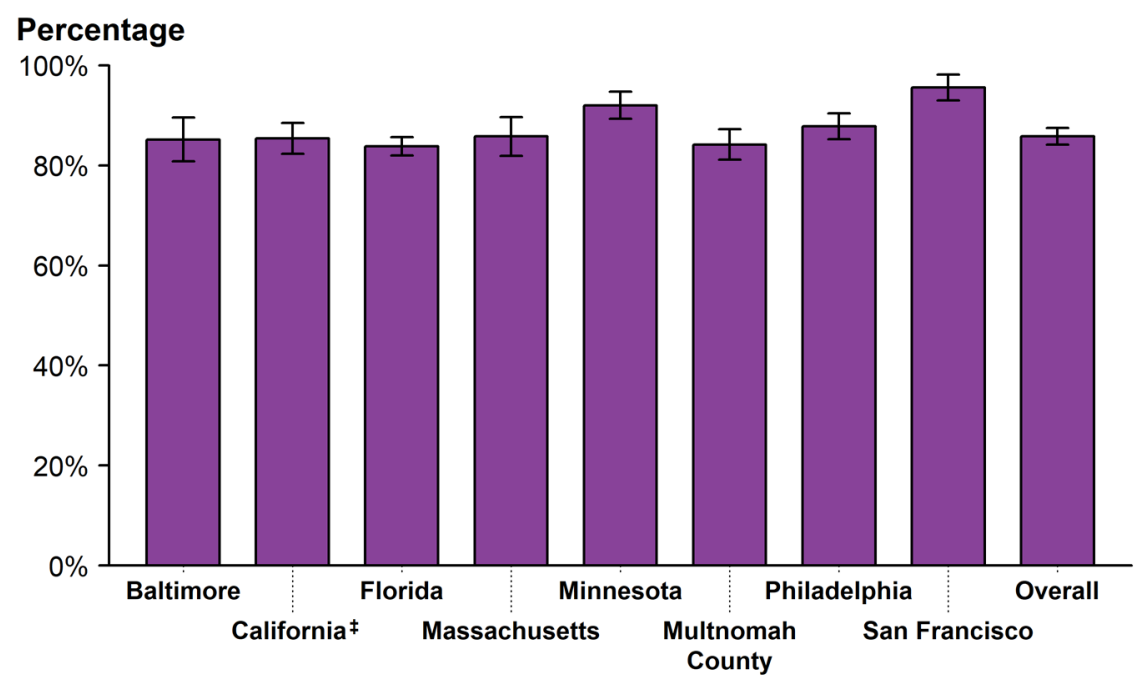

* In 2018, the recommended treatment for uncomplicated gonorrhea was treatment with $250 \mathrm{mg}$ dose of ceftriaxone plus $1 \mathrm{~g}$ dose of azithromycin.

${ }^{\dagger}$ Includes SSuN jurisdictions with all treatment information documented for $\geq 80 \%$ of cases.

${ }^{\ddagger}$ California data exclude San Francisco.

NOTE: See section A2.2 in the Appendix for SSUN methods.

ADAPTED FROM: Weston EJ, Workowski K, Torrone E, et al. Adherence to CDC recommendations for the treatment of uncomplicated gonorrhea - STD Surveillance Network (SSuN), United States, 2016. MMWR Morb Mortal Wkly Rep. 2018; 67:473-76. 


\section{Background}

Syphilis, a genital ulcerative disease caused by the bacterium Treponema pallidum, is associated with significant complications if left untreated and can facilitate the transmission and acquisition of HIV infection. ${ }^{1-3}$ Additionally, historical data demonstrate that untreated syphilis in pregnant women, if acquired during the four years before delivery, can lead to infection of the fetus in up to $80 \%$ of cases and may result in stillbirth or infant death in up to $40 \%$ of cases. $^{4}$

In 2000 and 2001, the national rate of reported primary and secondary (P\&S) syphilis cases was 2.1 cases per 100,000 population, the lowest rate since reporting began in 1941 (Figure 35, Table 1). However, the P\&S syphilis rate has increased almost every year since 2001 . This rise in the rate of reported P\&S syphilis has been primarily attributable to increased cases among men and, specifically, among gay, bisexual, and other men who have sex with men (MSM). MSM account for the majority of P\&S syphilis cases and estimated rates are substantially higher among MSM compared with women or men who have sex with women only (MSW). ${ }^{5}$ The number of cases among MSM has continued to increase, but within the last five years, cases among MSW and women have increased substantially as well. The increase in syphilis among women is of particular concern because it is associated with a striking and concurrent increase in congenital syphilis. These recent trends highlight the importance of national syphilis surveillance to understand the current epidemiology of syphilis in the United States and to focus prevention efforts.

\section{Interpreting Rates of Reported Cases of Syphilis}

Left untreated, infection with syphilis can span decades, progressing

\section{Figure 35. Syphilis - Rates of Reported Cases by Stage of Infection, United States, 1941-2018}

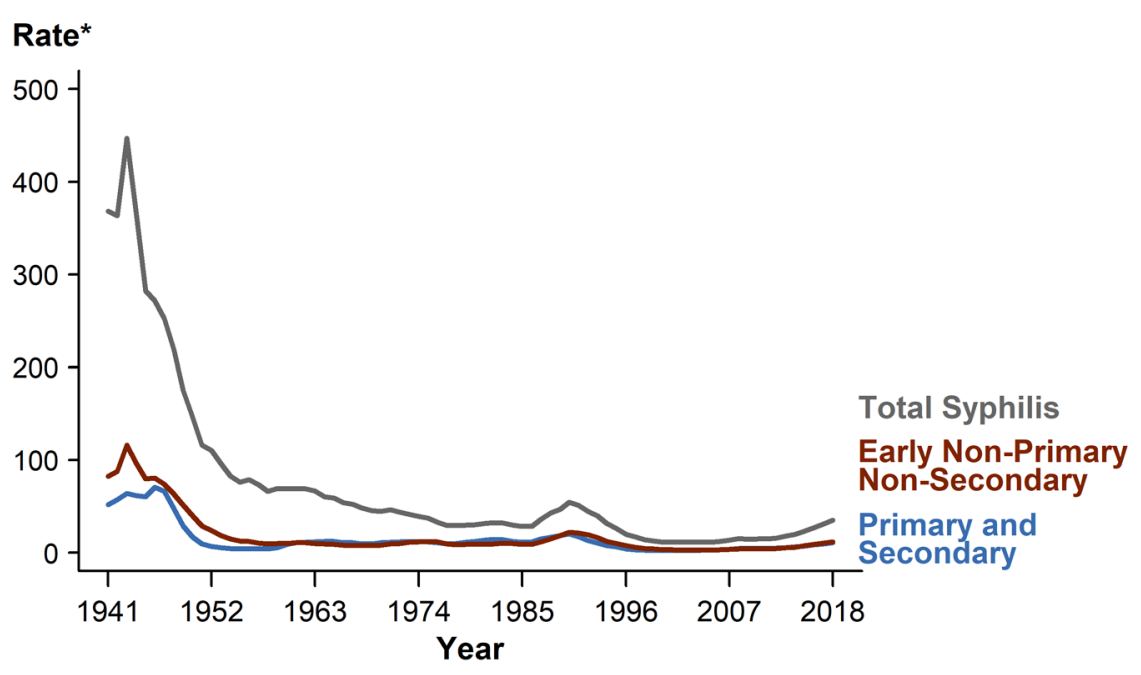

* Per 100,000

NOTE: See section A1.3 in the Appendix for more information on syphilis case reporting.

through multiple stages of infection. Case counts of all stages of syphilis represent the total burden of disease in the United States. The primary and secondary syphilis stages are the earliest stages of infection, reflect symptomatic disease, and are indicators of incident infection. ${ }^{6}$ For these reasons, trend analyses of syphilis primarily focus on reported cases and rates of reported cases of $\mathrm{P} \& \mathrm{~S}$ syphilis. When referring to "P\&S syphilis," case counts are the sum of both primary and secondary cases, and "rate of P\&S syphilis" refers to this sum per unit population.

Changes in reporting and screening practices can complicate interpretation of trends over time. To minimize the effect of changes in reporting over time, trend data in this report are restricted to jurisdictions that consistently report data of interest (e.g., sex of sex partners) for each year of a given time period. Details of these restrictions are provided in the pertinent text and figures.
Additionally, the case definitions for syphilis stages have changed over time, including a revision that took effect in January 2018. See Appendix A1.9 for more information on syphilis morbidity reporting and Appendix C1.4 for current syphilis case definition.

\section{Syphilis - All Stages (P\&S, Early Non-Primary Non-Secondary, Unknown Duration or Late, and Congenital)}

In 2018, the total case count of reported syphilis (all stages combined: P\&S, early non-primary non-secondary, unknown duration or late, and congenital) was the highest recorded since 1991. The total number of reported cases of syphilis (all stages) increased 13.3\% during 2017-2018 (from 101,584 cases to 115,045 cases) (Table 1). The number of reported cases of $\mathrm{P} \& \mathrm{~S}$ syphilis increased 14.4\% (from 30,644 to 
$35,063)$, the number of reported cases of early non-primary non-secondary syphilis increased 13.3\% (from 34,013 cases to 38,539 cases), the number of reported cases of unknown duration or late syphilis increased $11.5 \%$ (from 35,992 cases to 40,137 cases), and the number of reported cases of congenital syphilis increased $39.7 \%$ (from 935 to 1,306 ) (Table 1).

\section{P\&S Syphilis - United States}

In 2018 , a total of 35,063 cases of $P \& S$ syphilis were reported in the United States, yielding a rate of 10.8 cases per 100,000 population (Figure 35, Table 1). This rate represents a $14.9 \%$ increase compared with 2017 (9.4 cases per 100,000 population), and a $71.4 \%$ increase compared with 2014 (6.3 cases per 100,000 population).

\section{P\&S Syphilis by Region}

In 2018, the West had the highest rate of reported $\mathrm{P} \& \mathrm{~S}$ syphilis cases (15.0 cases per 100,000 population), followed by the South (11.1 cases per 100,000 population), the Northeast (8.7 cases per 100,000 population), and the Midwest ( 7.1 cases per 100,000 population) (Table 27). During 2017-2018, the P\&S syphilis rate increased $16.4 \%$ in the Midwest, $15.6 \%$ in the South, $15.4 \%$ in the West, and $10.1 \%$ in the Northeast (Figure 36, Table 27).

\section{P\&S Syphilis by State}

In 2018, rates of reported $\mathrm{P} \& \mathrm{~S}$ syphilis cases per 100,000 population ranged by state from 1.8 in Vermont to 22.7 in Nevada (Figure 37, Table 26). The rate of reported $P \& S$ syphilis cases in the District of Columbia was 40.2 cases per 100,000 population. During 2017-2018, P\&S syphilis rates increased in $74 \%$ (37/50) of states and the District of Columbia, and remained stable or decreased in $26 \%(13 / 50)$ of states (Table 27$)$.
P\&S Syphilis by Metropolitan Statistical Area

The overall rate of reported $\mathrm{P} \& \mathrm{~S}$

syphilis cases in the 50 most populous metropolitan statistical areas (MSAs) was 13.5 cases per 100,000 population in 2018 , which

\section{Figure 36. Primary and Secondary Syphilis - Rates of Reported Cases by Region, United States, 2009-2018}

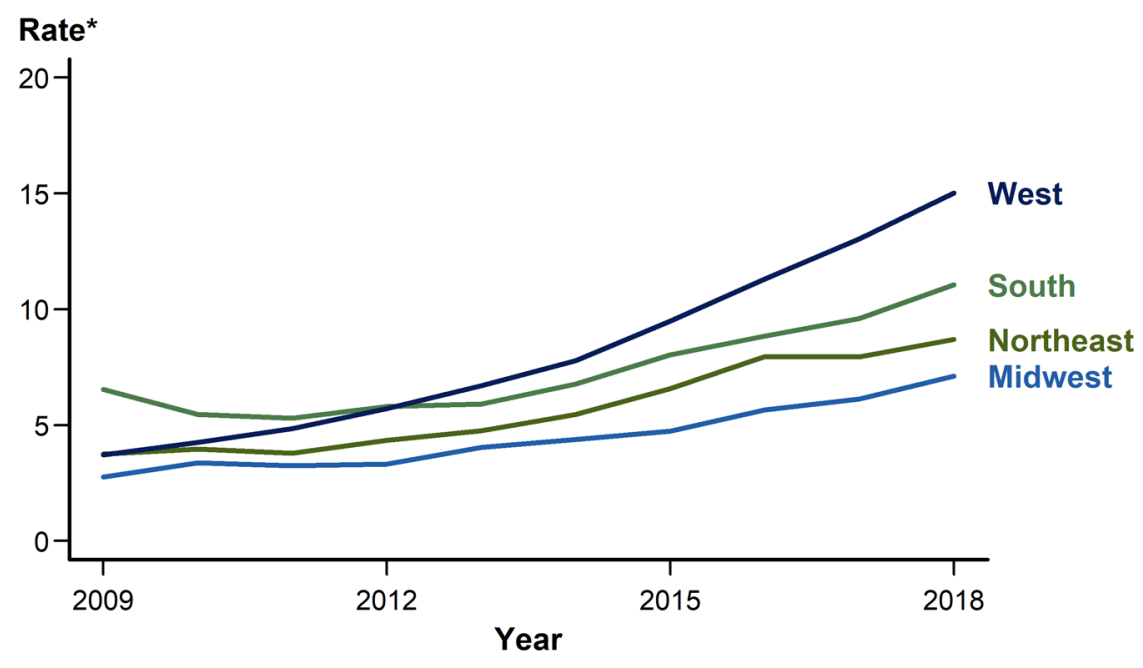

* Per 100,000

\section{Figure 37. Primary and Secondary Syphilis - Rates of Reported Cases by State and Territory, United States, 2018}

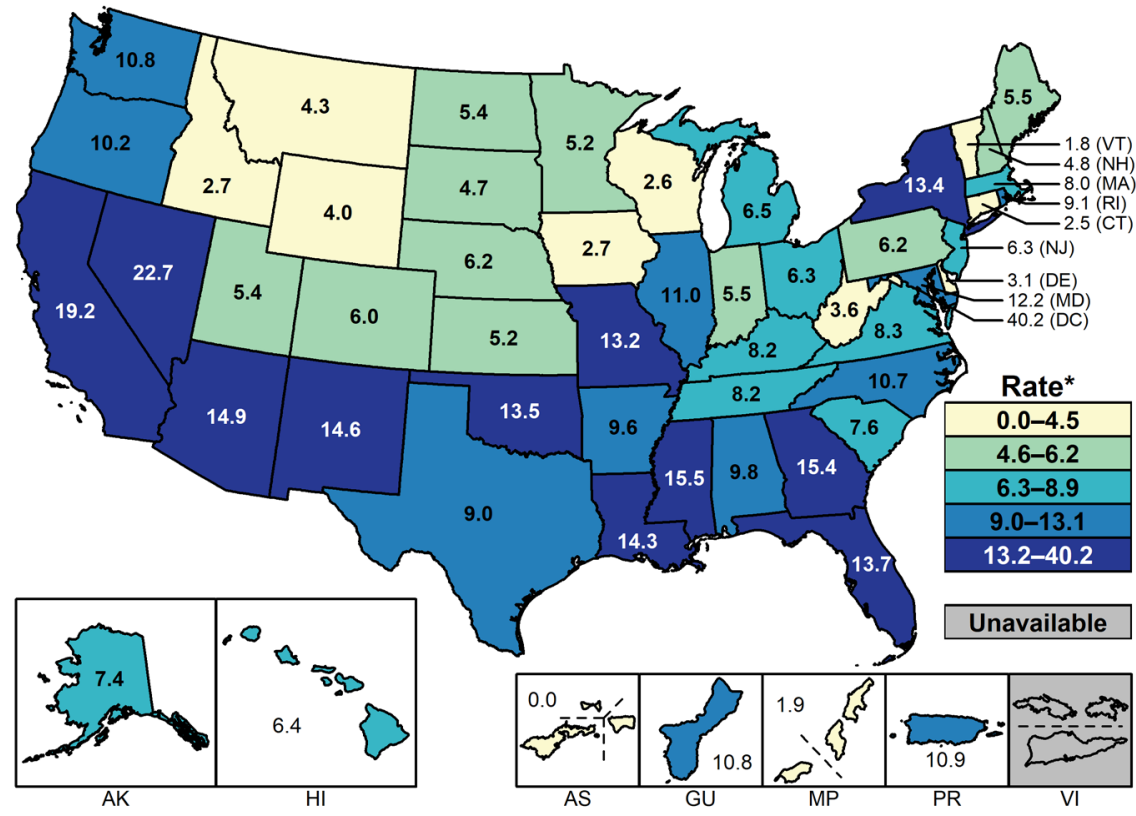

* Per 100,000 .

NOTE: Section A1.11 in the Appendix for more information on interpreting reported rates in US territories. 
represents a $12.5 \%$ increase since 2017 (12.0 cases per 100,000 population) (Table 30). Overall, in $2018,69.2 \%$ of reported $\mathrm{P} \& \mathrm{~S}$ syphilis cases $(71.9 \%$ of male cases and $53.0 \%$ of female cases) were reported by these 50 MSAs. The rate among women in these MSAs was 2.9 cases per 100,000 females, while the rate among men was 24.5 cases per 100,000 males (Tables 31 and 32).

\section{P\&S Syphilis by County}

In $2018,61.5 \%$ of reported P\&S syphilis cases occurred in 70 counties or independent cities (Table 33). Of 3,142 counties in the United States, $556(17.7 \%)$ had a P\&S syphilis rate of 8.8 cases per 100,000 population or greater, $557(17.7 \%)$ reported a rate from 4.2 to 8.7 cases per 100,000 population, $531(17.0 \%)$ reported a rate from $>0.0$ to 4.1 cases per 100,000 population, and 1,498 $(47.7 \%)$ counties reported no cases of P\&S syphilis in 2018 (Figure 38).

\section{P\&S Syphilis by Sex and Sex of Sex Partners}

As has been observed in previous years, in 2018, the rate of reported P\&S syphilis cases among men (18.7 cases per 100,000 males) was much higher than the rate among women (3.0 cases per 100,000 females), and men accounted for a large majority (85.7\%) of P\&S syphilis cases (Figure 39, Tables 28 and 29). Among men, the rate of P\&S syphilis has increased every year since 2000 , and during 2017-2018, the rate among men increased $11.3 \%$ (Figure 40, Table 29). In contrast, the $P \& S$ syphilis rate among women fluctuated between 0.8 and 1.7 cases per 100,000 females during 2000-2013, but has increased substantially since 2013 (Figure 40, Table 28). During 2014-2018, the $\mathrm{P} \& \mathrm{~S}$ syphilis rate among women more than doubled (172.7\% increase). During 2017-2018, the P\&S syphilis rate among women increased $30.4 \%$.

\section{Figure 38. Primary and Secondary Syphilis - Rates of Reported Cases by County, United States, 2018}

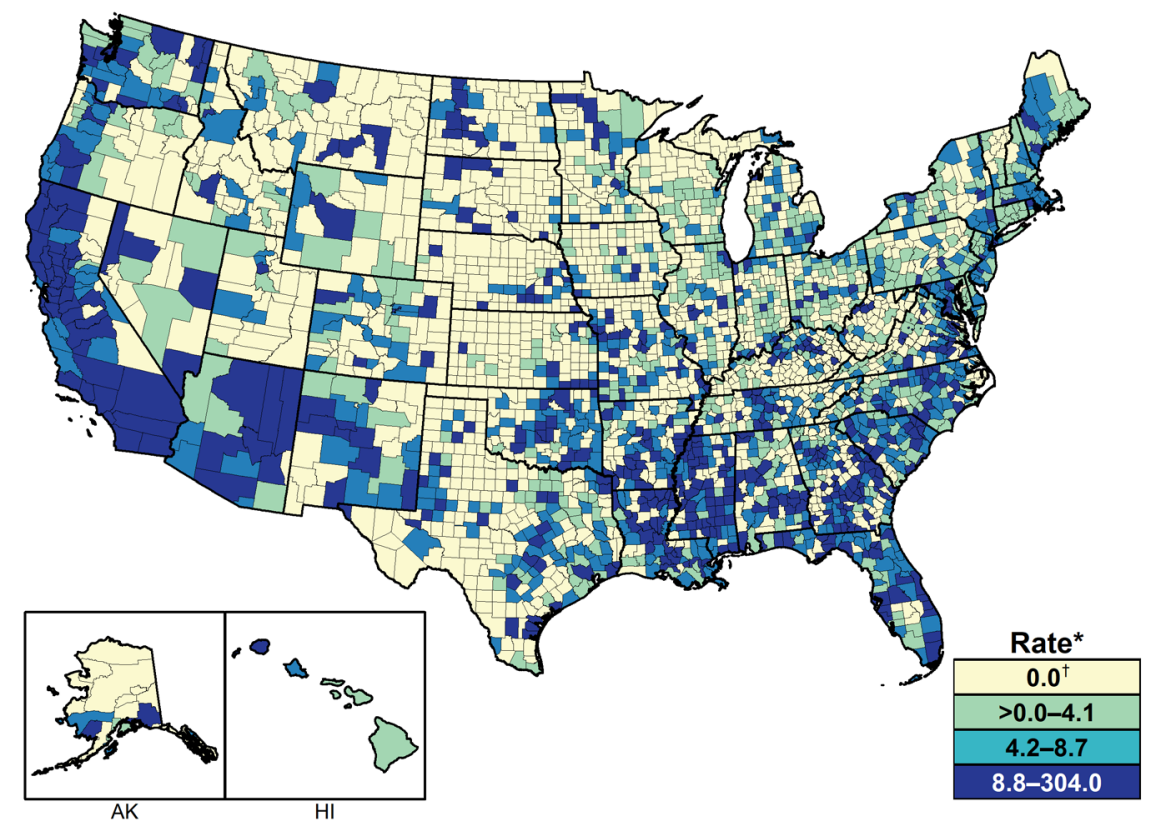

* Per 100,000 .

${ }^{\dagger}$ In 2018, 1,498 (47.7\%) of 3,142 counties in the United States reported no cases of primary and secondary syphilis. See section A1.4 in the Appendix for more information on county-level rates. Figure 39. Primary and Secondary Syphilis - Distribution of Cases
by Sex and Sex of Sex Partners, United States, 2018

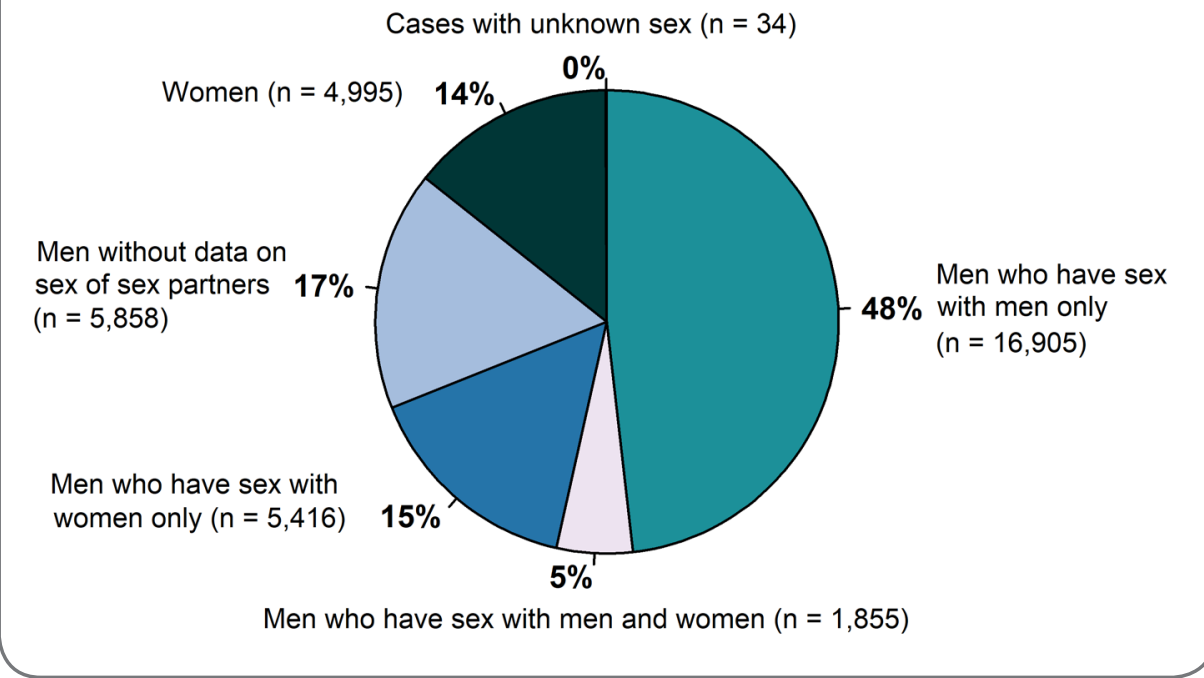

These increases in male and female P\&S syphilis rates were observed in every region of the country during
2017-2018. Among men, the rate increased $13.5 \%$ in the Midwest, $12.4 \%$ in the South, $11.5 \%$ in the 


\section{Figure 40. Primary and Secondary Syphilis - Rates of Reported Cases by Sex and Male-to-Female Rate Ratios, United States, 1990-2018}

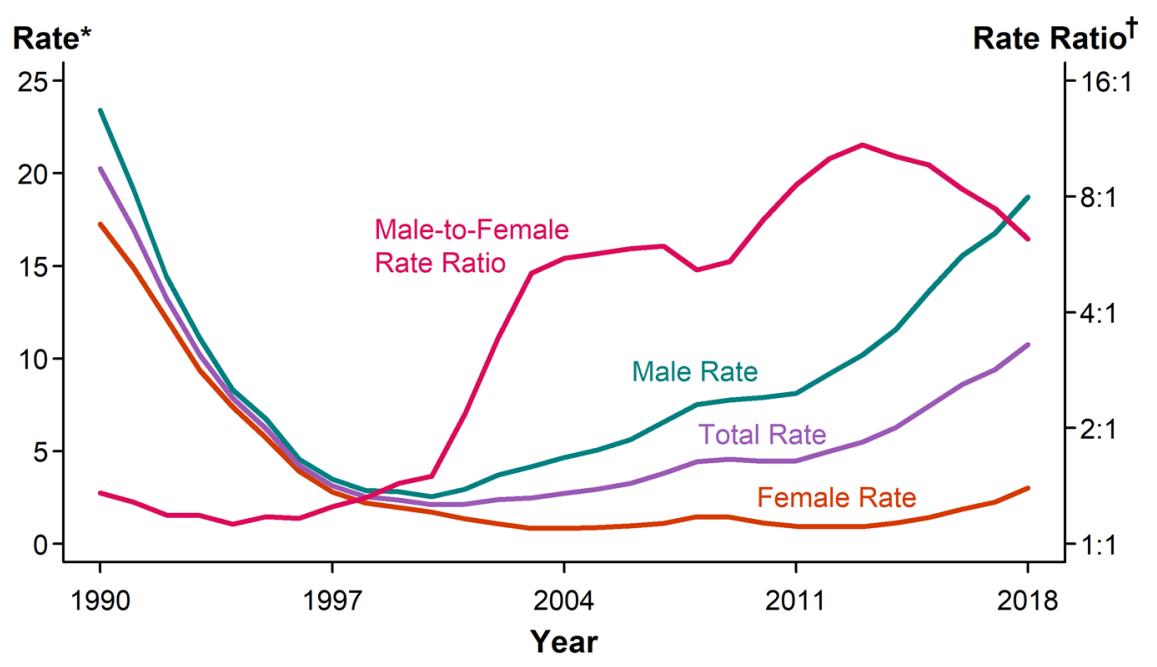

* Per 100,000 .

${ }^{\dagger}$ Log scale.
$16.3 \%$ among MSW, and $32.9 \%$ among women.

\section{P\&S Syphilis by Age}

As in previous years, in 2018, rates of reported P\&S syphilis cases were highest among persons aged 25-29 years (Figure 42, Table 34). In 2018, the highest rates were observed among men aged 25-29 years (55.7 cases per 100,000 males), $30-34$ years ( 45.8 cases per 100,000 males), and 20-24 years (44.6 cases per 100,000 males). The highest rates among women were among those aged 20-24 years (10.0 cases per 100,000 females) and those aged 25-29 years ( 9.4 cases per 100,000 females).

During 2017-2018, the overall rate of reported P\&S syphilis cases increased in all age groups among those aged 15 years or older (Figures 43 and 44, Table 34). Rates increased 14.9\% among those aged 15-19 years, $10.3 \%$ among those aged $20-24$ years, $12.6 \%$ among those aged 25 29 years, $21.2 \%$ among those aged
West, and $7.2 \%$ in the Northeast (Table 29). Among women, the largest increases were observed in the West (41.2\%), followed by the Northeast $(40.0 \%)$, the South $(30.8 \%)$ and the Midwest (30.8\%) (Table 28).

MSM continued to account for the majority of $\mathrm{P} \& \mathrm{~S}$ syphilis cases in 2018 (Figures 39 and 41). Of 35,063 reported P\&S syphilis cases in 2018, $18,760(53.5 \%)$ were among MSM, including 16,905 (48.2\%) cases among men who had sex with men only and $1,855(5.3 \%)$ cases among men who had sex with both men and women (Figure 39). Overall, $5,416(15.4 \%)$ cases were among MSW, 4,995 (14.2\%) were among women, 5,858 (16.7\%) were among men without information about sex of sex partners, and $34(0.1 \%)$ were cases reported with unknown sex. Among the 24,176 male cases with information on sex of sex partners, $77.6 \%$ occurred among MSM.

A total of 36 states were able to classify at least $70.0 \%$ of reported P\&S syphilis cases as MSM, MSW, or women each year during 2014 2018 (Figure 41). In these states, during 2017-2018, the number of cases increased 5.3\% among MSM,

\section{Figure 41. Primary and Secondary Syphilis - Reported Cases by Sex and Sex of Sex Partners, 36 States*, 2014-2018}

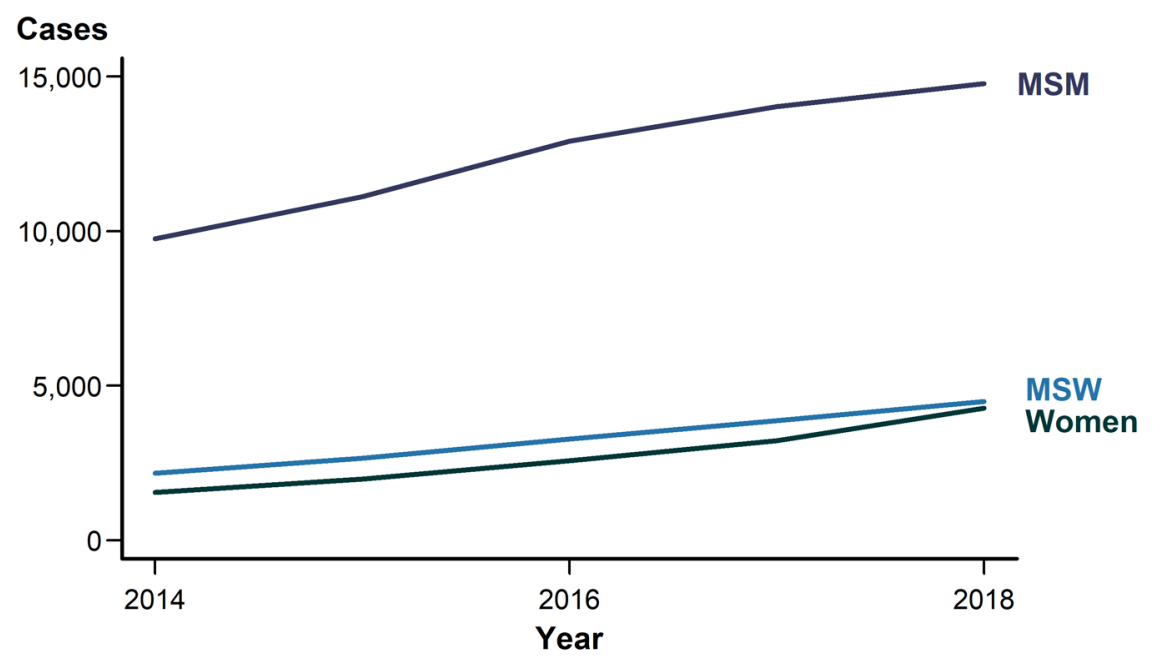

* 36 states were able to classify $\geq 70 \%$ of reported cases of primary and secondary syphilis as either MSM, MSW, or women for each year during 2014-2018. ACRONYMS: $M S M=$ Gay, bisexual, and other men who have sex with men; MSW = Men who have sex with women only. 

Figure 42. Primary and Secondary Syphilis - Rates of Reported
Cases by Age Group and Sex, United States, 2018

Male Rate*

60

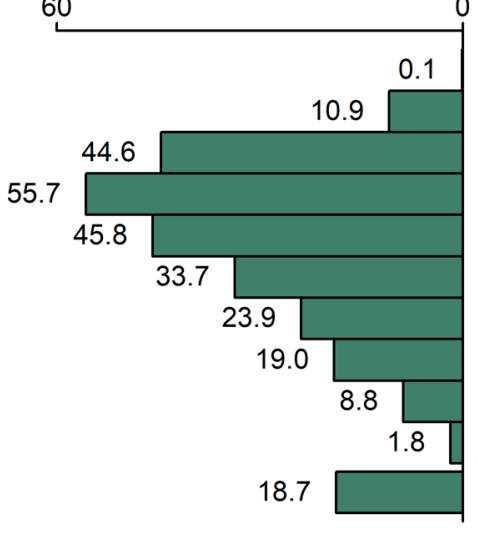

0 Age Group 0

10-14 0.1

$15-19 \square 4.3$

20-24

25-29

30-34

35-39

40-44

45-54

55-64

$65+$

Total

Female Rate* 60

* Per 100,000

30-34 years, $17.2 \%$ among those aged 35-39 years, 17.1\% among those aged $40-44$ years, $7.2 \%$ among those aged $45-54$ years, $21.1 \%$ among those aged 55-64 years, and $28.6 \%$ among those aged 65 or older.

In 2018, persons aged 15-44 years accounted for $80.6 \%$ of reported P\&S syphilis cases with known age.

\section{P\&S Syphilis by Race/ Hispanic Ethnicity}

In 2018, the rate of reported P\&S syphilis cases was highest among Blacks (28.1 cases per 100,000 population) (Table 35B). The P\&S syphilis rate among Blacks was 4.7 times the rate among Whites (6.0 cases per 100,000 population), the rate among Native Hawaiians/Other Pacific Islanders (NHOPI) (16.3 cases per 100,000 population) was 2.7 times the rate among Whites, the rate among American Indian/ Alaska Natives (AI/AN) (15.5 cases per 100,000 population) was 2.6 times the rate among Whites, the rate among Hispanics (13.0 cases per 100,000 population) was 2.2 times the rate among Whites, and the rate among Asians (4.6 cases per 100,000 population) was 0.8 times the rate among Whites.

During 2014-2018, the P\&S syphilis rate increased among all race/ Hispanic ethnicity groups (Figure 45). The greatest increases during 2017-2018 were observed among AI/ AN (40.9\%) and those who identified as Multirace (22.1\%), followed by
NHOPI (19.0\%), Whites (11.1\%), Blacks (17.1\%), Hispanics (13.0\%), and Asians (9.5\%).

\section{P\&S Syphilis and HIV Co- infection}

Reported cases of $\mathrm{P} \& \mathrm{~S}$ syphilis continue to be characterized by a high rate of HIV co-infection, particularly among MSM (Figure 46). Among 2018 P\&S syphilis cases with known HIV status, $41.6 \%$ of cases among MSM were HIV-positive, compared with $7.9 \%$ of cases among MSW, and $4.0 \%$ of cases among women.

\section{P\&S Syphilis by Reporting Source}

In $2018,16.3 \%$ of $P \& S$ syphilis cases were reported from STD clinics, $71.2 \%$ were reported from venues outside of STD clinics, and $12.5 \%$ of cases had an unknown reporting source (Table A2). During 2017-2018, the number of P\&S syphilis cases reported by STD clinics and by non-STD clinic settings increased (Figure 47). However, the proportion of $\mathrm{P} \& \mathrm{~S}$ syphilis cases that were reported by STD clinics has

\section{Figure 43. Primary and Secondary Syphilis - Rates of Reported Cases Among Females Aged 15-44 Years by Age Group, United States, 2009-2018}

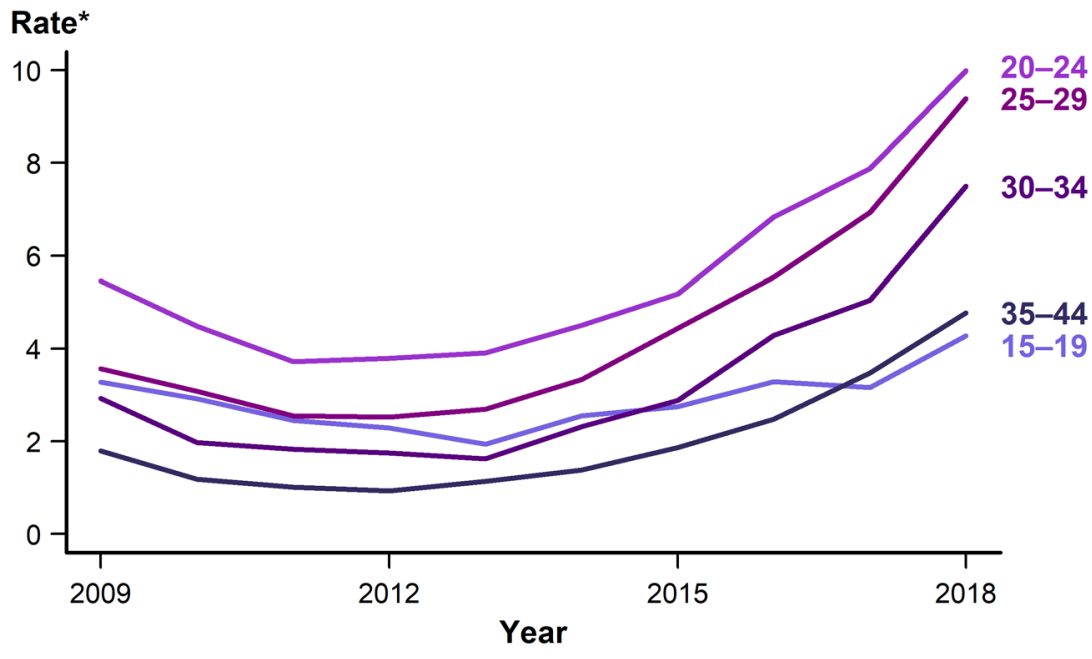

* Per 100,000 


\section{Figure 44. Primary and Secondary Syphilis - Rates of Reported Cases Among Males Aged 15-44 Years by Age Group, United States, 2009-2018}

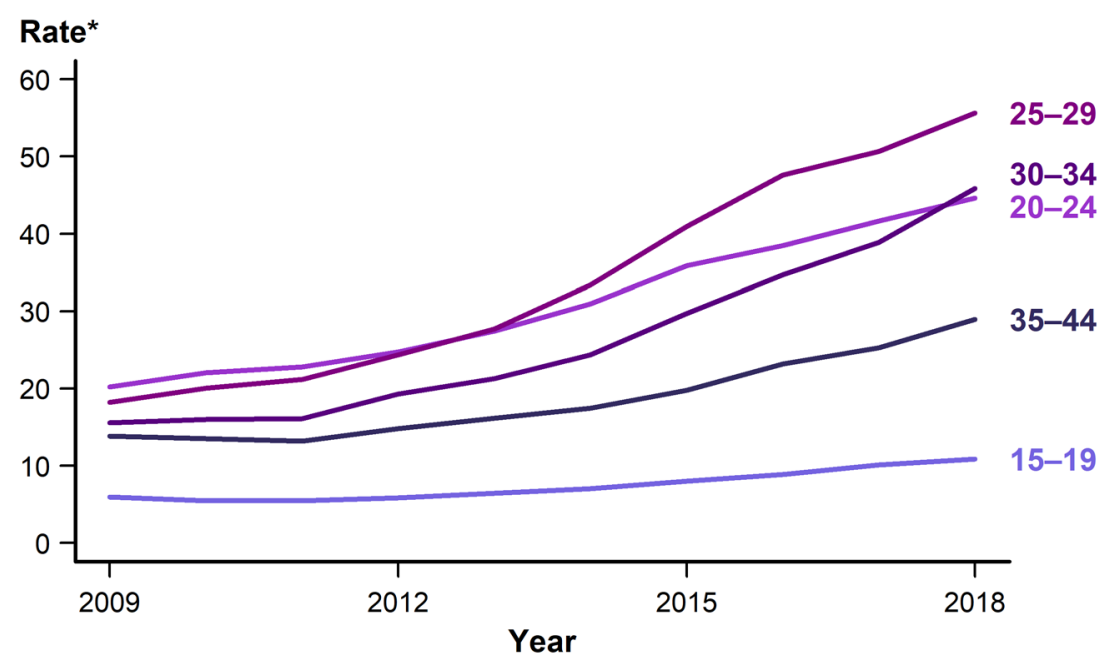

* Per 100,000.

\section{Figure 45. Primary and Secondary Syphilis - Rates of Reported Cases by Race/Hispanic Ethnicity, United States, 2014-2018}

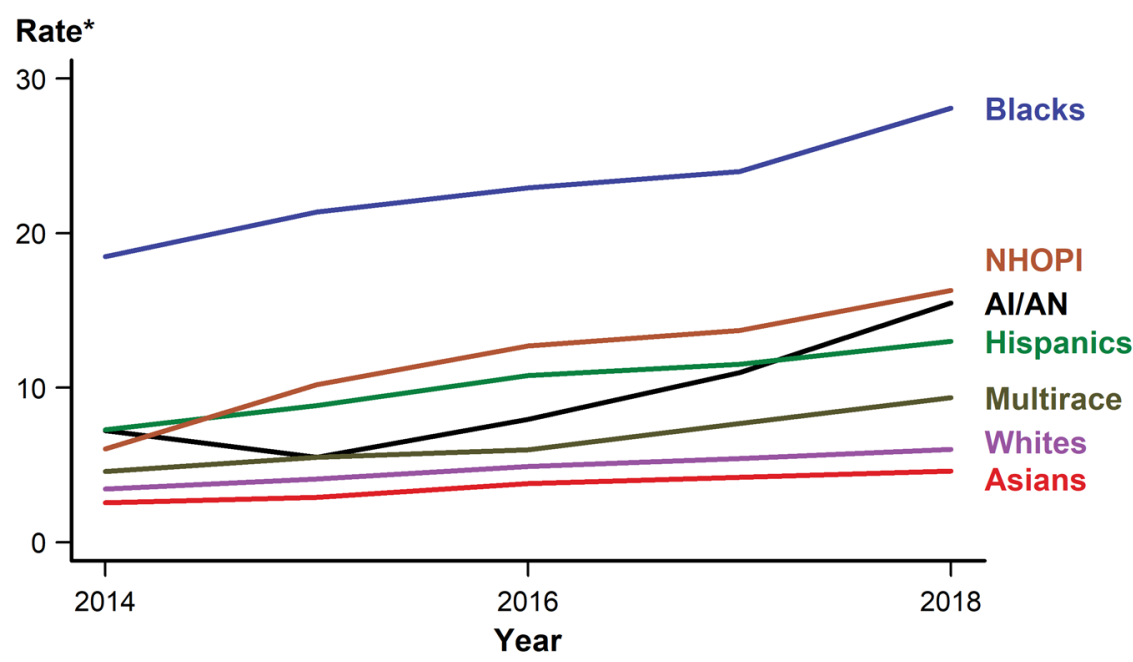

* Per 100,000 .

NOTE: See Section A1.5 in the Appendix for information on reporting STD case data for race/Hispanic ethnicity.

ACRONYMS: Al/AN = American Indians/Alaska Natives; NHOPI = Native Hawaiians/Other Pacific Islanders.

declined by nearly half over the last decade from $31.1 \%$ of cases in 2008 to $16.3 \%$ of cases in 2018 . In 2018 , private physicians/health maintenance organizations (HMOs) and STD clinics were the most common reporting sources for all reported $\mathrm{P} \& \mathrm{~S}$ syphilis cases, regardless of sex of sex partners. Of cases reported among MSM, private physicians/HMOs and STD clinics reported $30.4 \%$ and $22.4 \%$ of cases, respectively. Of cases reported among MSW, they reported $22.6 \%$ and $18.5 \%$, respectively, and of cases reported among women, they reported $25.8 \%$ and $12.2 \%$, respectively (Figure 48).

\section{Congenital Syphilis}

After decreasing from 10.5 to 8.4 reported congenital syphilis cases per 100,000 live births during 2008 2012 , the rate of reported congenital syphilis has subsequently increased each year since 2012 (Table 1). In 2018 , there were a total of 1,306 reported cases of congenital syphilis, including 78 syphilitic stillbirths and 16 infant deaths, and a national rate of 33.1 cases per 100,000 live births. This rate represents a $39.7 \%$ increase relative to 2017 (23.7 cases per 100,000 live births) and a $185.3 \%$ increase relative to 2014 (11.6 cases per 100,000 live births). As has been observed historically, this increase in the congenital syphilis rate has paralleled increases in P\&S syphilis among all women and reproductiveaged women during 2014-2018 (172.7\% and $165.4 \%$ increases, respectively) (Figure 49, Table 28).

During 2014-2018, the increase in reported congenital syphilis cases was primarily attributable to increases in the West and South. During this period, the congenital syphilis rate increased $278.9 \%$ in the West, $190.3 \%$ in the South, $84.8 \%$ in the Northeast, and $45.9 \%$ in the Midwest (Table 41). During 2017-2018, the congenital syphilis rate increased $49.5 \%$ in the South, $44.1 \%$ in the Northeast, $30.5 \%$ in the Midwest, and $29.3 \%$ in the West. In 2018, the highest congenital syphilis rates were reported from the West (48.5 cases per 100,000 live births), followed by the South (44.7 cases per 100,000 live births), Midwest (12.4 cases per 100,000 live births), and the Northeast ( 8.5 cases per 100,000 live births). In addition, rates were highest among Blacks (86.6 cases per 100,000 live births) and AI/AN (79.2 cases per 100,000 live births), followed by Hispanics 
Figure 46. Primary and Secondary Syphilis - Reported Cases by Sex and Sex of Sex Partners and HIV Status, United States, 2018

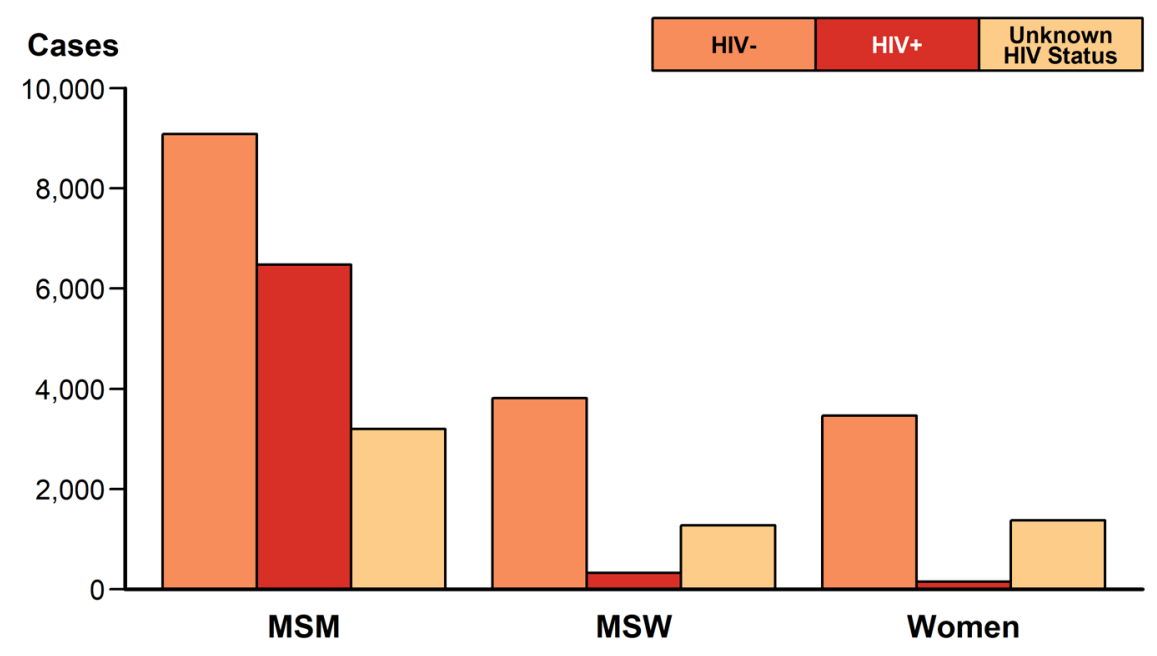

ACRONYMS: $M S M=$ Gay, bisexual, and other men who have sex with men; $M S W=$ Men who have sex with women only.

\section{Figure 47. Primary and Secondary Syphilis - Reported Cases by Reporting Source and Sex, United States, 2009-2018}

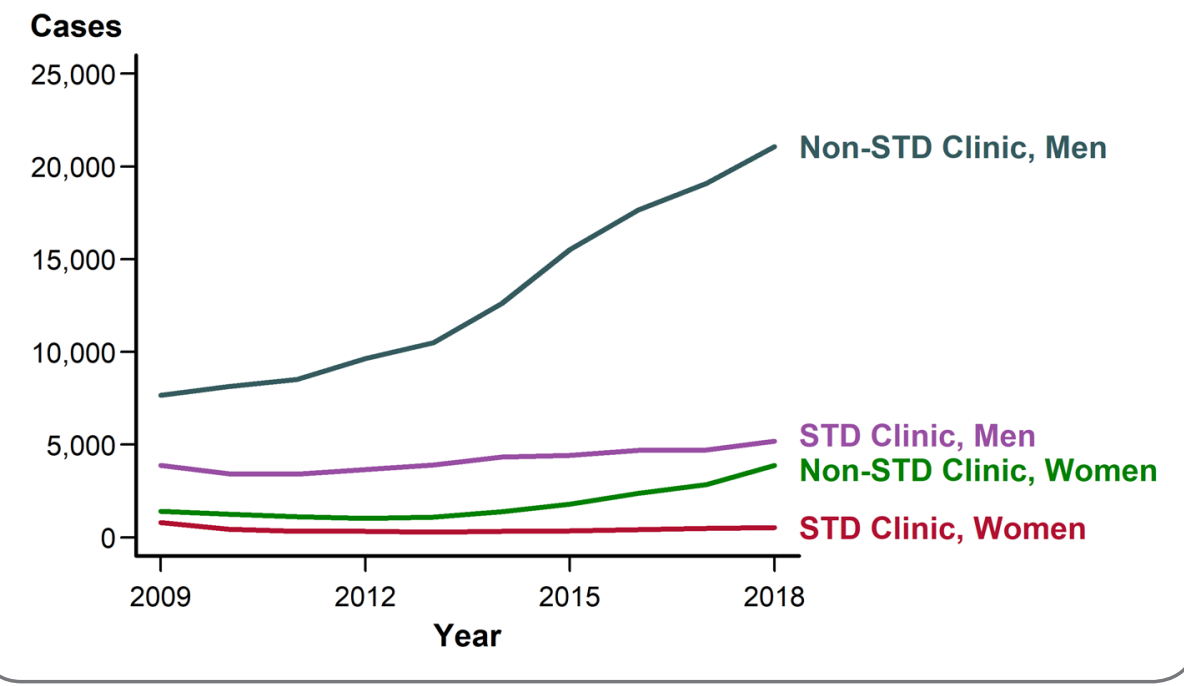

(44.7 cases per 100,000 live births), Whites (13.5 cases per 100,000 live births), and Asians/Pacific Islanders (9.2 cases per 100,000 live births) (Table 42).

\section{Syphilis among Special Populations}

More information about syphilis and congenital syphilis among race/
Hispanic ethnicity groups, women of reproductive age, adolescents, and MSM can be found in the Special Focus Profiles.

\section{Syphilis Summary}

The national rate of reported P\&S syphilis cases reached an historic low in 2000 and 2001, but has increased almost every year since then. This increase was largely attributable to an increase among men, and in particular among MSM. However, in the last five years, rates have increased among both men and women, and the $\mathrm{P} \& \mathrm{~S}$ syphilis rate among women has almost tripled. Rates of reported congenital syphilis cases also increased substantially during 20142018 and during 2017-2018. MSM continued to account for the majority of reported $\mathrm{P} \& \mathrm{~S}$ syphilis cases in 2018. Nationally, the highest rates of P\&S syphilis in 2018 were observed among men aged 20-34 years, among men in the West, and among Black men.

\section{References}

1. Jarzebowski W, Caumes E, Dupin N, et al. Effect of early syphilis infection on plasma viral load and CD4 cell count in human immunodeficiency virus- infected men: Results from the FHDH- ANRS CO4 cohort. Arch Intern Med. 2012;172(16):1237-1243.

2. Buchacz K, Patel P, Taylor M, et al. Syphilis increases HIV viral load and decreases CD4 cell counts in HIV-infected patients with new syphilis infections. AIDS. 2004;18(15):20752079.

3. Fleming DT, Wasserheit JN. From epidemiological synergy to public health policy and practice: The contribution of other sexually transmitted diseases to sexual transmission of HIV infection. Sex Transm Infect. 1999;75(1):317.

4. Ingraham NR. The value of penicillin alone in the prevention and treatment of congenital syphilis. Acta Derm Venereol. 1951;31(Suppl 24):60-88.

5. de Voux A, Kidd S, Grey JA, et al. Statespecific rates of primary and secondary syphilis among men who have sex with men - United States, 2015. MMWR Morb Mortal Wkly Rep. 2017;66(13):349-354.

6. Peterman TA, Kahn RH, Ciesielski CA, et al. Misclassification of the stages of syphilis: Implications for surveillance. Sex Transm Dis 2005;32(3):144-149. 
Figure 48. Primary and Secondary Syphilis - Percentage of Reported Cases* by Sex and Sex of Sex Partners and Selected Reporting Sources, United States, 2018

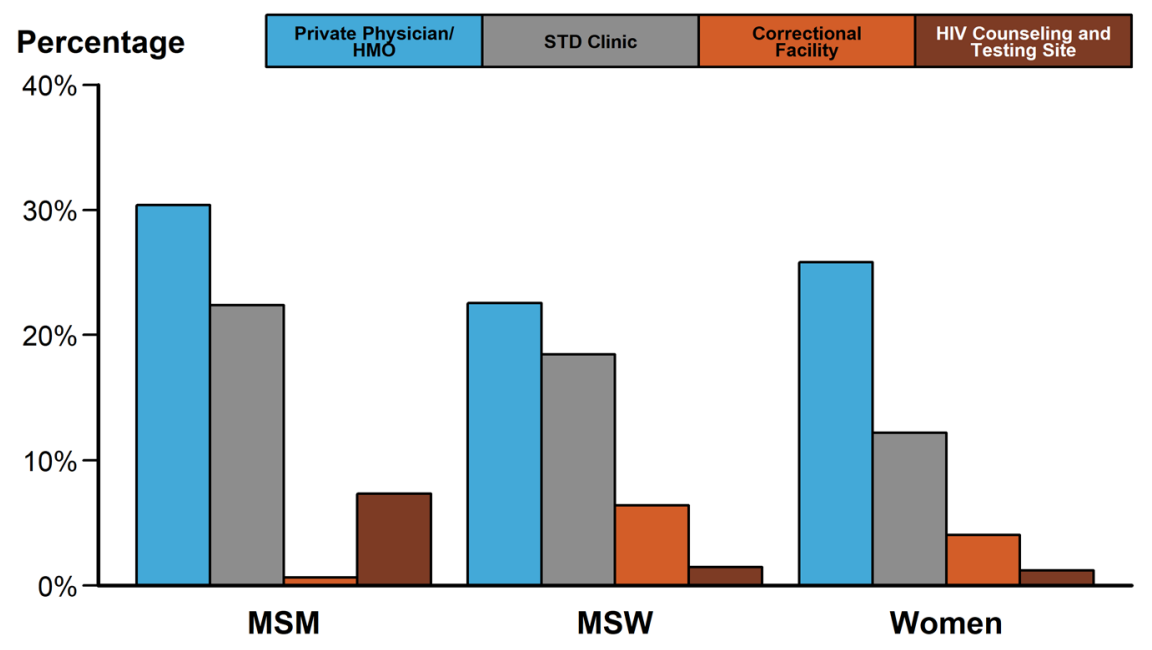

* Of all primary and secondary syphilis cases, $12.5 \%$ had a missing or unknown reporting source. Among all cases with a known reporting source, the reporting source categories presented represent $55.4 \%$ of cases.

ACRONYMS: $\mathrm{HMO}=$ Health maintenance organization; $\mathrm{MSM}=$ Gay, bisexual, and other men who have sex with men; MSW = Men who have sex with women only.

Figure 49. Congenital Syphilis - Reported Cases by Year of Birth and Rates of Reported Cases of Primary and Secondary Syphilis Among Females Aged 15-44 Years, United States, 2009-2018

CS Cases

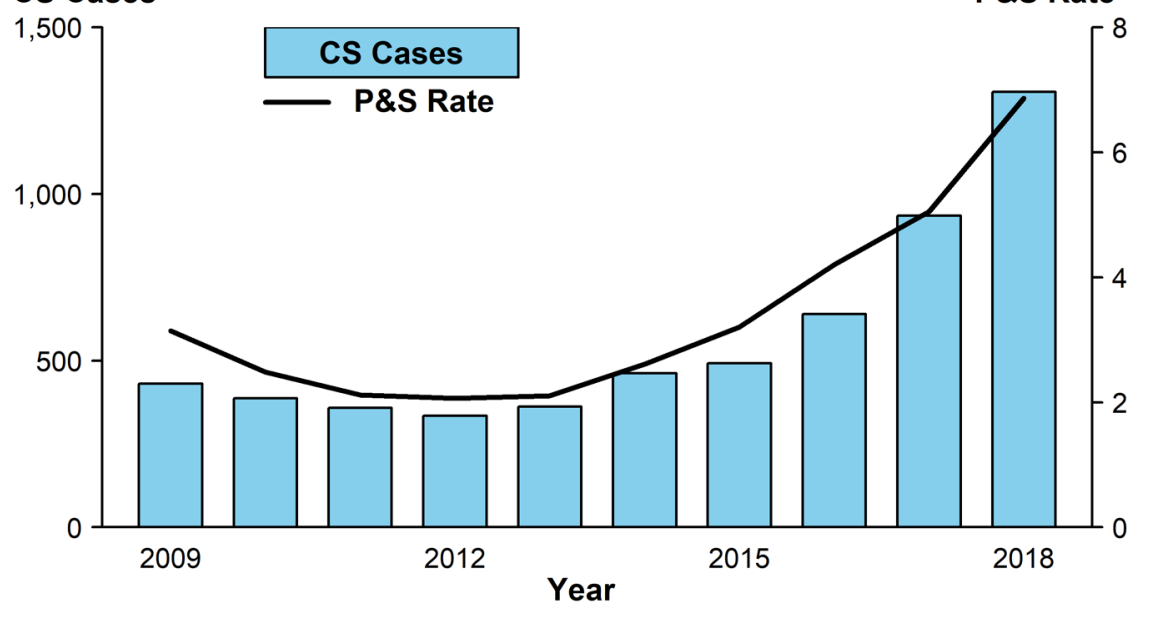

* Per 100,000

ACRONYMS: CS = Congenital syphilis; P\&S = Primary and secondary syphilis. 


\section{Chancroid}

Chancroid is caused by anogenital infection with the bacterium

Haemophilus ducreyi. Clinical manifestations of chancroid include anogenital ulcers, and inguinal lymphadenopathy or buboes in up to $50 \%$ of cases. ${ }^{1}$ Reported cases of chancroid peaked in 1947 and then declined rapidly through 1957 , presumably due to the increasing use of antibiotics such as sulfonamides and penicillin, which were introduced in the late 1930s and early 1940s (Figure 50, Table 1). ${ }^{2,3}$ Numerous localized outbreaks, some of which were linked to commercial sex work, were identified during 1981-1990.,5 Chancroid has declined since 1987; since 2000, the annual number of reported cases has been less than 100, and since 2011, the annual number of reported cases has been less than 20 . In 2018, only three cases of chancroid were reported in the United States (Table 43).

Although the overall decline in reported chancroid cases most likely reflects a decline in the incidence of this disease, these data should be interpreted with caution because $H$. ducreyi is difficult to culture and no molecular assays have been cleared by the Food and Drug Administration (FDA) for use in the United States. ${ }^{6}$

\section{Human Papillomavirus}

Human papillomavirus (HPV) is a common sexually transmitted infection in the United States. ${ }^{7}$ Over 40 distinct HPV types can infect the genital tract, ${ }^{8}$ although most infections are asymptomatic and appear to resolve spontaneously within a few years. ${ }^{9}$ Prevalence of genital infection with any HPV type was $42.5 \%$ among civilian, non-institutionalized adults aged $18-59$ years in the United States during 2013-2014. ${ }^{10}$ Among sexually active non-Hispanic

\section{Figure 50. Chancroid - Reported Cases by Year, United States, 1941-2018}

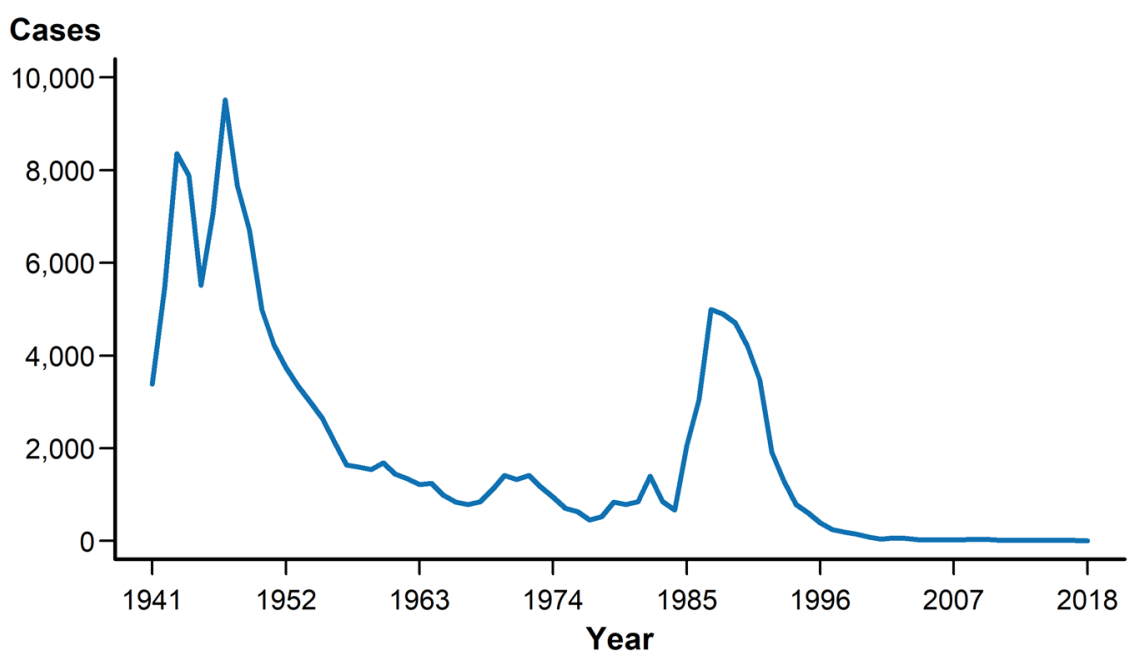

NOTE: See section A1.3 in the Appendix for more information on chancroid case reporting.

Whites and non-Hispanic Blacks, prevalence was significantly higher in males. ${ }^{11}$ Persistent infection with some HPV types can cause cancer and genital warts. ${ }^{12}$ HPV types 16 and 18 account for approximately $66 \%$ of cervical cancers in the United States, ${ }^{13}$ and approximately $25 \%$ of low-grade and $50 \%$ of high-grade cervical intraepithelial lesions, or dysplasia. ${ }^{14,15}$ HPV types 6 and 11 are responsible for approximately $90 \%$ of genital warts. ${ }^{16,17}$

Quadrivalent HPV vaccine, which targets HPV types 6,11,16, and 18, was licensed in the United States in mid-2006 for females ${ }^{18}$ and in late 2009 for males. ${ }^{19}$ Although a bivalent vaccine was also licensed for females,${ }^{20}$ almost all HPV vaccine administered in the United States through late 2014 was quadrivalent. ${ }^{21}$ A 9-valent vaccine, which protects against the quadrivalent and 5 additional oncogenic HPV types (types $31,33,45,52$, and 58), was licensed in late 2014 for males and females. ${ }^{22}$ All HPV vaccines have been recommended for routine use in United States females aged 11-12 years, with catch-up vaccination through age $26 .^{18,22}$ Since late 2011, routine use of the quadrivalent or 9-valent vaccine has been recommended for males aged 11-12, with catch-up vaccination through age 21;22-24 in June 2019, this age limit was extended to 26 years. ${ }^{25}$ Vaccination through age 26 has been recommended since late 2011 for gay, bisexual, and other men who have sex with men (MSM) and persons who are immunocompromised (including those infected with HIV). ${ }^{22-24}$ In October 2018, the FDA extended licensing approval of the 9-valent vaccine for women and men aged $27-45$ years,${ }^{26}$ and in June 2019 the CDC's Advisory Committee on Immunization Practices (ACIP) recommended that unvaccinated adults aged $27-45$ years discuss receiving the HPV vaccine with their health care providers. ${ }^{25}$ 
HPV vaccine uptake in the United States remains lower than the Healthy People 2020 goal of $80 \%$ coverage. ${ }^{27}$ A national survey conducted in 2018 found that $70 \%$ of girls aged $13-17$ years had received at least one dose of the HPV vaccine, and 54\% had received all doses in the series ${ }^{28}$ based on recommendations published in late $2016 .{ }^{24}$ Among boys, $66 \%$ of those aged 13-17 years received at least one dose and $49 \%$ received all recommended doses. ${ }^{28}$

A recent meta-analysis that included data from over 60 million individuals from 14 high-income countries, including the United States, showed a substantial impact of HPV vaccination on: genital HPV infections among adolescent girls and young women; high-grade cervical lesions among young women; and anogenital warts among adolescent boys and girls, and among young men and women. ${ }^{29}$ Although HPV infection is not a nationally notifiable condition in the United States, cervicovaginal prevalence of any quadrivalent HPV vaccine type has been estimated for civilian, non-institutionalized females aged 14-34 years using data from the National Health and Nutrition Examination Survey (NHANES; see Section A2.4 in the Appendix). ${ }^{30}$ Prevalence decreased significantly from 2003-2006 (the pre-vaccine era) to 2011-2014 in specimens from females aged 14-19 years (from $11.5 \%$ to $3.3 \%$ ) and $20-24$ years (from $18.5 \%$ to $7.2 \%$ ); these were the age groups most likely to benefit from HPV vaccination. Among women aged 25-34 years, vaccine-type HPV prevalence did not differ significantly between the two time periods. An NHANES analysis of 2013-2014 HPV prevalence from penile swab specimens found low prevalence of quadrivalent HPV vaccine types in young males, which the authors attributed to male vaccination and/ or herd protection from female vaccination. ${ }^{31}$
Health-care claims data from adolescents and adults with employer-provided private health insurance in the United States were used to examine the population effectiveness of HPV vaccination on clinical sequelae of HPV infection. Annual prevalence of high-grade histologically-detected cervical intraepithelial neoplasia grades 2 and 3 (CIN2+) during 2007-2014 was estimated using claims from 9 million females aged 15-39 years who received cervical cancer screening in a given calendar year. ${ }^{32}$ Prevalence of CIN2+ decreased significantly in females aged 15-19 and 20-24 years (Figure 51). Among those aged 15-19 years, annual percent change (APC) in CIN2+ prevalence was $-19.8 \%$ during 2007-2009 and -12.1\% during 2009-2014. For women aged 20-24 years, APC was $-6.7 \%$ during $2007-$ 2012, and $-12.5 \%$ during 2012-2014. No decreases in CIN2+ prevalence were observed among women aged 25-39 years. The observed decreases in high-grade cervical lesions only among young women provide ecologic evidence of population effectiveness of HPV vaccination on clinical sequelae of infection among privately-insured women in the United States.

Prevalence of anogenital warts was examined using health-care claims of privately-insured females and males aged 15-39 years during 2006-2014 (Figures 52A and 52B). ${ }^{33}$ Prevalence among adolescent females aged 15-19 years declined nonsignificantly during 2006-2008, and then significantly decreased through 2014 (APC=-14.1). Among women aged 20-24 years, anogenital wart prevalence was stable during 20062009, but declined significantly during 2009-2014 (APC=-12.9). Prevalence among women aged 2529 years also decreased significantly from 2009-2014 (APC=-6.0).

Prevalence increased or was stable during the entire period for women

\section{Figure 51. Cervical Intraepithelial Neoplasia Grades 2 and $3-$ Prevalence per 1000 Person-Years Among Female Enrollees in Private Health Plans Aged 15-39 Years, by Age Group and Year, 2007-2014}

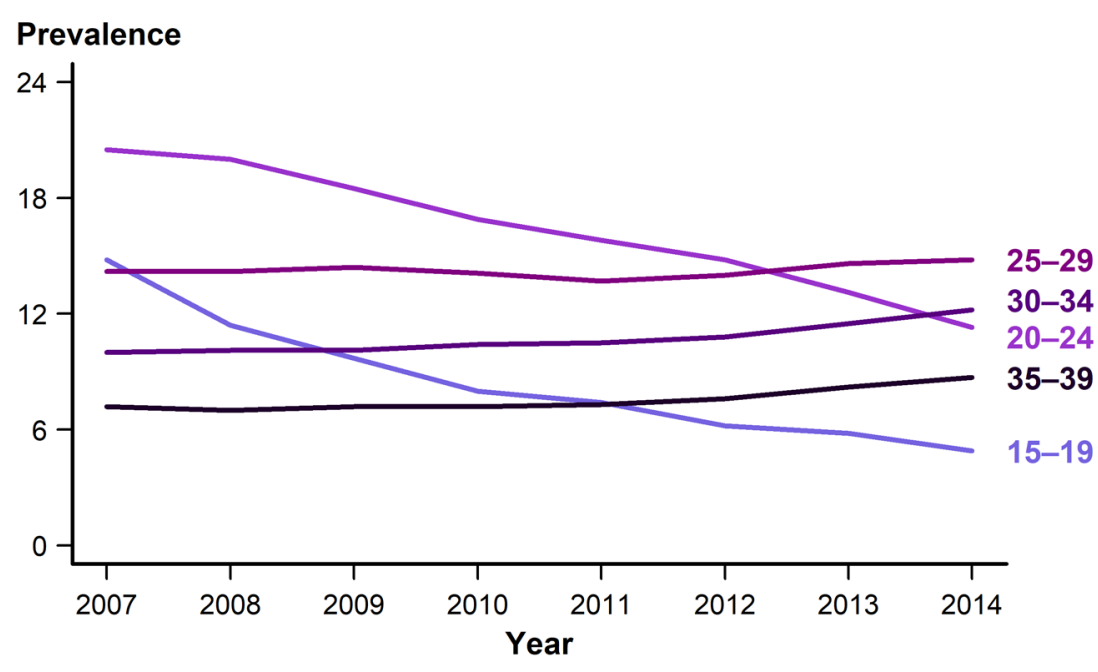

SOURCE: Flagg EW, Torrone EA, Weinstock H. Ecological association of human papillomavirus vaccination with cervical dysplasia prevalence in the United States, 2007-2014. Am J Public Health. 2016;106(12):2211-2218. 


\section{Figure 52. Anogenital Warts - Prevalence per 1000 Person-Years Among Enrollees in Private Health Plans Aged 15-39 Years by Sex, Age Group, and Year, 2006-2014}

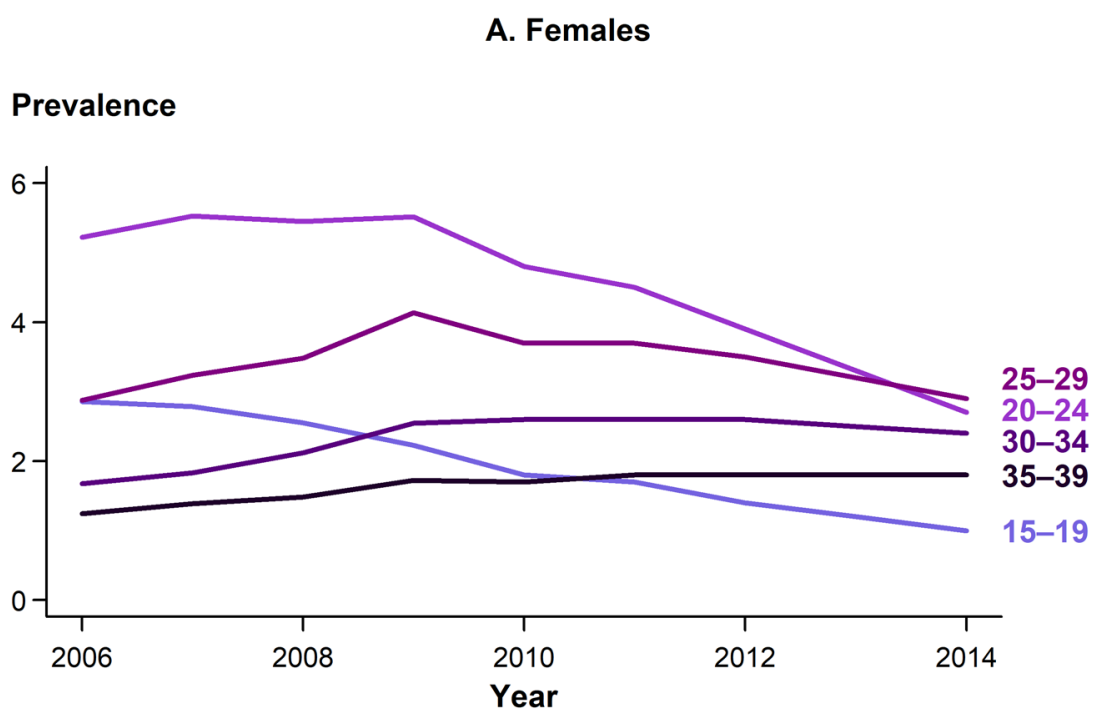

B. Males

\section{Prevalence}

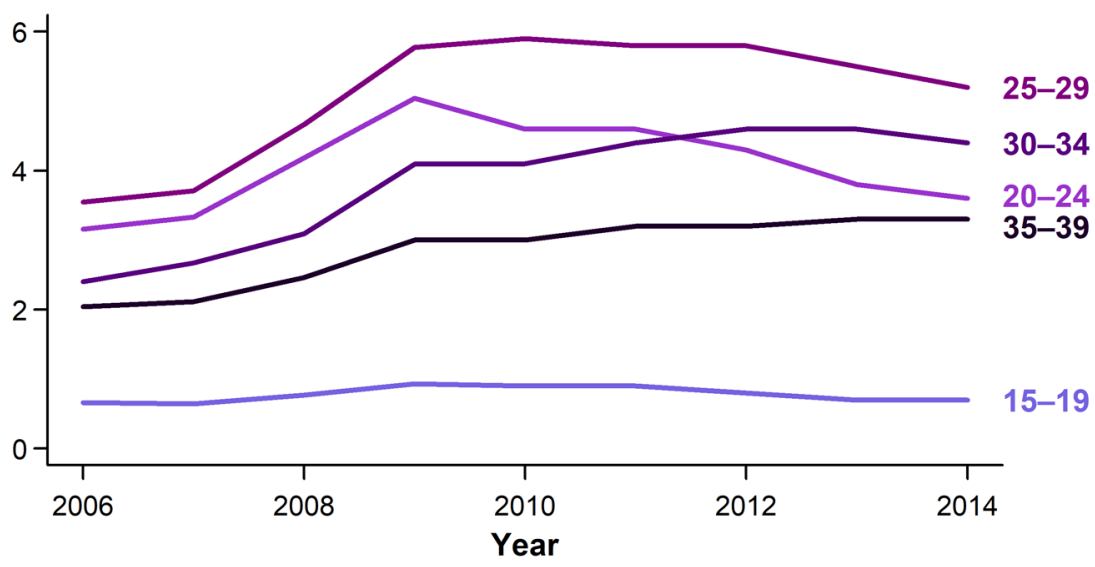

SOURCE: Flagg EW, Torrone EA. Declines in anogenital warts among age groups most likely to be impacted by human papillomavirus vaccination, United States, 2006-2014. Am $J$ Public Health. 2018;108(1):112-119.

aged 30-39 years. These declines in anogenital wart prevalence among females aged 15-29 years extend the observations of a previous study using claims from 2003 through 2010, in which decreased prevalence was found only among adolescent females aged $15-19$ years. ${ }^{34}$ The observed declines in prevalence among increasingly older age groups would be expected from including more years of observation after the initiation of routine HPV vaccination for females in 2006. Among males, anogenital wart prevalence increased significantly during 2006-2009 for all age groups except those aged 15-19 years. ${ }^{33}$ From 2009 to 2014, rates decreased somewhat among male adolescents aged $15-19$ years $(\mathrm{APC}=-5.4)$, but decreased significantly among men aged 20-24 years $(\mathrm{APC}=-6.5)$. Among those aged 25-29 years, prevalence declined non-significantly during 2010-2014 (APC=-1.7); prevalence increased or was stable throughout the entire period for men aged 30-39 years. The decreased prevalence observed among men aged 20-24 years is unlikely to be due to male vaccination for several reasons. Almost all men in this age group were aged 19 years or older since 2011, when HPV vaccine was first recommended for routine use in United States males ${ }^{23}$ and vaccination coverage in adult males through 2014 was extremely low. ${ }^{35}$ Also, the most likely sexual partners for men in this age group were females of a similar age or younger; ${ }^{36,37}$ therefore, the observed declines in anogenital wart prevalence among young men are consistent with herd protection from vaccination among females.

A study conducted in 27 clinics participating in the STD Surveillance Network (SSuN; see Section A2.2 in the Appendix) observed significant declines in prevalence of anogenital warts during 2010-2016 among women and men who have sex with women only (MSW) aged less than 40 years, and among MSM of all ages. ${ }^{38}$ Although some of the observed declines may be due to HPV vaccination, changes over time in the population of STD clinic patients or clinical practices, such as a decrease in physical examinations resulting in fewer anogenital warts diagnoses, may partially account for these findings.

\section{Pelvic Inflammatory Disease}

For information on pelvic inflammatory disease, see Special 
Focus Profiles, STDs in Women and Infants.

\section{Herpes Simplex Virus}

Herpes simplex virus (HSV) is among the most prevalent of sexually transmitted infections. ${ }^{7,39}$ Although most infections are subclinical, ${ }^{40}$ clinical manifestations are characterized by recurrent, painful genital and/or anal lesions..$^{41}$ Most genital HSV infections in the United States are caused by HSV type 2 (HSV-2), while HSV type 1 (HSV1) infections are typically orolabial and acquired during childhood; ${ }^{40,42}$ however, the prevalence of genital HSV-1 infections appears to be increasing among young adults. ${ }^{43,44}$

Genital HSV infection is not a nationally notifiable condition. Most persons with genital HSV infection have not received a diagnosis. ${ }^{45}$ The overall percentage of the HSV-2 seropositive NHANES population aged 14-49 years who reported never being told by a doctor or healthcare professional that they had genital herpes did not change significantly between 1988-1994 and 2007-2010, and remained high $(90.7 \%$ and $87.4 \%$, respectively). ${ }^{45}$

\section{NHANES data indicate the}

seroprevalence of HSV-2 in the civilian, non-institutionalized United States population has decreased from 1999-2000 to 2015-2016; ageadjusted seroprevalence declined from $18.0 \%$ in $1999-2000$ to $12.1 \%$ in 2015-2016 (Figure 53). ${ }^{46}$ Although these declines were observed among all race/Hispanic ethnicity groups, HSV-2 seroprevalence was highest among non-Hispanic Blacks throughout the entire time period.

A recent analysis of NHANES data showed that among men who reported having been diagnosed with genital HSV, $25.2 \%$ were HSV-1 positive and HSV-2 negative during 1999-2010; this percentage did not change

Figure 53. Herpes Simplex Virus Type 2 - National Estimates of Trends in Age-Adjusted Seroprevalence Among Persons Aged 14-49 Years by Race/Hispanic Ethnicity, National Health and Nutrition Examination Survey (NHANES), 1999-2000 through 2015-2016

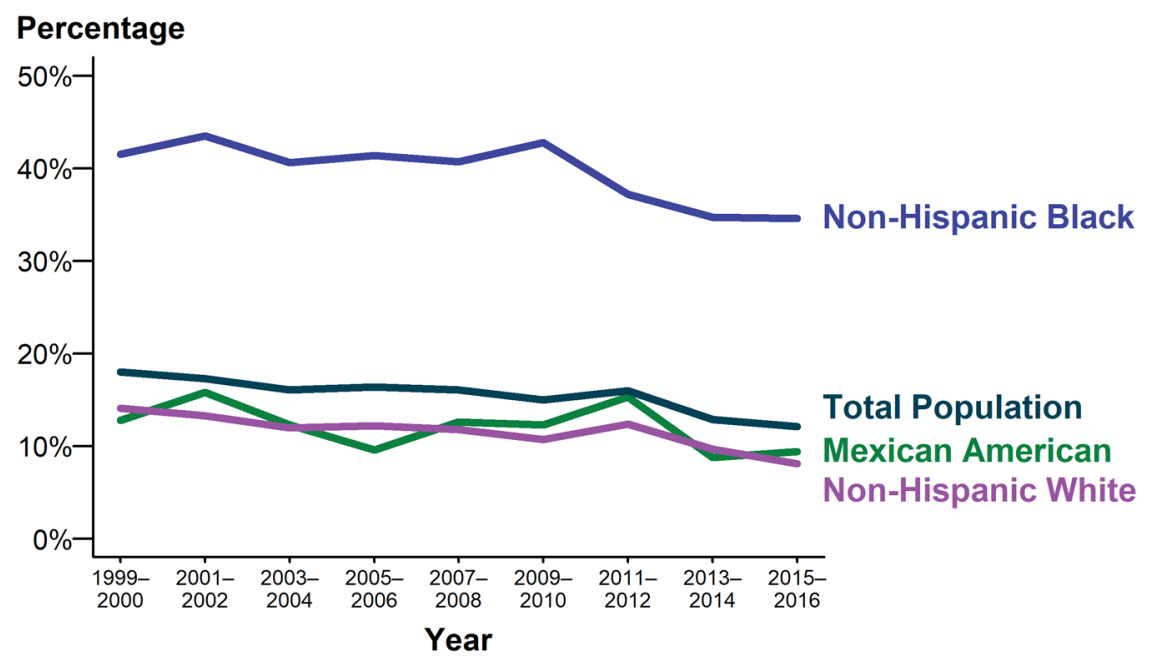

NOTE: Age-adjusted by the direct method to the 2000 US Census population, using age groups $14-19,20-29,30-39$, and $40-49$ years. Total population includes all race/Hispanic ethnicity groups, including those not shown separately. SOURCE: McQuillan G, Kruszon-Moran D, Flagg EW, et al. Prevalence of herpes simplex virus type 1 and type 2 in persons aged 14-49: United States, 2015-2016. NCHS data brief, no 304. Hyattsville, MD: National Center for Health Statistics. 2018.

significantly during $2011-2016 .{ }^{47} \mathrm{In}$ contrast, the percentage of diagnosed women who were HSV-1 positive and HSV-2 negative significantly increased from $16.5 \%$ during 1999 2010 to $31.6 \%$ during 2011-2016, while HSV-2 seropositivity in women decreased significantly, from $77.6 \%$ to $63.3 \%$. These findings indicate the percentage of genital HSV infections associated with HSV-1 has increased among women in the United States since 2010 .

NHANES data also show that among adolescents aged 14-19 years, HSV-1 seroprevalence has significantly decreased by almost $23 \%$, from $39.0 \%$ during $1999-2004$ to $30.1 \%$ during 2005-2010, indicating declining orolabial infection in this age group. ${ }^{42} \mathrm{HSV}-2$ seroprevalence in this age group was much lower, less than $2 \%$ in both time periods. ${ }^{42}$ Other studies have found that genital HSV-1 infections are increasing among young adults. ${ }^{43,44}$ This has been attributed, in part, to the decline in orolabial HSV-1 infections, because those who lack HSV-1 antibodies at sexual debut are more susceptible to genital HSV-1 infection. ${ }^{42,48}$ Increasingly common oral sex behavior among adolescents and young adults also has been suggested as a contributing factor. ${ }^{42,49}$ The absence of HSV-1 antibodies also increases the likelihood of developing symptomatic disease from newly-acquired (i.e., primary) genital HSV-2 infection. ${ }^{50}$ Young women may therefore be increasingly likely to first acquire HSV-1 infection genitally, or acquire a primary genital HSV-2 infection, during their childbearing years, ${ }^{48,51}$ and first-episode primary genital HSV infection during pregnancy increases the risk 
of neonatal HSV transmission. ${ }^{48,52}$ Another analysis of NHANES data found that among pregnant women with three or fewer lifetime sex partners, seronegativity for both HSV-1 and HSV-2 increased from 1999-2006 to $2007-2014,{ }^{53}$ raising the possibility that pregnant women with fewer sex partners may have increased risk of acquiring genital HSV during pregnancy and vertically transmitting HSV to their neonates.

For information on neonatal HSV infections, see Special Focus Profiles, STDs in Women and Infants.

\section{Trichomonas vaginalis}

Trichomonas vaginalis is a common sexually transmitted protozoal infection associated with adverse health outcomes such as preterm birth and symptomatic vaginitis. ${ }^{7,54-55}$ It is not a nationally notifiable condition, and trend data are limited to estimates of initial physician office visits for this condition from the National Disease and Therapeutic Index (NDTI; see Section A2.5 in the Appendix) (Figure 54, Table 44). Visits appear to be fairly stable since the 1990's; the number of initial visits for $T$. vaginalis infection in 2016 was 222,000 . NHANES data during 2013-2016 indicated prevalence of $T$. vaginalis in urine specimens obtained from participants aged 14-59 years was $0.5 \%$ among males and $2.1 \%$ among females; highest prevalences were observed among non-Hispanic Black males (3.4\%) and females $(9.6 \%) .{ }^{56} \mathrm{An}$ analysis of NHANES data during 2001-2004 from cervicovaginal swab specimens also found higher T. vaginalis prevalence among non-Hispanic Black females. ${ }^{57}$

Figure 54. Trichomonas vaginalis and Other Vaginal Infections Among Females — Initial Visits to Physicians' Offices, United States, 1966-2016

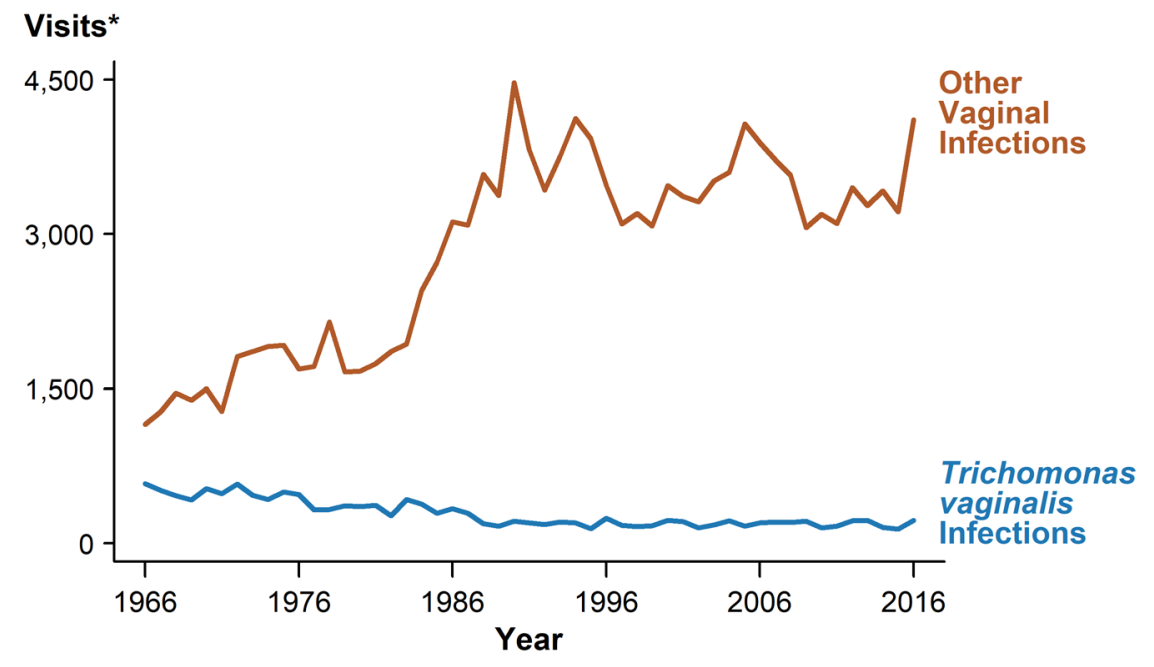

* In thousands.

NOTE: The relative standard errors for Trichomonas vaginalis infection estimates range from $23 \%$ to $17 \%$ and for other vaginal infection estimates range from $13 \%$ to $8 \%$. See Section A2.5 in the Appendix and Table 44.

SOURCE: National Disease and Therapeutic Index, IMS Health, Integrated Promotional Services $^{\text {TM }}$, IMS Health Report, 1966-2016.

\section{References}

1. Lewis DA. Epidemiology, clinical features, diagnosis and treatment of Haemophilus ducreyi - a disappearing pathogen? Expert Rev Anti Infect Ther. 2014;12(6):687-696.

2. Steen R. Eradicating chancroid. Bull World Health Organ. 2001;79:818-826.

3. Hall L. "The Cinderella of medicine": Sexuallytransmitted diseases in Britain in the nineteenth and twentieth centuries. Genitourin Med. 1993;69:314-319.

4. Schmid GP, Sanders LL, Blount JH, et al. Chancroid in the United States. Reestablishment of an old disease. JAMA. 1987;258(22):32653268.

5. Schulte JM, Martich FA, Schmid GP. Chancroid in the United States, 1981-1990: Evidence for underreporting of cases. MMWR Surveill Summ. 1992;41(No. SS-3):57-61.
6. Centers for Disease Control and Prevention. Sexually transmitted diseases treatment guidelines, 2015. MMWR Recomm Rep. 2015;64(No. RR-3):26-27.

7. Satterwhite CL, Torrone E, Meites E, et al. Sexually transmitted infections among US women and men: Prevalence and incidence estimates, 2008. Sex Transm Dis. 2013;40(3):187-193.

8. de Villiers E-M, Fauquet C, Broker TR, et al. Classification of papillomaviruses. Virology. 2004;324:17-27.

9. Insinga RP, Perez G, Wheeler CM, et al. Incident cervical HPV infections in young women: Transition probabilities for CIN and infection clearance. Cancer Epidemiol Biomarkers Prev. 2011;20(2):287-296. 
10. McQuillan G, Kruszon-Moran D, Markowitz LE, et al. Prevalence of HPV in adults aged 18-69: United States, 2011-2014. NCHS data brief, no 280. Hyattsville, MD: National Center for Health Statistics. 2017.

11. Lewis RM, Markowitz LE, Gargano JW, et al. Prevalence of genital human papillomavirus among sexually experienced males and females aged 14-59 years, United States, 2013-2014. J Infect Dis. 2018;217(6):869-877.

12. Doorbar J, Quint W, Banks L, et al. The biology and life-cycle of human papillomaviruses. Vaccine. 2012;30(Suppl 5):F55-F70.

13. Saraiya M, Unger ER, Thompson TD, et al. US assessment of HPV types in cancers: Implications for current and 9-valent HPV vaccines. $J$ Natl Cancer Inst. 2015;107(6):djv086.

14. Clifford GM, Rana RK, Franceschi S, et al. Human papillomavirus genotype distribution in low-grade cervical lesions: Comparison by geographic region and with cervical cancer. Cancer Epidemiol Biomarkers Prev. 2005;14(5):1157-1164.

15. Porras C, Rodriguez AC, Hildesheim A, et al. Human papillomavirus types by age in cervical cancer precursors: Predominance of human papillomavirus 16 in young women. Cancer Epidemiol Biomarkers Prev. 2009;18(3):863865.

16. Garland SM, Steben M, Sings HL, et al. Natural history of genital warts: Analysis of the placebo arm of 2 randomized phase III trials of a quadrivalent human papillomavirus (types 6, 11, 16, and 18) vaccine. J Infect Dis. 2009;199(6):805-814.

17. Gissmann L, Wolnik L, Ikenberg H, et al. Human papillomavirus types 6 and 11 DNA sequences in genital and laryngeal papillomas and in some cervical cancers. Proc Natl Acad Sci USA. 1983;80(2):560-563.

18. Markowitz LE, Dunne EF, Saraiya M, et al. Quadrivalent human papillomavirus vaccine. Recommendations of the Advisory Committee on Immunization Practices (ACIP). MMWR Recomm Rep. 2007;56(No. RR-2):1-24.

19. Centers for Disease Control and Prevention. FDA licensure of quadrivalent human papillomavirus vaccine (HPV4, Gardasil) for use in males and guidance from the Advisory Committee on Immunization Practices (ACIP). MMWR Morb Mortal Wkly Rep. 2010;59(20):630-632.

20. Centers for Disease Control and Prevention. FDA licensure of bivalent human papillomavirus vaccine (HPV2, Cervarix) for use in females and updated HPV vaccination recommendations from the Advisory Committee on Immunization Practices (ACIP). MMWR Morb Mortal Wkly Rep. 2010;59(20):626-629.
21. Stokley S, Jeyarajah J, Yankey D, et al. Human papillomavirus vaccination coverage among adolescents, 2007-2013, and postlicensure vaccine safety monitoring, 2006-2014 United States. MMWR Morb Mortal Wkly Rep. 2014;63(29):620-624.

22. Petrosky E, Bocchini Jr. JA, Hariri S, et al. Use of 9-valent human papillomavirus (HPV) vaccine: Updated HPV vaccination recommendations of the Advisory Committee on Immunization Practices. MMWR Morb Mortal Wkly Rep. 2015;64(11):300-304.

23. Centers for Disease Control and Prevention. Recommendations on the use of quadrivalent human papillomavirus vaccine in males Advisory Committee on Immunization Practices (ACIP), 2011. MMWR Morb Mortal Wkly Rep. 2011;60(50):1705-1708.

24. Meites E, Kempe A, Markowitz LE. Use of a 2-dose schedule for human papillomavirus vaccination - updated recommendations of the Advisory Committee on Immunization Practices. MMWR Morb Mortal Wkly Rep. 2016;65(49):1405-1408.

25. Meites E, Szilagyi PG, Chesson HW, et al. Human papillomavirus vaccination for adults: Updated recommendations of the Advisory Committee on Immunization Practices. MMWR Morb Mortal Wkly Rep. 2019;68(32):698-702.

26. US Food and Drug Administration. "Supplement Approval Letter - Biologics License Application (BLA) for Human Papillomavirus 9-valent Vaccine, Recombinant (GARDASIL ${ }^{\circledR}$ 9)". Letter to Merck Sharp \& Dohme Corp. October 5, 2018. US Food and Drug Administration. 2018. https://www.fda. gov/media/117053/download. Accessed July 2, 2019.

27. HealthyPeople.gov. Objectives IID-11.4 and IID-11.5. Healthy People 2020 Topics \& Objectives. Immunization and Infectious Diseases 2019; https://www.healthypeople. gov/2020/topics-objectives/topic/immunizationand-infectious-diseases/objectives. Accessed July 2, 2019.

28. Walker TY, Elam-Evans LD, Yankey D, et al. National, regional, state, and selected local area vaccination coverage among adolescents aged 13-17 years - United States, 2018. MMWR Morb Mortal Wkly Rep. 2019;68(33):718-723.

29. Drolet M, Benard E, Perez N, et al. Populationlevel impact and herd effects following the introduction of human papillomavirus vaccination programmes: Updated systematic review and meta-analysis. Lancet 2019;394(10197):497-509.

30. Oliver SE, Unger ER, Lewis R, et al. Prevalence of human papillomavirus among females after vaccine introduction - National Health and Nutrition Examination Survey, United States, 2003-2014. J Infect Dis. 2017;216(5):594-603.
31. Gargano JW, Unger ER, Liu G, et al. Prevalence of genital human papillomavirus in males, United States, 2013-2014. J Infect Dis. 2017;215(7):1070-1079.

32. Flagg EW, Torrone EA, Weinstock H. Ecological association of human papillomavirus vaccination with cervical dysplasia prevalence in the United States, 2007-2014. Am J Public Health. 2016;106(12):2211-2218.

33. Flagg EW, Torrone EA. Declines in anogenital warts among age groups most likely to be impacted by human papillomavirus vaccination, United States, 2006-2014. Am J Public Health. 2018;108(1):112-119.

34. Flagg EW, Schwartz R, Weinstock H. Prevalence of anogenital warts among participants in private health plans in the United States, 2003-2010: Potential impact of human papillomavirus vaccination. Am J Public Health 2013;103(8):1428-1435.

35. Williams WW, Lu P-J, O'Halloran A, et al. Surveillance of vaccination coverage among adult populations - United States, 2014. MMWR Surveill Summ. 2016;65(No. SS-1): $1-36$.

36. Ford K, Sohn W, Lepkowsk J. American adolescents: Sexual mixing patterns, bridge partners, and concurrency. Sex Transm Dis. 2002;29(1):13-19.

37. Aral SO, Hughes JP, Stoner B, et al. Sexual mixing patterns in the spread of gonococcal and chlamydial infections. Am J Public Health. 1999;89(6):825-833.

38. Mann LM, Llata E, Flagg EW, et al. Trends in the prevalence of anogenital warts among patients at sexually transmitted disease clinics - Sexually Transmitted Disease Surveillance Network, United States, 2010-2016. J Infect Dis. 2019;219(9):1389-1397.

39. Smith JS, Robinson NJ. Age-specific prevalence of infection with herpes simplex virus types 2 and 1: A global review. J Infect Dis. 2002;186(Suppl 1):S3-S28.

40. Corey L, Wald A. Genital Herpes. In: Holmes KK, Sparling FP, Stamm WE, et al., eds. Sexually Transmitted Diseases. 4th ed. New York, NY: McGraw-Hill; 2008:399-437.

41. Kimberlin DW, Rouse DJ. Genital herpes. $N$ Engl J Med. 2004;350(19):1970-1977.

42. Bradley H, Markowitz LE, Gibson T, et al. Seroprevalence of herpes simplex virus types 1 and 2 - United States, 1999-2010. J Infect Dis. 2014;209(3):325-333.

43. Bernstein DI, Bellamy AR, Hook EW III, et al. Epidemiology, clinical presentation, and antibody response to primary infection with herpes simplex virus type 1 and type 2 in young women. Clin Infect Dis. 2013;56(3):344-351. 
44. Roberts CM, Pfister JR, Spear SJ. Increasing proportion of herpes simplex virus type 1 as a cause of genital herpes infection in college students. Sex Transm Dis. 2003;30(10):797800.

45. Fanfair RN, Zaidi A, Taylor LD, et al. Trends in seroprevalence of herpes simplex virus type 2 among non-Hispanic Blacks and non-Hispanic Whites aged 14 to 49 years United States, 1988 to 2010. Sex Transm Dis. 2013;40(11):860-864.

46. McQuillan G, Kruszon-Moran D, Flagg EW, et al. Prevalence of herpes simplex virus type 1 and type 2 in persons aged 14-49: United States, 2015-2016. NCHS data brief, no 304. Hyattsville, MD: National Center for Health Statistics. 2018.

47. Flagg EW, Torrone EA. Seroprevalence of herpes simplex virus type 1 and type 2 infections among adults diagnosed with genital herpes: United States, 1999-2016. Sex Transm Dis. 2018;45(Supp1 2):S51.

48. Kimberlin DW. The scarlet H. $J$ Infect Dis. 2014;209(3):315-317.

49. Copen CE, Chandra A, Martinez G. Prevalence and timing of oral sex with opposite-sex partners among females and males aged 15-24 years: United States, 2007-2010. National health statistics reports; no 56. Hyattsville, MD: National Center for Health Statistics. 2012.

50. Langenberg AGM, Corey L, Ashley RL, et al. A prospective study of new infections with herpes simplex virus type 1 and type $2 . N$ Engl J Med. 1999;341:1432-1438.

51. Sampath A, Maduro G, Schillinger JA. Infant deaths due to herpes simplex virus, congenital syphilis, and HIV in New York City. Pediatrics. 2016;137(4):e20152387.
52. Brown ZA, Wald A, Morrow RA, et al. Effect of serologic status and cesarean delivery on transmission rates of herpes simplex virus from mother to infant. JAMA. 2003;289(2):203-209.

53. Patton ME, Bernstein K, Liu G, et al. Seroprevalence of herpes simplex virus types 1 and 2 among pregnant women and sexually active, non-pregnant women in the United States. Clin Infect Dis. 2018;67(10):1535-1542.

54. French JI, McGregor JA, Parker R. Readily treatable reproductive tract infections and preterm birth among Black women. Am J Obstet Gynecol. 2006;194:1717-1727.

55. Patel EU, Gaydos CA, Packman ZR, et al. Prevalence and correlates of Trichomonas vaginalis infection among men and women in the United States. Clin Infect Dis. 2018;67(2):211-217.

56. Flagg EW, Meites E, Phillips C, et al. Prevalence of Trichomonas vaginalis among males and females aged 14-59 years: United States, 2013-2016. Sex Transm Dis. 2019; Published ahead of print. DOI: 10.1097/ olq.0000000000001013.

57. Sutton M, Sternberg M, Koumans EH, et al. The prevalence of Trichomonas vaginalis infection among reproductive-age women in the United States, 2001-2004. Clin Infect Dis. 2007;45(10):1319-1326.

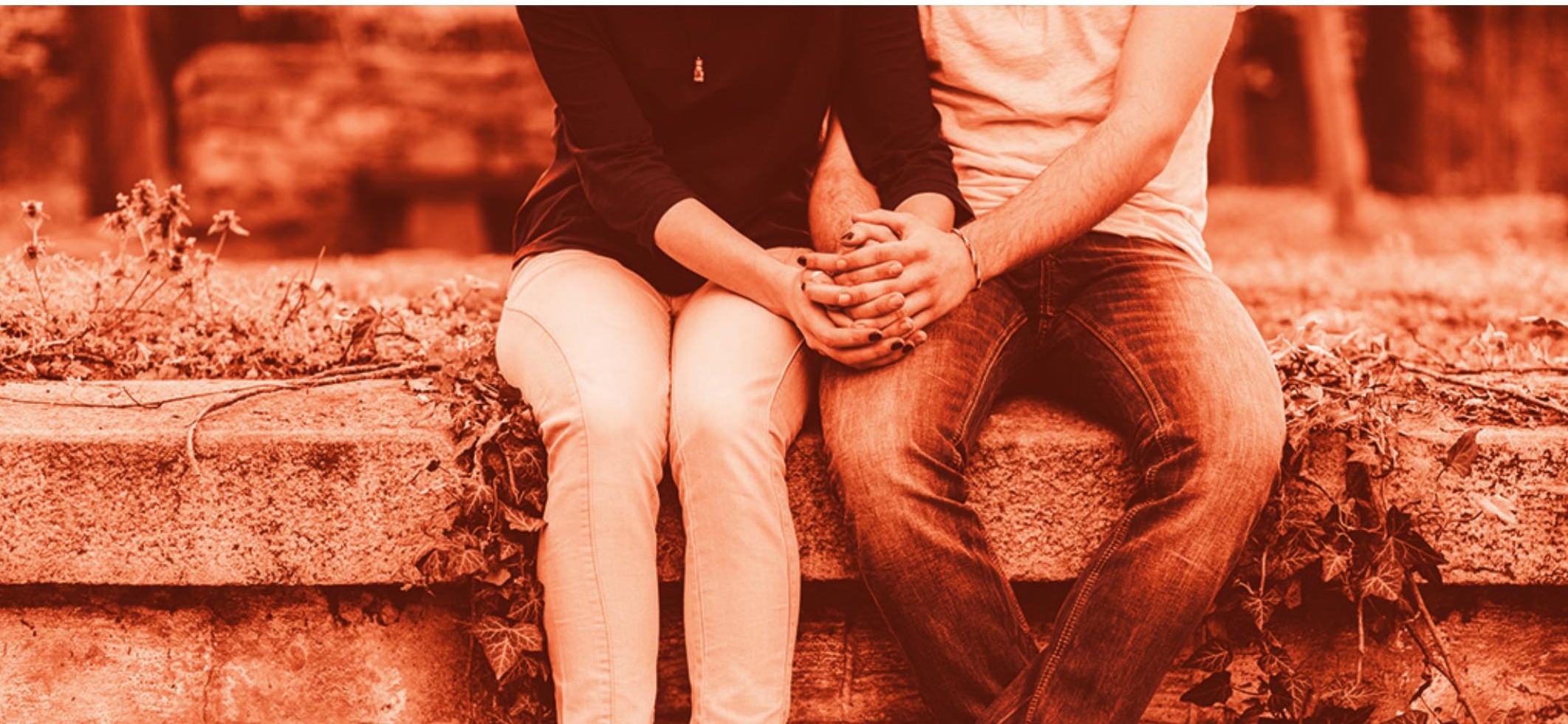




\section{Special Focus Profiles}

The Special Focus Profiles highlight trends and distribution of STDs in populations of particular interest to STD and HIV prevention programs in state and local health departments: women and infants, adolescents and young adults, racial and ethnic minority groups, and gay, bisexual, and other men who have sex with men (MSM).

\section{STDs in Women and Infants}

\section{Background}

Complications of sexually transmitted infections disproportionately affect women of all ages, with important implications for women of reproductive age. Undiagnosed and untreated STDs can lead to pelvic inflammatory disease (PID), ectopic pregnancy, as well as adverse fetal and neonatal outcomes. STDrelated morbidity disproportionately occurs in women for a number of reasons. Women are biologically more susceptible than men to the acquisition of some STDs and more likely to suffer from complications. It is also important to note that STDs are often asymptomatic in women, delaying diagnosis and treatment until there is a symptomatic complication. A female's sexual and reproductive health can also be interrelated to her particular social, cultural, and economic environment, creating conditions for risky sexual behaviors. Several factors, including the use of alcohol or recreational drugs inhibiting the ability to negotiate safer sexual practices, diminished gender power, high-levels of concurrency, poverty, homelessness or unstable housing, and partner violence may contribute to the challenges women face in protecting their sexual wellbeing. ${ }^{1-5}$ In some circumstances, maintaining the relationship with a partner may take a higher priority than STD risk reduction, affecting her reproductive health, as well as the health of her unborn baby. ${ }^{6} \mathrm{~A}$ woman can also be placed at risk for STDs through her partner's sexual encounter with an infected partner. Consequently, even a female who has only one partner may be obliged to practice safer sex, such as using condoms. $^{7}$

\section{Impact on Women and Fertility}

\section{Human Papillomavirus}

Human papillomavirus (HPV) is a common sexually transmitted infection in the United States. ${ }^{8,9}$ Although most HPV infections in women appear to be transient and may not result in clinically significant sequelae, ${ }^{10}$ high-risk HPV-type infections can cause abnormal changes in the uterine cervical epithelium, ${ }^{10,11}$ which are detected by cytological examination of Papanicolaou (Pap) smears. ${ }^{12}$ Persistent high-risk HPV-type 
infections may lead to cervical cancer precursors, which if undetected can result in cancer, ${ }^{11}$ and excisional treatment of cervical lesions can increase risk for future preterm delivery. ${ }^{13}$ Other low-risk HPV-type infections can cause genital warts, ${ }^{11,14}$ low-grade Pap smear abnormalities, ${ }^{11,15}$ laryngeal papillomas, ${ }^{16}$ and, rarely, recurrent respiratory papillomatosis in children born to infected mothers. ${ }^{17,18}$

Starting in 2006, HPV vaccines have been recommended for routine use in United States females aged 11-12 years, with catch-up vaccination through age 26. ${ }^{19,20} \mathrm{HPV}$ vaccination also has been recommended for routine use in males since $2011 .{ }^{20-23}$ In October 2018, the Food and Drug Administration (FDA) extended licensing approval of the vaccine for women and men aged 27-45 years, ${ }^{24}$ and in June 2019 the CDC's Advisory Committee on Immunization Practices (ACIP) recommended that unvaccinated adults aged $27-45$ years discuss receiving the HPV vaccine with their health care providers. ${ }^{23}$ For more information on HPV vaccination, see Other STDs.

A recent meta-analysis that included data from over 60 million individuals from 14 high-income countries, including the United States, showed a substantial impact of HPV vaccination on genital HPV infections and anogenital warts among adolescent girls and young women, and highgrade cervical lesions among young women. ${ }^{25}$ Cervicovaginal prevalence of any quadrivalent HPV vaccine type has been estimated for civilian, non-institutionalized, females aged 14-34 years using data from the National Health and Nutrition Examination Survey (NHANES). ${ }^{26}$ Prevalence decreased significantly from 2003-2006 (the pre-vaccine era) to 2011-2014 in specimens from females aged 14-19 years and 20-24 years, the age groups most likely to benefit from HPV vaccination. For more information on HPV infections, see Other STDs.

\section{Chlamydia}

Chlamydial infections in women are usually asymptomatic and screening is necessary to identify most infections. Routine chlamydia screening of sexually-active young women has been recommended by the CDC since 1993. ${ }^{27}$ Rates of reported cases of chlamydia among women increased steadily from the early 1990 s, likely reflecting expanded screening coverage and use of more sensitive diagnostic tests (Table 1). During 2011-2013, chlamydia case rates decreased from 643.4 to 619.0 cases per 100,000 females and then increased $11.9 \%$ over the next five years, resulting in a rate of 692.7 cases per 100,000 females in 2018 (Figure 1, Table 4).
Chlamydia rates are highest among young women, the population targeted for screening (Figures 5 and 6, Table 10). During 2017-2018, rates of reported chlamydia cases increased $1.3 \%$ and $0.8 \%$ among females aged 15-19 and 20-24 years, respectively (Figure 6). Regionally, among all women, chlamydia case rates were highest among women in the South, with a rate of 744.2 cases per 100,000 females in 2018 (Table 4). Rates of reported chlamydia cases exceeded gonorrhea case rates among women in all regions (Figures $\mathrm{A}$ and $\mathrm{C}$, Tables 4 and 15).

\section{Chlamydia Positivity in Selected Populations}

The STD Surveillance Network (SSuN) is an ongoing collaboration of state, county, and city health departments from 10 participating jurisdictions where demographic,

\section{Figure A. Chlamydia - Rates of Reported Cases Among Females by State and Territory, United States, 2018}

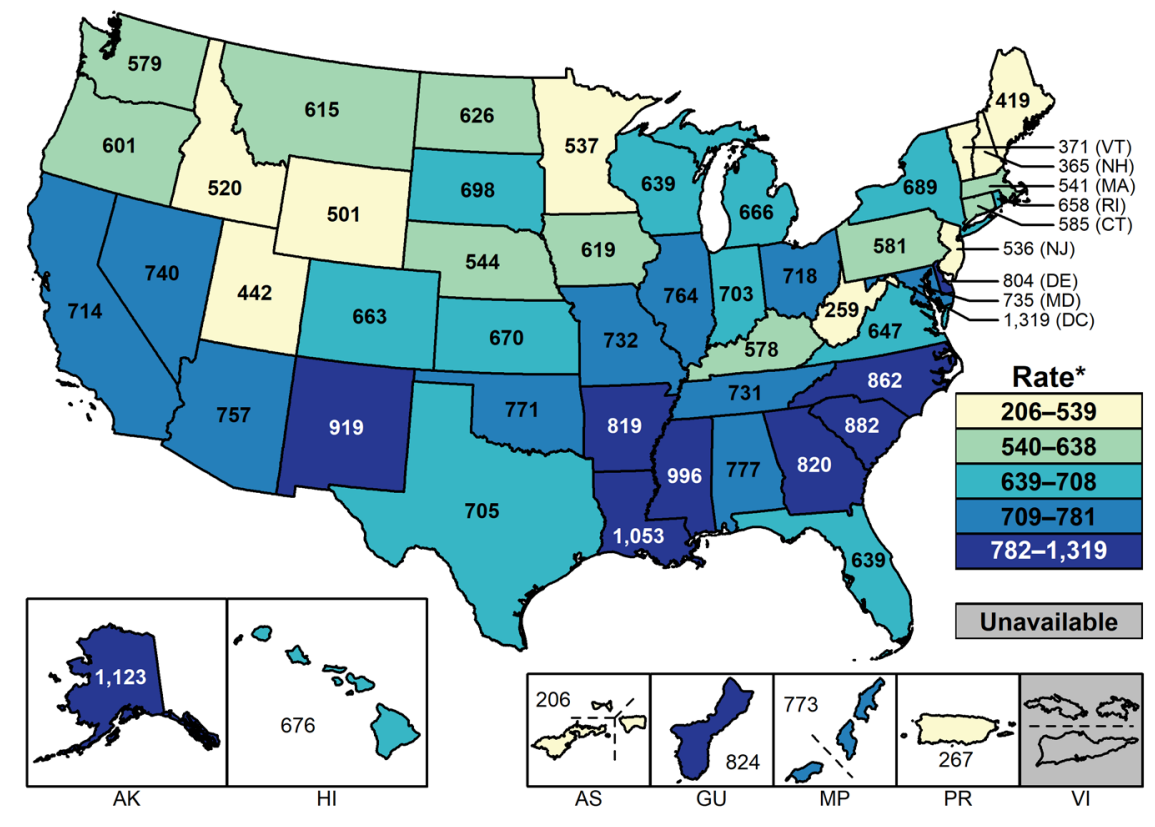

* Per $100,000$.

NOTE: See Sections A1.11 in the Appendix for more information on interpreting reported rates in US territories. 
Figure B. Chlamydia - Positivity Among Females Aged 14-39 Years by Race/Hispanic Ethnicity and Age Group in Clinics* Providing Family Planning and Reproductive Health Services, STD Surveillance Network (SSuN), 2018

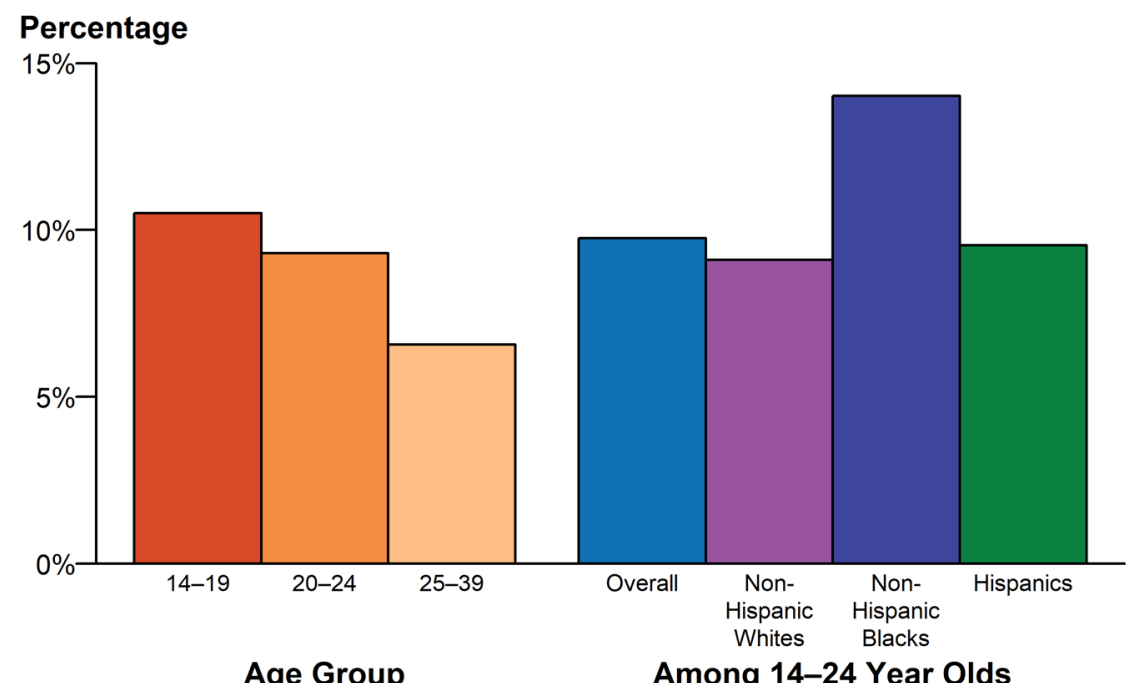

* Includes clinics $(n=26)$ that tested $>100$ females for chlamydia in 2018 and testing coverage was $>60 \%$.

NOTE: See section A2.2 in the Appendix for SSUN methods.

Figure C. Gonorrhea - Rates of Reported Cases Among Females by State and Territory, United States, 2018

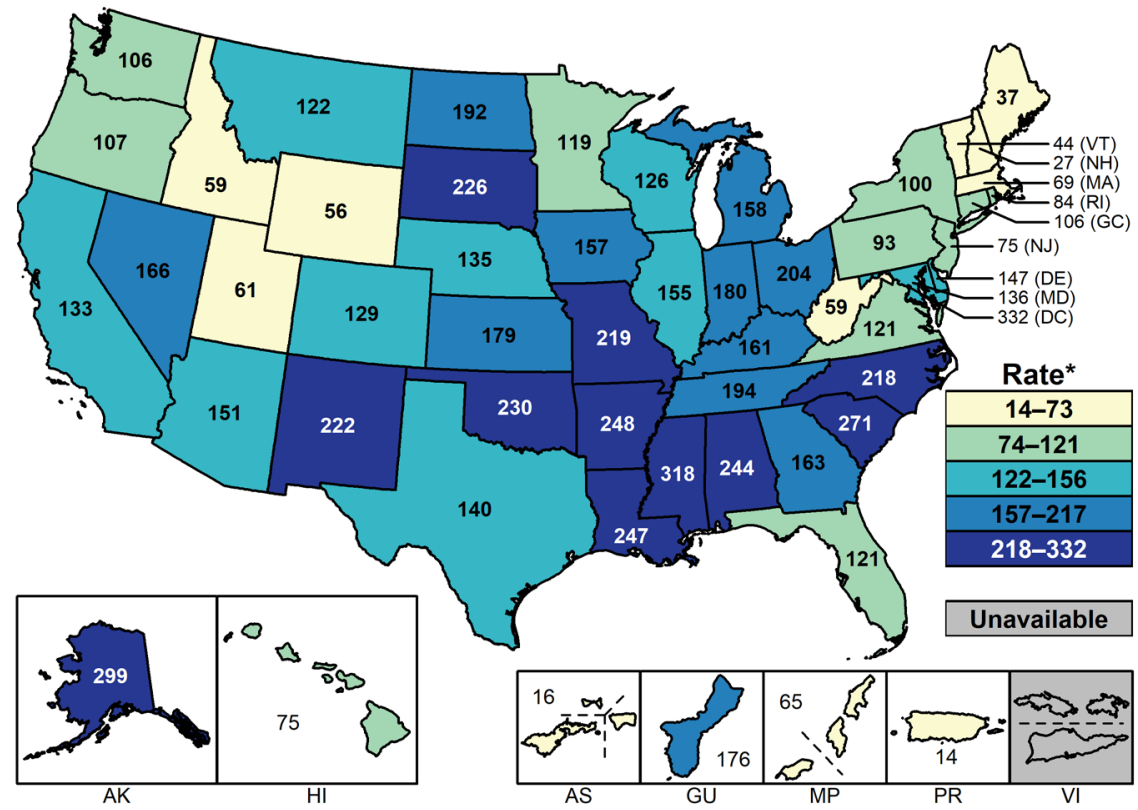

* Per 100,000 .

NOTE: See Sections A1.11 in the Appendix for more information on interpreting reported rates in US territories. clinical, and laboratory data are collected from women aged 15-44 years attending facilities that provide family planning and reproductive health services (See Section A2.2 of the Appendix). However, in the summer of 2018, data collection in these clinic settings ended and the results presented here only include data obtained from January through June 2018. Figure B shows chlamydia testing and positivity reported only among facilities that tested more than 100 females and more than $60 \%$ of young females aged 14-24 years. In 2018, the overall positivity of chlamydia among females aged 14-24 years was $9.8 \%$, but for females 14 19 years of age, chlamydia positivity was $10.5 \%$. For females between the ages of 14-24 years, chlamydia positivity among non-Hispanic Blacks was about 1.5 times those of nonHispanic Whites or Hispanics.

\section{Gonorrhea}

Like chlamydia, gonorrhea is often asymptomatic in women. Therefore, gonorrhea screening is an important strategy for the identification of gonorrhea among women. Large-scale screening programs for gonorrhea in women began in the 1970s. After an initial increase in cases detected through screening, rates of reported gonorrhea cases for both women and men declined steadily throughout the 1980s and early 1990s, and then declined more gradually in the late 1990s and the 2000s. However, more recently, there have been increases in overall cases (Figure 14, Table 1).

After reaching a 40-year low in 2009 (104.5 cases per 100,000 females), the rate of reported cases of gonorrhea for females increased slightly each year during 2009-2011, and then decreased during 2012-2014 (Figure 18). During 2015-2018, the gonorrhea rate among women increased $37.2 \%$ to 145.8 cases per 100,000 females (Figure 18, Table $15)$. 
The gonorrhea case rate among females was slightly higher than the rate among males during 2009-2012; however, the rate among males was higher than the rate among females during 2013-2018 (Figure 18, Tables 15 and 16). During 2014-2018, gonorrhea rates among females were highest among those aged 15-24 years (Figure 20, Table 21). For women in this age group, rates were highest among 19-year olds in 2018 (877.3 cases per 100,000 females) (Table 23).

\section{Pelvic Inflammatory Disease}

Data from studies suggest that as much as $10 \%$ of untreated chlamydial infections progress to clinically diagnosed PID and the risk with untreated gonococcal infection may be even higher. ${ }^{28-30} \mathrm{PID}$ is a major concern because it can result in inflammation and damage to the fallopian tubes, elevating the risk of infertility and ectopic pregnancy. Tubal factor infertility ranks among the most common causes of infertility, accounting for $30 \%$ of female infertility in the United States, ${ }^{31}$ and much of this damage results from previous episodes of PID. ${ }^{32}$ An important public health measure for preventing PID, and ultimately tubal factor infertility, is through the prevention and control of Chlamydia trachomatis and Neisseria gonorrhoeae. Strategies to improve the early detection and treatment of chlamydia, as demonstrated in randomized controlled trials,,$^{29,33}$ has been shown to reduce a woman's risk for PID and ultimately protect the fertility of women.

Accurate estimates of PID and tubal factor infertility resulting from chlamydial and gonococcal infections are difficult to obtain, in part because definitive diagnoses of these conditions can be complex. The National Disease and Therapeutic Index (NDTI; see Section A2.5 in the Appendix) provides estimates of initial visits to office-based, private physicians for PID. NDTI estimated that during 2007-2016 the number of initial visits to such physicians for PID among women aged 15-44 years decreased by $38.3 \%$ from 146,000 to 90,000 visits (Figure D). Similar declines have been observed in nationally representative data of emergency department (ED) visits from the Healthcare Cost and Utilization Project (HCUP), the largest collection of all-payer encounter level inpatient, ED, and ambulatory services data in the United States. According to an analysis using HCUP's Nationwide Emergency Department Sample (NEDS), the percentage of ED visits with a PID diagnosis decreased during 2006-2013 among females aged 15-44 years, with the largest decreases among females aged 15-19 years (Figure E).$^{34}$ It is not entirely clear what may be driving these declines, though several factors have been suggested, including earlier identification and treatment of chlamydia and gonorrhea infection and availability of single-dose therapies that increase adherence to treatment. ${ }^{35-37}$ While PID is declining nationally, it is still a major cause of morbidity in women.

Differences in self-reported lifetime diagnosis of PID by race/Hispanic ethnicity in reproductive age women have been observed in earlier research..$^{38}$ Data from the 2013-2014 cycle of NHANES indicates that non-Hispanic Black and non-Hispanic White women reporting a previous STI diagnosis had nearly equal selfreported lifetime PID prevalence (10.3\% vs. 10.0\%) (Figure F). ${ }^{39}$ However, the lifetime prevalence of PID among non-Hispanic Black women was 2.2 times that among non-Hispanic White women if no previous STI was diagnosed $(6.0 \%$

\section{Figure D. Pelvic Inflammatory Disease - Initial Visits to Physicians' Offices Among Females Aged 15-44 Years, United States, 2007-2016}

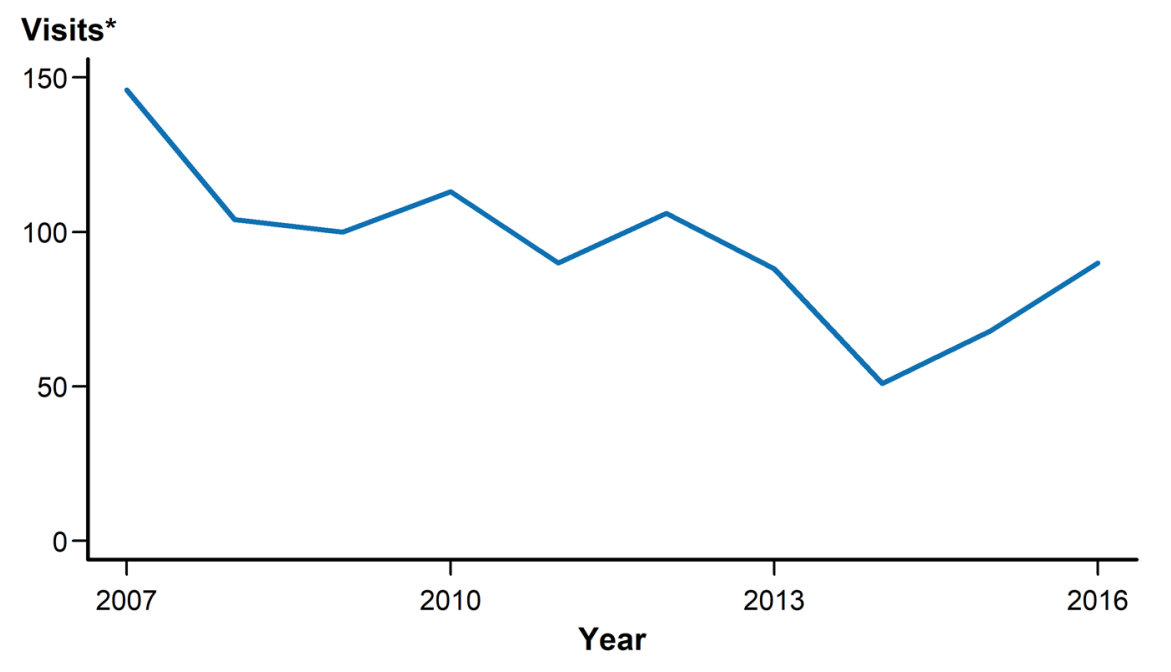

* In thousands.

NOTE: The relative standard errors for these estimates are $23 \%-16 \%$. See section A2.5 in the Appendix and Table 44.

SOURCE: National Disease and Therapeutic Index, IMS Health, Integrated Promotional Services $^{\mathrm{TM}}$, IMS Health Report, 1966-2016. 
Figure E. Estimated Percentage of Acute Pelvic Inflammatory Disease Emergency Department Visits Among Females Aged 15-44 Years by Age Group and Year, United States, 2006-2013

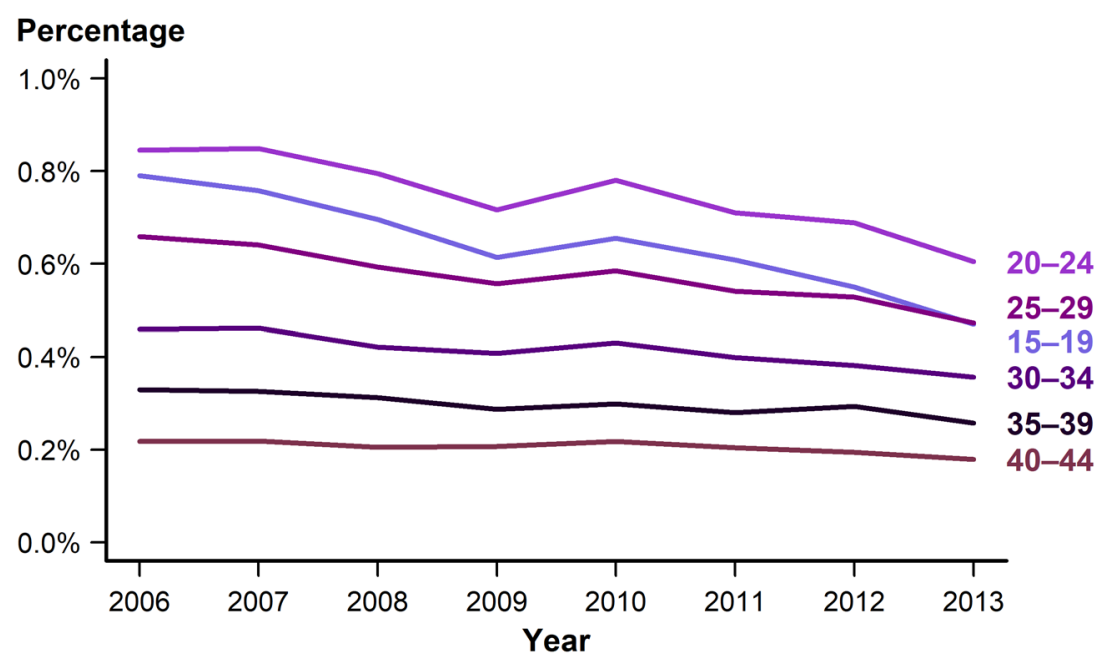

SOURCE: Kreisel, K, Flagg, EW, Torrone E. Trends in pelvic inflammatory disease emergency department visits, United States, 2006-2013. Am J Obstet Gynecol. 2018; 218(1): 117.e1-117.e10.

Figure F. Pelvic Inflammatory Disease - National Estimates of Lifetime Prevalence Among Sexually Experienced Women Aged 18-44 Years by Race/Hispanic Ethnicity and Previous STD Diagnosis, National Health and Nutrition Examination Survey (NHANES), 2013-2014

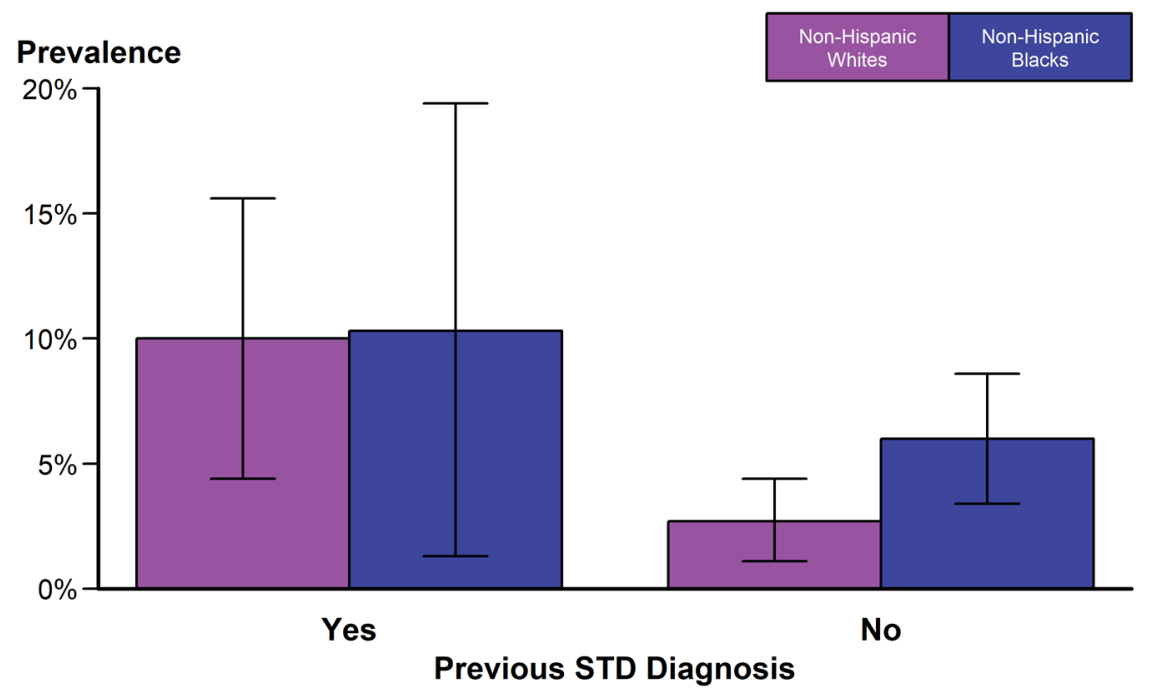

NOTE: Error bars indicate 95\% confidence intervals. Prevalence estimates among non-Hispanic Black women with a previous STD diagnosis have a relative standard error $>40 \%$ but $<50 \%$.

SOURCE: Kreisel, K, Torrone, E, Bernstein, K, et al. Prevalence of pelvic inflammatory disease in sexually experienced women of reproductive age — United States, 2013-2014. MMWR Morb Mortal Wkly Rep. 2017; 66(3):80-83. vs. $2.7 \%$ ). These findings suggest that PID is associated with previous STI diagnoses and it is therefore important for physicians to screen female patients for chlamydia and gonorrhea to reduce the incidence of PID. The racial disparities observed in PID diagnoses are consistent with the marked racial disparities observed for chlamydia and gonorrhea. However, because of the subjective methods by which PID is diagnosed, racial disparity data should be interpreted with caution.

\section{Impact on Pregnancy and Fetal Outcomes}

The sexually transmitted infections implicated in adverse pregnancy outcomes are broad and include viral, bacterial and protozoal infections. The spectrum of reproductive outcomes following STIs not only threatens the health of women and their ability to reproduce but can extend to detrimental effects on the fetus and neonates of infected individuals. Access to care and the provider's ability to assess risk, screen, and treat STIs are critical factors in improving obstetrical outcomes.

\section{Ectopic Pregnancy}

Ectopic pregnancy, defined as implantation of a fertilized ovum on any tissue other than the lining of the uterus, is a potentially lifethreatening condition that requires prompt evaluation and treatment. The ability to ascertain the number of ectopic pregnancies occurring in the United States has been affected by a shift in clinical management from an inpatient to an outpatient event, making inpatient hospital surveillance data sources unreliable. As a result, alternative surveillance methods, including data from large administrative claims, ${ }^{39,40}$ or emergency departments have been used to evaluate trends and assess the continued public health burden of this condition. Data from MarketScan Commercial Claims and Encounters Database, a large administrative 
claims database of United States commercial health plans, indicate that the ratio of ectopic pregnancy diagnoses to all live births among women with live births aged 15-44 years during the period of 2006-2017 have marginally increased across all age groups (Figure G). As in previous years, in 2017, rates of ectopic pregnancy were highest among women in the 35-44 year age groups.

\section{Neonatal Conjunctivitis}

Maternal infection with C. trachomatis or $N$. gonorrhoeae can also affect the infant, leading to conjunctivitis infections (termed ophthalmia neonatorum in the first four weeks of life), and, in the case of $C$. trachomatis, pneumonia. Although topical prophylaxis of infants at delivery may be effective for prevention of gonococcal ophthalmia neonatorum, prevention of neonatal pneumonia requires prenatal detection and treatment. The clinical presentation of conjunctivitis can be variable and these infections are especially important to treat promptly, as they can lead to visual impairment. ${ }^{41}$

During 2014-2018, 438 chlamydia or gonorrhea cases among infants aged $<1$ year with a specimen source of either 'eye' or 'conjunctiva' (conjunctivitis infections) were reported to CDC. The overall reported rate of chlamydial conjunctivitis in infants was relatively stable during 2014-2018, ranging from 1.4 to 2.3 cases per 100,000 live births (Figure $\mathrm{H})$. Similarly, the rate of gonococcal conjunctivitis in infants remained relatively constant and low during 2014-2018, ranging from $0.2-0.4$ cases per 100,000 live births. The rate of reported cases is heavily influenced by the completeness of reported data on specimen source. Of all cases reported to CDC of chlamydia or gonorrhea in infants aged $<1$ year during 2014-2018 ( $\mathrm{n}=2,348), 81.3 \%$ did not have a specimen source of either 'eye' or 'conjunctiva'; of those, $62.3 \%$ had a specimen source
Figure G. Ectopic Pregnancy - Ratio* Among Commercially Insured Females with Live Births Aged 15-44 Years by Age Group, 2006-2017

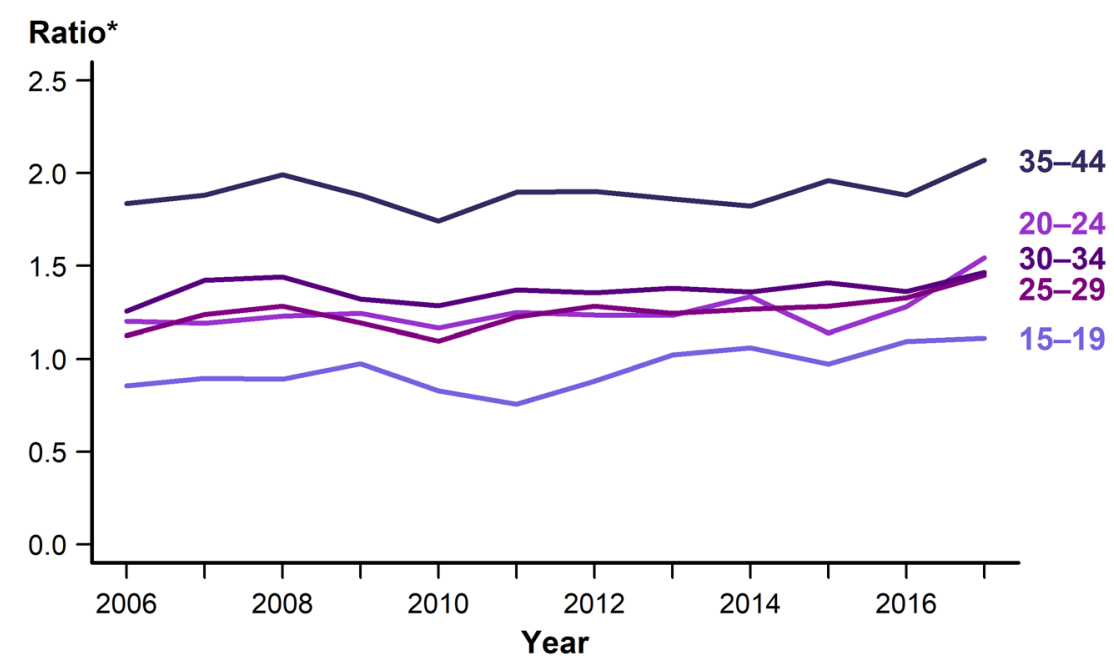

* Ratios represent the number of ectopic pregnancy diagnoses per 100,000 live births. SOURCE: MarketScan Commercial Claims and Encounters Database, Truven Health Analytics, Ann Arbor, MI, 2006-2017.

\section{Figure H. Chlamydia and Gonorrhea - Rates of Reported Cases Among Infants < 1 Year of Age by Year and Specimen Source, United States, 2014-2018}

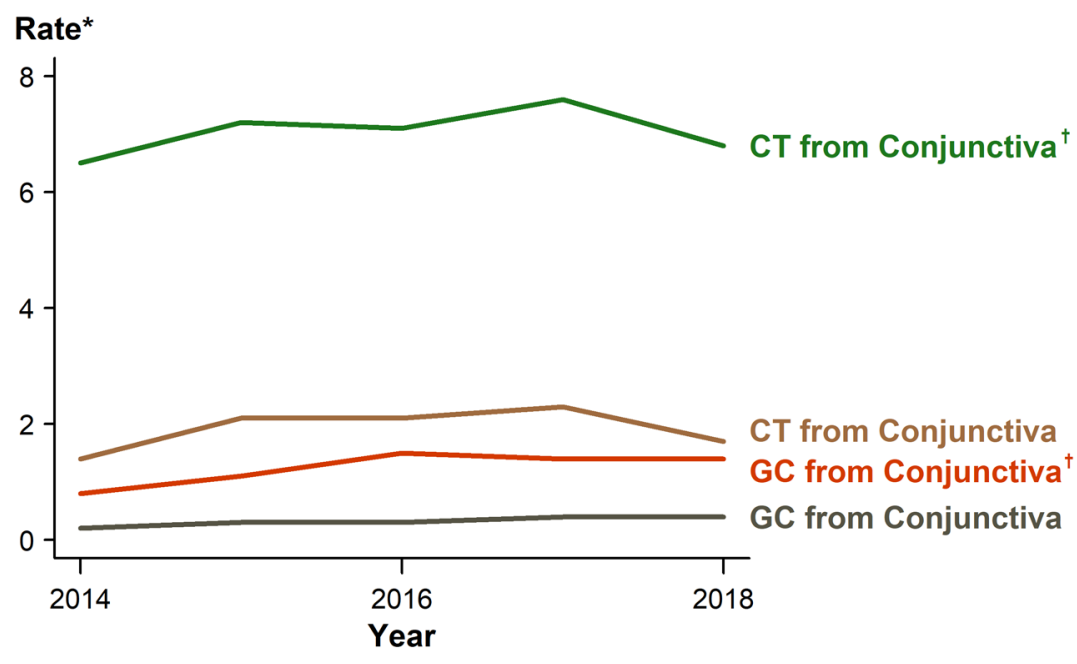

* Per 100,000 live births.

${ }^{\dagger}$ Includes cases with specimen source reported as missing, unknown, or other.

ADAPTED FROM: Kreisel K, Weston E, Braxton J, et al. Keeping an eye on chlamydia and gonorrhea conjunctivitis in infants in the United States, 2010-2015. Sex Transm Dis. 2017; 44(6): 356-358.

ACRONYMS: $\mathrm{CT}=$ Chlamydia; $\mathrm{GC}=$ Gonorrhea. 
of 'unknown' (46.6\%), 'other-not specified' (10.6\%), or was missing $(5.1 \%)$. When evaluating rates including these cases, the rate of chlamydia and gonorrhea infections follows similar trends but is higher in all years, indicating potential missed cases for surveillance (Figure H).

\section{Congenital Syphilis}

Syphilis is an important risk factor for adverse pregnancy outcomes. The consequences of untreated maternal infection can include fetal and infant death, preterm birth, and congenital infection in a proportion of surviving infants, resulting in both physical and mental developmental disabilities.

Most cases of congenital syphilis are preventable if women are screened for syphilis and treated early during prenatal care.

Trends in congenital syphilis usually mirror trends in primary and secondary (P\&S) syphilis among reproductive-aged women. After plateauing at a relatively low rate (0.9 cases per 100,000 females) during 2011-2013, the rate of reported cases of P\&S syphilis among all women has increased each year since then (Figure 49). During 2014-2018, the rate among women increased $172.7 \%$, from 1.1 to 3.0 cases per 100,000 females (Table 28). During this same period, the rate among reproductiveaged women (women aged 15-44 years) increased $165.4 \%$, from 2.6 to 6.9 cases per 100,000 females aged 15-44 years (Figure 49).

Similarly, the rate of reported congenital syphilis cases has increased each year since 2012 (Table 1). In 2018 , there were 1,306 reported cases of congenital syphilis, with a rate of 33.1 cases per 100,000 live births, the highest rate reported since 1995 . This increase in 2018 represents a $39.7 \%$ increase relative to 2017 and a $291.0 \%$ increase relative to 2012 (Table 41).

In 2018, the highest rates of reported $\mathrm{P} \& \mathrm{~S}$ syphilis cases among women and the highest rates of reported congenital syphilis cases were observed in the West and in the South (Figures I and J, Tables 28 and 41). The rate of $\mathrm{P} \& \mathrm{~S}$ syphilis among women increased in every region during 2017-2018. During 20172018 , the largest rate increase among women occurred in the West (41.2\%), followed by the Northeast $(40.0 \%)$, South (30.8\%), and Midwest (30.8\%) (Table 28). The congenital syphilis rate increased $49.5 \%$ in the South, $44.1 \%$ in the Northeast, $30.5 \%$ in the Midwest, and $29.3 \%$ in the West during 2017-2018 (Table 41).

Although most cases of congenital syphilis occur among infants whose mothers have had some prenatal care, late or limited prenatal care has been associated with congenital syphilis. Failure of health care providers to adhere to prenatal syphilis screening recommendations, as well as acquisition of infection during pregnancy after the initial screening test, also contribute to the occurrence of congenital syphilis.

\section{Neonatal Herpes Simplex Virus}

Herpes simplex virus (HSV) is among the most prevalent of sexually transmitted infections, ${ }^{8}$ and can have serious consequences for pregnant women and their infants..$^{42}$ Most genital HSV infections in the United States are caused by HSV type 2 (HSV-2), while HSV type 1 (HSV1) infections are typically orolabial and acquired during childhood. ${ }^{43,44}$ NHANES data show that HSV-1 seroprevalence has significantly decreased among adolescents, indicating declining orolabial infection; ${ }^{44} \mathrm{HSV}-2$ seroprevalence in this age group was much lower. ${ }^{44}$ Those who lack HSV-1 antibodies at sexual debut are more susceptible to genital HSV-1 infection, ${ }^{44,45}$ and are also at increased risk of developing symptomatic disease from newly-

\section{Figure I. Congenital Syphilis - Rates of Reported Cases by State and Territory, United States, 2018}

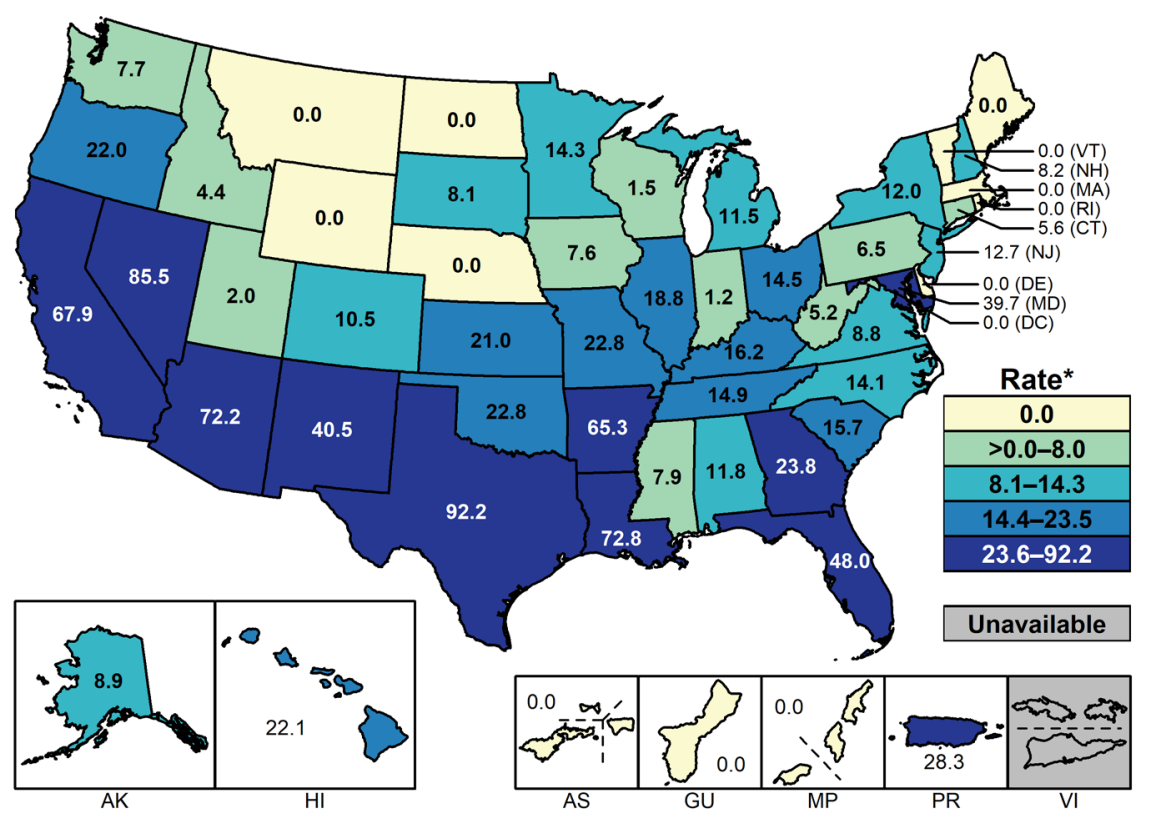

* Per 100,000 live births.

NOTE: See Section A1.11 in the Appendix for more information on interpreting rates for US territories. 


\section{Figure J. Primary and Secondary Syphilis - Rates of Reported Cases Among Females by State and Territory, United States, 2018}

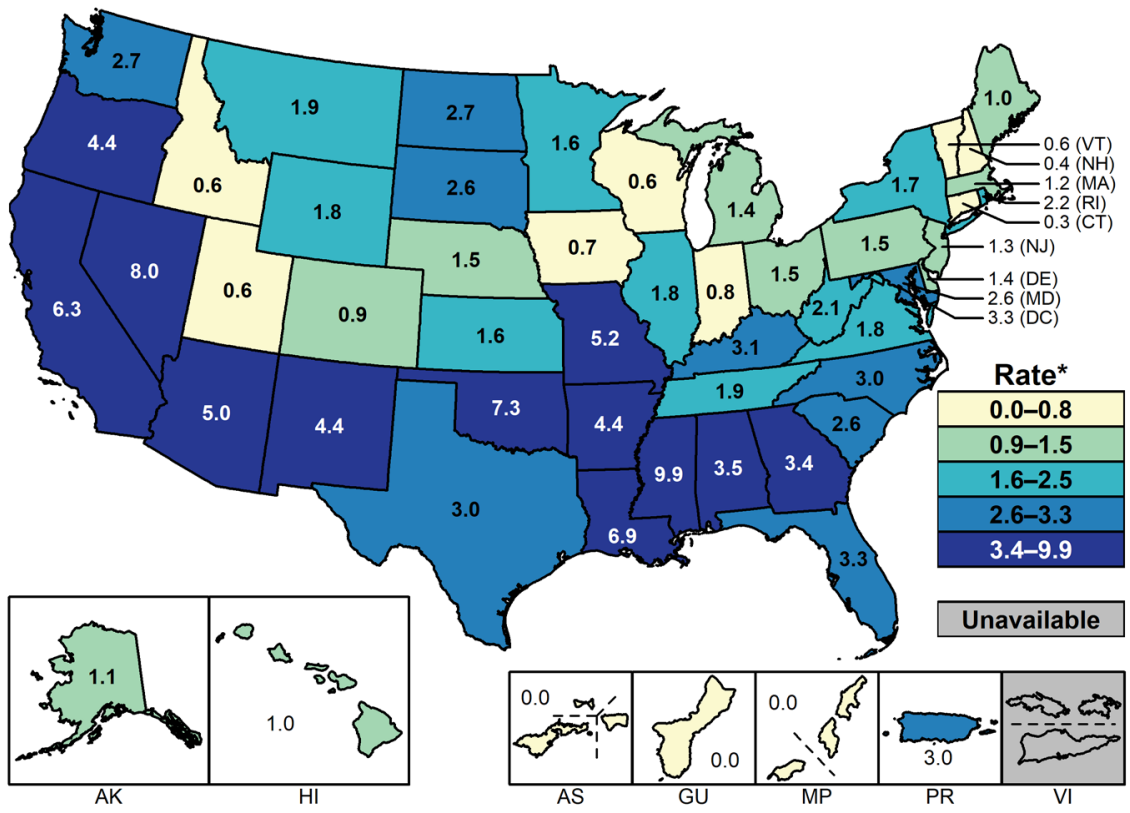

* Per 100,000

NOTE: See Sections A1.11 in the Appendix for more information on interpreting reported rates in US territories.

acquired (i.e., primary) genital HSV-2 infection. ${ }^{46}$ Young women may therefore be increasingly likely to first acquire HSV-1 infection genitally, or acquire a primary genital HSV-2 infection, during their child-bearing years $;{ }^{45,47}$ first-episode primary genital HSV infection during pregnancy increases the risk of neonatal HSV transmission, ${ }^{45,48}$ particularly if the mother acquires infection towards the end of her pregnancy ${ }^{45,49}$ Another analysis of NHANES data found that among pregnant women with three or fewer lifetime sex partners, seronegativity for both HSV-1 and HSV-2 increased from 1999-2006 to $2007-2014,{ }^{50}$ raising the possibility that pregnant women with fewer sex partners may have increased risk of acquiring genital HSV during pregnancy and vertically transmitting HSV to their neonates. For more information on genital HSV infections, see Other STDs.
Rates did not vary significantly by region or by race/Hispanic ethnicity; however prevalence was significantly higher among cases for which the expected primary payer was Medicaid (15.1 cases per 100,000 live births) compared with private insurance or managed health care (5.4 cases per 100,000 live births). A recent study using the HCUP National Inpatient Sample (NIS) found that incidence of neonatal HSV infection per 100,000 live births significantly increased from 2003 to 2014, from 7.9 during 2003-2005 to 10.0 during 2012$2014 .{ }^{55}$

In New York City, 76 cases of neonatal HSV infection were identified through population-based surveillance during a 4.5 year period (April 2006-September 2010), for an average annual incidence of 13.3 cases per 100,000 live births. ${ }^{47}$ Fortyone percent of the confirmed cases were infected with HSV-1. A review of certificates of death or stillbirth issued in New York City during 1981-2013 identified 34 deaths due to neonatal HSV infection, or 0.82 deaths per 100,000 live births. ${ }^{53}$

\section{Summary}

STDs are an important health priority and their substantial morbidity and mortality related to sequelae can often be overlooked in women. This is particularly true for reproductive aged women and their infants. The overall rate of reported female chlamydia cases has increased $11.4 \%$ over the last four years, much of that attributed to increased screening and more complete national reporting. Gonorrhea infections among females have also increased $45.2 \%$ to 145.8 cases per 100,000 females in recent years. Surveillance data continues to show that numbers and rates of chlamydia and gonorrhea cases are highest in females between the ages of 15 and 24, and certain races/ 
ethnicities are disproportionately impacted. Despite increases in reported cases of chlamydia and gonorrhea, available data suggest an overall decline in the incidence of PID, largely attributed to an increase in effective screening and treatment of chlamydial and gonococcal infections in adolescents and young women. In contrast to declining PID rates, data suggests rates of ectopic pregnancy have marginally increased over time.

\section{Mother to child transmission of} STDs can result in serious adverse consequences. Potential adverse neonatal outcomes include neonatal ophthalmia, neonatal pneumonia, and prematurity. The rate of congenital syphilis in the United States has increased every year since 2013. In 2018, there were 935 reported cases of congenital syphilis and the national congenital syphilis rate was 23.7 cases per 100,000 live births, the highest rate in two decades. Despite current recommended STD screening during pregnancy, some women may not have their infections treated during pregnancy because of a lack of or limited prenatal care.

\section{References}

1. Hess KL, Javanbakht M, Brown JM, et al. Intimate partner violence and sexually transmitted infections among young adult women. Sex Transm Dis. 2012;39: 366-71.

2. Sharpe TT, Voute C, Rose MA, et al. Social determinants of HIV/AIDS and sexually transmitted diseases among Black women: Implications for health equity. J of Women's Health. 2012;21:249-254.

3. Hogben M, Leichliter JS. Social determinants and sexually transmitted disease disparities. Sex Transm Dis. 2008;35(Suppl 12):S13-18.

4. McCree DH, Rompalo A. Biological and behavioral risk factors associated with STDs/ HIV in women: Implications for behavioral interventions. In: Aral SO, Douglas JM, Lipshutz JA, eds. Behavioral Interventions for Prevention and Control of Sexually Transmitted Diseases. New York, NY: Springer; 2007:310324.

5. Jolly DH, Mueller MP, Chen M, et al. Concurrency and other sexual risk behaviors among black young adults in a southeastern city. AIDS Educ Prev. 2016;28(1):59-76.
6. Pulerwitz J, Amaro H, De Jong W, et al. Relationship power, condom use and HIV risk among women in the USA. AIDS Care. 2002;14(6):789-800.

7. Swartzendruber A, Zenilman JM, Niccolai LM, et al. It takes 2: Partner attributes associated with sexually transmitted infections among adolescents. STD. 2013;40(5):372-378.

8. Satterwhite CL, Torrone E, Meites E, et al. Sexually transmitted infections among US women and men: Prevalence and incidence estimates, 2008. Sex Transm Dis. 2013;40(3):187-193

9. McQuillan G, Kruszon-Moran D, Markowitz LE, et al. Prevalence of HPV in adults aged 18-69: United States, 2011-2014. NCHS data brief, no 280. Hyattsville, MD: National Center for Health Statistics. 2017.

10. Insinga RP, Perez G, Wheeler CM, et al. Incident cervical HPV infections in young women: Transition probabilities for CIN and infection clearance. Cancer Epidemiol Biomarkers Prev. 2011;20(2):287-296.

11. Doorbar J, Quint W, Banks L, et al. The biology and life-cycle of human papillomaviruses. Vaccine. 2012;30(Suppl 5):F55-F70.

12. Saslow D, Solomon D, Lawson HW, et al. American Cancer Society, American Society for Colposcopy and Cervical Pathology, and American Society for Clinical Pathology screening guidelines for the prevention and early detection of cervical cancer. J Low Genit Tract Dis. 2012;16(3):175-204.

13. Wright TC, Massad S, Dunton CJ, et al. 2006 consensus guidelines for the management of women with cervical intraepithelial neoplasia or adenocarcinoma in situ. Am J Obstet Gynecol. 2007;197(4):340-345.

14. Garland SM, Steben M, Sings HL, et al. Natural history of genital warts: Analysis of the placebo arm of 2 randomized phase III trials of a quadrivalent human papillomavirus (types 6, 11, 16, and 18) vaccine. J Infect Dis. 2009;199(6):805-814.

15. Clifford GM, Rana RK, Franceschi S, et al. Human papillomavirus genotype distribution in low-grade cervical lesions: Comparison by geographic region and with cervical cancer. Cancer Epidemiol Biomarkers Prev. 2005;14(5):1157-1164.

16. Gissmann L, Wolnik L, Ikenberg H, et al. Human papillomavirus types 6 and 11 DNA sequences in genital and laryngeal papillomas and in some cervical cancers. Proc Natl Acad Sci USA. 1983;80(2):560-563.

17. Donne AJ, Clarke R. Recurrent respiratory papillomatosis: An uncommon but potentially devastating effect of human papillomavirus in children. Int J STD AIDS. 2010;21(6):381-385.

18. Armstrong LR, Preston EJ, Reichert M, et al. Incidence and prevalence of recurrent respiratory papillomatosis among children in Atlanta and Seattle. Clin Infect Dis. 2000;31(1):107-109.
19. Markowitz LE, Dunne EF, Saraiya M, et al. Quadrivalent human papillomavirus vaccine. Recommendations of the Advisory Committee on Immunization Practices (ACIP). MMWR Recomm Rep. 2007;56(No. RR-2):1-24.

20. Petrosky E, Bocchini Jr. JA, Hariri S, et al. Use of 9-valent human papillomavirus (HPV) vaccine: Updated HPV vaccination recommendations of the Advisory Committee on Immunization Practices. MMWR Morb Mortal Wkly Rep. 2015;64(11):300-304.

21. Centers for Disease Control and Prevention. Recommendations on the use of quadrivalent human papillomavirus vaccine in males Advisory Committee on Immunization Practices (ACIP), 2011. MMWR Morb Mortal Wkly Rep. 2011;60(50):1705-1708.

22. Meites E, Kempe A, Markowitz LE. Use of a 2-dose schedule for human papillomavirus vaccination - updated recommendations of the Advisory Committee on Immunization Practices. MMWR Morb Mortal Wkly Rep. 2016;65(49):1405-1408.

23. Meites E, Szilagyi PG, Chesson HW, et al. Human papillomavirus vaccination for adults: Updated recommendations of the Advisory Committee on Immunization Practices. $M M W R$ Morb Mortal Wkly Rep. 2019;68(32):698-702.

24. US Food and Drug Administration. "Supplement Approval Letter — Biologics License Application (BLA) for Human Papillomavirus 9-valent Vaccine, Recombinant (GARDASIL® 9)". Letter to Merck Sharp \& Dohme Corp. October 5, 2018. US Food and Drug Administration. 2018. https://www.fda. gov/media/117053/download. Accessed July 2, 2019.

25. Drolet M, Benard E, Perez N, et al. Populationlevel impact and herd effects following the introduction of human papillomavirus vaccination programmes: updated systematic review and meta-analysis. Lancet. 2019;394(10197):497-509.

26. Oliver SE, Unger ER, Lewis R, et al. Prevalence of human papillomavirus among females after vaccine introduction — National Health and Nutrition Examination Survey, United States, 2003-2014. J Infect Dis. 2017;216(5):594-603.

27. Centers for Disease Control and Prevention. Recommendations for the prevention and management of Chlamydia trachomatis infections, 1993. MMWR Recomm Rep. 1993;42(No. RR-12):1-39.

28. Haggerty CL, Gottlieb SL, Taylor BD, et al. Risk of sequelae after Chlamydia trachomatis genital infection in women. $J$ Infect Dis. 2010;201:S134-S155.

29. Oakeschott P, Kerry S, Aghaizu A, et al. Randomised controlled trial of screening for Chlamydia trachomatis to prevent pelvic inflammatory disease: The POPI (prevention of pelvic infection) trial. BMJ. 2010;340:c1642. 
30. Price MJ, Ades AE, De Angelis D, et al. Risk of pelvic inflammatory disease following Chlamydia trachomatis infection: Analysis of prospective studies with a multistate model. $\mathrm{Am}$ J Epidemiol. 2013;178(3):484-492.

31. Tsevat DG, Wiesenfeld HC, Parks C, et al. Sexually transmitted diseases and infertility. $A m$ J Obstet Gynecol. 2017;216(1):1-9.

32. Ness RB, Trautmann G, Richter HE, et al. Effectiveness of treatment strategies of some women with pelvic inflammatory disease: A randomized trial. Obste Gyneco. 2005;106(3):573-580.

33. Gottlieb SL, Xu F, Brunham RC. Screening and treating chlamydia genital infections to prevent PID: Interpretation of findings from randomized controlled trials. Sex Transm Dis. 2013;40(2):97-102.

34. Kreisel K, Flagg EW, Torrone E. Trends in pelvic inflammatory disease emergency department visits, United States, 2006-2013. Am J Obstet Gynecol. 2018;218:117.e111-110.

35. Bohm MK, Newman L, Satterwhite CL, et al. Pelvic inflammatory disease among privately insured women, United States, 2001-2005. Sex Transm Dis. 2010;37(3):131-136.

36. Whiteman MK, Kuklina E, Jamieson DJ, et al. Inpatient hospitalization for gynecologic disorders in the United States. Am J Obstet Gynecol. 2010;202(6):541.e541-546.

37. Owusu Edusei K Jr, Bohm MK, Chesson $\mathrm{HW}$, et al. Chlamydia screening and pelvic inflammatory disease: Insights from exploratory time-series analyses. Am J Prev Med. 2010;38(6):652-657.

38. Leichliter JS, Chandra A, Aral SO. Correlates of self-reported pelvic inflammatory disease treatment in sexually experienced reproductiveaged women in the United States, 1995 and 2006-2010. Sex Transm Dis. 2013;40(5):413-418.

39. Trabert B, Holt VL, Yu O, et al. Populationbased ectopic pregnancy trends, 1993-2007. Am J Prev Med. 2011;40(5):556-560.

40. Hoover KW, Tao G, Kent CK. Trends in the diagnosis and treatment of ectopic pregnancy in the United States. Obstet Gynecol. 2010;2010(115):3.

41. Kohlhoff SA, Hammerschlag MR. Gonococcal and chlamydial infections in infants and children. In: Sexually Transmitted Diseases. 4th ed. New York, NY: McGraw Hill; 2007:16131627.

42. Kimberlin DW. Herpes simplex virus infections of the newborn. Semin Perinatol. 2007;31(1):19-25.

43. Corey L, Wald A. Genital Herpes. In: Holmes KK, Sparling FP, Stamm WE, et al., eds. Sexually Transmitted Diseases. 4th ed. New York, NY: McGraw-Hill; 2008:399-437.

44. Bradley H, Markowitz LE, Gibson T, et al. Seroprevalence of herpes simplex virus types 1 and 2 - United States, 1999-2010. J Infect Dis 2014;209(3):325-333.
45. Kimberlin DW. The scarlet H. J Infect Dis. 2014;209(3):315-317.

46. Langenberg AGM, Corey L, Ashley RL, et al. A prospective study of new infections with herpes simplex virus type 1 and type $2 . N \mathrm{Engl} \mathrm{J} \mathrm{Med}$. 1999;341:1432-1438.

47. Sampath A, Maduro G, Schillinger JA. Infant deaths due to herpes simplex virus, congenital syphilis, and HIV in New York City. Pediatrics. 2016;137(4):e20152387.

48. Brown ZA, Wald A, Morrow RA, et al. Effect of serologic status and cesarean delivery on transmission rates of herpes simplex virus from mother to infant. JAMA. 2003;289(2):203-209.

49. Corey L, Wald A. Maternal and neonatal herpes simplex virus infections. $N$ Engl J Med. 2009;361(14):1376-1385.

50. Patton ME, Bernstein K, Liu G, et al. Seroprevalence of herpes simplex virus types 1 and 2 among pregnant women and sexually active, non-pregnant women in the United States. Clin Infect Dis. 2018;67(10):1535-1542.

51. Centers for Disease Control and Prevention. Neonatal herpes simplex virus infection following Jewish ritual circumcisions that included direct orogenital suction - New York City, 2000-2011. MMWR Morb Mortal Wkly Rep. 2012;61(22):405-409.

52. Dinh T-H, Dunne EF, Markowitz LE, et al. Assessing neonatal herpes reporting in the United States, 2000-2005. Sex Transm Dis. 2008;35(1):19-21.

53. Handel S, Klingler EJ, Washburn K, et al. Population-based surveillance for neonatal herpes in New York City, April 2006-September 2010. Sex Transm Dis. 2011;38(8):705-711.

54. Flagg EW, Weinstock H. Incidence of neonatal herpes simplex virus infections in the United States, 2006. Pediatrics. 2011;127(1):e1-e8.

55. Donda K, Sharma M, Amponsah JK, et al. Trends in the incidence, mortality, and cost of neonatal herpes simplex virus hospitalizations in the United States from 2003 to 2014. $J$ Perinatol. 2019;39(5):697-707. 


\section{STDs in Adolescents and Young Adults}

\section{Background}

Incidence and prevalence estimates suggest that young people aged 15-24 years acquire half of all new STDs $^{1}$ and that one in four sexuallyactive adolescent females has an STD, such as chlamydia or human papillomavirus (HPV). ${ }^{2}$ Compared with older adults, sexually-active adolescents aged $15-19$ years and young adults aged 20-24 years are at higher risk of acquiring STDs for a combination of behavioral, biological, and cultural reasons. For some STDs, such as chlamydia, adolescent females may have increased susceptibility to infection because of increased cervical ectopy. Cervical ectopy refers to columnar cells, which are typically found within the cervical canal, located on the outer surface of the cervix. Although this is a normal finding in adolescent and young adult females, these cells are more susceptible to infection. The higher prevalence of STDs among adolescents may also reflect multiple barriers to accessing quality STD prevention and management services, including inability to pay, lack of transportation, long waiting times, conflict between clinic hours and work and school schedules, embarrassment attached to seeking STD services, method of specimen collection, and concerns about confidentiality (e.g., Explanation of Benefits for services received mailed to parents or guardians). ${ }^{3}$

Traditionally, intervention efforts have targeted individual level factors associated with STD risk which do not address higher-level factors (e.g., peer norms and media influences) that may also influence behaviors. ${ }^{4}$ Interventions for atrisk adolescents and young adults that address underlying aspects of the social and cultural conditions affecting sexual risk-taking behaviors are needed, as are strategies designed to improve the underlying social conditions themselves. ${ }^{5,6}$ In addition, in designing STD programs, consideration should be given to the needs of adolescent and young adult populations including extended clinic hours, optimizing privacy in waiting rooms, and urine based specimen collection. ${ }^{3}$

\section{Chlamydia}

In 2018, there were $1,087,277$

reported cases of chlamydial infection among persons aged 15-24 years, representing $61.8 \%$ of all reported chlamydia cases. Among those aged 15-19 years, the rate of reported cases of chlamydia increased $1.8 \%$ during 2017-2018 (from 2,072.3 to $2,110.6$ cases per 100,000 population) (Table 10). Among those aged 20-24 years, the rate increased $1.6 \%$ during 2017-2018 (from 2,853.7 to 2,899.2 cases per 100,000 population) (Table $10)$.

Among females aged 15-24 years, the population targeted for chlamydia screening, the overall rate of reported cases of chlamydia in 2018 was $3,693.6$ cases per 100,000 females (Table 12A). While this was only a $1.0 \%$ rate increase from $2017(3,655.5$ cases per 100,000 females), it was an $11.8 \%$ increase from 2014 (3,305.2 cases per 100,000 females). Among males aged 15-24 years, the overall rate of reported cases of chlamydia (1,382.0 cases per 100,000 males $)$ increased 3.5\% from 2017 (1,335.6 cases per 100,000 males) and $30.9 \%$ from 2014 (1,055.4 cases per 100,000 males) (Table 12B). Rates varied by state for both males and females. The majority of states having the highest reported case rates were in the South (Figures K and L).

15-19 Year Old Females - In 2018, the rate of reported chlamydia cases among females aged 15-19 years was
$3,306.8$ cases per 100,000 females, a $1.3 \%$ increase from the 2017 rate of 3,264.8 cases per 100,000 females (Figures 5 and 6, Table 10). Increases in rates of reported cases of chlamydia during 2017-2018 were largest among 18-year old women $(2.9 \%)$ (Table 12A). During 20142018 , the overall rate of reported cases for females aged 15-19 years increased $12.1 \%$ (Table 10).

20-24 Year Old Females - In 2018, women aged 20-24 years had the highest rate of reported chlamydia cases $(4,064.6$ cases per 100,000 females) compared with any other age group for either sex (Figures 5 and 6 , Table 10). The rate of reported chlamydia cases among women aged 20-24 years remained relatively stable during 2017-2018 (0.8\% increase); however, within this age group, females aged 20 years had an increase of 3.3\% (Table 12A). During 2014-2018, the rate of reported chlamydia cases in 20-24 year old females increased 11.9\% (Table 10).

15-19 Year Old Males - In 2018, the rate of reported chlamydia cases among males aged $15-19$ years (959.0 cases per 100,000 males) increased $3.7 \%$ from 2017. During 2014-2018, the rate of reported chlamydia cases for males aged 15-19 years increased $32.8 \%$ (Figures 5 and 7, Table 10).

20-24 Year Old Males - In 2018, as in previous years, men aged 20-24 years had the highest rate of reported chlamydia cases among all males (1,784.5 cases per 100,000 males). The rate for men in this age group increased 3.3\% during 2017-2018 and $31.1 \%$ during $2014-2018$ (Figures 5 and 7, Table 10).

\section{Gonorrhea}

During 2017-2018, the rate of reported gonorrhea cases decreased 
Figure K. Chlamydia - Rates of Reported Cases Among Females Aged 15-24 Years by State and Territory, United States, 2018

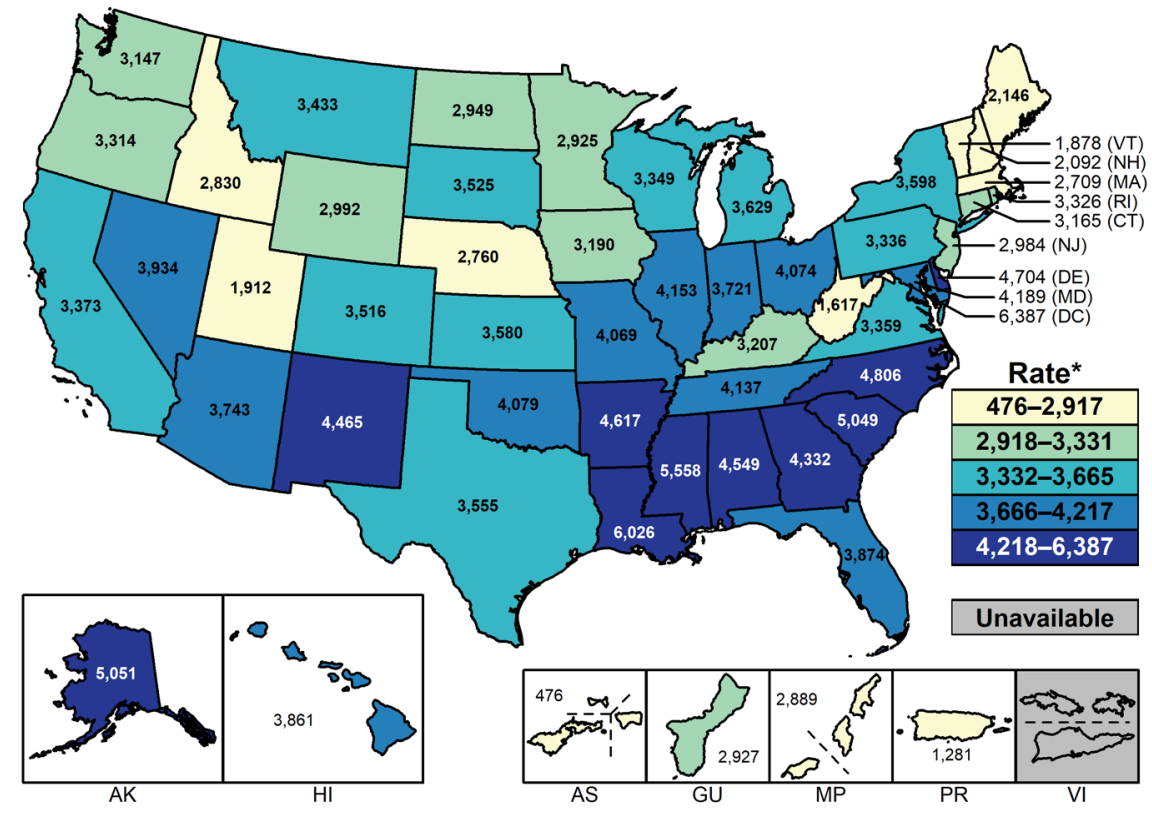

* Per 100,000 .

NOTE: See Sections A1.2 and A1.11 in the Appendix for more information on interpreting and estimating reported rates in US territories.

Figure L. Chlamydia - Rates of Reported Cases Among Males Aged 15-24 Years by State and Territory, United States, 2018

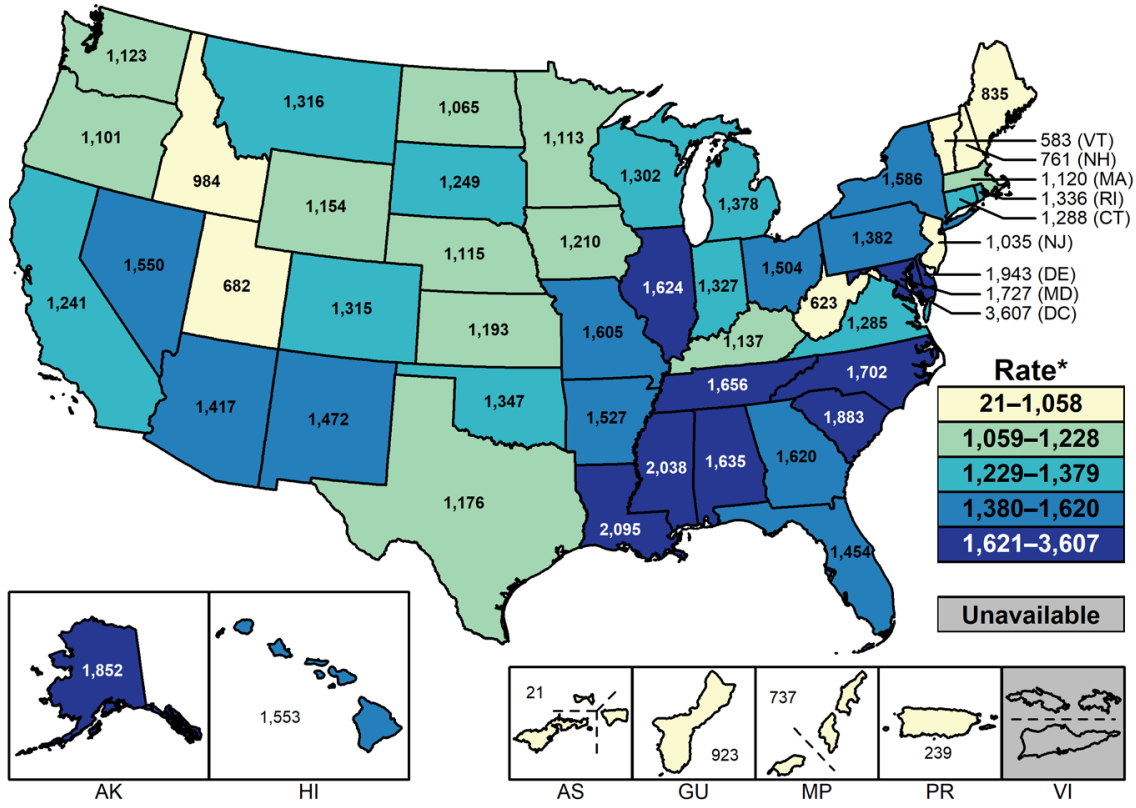

* Per 100,000 .

NOTE: See Sections A1.2 and A1.11 in the Appendix for more information on interpreting and estimating reported rates in US territories.
$1.3 \%$ for persons aged $15-19$ years and increased $1.2 \%$ for persons aged 20-24 years (Table 21). In 2018, among females aged 15-24 years, the rate was 627.0 cases per 100,000 females (Table 23). This was only a $0.1 \%$ increase from 2017 (626.3 cases per 100,000 females) but a $29.7 \%$ increase from 2014 (483.4 cases per 100,000 females). Among males aged 15-24 years, the rate was 525.6 cases per 100,000 males in 2018 . This was only a $0.4 \%$ increase from 2017 ( 523.5 cases per 100,000 males) but a 46.7\% increase from 2014 (358.3 cases per 100,000 males). For both females and males, rates varied by state. The majority of states with the highest reported case rates were in the South (Figures M and N).

\section{5-19 Year Old Females - In}

2018, females aged 15-19 years had the second highest rate of reported gonorrhea cases (548.1 cases per 100,000 females) compared with other age groups among females (Figures 19 and 20, Table 21). During 2017-2018, the rate of reported gonorrhea cases for females in this age group decreased $1.7 \%$ but increased $27.0 \%$ during 2014-2018 (Table 21).

20-24 Year Old Females - In 2018, women aged 20-24 years had the highest rate of reported gonorrhea cases $(702.6$ cases per 100,000 females) compared with other age groups among females (Figures 19 and 20, Table 21). During 20172018, the rate of reported gonorrhea for women in this age group only increased $1.5 \%$ but increased $32.3 \%$ during 2014-2018 (Table 21).

15-19 Year Old Males - In 2018, the rate of reported gonorrhea cases among males aged 15-19 years was 320.5 cases per 100,000 males (Figures 19 and 21, Table 21). During 2017-2018, the rate of reported gonorrhea cases for males in this age group decreased $0.9 \%$ but increased 44.1\% during 2014-2018 (Table 21). 
Figure M. Gonorrhea - Rates of Reported Cases Among Females Aged 15-24 Years by State and Territory, United States, 2018

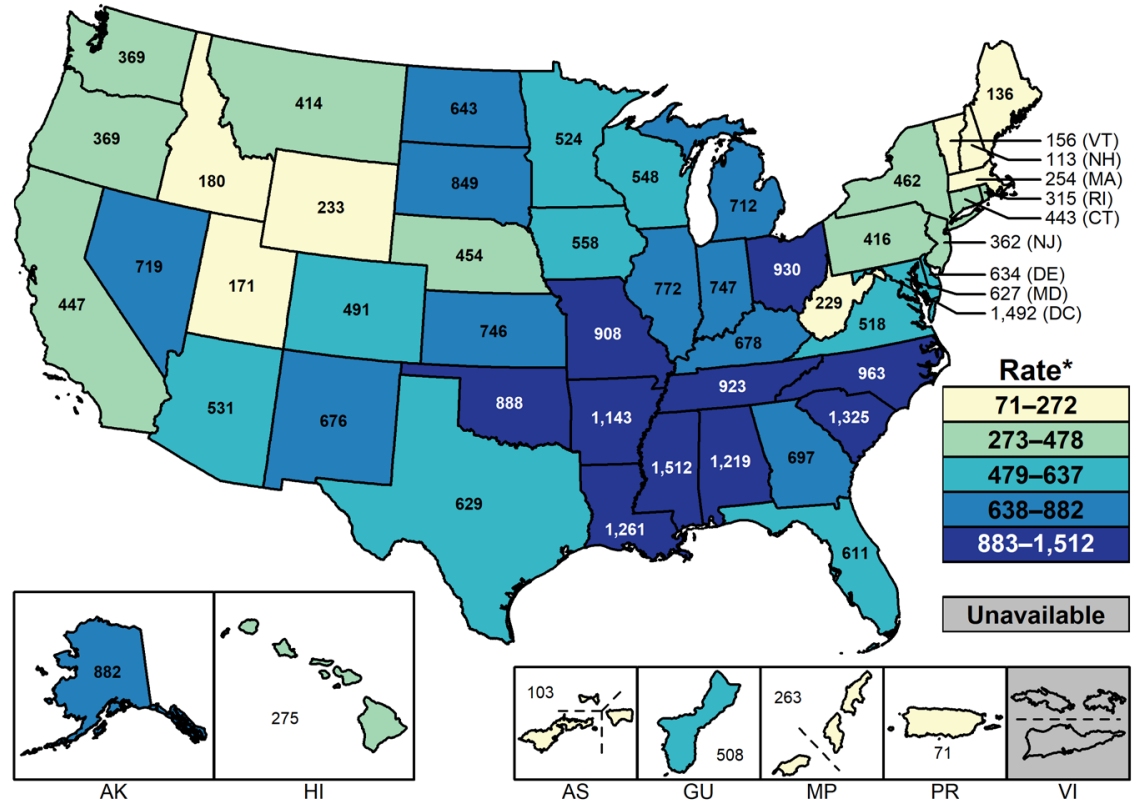

* Per 100,000 .

NOTE: See Sections A1.2 and A1.11 in the Appendix for more information on interpreting and estimating reported rates in US territories.

\section{Figure N. Gonorrhea - Rates of Reported Cases Among Males Aged 15-24 Years by State and Territory, United States, 2018}

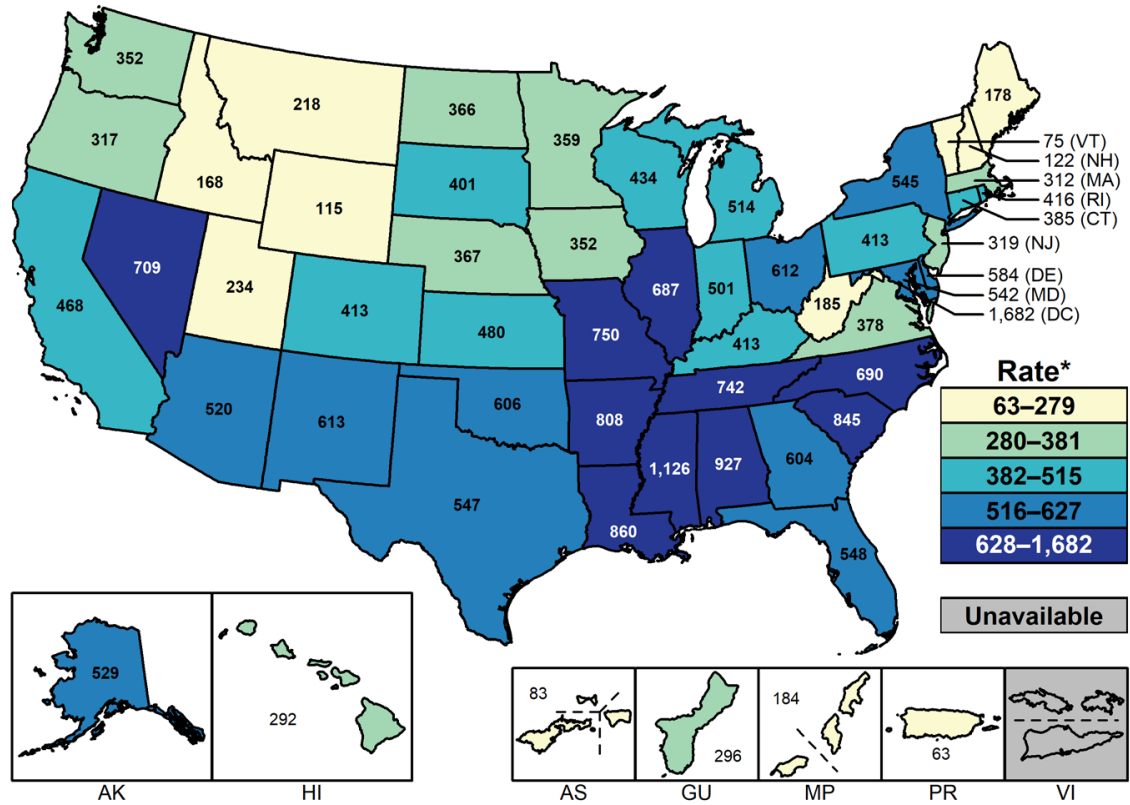

* Per 100,000 .

NOTE: See Sections A1.2 and A1.11 in the Appendix for more information on interpreting and estimating reported rates in US territories.
20-24 Year Old Males - In 2018, as in previous years, men aged $20-24$ years had the highest rate of reported gonorrhea cases ( 720.9 cases per 100,000 males) compared with any other age group for either sex (Figures 19 and 21, Table 21). During 20172018 , the rate of reported gonorrhea for men in this age group only increased $1.0 \%$ but increased $49.2 \%$ during 2014-2018 (Table 21).

\section{Primary and Secondary Syphilis}

In 2018, the rate of reported primary and secondary (P\&S) syphilis among females aged 15-24 years was 7.2 cases per 100,000 females, a $28.6 \%$ increase from 2017 (5.6 cases per 100,000 females) and a $100.0 \%$ increase from 2014 (3.6 cases per 100,000 females). Among males aged 15-24 years in 2018, the rate was 28.2 cases per 100,000 males, a $7.2 \%$ increase from 2017 (26.3 cases per 100,000 males) and a $44.6 \%$ increase from 2014 (19.5 cases per 100,000 males). During 2017-2018, the rate of reported $\mathrm{P} \& \mathrm{~S}$ syphilis cases increased $14.9 \%$ among persons aged $15-19$ years and $10.3 \%$ among persons aged 20-24 years (Table 34).

15-19 Year Old Females - In 2018, the rate of reported $\mathrm{P} \& \mathrm{~S}$ syphilis cases among females aged 15-19 years increased $34.4 \%$ from 2017 (from 3.2 to 4.3 cases per 100,000 females) and $72.0 \%$ from 2014 (2.5 cases per 100,000 females) (Figures 42 and 43, Table 34).

20-24 Year Old Females - In 2018, women aged 20-24 years had the highest rate of $\mathrm{P} \& \mathrm{~S}$ syphilis $(10.0$ cases per 100,000 females) compared with other age groups among females (Figures 42 and 43, Table 34). The P\&S syphilis rate among women in this age group has increased each year since 2011 and has increased $122 \%$ ( 4.5 cases per 100,000 females) (Figure 43, Table 34). During 20172018 , the rate increased $26.6 \%$. 
15-19 Year Old Males - In 2018, the rate of reported $\mathrm{P} \& \mathrm{~S}$ syphilis cases among males aged 15-19 years was 10.9 cases per 100,000 males (Figures 42 and 44, Table 34). The P\&S syphilis rate among males in this age group has increased each year since 2011, with an increase of 53.5\% from 2014 (7.1 cases per 100,000 males) and $7.9 \%$ from 2017 (10.1 cases per 100,000 males) (Figure 44, Table 34).

20-24 Year Old Males - In 2018, the rate of reported $\mathrm{P} \& \mathrm{~S}$ syphilis among males aged $20-24$ years was 44.6 cases per 100,000 males (Figures 42 and 44, Table 34). The P\&S syphilis rate among men in this age group has increased each year since 2006 , with a $44.3 \%$ increase from 2014 (30.9 cases per 100,000 males) and $7.0 \%$ from 2017 (41.7 cases per 100,000 males) (Figure 44, Table 34).

\section{Other STDs}

\section{Human papillomavirus}

Human papillomavirus (HPV) is a common sexually transmitted infection in the United States. ${ }^{1}$ Starting in 2006, HPV vaccines have been recommended for routine use in United States females aged 11-12 years, with catch-up vaccination through age 26. 7,8 Since late 2011, routine use of the HPV vaccine has been recommended for males aged 11-12 years, with catch-up vaccination through age $21 ;^{8-10}$ this age limit was recently extended to 26 years. ${ }^{11}$ Vaccination through age 26 has been recommended for gay, bisexual, and other men who have sex with men (MSM) and persons who are immunocompromised (including those infected with HIV). ${ }^{8}$ In October 2018, the Food and Drug Administration (FDA) extended licensing approval of the vaccine for women and men aged 27-45 years, ${ }^{12}$ and in June 2019, the CDC's Advisory Committee on Immunization Practices (ACIP) recommended that unvaccinated adults aged $27-45$ years discuss receiving the HPV vaccine with their health care providers. ${ }^{11}$ For more information on HPV vaccination, see Other STDs.

A recent meta-analysis that included data from over 60 million individuals from 14 high-income countries, including the United States, showed a substantial impact of HPV vaccination on: genital HPV infections among adolescent girls and young women; high-grade cervical lesions among young women; and anogenital warts among adolescent boys and girls, and among young men and women. ${ }^{13}$ Cervicovaginal prevalence of any quadrivalent HPV vaccine type has been estimated for civilian, non-institutionalized females aged 14-34 years using data from the National Health and Nutrition Examination Survey (NHANES; see Section A2.4 in the Appendix). ${ }^{14}$ Prevalence decreased significantly from 2003-2006 (the pre-vaccine era) to 2011-2014 in specimens from females aged 14-19 years and 20-24 years, the age groups most likely to benefit from HPV vaccination.

Health-care claims data from adolescents and adults with employerprovided private health insurance in the United States were used to examine the population effectiveness of HPV vaccination on two clinical sequelae of HPV infection: highgrade histologically-detected cervical intraepithelial neoplasia grades 2 and $3(\mathrm{CIN} 2+),{ }^{15}$ and anogenital warts. ${ }^{16}$ Prevalence of CIN2+ and of anogenital warts decreased significantly during 2007-2014 among females aged 15-19 and 20-24 years (Figures 51 and 52A); prevalence of anogenital warts also decreased significantly during 2009 2014 among women aged 25-29 years (Figure 52A). These declines provide ecologic evidence of population effectiveness of HPV vaccination in females. Anogenital wart prevalence also decreased significantly during
2009-2014 among men aged 20-24 years (Figure 52B); these declines among young men are consistent with herd protection from vaccination among females.

For more information on HPV infections, see Other STDs.

\section{Herpes simplex virus}

Herpes simplex virus (HSV) is among the most prevalent of sexually transmitted infections. ${ }^{1,17}$ Most genital HSV infections in the United States are caused by HSV type 2 (HSV-2), while HSV type 1 (HSV-1) infections are typically orolabial and acquired during childhood. ${ }^{18,19}$ NHANES data have shown that among adolescents aged 14-19 years, HSV1 seroprevalence has significantly decreased by almost $23 \%$, from $39.0 \%$ during $1999-2004$ to $30.1 \%$ during 2005-2010, indicating declining orolabial infection in this age group. ${ }^{19} \mathrm{HSV}-2$ seroprevalence in this age group was much lower in both time periods. ${ }^{19}$

Other studies have found that genital HSV-1 infections are increasing among young adults. ${ }^{20-22}$ This has been attributed, in part, to the decline in orolabial HSV-1 infections, because those who lack HSV-1 antibodies at sexual debut are more susceptible to genital HSV-1 infection. ${ }^{19,23}$ Increasingly common oral sex behavior among adolescents and young adults also has been suggested as a contributing factor. ${ }^{19,24}$

NHANES data have shown that among pregnant women with three or fewer lifetime sex partners, seronegativity for both HSV-1 and HSV-2 increased from 1999-2006 to 2007-2014, ${ }^{25}$ raising the possibility that pregnant women with fewer sex partners may have increased risk of acquiring genital HSV during pregnancy and vertically transmitting HSV to their neonates. 
For more information on genital HSV infections, see Other STDs. For information on neonatal HSV infections, see Special Focus Profiles, STDs in Women and Infants.

\section{National Job Training Program}

The National Job Training Program (NJTP) is an educational program for socioeconomically disadvantaged youth aged 16-24 years and is administered at more than 100 sites throughout the country. The NJTP screens participants for chlamydia and gonorrhea within two days of entry to the program. All of NJTP's chlamydia screening tests and the majority of gonorrhea screening tests are conducted by a single national contract laboratory (Center for Disease Detection, LLC, San Antonio, Texas).

To increase the stability of the 2018 estimates, chlamydia or gonorrhea prevalence data are presented when valid test results for 100 or more students per year are available for the population subgroup and state. Additional information about NJTP can be found in Section A2.1 in the Appendix.

Among females aged 16-24 years entering the program in 2018 in 41 states and the District of Columbia, the median state-specific chlamydia prevalence was $12.5 \%$ (range: $5.7 \%$ to $19.3 \%$ ) (Figure O). Among males aged 16-24 years entering the program in 2018 in all 50 states, the District of Columbia, and Puerto Rico, the median state-specific chlamydia prevalence was $6.6 \%$ (range: $1.0 \%$ to $13.3 \%$ ) (Figure $\mathrm{P}$ ).

Among females aged 16-24 years entering the program in 41 states and the District of Columbia, the median state-specific gonorrhea prevalence in 2018 was $2.2 \%$ (range: $0.4 \%$ to $7.6 \%$ ) (Figure Q). Among males aged 16-24 years entering the program in 46 states, the District of Columbia, and Puerto Rico, the median state-specific
Figure O. Chlamydia - Prevalence Among Females Aged 16-24 Years Entering the National Job Training Program (NJTP) by State and Territory of Residence, United States, 2018

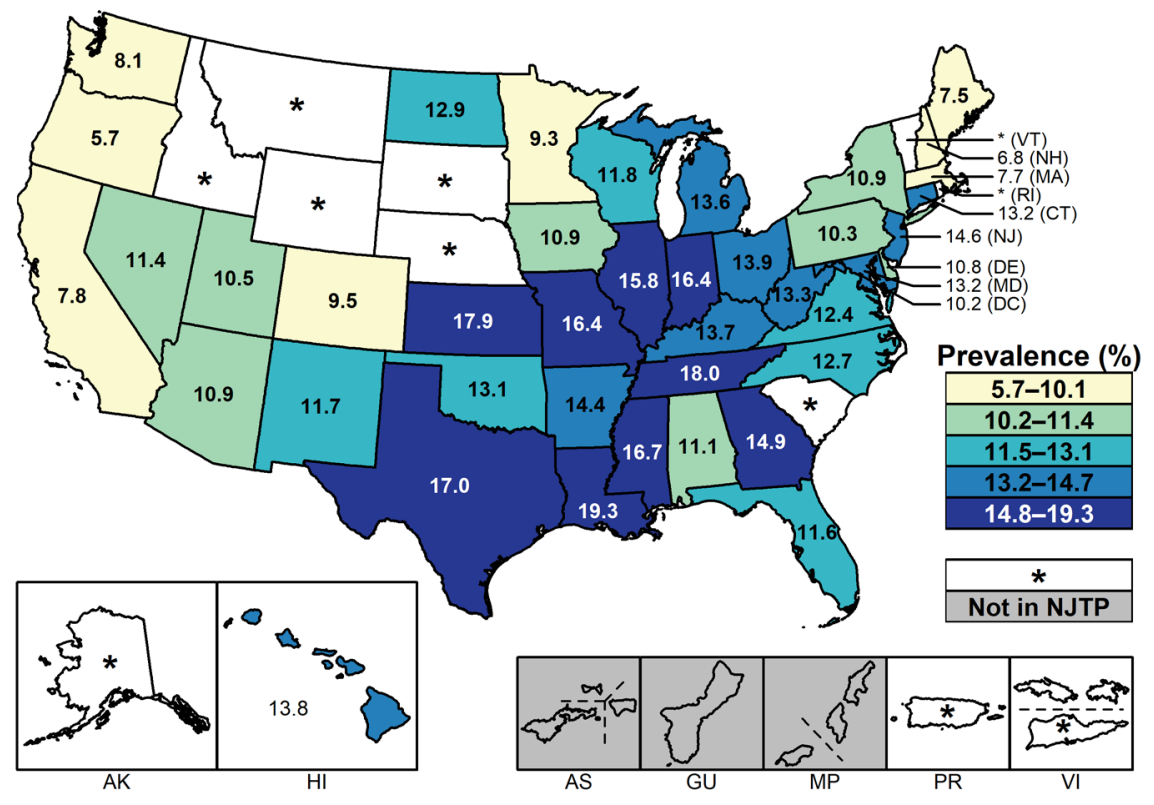

* Fewer than 100 females who resided in these states/territories and entered the NJTP were screened for chlamydia in 2018.

NOTE: See Section A2.1 in the Appendix for more information regarding NJTP methods.

Figure P. Chlamydia - Prevalence Among Males Aged 16-24 Years Entering the National Job Training Program (NJTP) by State and Territory of Residence, United States, 2018

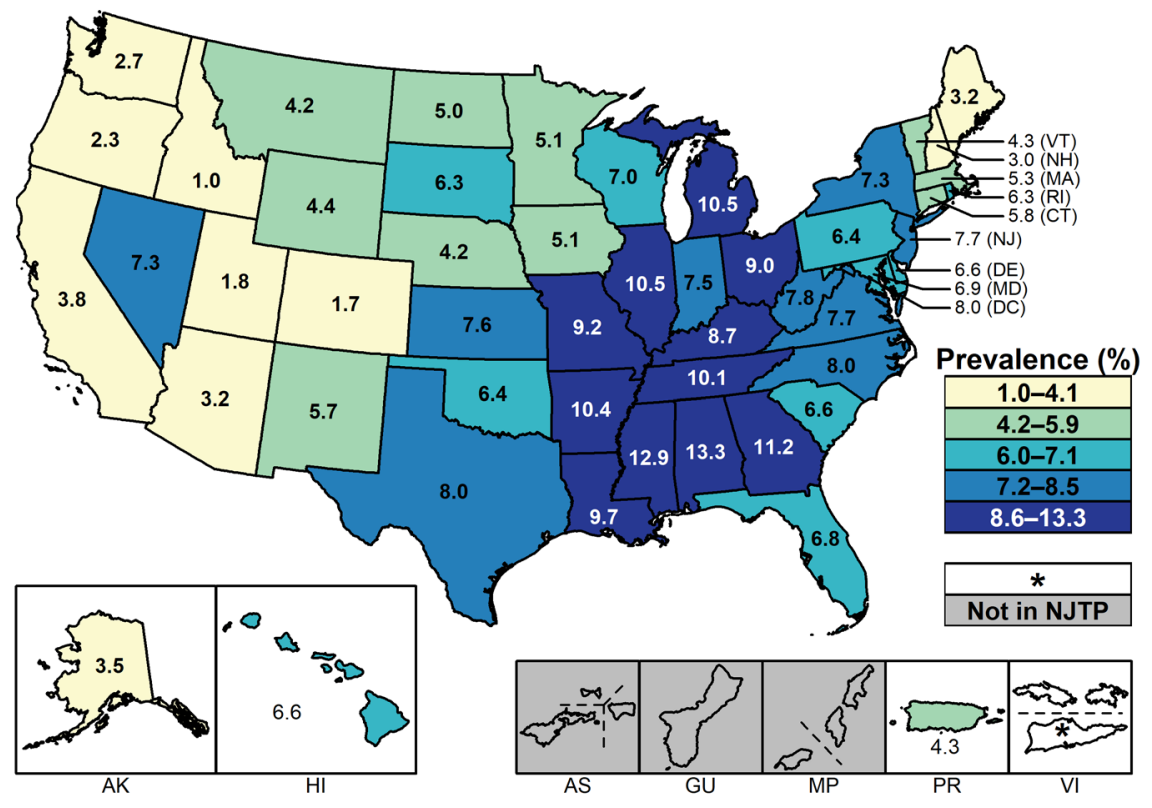

* Fewer than 100 males who resided in these states/territories and entered the NJTP were screened for chlamydia in 2018.

NOTE: See Section A2.1 in the Appendix for more information regarding NJTP methods. 


\section{Figure Q. Gonorrhea - Prevalence Among Females Aged 16-24 Years Entering the National Job Training Program (NJTP) by State and Territory of Residence, United States, 2018}

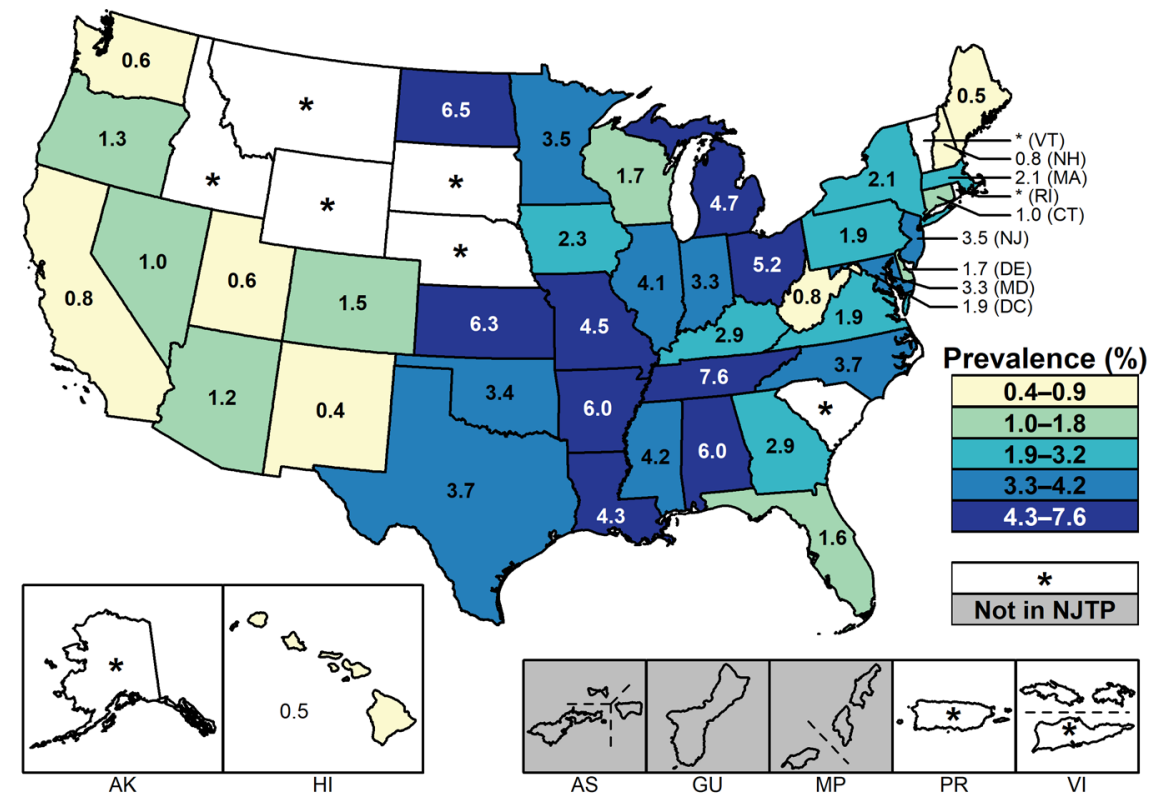

* Fewer than 100 females who resided in these states/territories and entered the NJTP were screened for gonorrhea in 2018.

NOTE: See Section A2.1 in the Appendix for more information regarding NJTP methods

gonorrhea prevalence in 2018 was $0.7 \%$ (range: $0.0 \%$ to $4.8 \%$ ) (Figure $\mathrm{R})$.

\section{Summary}

The rate of reported cases of chlamydia, gonorrhea, and $\mathrm{P} \& \mathrm{~S}$ syphilis increased for both sexes in 15-24 year olds during 2017-2018. For chlamydia, rates of reported cases are consistently highest among females aged 15-24 years, likely reflecting targeted screening of young women; however, rates of reported gonorrhea cases are consistently highest among males aged 15-24 years. These high rates among males aged 15-24 years likely reflect a combination of recent increased screening efforts in young men, including extra-genital screening, as well as increased incidence. Although rates of reported chlamydia and gonorrhea increased only marginally among both sexes during 2017-2018, the increases observed before 2017 were much more striking. The rate of reported chlamydia in females and males aged 15-24 years increased $1.0 \%$ and $3.5 \%$, respectively, during 2017-2018; however, increases of $11.8 \%$ and $30.9 \%$ were noted during 2014-2018 for females and males aged 15-24 years, respectively. Similarly, the rate of reported gonorrhea in females and males aged $15-24$ years increased $0.1 \%$ and $0.4 \%$, respectively, during 2017-2018; however, increases of $29.7 \%$ and $46.7 \%$ were noted during 2014-2018 for females and males aged 15-24 years, respectively. Whether these smaller increases in the past year compared to the past five years indicate a slowing to the increasing trend of rates of chlamydia and gonorrhea cannot be assessed with current data, and will take further years of case surveillance to determine. Similar to gonorrhea, rates of reported cases of P\&S syphilis have been consistently higher among adolescent and young adult males compared to females; however, the largest increase in P\&S syphilis has been observed in females aged 15-24 years. Rates of reported P\&S syphilis cases increased $28.6 \%$ and $7.2 \%$ during 2017-2018 and increased $100.0 \%$ and $44.6 \%$ during $2014-2018$ for females and males aged 15-24 years, respectively.

\section{References}

1. Satterwhite CL, Torrone E, Meites E, et al. Sexually transmitted infections among US women and men: Prevalence and incidence estimates, 2008. Sex Transm Dis. 2013;40(3):187-193.

2. Forhan SE, Gottlieb SL, Sternberg MR, et al. Prevalence of sexually transmitted infections among female adolescents aged 14 to 19 in the United States. Pediatrics. 2009;124(6):15051512 .

3. Tilson EC, Sanchez V, Ford CL, et al. Barriers to asymptomatic screening and other STD services for adolescents and young adults: Focus group discussions. BMC Public Health. 2004;4(1):21.

4. DiClemente RJ, Salazar LF, Crosby RA. A review of STD/HIV preventive interventions for adolescents: Sustaining effects using an ecological approach. J Pediatr Psychol. 2007;32(8):888-906.

5. Sieving RE, Bernat DH, Resnick MD, et al. A clinic-based youth development program to reduce sexual risk behaviors among adolescent girls: Prime time pilot study. Health Promot Pract. 2012;13(4):462-471.

6. Upchurch DM, Mason WM, Kusunoki Y, et al. Social and behavioral determinants of selfreported STD among adolescents. Perspect Sex Reprod Health. 2004;36(6):276-287.

7. Markowitz LE, Dunne EF, Saraiya M, et al. Quadrivalent human papillomavirus vaccine. Recommendations of the Advisory Committee on Immunization Practices (ACIP). $M M W R$ Recomm Rep. 2007;56(No. RR-2):1-24.

8. Petrosky E, Bocchini Jr. JA, Hariri S, et al. Use of 9-valent human papillomavirus (HPV) vaccine: Updated HPV vaccination recommendations of the Advisory Committee on Immunization Practices. MMWR Morb Mortal Wkly Rep. 2015;64(11):300-304.

9. Centers for Disease Control and Prevention. Recommendations on the use of quadrivalent human papillomavirus vaccine in males Advisory Committee on Immunization Practices (ACIP), 2011. MMWR Morb Mortal Wkly Rep. 2011;60(50):1705-1708. 


\section{Figure R. Gonorrhea - Prevalence Among Males Aged 16-24 Years Entering the National Job Training Program (NJTP) by State and Territory of Residence, United States, 2018}

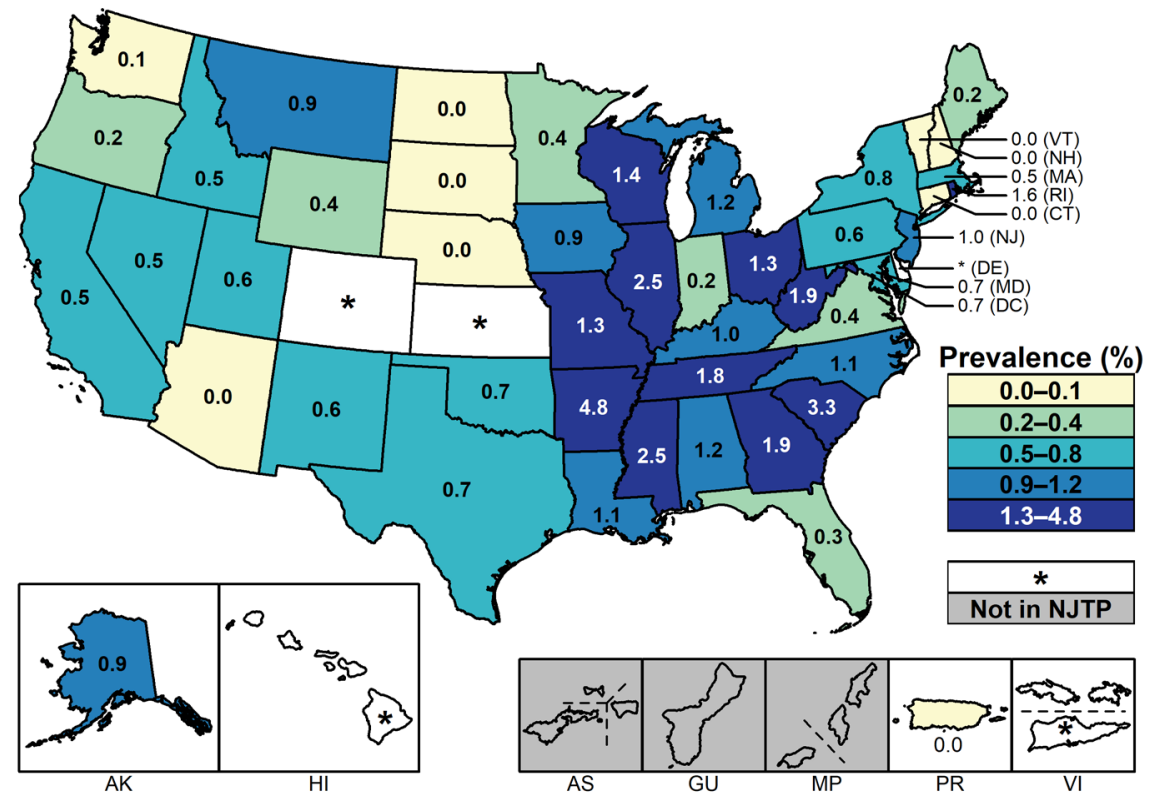

* Fewer than 100 males who resided in these states/territories and entered the NJTP were screened for gonorrhea in 2018.

NOTE: See Section A2.1 in the Appendix for more information regarding NJTP methods.

10. Meites E, Kempe A, Markowitz LE. Use of a 2-dose schedule for human papillomavirus vaccination - updated recommendations of the Advisory Committee on Immunization Practices. MMWR Morb Mortal Wkly Rep 2016;65(49):1405-1408.

11. Meites E, Szilagyi PG, Chesson HW, et al. Human papillomavirus vaccination for adults: Updated recommendations of the Advisory Committee on Immunization Practices. $M M W R$ Morb Mortal Wkly Rep. 2019; 68(32):698-702.

12. US Food and Drug Administration. "Supplement Approval Letter - Biologics License Application (BLA) for Human Papillomavirus 9-valent Vaccine, Recombinant (GARDASIL ${ }^{\circledR}$ 9)". Letter to Merck Sharp \& Dohme Corp. October 5, 2018. US Food and Drug Administration. 2018. https://www.fda. gov/media/117053/download. Accessed July 2, 2019 .

13. Drolet M, Benard E, Perez N, et al. Populationlevel impact and herd effects following the introduction of human papillomavirus vaccination programmes: Updated systematic review and meta-analysis. Lancet. 2019;394(10197):497-509.
14. Oliver SE, Unger ER, Lewis R, et al. Prevalence of human papillomavirus among females after vaccine introduction - National Health and Nutrition Examination Survey, United States, 2003-2014. J Infect Dis. 2017;216(5):594-603.

15. Flagg EW, Torrone EA, Weinstock $\mathrm{H}$. Ecological association of human papillomavirus vaccination with cervical dysplasia prevalence in the United States, 2007-2014. Am J Public Health. 2016;106(12):2211-2218.

16. Flagg EW, Torrone EA. Declines in anogenital warts among age groups most likely to be impacted by human papillomavirus vaccination, United States, 2006-2014. Am J Public Health. 2018;108(1):112-119.

17. Smith JS, Robinson NJ. Age-specific prevalence of infection with herpes simplex virus types 2 and 1: A global review. J Infect Dis. 2002;186(Suppl 1):S3-S28.

18. Corey L, Wald A. Genital Herpes. In: Holmes KK, Sparling FP, Stamm WE, et al., eds. Sexually Transmitted Diseases. 4th ed. New York, NY: McGraw-Hill; 2008:399-437.
19. Bradley H, Markowitz LE, Gibson T, et al. Seroprevalence of herpes simplex virus types 1 and 2 - United States, 1999-2010. J Infect Dis 2014;209(3):325-333.

20. Bernstein DI, Bellamy AR, Hook EW III, et al. Epidemiology, clinical presentation, and antibody response to primary infection with herpes simplex virus type 1 and type 2 in young women. Clin Infect Dis. 2013;56(3):344-351.

21. Roberts CM, Pfister JR, Spear SJ. Increasing proportion of herpes simplex virus type 1 as a cause of genital herpes infection in college students. Sex Transm Dis. 2003;30(10):797800.

22. Flagg EW, Torrone EA. Seroprevalence of herpes simplex virus type 1 and type 2 infections among adults diagnosed with genital herpes: United States, 1999-2016. Sex Transm Dis. 2018;45(Suppl 2):S51.

23. Kimberlin DW. The scarlet H. J Infect Dis. 2014;209(3):315-317.

24. Copen CE, Chandra A, Martinez G. Prevalence and timing of oral sex with opposite-sex partners among females and males aged 15-24 years: United States, 2007-2010. National health statistics reports; no 56. Hyattsville, MD: National Center for Health Statistics. 2012.

25. Patton ME, Bernstein K, Liu G, et al. Seroprevalence of herpes simplex virus types 1 and 2 among pregnant women and sexually active, non-pregnant women in the United States. Clin Infect Dis. 2018;67(10):1535-1542. 


\section{STDs in Racial and Ethnic Minorities}

\section{Background}

Disparities continue to persist in rates of STDs among some racial minority or Hispanic groups when compared with rates among Whites. ${ }^{1,2}$ This is also true across a wide variety of other health status indicators, providing evidence that race and Hispanic ethnicity in the United States are population characteristics strongly correlated with other factors affecting overall health status, such as income, employment, insurance coverage, and educational attainment. ${ }^{3-5}$ In 2017, the most recent year for which national data on poverty and insurance status are available, the overall proportion of the United States population living in poverty was $12.3 \%$ (or 39.7 million), a decrease of 0.4 percent from 2016. Although the overall poverty rate declined over the last few years, many Americans continue to face systemic challenges to achieving their full economic potential; the poverty rate in 2017 for Whites was 8.7\% (16.9 million), for Blacks it was $21.2 \%$ (8.9 million), and for Hispanics it was $18.3 \%$ (10.7 million). Significant differences by race/Hispanic ethnicity in the proportion of the population living in poverty persisted in 2017 and were even more acute for family households headed by women (25.7\% versus $9.3 \%$ for all family households), regardless of other factors. ${ }^{3}$ Those who cannot afford basic necessities often have trouble accessing and affording quality health care, including sexual health services. ${ }^{6}$

Access to, and routine use of, quality health care including STD prevention and treatment is key to reducing STD disparities in the United States. Of the estimated 19 million new cases of STIs that occur each year, approximately half of all cases occur among people aged $15-24$ years. $^{7}$ However, the overall proportion of adults without health insurance decreased from $13.3 \%$ in 2013 to $8.8 \%$ (or 28.2 million) in 2016 and remains unchanged at $8.8 \%$ (28.5 million) in 2017. By age groupings, the highest proportion of the population lacking health insurance in 2017 were 19-25 and 26-34 year olds ( $14.0 \%$ and $15.6 \%$, respectively), demonstrating that many people in the United States continue to struggle to afford full, routine access to health care. ${ }^{8}$ Among all races or ethnic groups in the United States, Hispanics had the lowest rate of health insurance coverage in 2017 at $84.0 \%$ (unchanged from 2016). ${ }^{8}$

Even when health care is readily available to racial and ethnic minority populations, fear and distrust of health care institutions can negatively affect the health care-seeking experience. Social and cultural discrimination, language barriers, provider bias, or the perception that these may exist, likely discourage some people from seeking care. ${ }^{9,10}$ Moreover, the quality of care can differ substantially for minority patients. ${ }^{11}$ Broader inequities in social and economic conditions for minority communities are reflected in the profound disparities observed in the incidence of STDs by race/ Hispanic ethnicity.

In communities where STD prevalence is higher because of these and other factors, people may experience difficulties reducing their risk for STIs. With each sexual encounter, they face a greater chance of encountering an infected partner than those in lower prevalence settings do, regardless of similar sexual behavior patterns. ${ }^{2}$ Acknowledging inequities in STD rates by race and Hispanic ethnicity is a critical first step toward empowering affected groups and the public health community to collaborate in addressing systemic inequities in the burden of disease - with the ultimate goal of minimizing the health impacts of STDs on individuals and populations.

\section{STD Reporting Practices}

Surveillance data are based on cases of STDs reported to state and local health departments (see Section A.1 in the Appendix). In many state and local health departments, electronic laboratory reporting is increasingly becoming the primary source of initial case notifications. Laboratory reports are often missing race and Hispanic ethnicity of the patient; ascertainment of information on race and Hispanic ethnicity is therefore a function of active follow-up or dependent on previous information available about the patient in existing health department surveillance databases. Prevalence data from populationbased surveys, such as the National Health and Nutrition Examination Survey (NHANES) and the National Longitudinal Study of Adolescent Health, confirm the existence of marked disparities in some minority populations (see Other STDs below) for both nationally notifiable and nonnationally notifiable STDs. ${ }^{12,13}$

\section{Method of Classifying Race/ Hispanic Ethnicity}

Interpretation of racial and ethnic disparities among persons with STDs is influenced by data collection methods and by the categories by which these data are displayed. Race/Hispanic ethnicity data in this report are presented in Office of Management and Budget (OMB) race and Hispanic ethnicity categories according to the 1997 revised OMB standards..$^{14}$ As of 2018, most reporting jurisdictions are locally compliant with OMB standards and report in the standard categories, including the ability to collect more than one race per person. However, 
a small number of jurisdictions reported race in pre-1997 categories; while other jurisdictions continued to be unable to report more than one race per person in 2018. All race/ Hispanic ethnicity data reported by jurisdictions are summarized in tables, charts and interpretative text in this report regardless of local compliance with the 1997 OMB standards. No redistribution of cases is done; cases missing race/ Hispanic ethnicity are not included in the calculation of rates by race/ Hispanic ethnicity. Caution should be used in interpreting and comparing rates for individual race groups or by Hispanic ethnicity. Because missing cases are excluded, rates presented underestimate the likely actual rate of reported cases for specific groups. See Section A1.5 of the Appendix for additional information on reporting of race/Hispanic ethnicity.

\section{Completeness of Race/ Hispanic Ethnicity Data in 2018}

Chlamydia $-28.5 \%$ of chlamydia case reports were missing race/ Hispanic ethnicity data, ranging by jurisdiction from $0.1 \%$ to $94.4 \%$

(Table A1).

Gonorrhea - 19.6\% of gonorrhea case reports were missing information on race/Hispanic ethnicity, ranging by jurisdiction from $0.0 \%$ to $92.0 \%$ (Table A1).

Syphilis - 4.5\% of all primary and secondary $(\mathrm{P} \& \mathrm{~S})$ syphilis case reports were missing information on race/Hispanic ethnicity, ranging by jurisdiction from $0.0 \%$ to $46.7 \%$ missing (Table A1).

\section{Chlamydia}

During 2014-2018, rates of reported chlamydia cases increased among all racial and Hispanic ethnicity groups. Specifically, rates increased 7.0\% among American Indians/
Alaska Natives (AI/AN), 8.1\% among Hispanics, 9.0\% among Blacks, $17.6 \%$ among Whites, $20.1 \%$ among Native Hawaiians/Other Pacific Islanders (NHOPI), 29.3\% among Asians, and 59.7\% among Multirace (Figure 8).

Blacks - In 2018, the overall rate of reported chlamydia cases among Blacks in the United States was $1,192.5$ cases per 100,000 population (Figure 8, Table 11B). The rate of reported chlamydia cases among Black females was five times the rate among White females $(1,411.1$ and 281.7 cases per 100,000 population, respectively; Figure S and Table 11B). The rate of reported chlamydia cases among Black males was 6.8 times the rate among White males (952.3 and 140.4 cases per 100,000 population, respectively). Rates of reported cases of chlamydia were highest for Blacks aged 15-19 and 20-24 years in 2018 (Table 11B). The rate of reported chlamydia cases among Black females aged $15-19$ years $(6,817.3$ cases per 100,000 population) was 4.5 times the rate among White females in the same age group $(1,520.1$ cases per 100,000 population). The rate of reported chlamydia cases among Black females aged 20-24 years was 3.7 times the rate among White females in the same age group $(7,087.7$ and $1,935.8$ cases per 100,000 population, respectively). Among females aged 15-24 years, the population targeted for screening, rates were highest among Blacks in all US regions (Figure T).

Similar racial disparities in reported chlamydia rates exist among males. Among males aged 15-19 years, the rate of reported chlamydia cases among Blacks was 9.1 times the rate among Whites $(2,668.6$ and 293.0 cases per 100,000 population, respectively; Table 11B). The rate of reported chlamydia cases among Black males aged 20-24 years was 5.3 times the rate among White males of the same age group $(3,867.1$ and 732.6 cases per 100,000 population, respectively).

\section{Figure S. Chlamydia - Rates of Reported Cases by Race/Hispanic Ethnicity and Sex, United States, 2018}

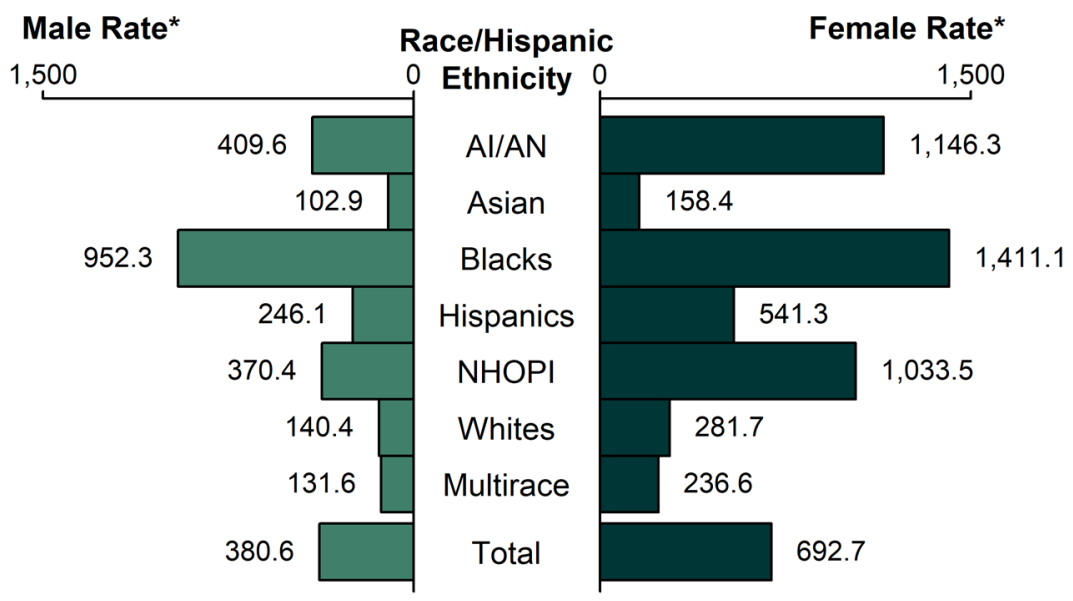

* Per 100,000 .

NOTE: See Section A1.5 in the Appendix for information on race/Hispanic ethnicity in STD case reporting.

ACRONYMS: Al/AN = American Indians/Alaska Natives; $\mathrm{NHOPI} \mathrm{=} \mathrm{Native}$ Hawaiians/Other Pacific Islanders. 


\section{Figure T. Chlamydia - Rate Ratios* Among Females Aged 15-24 Years by Race/Hispanic Ethnicity and Region, United States, 2018}

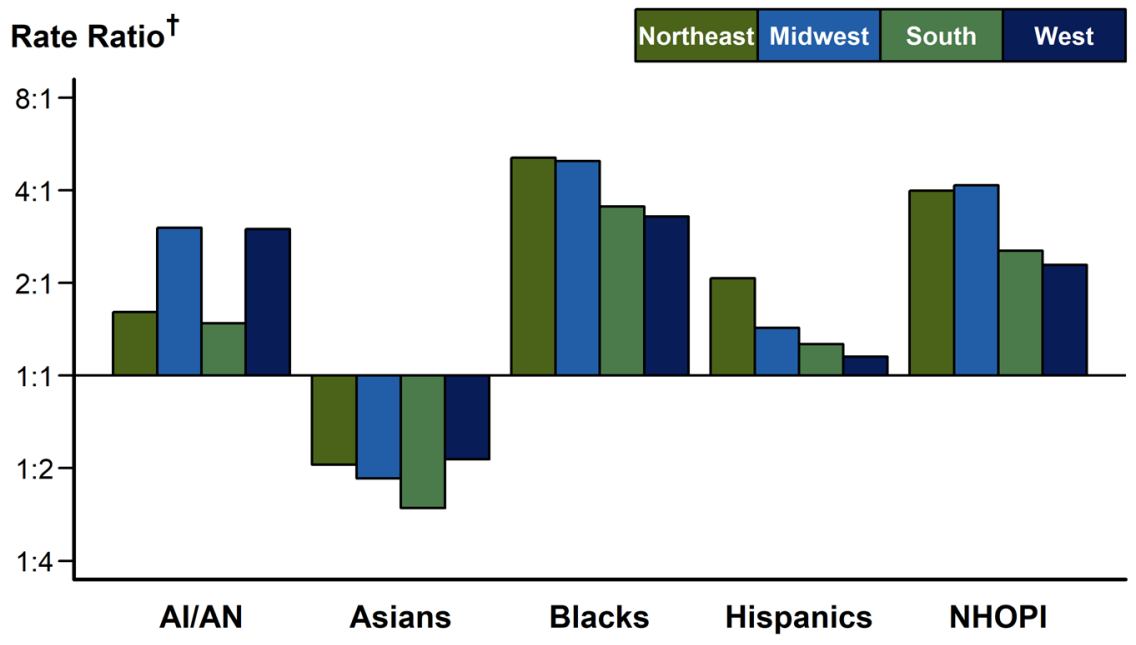

${ }^{*}$ For the rate ratios, Whites are the reference population.

${ }^{\dagger} Y$-axis is log scale.

NOTE: See Section A1.5 in the Appendix for information on reporting STD case data for race/Hispanic ethnicity.

ACRONYMS: Al/AN = American Indians/Alaska Natives; $\mathrm{NHOPI}=$ Native

Hawaiians/Other Pacific Islanders.

\section{American Indians/Alaska Natives}

— In 2018, the rate of reported chlamydia cases among AI/AN was 784.8 cases per 100,000 population (Table 11B). Overall, the rate of reported chlamydia cases among AI/ AN in the United States was 3.7 times the rate among Whites.

\section{Native Hawaiians/Other Pacific}

Islanders - In 2018, the rate of reported chlamydia cases among NHOPI was 700.8 cases per 100,000 population (Table 11B). The overall rate of reported chlamydia cases among NHOPI was 3.3 times the rate among Whites and 5.3 times the rate among Asians.

Hispanics - In 2018, the rate of reported chlamydia cases among Hispanics was 392.6 cases per 100,000 population, which was 1.9 times the rate among Whites (Table 11B).
Asians - In 2018, the rate of reported chlamydia cases among Asians was 132.1 cases per 100,000 population (Table 11B). The overall rate of reported chlamydia cases among Whites was 1.6 times the rate among Asians.

\section{Gonorrhea}

During 2014-2018, rates of reported gonorrhea cases increased $119.5 \%$ among Multirace persons (43.0 to 94.4 cases per 100,000 population), 99.4\% among Asians (17.6 to 35.1 cases per 100,000 population), $90.3 \%$ among NHOPI (95.3 to 181.4 cases per 100,000 population), $89.1 \%$ among Whites (37.6 to 71.1 cases per 100,000 population), $84.2 \%$ among AI/AN (178.9 to 329.5 cases per 100,000 population), $66.0 \%$ among Hispanics (69.8 to 115.9 cases per 100,000 population), and $38.8 \%$ among Blacks (395.4 to 548.9 cases per 100,000 population) (Figure 22).
Blacks - In 2018, the overall rate of reported gonorrhea cases among Blacks in the United States was 7.7 times the rate among Whites (Table 22B). This disparity was similar for Black males (8.5 times the rate among White males) and Black females (6.9 times the rate among White females) (Figure U, Table 22B). As in previous years, the disparity in gonorrhea rates for Blacks in 2018 was larger in the Midwest and Northeast than in the South and West (Figure V).

Considering Hispanic ethnicity and all race and age categories, rates of reported gonorrhea cases were highest for Blacks aged 20-24, 15-19, and 25-29 years in 2018 (Table 22B). The rate of reported gonorrhea cases among Black females aged 20-24 years $(2,040.3$ cases per 100,000 population) was 6.9 times the rate among White females in the same age group (297.5 cases per 100,000 population). The rate of reported gonorrhea cases among Black females aged $15-19$ years $(1,756.4$ cases per 100,000 population) was 8.8 times the rate among White females in the same age group (200.1 cases per 100,000 population). Among Black males aged 20-24 years, the rate of reported gonorrhea cases $(2,212.1$ cases per 100,000 population) was 9.4 times the rate among White males in the same age group (236.3 cases per 100,000 population). The rate of reported gonorrhea cases among Black males aged $25-29$ years $(1,860.7$ cases per 100,000 population) was 7.0 times the rate among White males in the same age group (265.5 cases per 100,000 population).

American Indians/Alaska Natives - In 2018, the rate of reported gonorrhea cases among AI/AN (329.5 cases per 100,000 population) was 4.6 times the rate among Whites (Table 22B). The disparity between gonorrhea rates for $\mathrm{AI} / \mathrm{AN}$ and Whites was larger for AI/AN females (6.3 times the rate among White females) 
Figure U. Gonorrhea - Rates of Reported Cases by Race/Hispanic Ethnicity and Sex, United States, 2018

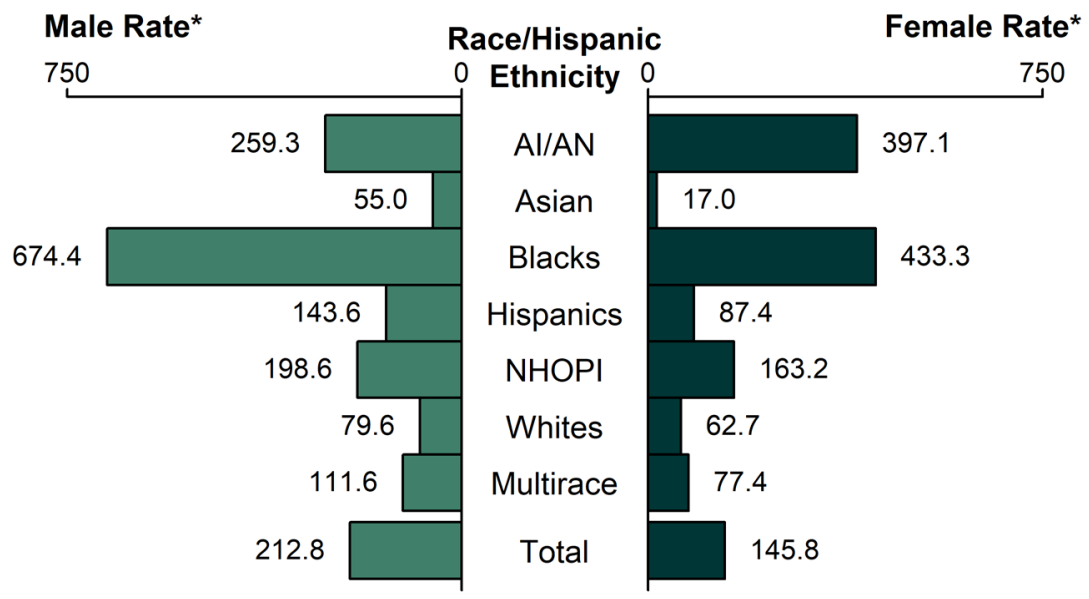

${ }^{*}$ Per 100,000 .

NOTE: See Section A1.5 in the Appendix for information on race/Hispanic ethnicity in STD case reporting.

ACRONYMS: AI/AN = American Indians/Alaska Natives; $\mathrm{NHOPI}=$ Native

Hawaiians/Other Pacific Islanders.

\section{Figure V. Gonorrhea - Rate Ratios* by Race/Hispanic Ethnicity and Region, United States, 2018}

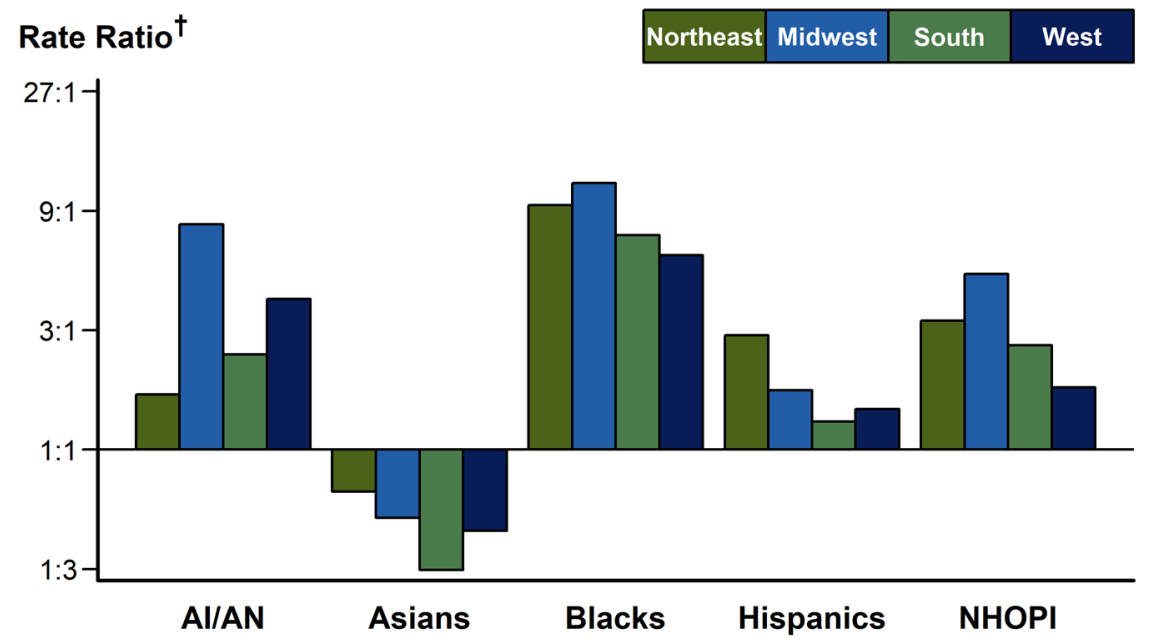

${ }^{*}$ For the rate ratios, Whites are the reference population.

${ }^{\dagger} \mathrm{Y}$-axis is log scale.

NOTE: See Section A1.5 in the Appendix for information on race/Hispanic ethnicity in STD case reporting.

ACRONYMS: Al/AN = American Indians/Alaska Natives; NHOPI = Native

Hawaiians/Other Pacific Islanders. than for $\mathrm{AI} / \mathrm{AN}$ males (3.3 times the rate among White males) (Figure $\mathrm{U}$, Table 22B). The disparity in gonorrhea rates for AI/AN in 2018 was larger in the Midwest than in the West, Northeast, and South (Figure V).

\section{Native Hawaiians/Other Pacific} Islanders - In 2018, the rate of reported gonorrhea cases among NHOPI (181.4 cases per 100,000 population) was 2.6 times the rate among Whites (Table 22B). This disparity was similar for NHOPI females (2.6 times the rate among White females) and NHOPI males (2.5 times the rate among White males) (Figure U, Table 22B). The disparity in gonorrhea rates for NHOPI in 2018 was higher in the Midwest than in the West, Northeast, and South (Figure V).

Hispanics - In 2018, the rate of reported gonorrhea cases among Hispanics was 115.9 cases per 100,000 population, which was 1.6 times the rate among Whites (Table 22B). This disparity was similar for Hispanic females (1.4 times the rate among White females) and Hispanic males (1.8 times the rate among White males) (Figure U, Table 22B). The disparity in gonorrhea rates for Hispanics in 2018 was higher in the Northeast than in the Midwest, South, and West (Figure V).

Asians - In 2018, the rate of reported gonorrhea cases among Asians (35.1 cases per 100,000 population) was 0.5 times the rate among Whites (Table 22B). This difference was larger for Asian females ( 0.3 times the rate among White females) than for Asian males (0.7 times the rate among White males) (Figure U, Table 22B). In 2018, gonorrhea rates among Asians were lower than rates among Whites in all four regions of the United States (Figure V). 


\section{Primary and Secondary Syphilis}

During 2014-2018, rates of reported P\&S syphilis cases increased for all race/Hispanic ethnicity groups, more than doubling for some groups (Figure 45). The rate of reported $\mathrm{P} \& \mathrm{~S}$ syphilis cases increased $171.7 \%$ among NHOPI (6.0 to 16.3 cases per 100,000 population), $115.3 \%$ among $\mathrm{AI} / \mathrm{AN}$ ( 7.2 to 15.5 cases per 100,000 population), $104.3 \%$ among those who identified as Multirace (4.6 to 9.4 cases per 100,000 population), $78.1 \%$ among Hispanics (7.3 to 13.0 cases per 100,000 population), $76.9 \%$ among Asians (2.6 to 4.6 cases per 100,000 population), $71.4 \%$ among Whites (3.5 to 6.0 cases per 100,000 population), and $51.9 \%$ among Blacks (18.5 to 28.1 cases per 100,000 population). Across all race/Hispanic ethnicity groups, MSM accounted for the highest proportion of $\mathrm{P} \& S$ syphilis cases (Figure Z).

Blacks - In 2018, 34.7\% of reported P\&S syphilis cases with known race/Hispanic ethnicity information occurred among Blacks (Table 35A). The rate of reported $\mathrm{P} \& \mathrm{~S}$ syphilis cases among Blacks was 4.7 times the rate among Whites (28.1 versus 6.0 cases per 100,000 population, respectively) (Table 35B). This disparity was similar for Black females and males (Figure W). Similar disparities were seen in all regions of the United States (Figure X).

Hispanics - In 2018, 23.3\% of reported $\mathrm{P} \& \mathrm{~S}$ syphilis cases with known race/Hispanic ethnicity information occurred among Hispanics (Table 35A). The rate of reported $\mathrm{P} \& \mathrm{~S}$ syphilis cases among Hispanics was 2.2 times the rate among Whites (13.0 versus 6.0 cases per 100,000 population, respectively) (Table 35B). This disparity was greater for Hispanic males (2.2 times the rate among White males) than Hispanic females (1.7 times the rate among White females) (Figure W).

\section{Figure W. Primary and Secondary Syphilis - Rates of Reported Cases by Race/Hispanic Ethnicity and Sex, United States, 2018}

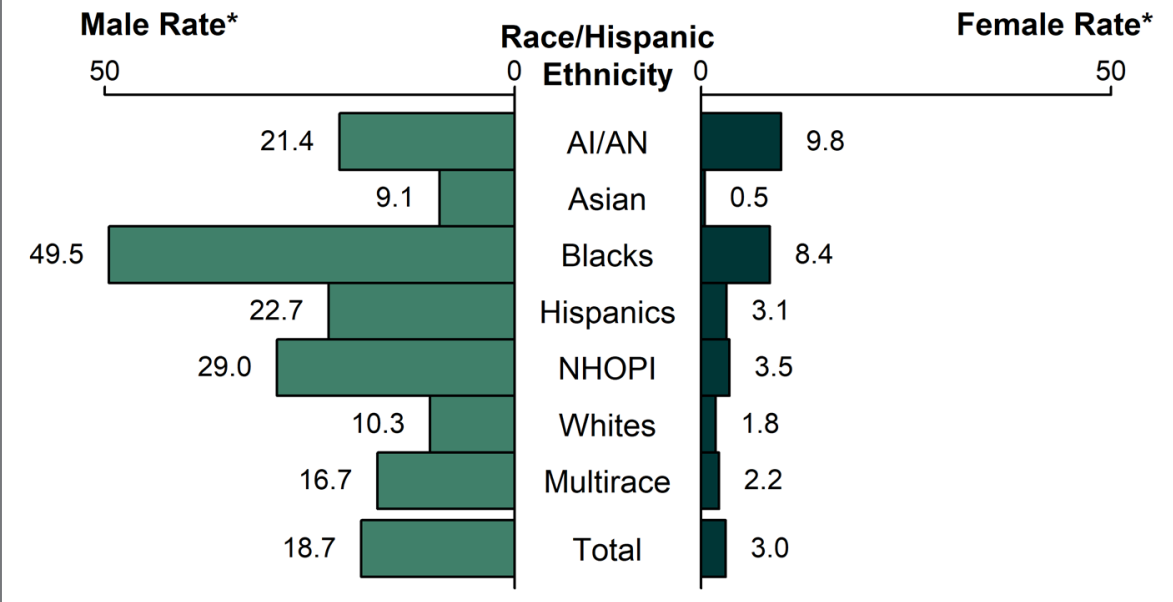

* Per 100,000 .

NOTE: See Section A1.5 in the Appendix for information on reporting STD case data for race/Hispanic ethnicity.

ACRONYMS: AI/AN = American Indians/Alaska Natives; $\mathrm{NHOPI}=$ Native Hawaiians/Other Pacific Islanders

Native Hawaiians/Other Pacific Islanders - In 2018, the rate of reported P\&S syphilis cases among NHOPI was 2.7 times the rate among Whites (16.3 versus 6.0 cases per 100,000 population, respectively) (Table 35B). This disparity was greater for NHOPI males (2.8 times the rate among White males) than NHOPI females (1.9 times the rate among White females) (Figure W).

American Indians/Alaska Natives

- In 2018, the rate of reported P\&S syphilis cases among AI/AN was 2.6 times the rate among Whites (15.5 versus 6.0 cases per 100,000 population, respectively) (Table 35B). This disparity was greater for AI/AN females (5.4 times the rate among White females) than for AI/AN males (2.1 times the rate among White males) (Figure W).

Asians - In 2018, the rate of reported $\mathrm{P} \& \mathrm{~S}$ syphilis cases among Asians was 0.8 times the rate among Whites (4.6 versus 6.0 cases per 100,000 population, respectively) (Table 35B). This disparity was greater for Asian females (0.3 times the rate among White females) than for Asian males (0.9 times the rate among White males) (Figure W). Similar disparities were seen in all regions of the United States (Figure $\mathrm{X})$.

\section{Congenital Syphilis}

Race/Hispanic ethnicity for cases of congenital syphilis are assigned according to the mother's race/ Hispanic ethnicity information. During 2014-2018, rates of reported congenital syphilis cases increased for all race/Hispanic ethnicity groups (Figure Y). The rate of reported congenital syphilis increased $500.0 \%$ among AI/AN (13.2 to 79.2 cases per 100,000 live births), $275.0 \%$ among Whites (3.6 to 13.5 cases per 100,000 live births), 263.4\% among Hispanics (12.3 to 44.7 cases per 100,000 live births), 126.7\% among Blacks (38.2 to 86.6 cases per 100,000 live births), and $31.4 \%$ among Asian/Pacific Islanders ( 7.0 to 9.2 cases per 100,000 live births). 
Figure X. Primary and Secondary Syphilis - Rate Ratios* by Sex, Race/Hispanic Ethnicity, and Region, United States, 2018

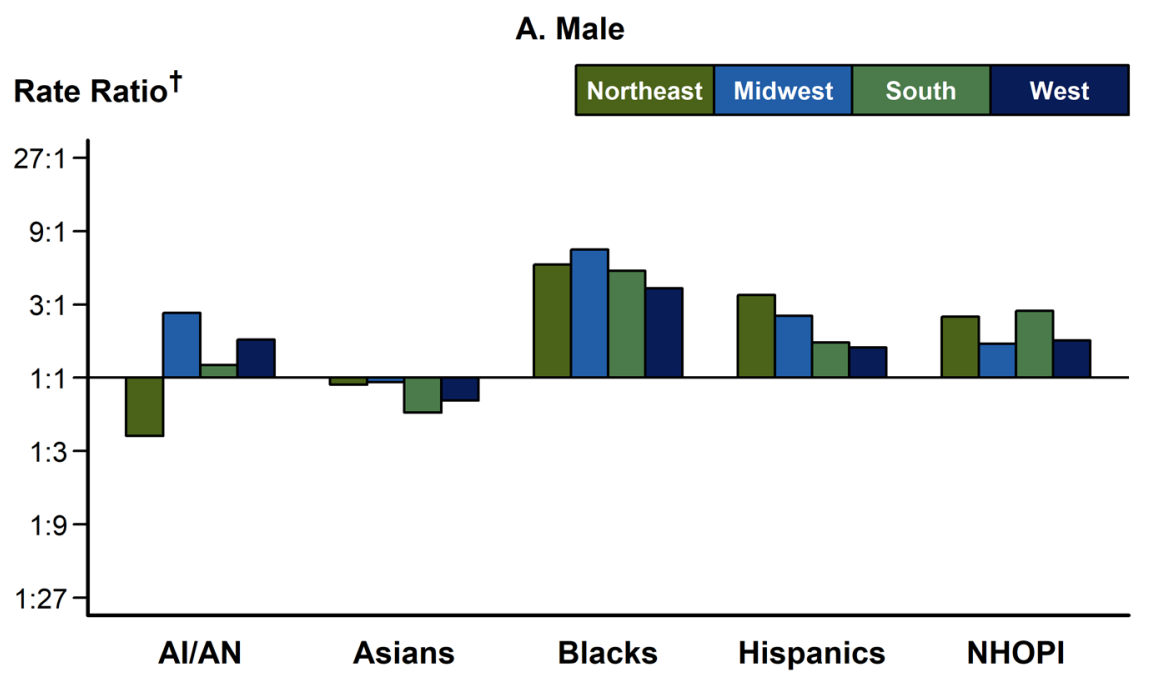

B. Female

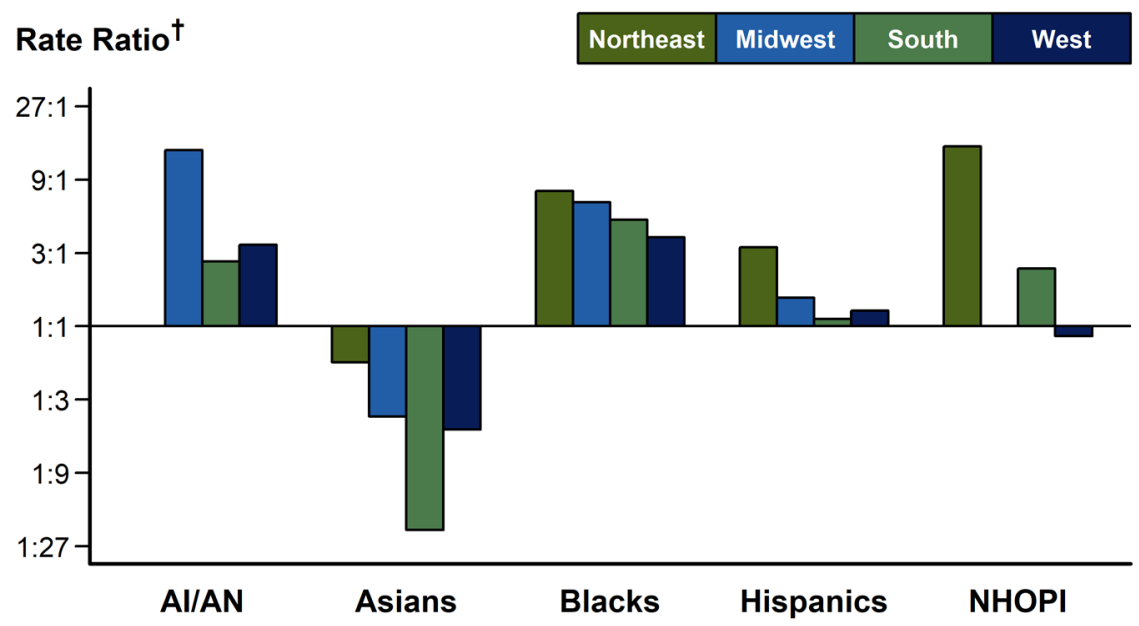

${ }^{*}$ For the rate ratios, Whites are the reference population.

${ }^{\dagger} \mathrm{Y}$-axis is log scale.

NOTE: See Section A1.5 in the Appendix for information on reporting STD case data for race/Hispanic ethnicity.

ACRONYMS: Al/AN = American Indians/Alaska Natives; NHOPI = Native

Hawaiians/Other Pacific Islanders.

In $2018,40.4 \%$ of reported congenital syphilis cases with known race/ Hispanic ethnicity information occurred among Blacks, 32.6\% occurred among Hispanics, and $22.7 \%$ occurred among Whites (Table 42). Disparities persist across race/ Hispanic ethnicity groups. The rate of reported cases of congenital syphilis among Blacks was 6.4 times the rate among Whites (86.6 versus 13.5 cases per 100,000 live births, respectively). The rate of reported cases of congenital syphilis among AI/AN (79.2 cases per 100,000 live births) was 5.9 times the rate among Whites, the rate among Hispanics (44.7 cases per 100,000 live births) was 3.3 times the rate among Whites, and the rate among Asians/Pacific Islanders (9.2 cases per 100,000 live births) was 0.7 times the rate among Whites.

\section{Other STDs}

Data from the National Health and Nutrition Examination Survey (NHANES; see Section A2.4 in the Appendix) indicate the seroprevalence of herpes simplex virus type 2 (HSV-2) among the civilian, noninstitutionalized population in the United States has decreased from 1999-2000 to 2015-2016 for all race/ Hispanic ethnicity groups (Figure 53) ${ }^{15}$ however, HSV-2 seroprevalence was highest among non-Hispanic Blacks throughout the entire time period. For more information on HSV infections, see Other STDs.

Trichomonas vaginalis prevalence in urine specimens obtained from NHANES participants aged 14-59 years during 2013-2016 indicated a prevalence of $0.5 \%$ among males and $2.1 \%$ among females; highest rates were observed among nonHispanic Black males (3.4\%) and females $(9.6 \%) .{ }^{16} \mathrm{An}$ analysis of NHANES data from 2001-2004 from cervicovaginal swab specimens also found higher $T$. vaginalis prevalence among non-Hispanic Black females. ${ }^{17}$ For more information on $T$. vaginalis infections, see Other STDs.

\section{Summary}

Inequities in the burden of disease for chlamydia, gonorrhea, syphilis and other STDs by race/Hispanic ethnicity continue to persist at unacceptable levels in the United States. These disparities are not explained by individual or populationlevel behavioral differences; rather they result in large measure from stubbornly entrenched systemic, societal, and cultural barriers to STD diagnoses, treatment and preventive services accessible to some groups 


\section{Figure Y. Congenital Syphilis - Rates of Reported Cases by Year of Birth and Race/Hispanic Ethnicity of Mother, United States, 2009-2018}

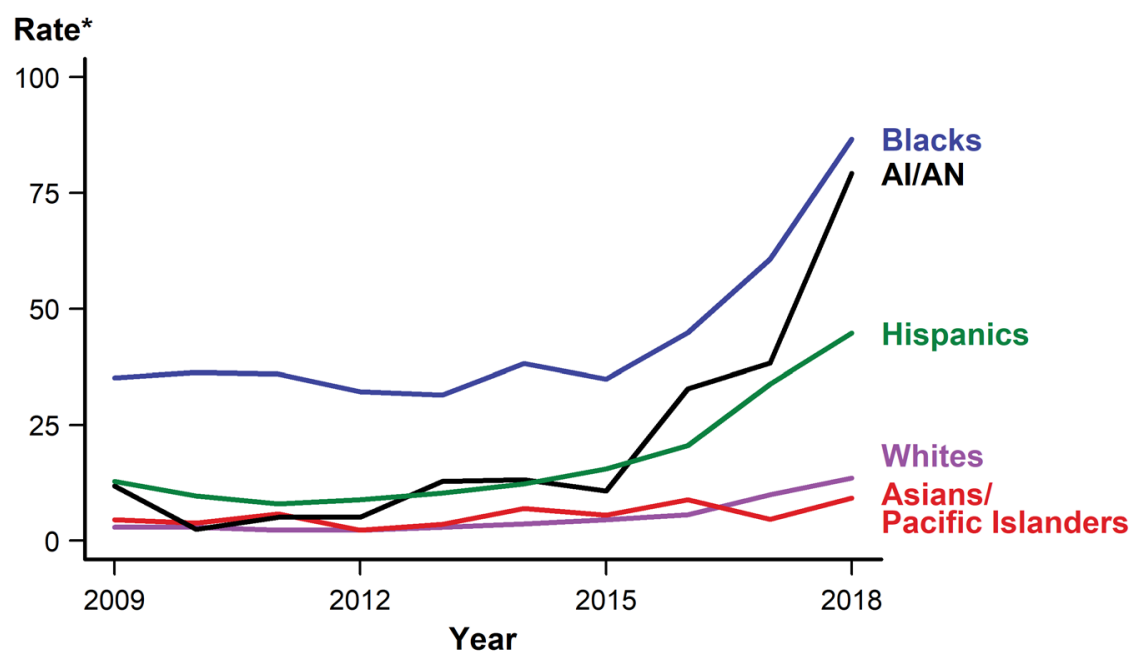

* Per 100,000 live births.

NOTE: National Center for Health Statistics bridged race categories are presented to allow the display of data across several years. See Section A1.5 in the Appendix for information on reporting STD case data for race/Hispanic ethnicity.

ACRONYMS: Al/AN = American Indians/Alaska Natives.

\section{Figure Z. Primary and Secondary Syphilis - Reported Cases* by Sex and Sex of Sex Partners and Race/Hispanic Ethnicity, United States, 2018}

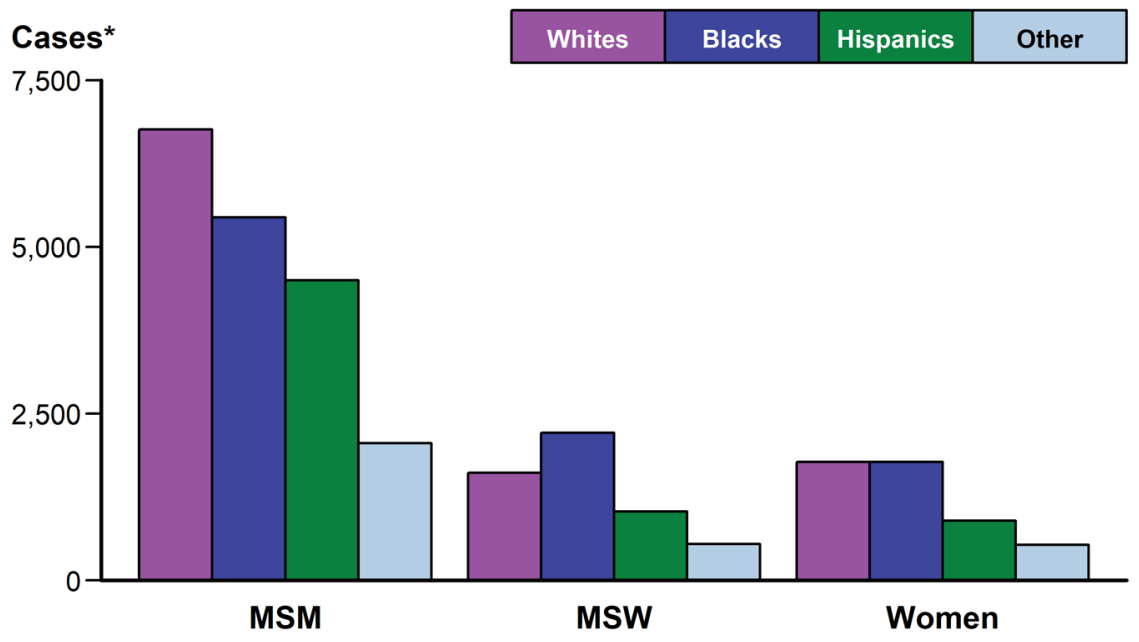

* Of all reported cases of primary and secondary syphilis, $16.7 \%$ were among men without data on sex of sex partners, and $<0.1 \%$ were cases with unknown sex; $6.1 \%$ of all cases had missing or unknown race/Hispanic ethnicity. Cases with missing or unknown race/Hispanic ethnicity are included in the "Other" category.

NOTE: See Section A1.5 in the Appendix for information on reporting STD case data for race/Hispanic ethnicity

ACRONYMS: MSM = Gay, bisexual, and other men who have sex with men; MSW = Men who have sex with women only. on a routine basis. While incremental progress has been achieved in recent years in reducing the magnitude of disparities in some STDs, especially for Blacks, much more needs to be done to address these issues through individual, group, and structural-level health care interventions. Continued monitoring of differences across groups in reported case incidence is also critical to the success of these efforts, including a sharpened focus on ascertainment of race/Hispanic ethnicity in categories congruent with the 1997 OMB guidance for persons diagnosed and reported with STDs. ${ }^{14}$

\section{References}

1. Newman LM, Berman SM. Epidemiology of STD Disparities in African American Communities. Sex Transm Dis. 2008;35(12):S4 S12.

2. Hogben M, Leichliter JS. Social determinants and sexually transmitted disease disparities. Sex Transm Dis. 2008;35(Suppl 12):S13-18.

3. Semega JL, Fontenot KR, Kollar MA. U.S. Census Bureau, Current Population Reports, P60-259, Income and Poverty in the United States: 2016. Washington, DC: U.S. Government Printing Office;2017. Available at: https://www.census.gov/content/dam/Census/ library/publications/2018/demo/p60-263.pdf. Accessed July30, 2019.

4. Harling G, Subramanian SV, Barnighausen T, et al. Socioeconomic disparities in sexually transmitted infections among young adults in the United States: Examining the interaction between Income and race/ethnicity. Sex Transm Dis. 2013;40(7):575-581.

5. Centers for Disease Control and Prevention. CDC Health Disparities and Inequalities Report — United States 2013. MMWR Morb Mortal Wkly Rep. 2013;62(Suppl 3).

6. Institute of Medicine. Summary: The hidden epidemic: Confronting sexually transmitted diseases. Washington (DC): National Academy Press; 1997.

7. Satterwhite CL, Torrone E, Meites E, et al. Sexually transmitted infections among US women and men: Prevalence and incidence estimates, 2008. Sex Transm Dis. 2013;40(3):187-193.

8. Berchick ER, Hood E, Barnett JC. Current Population Reports, P60-264, Health Insurance Coverage in the United States: 2017. Washington, DC: U.S. Government Printing Office;2017. Available at: https:// www.census.gov/content/dam/Census/library/ publications/2018/demo/p60-264.pdf Accessed July 30, 2019. 
9. Pérez-Escamilla R. Health care access among Latinos: Implications for social and health care reform. J Hispanic High Educ. 2010;9(1):4360.

10. Berk ML, Schur CL. The effect of fear on access to care among undocumented Latino immigrants. J Immigr Health. 2001;3(3):151156.

11. Institute of Medicine. Unequal treatment: Confronting racial and ethnic disparities in health care. Washington, DC: National Academies Press; 2002.

12. Datta SD, Sternberg M, Johnson RE, et al. Gonorrhea and chlamydia in the United States among persons 14 to 39 years of age, 1999 to 2002. Ann Intern Med. 2007;147(2):89-96.

13. Miller WC, Ford CA, Morris M, et al. Prevalence of chlamydial and gonococcal infections among young adults in the United States. JAMA. 2004;291(18):2229-2236.

14. Office of Management and Budget. Provisional guidance on the implementation of the 1997 standards for federal data on race and ethnicity. In:1997. Available at: https://www.whitehouse. gov/wp-content/uploads/2017/11/ProvisionalGuidance-jan16-2001-1.pdf. Accessed August $1,2019$.

15. McQuillan G, Kruszon-Moran D, Flagg EW, et al. Prevalence of herpes simplex virus type 1 and type 2 in persons aged 14-49: United States, 2015-2016. NCHS data brief, no 304. Hyattsville, MD: National Center for Health Statistics. 2018.

16. Flagg EW, Meites E, Phillips C, et al. Prevalence of Trichomonas vaginalis among males and females aged 14-59 years: United States, 2013-2016. Sex Transm Dis. Published online 27 May 2019. DOI: 10.1097/ OLQ.0000000000001013.

17. Sutton M, Sternberg M, Koumans EH, et al. The prevalence of Trichomonas vaginalis infection among reproductive-age women in the United States, 2001-2004. Clin Infect Dis. 2007;45(10):1319-1326. 


\section{Background}

The incidence of many STDs in gay, bisexual, and other men who have sex with men (MSM) - including primary and secondary (P\&S) syphilis and antimicrobial-resistant gonorrhea - is greater than that reported in women and men who have sex with women only (MSW). ${ }^{1-6}$ In addition to the negative effects of untreated STDs, elevated STD burden is of concern because it may indicate high risk for subsequent HIV infection. Annual increases in reported STD cases could reflect increased frequency of behaviors that transmit both STDs and HIV (e.g., condomless anal sex), and having an STD increases the risk of acquisition or transmission of HIV. $^{7-14}$

The relatively high incidence of STD infection among MSM may be related to multiple factors, including individual behaviors and sexual network characteristics. ${ }^{15-17}$ The number of lifetime or recent sex partners, rate of partner exchange, and frequency of condomless sex each influence an individual's probability of exposure to STDs. ${ }^{15}$ However, MSM network characteristics such as high prevalence of STDs, interconnectedness and concurrency of sex partners, and possibly limited access to healthcare also affect the risk of acquiring an STD. ${ }^{15,18}$ Furthermore, experiences of stigmaverbal harassment, discrimination, or physical assault based on attraction to men - are associated with increased sexual risk behavior among MSM. ${ }^{19}$

Disparities among MSM reflect those observed in the general population, with disproportionate incidence of STDs reported among racial minority and Hispanic MSM, MSM of lower socioeconomic status, and young MSM. ${ }^{20-24}$ The higher burden of STDs among MSM with these characteristics, relative to the general population of MSM, may suggest distinct mixing patterns in their sexual networks, reduced access to screening and treatment, and differential experiences of stigma and discrimination, rather than greater numbers of sexual partners or frequency of condomless sex..$^{15,21-22 \text {, }}$ ${ }^{24-26}$ Furthermore, disparities may be more pronounced for racial minority and Hispanic MSM who are also unemployed, young, and/or of lower socioeconomic status. ${ }^{26-27}$

With the exception of reported syphilis cases, nationally notifiable STD surveillance data do not routinely include information on sex of sex partners, and these data are missing for the majority of gonorrhea and chlamydia cases reported to CDC. Therefore, trends in STDs among MSM in the United States are based on findings from sentinel and enhanced surveillance systems. Testing strategies are also evolving to include more extragenital STD screening, which may increase detection of asymptomatic infections. Until recently, testing for gonorrhea and chlamydia in MSM largely focused on detecting urethral infections, which are more likely to be symptomatic than pharyngeal or rectal infections. ${ }^{28}$

For data reported in this chapter, MSM were defined as men who either reported having one or more male sex partners or who self-reported as gay/ homosexual or bisexual. MSW were defined as men who reported having sex with women only or who did not report the sex of their sex partners, but reported that they considered themselves straight/heterosexual. Data presented in this chapter are derived from the National Notifiable Diseases Surveillance System (NNDSS), the Gonococcal Isolate Surveillance Project (GISP), and the STD Surveillance Network (SSuN).

\section{Nationally Notifiable Diseases Surveillance System}

MSM accounted for $64.3 \%$ of reported P\&S syphilis cases among women or men with information about sex of sex partners in 2018 (Figure 39). Among men exclusively, MSM accounted for $77.6 \%$ of reported cases with information on sex of sex partners. Of MSM P\&S syphilis cases, $36.0 \%$ were White, $29.0 \%$ were Black, and $24.0 \%$ were Hispanic (Figure Z). Relative to the percentage of the US population that is White $(60.4 \%)$, Black (12.5\%), and Hispanic (18.3\%), ${ }^{29}$ this represents a higher burden of disease for nonWhite MSM, which was also evident among MSW and women. In addition, among MSM P\&S syphilis cases with known HIV status in 2017, 35.5\% were also reported to be HIV-positive (Figure 46).

In 2018, 44 states provided data to classify at least $70 \%$ of cases as MSM, MSW, or women. Among these areas, estimated rates of $\mathrm{P} \& \mathrm{~S}$ syphilis cases in MSM ranged from 108.9 cases per 100,000 MSM in Vermont to 962.3 cases per 100,000 MSM in Mississippi, with 28 states $(64 \%)$ estimated to have rates between 200 and 500 cases per 100,000 MSM (Figure AA).

When examining reported $\mathrm{P} \& \mathrm{~S}$ syphilis cases over time, 36 states were able to classify at least $70 \%$ of reported $\mathrm{P} \& \mathrm{~S}$ syphilis cases as MSM, MSW, or women each year during 2014-2018. In these states, cases among MSM increased 5.3\% during 2017-2018 and 51.5\% during 20142018 (Figure 41). However, despite the increase in cases, the percentage of $\mathrm{P} \& \mathrm{~S}$ syphilis cases that were attributed to MSM in those states decreased from $72.4 \%$ in 2014 to $62.7 \%$ in 2018 due to larger relative 
Figure AA. Primary and Secondary Syphilis - Estimated Rates of Reported Cases Among MSM by State, United States, 2018

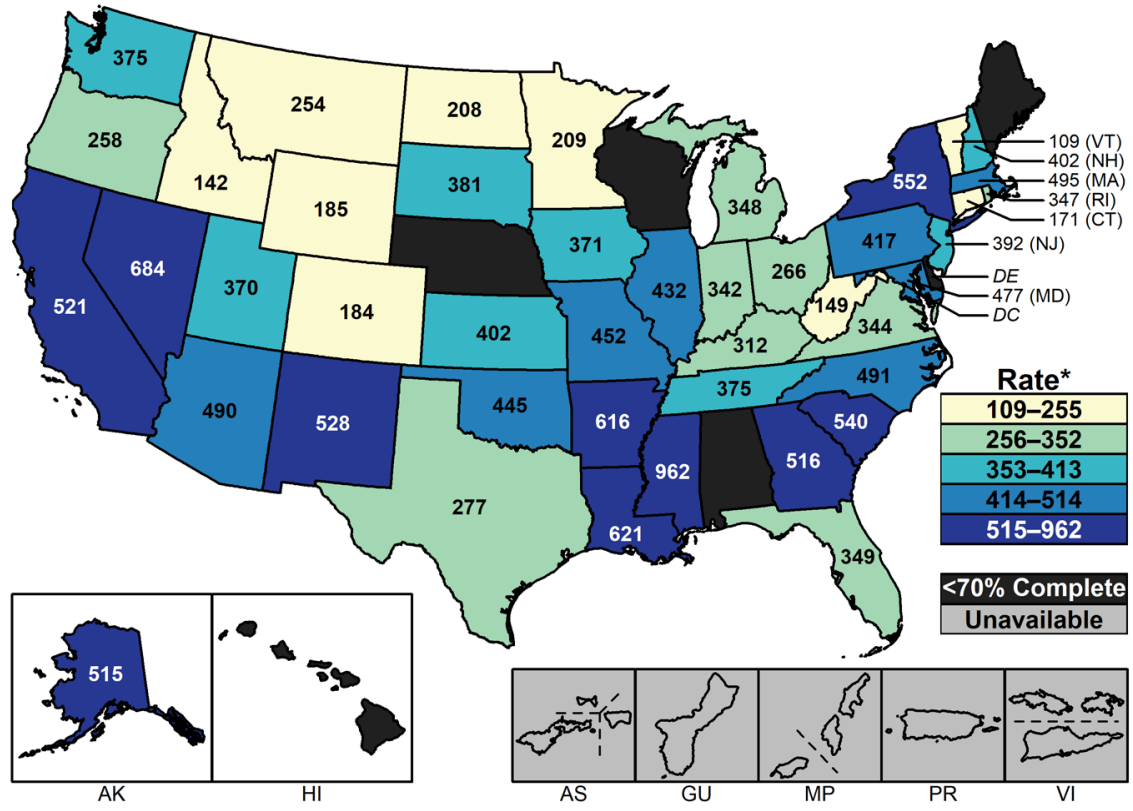

* Per 100,000 .

NOTE: States reporting less than $70 \%$ of cases identified as MSM, MSW, or women in 2018 are suppressed. See Section A1.2 in the Appendix for information on estimating MSM population sizes for rate denominators.

ACRONYMS: $M S M=$ Gay, bisexual, and other men who have sex with men; $M S W=$ Men who have sex with women only. of $38.5 \%$ in 2017 (Figure BB). In 2018 , this proportion was $37.2 \%$. The reason for this increase over time is unclear, but might reflect changes in the epidemiology of gonorrhea or in healthcare-seeking behavior of men infected with gonorrhea. GISP has demonstrated that gonococcal isolates from MSM are more likely to exhibit antimicrobial resistance than isolates from MSW. ${ }^{3,4}$ In 2018, the proportion of isolates with elevated azithromycin minimum inhibitory concentrations (MICs) $(\geq 2.0 \mu \mathrm{g} / \mathrm{mL})$ and elevated ceftriaxone MICs $(\geq 0.125 \mu \mathrm{g} / \mathrm{mL})$ was higher in isolates from MSM than from MSW (Figure CC). For azithromycin, $8.2 \%$ of isolates from MSM had elevated MICs compared to $2.4 \%$ in MSW. For ceftriaxone, the proportion was slightly higher at $0.22 \%$ in MSM compared to $0.16 \%$ in MSW.

Information on the antimicrobial susceptibility criteria used in GISP can be found in Section A2.3 of the Appendix. More information about GISP and additional data can be found at: https://www.cdc.gov/std/GISP. increases in reported P\&S syphilis cases among MSW and women.

A description of the methods for estimating MSM population sizes for syphilis rate denominators can be found in Section A1.2 of the Appendix. More information about syphilis can be found in the Syphilis section of the National Profile.

\section{Gonococcal Isolate Surveillance Project}

GISP is a national sentinel surveillance system designed to monitor trends in antimicrobial susceptibilities of Neisseria gonorrhoeae strains in the United States. ${ }^{3}$ Overall, the proportion of isolates collected in selected STD clinics participating in GISP that were from MSM increased steadily, from 3.9\% in 1989 to a high

\section{Figure BB. Neisseria gonorrhoeae - Percentage of Urethral Isolates Obtained from MSM Attending STD Clinics, Gonococcal Isolate Surveillance Project (GISP), 1989-2018}

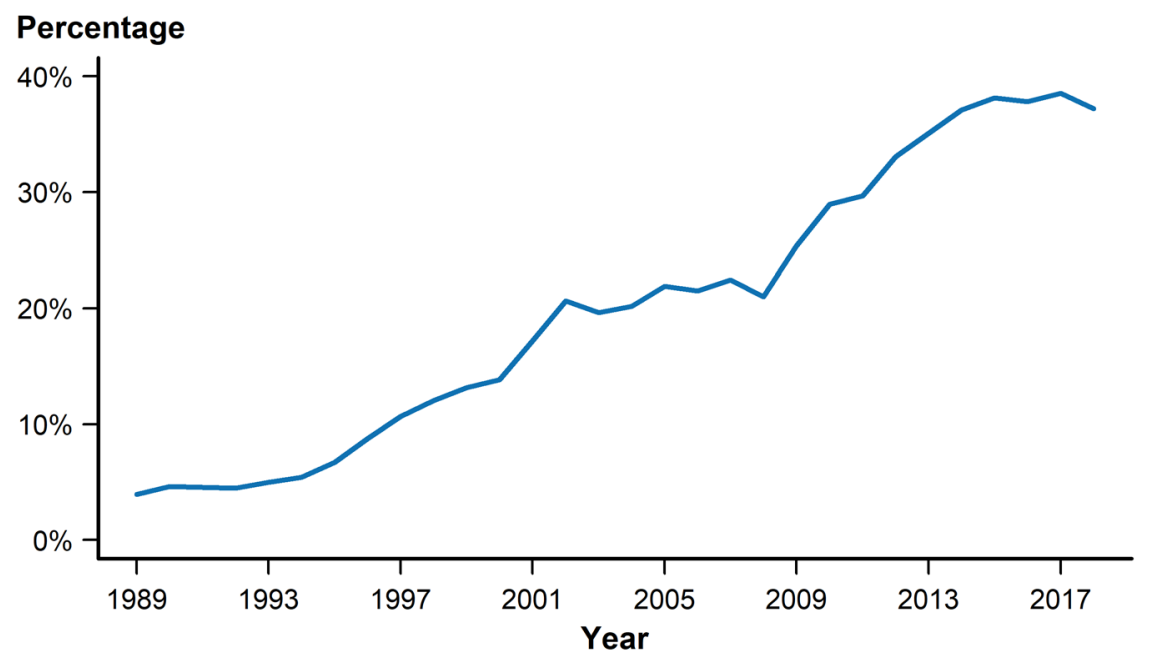

ACRONYMS: MSM = Gay, bisexual, and other men who have sex with men. 
Figure CC. Neisseria gonorrhoeae - Percentage of Urethral Isolates with Elevated Minimum Inhibitory Concentrations (MICs) to Azithromycin $^{*}$ and Ceftriaxone ${ }^{\dagger}$ by Sex and Sex of Sex Partners, Gonococcal Isolate Surveillance Project (GISP), 2009-2018

\section{A. Azithromycin}

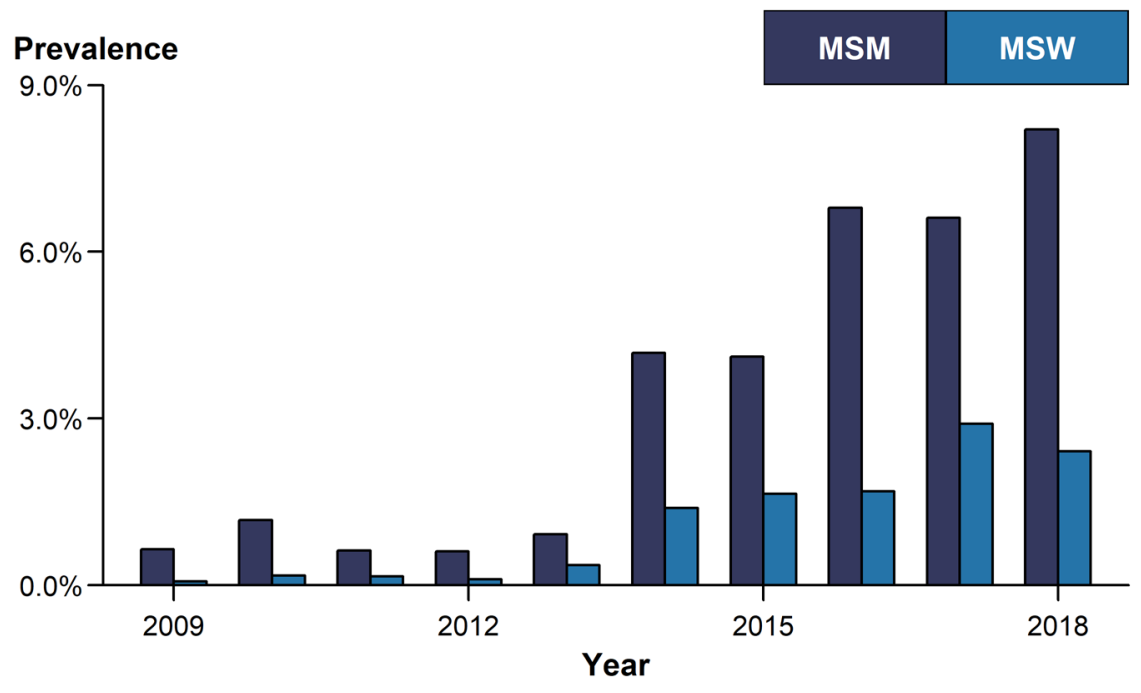

\section{B. Ceftriaxone}

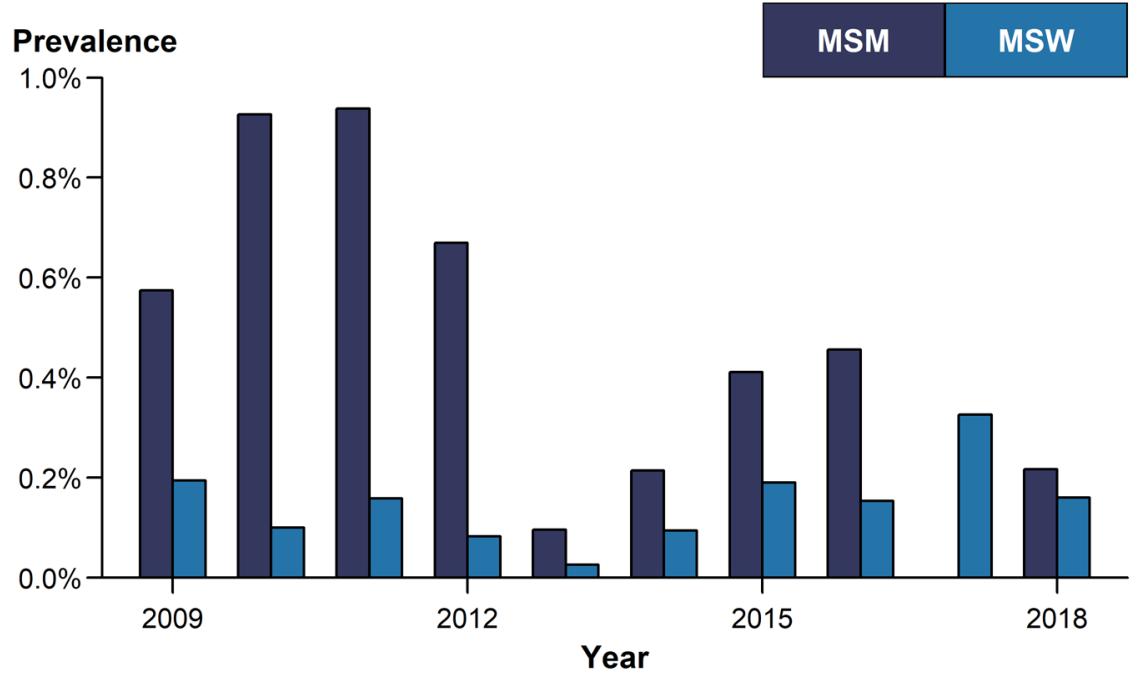

* Elevated Azithromycin MIC: $\geq 2.0 \mu \mathrm{g} / \mathrm{mL}$.

${ }^{\dagger}$ Elevated Ceftriaxone MIC: $\geq 0.125 \mu \mathrm{g} / \mathrm{mL}$.

ACRONYMS: $M S M=$ Gay, bisexual, and other men who have sex with men; MSW = Men who have sex with women only.

\section{STD Surveillance Network}

The STD Surveillance Network $(\mathrm{SSuN})$ is an ongoing collaboration of state, county, and city health departments conducting sentinel and enhanced surveillance activities. These include collecting enhanced clinical and behavioral information among all patients attending selected STD clinics, among women aged
15-44 years in selected reproductive health clinics, and conducting enhanced patient and provider investigations on a representative sample of gonorrhea cases diagnosed and reported from all reporting sources in their jurisdiction (See Section A2.2 of the Appendix). Estimated rates of reported gonorrhea among MSM based on SSuN data are provided in the Gonorrhea section of the National Profile (Figure 25).

\section{Urethral and Extragenital Gonorrhea and Chlamydia in STD Clinics, 2018}

In 2018, 31,650 unique MSM presented for care in 18 STD clinics in nine $\mathrm{SSuN}$ jurisdictions. In total, 26,159 unique MSM were tested for urogenital gonorrhea and/or chlamydia $(26,151$ for gonorrhea and 26,087 for chlamydia) (Figure DD). Urogenital testing varied between jurisdictions, with the proportion tested for urogenital gonorrhea ranging from $49.7 \%-94.7 \%$ and the proportion tested for urogenital chlamydia ranging from $48.7 \%$ to $94.7 \%$. However, the proportion tested for urogenital gonorrhea and for urogenital chlamydia was similar within each jurisdiction. When examining positivity among MSM tested for each disease, urogenital gonorrhea positivity was higher than urogenital chlamydia positivity in seven of the nine jurisdictions: Baltimore, Miami, Multnomah County, New York City, Philadelphia, San Francisco, and Seattle. Urogenital chlamydia positivity was equal or higher than urogenital gonorrhea positivity in Massachusetts and Minnesota. The median urogenital positivity for gonorrhea among MSM was $7.9 \%$ (range: $4.3 \%-13.3 \%$ ) and for chlamydia was $5.8 \%$ (range: $4.3 \%-$ $11.1 \%$ ) across the nine jurisdictions.

In 2018, there were 20,838 unique MSM tested for rectal gonorrhea and/ 
Figure DD. Gonorrhea and Chlamydia - Proportion* of MSM STD Clinic Patients Tested and Testing Positive ${ }^{\dagger}$ for Urogenital Gonorrhea and Chlamydia by Jurisdiction, STD Surveillance Network (SSuN), 2018

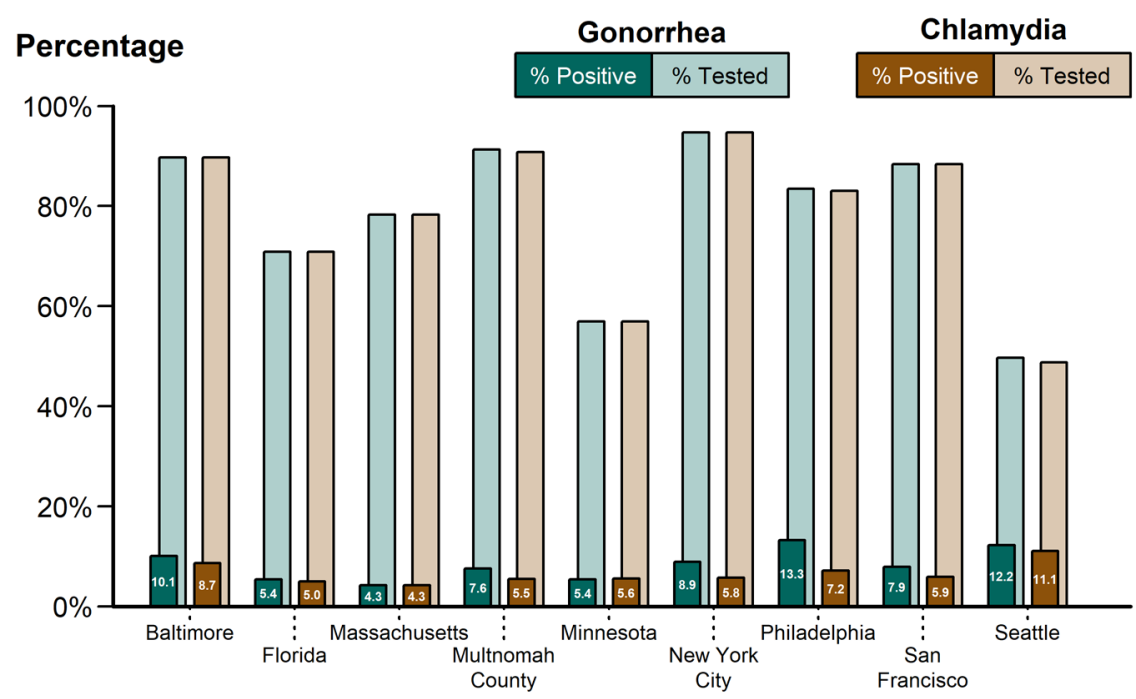

* Results based on data obtained from unique patients with known sex of sex partners tested for urogenital gonorrhea $(n=26,151)$ and for urogenital chlamydia $(n=26,087) \geq 1$ time in 2018.

${ }^{\dagger}$ Percent positive among those tested for urogenital gonorrhea or chlamydia. NOTE: See section A2.2 in the Appendix for SSuN methods.

ACRONYMS: MSM = Gay, bisexual, and other men who have sex with men.

Figure EE. Gonorrhea and Chlamydia - Proportion* of MSM STD Clinic Patients Tested and Testing Positive ${ }^{\dagger}$ for Rectal Gonorrhea and Chlamydia by Jurisdiction, STD Surveillance Network (SSuN), 2018

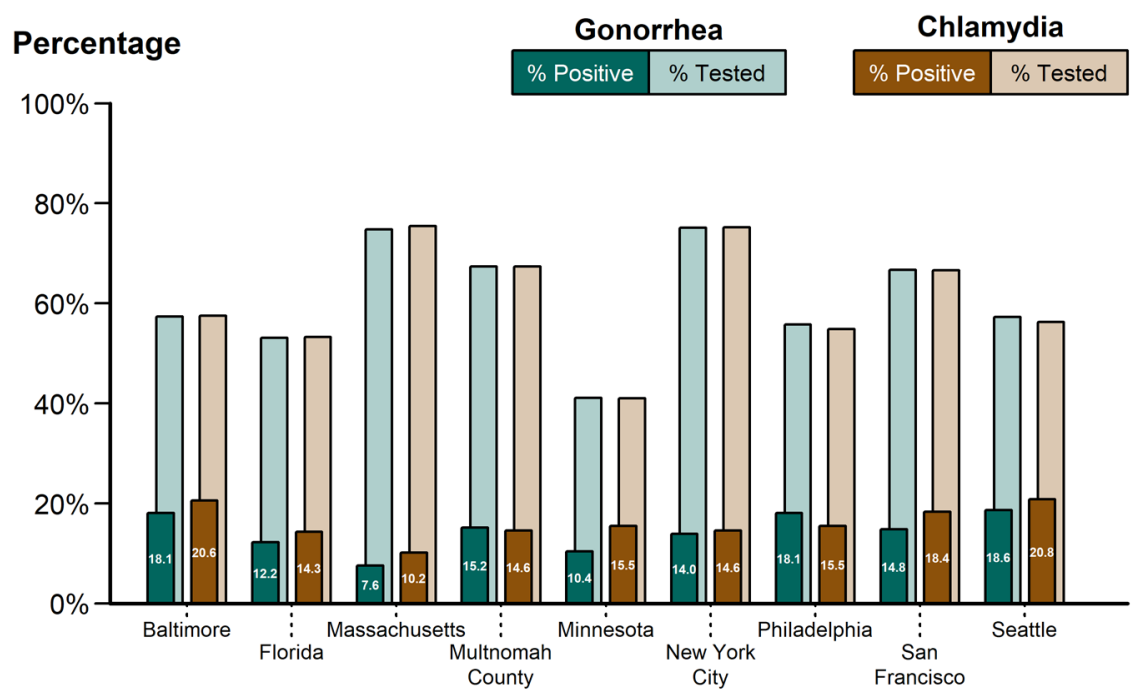

* Results based on data obtained from unique patients with known sex of sex partners tested for rectal gonorrhea $(n=20,798)$ and for rectal chlamydia $(n=20,755) \geq 1$ time in 2018.

${ }^{\dagger}$ Percent positive among those tested for rectal gonorrhea or chlamydia. NOTE: See section A2.2 in the Appendix for SSUN methods.

ACRONYMS: $M S M=$ Gay, bisexual, and other men who have sex with men. or chlamydia $(20,798$ for gonorrhea and 20,755 for chlamydia) (Figure EE). When compared to urogenital testing, rectal testing occurs less frequently in the majority of jurisdictions. The median positivity for rectal gonorrhea among MSM was $14.8 \%$ (range: $7.6 \%-18.1 \%$ ) and for rectal chlamydia was $16.1 \%$ (range: $10.2 \%-20.8 \%$ ) in SSuN jurisdictions.

During 2018, 23,739 MSM were tested at the pharyngeal site for gonorrhea and/or chlamydia $(23,695$ for gonorrhea and 21,767 for chlamydia) in eight of the $10 \mathrm{SSuN}$ jurisdictions where data was available (Figure FF). Pharyngeal testing varied between $52.2 \%-86.5 \%$ for gonorrhea and between 68.2-76.6\% for chlamydia. Among MSM who were tested at the pharyngeal site for gonorrhea, the median positivity was $12.9 \%$ (range: $8.0 \%-19.8 \%$ ). In contrast, MSM tested at the pharyngeal site for chlamydia was $2.7 \%$ (range: $2.0 \%-3.1 \%$ ). Pharyngeal chlamydia testing data was not available for Minnesota.

\section{HIV Status and STDs in STD Clinics, 2018}

Among HIV-positive MSM attending SSuN STD clinics in seven of the 10 SSuN jurisdictions in 2018, urogenital chlamydia positivity was $6.1 \%$ and urogenital gonorrhea positivity was $12.7 \%$ (compared to $6.7 \%$ and $7.6 \%$, respectively, among HIV-negative MSM) (Figure GG). Among HIVpositive MSM, 7.0\% were diagnosed with P\&S syphilis compared to $3.4 \%$ of HIV-negative MSM. Percentages represent the overall average of the mean value by jurisdiction.

\section{Anogenital Warts in STD Clinics, 2010-2016}

Human papillomavirus (HPV) is a common sexually transmitted infection in the United States. ${ }^{31}$ MSM are at elevated risk for clinical sequelae of HPV infection, including 


\section{Figure FF. Gonorrhea and Chlamydia - Proportion* of MSM STD Clinic Patients Tested and Testing Positive ${ }^{\dagger}$ for Pharyngeal Gonorrhea and Chlamydia by Jurisdiction, STD Surveillance Network (SSuN), 2018}

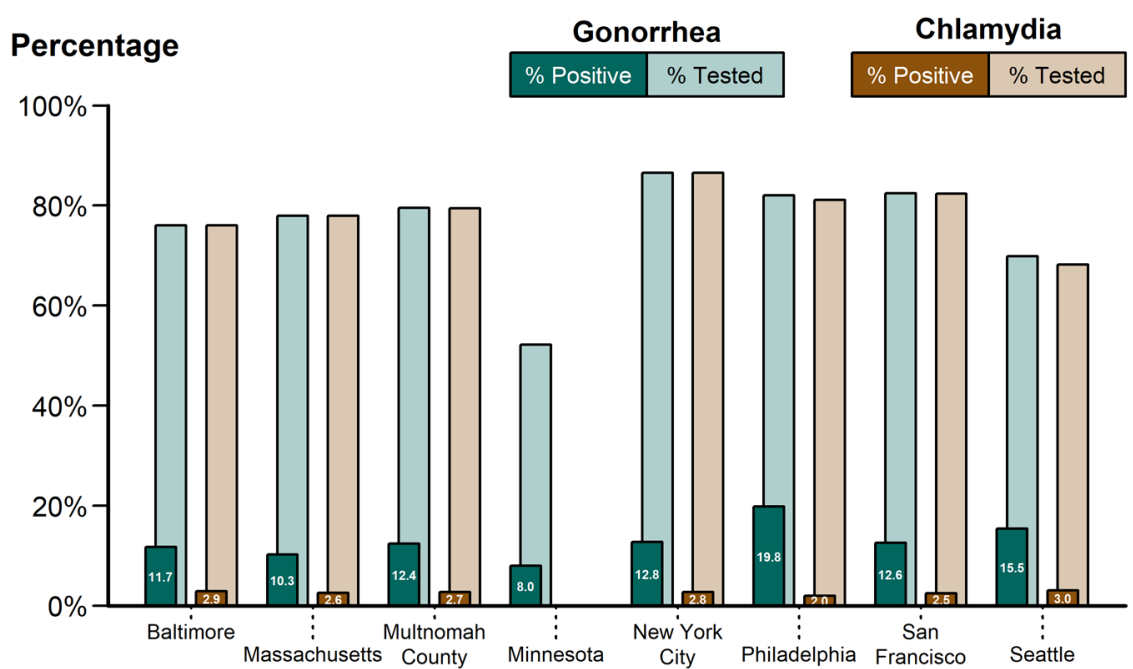

* Results based on data obtained from unique patients with known sex of sex partners tested for pharyngeal gonorrhea $(n=23,695)$ and for pharyngeal chlamydia $(n=21,767) \geq 1$ time in 2018.

${ }^{\dagger}$ Percent positive among those tested for pharyngeal gonorrhea or chlamydia NOTE: See section A2.2 in the Appendix for SSUN methods.

ACRONYMS: MSM = Gay, bisexual, and other men who have sex with men.

anal intraepithelial neoplasia, anal cancer, and anogenital warts. ${ }^{32,33}$ Since late 2011, routine use of HPV vaccine has been recommended for males aged 11-12 years, with catchup vaccination through age $21 ; 34-36$ this age limit was recently extended to 26 years. ${ }^{37}$ Vaccination through age 26 has been recommended since late 2011 for MSM and persons who are immunocompromised (including those infected with HIV). ${ }^{34}$ For more information on HPV infections and HPV vaccination, see Other STDs.

An analysis of data from 27 clinics participating in SSuN observed significant declines in prevalence of anogenital warts during 2010-2016 among MSM of all ages. ${ }^{38}$ Although some of the observed declines may be due to HPV vaccination, changes over time in attributes of STD clinic patients or clinical practices, such as a decrease in physical examinations resulting in fewer anogenital warts diagnoses, may partially account for these findings.

\section{Summary}

The number of reported P\&S syphilis cases among MSM continued to rise in 2018, and the majority of P\&S syphilis cases remained among MSM. Furthermore, the proportion of GISP isolates with elevated MICs to antimicrobials currently used to treat gonorrhea was higher among MSM than among MSW. Beyond STD burden in the general MSM population, the data indicated heterogeneity of STD prevalence among MSM according to geography, race/Hispanic ethnicity, and HIV status. State-specific P\&S syphilis rate estimates among MSM varied from 108.9 to 962.3 cases per 100,000 MSM. Reported P\&S syphilis was disproportionately prevalent among
Black and Hispanic MSM, and data from MSM who attended SSuN clinics suggested that $\mathrm{P} \& \mathrm{~S}$ syphilis and urogenital gonorrhea may be more prevalent among MSM living with diagnosed HIV infection than among HIV-negative MSM.

\section{References}

1. An Q, Wejnert C, Bernstein K, et al. Syphilis screening and diagnosis among men who have sex with men, 2008-2014, 20 US cities. JAIDS 2017;75(Suppl 3):S363-S369.

2. de Voux A, Kidd S, Grey JA, et al. Statespecific rates of primary and secondary syphilis among men who have sex with men - United States, 2015. MMWR Morb Mortal Wkly Rep. 2017;66(13):349-354.

3. Kirkcaldy RD, Harvey A, Papp JR, et al. Neisseria gonorrhoeae antimicrobial susceptibility surveillance - the Gonococcal Isolate Surveillance Project, 27 sites, United States, 2014. MMWR Surveill Summ. 2016;65(No. SS-7):1-19.

4. Kirkcaldy RD, Zaidi A, Hook EW 3rd, et al. Neisseria gonorrhoeae antimicrobial resistance among men who have sex with men and men who have sex exclusively with women: The Gonococcal Isolate Surveillance Project, 2005-2010. Ann Intern Med. 2013;158(5 Pt 1):321-328.

5. Patton ME, Su JR, Nelson R, et al. Primary and secondary syphilis — United States, 2005-2013. MMWR Morb Mortal Wkly Rep. 2014;63(18):402-406.

6. Peterman TA, Su J, Bernstein KT, et al. Syphilis in the United States: on the rise? Expert Rev Anti Infect Ther. 2015;13(2):161-168.

7. Solomon MM, Mayer KH. Evolution of the syphilis epidemic among men who have sex with men. Sex Health. 2014;12(2):96-102.

8. Buchacz K, Patel P, Taylor M, et al. Syphilis increases HIV viral load and decreases CD4 cell counts in HIV-infected patients with new syphilis infections. AIDS. 2004;18(15):20752079.

9. Fleming DT, Wasserheit JN. From epidemiological synergy to public health policy and practice: The contribution of other sexually transmitted diseases to sexual transmission of HIV infection. Sex Transm Infect. 1999;75(1):317.

10. Jarzebowski W, Caumes E, Dupin N, et al. Effect of early syphilis infection on plasma viral load and CD4 cell count in human immunodeficiency virus- infected men: Results from the FHDH- ANRS CO4 cohort. Arch Intern Med. 2012;172(16):1237-1243. 


\section{Figure GG. Proportion of MSM Attending STD Clinics with Primary and Secondary Syphilis*, Urogenital Gonorrhea, or Urogenital Chlamydia by Known HIV Status, STD Surveillance Network (SSuN), 2018}

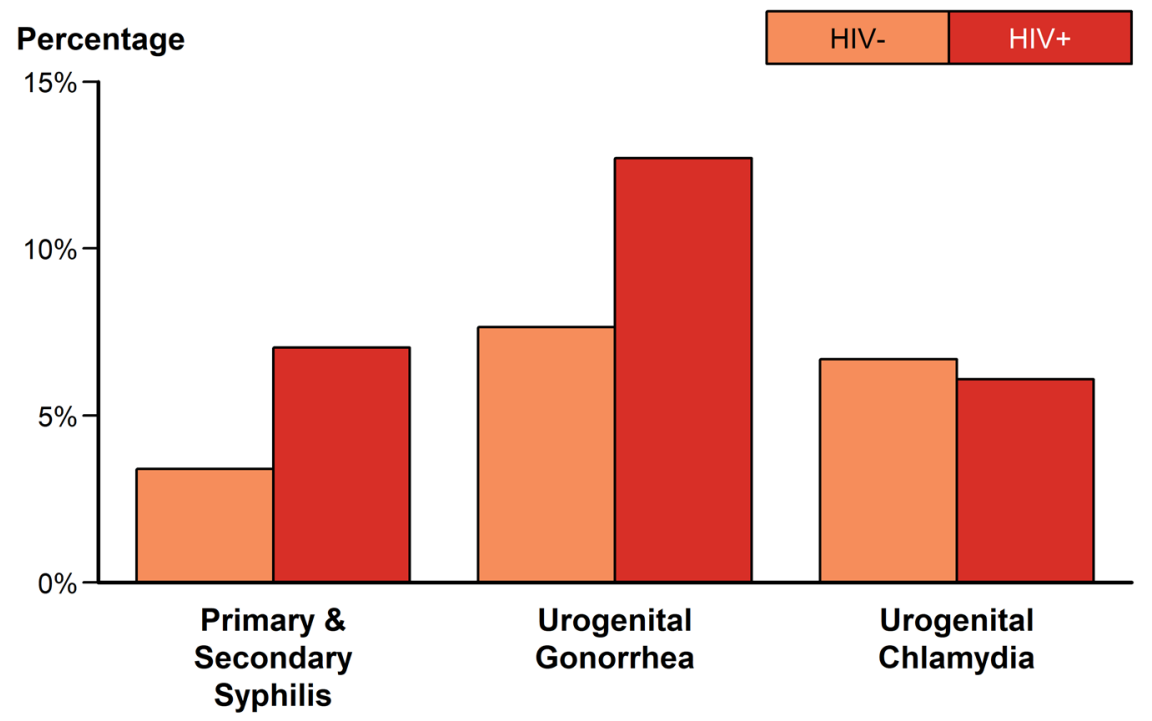

* Includes SSUN jurisdictions that reported data on at least 20 patients with a diagnosis of primary and secondary syphilis in 2018.

NOTE: See section A2.2 in the Appendix for SSUN methods.

ACRONYMS: $M S M=$ Gay, bisexual, and other men who have sex with men.

11. Katz DA, Dombrowski JC, Bell TR, et al. HIV incidence among men who have sex with men after diagnosis with sexually transmitted infections. Sex Transm Dis. 2016;43(4):249254.

12. Kelley CF, Vaughan AS, Luisi N, et al. The effect of high rates of bacterial sexually transmitted infections on HIV incidence in a cohort of black and white men who have sex with men in Atlanta, Georgia. AIDS Res Hum Retroviruses. 2015;31(6):587-592.

13. Pathela P, Braunstein SL, Blank S, et al. HIV incidence among men with and those without sexually transmitted rectal infections: Estimates from matching against and HIV case registry. Clin Infect Dis. 2013;57(8):1203-1209.

14. Solomon MM, Mayer KH, Glidden DV, et al. Syphilis predicts HIV incidence among men and transgender women who have sex with men in a preexposure prophylaxis trial. Clin Infect Dis. 2014;59(7):1020-1026.

15. Glick SN, Morris M, Foxman B, et al. A comparison of sexual behavior patterns among men who have sex with men and heterosexual men and women. J Acquir Immune Defic Syndr. 2012;60(1):83-90.
16. Paz-Bailey G, Mendoza MCB, Finlayson T, et al. Trends in condom use among MSM in the United States: The role of antiretroviral therapy and seroadaptive strategies. AIDS. 2016;30(12):1985-1990.

17. Spicknall IH, Gift TL, Bernstein KT, et al. Sexual networks and infection transmission networks among men who have sex with men as causes of disparity and targets of prevention. Sex Transm Infect. 2017;93(5):307-308.

18. Alvy LM, McKirnan DJ, Du Bois SN, et al. Health care disparities and behavioral health among men who have sex with men. J Gay Lesbian Soc Serv. 2011;23(4):507-522.

19. Balaji AB, Bowles KE, Hess KL, et al. Association between enacted stigma and HIVrelated risk behavior among MSM, National HIV Behavioral Surveillance System, 2011. AIDS Behav. 2017;21(1):227-237.

20. Brewer TH, Schillinger J, Lewis FM, et al. Infectious syphilis among adolescent and young adult men: Implications for human immunodeficiency virus transmission and public health interventions. Sex Transm Dis. 2011;38(5):367-371.
21. Jeffries WL, Marks G, Lauby J. Homophobia is associated with sexual behavior that increases risk of acquiring and transmitting HIV infection among black men who have sex with men. AIDS Behav. 2013;17(4):1442-1453.

22. McKirnan DJ, Du Bois SN, Alvy LM, et al. Health care access and health behaviors among men who have sex with men: The cost of health disparities. Health Educ Behav. 2013;40(1):3241.

23. Su JR, Beltrami JF, Zaidi AA, et al. Primary and secondary syphilis among black and Hispanic men who have sex with men: Case report data from 27 States. Ann Intern Med. 2011;155(3):145-151.

24. Díaz RM, Ayala G, Bein E. Sexual risk as an outcome of social oppression: Data from a probability sample of Latino gay men in three US cities. Cultur Divers Ethnic Minor Psychol. 2004;10(3):255-267.

25. Millett GA, Flores SA, Peterson JL, et al. Explaining disparities in HIV infection among black and white men who have sex with men: A meta-analysis of HIV risk behaviors. AIDS. 2007;21(15):2083-2091.

26. Sullivan PS, Peterson J, Rosenberg ES, et al. Understanding racial HIV/STI disparities in Black and White men who have sex with men: A multilevel approach. PLoS One. 2014;9(3):e90514.

27. Mayer KH, Wang L, Koblin B, et al. Concomitant socioeconomic, behavioral, and biological factors associated with the disproportionate HIV infection burden among black men who have sex with men in 6 US cities. PLoS One. 2014;9(1):e87298.

28. Patton ME, Kidd S, Llata E, et al. Extragenital gonorrhea and chlamydia testing and infection among men who have sex with men - STD Surveillance Network, United States, 2010 2012. Clin Infect Dis. 2014;58(11):1564-1570.

29. U.S. Census Bureau. Annual Estimates of the Resident Population by Sex, Race, and Hispanic Origin for the United States, States, and Counties: April 1, 2010 to July 1, 2018, Table PEPSR6H; generated by Jeremy Grey; using American FactFinder. 2019. Available at: https://factfinder.census.gov/faces/nav/jsf/pages/ index.xhtml. Accessed August 1, 2019.

30. Rietmeijer K, Donnelly J, Bernstein K, et al. Here comes the SSuN — early experiences with the STD Surveillance Network. Pub Health Rep. 2009;124(Suppl 2):72-77.

31. Satterwhite CL, Torrone E, Meites E, et al. Sexually transmitted infections among US women and men: Prevalence and incidence estimates, 2008. Sex Transm Dis. 2013;40(3):187-193. 
32. Jin F, Prestage GP, Kippax SC, et al. Risk factors for genital and anal warts in a prospective cohort of HIV-negative homosexual men: the HIM study. Sex Transm Dis. 2007;34(7):488-493.

33. Chin-Hong PV, Palefsky JM. Natural history and clinical management of anal human papillomavirus disease in men and women infected with human immunodeficiency virus. Clin Infect Dis. 2002;35(9):1127-1134.

34. Petrosky E, Bocchini Jr. JA, Hariri S, et al. Use of 9-valent human papillomavirus (HPV) vaccine: Updated HPV vaccination recommendations of the Advisory Committee on Immunization Practices. MMWR Morb Mortal Wkly Rep. 2015;64(11):300-304.

35. Centers for Disease Control and Prevention. Recommendations on the use of quadrivalent human papillomavirus vaccine in males Advisory Committee on Immunization Practices (ACIP), 2011. MMWR Morb Mortal Wkly Rep. 2011;60(50):1705-1708.

36. Meites E, Kempe A, Markowitz LE. Use of a 2-dose schedule for human papillomavirus vaccination - updated recommendations of the Advisory Committee on Immunization Practices. MMWR Morb Mortal Wkly Rep. 2016;65(49):1405-1408.
37. Meites E, Szilagyi PG, Chesson HW, et al. Human papillomavirus vaccination for adults: Updated recommendations of the Advisory Committee on Immunization Practices. MMWR Morb Mortal Wkly Rep. 2019; 68(32):698-702.

38. Mann LM, Llata E, Flagg EW, et al. Trends in the prevalence of anogenital warts among patients at sexually transmitted disease clinics - Sexually Transmitted Disease Surveillance Network, United States, 2010-2016. J Infect Dis. 2019;219(9):1389-1397

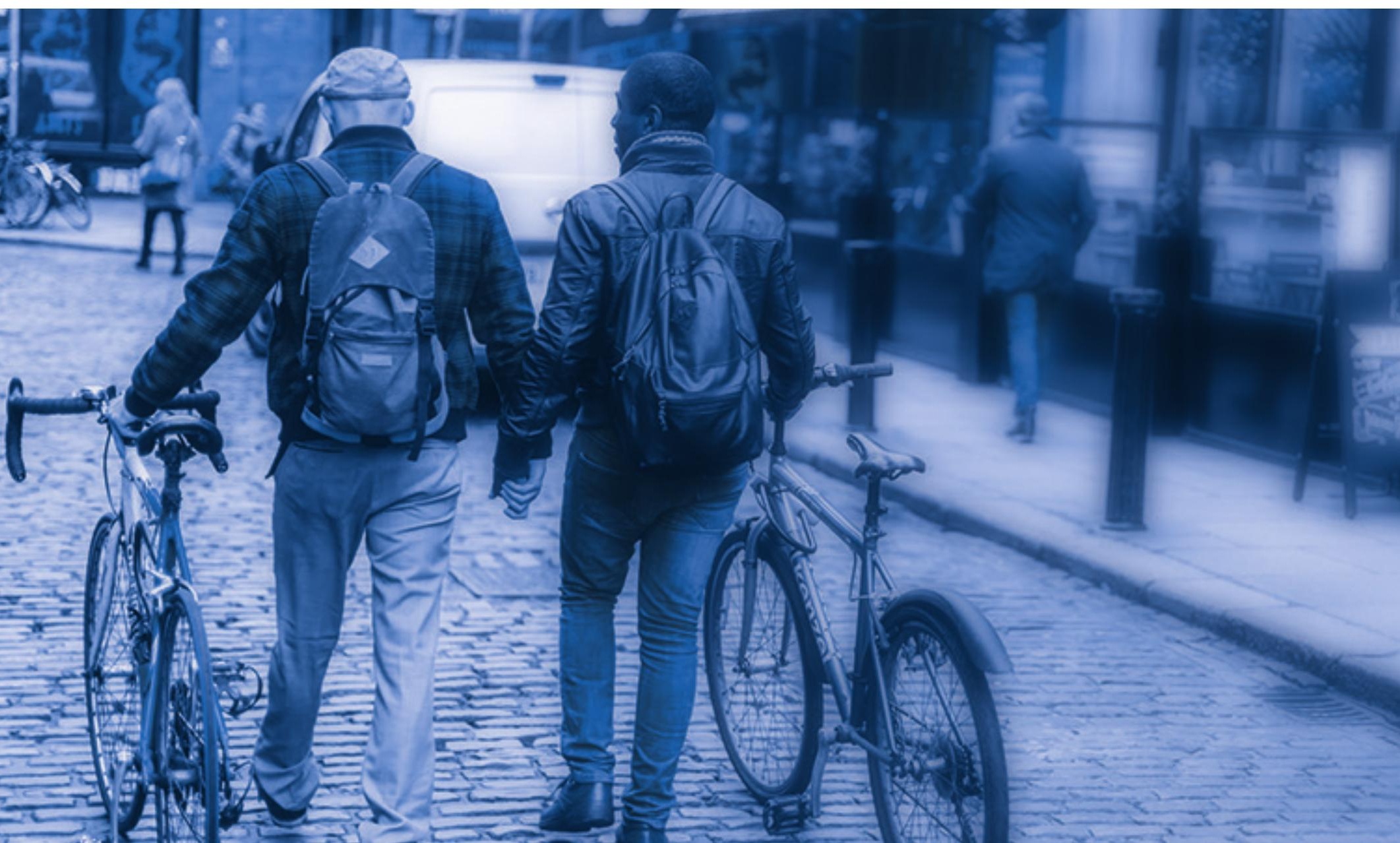




\section{Tables}

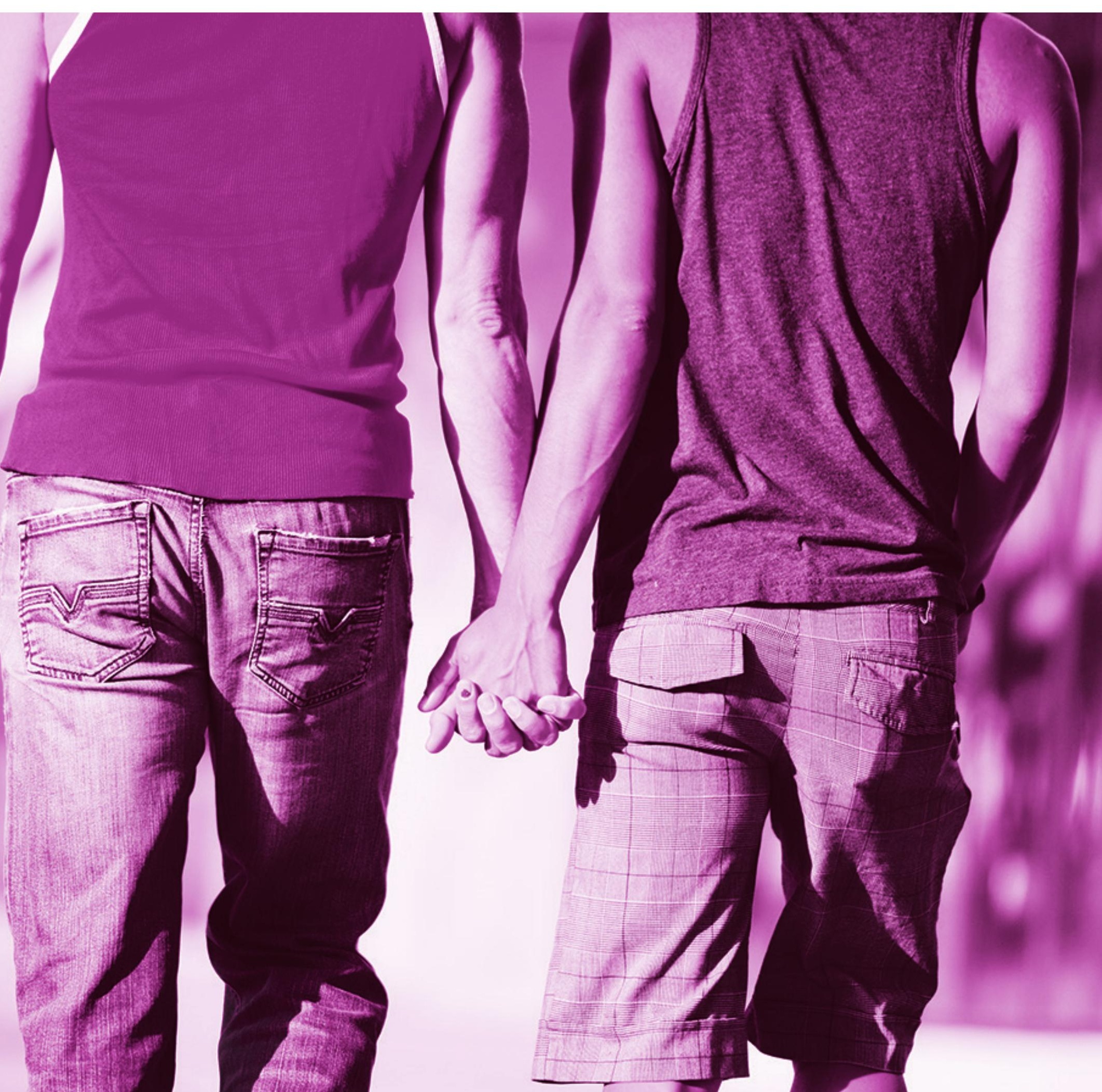


Table 1. Sexually Transmitted Diseases - Reported Cases and Rates of Reported Cases*, United States, 19412018

\begin{tabular}{|c|c|c|c|c|c|c|c|c|c|c|c|c|c|c|c|c|}
\hline \multirow[b]{3}{*}{ Year $^{+}$} & \multicolumn{10}{|c|}{ Syphilis } & \multirow{2}{*}{\multicolumn{2}{|c|}{ Chlamydia }} & \multirow{2}{*}{\multicolumn{2}{|c|}{ Gonorrhea }} & \multirow{2}{*}{\multicolumn{2}{|c|}{ Chancroid }} \\
\hline & \multicolumn{2}{|c|}{ All Stages ${ }^{\ddagger}$} & \multicolumn{2}{|c|}{$\begin{array}{c}\text { Primary and } \\
\text { Secondary }\end{array}$} & \multicolumn{2}{|c|}{$\begin{array}{l}\text { Early } \\
\text { Latent }\end{array}$} & \multicolumn{2}{|c|}{$\begin{array}{l}\text { Unk. Duration } \\
\text { or Late }{ }^{\S}\end{array}$} & \multicolumn{2}{|c|}{ Congenital } & & & & & & \\
\hline & Cases & Rate & Cases & Rate & Cases & Rate & Cases & Rate & Cases & Rate\| & Cases & Rate & Cases & Rate & Cases & Rate \\
\hline 1941 & 485,560 & 368.2 & 68,231 & 51.7 & 109,018 & 82.6 & 202,984 & 153.9 & 17,600 & 651.1 & NR & - & 193,468 & 146.7 & 3,384 & 2.5 \\
\hline 1942 & 479,601 & 363.4 & 75,312 & 57.0 & 116,245 & 88.0 & 202,064 & 153.1 & 16,918 & 566.0 & NR & - & 212,403 & 160.9 & 5,477 & 4.1 \\
\hline 1943 & 575,593 & 447.0 & 82,204 & 63.8 & 149,390 & 116.0 & 251,958 & 195.7 & 16,164 & 520.7 & NR & - & 275,070 & 213.6 & 8,354 & 6.4 \\
\hline 1944 & 467,755 & 367.9 & 78,443 & 61.6 & 123,038 & 96.7 & 202,848 & 159.6 & 13,578 & 462.0 & NR & - & 300,676 & 236.5 & 7,878 & 6.1 \\
\hline 1945 & 359,114 & 282.3 & 77,007 & 60.5 & 101,719 & 79.9 & 142,187 & 111.8 & 12,339 & 431.7 & NR & - & 287,181 & 225.8 & 5,515 & 4.3 \\
\hline 1946 & 363,647 & 271.7 & 94,957 & 70.9 & 107,924 & 80.6 & 125,248 & 93.6 & 12,106 & 354.9 & NR & - & 368,020 & 275.0 & 7,091 & 5.2 \\
\hline 1947 & 355,592 & 252.3 & 93,545 & 66.4 & 104,124 & 73.9 & 122,089 & 86.6 & 12,200 & 319.6 & NR & - & 380,666 & 270.0 & 9,515 & 6.7 \\
\hline 1948 & 314,313 & 218.2 & 68,174 & 47.3 & 90,598 & 62.9 & 123,312 & 85.6 & 13,931 & 383.0 & NR & - & 345,501 & 239.8 & 7,661 & 5.3 \\
\hline 1949 & 256,463 & 175.3 & 41,942 & 28.7 & 75,045 & 51.3 & 116,397 & 79.5 & 13,952 & 382.4 & NR & - & 317,950 & 217.3 & 6,707 & 4.6 \\
\hline 1950 & 217,558 & 146.0 & 23,939 & 16.7 & 59,256 & 39.7 & 113,569 & 70.2 & 13,377 & 368.3 & NR & - & 286,746 & 192.5 & 4,977 & 3.3 \\
\hline 1951 & 174,924 & 116.1 & 14,485 & 9.6 & 43,316 & 28.7 & 98,311 & 65.2 & 11,094 & 290.4 & NR & - & 254,470 & 168.9 & 4,233 & 2.8 \\
\hline 1952 & 167,762 & 110.2 & 10,449 & 6.9 & 36,454 & 24.0 & 105,238 & 69.1 & 8,553 & 218.8 & NR & - & 244,957 & 160.8 & 3,738 & 2.5 \\
\hline 1953 & 148,573 & 95.9 & 8,637 & 5.6 & 28,295 & 18.3 & 98,870 & 63.8 & 7,675 & 193.9 & NR & - & 238,340 & 153.9 & 3,338 & 2.2 \\
\hline 1954 & 130,697 & 82.9 & 7,147 & 4.5 & 23,861 & 15.1 & 89,123 & 56.5 & 6,676 & 164.0 & NR & - & 242,050 & 153.5 & 3,003 & 1.9 \\
\hline 1955 & 122,392 & 76.2 & 6,454 & 4.0 & 20,054 & 12.5 & 86,526 & 53.8 & 5,354 & 130.7 & NR & - & 236,197 & 147.0 & 2,649 & 1.7 \\
\hline 1956 & 130,201 & 78.7 & 6,392 & 3.9 & 19,783 & 12.0 & 95,097 & 57.5 & 5,491 & 130.4 & NR & - & 224,346 & 135.7 & 2,135 & 1.3 \\
\hline 1957 & 123,758 & 73.5 & 6,576 & 3.9 & 17,796 & 10.6 & 91,309 & 54.2 & 5,288 & 123.0 & NR & - & 214,496 & 127.4 & 1,637 & 1.0 \\
\hline 1958 & 113,884 & 66.4 & 7,176 & 4.2 & 16,556 & 9.7 & 83,027 & 48.4 & 4,866 & 114.6 & NR & - & 232,386 & 135.6 & 1,595 & 0.9 \\
\hline 1959 & 120,824 & 69.2 & 9,799 & 5.6 & 17,025 & 9.8 & 86,740 & 49.7 & 5,130 & 119.7 & NR & - & 240,254 & 137.6 & 1,537 & 0.9 \\
\hline 1960 & 122,538 & 68.8 & 16,145 & 9.1 & 18,017 & 10.1 & 81,798 & 45.9 & 4,416 & 103.7 & NR & - & 258,933 & 145.4 & 1,680 & 0.9 \\
\hline 1961 & 124,658 & 68.8 & 19,851 & 11.0 & 19,486 & 10.8 & 79,304 & 43.8 & 4,163 & 97.5 & NR & - & 264,158 & 145.8 & 1,438 & 0.8 \\
\hline 1962 & 126,245 & 68.7 & 21,067 & 11.5 & 19,585 & 10.7 & 79,533 & 43.3 & 4,070 & 97.7 & NR & - & 263,714 & 143.6 & 1,344 & 0.7 \\
\hline 1963 & 124,137 & 66.5 & 22,251 & 11.9 & 18,235 & 9.8 & 78,076 & 41.8 & 4,031 & 98.4 & NR & - & 278,289 & 149.0 & 1,220 & 0.7 \\
\hline 1964 & 114,325 & 60.4 & 22,969 & 12.1 & 17,781 & 9.4 & 68,629 & 36.3 & 3,516 & 87.3 & NR & - & 300,666 & 158.9 & 1,247 & 0.7 \\
\hline 1965 & 112,842 & 58.9 & 23,338 & 12.2 & 17,458 & 9.1 & 67,317 & 35.1 & 3,564 & 94.8 & NR & - & 324,925 & 169.5 & 982 & 0.5 \\
\hline 1966 & 105,159 & 54.2 & 21,414 & 11.0 & 15,950 & 8.2 & 63,541 & 32.7 & 3,170 & 87.9 & NR & - & 351,738 & 181.2 & 838 & 0.4 \\
\hline 1967 & 102,581 & 52.2 & 21,053 & 10.7 & 15,554 & 7.9 & 61,975 & 31.5 & 2,894 & 82.2 & NR & - & 404,836 & 205.9 & 784 & 0.4 \\
\hline 1968 & 96,271 & 48.4 & 19,019 & 9.6 & 15,150 & 7.6 & 58,564 & 29.4 & 2,381 & 68.0 & NR & - & 464,543 & 233.4 & 845 & 0.4 \\
\hline 1969 & 92,162 & 45.7 & 19,130 & 9.5 & 15,402 & 7.6 & 54,587 & 27.1 & 2,074 & 57.6 & NR & - & 534,872 & 265.4 & 1,104 & 0.5 \\
\hline 1970 & 91,382 & 44.8 & 21,982 & 10.8 & 16,311 & 8.0 & 50,348 & 24.7 & 1,953 & 52.3 & NR & - & 600,072 & 294.2 & 1,416 & 0.7 \\
\hline 1971 & 95,997 & 46.4 & 23,783 & 11.5 & 19,417 & 9.4 & 49,993 & 24.2 & 2,052 & 57.7 & NR & - & 670,268 & 324.1 & 1,320 & 0.6 \\
\hline 1972 & 91,149 & 43.6 & 24,429 & 11.7 & 20,784 & 9.9 & 43,456 & 20.8 & 1,758 & 54.0 & NR & - & 767,215 & 366.6 & 1,414 & 0.7 \\
\hline 1973 & 87,469 & 41.4 & 24,825 & 11.7 & 23,584 & 11.2 & 37,054 & 17.5 & 1,527 & 48.7 & NR & - & 842,621 & 398.7 & 1,165 & 0.6 \\
\hline 1974 & 83,771 & 39.3 & 25,385 & 11.9 & 25,124 & 11.8 & 31,854 & 14.9 & 1,138 & 36.0 & NR & - & 906,121 & 424.7 & 945 & 0.4 \\
\hline 1975 & 80,356 & 37.3 & 25,561 & 11.9 & 26,569 & 12.3 & 27,096 & 12.6 & 916 & 29.1 & NR & - & 999,937 & 464.1 & 700 & 0.3 \\
\hline 1976 & 71,761 & 33.0 & 23,731 & 10.9 & 25,363 & 11.7 & 21,905 & 10.1 & 626 & 19.8 & NR & - & $1,001,994$ & 460.6 & 628 & 0.3 \\
\hline 1977 & 64,621 & 29.4 & 20,399 & 9.3 & 21,329 & 9.7 & 22,313 & 10.2 & 463 & 13.9 & NR & - & $1,002,219$ & 456.0 & 455 & 0.2 \\
\hline 1978 & 64,875 & 29.2 & 21,656 & 9.8 & 19,628 & 8.8 & 23,038 & 10.4 & 434 & 13.0 & NR & - & $1,013,436$ & 456.3 & 521 & 0.2 \\
\hline 1979 & 67,049 & 29.9 & 24,874 & 11.1 & 20,459 & 9.1 & 21,301 & 9.5 & 332 & 9.5 & NR & - & $1,004,058$ & 447.1 & 840 & 0.4 \\
\hline 1980 & 68,832 & 30.3 & 27,204 & 12.0 & 20,297 & 8.9 & 20,979 & 9.2 & 277 & 7.7 & NR & - & $1,004,029$ & 442.1 & 788 & 0.3 \\
\hline 1981 & 72,799 & 31.7 & 31,266 & 13.6 & 21,033 & 9.2 & 20,168 & 8.8 & 287 & 7.9 & NR & - & 990,864 & 431.8 & 850 & 0.4 \\
\hline 1982 & 75,579 & 32.6 & 33,613 & 14.5 & 21,894 & 9.5 & 19,779 & 8.5 & 259 & 7.0 & NR & - & 960,633 & 414.7 & 1,392 & 0.6 \\
\hline 1983 & 74,637 & 31.9 & 32,698 & 14.0 & 23,738 & 10.2 & 17,896 & 7.7 & 239 & 6.6 & NR & - & 900,435 & 385.1 & 847 & 0.4 \\
\hline
\end{tabular}




\begin{tabular}{|c|c|c|c|c|c|c|c|c|c|c|c|c|c|c|c|c|}
\hline \multirow[b]{3}{*}{ Year $^{+}$} & \multicolumn{10}{|c|}{ Syphilis } & & & & & & \\
\hline & \multicolumn{2}{|c|}{ All Stages ${ }^{*}$} & \multicolumn{2}{|c|}{$\begin{array}{l}\text { Primary and } \\
\text { Secondary }\end{array}$} & \multicolumn{2}{|c|}{$\begin{array}{l}\text { Early } \\
\text { Latent }\end{array}$} & \multicolumn{2}{|c|}{$\begin{array}{l}\text { Unk. Duration } \\
\text { or Late }\end{array}$} & \multicolumn{2}{|c|}{ Congenital } & \multicolumn{2}{|c|}{ Chlamydia } & \multicolumn{2}{|c|}{ Gonorrhea } & \multicolumn{2}{|c|}{ Chancroid } \\
\hline & Cases & Rate & Cases & Rate & Cases & Rate & Cases & Rate & Cases & Rate\| & Cases & Rate & Cases & Rate & Cases & Rate \\
\hline 1984 & 69,872 & 29.6 & 28,607 & 12.1 & 23,131 & 9.8 & 17,829 & 7.6 & 305 & 8.3 & 7,594 & 6.5 & 878,556 & 372.5 & 665 & 0.3 \\
\hline 1985 & 67,563 & 28.4 & 27,131 & 11.4 & 21,689 & 9.1 & 18,414 & 7.7 & 329 & 8.7 & 25,848 & 17.4 & 911,419 & 383.0 & 2,067 & 0.9 \\
\hline 1986 & 67,779 & 28.2 & 27,667 & 11.5 & 21,656 & 9.0 & 18,046 & 7.5 & 410 & 10.9 & 58,001 & 35.2 & 892,229 & 371.5 & 3,045 & 1.3 \\
\hline 1987 & 87,286 & 36.0 & 35,585 & 14.7 & 28,233 & 11.7 & 22,988 & 9.5 & 480 & 12.6 & 91,913 & 50.8 & 787,532 & 325.0 & 4,986 & 2.1 \\
\hline 1988 & 104,546 & 42.8 & 40,474 & 16.6 & 35,968 & 14.7 & 27,363 & 11.2 & 741 & 19.0 & 157,854 & 87.1 & 738,160 & 301.9 & 4,891 & 2.0 \\
\hline 1989 & 115,089 & 46.6 & 45,826 & 18.6 & 45,394 & 18.4 & 22,032 & 8.9 & 1,837 & 45.5 & 200,904 & 102.5 & 733,294 & 297.1 & 4,697 & 1.9 \\
\hline 1990 & 135,590 & 54.3 & 50,578 & 20.3 & 55,397 & 22.2 & 25,750 & 10.3 & 3,865 & 92.9 & 323,663 & 160.2 & 690,042 & 276.4 & 4,212 & 1.7 \\
\hline 1991 & 128,719 & 50.9 & 42,950 & 17.0 & 53,855 & 21.3 & 27,490 & 10.9 & 4,424 & 107.6 & 381,228 & 179.7 & 621,918 & 245.8 & 3,476 & 1.4 \\
\hline 1992 & 114,730 & 44.7 & 34,009 & 13.3 & 49,929 & 19.5 & 26,725 & 10.4 & 4,067 & 100.0 & 409,694 & 182.3 & 502,858 & 196.0 & 1,906 & 0.7 \\
\hline 1993 & 102,612 & 39.5 & 26,527 & 10.2 & 41,919 & 16.1 & 30,746 & 11.8 & 3,420 & 85.5 & 405,332 & 178.0 & 444,649 & 171.1 & 1,292 & 0.5 \\
\hline 1994 & 82,713 & 31.4 & 20,641 & 7.8 & 32,017 & 12.2 & 27,603 & 10.5 & 2,452 & 62.0 & 451,785 & 192.5 & 419,602 & 163.9 & 782 & 0.3 \\
\hline 1995 & 69,359 & 26.0 & 16,543 & 6.2 & 26,657 & 10.0 & 24,296 & 9.1 & 1,863 & 47.8 & 478,577 & 187.8 & 392,651 & 147.5 & 607 & 0.2 \\
\hline 1996 & 53,240 & 19.8 & 11,405 & 4.2 & 20,187 & 7.5 & 20,366 & 7.6 & 1,282 & 32.9 & 492,631 & 190.6 & 328,169 & 121.8 & 386 & 0.1 \\
\hline 1997 & 46,716 & 17.1 & 8,556 & 3.1 & 16,631 & 6.1 & 20,447 & 7.5 & 1,082 & 27.9 & 537,904 & 205.5 & 327,665 & 120.2 & 246 & 0.1 \\
\hline 1998 & 38,289 & 13.9 & 7,007 & 2.5 & 12,696 & 4.6 & 17,743 & 6.4 & 843 & 21.4 & 614,250 & 231.8 & 356,492 & 129.2 & 189 & 0.1 \\
\hline 1999 & 35,386 & 12.7 & 6,617 & 2.4 & 11,534 & 4.1 & 16,655 & 6.0 & 580 & 14.6 & 662,647 & 247.2 & 360,813 & 129.3 & 110 & 0.0 \\
\hline 2000 & 31,618 & 11.2 & 5,979 & 2.1 & 9,465 & 3.4 & 15,594 & 5.5 & 580 & 14.3 & 709,452 & 251.4 & 363,136 & 128.7 & 78 & 0.0 \\
\hline 2001 & 32,286 & 11.3 & 6,103 & 2.1 & 8,701 & 3.0 & 16,976 & 5.9 & 506 & 12.6 & 783,242 & 274.5 & 361,705 & 126.8 & 38 & 0.0 \\
\hline 2002 & 32,919 & 11.4 & 6,862 & 2.4 & 8,429 & 2.9 & 17,168 & 6.0 & 460 & 11.4 & 834,555 & 289.4 & 351,852 & 122.0 & 48 & 0.0 \\
\hline 2003 & 34,289 & 11.8 & 7,177 & 2.5 & 8,361 & 2.9 & 18,319 & 6.3 & 432 & 10.6 & 877,478 & 301.7 & 335,104 & 115.2 & 54 & 0.0 \\
\hline 2004 & 33,423 & 11.4 & 7,980 & 2.7 & 7,768 & 2.6 & 17,300 & 5.9 & 375 & 9.1 & 929,462 & 316.5 & 330,132 & 112.4 & 30 & 0.0 \\
\hline 2005 & 33,288 & 11.2 & 8,724 & 2.9 & 8,176 & 2.8 & 16,049 & 5.4 & 339 & 8.2 & 976,445 & 329.4 & 339,593 & 114.6 & 17 & 0.0 \\
\hline 2006 & 36,958 & 12.3 & 9,756 & 3.3 & 9,186 & 3.1 & 17,644 & 5.9 & 372 & 8.7 & $1,030,911$ & 344.3 & 358,366 & 119.7 & 19 & 0.0 \\
\hline 2007 & 40,925 & 13.6 & 11,466 & 3.8 & 10,768 & 3.6 & 18,256 & 6.1 & 435 & 10.1 & $1,108,374$ & 367.5 & 355,991 & 118.0 & 23 & 0.0 \\
\hline 2008 & 46,292 & 15.2 & 13,500 & 4.4 & 12,401 & 4.1 & 19,945 & 6.6 & 446 & 10.5 & $1,210,523$ & 398.1 & 336,742 & 110.7 & 25 & 0.0 \\
\hline 2009 & 44,832 & 14.6 & 13,997 & 4.6 & 13,066 & 4.3 & 17,338 & 5.6 & 431 & 10.4 & $1,244,180$ & 405.3 & 301,174 & 98.1 & 28 & 0.0 \\
\hline 2010 & 45,844 & 14.8 & 13,774 & 4.5 & 13,604 & 4.4 & 18,079 & 5.9 & 387 & 9.7 & $1,307,893$ & 423.6 & 309,341 & 100.2 & 24 & 0.0 \\
\hline 2011 & 46,040 & 14.8 & 13,970 & 4.5 & 13,136 & 4.2 & 18,576 & 6.0 & 358 & 9.1 & $1,412,791$ & 453.4 & 321,849 & 103.3 & 8 & 0.0 \\
\hline 2012 & 49,915 & 15.9 & 15,667 & 5.0 & 14,503 & 4.6 & 19,411 & 6.2 & 334 & 8.4 & $1,422,976$ & 453.3 & 334,826 & 106.7 & 15 & 0.0 \\
\hline 2013 & 56,485 & 17.9 & 17,375 & 5.5 & 16,929 & 5.4 & 21,819 & 6.9 & 362 & 9.2 & $1,401,906$ & 443.5 & 333,004 & 105.3 & 10 & 0.0 \\
\hline 2014 & 63,454 & 19.9 & 19,999 & 6.3 & 19,452 & 6.1 & 23,541 & 7.4 & 462 & 11.6 & $1,441,789$ & 452.2 & 350,062 & 109.8 & 6 & 0.0 \\
\hline 2015 & 74,707 & 23.2 & 23,872 & 7.4 & 24,173 & 7.5 & 26,170 & 8.1 & 492 & 12.4 & $1,526,658$ & 475.0 & 395,216 & 123.0 & 11 & 0.0 \\
\hline 2016 & 88,053 & 27.3 & 27,814 & 8.6 & 28,924 & 9.0 & 30,676 & 9.5 & 639 & 16.2 & $1,598,354$ & 494.7 & 468,514 & 145.0 & 7 & 0.0 \\
\hline 2017 & 101,584 & 31.2 & 30,644 & 9.4 & 34,013 & 10.4 & 35,992 & 11.1 & 935 & 23.7 & $1,708,569$ & 524.6 & 555,608 & 170.6 & 7 & 0.0 \\
\hline 2018 & 115,045 & 35.3 & 35,063 & 10.8 & 38,539 & 11.8 & 40,137 & 12.3 & 1,306 & 33.1 & $1,758,668$ & 539.9 & 583,405 & 179.1 & 3 & 0.0 \\
\hline
\end{tabular}

* Per 100,000.

${ }^{\dagger}$ For 1941-1946, data were reported for the federal fiscal year ending June 30 of the year indicated. From 1947 to the present, data were reported for the calendar year ending December 31. For 1941-1958, data for Alaska and Hawaii were not included.

${ }^{\ddagger}$ Includes stage of syphilis not stated.

${ }^{5}$ The case classification of 'Unknown duration or late syphilis' went into effect in January of 2018. Prior to 2018, cases in this category include cases classified as late latent syphilis, latent syphilis of unknown duration, late syphilis with clinical manifestations, and neurosyphilis. See Appendix A1.9 for a detailed explanation of changes to the syphilis case definition.

II Rates include all cases of congenitally acquired syphilis per 100,000 live births. As of 1995, cases of congenital syphilis are obtained in hardcopy and electronic format on the basis of case reporting form CDC 73.126.

$\mathrm{NR}=$ No report.

NOTE: Adjustments to the number of cases reported from state health departments were made for hardcopy forms and for electronic data submissions through June 19, 2019. The number of cases and the rates shown here supersede those published in previous reports. See Appendix A1.1 for more information. Cases and rates shown in this table exclude US territories. Case definitions have changed over time. See Section C.1 in the Appendix for more information. 
Table 2. Chlamydia - Reported Cases and Rates of Reported Cases by State, Ranked by Rates, United States, 2018

\begin{tabular}{|c|c|c|c|}
\hline Rank* & State & Cases & Rate per 100,000 Population \\
\hline 1 & Alaska & 6,159 & 832.5 \\
\hline 2 & Louisiana & 36,293 & 774.8 \\
\hline 3 & Mississippi & 22,086 & 740.1 \\
\hline 4 & South Carolina & 33,910 & 674.9 \\
\hline 5 & New Mexico & 14,000 & 670.5 \\
\hline 6 & North Carolina & 66,553 & 647.8 \\
\hline 7 & Georgia & 65,936 & 632.2 \\
\hline 8 & Delaware & 6,038 & 627.7 \\
\hline 9 & Illinois & 77,325 & 604.0 \\
\hline 10 & New York & 119,571 & 602.4 \\
\hline 11 & Arkansas & 17,663 & 587.9 \\
\hline 12 & Maryland & 35,482 & 586.3 \\
\hline 13 & California & 231,415 & 585.3 \\
\hline 14 & Nevada & 17,508 & 584.0 \\
\hline 15 & Alabama & 28,437 & 583.4 \\
\hline 16 & Arizona & 40,807 & 581.6 \\
\hline 17 & Tennessee & 38,212 & 569.0 \\
\hline 18 & Missouri & 34,728 & 568.1 \\
\hline 19 & Oklahoma & 21,974 & 559.0 \\
\hline 20 & Ohio & 63,220 & 542.3 \\
\hline \multirow[t]{2}{*}{21} & Hawaii & 7,735 & 541.8 \\
\hline & US TOTAL ${ }^{+}$ & $1,758,668$ & 539.9 \\
\hline 22 & Indiana & 34,926 & 523.9 \\
\hline 23 & Colorado & 29,124 & 519.4 \\
\hline 24 & Rhode Island & 5,487 & 517.8 \\
\hline 25 & Texas & 146,510 & 517.6 \\
\hline 26 & South Dakota & 4,432 & 509.6 \\
\hline 27 & Michigan & 50,592 & 507.8 \\
\hline 28 & Virginia & 42,965 & 507.3 \\
\hline 29 & Florida & 104,758 & 499.2 \\
\hline 30 & Kansas & 14,231 & 488.5 \\
\hline 31 & Wisconsin & 28,027 & 483.6 \\
\hline 32 & Montana & 4,917 & 468.1 \\
\hline 33 & lowa & 14,682 & 466.7 \\
\hline 34 & North Dakota & 3,525 & 466.6 \\
\hline 35 & Connecticut & 16,732 & 466.3 \\
\hline 36 & Washington & 34,449 & 465.2 \\
\hline 37 & Oregon & 19,224 & 464.0 \\
\hline 38 & Pennsylvania & 59,340 & 463.4 \\
\hline 39 & Massachusetts & 30,460 & 444.0 \\
\hline 40 & Kentucky & 19,440 & 436.4 \\
\hline 41 & Minnesota & 23,569 & 422.6 \\
\hline 42 & Nebraska & 8,026 & 418.0 \\
\hline 43 & New Jersey & 36,514 & 405.5 \\
\hline 44 & Idaho & 6,572 & 382.8 \\
\hline 45 & Wyoming & 2,169 & 374.4 \\
\hline 46 & Utah & 10,541 & 339.8 \\
\hline 47 & Maine & 4,345 & 325.2 \\
\hline 48 & New Hampshire & 3,734 & 278.1 \\
\hline 49 & Vermont & 1,712 & 274.5 \\
\hline 50 & West Virginia & 3,599 & 198.2 \\
\hline
\end{tabular}

* States were ranked by rate, then by case count, then in alphabetical order, with rates shown rounded to the nearest tenth.

${ }^{\dagger}$ Total includes cases reported by the District of Columbia with 9,014 cases and a rate of 1,298.9 cases per 100,000 population, but excludes territories. 
Table 3. Chlamydia - Reported Cases and Rates of Reported Cases by State/Territory and Region in Alphabetical Order, United States, 2014-2018

\begin{tabular}{|c|c|c|c|c|c|c|c|c|c|c|}
\hline \multirow[b]{2}{*}{ State/Territory } & \multicolumn{5}{|c|}{ Cases } & \multicolumn{5}{|c|}{ Rates per 100,000 Population } \\
\hline & 2014 & 2015 & 2016 & 2017 & 2018 & 2014 & 2015 & 2016 & 2017 & 2018 \\
\hline Alabama & 29,010 & 26,359 & 26,901 & 29,935 & 28,437 & 598.2 & 542.5 & 553.1 & 614.1 & 583.4 \\
\hline Alaska & 5,789 & 5,660 & 5,698 & 5,934 & 6,159 & 785.8 & 766.5 & 768.0 & 802.1 & 832.5 \\
\hline Arizona & 32,397 & 32,387 & 34,923 & 39,598 & 40,807 & 481.3 & 474.3 & 503.9 & 564.4 & 581.6 \\
\hline Arkansas & 15,605 & 16,166 & 16,737 & 17,320 & 17,663 & 526.1 & 542.8 & 560.1 & 576.5 & 587.9 \\
\hline California & 176,308 & 189,170 & 198,155 & 218,785 & 231,415 & 454.4 & 483.3 & 504.9 & 553.4 & 585.3 \\
\hline Colorado & 21,863 & 23,857 & 25,569 & 26,995 & 29,124 & 408.2 & 437.2 & 461.5 & 481.4 & 519.4 \\
\hline Connecticut & 13,382 & 13,126 & 13,911 & 17,750 & 16,732 & 372.1 & 365.5 & 389.0 & 494.7 & 466.3 \\
\hline Delaware & 4,473 & 4,605 & 5,365 & 5,392 & 6,038 & 478.1 & 486.8 & 563.5 & 560.5 & 627.7 \\
\hline District of Columbia & 5,293 & 7,894 & 7,283 & 9,107 & 9,014 & 803.3 & $1,174.3$ & $1,069.2$ & $1,312.3$ & $1,298.9$ \\
\hline Florida & 84,194 & 90,468 & 94,742 & 100,018 & 104,758 & 423.2 & 446.3 & 459.6 & 476.6 & 499.2 \\
\hline Georgia & 51,945 & 57,639 & 62,776 & 65,104 & 65,936 & 514.4 & 564.3 & 608.9 & 624.2 & 632.2 \\
\hline Hawaii & 6,419 & 7,074 & 6,902 & 6,850 & 7,735 & 452.2 & 494.1 & 483.1 & 479.8 & 541.8 \\
\hline Idaho & 5,442 & 5,631 & 5,897 & 6,200 & 6,572 & 333.0 & 340.3 & 350.4 & 361.1 & 382.8 \\
\hline Illinois & 66,536 & 69,610 & 72,201 & 75,518 & 77,325 & 516.6 & 541.3 & 564.0 & 589.9 & 604.0 \\
\hline Indiana & 28,519 & 28,886 & 30,847 & 34,278 & 34,926 & 432.3 & 436.4 & 465.0 & 514.2 & 523.9 \\
\hline lowa & 11,804 & 12,085 & 12,983 & 13,893 & 14,682 & 379.9 & 386.9 & 414.2 & 441.6 & 466.7 \\
\hline Kansas & 11,116 & 11,464 & 12,160 & 13,554 & 14,231 & 382.8 & 393.7 & 418.3 & 465.3 & 488.5 \\
\hline Kentucky & 17,664 & 17,444 & 18,286 & 19,320 & 19,440 & 400.2 & 394.2 & 412.1 & 433.7 & 436.4 \\
\hline Louisiana & 28,955 & 32,325 & 31,727 & 34,756 & 36,293 & 622.7 & 692.1 & 677.7 & 742.0 & 774.8 \\
\hline Maine & 3,530 & 3,965 & 4,156 & 4,555 & 4,345 & 265.4 & 298.3 & 312.1 & 341.0 & 325.2 \\
\hline Maryland & 27,424 & 27,450 & 30,658 & 33,416 & 35,482 & 458.9 & 457.0 & 509.6 & 552.1 & 586.3 \\
\hline Massachusetts & 21,271 & 24,100 & 26,807 & 29,315 & 30,460 & 315.3 & 354.7 & 393.5 & 427.3 & 444.0 \\
\hline Michigan & 44,256 & 46,486 & 45,936 & 50,595 & 50,592 & 446.6 & 468.5 & 462.7 & 507.9 & 507.8 \\
\hline Minnesota & 19,907 & 21,243 & 22,685 & 23,539 & 23,569 & 364.8 & 387.0 & 411.0 & 422.1 & 422.6 \\
\hline Mississippi & 19,605 & 17,371 & 20,112 & 21,149 & 22,086 & 654.8 & 580.5 & 672.9 & 708.7 & 740.1 \\
\hline Missouri & 27,981 & 28,948 & 30,843 & 32,683 & 34,728 & 461.5 & 475.8 & 506.2 & 534.6 & 568.1 \\
\hline Montana & 4,193 & 4,184 & 4,416 & 4,560 & 4,917 & 409.6 & 405.1 & 423.6 & 434.1 & 468.1 \\
\hline Nebraska & 7,499 & 7,956 & 8,197 & 8,595 & 8,026 & 398.6 & 419.6 & 429.8 & 447.6 & 418.0 \\
\hline Nevada & 11,841 & 12,925 & 14,649 & 16,260 & 17,508 & 417.1 & 447.1 & 498.3 & 542.4 & 584.0 \\
\hline New Hampshire & 3,586 & 3,095 & 3,467 & 4,412 & 3,734 & 270.3 & 232.6 & 259.7 & 328.6 & 278.1 \\
\hline New Jersey & 29,904 & 31,337 & 34,519 & 35,239 & 36,514 & 334.6 & 349.8 & 385.9 & 391.3 & 405.5 \\
\hline New Mexico & 11,558 & 12,632 & 13,108 & 13,560 & 14,000 & 554.2 & 605.8 & 629.9 & 649.4 & 670.5 \\
\hline New York & 98,814 & 103,615 & 109,433 & 116,814 & 119,571 & 500.4 & 523.4 & 554.2 & 588.5 & 602.4 \\
\hline North Carolina & 47,147 & 64,376 & 58,006 & 62,876 & 66,553 & 474.1 & 641.0 & 571.7 & 612.0 & 647.8 \\
\hline North Dakota & 3,451 & 3,159 & 3,455 & 3,278 & 3,525 & 466.7 & 417.3 & 455.8 & 433.9 & 466.6 \\
\hline Ohio & 54,858 & 56,726 & 60,496 & 61,389 & 63,220 & 473.2 & 488.5 & 520.9 & 526.6 & 542.3 \\
\hline Oklahoma & 20,662 & 21,025 & 21,449 & 21,752 & 21,974 & 532.8 & 537.5 & 546.7 & 553.4 & 559.0 \\
\hline Oregon & 15,508 & 16,305 & 17,425 & 18,634 & 19,224 & 390.6 & 404.7 & 425.7 & 449.8 & 464.0 \\
\hline Pennsylvania & 50,536 & 53,460 & 56,930 & 56,447 & 59,340 & 395.2 & 417.6 & 445.3 & 440.8 & 463.4 \\
\hline Rhode Island & 4,349 & 4,575 & 4,936 & 5,282 & 5,487 & 412.2 & 433.1 & 467.2 & 498.5 & 517.8 \\
\hline South Carolina & 28,087 & 27,538 & 28,179 & 32,235 & 33,910 & 581.2 & 562.4 & 568.0 & 641.6 & 674.9 \\
\hline South Dakota & 4,166 & 3,949 & 4,331 & 4,437 & 4,432 & 488.3 & 460.0 & 500.4 & 510.2 & 509.6 \\
\hline Tennessee & 30,793 & 31,272 & 32,304 & 35,087 & 38,212 & 470.2 & 473.8 & 485.7 & 522.4 & 569.0 \\
\hline Texas & 131,219 & 141,158 & 142,952 & 151,533 & 146,510 & 486.8 & 513.9 & 513.1 & 535.4 & 517.6 \\
\hline Utah & 8,223 & 8,633 & 9,457 & 10,135 & 10,541 & 279.4 & 288.2 & 309.9 & 326.7 & 339.8 \\
\hline Vermont & 2,237 & 1,901 & 1,690 & 1,858 & 1,712 & 357.0 & 303.7 & 270.6 & 297.9 & 274.5 \\
\hline Virginia & 36,048 & 35,349 & 39,666 & 42,374 & 42,965 & 432.9 & 421.7 & 471.6 & 500.3 & 507.3 \\
\hline Washington & 26,577 & 28,699 & 31,254 & 32,231 & 34,449 & 376.4 & 400.2 & 428.8 & 435.2 & 465.2 \\
\hline West Virginia & 4,719 & 4,958 & 4,821 & 4,140 & 3,599 & 255.0 & 268.9 & 263.3 & 228.0 & 198.2 \\
\hline Wisconsin & 23,154 & 24,381 & 26,894 & 27,740 & 28,027 & 402.1 & 422.4 & 465.4 & 478.6 & 483.6 \\
\hline Wyoming & 1,972 & 2,037 & 2,060 & 2,142 & 2,169 & 337.6 & 347.5 & 351.8 & 369.7 & 374.4 \\
\hline US TOTAL & $1,441,789$ & $1,526,658$ & $1,598,354$ & $1,708,569$ & $1,758,668$ & 452.2 & 475.0 & 494.7 & 524.6 & 539.9 \\
\hline Northeast & 227,609 & 239,174 & 255,849 & 271,672 & 277,895 & 405.3 & 424.9 & 455.2 & 481.1 & 492.1 \\
\hline Midwest & 303,247 & 314,893 & 331,028 & 349,499 & 357,283 & 447.6 & 463.7 & 487.2 & 512.6 & 524.0 \\
\hline South & 582,843 & 623,397 & 641,964 & 685,514 & 698,870 & 486.6 & 514.4 & 524.8 & 554.4 & 565.2 \\
\hline West & 328,090 & 349,194 & 369,513 & 401,884 & 424,620 & 436.4 & 459.2 & 482.0 & 519.2 & 548.5 \\
\hline American Samoa & NR & NR & NR & NR & 55 & - & - & - & - & 106.8 \\
\hline Guam & 839 & 881 & 934 & 1,107 & 944 & 521.1 & 544.5 & 559.6 & 661.5 & 564.1 \\
\hline Northern Mariana Islands & NR & NR & NR & NR & 246 & - & - & - & - & 470.7 \\
\hline Puerto Rico & 4,899 & 5,295 & 7,198 & 5,961 & 5,942 & 138.1 & 152.4 & 211.0 & 177.8 & 177.3 \\
\hline Virgin Islands & 791 & 743 & 571 & 458 & NR & 759.3 & 721.7 & 530.1 & 427.0 & - \\
\hline TERRITORIES & 6,529 & 6,919 & 8,703 & 7,526 & 7,187 & 171.2 & 185.1 & 236.1 & 207.5 & 198.4 \\
\hline TOTAL & $1,448,318$ & $1,533,577$ & $1,607,057$ & $1,716,095$ & $1,765,855$ & 448.9 & 471.6 & 491.7 & 521.1 & 536.2 \\
\hline
\end{tabular}

$\mathrm{NR}=$ No report.

NOTE: See Section A1.11 in the Appendix for more information on interpreting case counts and rates in US territories. 
Table 4. Chlamydia Among Females - Reported Cases and Rates of Reported Cases by State/Territory and Region in Alphabetical Order, United States, 2014-2018

\begin{tabular}{|c|c|c|c|c|c|c|c|c|c|c|}
\hline \multirow[b]{2}{*}{ State/Territory } & \multicolumn{5}{|c|}{ Cases } & \multicolumn{5}{|c|}{ Rates per 100,000 Population } \\
\hline & 2014 & 2015 & 2016 & 2017 & 2018 & 2014 & 2015 & 2016 & 2017 & 2018 \\
\hline Alabama & 20,619 & 18,674 & 19,131 & 20,993 & 19,537 & 825.0 & 745.2 & 762.9 & 834.7 & 776.8 \\
\hline Alaska & 3,940 & 3,786 & 3,807 & 3,941 & 3,965 & $1,127.5$ & $1,083.6$ & $1,076.1$ & $1,116.4$ & $1,123.2$ \\
\hline Arizona & 22,747 & 22,299 & 23,693 & 26,453 & 26,717 & 671.5 & 648.9 & 679.2 & 749.8 & 757.3 \\
\hline Arkansas & 11,625 & 12,088 & 12,216 & 12,453 & 12,513 & 770.4 & 797.7 & 803.5 & 814.9 & 818.8 \\
\hline California & 115,339 & 121,387 & 123,906 & 135,040 & 142,006 & 590.5 & 616.1 & 627.2 & 679.0 & 714.0 \\
\hline Colorado & 14,906 & 16,151 & 16,945 & 17,251 & 18,474 & 559.4 & 595.4 & 615.1 & 619.5 & 663.4 \\
\hline Connecticut & 9,512 & 9,089 & 9,495 & 11,487 & 10,751 & 516.4 & 494.2 & 518.6 & 625.5 & 585.4 \\
\hline Delaware & 3,084 & 3,118 & 3,678 & 3,562 & 3,992 & 638.6 & 638.8 & 748.5 & 717.5 & 804.1 \\
\hline District of Columbia & 3,709 & 4,632 & 4,018 & 4,754 & 4,812 & $1,071.1$ & $1,309.9$ & $1,122.5$ & $1,303.3$ & $1,319.2$ \\
\hline Florida & 58,800 & 62,048 & 63,415 & 66,173 & 68,509 & 578.2 & 598.4 & 601.5 & 616.8 & 638.6 \\
\hline Georgia & 36,871 & 40,302 & 43,377 & 44,080 & 43,903 & 713.1 & 769.9 & 820.0 & 823.3 & 820.0 \\
\hline Hawaii & 4,469 & 4,720 & 4,480 & 4,424 & 4,811 & 637.1 & 668.3 & 630.1 & 621.8 & 676.2 \\
\hline Idaho & 3,895 & 3,963 & 4,022 & 4,198 & 4,452 & 477.3 & 479.7 & 479.0 & 490.1 & 519.8 \\
\hline Illinois & 46,516 & 47,268 & 48,128 & 49,336 & 49,746 & 709.1 & 722.1 & 739.3 & 757.9 & 764.2 \\
\hline Indiana & 20,586 & 20,385 & 21,664 & 23,595 & 23,768 & 615.0 & 607.1 & 644.1 & 698.1 & 703.3 \\
\hline lowa & 8,385 & 8,372 & 8,872 & 9,261 & 9,793 & 536.1 & 532.6 & 563.1 & 585.8 & 619.4 \\
\hline Kansas & 8,276 & 8,325 & 8,688 & 9,472 & 9,794 & 568.1 & 571.0 & 595.3 & 648.2 & 670.3 \\
\hline Kentucky & 12,404 & 12,140 & 12,345 & 13,035 & 13,055 & 553.7 & 540.5 & 548.6 & 576.8 & 577.7 \\
\hline Louisiana & 21,297 & 23,351 & 22,942 & 24,544 & 25,225 & 896.4 & 978.3 & 959.0 & $1,024.9$ & $1,053.3$ \\
\hline Maine & 2,478 & 2,735 & 2,795 & 2,959 & 2,854 & 365.0 & 403.4 & 411.7 & 434.3 & 418.9 \\
\hline Maryland & 19,162 & 18,612 & 20,145 & 21,957 & 22,912 & 622.1 & 601.3 & 649.4 & 704.2 & 734.8 \\
\hline Massachusetts & 14,000 & 15,588 & 17,299 & 18,716 & 19,096 & 402.9 & 445.6 & 493.4 & 530.3 & 541.0 \\
\hline Michigan & 31,470 & 32,425 & 31,497 & 34,120 & 33,687 & 624.2 & 642.8 & 624.5 & 674.5 & 665.9 \\
\hline Minnesota & 13,484 & 14,112 & 14,967 & 15,338 & 15,021 & 491.2 & 511.4 & 539.9 & 547.8 & 536.5 \\
\hline Mississippi & 14,008 & 12,335 & 14,123 & 14,740 & 15,325 & 909.9 & 800.7 & 917.1 & 958.2 & 996.3 \\
\hline Missouri & 19,549 & 19,926 & 20,757 & 21,701 & 22,787 & 632.8 & 643.1 & 669.4 & 697.5 & 732.4 \\
\hline Montana & 2,878 & 2,846 & 2,962 & 3,030 & 3,206 & 564.9 & 554.1 & 572.1 & 581.0 & 614.7 \\
\hline Nebraska & 5,110 & 5,409 & 5,527 & 5,660 & 5,233 & 540.9 & 568.8 & 577.9 & 588.4 & 544.0 \\
\hline Nevada & 8,039 & 8,743 & 9,849 & 10,473 & 11,057 & 569.2 & 607.0 & 671.8 & 700.9 & 740.0 \\
\hline New Hampshire & 2,452 & 2,089 & 2,316 & 2,917 & 2,474 & 365.2 & 310.4 & 343.7 & 430.4 & 365.0 \\
\hline New Jersey & 21,556 & 22,274 & 24,021 & 23,811 & 24,688 & 471.0 & 485.7 & 524.9 & 516.6 & 535.6 \\
\hline New Mexico & 8,395 & 9,227 & 9,306 & 9,328 & 9,683 & 797.5 & 877.4 & 886.0 & 885.1 & 918.8 \\
\hline New York & 65,114 & 66,164 & 67,602 & 70,379 & 70,348 & 640.6 & 649.7 & 665.5 & 689.2 & 688.9 \\
\hline North Carolina & 35,494 & 47,178 & 41,085 & 43,580 & 45,450 & 696.0 & 915.9 & 788.0 & 826.6 & 862.1 \\
\hline North Dakota & 2,202 & 2,028 & 2,187 & 2,127 & 2,303 & 610.9 & 551.0 & 592.7 & 577.8 & 625.7 \\
\hline Ohio & 39,033 & 39,825 & 41,797 & 42,132 & 42,674 & 659.4 & 671.9 & 705.7 & 708.6 & 717.8 \\
\hline Oklahoma & 14,855 & 14,904 & 14,933 & 15,183 & 15,282 & 758.7 & 754.9 & 754.3 & 765.5 & 770.5 \\
\hline Oregon & 10,545 & 11,075 & 11,542 & 12,203 & 12,567 & 525.6 & 544.0 & 558.5 & 583.9 & 601.4 \\
\hline Pennsylvania & 34,170 & 35,201 & 37,030 & 36,201 & 37,938 & 523.0 & 538.4 & 567.7 & 554.0 & 580.6 \\
\hline Rhode Island & 3,037 & 3,064 & 3,278 & 3,454 & 3,584 & 558.5 & 562.9 & 603.3 & 634.2 & 658.0 \\
\hline South Carolina & 20,581 & 19,743 & 19,783 & 22,343 & 22,822 & 828.8 & 784.3 & 774.8 & 863.8 & 882.3 \\
\hline South Dakota & 2,942 & 2,831 & 3,072 & 3,094 & 3,008 & 694.1 & 663.8 & 715.8 & 718.4 & 698.4 \\
\hline Tennessee & 21,203 & 21,112 & 21,714 & 23,348 & 25,151 & 631.6 & 624.1 & 637.0 & 678.7 & 731.1 \\
\hline Texas & 96,959 & 102,141 & 101,618 & 105,995 & 100,417 & 714.3 & 738.5 & 724.2 & 744.2 & 705.0 \\
\hline Utah & 5,414 & 5,704 & 6,031 & 6,606 & 6,808 & 370.0 & 383.1 & 398.0 & 428.9 & 442.0 \\
\hline Vermont & 1,613 & 1,352 & 1,171 & 1,242 & 1,171 & 507.7 & 425.8 & 370.4 & 393.8 & 371.3 \\
\hline Virginia & 24,754 & 23,859 & 26,146 & 27,606 & 27,849 & 585.1 & 560.3 & 611.6 & 641.5 & 647.2 \\
\hline Washington & 18,193 & 19,047 & 20,276 & 20,515 & 21,432 & 515.1 & 531.2 & 556.0 & 554.1 & 578.9 \\
\hline West Virginia & 3,356 & 3,449 & 3,330 & 2,775 & 2,374 & 358.4 & 369.6 & 359.9 & 302.5 & 258.8 \\
\hline Wisconsin & 16,063 & 16,660 & 18,382 & 18,667 & 18,617 & 554.3 & 573.7 & 632.7 & 640.9 & 639.2 \\
\hline Wyoming & 1,352 & 1,387 & 1,356 & 1,399 & 1,422 & 472.5 & 482.9 & 473.2 & 492.8 & 500.9 \\
\hline US TOTAL & $1,006,441$ & $1,045,143$ & $1,072,719$ & $1,127,651$ & $1,145,063$ & 621.6 & 640.4 & 653.9 & 682.1 & 692.7 \\
\hline Northeast & 153,932 & 157,556 & 165,007 & 171,166 & 172,904 & 534.4 & 546.0 & 572.8 & 591.4 & 597.5 \\
\hline Midwest & 213,616 & 217,566 & 225,538 & 234,503 & 236,431 & 621.9 & 632.2 & 655.5 & 679.3 & 684.9 \\
\hline South & 418,781 & 439,686 & 443,999 & 467,121 & 469,128 & 685.9 & 711.7 & 711.8 & 741.0 & 744.2 \\
\hline West & 220,112 & 230,335 & 238,175 & 254,861 & 266,600 & 583.6 & 604.0 & 619.4 & 656.7 & 687.0 \\
\hline American Samoa & NR & NR & NR & NR & 53 & - & - & - & - & 206.0 \\
\hline Guam & 595 & 618 & 654 & 753 & 670 & 749.6 & 774.4 & 806.8 & 926.6 & 824.5 \\
\hline Northern Mariana Islands & NR & NR & NR & NR & 189 & - & - & - & - & 772.6 \\
\hline Puerto Rico & 3,770 & 3,950 & 5,551 & 4,702 & 4,697 & 204.4 & 217.4 & 310.5 & 267.2 & 267.0 \\
\hline Virgin Islands & 590 & 563 & 405 & 318 & NR & $1,060.7$ & $1,020.6$ & 720.9 & 567.6 & - \\
\hline TERRITORIES & 4,955 & 5,131 & 6,610 & 5,773 & 5,609 & 250.4 & 262.9 & 343.4 & 304.4 & 296.6 \\
\hline TOTAL & $1,011,396$ & $1,050,274$ & $1,079,329$ & $1,133,424$ & $1,150,672$ & 617.1 & 636.0 & 650.3 & 677.9 & 688.2 \\
\hline
\end{tabular}

$\mathrm{NR}=$ No report.

NOTE: Cases reported with unknown sex are not included in this table. See Section A1.11 in the Appendix for more information on interpreting case counts and rates in US territories. 
Table 5. Chlamydia Among Males - Reported Cases and Rates of Reported Cases by State/Territory and Region in Alphabetical Order, United States, 2014-2018

\begin{tabular}{|c|c|c|c|c|c|c|c|c|c|c|}
\hline \multirow[b]{2}{*}{ State/Territory } & \multicolumn{5}{|c|}{ Cases } & \multicolumn{5}{|c|}{ Rates per 100,000 Population } \\
\hline & 2014 & 2015 & 2016 & 2017 & 2018 & 2014 & 2015 & 2016 & 2017 & 2018 \\
\hline Alabama & 8,318 & 7,549 & 7,585 & 8,837 & 8,807 & 354.0 & 320.8 & 322.0 & 374.5 & 373.2 \\
\hline Alaska & 1,849 & 1,871 & 1,891 & 1,993 & 2,189 & 477.4 & 480.9 & 487.2 & 515.3 & 565.9 \\
\hline Arizona & 9,650 & 10,028 & 11,217 & 13,055 & 13,972 & 288.6 & 295.7 & 325.8 & 374.3 & 400.5 \\
\hline Arkansas & 3,964 & 4,078 & 4,521 & 4,866 & 5,147 & 272.0 & 278.8 & 308.0 & 329.7 & 348.7 \\
\hline California & 60,687 & 67,475 & 73,625 & 83,273 & 88,787 & 314.9 & 347.0 & 377.7 & 423.8 & 451.9 \\
\hline Colorado & 6,957 & 7,706 & 8,624 & 9,744 & 10,650 & 258.5 & 280.9 & 309.6 & 345.2 & 377.3 \\
\hline Connecticut & 3,757 & 3,926 & 4,268 & 5,618 & 5,829 & 214.1 & 224.1 & 244.5 & 320.7 & 332.7 \\
\hline Delaware & 1,389 & 1,487 & 1,687 & 1,830 & 2,031 & 306.8 & 324.8 & 366.2 & 393.1 & 436.3 \\
\hline District of Columbia & 1,555 & 3,108 & 3,112 & 4,279 & 4,135 & 497.4 & 975.5 & 962.8 & $1,299.8$ & $1,256.1$ \\
\hline Florida & 25,239 & 28,332 & 31,275 & 33,811 & 36,219 & 259.6 & 286.1 & 310.6 & 329.6 & 353.1 \\
\hline Georgia & 14,736 & 17,212 & 19,338 & 20,837 & 21,691 & 299.1 & 345.6 & 385.2 & 410.5 & 427.4 \\
\hline Hawaii & 1,950 & 2,352 & 2,421 & 2,426 & 2,911 & 271.6 & 324.2 & 337.4 & 338.8 & 406.5 \\
\hline Idaho & 1,547 & 1,663 & 1,869 & 1,990 & 2,102 & 189.0 & 200.7 & 221.6 & 231.3 & 244.3 \\
\hline Illinois & 19,908 & 21,966 & 24,008 & 26,089 & 27,555 & 315.0 & 347.9 & 381.6 & 414.6 & 437.9 \\
\hline Indiana & 7,921 & 8,492 & 9,174 & 10,673 & 11,131 & 243.8 & 260.3 & 280.6 & 324.7 & 338.6 \\
\hline lowa & 3,419 & 3,712 & 4,111 & 4,631 & 4,889 & 221.6 & 239.2 & 263.7 & 296.0 & 312.4 \\
\hline Kansas & 2,840 & 3,139 & 3,472 & 4,082 & 4,437 & 196.2 & 215.9 & 239.8 & 281.1 & 305.6 \\
\hline Kentucky & 5,194 & 5,273 & 5,590 & 6,194 & 6,249 & 239.0 & 242.0 & 255.7 & 282.3 & 284.8 \\
\hline Louisiana & 7,655 & 8,974 & 8,784 & 10,212 & 11,068 & 336.6 & 392.9 & 383.7 & 446.0 & 483.4 \\
\hline Maine & 1,050 & 1,230 & 1,356 & 1,596 & 1,491 & 161.2 & 188.9 & 207.8 & 243.8 & 227.8 \\
\hline Maryland & 8,237 & 8,780 & 10,479 & 11,449 & 12,539 & 284.4 & 301.6 & 359.6 & 390.2 & 427.3 \\
\hline Massachusetts & 7,197 & 8,406 & 9,433 & 10,517 & 11,231 & 220.0 & 255.0 & 285.4 & 315.8 & 337.2 \\
\hline Michigan & 12,723 & 14,015 & 14,417 & 16,420 & 16,844 & 261.3 & 287.3 & 295.1 & 334.8 & 343.5 \\
\hline Minnesota & 6,414 & 7,122 & 7,703 & 8,183 & 8,527 & 236.5 & 260.9 & 280.4 & 294.7 & 307.1 \\
\hline Mississippi & 5,588 & 5,018 & 5,955 & 6,363 & 6,723 & 384.2 & 345.6 & 411.0 & 440.1 & 465.0 \\
\hline Missouri & 8,432 & 9,022 & 10,086 & 10,982 & 11,941 & 283.5 & 302.2 & 337.1 & 365.8 & 397.7 \\
\hline Montana & 1,314 & 1,338 & 1,454 & 1,528 & 1,710 & 255.6 & 257.6 & 277.1 & 288.9 & 323.3 \\
\hline Nebraska & 2,357 & 2,531 & 2,649 & 2,927 & 2,789 & 251.6 & 267.8 & 278.6 & 305.5 & 291.1 \\
\hline Nevada & 3,786 & 4,152 & 4,777 & 5,741 & 6,381 & 265.4 & 286.2 & 324.1 & 381.8 & 424.3 \\
\hline New Hampshire & 1,130 & 1,006 & 1,150 & 1,494 & 1,259 & 172.4 & 153.0 & 174.0 & 224.7 & 189.3 \\
\hline New Jersey & 8,272 & 9,025 & 10,435 & 11,362 & 11,817 & 189.6 & 206.4 & 238.9 & 258.4 & 268.8 \\
\hline New Mexico & 3,148 & 3,400 & 3,794 & 4,229 & 4,313 & 304.8 & 329.0 & 368.1 & 408.9 & 417.1 \\
\hline New York & 33,634 & 37,346 & 41,722 & 46,349 & 49,175 & 351.0 & 388.6 & 435.2 & 480.9 & 510.2 \\
\hline North Carolina & 11,638 & 17,195 & 16,918 & 19,295 & 21,103 & 240.2 & 351.5 & 343.0 & 385.8 & 421.9 \\
\hline North Dakota & 1,249 & 1,131 & 1,268 & 1,151 & 1,222 & 329.5 & 290.9 & 326.0 & 297.2 & 315.5 \\
\hline Ohio & 15,825 & 16,901 & 18,699 & 19,257 & 20,546 & 278.9 & 297.2 & 328.5 & 337.1 & 359.6 \\
\hline Oklahoma & 5,802 & 6,121 & 6,516 & 6,569 & 6,692 & 302.2 & 316.0 & 335.2 & 337.3 & 343.6 \\
\hline Oregon & 4,953 & 5,223 & 5,876 & 6,412 & 6,638 & 252.2 & 262.0 & 289.9 & 312.3 & 323.3 \\
\hline Pennsylvania & 16,315 & 18,201 & 19,840 & 20,184 & 21,350 & 260.9 & 290.5 & 316.9 & 321.8 & 340.4 \\
\hline Rhode Island & 1,312 & 1,511 & 1,656 & 1,825 & 1,903 & 256.6 & 295.1 & 322.8 & 354.4 & 369.5 \\
\hline South Carolina & 7,376 & 7,705 & 8,286 & 9,807 & 10,976 & 314.0 & 323.9 & 344.1 & 402.3 & 450.3 \\
\hline South Dakota & 1,224 & 1,118 & 1,259 & 1,343 & 1,424 & 285.1 & 258.8 & 288.6 & 306.0 & 324.4 \\
\hline Tennessee & 9,587 & 10,158 & 10,584 & 11,736 & 13,057 & 300.3 & 315.7 & 326.4 & 358.2 & 398.6 \\
\hline Texas & 34,110 & 38,539 & 40,992 & 45,170 & 45,450 & 254.9 & 282.6 & 296.4 & 321.2 & 323.2 \\
\hline Utah & 2,808 & 2,929 & 3,424 & 3,516 & 3,733 & 189.8 & 194.3 & 222.9 & 225.1 & 239.0 \\
\hline Vermont & 622 & 549 & 518 & 611 & 527 & 201.4 & 177.9 & 167.9 & 198.2 & 171.0 \\
\hline Virginia & 11,244 & 11,460 & 13,395 & 14,311 & 14,924 & 274.6 & 277.8 & 323.8 & 343.5 & 358.2 \\
\hline Washington & 8,384 & 9,651 & 10,975 & 11,713 & 13,006 & 237.5 & 269.2 & 301.4 & 316.3 & 351.2 \\
\hline West Virginia & 1,363 & 1,509 & 1,491 & 1,365 & 1,224 & 149.1 & 165.6 & 164.6 & 151.9 & 136.2 \\
\hline Wisconsin & 7,077 & 7,703 & 8,487 & 9,058 & 9,386 & 247.5 & 268.6 & 295.4 & 314.2 & 325.6 \\
\hline Wyoming & 619 & 643 & 704 & 741 & 747 & 207.7 & 215.1 & 235.5 & 250.8 & 252.8 \\
\hline US TOTAL & 433,325 & 478,981 & 522,870 & 577,644 & 610,447 & 276.1 & 302.7 & 328.7 & 360.1 & 380.6 \\
\hline Northeast & 73,289 & 81,200 & 90,378 & 99,556 & 104,582 & 268.0 & 296.1 & 329.8 & 361.6 & 379.9 \\
\hline Midwest & 89,389 & 96,852 & 105,333 & 114,796 & 120,691 & 267.7 & 289.2 & 314.1 & 341.1 & 358.6 \\
\hline South & 162,995 & 182,498 & 196,508 & 216,931 & 228,035 & 277.6 & 307.2 & 327.8 & 357.9 & 376.2 \\
\hline West & 107,652 & 118,431 & 130,651 & 146,361 & 157,139 & 287.3 & 312.4 & 342.0 & 379.2 & 407.1 \\
\hline American Samoa & NR & NR & NR & NR & 2 & - & - & - & - & 7.8 \\
\hline Guam & 244 & 263 & 280 & 354 & 274 & 298.9 & 320.8 & 326.2 & 411.2 & 318.2 \\
\hline Northern Mariana Islands & NR & NR & NR & NR & 57 & - & - & - & - & 205.0 \\
\hline Puerto Rico & 1,126 & 1,319 & 1,647 & 1,255 & 1,240 & 66.1 & 79.6 & 101.4 & 78.8 & 77.9 \\
\hline Virgin Islands & 201 & 180 & 166 & 140 & NR & 414.0 & 376.7 & 322.1 & 273.2 & - \\
\hline TERRITORIES & 1,571 & 1,762 & 2,093 & 1,749 & 1,573 & 85.6 & 98.6 & 118.8 & 101.1 & 90.8 \\
\hline TOTAL & 434,896 & 480,743 & 524,963 & 579,393 & 612,020 & 273.9 & 300.4 & 326.4 & 357.3 & 377.5 \\
\hline
\end{tabular}

$\mathrm{NR}=$ No report.

NOTE: Cases reported with unknown sex are not included in this table. See Section A1.11 in the Appendix for more information on interpreting case counts and rates in US territories. 
Table 6. Chlamydia - Reported Cases and Rates of Reported Cases in Selected Metropolitan Statistical Areas (MSAs)* in Alphabetical Order, United States, 2014-2018

\begin{tabular}{|c|c|c|c|c|c|c|c|c|c|c|}
\hline \multirow[b]{2}{*}{ MSAs } & \multicolumn{5}{|c|}{ Cases } & \multicolumn{5}{|c|}{ Rates per 100,000 Population } \\
\hline & 2014 & 2015 & 2016 & 2017 & 2018 & 2014 & 2015 & 2016 & 2017 & 2018 \\
\hline Atlanta-Sandy Springs-Roswell, GA & 25,744 & $19,106^{\dagger}$ & 33,273 & 34,189 & $32,883^{+}$ & 458.5 & $334.6^{+}$ & 574.7 & 581.0 & $558.8^{\dagger}$ \\
\hline Austin-Round Rock, TX & 10,920 & 11,679 & 12,299 & 13,015 & 12,845 & 561.9 & 583.7 & 598.1 & 615.1 & 607.1 \\
\hline Baltimore-Columbia-Towson, MD & 14,095 & 14,016 & 15,829 & 16,766 & 17,466 & 505.9 & 501.0 & 565.5 & 597.0 & 622.0 \\
\hline Birmingham-Hoover, AL & 6,309 & 5,839 & $4,982^{\ddagger}$ & 7,388 & 6,391 & 551.6 & 509.7 & $434.2^{\ddagger}$ & 642.5 & 555.8 \\
\hline Boston-Cambridge-Newton, MA-NH & $14,264^{\dagger}$ & $14,378^{\dagger}$ & $15,880^{\dagger}$ & $18,232^{\dagger}$ & 21,110 & $301.4^{+}$ & $301.2^{+}$ & $331.2^{+}$ & $377.0^{+}$ & 436.5 \\
\hline Buffalo-Cheektowaga-Niagara Falls, NY & 5,841 & 5,900 & 6,252 & 6,584 & 6,443 & 514.0 & 519.7 & 551.9 & 579.1 & 566.7 \\
\hline Charlotte-Concord-Gastonia, NC-SC & 11,766 & 16,284 & 14,314 & 15,901 & 16,983 & 494.3 & 671.1 & 578.5 & 629.7 & 672.5 \\
\hline Chicago-Naperville-Elgin, IL-IN-WI & 51,457 & 54,248 & 56,478 & 59,342 & 60,221 & 538.6 & 568.0 & 593.7 & 622.5 & 631.7 \\
\hline Cincinnati, $\mathrm{OH}-\mathrm{KY}-\mathrm{IN}$ & 10,516 & 11,219 & 11,392 & 11,205 & 11,220 & 489.2 & 519.9 & 526.2 & 514.2 & 514.9 \\
\hline Cleveland-Elyria, $\mathrm{OH}$ & 11,363 & 11,312 & 12,475 & 13,773 & 13,771 & 550.6 & 548.9 & 606.9 & 669.0 & 668.9 \\
\hline Columbus, $\mathrm{OH}$ & 10,258 & 11,327 & 12,113 & 11,921 & 12,511 & 514.3 & 560.3 & 593.3 & 573.5 & 601.9 \\
\hline Dallas-Fort Worth-Arlington, TX & 30,549 & 35,900 & 32,771 & 37,263 & 36,756 & 439.3 & 505.4 & 453.1 & 503.6 & 496.7 \\
\hline Denver-Aurora-Lakewood, CO & 13,346 & 13,942 & 14,282 & 15,520 & 16,928 & 484.6 & 495.4 & 500.6 & 537.4 & 586.1 \\
\hline Detroit-Warren-Dearborn, MI & 21,012 & 22,238 & 21,966 & 24,278 & 24,101 & 489.0 & 516.9 & 511.1 & 562.9 & 558.8 \\
\hline Hartford-West Hartford-East Hartford, CT & 4,713 & 4,689 & 4,898 & $6,153^{+}$ & $5,735^{+}$ & 388.1 & 387.1 & 405.9 & $508.4^{+}$ & $473.9^{+}$ \\
\hline Houston-The Woodlands-Sugar Land, TX & 30,554 & 32,823 & 35,594 & 35,807 & 35,960 & 470.8 & 493.1 & 525.6 & 519.5 & 521.7 \\
\hline Indianapolis-Carmel-Anderson, IN & 11,952 & 11,544 & 12,794 & 13,701 & 13,709 & 606.3 & 580.4 & 638.3 & 675.4 & 675.8 \\
\hline Jacksonville, FL & 7,391 & 8,012 & 8,434 & 8,763 & 9,133 & 520.8 & 552.7 & 570.6 & 582.3 & 606.9 \\
\hline Kansas City, MO-KS & 9,866 & 10,240 & 11,043 & 11,868 & 12,859 & 476.4 & 490.5 & 524.7 & 557.5 & 604.0 \\
\hline Las Vegas-Henderson-Paradise, NV & 9,485 & 10,049 & 11,362 & 11,898 & 13,695 & 458.3 & 475.2 & 527.1 & 539.8 & 621.3 \\
\hline Los Angeles-Long Beach-Anaheim, CA & 64,263 & 68,285 & 71,943 & 78,312 & 82,143 & 484.6 & 511.9 & 540.5 & 586.4 & 615.1 \\
\hline Louisville-Jefferson County, KY-IN & 6,751 & 6,735 & 6,881 & 7,086 & 6,311 & 531.7 & 526.8 & 536.1 & 547.6 & 487.7 \\
\hline Memphis, TN-MS-AR & 10,554 & 10,342 & 10,365 & 11,718 & 12,555 & 785.7 & 769.4 & 771.9 & 869.1 & 931.2 \\
\hline Miami-Fort Lauderdale-West Palm Beach, FL & 24,599 & 26,746 & 28,070 & 29,430 & 30,742 & 414.8 & 444.9 & 462.7 & 477.9 & 499.2 \\
\hline Milwaukee-Waukesha-West Allis, WI & 10,303 & 10,645 & 11,891 & 12,187 & 12,441 & 655.3 & 675.6 & 756.2 & 773.2 & 789.3 \\
\hline Minneapolis-St. Paul-Bloomington, MN-WI & $13,589^{\dagger}$ & $14,709^{+}$ & 15,584 & 16,901 & 16,617 & $388.8^{+}$ & $417.3^{+}$ & 438.9 & 469.4 & 461.5 \\
\hline Nashville-Davidson-Murfreesboro-Franklin, TN & 7,878 & 8,066 & 8,196 & 8,928 & 10,056 & 439.5 & 440.7 & 439.4 & 469.1 & 528.4 \\
\hline New Orleans-Metairie, LA & 8,595 & 9,291 & 9,626 & 9,850 & 10,558 & 686.6 & 735.7 & 758.6 & 772.1 & 827.6 \\
\hline New York-Newark-Jersey City, NY-NJ-PA & 93,515 & 97,835 & 105,463 & 112,808 & 115,654 & 465.4 & 484.8 & 523.3 & 555.1 & 569.1 \\
\hline Oklahoma City, OK & 7,293 & 7,633 & 7,693 & 8,198 & 8,449 & 545.6 & 561.9 & 560.2 & 592.5 & 610.6 \\
\hline Orlando-Kissimmee-Sanford, FL & 11,001 & 12,026 & 12,492 & 13,870 & 14,774 & 473.9 & 503.8 & 511.7 & 552.6 & 588.6 \\
\hline Philadelphia-Camden-Wilmington, PA-NJ-DE-MD & 33,376 & 34,910 & 38,182 & 38,661 & 38,884 & 551.6 & 575.1 & 629.0 & 634.2 & 637.8 \\
\hline Phoenix-Mesa-Scottsdale, AZ & 21,576 & 21,795 & 23,567 & 26,939 & 28,252 & 480.6 & 476.4 & 505.6 & 568.7 & 596.4 \\
\hline Pittsburgh, PA & 8,059 & 8,604 & 8,623 & 7,801 & 8,623 & 342.1 & 365.7 & 368.1 & 334.3 & 369.6 \\
\hline Portland-Vancouver-Hillsboro, OR-WA & 9,283 & 9,982 & 11,052 & 11,461 & 11,830 & 395.3 & 417.8 & 455.8 & 467.2 & 482.2 \\
\hline Providence-Warwick, RI-MA & $5,695^{+}$ & $5,907^{+}$ & $6,668^{\dagger}$ & $6,977^{+}$ & 7,537 & $353.9^{\dagger}$ & $366.2^{+}$ & $412.9^{\dagger}$ & $430.4^{+}$ & 464.9 \\
\hline Raleigh, NC & 5,126 & 6,911 & 6,493 & 7,243 & 7,710 & 412.4 & 542.6 & 498.3 & 542.5 & 577.5 \\
\hline Richmond, VA & 7,817 & 7,878 & 8,592 & 8,562 & 8,845 & 620.4 & 619.7 & 670.4 & 661.6 & 683.4 \\
\hline Riverside-San Bernardino-Ontario, CA & 19,560 & 20,778 & 20,081 & 24,294 & 24,786 & 440.4 & 462.8 & 443.5 & 530.4 & 541.1 \\
\hline Sacramento-Roseville-Arden-Arcade, CA & 9,674 & 10,621 & 10,892 & 12,408 & 14,107 & 431.0 & 467.0 & 474.3 & 533.7 & 606.8 \\
\hline Salt Lake City, UT & 4,423 & 4,751 & 5,264 & 5,516 & 5,472 & 383.5 & 406.0 & 443.8 & 458.5 & 454.8 \\
\hline San Antonio-New Braunfels, TX & 11,573 & 14,465 & 15,149 & 14,580 & 12,199 & 497.0 & 606.7 & 623.5 & 589.3 & 493.1 \\
\hline San Diego-Carlsbad, CA & 15,754 & 17,378 & 18,937 & 20,832 & 21,912 & 482.7 & 526.7 & 570.8 & 624.1 & 656.5 \\
\hline San Francisco-Oakland-Hayward, CA & 20,377 & 23,519 & 24,894 & 27,850 & 29,286 & 443.6 & 505.1 & 532.0 & 589.1 & 619.5 \\
\hline San Jose-Sunnyvale-Santa Clara, CA & 6,278 & 6,898 & 7,166 & 7,877 & 8,553 & 321.5 & 348.9 & 362.1 & 394.2 & 428.0 \\
\hline Seattle-Tacoma-Bellevue, WA & 13,861 & 15,257 & 16,886 & 17,832 & 18,744 & 377.5 & 408.6 & 444.5 & 461.1 & 484.7 \\
\hline St. Louis, MO-IL & 14,711 & 14,961 & 15,512 & 16,469 & 17,222 & 524.2 & 532.1 & 552.6 & 586.6 & 613.5 \\
\hline Tampa-St. Petersburg-Clearwater, FL & 12,952 & 13,472 & 13,996 & 14,512 & 15,454 & 444.2 & 452.8 & 461.6 & 469.4 & 499.9 \\
\hline Virginia Beach-Norfolk-Newport News, VA-NC & 12,192 & 11,281 & 13,223 & 13,571 & 14,137 & 710.2 & 654.0 & 765.7 & 786.6 & 819.4 \\
\hline Washington-Arlington-Alexandria, DC-VA-MD-WV & 18,342 & 18,890 & 21,269 & 33,134 & 34,211 & 304.0 & 309.8 & 346.9 & 533.0 & 550.3 \\
\hline SELECTED MSAs TOTAL & 820,371 & 865,316 & 923,081 & 999,374 & $1,028,784$ & 469.1 & 489.6 & 518.8 & 555.7 & 572.1 \\
\hline
\end{tabular}

* MSAs were selected on the basis of the largest population in the 2010 US Census.

† The variable used to identify county, which is used to classify cases into MSAs, was complete for $\leq 95 \%$ of cases in a state contributing data to this MSA. See Section A1.4 in the Appendix for more information.

₹ 2016 county data for Alabama have been corrected and may not match previous reports.

NOTE: County was misclassified in the 2017 District of Columbia STD morbidity data resulting in inaccurate county-level case counts and rates. 
Table 7. Chlamydia Among Females - Reported Cases and Rates of Reported Cases in Selected Metropolitan Statistical Areas (MSAs)* in Alphabetical Order, United States, 2014-2018

\begin{tabular}{|c|c|c|c|c|c|c|c|c|c|c|}
\hline \multirow[b]{2}{*}{ MSAs } & \multicolumn{5}{|c|}{ Cases } & \multicolumn{5}{|c|}{ Rates per 100,000 Population } \\
\hline & 2014 & 2015 & 2016 & 2017 & 2018 & 2014 & 2015 & 2016 & 2017 & 2018 \\
\hline Atlanta-Sandy Springs-Roswell, GA & 17,564 & $12,640^{\dagger}$ & 22,235 & 22,204 & $21,168^{\dagger}$ & 607.2 & $429.0^{+}$ & 743.6 & 730.5 & $696.4^{\dagger}$ \\
\hline Austin-Round Rock, TX & 7,513 & 7,779 & 7,827 & 8,216 & 7,820 & 773.7 & 777.8 & 761.8 & 778.6 & 741.1 \\
\hline Baltimore-Columbia-Towson, MD & 9,780 & 9,453 & 10,359 & 11,007 & 11,145 & 678.0 & 652.7 & 714.5 & 757.0 & 766.5 \\
\hline Birmingham-Hoover, AL & 4,300 & 3,940 & $3,386^{\ddagger}$ & 4,985 & 4,126 & 724.5 & 662.3 & $568.9^{\ddagger}$ & 835.2 & 691.2 \\
\hline Boston-Cambridge-Newton, MA-NH & $9,243^{+}$ & $9,137^{\dagger}$ & $9,987^{+}$ & $11,314^{+}$ & 12,904 & $379.6^{+}$ & $372.0^{+}$ & $405.1^{+}$ & $455.2^{+}$ & 519.2 \\
\hline Buffalo-Cheektowaga-Niagara Falls, NY & 4,077 & 4,035 & 4,142 & 4,383 & 4,233 & 696.2 & 690.1 & 710.1 & 748.2 & 722.6 \\
\hline Charlotte-Concord-Gastonia, NC-SC & 8,633 & 11,672 & 10,033 & 10,810 & 11,340 & 705.1 & 935.2 & 786.8 & 831.3 & 872.0 \\
\hline Chicago-Naperville-Elgin, IL-IN-WI & 35,696 & 36,547 & 37,102 & 38,191 & 38,003 & 730.9 & 749.1 & 764.1 & 785.1 & 781.2 \\
\hline Cincinnati, OH-KY-IN & 7,724 & 8,050 & 8,052 & 7,848 & 7,747 & 703.9 & 731.0 & 729.4 & 706.5 & 697.4 \\
\hline Cleveland-Elyria, $\mathrm{OH}$ & 7,914 & 7,815 & 8,601 & 9,167 & 9,098 & 740.8 & 732.5 & 809.0 & 861.2 & 854.8 \\
\hline Columbus, $\mathrm{OH}$ & 6,895 & 7,704 & 8,027 & 7,864 & 8,121 & 680.3 & 750.2 & 774.2 & 744.9 & 769.2 \\
\hline Dallas-Fort Worth-Arlington, TX & 22,213 & 25,902 & 22,719 & 25,893 & 24,762 & 628.6 & 717.3 & 617.7 & 689.0 & 658.9 \\
\hline Denver-Aurora-Lakewood, CO & 9,020 & 9,265 & 9,360 & 9,652 & 10,514 & 652.7 & 656.7 & 654.8 & 668.2 & 727.9 \\
\hline Detroit-Warren-Dearborn, MI & 14,822 & 15,410 & 14,957 & 16,198 & 15,818 & 670.3 & 696.5 & 677.4 & 731.7 & 714.5 \\
\hline Hartford-West Hartford-East Hartford, CT & 3,349 & 3,226 & 3,307 & $3,945^{+}$ & $3,722^{+}$ & 538.2 & 520.0 & 535.5 & $636.9^{+}$ & $600.9^{\dagger}$ \\
\hline Houston-The Woodlands-Sugar Land, TX & 22,832 & 23,828 & 25,470 & 25,253 & 24,769 & 699.6 & 711.6 & 747.1 & 728.0 & 714.0 \\
\hline Indianapolis-Carmel-Anderson, IN & 8,398 & 7,816 & 8,604 & 9,090 & 9,102 & 832.9 & 768.7 & 839.5 & 876.6 & 877.7 \\
\hline Jacksonville, FL & 5,238 & 5,637 & 5,757 & 5,905 & 6,152 & 719.3 & 758.6 & 759.4 & 765.5 & 797.6 \\
\hline Kansas City, MO-KS & 6,991 & 7,108 & 7,544 & 7,947 & 8,461 & 662.4 & 668.8 & 703.9 & 733.4 & 780.9 \\
\hline Las Vegas-Henderson-Paradise, NV & 6,486 & 6,885 & 7,697 & 7,642 & 8,619 & 627.4 & 650.6 & 712.5 & 691.9 & 780.3 \\
\hline Los Angeles-Long Beach-Anaheim, CA & 40,401 & 42,385 & 43,278 & 46,649 & 48,445 & 600.7 & 626.6 & 641.1 & 689.1 & 715.6 \\
\hline Louisville-Jefferson County, KY-IN & 4,827 & 4,686 & 4,735 & 4,741 & 4,198 & 742.6 & 716.8 & 721.7 & 717.0 & 634.9 \\
\hline Memphis, TN-M & 7,758 & 7,238 & 7,324 & 8,164 & 8,657 & $1,109.2$ & $1,033.5$ & $1,046.4$ & $1,161.4$ & $1,231.6$ \\
\hline Miami-Fort Lauderdale-West Palm Beach, FL & 16,473 & 17,461 & 17,881 & 18,277 & 18,770 & 539.6 & 563.6 & 572.6 & 577.3 & 592.8 \\
\hline Milwaukee-Waukesha-West Allis, WI & 7,183 & 7,242 & 8,013 & 8,155 & 8,181 & 890.0 & 895.8 & 994.2 & $1,009.8$ & $1,013.0$ \\
\hline Minneapolis-St. Paul-Bloomington, MN-WI & $8,957^{+}$ & $9,497^{+}$ & 9,958 & 10,694 & 10,265 & $507.0^{\dagger}$ & $533.5^{+}$ & 555.8 & 589.0 & 565.4 \\
\hline Nashville-Davids & 5,278 & 5,322 & 5,374 & 5,846 & 6,487 & 574.5 & 567.4 & 562.0 & 600.6 & 666.4 \\
\hline New Orleans-Metairie, LA & 6,301 & 6,710 & 6,843 & 6,665 & 6,953 & 975.3 & $1,028.6$ & $1,044.5$ & $1,010.1$ & $1,053.7$ \\
\hline New York-Newark-Jersey City, NY-NJ-PA & 62,097 & 62,905 & 65,594 & 68,082 & 68,215 & 598.4 & 603.9 & 630.7 & 649.2 & 650.5 \\
\hline Oklahoma City, OK & 5,255 & 5,420 & 5,308 & 5,669 & 5,786 & 775.0 & 786.4 & 762.5 & 808.7 & 825.4 \\
\hline Orlando-Kissimmee-Sanfo & 8,021 & 8,505 & 8,593 & 9,252 & 9,778 & 675.8 & 696.6 & 688.5 & 721.2 & 762.2 \\
\hline Philadelphia-Camden-Wilmingto & 22,317 & 22,967 & 24,813 & 24,713 & 24,445 & 713.7 & 732.2 & 791.6 & 784.9 & 776.4 \\
\hline Phoenix-Mesa-Scottsdale, AZ & 14,841 & 14,607 & 15,621 & 17,504 & 18,009 & 656.9 & 634.3 & 665.8 & 734.9 & 756.1 \\
\hline Pittsburgh, PA & 5,509 & 5,681 & 5,551 & 4,923 & 5,443 & 454.6 & 469.9 & 461.8 & 411.2 & 454.6 \\
\hline Portland-Vancouver-Hillsboro, OR-WA & 6,158 & 6,559 & 7,076 & 7,178 & 7,388 & 518.4 & 542.7 & 577.2 & 579.2 & 596.2 \\
\hline Providence-Warwick, RI-MA & $3,945^{+}$ & $3,989^{+}$ & $4,501^{+}$ & $4,622^{+}$ & 4,980 & $475.6^{\dagger}$ & $479.9^{\dagger}$ & $541.6^{+}$ & $554.1^{+}$ & 597.1 \\
\hline Raleigh, NC & 3,502 & 4,685 & 4,244 & 4,721 & 5,017 & 550.1 & 718.3 & 634.9 & 690.4 & 733.7 \\
\hline Richmond, VA & 5,311 & 5,330 & 5,725 & 5,653 & 5,780 & 815.9 & 811.1 & 863.5 & 844.8 & 863.8 \\
\hline Riverside-San Bernardino-Ontario, CA & 13,988 & 14,693 & 13,893 & 16,830 & 17,013 & 626.6 & 651.4 & 610.5 & 731.6 & 739.6 \\
\hline Sacramento-Roseville-Arden-Arcade, CA & 6,686 & 7,284 & 7,282 & 8,123 & 9,184 & 583.6 & 627.3 & 620.5 & 683.9 & 773.3 \\
\hline Salt Lake City, UT & 2,873 & 3,067 & 3,275 & 3,520 & 3,426 & 500.3 & 526.4 & 554.6 & 587.7 & 572.1 \\
\hline San Antonio-New Braunfels, TX & 8,158 & 10,005 & 10,466 & 9,924 & 8,140 & 690.9 & 828.4 & 850.9 & 793.4 & 650.8 \\
\hline San Diego-Carlsbad, CA & 10,211 & 11,154 & 11,690 & 12,791 & 13,410 & 628.9 & 680.1 & 708.7 & 771.1 & 808.5 \\
\hline San Francisco-Oakland-Hayward, CA & 11,509 & 12,508 & 12,900 & 13,965 & 14,362 & 494.3 & 530.5 & 544.7 & 584.3 & 600.9 \\
\hline San Jose-Sunnyvale-Santa Clara, CA & 4,100 & 4,328 & 4,445 & 4,754 & 5,171 & 422.3 & 440.8 & 453.4 & 480.8 & 522.9 \\
\hline Seattle-Tacoma-Bellevue, WA & 8,751 & 9,306 & 10,085 & 10,454 & 10,535 & 476.5 & 498.4 & 530.8 & 541.1 & 545.3 \\
\hline St. Louis, MO-IL & 10,271 & 10,158 & 10,417 & 11,016 & 11,421 & 709.9 & 701.1 & 720.8 & 762.1 & 790.1 \\
\hline Tampa-St. Petersburg-Clearwater, FL & 9,066 & 9,269 & 9,360 & 9,743 & 10,288 & 602.5 & 603.7 & 598.4 & 611.6 & 645.8 \\
\hline Virginia Beach-Norfolk-Newport News, VA-NC & 8,425 & 7,677 & 8,791 & 8,873 & 9,140 & 966.9 & 877.4 & $1,002.4$ & $1,012.3$ & $1,042.7$ \\
\hline Washington-Arlington-Alexandria, DC-VA-MD-WV & 12,501 & 12,517 & 13,635 & 20,072 & 20,831 & 405.0 & 401.3 & 434.6 & 631.7 & 655.6 \\
\hline SELECTED MSAs TOTAL & 559,361 & 577,004 & 601,837 & 639,087 & 647,342 & 626.7 & 639.8 & 663.0 & 697.0 & 706.0 \\
\hline
\end{tabular}

* MSAs were selected on the basis of the largest population in the 2010 US Census.

t The variable used to identify county, which is used to classify cases into MSAs, was complete for $\leq 95 \%$ of cases in a state contributing data to this MSA. See Section A1.4 in the Appendix for more information.

₹ 2016 county data for Alabama have been corrected and may not match previous reports.

NOTE: Cases reported with unknown sex are not included in this table. County was misclassified in the 2017 District of Columbia STD morbidity data resulting in inaccurate county-level case counts and rates. 
Table 8. Chlamydia Among Males - Reported Cases and Rates of Reported Cases in Selected Metropolitan Statistical Areas (MSAs)* in Alphabetical Order, United States, 2014-2018

\begin{tabular}{|c|c|c|c|c|c|c|c|c|c|c|}
\hline \multirow[b]{2}{*}{ MSAs } & \multicolumn{5}{|c|}{ Cases } & \multicolumn{5}{|c|}{ Rates per 100,000 Population } \\
\hline & 2014 & 2015 & 2016 & 2017 & 2018 & 2014 & 2015 & 2016 & 2017 & 2018 \\
\hline Atlanta-Sandy Springs-Roswell, GA & 7,979 & $6,429^{\dagger}$ & 11,000 & 11,888 & $11,533^{\dagger}$ & 293.2 & $232.6^{+}$ & 392.9 & 417.9 & $405.4^{+}$ \\
\hline Austin-Round Rock, TX & 3,372 & 3,871 & 4,444 & 4,775 & 4,964 & 346.8 & 386.8 & 431.9 & 450.2 & 468.0 \\
\hline Baltimore-Columbia-Towson, MD & 4,294 & 4,514 & 5,446 & 5,755 & 6,297 & 319.6 & 334.6 & 403.7 & 425.0 & 465.0 \\
\hline Birmingham-Hoover, AL & 1,990 & 1,886 & $1,573^{\ddagger}$ & 2,377 & 2,231 & 361.6 & 342.4 & $284.9^{\ddagger}$ & 429.9 & 403.5 \\
\hline Boston-Cambridge-Newton, MA-NH & $4,988^{\dagger}$ & $5,211^{+}$ & $5,861^{\dagger}$ & $6,887^{\dagger}$ & 8,165 & $217.2^{+}$ & $224.8^{+}$ & $251.7^{\dagger}$ & $292.9^{\dagger}$ & 347.3 \\
\hline Buffalo-Cheektowaga-Niagara Falls, NY & 1,764 & 1,865 & 2,110 & 2,201 & 2,210 & 320.3 & 338.8 & 384.0 & 399.4 & 401.1 \\
\hline Charlotte-Concord-Gastonia, NC-SC & 3,125 & 4,607 & 4,278 & 5,085 & 5,631 & 270.4 & 391.0 & 356.7 & 415.1 & 459.7 \\
\hline Chicago-Naperville-Elgin, IL-IN-WI & 15,679 & 17,517 & 19,320 & 21,071 & 22,204 & 335.7 & 374.9 & 414.8 & 451.3 & 475.6 \\
\hline Cincinnati, $\mathrm{OH}-\mathrm{KY}-\mathrm{IN}$ & 2,787 & 3,168 & 3,333 & 3,352 & 3,467 & 264.9 & 299.9 & 314.1 & 313.8 & 324.6 \\
\hline Cleveland-Elyria, $\mathrm{OH}$ & 3,449 & 3,497 & 3,874 & 4,606 & 4,673 & 346.5 & 351.8 & 390.3 & 463.2 & 469.9 \\
\hline Columbus, $\mathrm{OH}$ & 3,363 & 3,623 & 4,086 & 4,057 & 4,390 & 342.8 & 364.2 & 406.7 & 396.6 & 429.1 \\
\hline Dallas-Fort Worth-Arlington, TX & 8,313 & 9,941 & 10,006 & 11,342 & 11,936 & 243.0 & 284.7 & 281.4 & 311.5 & 327.8 \\
\hline Denver-Aurora-Lakewood, CO & 4,326 & 4,677 & 4,922 & 5,868 & 6,414 & 315.2 & 333.3 & 345.8 & 406.4 & 444.3 \\
\hline Detroit-Warren-Dearborn, MI & 6,153 & 6,795 & 6,992 & 8,048 & 8,246 & 295.0 & 325.2 & 334.6 & 383.4 & 392.8 \\
\hline Hartford-West Hartford-East Hartford, CT & 1,333 & 1,443 & 1,541 & $1,982^{+}$ & $1,987^{+}$ & 225.1 & 244.2 & 261.5 & $335.5^{+}$ & $336.3^{\dagger}$ \\
\hline Houston-The Woodlands-Sugar Land, TX & 7,700 & 8,939 & 10,004 & 10,434 & 11,106 & 238.6 & 270.2 & 297.4 & 304.8 & 324.4 \\
\hline Indianapolis-Carmel-Anderson, IN & 3,544 & 3,723 & 4,185 & 4,604 & 4,593 & 368.0 & 383.0 & 427.3 & 464.3 & 463.2 \\
\hline Jacksonville, FL & 2,138 & 2,367 & 2,672 & 2,854 & 2,974 & 309.4 & 335.1 & 371.1 & 389.0 & 405.4 \\
\hline Kansas City, MO-KS & 2,875 & 3,132 & 3,499 & 3,921 & 4,398 & 283.1 & 305.6 & 338.8 & 375.1 & 420.7 \\
\hline Las Vegas-Henderson-Paradise, NV & 2,986 & 3,139 & 3,646 & 4,228 & 5,038 & 288.3 & 297.1 & 339.1 & 384.5 & 458.2 \\
\hline Los Angeles-Long Beach-Anaheim, CA & 23,766 & 25,764 & 28,299 & 31,501 & 33,493 & 363.6 & 391.8 & 431.4 & 478.4 & 508.7 \\
\hline Louisville-Jefferson County, KY-IN & 1,896 & 2,031 & 2,131 & 2,323 & 2,073 & 305.9 & 325.1 & 339.7 & 367.2 & 327.6 \\
\hline Memphis, TN-MS-AR & 2,795 & 3,104 & 3,035 & 3,546 & 3,897 & 434.1 & 482.2 & 472.1 & 549.5 & 603.9 \\
\hline Miami-Fort Lauderdale-West Palm Be & 8,086 & 9,270 & 10,172 & 11,141 & 11,965 & 281.1 & 318.1 & 345.6 & 372.3 & 399.8 \\
\hline Milwaukee-Waukesha-West Allis, WI & 3,111 & 3,394 & 3,860 & 4,021 & 4,248 & 406.6 & 442.3 & 503.6 & 523.1 & 552.7 \\
\hline Minneapolis-St. Paul-Bloomington, MN-WI & $4,623^{\dagger}$ & $5,206^{+}$ & 5,614 & 6,192 & 6,335 & $267.5^{+}$ & $298.4^{+}$ & 319.1 & 346.9 & 354.9 \\
\hline Nashville-Davidson-Murfreesboro-Franklin, TN & 2,598 & 2,744 & 2,822 & 3,081 & 3,565 & 297.3 & 307.5 & 310.4 & 331.4 & 383.5 \\
\hline New Orleans-Metairie, LA & 2,294 & 2,581 & 2,783 & 3,185 & 3,605 & 378.7 & 422.7 & 453.4 & 517.1 & 585.3 \\
\hline New York-Newark-Jersey City, NY-N & 31,310 & 34,799 & 39,725 & 44,597 & 47,384 & 322.3 & 356.3 & 407.3 & 453.5 & 481.8 \\
\hline Oklahoma City, OK & 2,036 & 2,213 & 2,385 & 2,529 & 2,663 & 309.1 & 330.7 & 352.3 & 370.4 & 390.0 \\
\hline Orlando-Kissimmee-Sanford, FL & 2,964 & 3,511 & 3,896 & 4,616 & 4,992 & 261.2 & 301.0 & 326.5 & 376.2 & 406.9 \\
\hline Philadelphia-Camden-Wilmington, PA-NJ-DE-MD & 11,010 & 11,912 & 13,315 & 13,889 & 14,401 & 376.5 & 406.1 & 453.5 & 471.2 & 488.6 \\
\hline Phoenix-Mesa-Scottsdale, AZ & 6,735 & 7,173 & 7,944 & 9,354 & 10,133 & 302.0 & 315.7 & 343.1 & 397.1 & 430.2 \\
\hline Pittsburgh, PA & 2,541 & 2,909 & 3,062 & 2,876 & 3,172 & 222.1 & 254.3 & 268.5 & 253.2 & 279.2 \\
\hline Portland-Vancouver-Hillsboro, OR-WA & 3,124 & 3,416 & 3,970 & 4,271 & 4,426 & 269.2 & 289.3 & 331.1 & 351.8 & 364.6 \\
\hline Providence-Warwick, RI-MA & $1,744^{+}$ & $1,913^{+}$ & $2,163^{+}$ & $2,350^{+}$ & 2,547 & $223.6^{+}$ & $244.7^{\dagger}$ & $276.0^{+}$ & $298.6^{+}$ & 323.6 \\
\hline Raleigh, NC & 1,622 & 2,226 & 2,248 & 2,522 & 2,693 & 267.5 & 358.3 & 354.3 & 387.2 & 413.5 \\
\hline Richmond, VA & 2,503 & 2,540 & 2,827 & 2,797 & 2,992 & 410.9 & 413.5 & 456.9 & 447.5 & 478.7 \\
\hline Riverside-San Bernardino-Ontario, CA & 5,542 & 6,070 & 6,141 & 7,415 & 7,730 & 250.8 & 271.8 & 272.7 & 325.2 & 339.0 \\
\hline Sacramento-Roseville-Arden-Arcade, CA & 2,976 & 3,322 & 3,580 & 4,255 & 4,838 & 270.9 & 298.5 & 318.9 & 374.2 & 425.4 \\
\hline Salt Lake City, UT & 1,550 & 1,684 & 1,989 & 1,991 & 2,046 & 267.7 & 286.6 & 333.9 & 329.5 & 338.6 \\
\hline San Antonio-New Braunfels, TX & 3,412 & 4,459 & 4,683 & 4,652 & 4,030 & 297.2 & 379.1 & 390.4 & 380.3 & 329.5 \\
\hline San Diego-Carlsbad, CA & 5,508 & 6,190 & 7,195 & 8,017 & 8,475 & 335.9 & 373.0 & 431.3 & 477.5 & 504.8 \\
\hline San Francisco-Oakland-Hayward, CA & 8,823 & 10,961 & 11,936 & 13,788 & 14,790 & 389.4 & 476.9 & 516.5 & 589.9 & 632.8 \\
\hline San Jose-Sunnyvale-Santa Clara, CA & 2,163 & 2,565 & 2,717 & 3,109 & 3,373 & 220.3 & 257.8 & 272.1 & 307.9 & 334.1 \\
\hline Seattle-Tacoma-Bellevue, WA & 5,110 & 5,951 & 6,799 & 7,375 & 8,200 & 278.5 & 318.8 & 358.0 & 381.1 & 423.7 \\
\hline St. Louis, MO-IL & 4,429 & 4,762 & 5,091 & 5,450 & 5,801 & 325.8 & 349.4 & 373.9 & 400.2 & 425.9 \\
\hline Tampa-St. Petersburg-Clearwater, FL & 3,835 & 4,195 & 4,632 & 4,768 & 5,166 & 271.8 & 291.3 & 315.5 & 318.2 & 344.8 \\
\hline Virginia Beach-Norfolk-Newport News, VA-NC & 3,748 & 3,595 & 4,406 & 4,593 & 4,959 & 443.4 & 423.0 & 518.4 & 541.2 & 584.3 \\
\hline Washington-Arlington-Alexandria, DC-VA-MD-WV & 5,813 & 6,359 & 7,595 & 12,856 & 13,275 & 197.2 & 213.5 & 253.6 & 423.0 & 436.8 \\
\hline SELECTED MSAs TOTAL & 259,825 & 287,163 & 319,807 & 358,395 & 379,724 & 303.4 & 331.8 & 367.0 & 406.7 & 430.9 \\
\hline
\end{tabular}

* MSAs were selected on the basis of the largest population in the 2010 US Census.

${ }^{\dagger}$ The variable used to identify county, which is used to classify cases into MSAs, was complete for $\leq 95 \%$ of cases in a state contributing data to this MSA. See Section A1.4 in the Appendix for more information.

₹ 2016 county data for Alabama have been corrected and may not match previous reports.

NOTE: Cases reported with unknown sex are not included in this table. County was misclassified in the 2017 District of Columbia STD morbidity data resulting in inaccurate county-level case counts and rates. 
Table 9. Chlamydia - Reported Cases and Rates of Reported Cases in Counties and Independent Cities* Ranked by Number of Reported Cases, United States, 2018

\begin{tabular}{|c|c|c|c|c|}
\hline Rank* & County/Independent City & Cases & Rate per 100,000 Population & Cumulative Percentage \\
\hline 1 & Los Angeles County, CA & 67,878 & 667.9 & 4 \\
\hline 2 & Cook County, IL & 43,271 & 830.3 & 6 \\
\hline 3 & Harris County, TX & 27,415 & 589.2 & 8 \\
\hline 4 & Maricopa County, AZ & 26,464 & 614.4 & 9 \\
\hline 5 & San Diego County, CA & 21,912 & 656.5 & 11 \\
\hline 6 & Kings County, NY & 21,656 & 817.6 & 12 \\
\hline 7 & Philadelphia County, PA & 20,206 & $1,278.2$ & 13 \\
\hline 8 & Dallas County, TX & 18,088 & 690.9 & 14 \\
\hline 9 & Bronx County, NY & 17,689 & $1,202.4$ & 15 \\
\hline 10 & New York County, NY & 17,218 & $1,034.3$ & 16 \\
\hline 11 & Wayne County, MI & 15,114 & 861.9 & 17 \\
\hline 12 & Orange County, CA & 14,265 & 447.1 & 18 \\
\hline 13 & Queens County, NY & 14,059 & 596.1 & 18 \\
\hline 14 & Clark County, NV & 13,695 & 621.3 & 19 \\
\hline 15 & Miami-Dade County, FL & 13,395 & 486.8 & 20 \\
\hline 16 & San Bernardino County, CA & 13,333 & 618.0 & 21 \\
\hline 17 & Sacramento County, CA & 11,457 & 748.5 & 21 \\
\hline 18 & Riverside County, CA & 11,453 & 472.6 & 22 \\
\hline 19 & Broward County, FL & 11,322 & 584.9 & 23 \\
\hline 20 & Milwaukee County, WI & 11,195 & $1,175.8$ & 23 \\
\hline 21 & Cuyahoga County, $\mathrm{OH}$ & 11,025 & 883.0 & 24 \\
\hline 22 & Bexar County, TX & 10,544 & 538.3 & 25 \\
\hline 23 & Marion County, IN & 10,408 & $1,095.5$ & 25 \\
\hline 24 & King County, WA & 10,233 & 467.5 & 26 \\
\hline 25 & Franklin County, $\mathrm{OH}$ & 10,161 & 786.5 & 26 \\
\hline 26 & Orange County, FL & 9,885 & 732.8 & 27 \\
\hline 27 & Shelby County, TN & 9,724 & $1,037.8$ & 27 \\
\hline 28 & Alameda County, CA & 9,701 & 583.3 & 28 \\
\hline 29 & Tarrant County, TX & 9,575 & 466.1 & 29 \\
\hline 30 & San Francisco County, CA & 9,491 & $1,073.2$ & 29 \\
\hline 31 & Mecklenburg County, NC & 9,182 & 852.7 & 30 \\
\hline 32 & Washington, D.C. & 9,014 & $1,298.9$ & 30 \\
\hline 33 & Travis County, TX & 8,992 & 733.0 & 31 \\
\hline 34 & Hillsborough County, FL & 8,782 & 623.5 & 31 \\
\hline 35 & Fulton County, GA & 8,376 & 804.3 & 32 \\
\hline 36 & Santa Clara County, CA & 8,315 & 429.0 & 32 \\
\hline 37 & Baltimore (City), MD & 8,013 & $1,310.1$ & 33 \\
\hline 38 & Prince George's County, MD & 8,013 & 877.9 & 33 \\
\hline 39 & Hennepin County, MN & 7,866 & 628.3 & 33 \\
\hline 40 & Denver County, CO & 7,466 & $1,059.6$ & 34 \\
\hline 41 & Fresno County, CA & 7,129 & 720.6 & 34 \\
\hline 42 & Duval County, FL & 7,112 & 758.3 & 35 \\
\hline 43 & Hamilton County, $\mathrm{OH}$ & 6,954 & 854.5 & 35 \\
\hline 44 & Kern County, CA & 6,948 & 777.9 & 35 \\
\hline 45 & Suffolk County, MA & 6,934 & 869.0 & 36 \\
\hline 46 & Pima County, AZ & 6,688 & 653.9 & 36 \\
\hline 47 & Wake County, NC & 6,482 & 604.5 & 37 \\
\hline 48 & Essex County, NJ & 6,479 & 801.6 & 37 \\
\hline 49 & St. Louis County, MO & 6,450 & 647.1 & 37 \\
\hline 50 & Jackson County, MO & 6,437 & 921.0 & 38 \\
\hline 51 & Contra Costa County, CA & 6,189 & 539.4 & 38 \\
\hline 52 & Palm Beach County, FL & 6,025 & 409.5 & 38 \\
\hline 53 & Pierce County, WA & 5,848 & 667.0 & 39 \\
\hline 54 & Oklahoma County, OK & 5,820 & 738.6 & 39 \\
\hline 55 & DeKalb County, GA & 5,792 & 768.9 & 39 \\
\hline 56 & Honolulu County, HI & 5,721 & 578.7 & 40 \\
\hline 57 & El Paso County, TX & 5,700 & 678.2 & 40 \\
\hline 58 & Allegheny County, PA & 5,660 & 462.8 & 40 \\
\hline 59 & Multnomah County, OR & 5,501 & 681.2 & 41 \\
\hline 60 & Erie County, NY & 5,449 & 588.7 & 41 \\
\hline 61 & Middlesex County, MA & 5,411 & 337.6 & 41 \\
\hline 62 & Davidson County, TN & 5,372 & 777.2 & 42 \\
\hline 63 & Monroe County, NY & 5,347 & 715.2 & 42 \\
\hline 64 & Salt Lake County, UT & 5,286 & 465.5 & 42 \\
\hline 65 & Guilford County, NC & 5,140 & 975.4 & 43 \\
\hline 66 & Suffolk County, NY & 5,127 & 343.4 & 43 \\
\hline 67 & Jefferson County, AL & 5,121 & 776.9 & 43 \\
\hline 68 & Bernalillo County, NM & 5,050 & 746.2 & 43 \\
\hline 69 & Orleans Parish, LA & 4,974 & $1,264.7$ & 44 \\
\hline 70 & Hartford County, CT & 4,858 & 542.6 & 44 \\
\hline
\end{tabular}

* The top 70 counties and independent cities ranked in descending order by number of cases reported in 2018 then by rate are displayed.

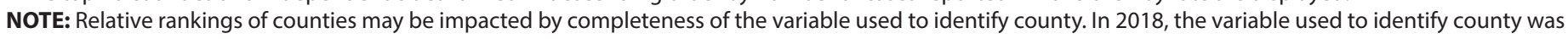
complete for $\leq 95 \%$ of cases in Connecticut, Georgia, Hawaii, and South Dakota. See Section A1.4 in the Appendix for more information. 
Table 10. Chlamydia - Reported Cases and Rates of Reported Cases by Age Group and Sex, United States, 2014-2018

\begin{tabular}{|c|c|c|c|c|c|c|c|c|}
\hline & \multirow{2}{*}{$\begin{array}{l}\text { Age } \\
\text { Group }\end{array}$} & \multicolumn{4}{|c|}{ Cases } & \multicolumn{3}{|c|}{ Rates per 100,000 Population* } \\
\hline & & Total & Male & Female & Unknown Sex & Total & Male & Female \\
\hline \multirow{14}{*}{$\underset{\vec{A}}{\mathrm{O}}$} & $0-4$ & 603 & 200 & 388 & 15 & 3.0 & 2.0 & 4.0 \\
\hline & $5-9$ & 181 & 26 & 152 & 3 & 0.9 & 0.2 & 1.5 \\
\hline & $10-14$ & 11,406 & 1,342 & 10,041 & 23 & 55.2 & 12.7 & 99.2 \\
\hline & $15-19$ & 381,717 & 77,908 & 303,294 & 515 & 1,811.9 & 722.4 & $2,949.3$ \\
\hline & $20-24$ & 566,385 & 159,804 & 405,876 & 705 & $2,472.0$ & $1,361.3$ & $3,632.7$ \\
\hline & $25-29$ & 253,825 & 91,729 & 161,793 & 303 & $1,154.4$ & 821.8 & $1,494.4$ \\
\hline & $30-34$ & 113,208 & 45,990 & 67,060 & 158 & 525.9 & 425.5 & 625.6 \\
\hline & $35-39$ & 52,536 & 22,894 & 29,545 & 97 & 263.7 & 230.3 & 296.0 \\
\hline & $40-44$ & 27,426 & 13,711 & 13,662 & 53 & 133.2 & 134.2 & 131.7 \\
\hline & $45-54$ & 24,773 & 14,318 & 10,424 & 31 & 57.0 & 66.8 & 47.3 \\
\hline & $55-64$ & 6,527 & 3,911 & 2,603 & 13 & 16.3 & 20.2 & 12.5 \\
\hline & $65+$ & 1,449 & 871 & 570 & 8 & 3.1 & 4.3 & 2.2 \\
\hline & Unknown Age & 1,753 & 621 & 1,033 & 99 & & & \\
\hline & TOTAL & $1,441,789$ & 433,325 & $1,006,441$ & 2,023 & 452.2 & 276.1 & 621.6 \\
\hline \multirow{14}{*}{ v } & $0-4$ & 518 & 196 & 322 & 0 & 2.6 & 1.9 & 3.3 \\
\hline & $5-9$ & 148 & 18 & 130 & 0 & 0.7 & 0.2 & 1.3 \\
\hline & $10-14$ & 10,642 & 1,216 & 9,394 & 32 & 51.6 & 11.6 & 93.0 \\
\hline & $15-19$ & 391,396 & 82,775 & 307,937 & 684 & $1,854.2$ & 766.6 & $2,986.5$ \\
\hline & $20-24$ & 589,963 & 172,313 & 416,772 & 878 & $2,594.5$ & $1,476.8$ & $3,764.4$ \\
\hline & $25-29$ & 280,429 & 104,679 & 175,291 & 459 & $1,248.5$ & 917.5 & $1,586.0$ \\
\hline & $30-34$ & 123,866 & 52,019 & 71,653 & 194 & 571.5 & 477.7 & 664.3 \\
\hline & $35-39$ & 59,905 & 27,180 & 32,621 & 104 & 294.0 & 267.2 & 319.8 \\
\hline & $40-44$ & 30,379 & 15,210 & 15,118 & 51 & 150.3 & 151.6 & 148.4 \\
\hline & $45-54$ & 28,833 & 17,011 & 11,764 & 58 & 66.8 & 79.9 & 53.7 \\
\hline & $55-64$ & 7,756 & 4,901 & 2,840 & 15 & 19.0 & 24.9 & 13.4 \\
\hline & $65+$ & 1,596 & 1,043 & 546 & 7 & 3.3 & 4.9 & 2.0 \\
\hline & Unknown Age & 1,227 & 420 & 755 & 52 & & & \\
\hline & TOTAL & $1,526,658$ & 478,981 & $1,045,143$ & 2,534 & 475.0 & 302.7 & 640.4 \\
\hline \multirow{14}{*}{$\frac{\text { o }}{a}$} & $0-4$ & 597 & 225 & 368 & 4 & 3.0 & 2.2 & 3.8 \\
\hline & $5-9$ & 188 & 25 & 161 & 2 & 0.9 & 0.2 & 1.6 \\
\hline & $10-14$ & 10,571 & 1,341 & 9,206 & 24 & 51.3 & 12.7 & 91.2 \\
\hline & $15-19$ & 407,230 & 89,899 & 316,639 & 692 & 1,927.3 & 832.3 & $3,065.8$ \\
\hline & $20-24$ & 601,173 & 181,857 & 418,388 & 928 & $2,686.1$ & $1,582.6$ & $3,842.0$ \\
\hline & $25-29$ & 298,176 & 114,484 & 183,222 & 470 & 1,302.6 & 984.3 & $1,627.3$ \\
\hline & $30-34$ & 133,062 & 58,583 & 74,226 & 253 & 610.8 & 534.1 & 686.1 \\
\hline & $35-39$ & 66,669 & 31,671 & 34,872 & 126 & 320.9 & 305.2 & 335.4 \\
\hline & $40-44$ & 32,548 & 16,784 & 15,705 & 59 & 165.2 & 171.7 & 158.3 \\
\hline & $45-54$ & 32,316 & 19,569 & 12,683 & 64 & 75.5 & 92.7 & 58.5 \\
\hline & $55-64$ & 9,321 & 5,942 & 3,354 & 25 & 22.5 & 29.7 & 15.6 \\
\hline & $65+$ & 1,772 & 1,161 & 597 & 14 & 3.6 & 5.3 & 2.2 \\
\hline & Unknown Age & 4,731 & 1,329 & 3,298 & 104 & & & \\
\hline & TOTAL & $1,598,354$ & 522,870 & $1,072,719$ & 2,765 & 494.7 & 328.7 & 653.9 \\
\hline \multirow{14}{*}{$\underset{\mathbf{v}}{\mathrm{v}}$} & $0-4$ & 514 & 188 & 323 & 3 & 2.6 & 1.8 & 3.3 \\
\hline & $5-9$ & 167 & 9 & 158 & 0 & 0.8 & 0.1 & 1.6 \\
\hline & $10-14$ & 10,726 & 1,252 & 9,454 & 20 & 51.6 & 11.8 & 92.9 \\
\hline & $15-19$ & 437,904 & 99,864 & 337,290 & 750 & $2,072.3$ & 924.6 & $3,264.8$ \\
\hline & $20-24$ & 631,207 & 195,971 & 434,050 & 1,186 & $2,853.7$ & $1,726.7$ & $4,030.4$ \\
\hline & $25-29$ & 321,857 & 127,007 & 194,267 & 583 & 1,377.2 & $1,067.1$ & $1,694.0$ \\
\hline & $30-34$ & 144,451 & 65,690 & 78,502 & 259 & 657.4 & 592.4 & 721.3 \\
\hline & $35-39$ & 74,202 & 36,427 & 37,621 & 154 & 349.5 & 343.1 & 354.4 \\
\hline & $40-44$ & 36,332 & 19,310 & 16,940 & 82 & 185.0 & 198.0 & 171.3 \\
\hline & $45-54$ & 36,229 & 22,431 & 13,713 & 85 & 85.5 & 107.3 & 63.9 \\
\hline & $55-64$ & 11,356 & 7,490 & 3,840 & 26 & 27.0 & 37.0 & 17.7 \\
\hline & $65+$ & 2,178 & 1,461 & 698 & 19 & 4.3 & 6.5 & 2.5 \\
\hline & Unknown Age & 1,446 & 544 & 795 & 107 & & & \\
\hline & TOTAL & $1,708,569$ & 577,644 & $1,127,651$ & 3,274 & 524.6 & 360.1 & 682.1 \\
\hline \multirow{14}{*}{$\underset{\infty}{0}$} & $0-4$ & 496 & 186 & 300 & 10 & 2.5 & 1.8 & 3.1 \\
\hline & $5-9$ & 144 & 19 & 125 & 0 & 0.7 & 0.2 & 1.3 \\
\hline & $10-14$ & 10,905 & 1,438 & 9,450 & 17 & 52.5 & 13.6 & 92.9 \\
\hline & $15-19$ & 446,008 & 103,582 & 341,635 & 791 & $2,110.6$ & 959.0 & $3,306.8$ \\
\hline & $20-24$ & 641,269 & 202,528 & 437,732 & 1,009 & $2,899.2$ & $1,784.5$ & $4,064.6$ \\
\hline & $25-29$ & 333,561 & 135,059 & 197,966 & 536 & $1,427.3$ & $1,134.7$ & $1,726.2$ \\
\hline & $30-34$ & 154,132 & 72,222 & 81,645 & 265 & 701.5 & 651.3 & 750.2 \\
\hline & $35-39$ & 78,094 & 39,320 & 38,635 & 139 & 367.8 & 370.4 & 363.9 \\
\hline & $40-44$ & 38,657 & 21,111 & 17,462 & 84 & 196.8 & 216.5 & 176.6 \\
\hline & $45-54$ & 38,323 & 24,067 & 14,173 & 83 & 90.4 & 115.1 & 66.0 \\
\hline & $55-64$ & 12,536 & 8,474 & 4,029 & 33 & 29.9 & 41.8 & 18.5 \\
\hline & $65+$ & 2,331 & 1,676 & 640 & 15 & 4.6 & 7.4 & 2.3 \\
\hline & Unknown Age & 2,212 & 765 & 1,271 & 176 & & & \\
\hline & TOTAL & $1,758,668$ & 610,447 & $1,145,063$ & 3,158 & 539.9 & 380.6 & 692.7 \\
\hline
\end{tabular}

* No population data are available for unknown sex and age; therefore, rates are not calculated. NOTE: Cases in the 0-4 age group may include cases due to perinatal transmission. 
Table 11A. Chlamydia - Reported Cases by Race/Hispanic Ethnicity, Age Group, and Sex, United States, 2018

\begin{tabular}{|c|c|c|c|c|c|c|c|c|c|c|c|c|}
\hline \multirow{2}{*}{$\begin{array}{l}\text { Age } \\
\text { Group }\end{array}$} & \multicolumn{3}{|c|}{$\begin{array}{l}\text { American Indians/ } \\
\text { Alaska Natives }\end{array}$} & \multicolumn{3}{|c|}{ Asians } & \multicolumn{3}{|c|}{ Blacks } & \multicolumn{3}{|c|}{$\begin{array}{l}\text { Native Hawaiians/ } \\
\text { Other Pacific Islanders }\end{array}$} \\
\hline & Total* & Male & Female & Total* & Male & Female & Total* & Male & Female & Total* & Male & Female \\
\hline $0-4$ & 5 & 3 & 2 & 3 & 0 & 3 & 101 & 44 & 57 & 2 & 0 & 2 \\
\hline $5-9$ & 4 & 0 & 4 & 1 & 0 & 1 & 41 & 5 & 36 & 0 & 0 & 0 \\
\hline $10-14$ & 159 & 14 & 145 & 41 & 3 & 38 & 4,111 & 678 & 3,433 & 13 & 0 & 13 \\
\hline $15-19$ & 4,416 & 868 & 3,539 & 3,530 & 532 & 2,996 & 139,846 & 40,167 & 99,598 & 839 & 126 & 710 \\
\hline $20-24$ & 5,889 & 1,388 & 4,493 & 8,338 & 2,362 & 5,967 & 175,267 & 63,118 & 112,044 & 1,484 & 358 & 1,126 \\
\hline $25-29$ & 3,921 & 1,092 & 2,824 & 5,201 & 2,380 & 2,814 & 92,982 & 40,198 & 52,725 & 874 & 255 & 618 \\
\hline $30-34$ & 2,247 & 713 & 1,528 & 3,039 & 1,538 & 1,495 & 37,778 & 19,462 & 18,295 & 435 & 150 & 285 \\
\hline $35-39$ & 1,223 & 408 & 813 & 1,727 & 840 & 885 & 17,124 & 9,765 & 7,340 & 216 & 94 & 122 \\
\hline $40-44$ & 514 & 166 & 347 & 976 & 539 & 437 & 7,607 & 4,839 & 2,761 & 92 & 51 & 41 \\
\hline $45-54$ & 358 & 137 & 221 & 1,029 & 581 & 448 & 6,960 & 4,845 & 2,110 & 59 & 30 & 29 \\
\hline $55-64$ & 102 & 49 & 53 & 320 & 177 & 142 & 2,368 & 1,589 & 778 & 19 & 8 & 11 \\
\hline $65+$ & 17 & 7 & 10 & 73 & 51 & 22 & 352 & 255 & 97 & 5 & 1 & 4 \\
\hline Unknown Age & 6 & 3 & 2 & 34 & 9 & 25 & 248 & 120 & 115 & 4 & 1 & 3 \\
\hline TOTAL & 18,861 & 4,848 & 13,981 & 24,312 & 9,012 & 15,273 & 484,785 & 185,085 & 299,389 & 4,042 & 1,074 & 2,964 \\
\hline
\end{tabular}

\begin{tabular}{|c|c|c|c|c|c|c|c|c|c|c|c|c|}
\hline \multirow{2}{*}{$\begin{array}{l}\text { Age } \\
\text { Group }\end{array}$} & \multicolumn{3}{|c|}{ Whites } & \multicolumn{3}{|c|}{ Multirace } & \multicolumn{3}{|c|}{ Hispanics } & \multicolumn{3}{|c|}{$\begin{array}{l}\text { Other/ } \\
\text { Unknown }\end{array}$} \\
\hline & Total* & Male & Female & Total* & Male & Female & Total* & Male & Female & Total* & Male & Female \\
\hline $0-4$ & 98 & 37 & 61 & 1 & 1 & 0 & 56 & 28 & 28 & 230 & 73 & 147 \\
\hline $5-9$ & 20 & 2 & 18 & 2 & 0 & 2 & 20 & 1 & 19 & 56 & 11 & 45 \\
\hline $10-14$ & 1,830 & 131 & 1,699 & 116 & 5 & 111 & 1,462 & 182 & 1,280 & 3,173 & 425 & 2,731 \\
\hline $15-19$ & 100,212 & 16,921 & 83,231 & 3,538 & 627 & 2,910 & 55,594 & 11,069 & 44,475 & 138,033 & 33,272 & 104,176 \\
\hline $20-24$ & 156,960 & 44,878 & 111,981 & 4,489 & 1,201 & 3,287 & 81,588 & 22,447 & 59,078 & 207,254 & 66,776 & 139,756 \\
\hline $25-29$ & 77,681 & 30,430 & 47,199 & 2,284 & 1,009 & 1,272 & 45,337 & 17,032 & 28,249 & 105,281 & 42,663 & 62,265 \\
\hline $30-34$ & 37,837 & 17,630 & 20,180 & 1,129 & 676 & 453 & 22,479 & 9,893 & 12,565 & 49,188 & 22,160 & 26,844 \\
\hline $35-39$ & 19,425 & 9,947 & 9,464 & 557 & 388 & 167 & 12,083 & 5,654 & 6,417 & 25,739 & 12,224 & 13,427 \\
\hline $40-44$ & 9,522 & 5,400 & 4,113 & 296 & 221 & 75 & 6,139 & 3,087 & 3,040 & 13,511 & 6,808 & 6,648 \\
\hline $45-54$ & 10,623 & 7,546 & 3,071 & 316 & 286 & 30 & 5,151 & 3,022 & 2,115 & 13,827 & 7,620 & 6,149 \\
\hline $55-64$ & 3,929 & 3,145 & 779 & 89 & 78 & 11 & 1,143 & 693 & 448 & 4,566 & 2,735 & 1,807 \\
\hline $65+$ & 733 & 596 & 137 & 8 & 7 & 1 & 151 & 104 & 47 & 992 & 655 & 322 \\
\hline Unknown Age & 757 & 254 & 495 & 3 & 3 & 0 & 195 & 65 & 129 & 965 & 310 & 502 \\
\hline TOTAL & 419,627 & 136,917 & 282,428 & 12,828 & 4,502 & 8,319 & 231,398 & 73,277 & 157,890 & 562,815 & 195,732 & 364,819 \\
\hline
\end{tabular}

* Total includes cases reported with unknown sex.

NOTE: These tables should be used only for race/Hispanic ethnicity comparisons. See Table 10 for age-specific cases and rates and Tables 3-5 for total and sexspecific cases and rates. Cases in the $0-4$ age group may include cases due to perinatal transmission. 
Table 11B. Chlamydia - Rates of Reported Cases* by Race/Hispanic Ethnicity, Age Group, and Sex, United States, 2018

\begin{tabular}{|c|c|c|c|c|c|c|c|c|c|c|c|c|}
\hline \multirow{2}{*}{$\begin{array}{l}\text { Age } \\
\text { Group }\end{array}$} & \multicolumn{3}{|c|}{$\begin{array}{l}\text { American Indians/ } \\
\text { Alaska Natives }\end{array}$} & \multicolumn{3}{|c|}{ Asians } & \multicolumn{3}{|c|}{ Blacks } & \multicolumn{3}{|c|}{$\begin{array}{l}\text { Native Hawaiians/ } \\
\text { Other Pacific Islanders }\end{array}$} \\
\hline & Total $^{+}$ & Male & Female & Total $^{\dagger}$ & Male & Female & Total $^{\dagger}$ & Male & Female & Total $^{\dagger}$ & Male & Female \\
\hline $0-4$ & 3.0 & 3.6 & 2.5 & 0.3 & 0.0 & 0.6 & 3.7 & 3.2 & 4.2 & 4.9 & 0.0 & 10.0 \\
\hline $5-9$ & 2.3 & 0.0 & 4.7 & 0.1 & 0.0 & 0.2 & 1.5 & 0.4 & 2.6 & 0.0 & 0.0 & 0.0 \\
\hline $10-14$ & 90.0 & 15.6 & 166.4 & 3.9 & 0.6 & 7.3 & 146.0 & 47.5 & 246.9 & 31.5 & 0.0 & 63.6 \\
\hline $15-19$ & $2,445.7$ & 945.2 & $3,988.2$ & 327.9 & 98.3 & 559.5 & $4,714.8$ & $2,668.6$ & $6,817.3$ & $2,061.6$ & 603.8 & $3,580.8$ \\
\hline $20-24$ & $3,131.2$ & $1,447.2$ & $4,875.1$ & 646.1 & 361.7 & 935.8 & $5,454.9$ & $3,867.1$ & $7,087.7$ & $3,375.6$ & $1,596.6$ & $5,227.5$ \\
\hline $25-29$ & $1,992.9$ & $1,086.0$ & $2,935.5$ & 328.7 & 305.8 & 349.9 & $2,740.6$ & $2,371.7$ & $3,105.5$ & $1,686.0$ & 948.4 & $2,476.8$ \\
\hline $30-34$ & $1,341.2$ & 848.9 & $1,828.9$ & 190.7 & 201.3 & 180.2 & $1,325.6$ & $1,406.1$ & $1,248.1$ & 866.4 & 583.9 & $1,162.6$ \\
\hline $35-39$ & 797.8 & 535.3 & $1,054.8$ & 114.4 & 119.1 & 110.0 & 628.1 & 753.0 & 513.6 & 473.6 & 402.2 & 548.6 \\
\hline $40-44$ & 366.2 & 240.2 & 486.9 & 67.9 & 80.4 & 56.9 & 306.3 & 414.3 & 209.8 & 237.7 & 259.1 & 215.6 \\
\hline $45-54$ & 119.5 & 94.0 & 143.6 & 40.4 & 49.0 & 32.9 & 132.6 & 196.8 & 75.7 & 82.8 & 85.4 & 80.4 \\
\hline $55-64$ & 35.2 & 36.0 & 34.4 & 15.7 & 19.2 & 12.7 & 49.1 & 72.1 & 29.7 & 32.1 & 27.7 & 36.3 \\
\hline $65+$ & 6.2 & 5.7 & 6.7 & 3.2 & 5.2 & 1.7 & 7.7 & 13.8 & 3.5 & 9.5 & 4.0 & 14.2 \\
\hline \multicolumn{13}{|c|}{ Unknown Age } \\
\hline TOTAL & 784.8 & 409.6 & $1,146.3$ & 132.1 & 102.9 & 158.4 & $1,192.5$ & 952.3 & 1,411.1 & 700.8 & 370.4 & $1,033.5$ \\
\hline
\end{tabular}

\begin{tabular}{|c|c|c|c|c|c|c|c|c|c|}
\hline \multirow{2}{*}{$\begin{array}{l}\text { Age } \\
\text { Group }\end{array}$} & \multicolumn{3}{|c|}{ Whites } & \multicolumn{3}{|c|}{ Multirace } & \multicolumn{3}{|c|}{ Hispanics } \\
\hline & Total $^{\dagger}$ & Male & Female & Total $^{\dagger}$ & Male & Female & Total $^{\dagger}$ & Male & Female \\
\hline $0-4$ & 1.0 & 0.7 & 1.3 & 0.1 & 0.2 & 0.0 & 1.1 & 1.1 & 1.1 \\
\hline $5-9$ & 0.2 & 0.0 & 0.4 & 0.2 & 0.0 & 0.4 & 0.4 & 0.0 & 0.7 \\
\hline $10-14$ & 17.1 & 2.4 & 32.6 & 14.0 & 1.2 & 27.3 & 28.3 & 6.9 & 50.5 \\
\hline $15-19$ & 890.7 & 293.0 & $1,520.1$ & 492.5 & 172.2 & 821.3 & $1,134.9$ & 442.3 & $1,856.0$ \\
\hline $20-24$ & $1,317.8$ & 732.6 & $1,935.8$ & 705.8 & 373.7 & $1,044.8$ & $1,687.1$ & 898.5 & $2,527.0$ \\
\hline $25-29$ & 605.6 & 465.8 & 749.8 & 434.2 & 392.4 & 473.1 & 946.0 & 678.2 & $1,238.3$ \\
\hline $30-34$ & 305.7 & 281.6 & 329.9 & 278.2 & 351.2 & 212.3 & 496.4 & 416.0 & 584.5 \\
\hline $35-39$ & 161.5 & 164.1 & 158.6 & 157.8 & 233.7 & 89.3 & 273.5 & 247.2 & 301.2 \\
\hline $40-44$ & 85.0 & 95.9 & 73.8 & 102.8 & 163.2 & 49.2 & 151.4 & 149.8 & 152.4 \\
\hline $45-54$ & 39.6 & 56.5 & 22.8 & 62.7 & 120.4 & 11.3 & 74.7 & 86.9 & 61.9 \\
\hline $55-64$ & 13.2 & 21.7 & 5.1 & 21.3 & 39.3 & 5.0 & 24.3 & 30.4 & 18.5 \\
\hline $65+$ & 1.9 & 3.4 & 0.6 & 2.1 & 4.1 & 0.5 & 3.6 & 5.7 & 2.0 \\
\hline \multicolumn{10}{|c|}{ Unknown Age } \\
\hline TOTAL & 212.1 & 140.4 & 281.7 & 184.9 & 131.6 & 236.6 & 392.6 & 246.1 & 541.3 \\
\hline
\end{tabular}

* Per 100,000.

${ }^{\dagger}$ Total includes cases reported with unknown sex.

NOTE: These tables should be used only for race/Hispanic ethnicity comparisons. See Table 10 for age-specific cases and rates and Tables 3-5 for total and sexspecific cases and rates. Cases in the 0-4 age group may include cases due to perinatal transmission. No population data exist for unknown sex, unknown age, or other/unknown race; therefore rates are not calculated. 
Table 12A. Chlamydia - Reported Cases and Rates of Reported Cases Among Females Aged 15-24 Years by Age, United States, 2014-2018

\begin{tabular}{|c|c|c|c|c|c|c|c|c|c|c|}
\hline \multirow[b]{2}{*}{ Ages } & \multicolumn{5}{|c|}{ Cases } & \multicolumn{5}{|c|}{ Rates per 100,000 Females } \\
\hline & 2014 & 2015 & 2016 & 2017 & 2018 & 2014 & 2015 & 2016 & 2017 & 2018 \\
\hline 15 & 20,096 & 19,643 & 19,704 & 20,247 & 19,518 & 987.4 & 945.9 & 958.9 & $1,004.4$ & 968.3 \\
\hline 16 & 38,507 & 37,786 & 39,066 & 40,681 & 40,001 & $1,891.0$ & $1,847.7$ & $1,874.5$ & $1,968.3$ & $1,935.4$ \\
\hline 17 & 58,940 & 60,149 & 61,406 & 65,496 & 66,329 & $2,880.9$ & $2,935.4$ & $2,988.7$ & $3,120.8$ & $3,160.5$ \\
\hline 18 & 87,040 & 89,481 & 93,174 & 98,907 & 101,783 & $4,224.2$ & 4,339.6 & $4,521.3$ & $4,774.1$ & $4,912.9$ \\
\hline 19 & 98,711 & 100,878 & 103,289 & 111,959 & 114,004 & $4,688.0$ & $4,854.1$ & 4,980.6 & $5,387.4$ & $5,485.8$ \\
\hline 20 & 98,480 & 99,861 & 100,524 & 107,397 & 110,939 & $4,581.9$ & $4,703.6$ & $4,812.4$ & $5,140.2$ & $5,309.8$ \\
\hline 21 & 94,204 & 95,927 & 96,723 & 100,160 & 102,127 & $4,323.2$ & $4,427.2$ & $4,532.8$ & $4,759.7$ & $4,853.2$ \\
\hline 22 & 82,581 & 84,740 & 84,813 & 86,678 & 86,274 & $3,679.3$ & $3,855.7$ & $3,892.5$ & $4,030.4$ & $4,011.6$ \\
\hline 23 & 71,535 & 73,686 & 73,054 & 75,046 & 73,729 & $3,112.0$ & $3,254.1$ & $3,304.7$ & $3,416.6$ & $3,356.6$ \\
\hline 24 & 59,076 & 62,558 & 63,274 & 64,769 & 64,663 & $2,567.1$ & $2,697.2$ & $2,777.9$ & $2,906.1$ & $2,901.4$ \\
\hline Total & 709,170 & 724,709 & 735,027 & 771,340 & 779,367 & $3,305.2$ & $3,389.3$ & $3,464.1$ & $3,655.5$ & $3,693.6$ \\
\hline
\end{tabular}

NOTE: Cases reported with unknown sex are not included in this table.

Table 12B. Chlamydia - Reported Cases and Rates of Reported Cases Among Males Aged 15-24 Years by Age, United States, 2014-2018

\begin{tabular}{|c|c|c|c|c|c|c|c|c|c|c|}
\hline \multirow[b]{2}{*}{ Ages } & \multicolumn{5}{|c|}{ Cases } & \multicolumn{5}{|c|}{ Rates per 100,000 Males } \\
\hline & 2014 & 2015 & 2016 & 2017 & 2018 & 2014 & 2015 & 2016 & 2017 & 2018 \\
\hline 15 & 3,921 & 3,773 & 4,074 & 4,144 & 4,088 & 184.2 & 173.6 & 190.2 & 197.4 & 194.8 \\
\hline 16 & 8,813 & 9,286 & 9,997 & 10,782 & 10,573 & 413.6 & 434.1 & 458.3 & 500.1 & 490.4 \\
\hline 17 & 15,276 & 16,244 & 17,690 & 19,665 & 19,972 & 714.2 & 757.2 & 822.6 & 894.7 & 908.7 \\
\hline 18 & 21,737 & 23,232 & 25,214 & 28,502 & 30,184 & $1,004.0$ & $1,077.5$ & $1,168.1$ & $1,313.8$ & $1,391.3$ \\
\hline 19 & 28,161 & 30,240 & 32,924 & 36,771 & 38,765 & $1,268.1$ & $1,384.4$ & $1,517.5$ & $1,688.0$ & $1,779.5$ \\
\hline 20 & 32,581 & 34,896 & 37,391 & 41,038 & 43,337 & $1,435.6$ & $1,557.6$ & $1,701.6$ & 1,876.1 & $1,981.2$ \\
\hline 21 & 34,115 & 36,611 & 38,743 & 42,212 & 43,800 & $1,476.4$ & $1,598.7$ & 1,719.6 & $1,906.6$ & $1,978.3$ \\
\hline 22 & 33,793 & 35,981 & 37,628 & 40,515 & 41,619 & $1,427.2$ & $1,543.1$ & $1,634.5$ & $1,784.7$ & $1,833.3$ \\
\hline 23 & 31,260 & 33,768 & 35,462 & 37,373 & 38,096 & $1,302.6$ & $1,414.2$ & $1,514.0$ & $1,611.7$ & $1,642.9$ \\
\hline 24 & 28,055 & 31,057 & 32,633 & 34,833 & 35,676 & $1,173.2$ & $1,284.4$ & $1,361.8$ & $1,476.7$ & $1,512.4$ \\
\hline Total & 237,712 & 255,088 & 271,756 & 295,835 & 306,110 & $1,055.4$ & $1,135.5$ & $1,219.0$ & $1,335.6$ & $1,382.0$ \\
\hline
\end{tabular}

NOTE: Cases reported with unknown sex are not included in this table. 
Table 13. Gonorrhea - Reported Cases and Rates of Reported Cases by State, Ranked by Rates, United States, 2018

\begin{tabular}{|c|c|c|c|}
\hline Rank* & State & Cases & Rate per 100,000 Population \\
\hline 1 & Mississippi & 9,749 & 326.7 \\
\hline 2 & Alaska & 2,247 & 303.7 \\
\hline 3 & South Carolina & 13,801 & 274.7 \\
\hline 5 & Louisiana & 12,043 & 257.1 \\
\hline 6 & New Mexico & 5,268 & 252.3 \\
\hline 7 & Missouri & 15,090 & 246.8 \\
\hline 9 & North Carolina & 23,725 & 230.9 \\
\hline 10 & Oklahoma & 8,998 & 228.9 \\
\hline 11 & Tennessee & 14,627 & 217.8 \\
\hline 12 & Nevada & 6,475 & 216.0 \\
\hline 13 & Ohio & 25,146 & 215.7 \\
\hline 14 & California & 79,192 & 200.3 \\
\hline 18 & New York & 37,262 & 187.7 \\
\hline 19 & Arizona & 12,870 & 183.4 \\
\hline 20 & Indiana & 12,193 & 182.9 \\
\hline 21 & North Dakota & 1,369 & 181.2 \\
\hline \multirow[t]{2}{*}{22} & Kansas & 5,256 & 180.4 \\
\hline & US TOTAL ${ }^{+}$ & 583,405 & 179.1 \\
\hline 23 & Delaware & 1,691 & 175.8 \\
\hline 24 & Maryland & 10,305 & 170.3 \\
\hline 25 & Kentucky & 7,470 & 167.7 \\
\hline 26 & Michigan & 16,688 & 167.5 \\
\hline 27 & Texas & 47,231 & 166.9 \\
\hline 35 & Connecticut & 4,959 & 138.2 \\
\hline 36 & Wisconsin & 7,882 & 136.0 \\
\hline 37 & Minnesota & 7,542 & 135.2 \\
\hline 38 & Rhode Island & 1,336 & 126.1 \\
\hline 39 & Pennsylvania & 15,887 & 124.1 \\
\hline 40 & Massachusetts & 8,076 & 117.7 \\
\hline 41 & Montana & 1,181 & 112.4 \\
\hline 42 & Hawaii & 1,495 & 104.7 \\
\hline 43 & New Jersey & 9,067 & 100.7 \\
\hline 44 & Utah & 2,895 & 93.3 \\
\hline 45 & Idaho & 1,134 & 66.0 \\
\hline 46 & West Virginia & 1,143 & 62.9 \\
\hline 47 & Wyoming & 311 & 53.7 \\
\hline 48 & Maine & 710 & 53.1 \\
\hline 49 & New Hampshire & 594 & 44.2 \\
\hline 50 & Vermont & 268 & 43.0 \\
\hline
\end{tabular}

* States were ranked by rate, then by case count, then in alphabetical order, with rates shown rounded to the nearest tenth.

${ }^{\dagger}$ Total includes cases reported by the District of Columbia with 4,240 cases and a rate of 611.0 cases per 100,000 population, but excludes territories. 
Table 14. Gonorrhea - Reported Cases and Rates of Reported Cases by State/Territory and Region in Alphabetical Order, United States, 2014-2018

\begin{tabular}{|c|c|c|c|c|c|c|c|c|c|c|}
\hline \multirow[b]{2}{*}{ State/Territory } & \multicolumn{5}{|c|}{ Cases } & \multicolumn{5}{|c|}{ Rates per 100,000 Population } \\
\hline & 2014 & 2015 & 2016 & 2017 & 2018 & 2014 & 2015 & 2016 & 2017 & 2018 \\
\hline Alabama & 7,677 & 7,196 & 8,408 & 11,948 & 12,742 & 158.3 & 148.1 & 172.9 & 245.1 & 261.4 \\
\hline Alaska & 1,341 & 1,113 & 1,454 & 2,189 & 2,247 & 182.0 & 150.7 & 196.0 & 295.9 & 303.7 \\
\hline Arizona & 7,750 & 8,245 & 10,330 & 12,502 & 12,870 & 115.1 & 120.8 & 149.0 & 178.2 & 183.4 \\
\hline Arkansas & 4,539 & 4,780 & 5,732 & 6,710 & 7,300 & 153.0 & 160.5 & 191.8 & 223.3 & 243.0 \\
\hline California & 45,408 & 54,135 & 64,551 & 75,348 & 79,192 & 117.0 & 138.3 & 164.5 & 190.6 & 200.3 \\
\hline Colorado & 3,170 & 4,387 & 5,975 & 8,478 & 8,894 & 59.2 & 80.4 & 107.8 & 151.2 & 158.6 \\
\hline Connecticut & 2,333 & 2,088 & 2,731 & 3,913 & 4,959 & 64.9 & 58.1 & 76.4 & 109.1 & 138.2 \\
\hline Delaware & 1,279 & 1,310 & 1,702 & 1,784 & 1,691 & 136.7 & 138.5 & 178.8 & 185.5 & 175.8 \\
\hline District of Columbia & 1,883 & 2,742 & 3,226 & 4,563 & 4,240 & 285.8 & 407.9 & 473.6 & 657.5 & 611.0 \\
\hline Florida & 20,944 & 24,125 & 28,162 & 31,683 & 32,644 & 105.3 & 119.0 & 136.6 & 151.0 & 155.6 \\
\hline Georgia & 13,770 & 15,982 & 20,553 & 22,667 & 20,867 & 136.4 & 156.5 & 199.3 & 217.3 & 200.1 \\
\hline Hawaii & 1,020 & 1,239 & 1,467 & 1,358 & 1,495 & 71.9 & 86.5 & 102.7 & 95.1 & 104.7 \\
\hline Idaho & 443 & 472 & 635 & 987 & 1,134 & 27.1 & 28.5 & 37.7 & 57.5 & 66.0 \\
\hline Illinois & 15,970 & 17,130 & 21,199 & 23,859 & 25,422 & 124.0 & 133.2 & 165.6 & 186.4 & 198.6 \\
\hline Indiana & 7,289 & 7,843 & 9,451 & 11,835 & 12,193 & 110.5 & 118.5 & 142.5 & 177.5 & 182.9 \\
\hline lowa & 1,641 & 2,247 & 2,600 & 3,758 & 4,839 & 52.8 & 71.9 & 82.9 & 119.5 & 153.8 \\
\hline Kansas & 2,568 & 2,536 & 3,353 & 4,545 & 5,256 & 88.4 & 87.1 & 115.3 & 156.0 & 180.4 \\
\hline Kentucky & 4,353 & 4,678 & 5,812 & 7,417 & 7,470 & 98.6 & 105.7 & 131.0 & 166.5 & 167.7 \\
\hline Louisiana & 9,002 & 10,282 & 10,782 & 12,017 & 12,043 & 193.6 & 220.1 & 230.3 & 256.5 & 257.1 \\
\hline Maine & 237 & 417 & 451 & 620 & 710 & 17.8 & 31.4 & 33.9 & 46.4 & 53.1 \\
\hline Maryland & 6,108 & 6,858 & 9,523 & 10,978 & 10,305 & 102.2 & 114.2 & 158.3 & 181.4 & 170.3 \\
\hline Massachusetts & 3,817 & 3,817 & 4,980 & 7,737 & 8,076 & 56.6 & 56.2 & 73.1 & 112.8 & 117.7 \\
\hline Michigan & 9,688 & 10,330 & 12,450 & 15,742 & 16,688 & 97.8 & 104.1 & 125.4 & 158.0 & 167.5 \\
\hline Minnesota & 4,073 & 4,097 & 5,104 & 6,519 & 7,542 & 74.6 & 74.6 & 92.5 & 116.9 & 135.2 \\
\hline Mississippi & 5,625 & 5,775 & 7,157 & 9,258 & 9,749 & 187.9 & 193.0 & 239.5 & 310.2 & 326.7 \\
\hline Missouri & 7,387 & 8,942 & 11,479 & 13,086 & 15,090 & 121.8 & 147.0 & 188.4 & 214.0 & 246.8 \\
\hline Montana & 434 & 844 & 867 & 782 & 1,181 & 42.4 & 81.7 & 83.2 & 74.4 & 112.4 \\
\hline Nebraska & 1,459 & 1,703 & 2,156 & 2,653 & 2,696 & 77.5 & 89.8 & 113.1 & 138.2 & 140.4 \\
\hline Nevada & 3,188 & 3,630 & 4,380 & 5,520 & 6,475 & 112.3 & 125.6 & 149.0 & 184.1 & 216.0 \\
\hline New Hampshire & 226 & 245 & 456 & 513 & 594 & 17.0 & 18.4 & 34.2 & 38.2 & 44.2 \\
\hline New Jersey & 6,636 & 7,228 & 8,162 & 9,439 & 9,067 & 74.2 & 80.7 & 91.3 & 104.8 & 100.7 \\
\hline New Mexico & 2,246 & 2,489 & 3,516 & 4,489 & 5,268 & 107.7 & 119.4 & 169.0 & 215.0 & 252.3 \\
\hline New York & 20,758 & 25,561 & 29,000 & 34,099 & 37,262 & 105.1 & 129.1 & 146.9 & 171.8 & 187.7 \\
\hline North Carolina & 14,415 & 19,809 & 19,687 & 22,871 & 23,725 & 145.0 & 197.2 & 194.0 & 222.6 & 230.9 \\
\hline North Dakota & 694 & 684 & 1,000 & 966 & 1,369 & 93.8 & 90.4 & 131.9 & 127.9 & 181.2 \\
\hline Ohio & 16,237 & 16,564 & 20,487 & 23,967 & 25,146 & 140.0 & 142.6 & 176.4 & 205.6 & 215.7 \\
\hline Oklahoma & 6,137 & 6,542 & 7,574 & 9,081 & 8,998 & 158.2 & 167.3 & 193.0 & 231.0 & 228.9 \\
\hline Oregon & 2,320 & 3,232 & 4,353 & 5,022 & 5,913 & 58.4 & 80.2 & 106.3 & 121.2 & 142.7 \\
\hline Pennsylvania & 12,710 & 12,791 & 14,603 & 15,244 & 15,887 & 99.4 & 99.9 & 114.2 & 119.0 & 124.1 \\
\hline Rhode Island & 590 & 580 & 716 & 1,087 & 1,336 & 55.9 & 54.9 & 67.8 & 102.6 & 126.1 \\
\hline South Carolina & 8,253 & 8,206 & 9,194 & 12,623 & 13,801 & 170.8 & 167.6 & 185.3 & 251.2 & 274.7 \\
\hline South Dakota & 892 & 1,048 & 1,269 & 1,290 & 1,689 & 104.6 & 122.1 & 146.6 & 148.3 & 194.2 \\
\hline Tennessee & 7,199 & 8,386 & 10,179 & 12,426 & 14,627 & 109.9 & 127.1 & 153.0 & 185.0 & 217.8 \\
\hline Texas & 35,322 & 39,717 & 42,472 & 47,409 & 47,231 & 131.0 & 144.6 & 152.4 & 167.5 & 166.9 \\
\hline Utah & 1,441 & 1,562 & 2,100 & 2,543 & 2,895 & 49.0 & 52.1 & 68.8 & 82.0 & 93.3 \\
\hline Vermont & 84 & 155 & 126 & 203 & 268 & 13.4 & 24.8 & 20.2 & 32.5 & 43.0 \\
\hline Virginia & 8,250 & 8,099 & 11,084 & 12,596 & 11,776 & 99.1 & 96.6 & 131.8 & 148.7 & 139.0 \\
\hline Washington & 6,221 & 7,171 & 8,174 & 9,915 & 11,207 & 88.1 & 100.0 & 112.2 & 133.9 & 151.3 \\
\hline West Virginia & 841 & 769 & 919 & 1,296 & 1,143 & 45.5 & 41.7 & 50.2 & 71.4 & 62.9 \\
\hline Wisconsin & 4,078 & 5,260 & 6,498 & 7,661 & 7,882 & 70.8 & 91.1 & 112.4 & 132.2 & 136.0 \\
\hline Wyoming & 116 & 175 & 275 & 412 & 311 & 19.9 & 29.9 & 47.0 & 71.1 & 53.7 \\
\hline US TOTAL & 350,062 & 395,216 & 468,514 & 555,608 & 583,405 & 109.8 & 123.0 & 145.0 & 170.6 & 179.1 \\
\hline Northeast & 47,391 & 52,882 & 61,225 & 72,855 & 78,159 & 84.4 & 94.0 & 108.9 & 129.0 & 138.4 \\
\hline Midwest & 71,976 & 78,384 & 97,046 & 115,881 & 125,812 & 106.2 & 115.4 & 142.8 & 170.0 & 184.5 \\
\hline South & 155,597 & 175,256 & 202,166 & 237,327 & 240,352 & 129.9 & 144.6 & 165.3 & 191.9 & 194.4 \\
\hline West & 75,098 & 88,694 & 108,077 & 129,545 & 139,082 & 99.9 & 116.6 & 141.0 & 167.3 & 179.7 \\
\hline American Samoa & NR & NR & NR & NR & 13 & - & - & - & - & 25.2 \\
\hline Guam & 99 & 147 & 133 & 202 & 261 & 61.5 & 90.9 & 79.7 & 120.7 & 156.0 \\
\hline Northern Mariana Islands & NR & NR & NR & NR & 34 & - & - & - & - & 65.1 \\
\hline Puerto Rico & 454 & 620 & 744 & 588 & 557 & 12.8 & 17.8 & 21.8 & 17.5 & 16.6 \\
\hline Virgin Islands & 84 & 52 & 35 & 15 & NR & 80.6 & 50.5 & 32.5 & 14.0 & - \\
\hline TERRITORIES & 637 & 819 & 912 & 805 & 865 & 16.7 & 21.9 & 24.7 & 22.2 & 23.9 \\
\hline TOTAL & 350,699 & 396,035 & 469,426 & 556,413 & 584,270 & 108.7 & 121.8 & 143.6 & 168.9 & 177.4 \\
\hline
\end{tabular}

$\mathrm{NR}=$ No report.

NOTE: See Section A1.11 in the Appendix for more information on interpreting case counts and rates in US territories. 
Table 15. Gonorrhea Among Females - Reported Cases and Rates of Reported Cases by State/Territory and Region in Alphabetical Order, United States, 2014-2018

\begin{tabular}{|c|c|c|c|c|c|c|c|c|c|c|}
\hline \multirow[b]{2}{*}{ State/Territory } & \multicolumn{5}{|c|}{ Cases } & \multicolumn{5}{|c|}{ Rates per 100,000 Population } \\
\hline & 2014 & 2015 & 2016 & 2017 & 2018 & 2014 & 2015 & 2016 & 2017 & 2018 \\
\hline Alabama & 4,090 & 3,629 & 4,088 & 5,693 & 6,134 & 163.6 & 144.8 & 163.0 & 226.4 & 243.9 \\
\hline Alaska & 665 & 567 & 738 & 1,090 & 1,057 & 190.3 & 162.3 & 208.6 & 308.8 & 299.4 \\
\hline Arizona & 3,564 & 3,505 & 4,315 & 5,219 & 5,332 & 105.2 & 102.0 & 123.7 & 147.9 & 151.1 \\
\hline Arkansas & 2,527 & 2,510 & 2,964 & 3,525 & 3,788 & 167.5 & 165.6 & 195.0 & 230.7 & 247.9 \\
\hline California & 16,009 & 18,404 & 20,914 & 24,599 & 26,425 & 82.0 & 93.4 & 105.9 & 123.7 & 132.9 \\
\hline Colorado & 1,318 & 1,832 & 2,323 & 3,465 & 3,589 & 49.5 & 67.5 & 84.3 & 124.4 & 128.9 \\
\hline Connecticut & 1,108 & 851 & 1,165 & 1,660 & 1,938 & 60.1 & 46.3 & 63.6 & 90.4 & 105.5 \\
\hline Delaware & 693 & 641 & 799 & 757 & 728 & 143.5 & 131.3 & 162.6 & 152.5 & 146.6 \\
\hline District of Columbia & 858 & 874 & 857 & 1,287 & 1,211 & 247.8 & 247.2 & 239.4 & 352.8 & 332.0 \\
\hline Florida & 9,228 & 10,078 & 11,488 & 12,769 & 12,936 & 90.7 & 97.2 & 109.0 & 119.0 & 120.6 \\
\hline Georgia & 6,552 & 7,322 & 9,156 & 9,873 & 8,718 & 126.7 & 139.9 & 173.1 & 184.4 & 162.8 \\
\hline Hawaii & 350 & 446 & 551 & 476 & 531 & 49.9 & 63.2 & 77.5 & 66.9 & 74.6 \\
\hline Idaho & 196 & 197 & 237 & 357 & 509 & 24.0 & 23.8 & 28.2 & 41.7 & 59.4 \\
\hline Illinois & 7,559 & 7,698 & 8,920 & 9,750 & 10,064 & 115.2 & 117.6 & 137.0 & 149.8 & 154.6 \\
\hline Indiana & 3,819 & 3,984 & 4,811 & 5,931 & 6,083 & 114.1 & 118.6 & 143.0 & 175.5 & 180.0 \\
\hline lowa & 862 & 1,122 & 1,267 & 1,887 & 2,476 & 55.1 & 71.4 & 80.4 & 119.4 & 156.6 \\
\hline Kansas & 1,464 & 1,262 & 1,695 & 2,269 & 2,615 & 100.5 & 86.6 & 116.1 & 155.3 & 179.0 \\
\hline Kentucky & 2,270 & 2,242 & 2,716 & 3,478 & 3,643 & 101.3 & 99.8 & 120.7 & 153.9 & 161.2 \\
\hline Louisiana & 5,049 & 5,535 & 5,493 & 5,978 & 5,904 & 212.5 & 231.9 & 229.6 & 249.6 & 246.5 \\
\hline Maine & 98 & 143 & 134 & 225 & 250 & 14.4 & 21.1 & 19.7 & 33.0 & 36.7 \\
\hline Maryland & 2,793 & 3,090 & 3,944 & 4,629 & 4,227 & 90.7 & 99.8 & 127.1 & 148.5 & 135.6 \\
\hline Massachusetts & 1,215 & 1,027 & 1,390 & 2,495 & 2,431 & 35.0 & 29.4 & 39.6 & 70.7 & 68.9 \\
\hline Michigan & 5,129 & 5,191 & 6,201 & 7,604 & 7,981 & 101.7 & 102.9 & 122.9 & 150.3 & 157.8 \\
\hline Minnesota & 1,802 & 1,675 & 2,214 & 2,939 & 3,345 & 65.6 & 60.7 & 79.9 & 105.0 & 119.5 \\
\hline Mississippi & 2,987 & 3,131 & 3,665 & 4,563 & 4,888 & 194.0 & 203.2 & 238.0 & 296.6 & 317.8 \\
\hline Missouri & 3,620 & 4,187 & 5,228 & 5,997 & 6,803 & 117.2 & 135.1 & 168.6 & 192.7 & 218.7 \\
\hline Montana & 221 & 462 & 493 & 427 & 638 & 43.4 & 89.9 & 95.2 & 81.9 & 122.3 \\
\hline Nebraska & 770 & 870 & 1,055 & 1,285 & 1,302 & 81.5 & 91.5 & 110.3 & 133.6 & 135.4 \\
\hline Nevada & 1,294 & 1,402 & 1,611 & 1,972 & 2,477 & 91.6 & 97.3 & 109.9 & 132.0 & 165.8 \\
\hline New Hampshire & 91 & 65 & 132 & 179 & 184 & 13.6 & 9.7 & 19.6 & 26.4 & 27.1 \\
\hline New Jersey & 3,082 & 3,110 & 3,338 & 3,731 & 3,446 & 67.3 & 67.8 & 72.9 & 80.9 & 74.8 \\
\hline New Mexico & 961 & 1,087 & 1,542 & 2,073 & 2,343 & 91.3 & 103.4 & 146.8 & 196.7 & 222.3 \\
\hline New York & 7,077 & 8,593 & 8,709 & 9,649 & 10,202 & 69.6 & 84.4 & 85.7 & 94.5 & 99.9 \\
\hline North Carolina & 7,759 & 10,064 & 9,527 & 11,013 & 11,480 & 152.2 & 195.4 & 182.7 & 208.9 & 217.8 \\
\hline North Dakota & 385 & 375 & 516 & 528 & 708 & 106.8 & 101.9 & 139.8 & 143.4 & 192.3 \\
\hline Ohio & 8,735 & 8,466 & 10,130 & 11,736 & 12,146 & 147.6 & 142.8 & 171.0 & 197.4 & 204.3 \\
\hline Oklahoma & 3,451 & 3,580 & 4,052 & 4,721 & 4,556 & 176.3 & 181.3 & 204.7 & 238.0 & 229.7 \\
\hline Oregon & 786 & 1,158 & 1,519 & 1,920 & 2,244 & 39.2 & 56.9 & 73.5 & 91.9 & 107.4 \\
\hline Pennsylvania & 6,164 & 5,889 & 6,135 & 5,947 & 6,050 & 94.3 & 90.1 & 94.1 & 91.0 & 92.6 \\
\hline Rhode Island & 218 & 172 & 221 & 357 & 457 & 40.1 & 31.6 & 40.7 & 65.5 & 83.9 \\
\hline South Carolina & 4,527 & 4,401 & 4,709 & 6,470 & 7,016 & 182.3 & 174.8 & 184.4 & 250.1 & 271.2 \\
\hline South Dakota & 557 & 621 & 757 & 722 & 974 & 131.4 & 145.6 & 176.4 & 167.6 & 226.1 \\
\hline Tennessee & 3,419 & 3,809 & 4,681 & 5,667 & 6,681 & 101.8 & 112.6 & 137.3 & 164.7 & 194.2 \\
\hline Texas & 17,253 & 17,843 & 18,620 & 20,963 & 19,982 & 127.1 & 129.0 & 132.7 & 147.2 & 140.3 \\
\hline Utah & 565 & 507 & 717 & 865 & 942 & 38.6 & 34.1 & 47.3 & 56.2 & 61.2 \\
\hline Vermont & 35 & 85 & 32 & 87 & 140 & 11.0 & 26.8 & 10.1 & 27.6 & 44.4 \\
\hline Virginia & 4,361 & 4,007 & 5,056 & 5,734 & 5,208 & 103.1 & 94.1 & 118.3 & 133.2 & 121.0 \\
\hline Washington & 2,504 & 2,797 & 2,943 & 3,488 & 3,922 & 70.9 & 78.0 & 80.7 & 94.2 & 105.9 \\
\hline West Virginia & 461 & 365 & 422 & 618 & 542 & 49.2 & 39.1 & 45.6 & 67.4 & 59.1 \\
\hline Wisconsin & 2,046 & 2,557 & 3,189 & 3,696 & 3,670 & 70.6 & 88.1 & 109.8 & 126.9 & 126.0 \\
\hline Wyoming & 61 & 86 & 120 & 224 & 158 & 21.3 & 29.9 & 41.9 & 78.9 & 55.7 \\
\hline US TOTAL & 162,608 & 173,514 & 197,499 & 232,587 & 241,074 & 100.4 & 106.3 & 120.4 & 140.7 & 145.8 \\
\hline Northeast & 19,088 & 19,935 & 21,256 & 24,330 & 25,098 & 66.3 & 69.1 & 73.8 & 84.1 & 86.7 \\
\hline Midwest & 36,748 & 38,008 & 45,983 & 54,344 & 58,167 & 107.0 & 110.4 & 133.6 & 157.4 & 168.5 \\
\hline South & 78,278 & 83,121 & 92,237 & 107,738 & 107,642 & 128.2 & 134.5 & 147.9 & 170.9 & 170.7 \\
\hline West & 28,494 & 32,450 & 38,023 & 46,175 & 50,167 & 75.6 & 85.1 & 98.9 & 119.0 & 129.3 \\
\hline American Samoa & NR & NR & NR & NR & 4 & - & - & - & - & 15.5 \\
\hline Guam & 47 & 67 & 59 & 97 & 143 & 59.2 & 84.0 & 72.8 & 119.4 & 176.0 \\
\hline Northern Mariana Islands & NR & NR & NR & NR & 16 & - & - & - & - & 65.4 \\
\hline Puerto Rico & 161 & 259 & 313 & 230 & 238 & 8.7 & 14.3 & 17.5 & 13.1 & 13.5 \\
\hline Virgin Islands & 54 & 28 & 14 & 5 & NR & 97.1 & 50.8 & 24.9 & 8.9 & - \\
\hline TERRITORIES & 262 & 354 & 386 & 332 & 401 & 13.2 & 18.1 & 20.1 & 17.5 & 21.2 \\
\hline TOTAL & 162,870 & 173,868 & 197,885 & 232,919 & 241,475 & 99.4 & 105.3 & 119.2 & 139.3 & 144.4 \\
\hline
\end{tabular}

NR $=$ No report.

NOTE: Cases reported with unknown sex are not included in this table. See Section A1.11 in the Appendix for more information on interpreting case counts and rates in US territories. 
Table 16. Gonorrhea Among Males - Reported Cases and Rates of Reported Cases by State/Territory and Region in Alphabetical Order, United States, 2014-2018

\begin{tabular}{|c|c|c|c|c|c|c|c|c|c|c|}
\hline \multirow[b]{2}{*}{ State/Territory } & \multicolumn{5}{|c|}{ Cases } & \multicolumn{5}{|c|}{ Rates per 100,000 Population } \\
\hline & 2014 & 2015 & 2016 & 2017 & 2018 & 2014 & 2015 & 2016 & 2017 & 2018 \\
\hline Alabama & 3,563 & 3,519 & 4,265 & 6,214 & 6,560 & 151.6 & 149.5 & 181.1 & 263.3 & 278.0 \\
\hline Alaska & 676 & 546 & 716 & 1,099 & 1,189 & 174.5 & 140.4 & 184.5 & 284.1 & 307.4 \\
\hline Arizona & 4,186 & 4,724 & 6,011 & 7,256 & 7,509 & 125.2 & 139.3 & 174.6 & 208.0 & 215.3 \\
\hline Arkansas & 2,007 & 2,270 & 2,768 & 3,184 & 3,511 & 137.7 & 155.2 & 188.6 & 215.7 & 237.9 \\
\hline California & 29,310 & 35,644 & 43,259 & 50,577 & 52,553 & 152.1 & 183.3 & 221.9 & 257.4 & 267.5 \\
\hline Colorado & 1,852 & 2,555 & 3,652 & 5,013 & 5,305 & 68.8 & 93.1 & 131.1 & 177.6 & 188.0 \\
\hline Connecticut & 1,219 & 1,237 & 1,564 & 2,246 & 3,003 & 69.5 & 70.6 & 89.6 & 128.2 & 171.4 \\
\hline Delaware & 586 & 669 & 903 & 1,027 & 958 & 129.4 & 146.1 & 196.0 & 220.6 & 205.8 \\
\hline District of Columbia & 1,011 & 1,817 & 2,298 & 3,254 & 2,989 & 323.4 & 570.3 & 710.9 & 988.5 & 908.0 \\
\hline Florida & 11,686 & 14,039 & 16,661 & 18,904 & 19,704 & 120.2 & 141.8 & 165.4 & 184.3 & 192.1 \\
\hline Georgia & 7,137 & 8,631 & 11,378 & 12,741 & 12,075 & 144.9 & 173.3 & 226.6 & 251.0 & 237.9 \\
\hline Hawaii & 669 & 793 & 914 & 882 & 959 & 93.2 & 109.3 & 127.4 & 123.2 & 133.9 \\
\hline Idaho & 247 & 275 & 396 & 629 & 622 & 30.2 & 33.2 & 46.9 & 73.1 & 72.3 \\
\hline Illinois & 8,386 & 9,335 & 12,255 & 14,057 & 15,351 & 132.7 & 147.8 & 194.8 & 223.4 & 244.0 \\
\hline Indiana & 3,465 & 3,854 & 4,636 & 5,899 & 6,101 & 106.6 & 118.2 & 141.8 & 179.5 & 185.6 \\
\hline lowa & 779 & 1,122 & 1,332 & 1,870 & 2,363 & 50.5 & 72.3 & 85.4 & 119.5 & 151.0 \\
\hline Kansas & 1,104 & 1,274 & 1,658 & 2,276 & 2,641 & 76.3 & 87.6 & 114.5 & 156.8 & 181.9 \\
\hline Kentucky & 2,068 & 2,430 & 3,006 & 3,906 & 3,762 & 95.2 & 111.5 & 137.5 & 178.0 & 171.4 \\
\hline Louisiana & 3,953 & 4,747 & 5,289 & 6,039 & 6,139 & 173.8 & 207.9 & 231.0 & 263.8 & 268.1 \\
\hline Maine & 137 & 274 & 316 & 392 & 459 & 21.0 & 42.1 & 48.4 & 59.9 & 70.1 \\
\hline Maryland & 3,304 & 3,755 & 5,573 & 6,346 & 6,074 & 114.1 & 129.0 & 191.2 & 216.3 & 207.0 \\
\hline Massachusetts & 2,590 & 2,768 & 3,575 & 5,206 & 5,578 & 79.2 & 84.0 & 108.1 & 156.3 & 167.5 \\
\hline Michigan & 4,551 & 5,129 & 6,245 & 8,121 & 8,700 & 93.5 & 105.1 & 127.8 & 165.6 & 177.4 \\
\hline Minnesota & 2,260 & 2,420 & 2,881 & 3,568 & 4,187 & 83.3 & 88.6 & 104.9 & 128.5 & 150.8 \\
\hline Mississippi & 2,637 & 2,638 & 3,486 & 4,681 & 4,846 & 181.3 & 181.7 & 240.6 & 323.7 & 335.2 \\
\hline Missouri & 3,767 & 4,755 & 6,251 & 7,088 & 8,287 & 126.7 & 159.3 & 208.9 & 236.1 & 276.0 \\
\hline Montana & 213 & 381 & 374 & 355 & 543 & 41.4 & 73.4 & 71.3 & 67.1 & 102.7 \\
\hline Nebraska & 686 & 833 & 1,097 & 1,366 & 1,393 & 73.2 & 88.1 & 115.4 & 142.6 & 145.4 \\
\hline Nevada & 1,892 & 2,218 & 2,763 & 3,539 & 3,985 & 132.6 & 152.9 & 187.4 & 235.3 & 265.0 \\
\hline New Hampshire & 135 & 180 & 324 & 334 & 410 & 20.6 & 27.4 & 49.0 & 50.2 & 61.7 \\
\hline New Jersey & 3,544 & 4,108 & 4,810 & 5,699 & 5,617 & 81.2 & 94.0 & 110.1 & 129.6 & 127.8 \\
\hline New Mexico & 1,284 & 1,401 & 1,971 & 2,412 & 2,925 & 124.3 & 135.6 & 191.2 & 233.2 & 282.8 \\
\hline New York & 13,624 & 16,893 & 20,224 & 24,383 & 27,025 & 142.2 & 175.8 & 210.9 & 253.0 & 280.4 \\
\hline North Carolina & 6,652 & 9,744 & 10,160 & 11,857 & 12,245 & 137.3 & 199.2 & 206.0 & 237.1 & 244.8 \\
\hline North Dakota & 309 & 309 & 484 & 438 & 661 & 81.5 & 79.5 & 124.4 & 113.1 & 170.7 \\
\hline Ohio & 7,502 & 8,098 & 10,357 & 12,231 & 13,000 & 132.2 & 142.4 & 182.0 & 214.1 & 227.5 \\
\hline Oklahoma & 2,685 & 2,962 & 3,521 & 4,360 & 4,442 & 139.8 & 152.9 & 181.1 & 223.9 & 228.1 \\
\hline Oregon & 1,532 & 2,073 & 2,834 & 3,095 & 3,663 & 78.0 & 104.0 & 139.8 & 150.8 & 178.4 \\
\hline Pennsylvania & 6,543 & 6,892 & 8,449 & 9,285 & 9,821 & 104.6 & 110.0 & 134.9 & 148.1 & 156.6 \\
\hline Rhode Island & 372 & 408 & 495 & 729 & 879 & 72.7 & 79.7 & 96.5 & 141.6 & 170.7 \\
\hline South Carolina & 3,689 & 3,781 & 4,436 & 6,122 & 6,744 & 157.0 & 158.9 & 184.2 & 251.1 & 276.7 \\
\hline South Dakota & 335 & 427 & 512 & 568 & 715 & 78.0 & 98.8 & 117.4 & 129.4 & 162.9 \\
\hline Tennessee & 3,778 & 4,577 & 5,497 & 6,758 & 7,946 & 118.4 & 142.3 & 169.5 & 206.3 & 242.6 \\
\hline Texas & 18,035 & 21,792 & 23,779 & 26,344 & 27,117 & 134.8 & 159.8 & 171.9 & 187.3 & 192.8 \\
\hline Utah & 876 & 1,055 & 1,383 & 1,672 & 1,953 & 59.2 & 70.0 & 90.0 & 107.1 & 125.1 \\
\hline Vermont & 49 & 70 & 94 & 116 & 126 & 15.9 & 22.7 & 30.5 & 37.6 & 40.9 \\
\hline Virginia & 3,879 & 4,085 & 5,996 & 6,779 & 6,525 & 94.7 & 99.0 & 144.9 & 162.7 & 156.6 \\
\hline Washington & 3,717 & 4,374 & 5,231 & 6,425 & 7,279 & 105.3 & 122.0 & 143.7 & 173.5 & 196.5 \\
\hline West Virginia & 380 & 404 & 497 & 678 & 601 & 41.6 & 44.3 & 54.9 & 75.4 & 66.9 \\
\hline Wisconsin & 2,027 & 2,697 & 3,302 & 3,961 & 4,208 & 70.9 & 94.1 & 114.9 & 137.4 & 146.0 \\
\hline Wyoming & 55 & 88 & 155 & 188 & 153 & 18.5 & 29.4 & 51.8 & 63.6 & 51.8 \\
\hline US TOTAL & 186,943 & 221,070 & 270,033 & 322,169 & 341,401 & 119.1 & 139.7 & 169.7 & 200.8 & 212.8 \\
\hline Northeast & 28,213 & 32,830 & 39,851 & 48,390 & 52,918 & 103.2 & 119.7 & 145.4 & 175.8 & 192.2 \\
\hline Midwest & 35,171 & 40,253 & 51,010 & 61,443 & 67,607 & 105.3 & 120.2 & 152.1 & 182.5 & 200.9 \\
\hline South & 77,050 & 91,860 & 109,513 & 129,194 & 132,238 & 131.2 & 154.6 & 182.7 & 213.1 & 218.2 \\
\hline West & 46,509 & 56,127 & 69,659 & 83,142 & 88,638 & 124.1 & 148.1 & 182.3 & 215.4 & 229.6 \\
\hline American Samoa & NR & NR & $N R$ & NR & 9 & - & - & - & - & 34.9 \\
\hline Guam & 52 & 80 & 74 & 105 & 118 & 63.7 & 97.6 & 86.2 & 122.0 & 137.1 \\
\hline Northern Mariana Islands & NR & NR & NR & NR & 18 & - & - & - & - & 64.7 \\
\hline Puerto Rico & 293 & 359 & 431 & 356 & 318 & 17.2 & 21.7 & 26.5 & 22.4 & 20.0 \\
\hline Virgin Islands & 30 & 24 & 21 & 10 & NR & 61.8 & 50.2 & 40.8 & 19.5 & - \\
\hline TERRITORIES & 375 & 463 & 526 & 471 & 463 & 20.4 & 25.9 & 29.9 & 27.2 & 26.7 \\
\hline TOTAL & 187,318 & 221,533 & 270,559 & 322,640 & 341,864 & 118.0 & 138.4 & 168.2 & 199.0 & 210.8 \\
\hline
\end{tabular}

$\mathrm{NR}=$ No report.

NOTE: Cases reported with unknown sex are not included in this table. See Section A1.11 in the Appendix for more information on interpreting case counts and rates in US territories. 
Table 17. Gonorrhea - Reported Cases and Rates of Reported Cases in Selected Metropolitan Statistical Areas (MSAs)* in Alphabetical Order, United States, 2014-2018

\begin{tabular}{|c|c|c|c|c|c|c|c|c|c|c|}
\hline \multirow[b]{2}{*}{ MSAs } & \multicolumn{5}{|c|}{ Cases } & \multicolumn{5}{|c|}{ Rates per 100,000 Population } \\
\hline & 2014 & 2015 & 2016 & 2017 & 2018 & 2014 & 2015 & 2016 & 2017 & 2018 \\
\hline Atlanta-Sandy Springs-Roswell, GA & 7,256 & $6,471^{+}$ & 11,670 & 12,373 & $10,383^{\dagger}$ & 129.2 & $113.3^{\dagger}$ & 201.6 & 210.3 & $176.4^{\dagger}$ \\
\hline Austin-Round Rock, TX & 2,860 & 3,199 & 3,670 & 4,414 & 4,336 & 147.2 & 159.9 & 178.5 & 208.6 & 204.9 \\
\hline Baltimore-Columbia-Towson, MD & 3,459 & 4,179 & 5,854 & 6,892 & 5,998 & 124.2 & 149.4 & 209.2 & 245.4 & 213.6 \\
\hline Birmingham-Hoover, AL & 1,957 & 2,088 & $2,025^{\ddagger}$ & 3,486 & 3,360 & 171.1 & 182.3 & $176.5^{\ddagger}$ & 303.2 & 292.2 \\
\hline Boston-Cambridge-Newton, MA-NH & $2,716^{+}$ & $2,487^{\dagger}$ & $3,002^{+}$ & $4,385^{+}$ & 5,362 & $57.4^{+}$ & $52.1^{\dagger}$ & $62.6^{+}$ & $90.7^{\dagger}$ & 110.9 \\
\hline Buffalo-Cheektowaga-Niagara Falls, NY & 1,342 & 1,982 & 2,180 & 2,448 & 2,553 & 118.1 & 174.6 & 192.4 & 215.3 & 224.6 \\
\hline Charlotte-Concord-Gastonia, NC-SC & 3,645 & 4,673 & 4,749 & 5,411 & 5,762 & 153.1 & 192.6 & 191.9 & 214.3 & 228.2 \\
\hline Chicago-Naperville-Elgin, IL-IN-WI & 12,630 & 13,529 & 16,634 & 18,558 & 19,578 & 132.2 & 141.6 & 174.9 & 194.7 & 205.4 \\
\hline Cincinnati, $\mathrm{OH}-\mathrm{KY}-\mathrm{IN}$ & 3,346 & 3,713 & 4,096 & 4,716 & $4,985^{+}$ & 155.7 & 172.1 & 189.2 & 216.4 & $228.8^{\dagger}$ \\
\hline Cleveland-Elyria, $\mathrm{OH}$ & 3,802 & 3,428 & 4,205 & 5,843 & 5,400 & 184.2 & 166.3 & 204.6 & 283.8 & 262.3 \\
\hline Columbus, $\mathrm{OH}$ & 3,260 & 3,676 & 4,821 & 5,197 & 5,657 & 163.4 & 181.8 & 236.1 & 250.0 & 272.1 \\
\hline Dallas-Fort Worth-Arlington, TX & 9,195 & 11,334 & 11,092 & 12,846 & 13,245 & 132.2 & 159.6 & 153.3 & 173.6 & 179.0 \\
\hline Denver-Aurora-Lakewood, CO & 2,016 & 2,838 & 3,848 & 5,408 & 5,270 & 73.2 & 100.8 & 134.9 & 187.2 & 182.5 \\
\hline Detroit-Warren-Dearborn, MI & 5,311 & 5,494 & 6,816 & 8,668 & 8,817 & 123.6 & 127.7 & 158.6 & 201.0 & 204.4 \\
\hline Hartford-West Hartford-East Hartford, CT & 894 & $726^{+}$ & $963^{+}$ & 1,831 & $1,983^{+}$ & 73.6 & $59.9^{\dagger}$ & $79.8^{+}$ & 151.3 & $163.8^{\dagger}$ \\
\hline Houston-The Woodlands-Sugar Land, TX & 8,299 & 9,290 & 10,378 & 10,789 & 10,831 & 127.9 & 139.6 & 153.2 & 156.5 & 157.1 \\
\hline Indianapolis-Carmel-Anderson, IN & 3,759 & 3,716 & 4,808 & 5,430 & 4,902 & 190.7 & 186.8 & 239.9 & 267.7 & 241.6 \\
\hline Jacksonville, FL & 2,608 & 2,740 & 3,168 & 3,721 & 4,054 & 183.8 & 189.0 & 214.3 & 247.2 & 269.4 \\
\hline Kansas City, MO-KS & 2,642 & 2,943 & 4,009 & 5,274 & 5,657 & 127.6 & 141.0 & 190.5 & 247.7 & 265.7 \\
\hline Las Vegas-Henderson-Paradise, NV & 2,653 & 2,975 & 3,653 & 4,430 & 5,294 & 128.2 & 140.7 & 169.5 & 201.0 & 240.2 \\
\hline Los Angeles-Long Beach-Anaheim, CA & 17,130 & 19,867 & 25,438 & 29,669 & 31,206 & 129.2 & 148.9 & 191.1 & 222.2 & 233.7 \\
\hline Louisville-Jefferson County, KY-IN & 1,962 & 2,187 & 2,957 & 3,413 & $2,663^{+}$ & 154.5 & 171.1 & 230.4 & 263.8 & $205.8^{\dagger}$ \\
\hline Memphis, TN-MS-AR & 2,625 & 3,143 & 3,746 & 4,653 & 5,458 & 195.4 & 233.8 & 279.0 & 345.1 & 404.8 \\
\hline Miami-Fort Lauderdale-West Palm Bea & 6,128 & 6,905 & 7,984 & 8,848 & 9,618 & 103.3 & 114.8 & 131.6 & 143.7 & 156.2 \\
\hline Milwaukee-Waukesha-West Allis, WI & 2,584 & 3,719 & 4,454 & 4,910 & 4,748 & 164.4 & 236.0 & 283.2 & 311.5 & 301.2 \\
\hline Minneapolis-St. Paul-Bloomington, MN-WI & 3,341 & 3,289 & 4,123 & 5,260 & 5,834 & 95.6 & 93.3 & 116.1 & 146.1 & 162.0 \\
\hline Nashville-Davidson-Murfreesboro-Franklin, TN & 1,922 & 2,200 & 2,695 & 2,706 & 3,102 & 107.2 & 120.2 & 144.5 & 142.2 & 163.0 \\
\hline New Orleans-Metairie, LA & 2,667 & 2,929 & 3,414 & 3,638 & 3,584 & 213.0 & 231.9 & 269.1 & 285.2 & 280.9 \\
\hline New York-Newark-Jersey City, NY-NJ-PA & 20,054 & 23,721 & 26,186 & 32,018 & 34,772 & 99.8 & 117.5 & 129.9 & 157.6 & 171.1 \\
\hline Oklahoma City, OK & 2,366 & 2,403 & 2,953 & 3,418 & 3,596 & 177.0 & 176.9 & 215.0 & 247.0 & 259.9 \\
\hline Orlando-Kissimmee-Sanford, FL & 2,571 & 3,073 & 3,393 & 4,290 & 4,307 & 110.8 & 128.7 & 139.0 & 170.9 & 171.6 \\
\hline Philadelphia-Camden-Wilmington, PA-NJ-DE-MD & 9,618 & 9,724 & 11,394 & 12,096 & 12,123 & 158.9 & 160.2 & 187.7 & 198.4 & 198.9 \\
\hline Phoenix-Mesa-Scottsdale, AZ & 5,944 & 6,495 & 8,086 & 9,433 & 9,448 & 132.4 & 142.0 & 173.5 & 199.1 & 199.4 \\
\hline Pittsburgh, PA & 2,602 & 2,422 & 2,601 & 2,370 & 2,629 & 110.4 & 102.9 & 111.0 & 101.6 & 112.7 \\
\hline Portland-Vancouver-Hillsborc & 1,499 & 2,386 & 3,177 & 3,444 & 4,238 & 63.8 & 99.9 & 131.0 & 140.4 & 172.8 \\
\hline Providence-Warwick, RI-MA & $913^{+}$ & $834^{+}$ & $1,108^{\dagger}$ & $1,561^{\dagger}$ & 1,956 & $56.7^{\dagger}$ & $51.7^{+}$ & $68.6^{+}$ & $96.3^{+}$ & 120.7 \\
\hline Raleigh, NC & 1,408 & 2,018 & 1,915 & 2,491 & 2,554 & 113.3 & 158.5 & 147.0 & 186.6 & 191.3 \\
\hline Richmond, VA & 2,173 & 2,200 & 3,198 & 3,200 & 2,977 & 172.5 & 173.0 & 249.5 & 247.3 & 230.0 \\
\hline Riverside-San Bernardino-Ontario, CA & 4,292 & 4,904 & 5,958 & 7,329 & 7,977 & 96.6 & 109.2 & 131.6 & 160.0 & 174.1 \\
\hline Sacramento-Roseville-Ard & 2,616 & 3,317 & 3,402 & 4,057 & 4,402 & 116.6 & 145.9 & 148.1 & 174.5 & 189.3 \\
\hline Salt Lake City, UT & 1,026 & 1,078 & 1,462 & 1,693 & 1,933 & 89.0 & 92.1 & 123.3 & 140.7 & 160.7 \\
\hline San Antonio-New Braunfels, TX & 3,155 & 4,160 & 4,779 & 4,891 & 4,304 & 135.5 & 174.5 & 196.7 & 197.7 & 174.0 \\
\hline San Diego-Carlsbad, CA & 3,420 & 3,691 & 4,989 & 5,973 & 6,184 & 104.8 & 111.9 & 150.4 & 179.0 & 185.3 \\
\hline San Francisco-Oakland-H & 7,110 & 9,330 & 10,669 & 12,169 & 12,642 & 154.8 & 200.4 & 228.0 & 257.4 & 267.4 \\
\hline San Jose-Sunnyvale-Santa Clara, CA & 1,552 & 1,857 & 1,976 & 2,528 & 2,230 & 79.5 & 93.9 & 99.9 & 126.5 & 111.6 \\
\hline Seattle-Tacoma-Bellevue, WA & 3,931 & 4,766 & 5,149 & 6,667 & 7,190 & 107.1 & 127.7 & 135.5 & 172.4 & 185.9 \\
\hline St. Louis, MO-IL & 4,346 & 5,257 & 6,558 & 6,699 & 7,260 & 154.9 & 187.0 & 233.6 & 238.6 & 258.6 \\
\hline Tampa-St. Petersburg-Clearwater, FL & 3,455 & 3,916 & 4,408 & 4,661 & 4,352 & 118.5 & 131.6 & 145.4 & 150.8 & 140.8 \\
\hline Virginia Beach-Norfolk-Newport News, VA-NC & 3,206 & 3,300 & 4,320 & 4,785 & 4,505 & 186.8 & 191.3 & 250.2 & 277.4 & 261.1 \\
\hline Washington-Arlington-Alexandria, DC-VA-MD-WV & 2,974 & 3,008 & 4,335 & 9,682 & 9,313 & 49.3 & 49.3 & 70.7 & 155.7 & 149.8 \\
\hline SELECTED MSAs TOTAL & 212,240 & 239,580 & 288,468 & 340,672 & 350,532 & 121.4 & 135.6 & 162.1 & 189.4 & 194.9 \\
\hline
\end{tabular}

* MSAs were selected on the basis of the largest population in the 2010 US Census.

${ }^{\dagger}$ The variable used to identify county, which is used to classify cases into MSAs, was complete for $\leq 95 \%$ of cases in a state contributing data to this MSA. See Section A1.4 in the Appendix for more information.

₹ 2016 county data for Alabama have been corrected and may not match previous reports.

NOTE: County was misclassified in the 2017 District of Columbia STD morbidity data resulting in inaccurate county-level case counts and rates. 
Table 18. Gonorrhea Among Females - Reported Cases and Rates of Reported Cases in Selected Metropolitan Statistical Areas (MSAs)* in Alphabetical Order, United States, 2014-2018

\begin{tabular}{|c|c|c|c|c|c|c|c|c|c|c|}
\hline \multirow[b]{2}{*}{ MSAs } & \multicolumn{5}{|c|}{ Cases } & \multicolumn{5}{|c|}{ Rates per 100,000 Population } \\
\hline & 2014 & 2015 & 2016 & 2017 & 2018 & 2014 & 2015 & 2016 & 2017 & 2018 \\
\hline Atlanta-Sandy Springs-Roswell, GA & 3,030 & $2,578^{+}$ & 4,696 & 4,723 & $3,683^{\dagger}$ & 104.7 & $87.5^{+}$ & 157.0 & 155.4 & $121.2^{\dagger}$ \\
\hline Austin-Round Rock, TX & 1,213 & 1,039 & 1,162 & 1,522 & 1,326 & 124.9 & 103.9 & 113.1 & 144.2 & 125.7 \\
\hline Baltimore-Columbia-Towson, MD & 1,608 & 1,964 & 2,492 & 3,059 & 2,538 & 111.5 & 135.6 & 171.9 & 210.4 & 174.6 \\
\hline Birmingham-Hoover, AL & 970 & 999 & $943^{\ddagger}$ & 1,644 & 1,494 & 163.4 & 167.9 & $158.4^{\ddagger}$ & 275.4 & 250.3 \\
\hline Boston-Cambridge-Newton, MA-NH & $844^{+}$ & $601^{+}$ & $720^{+}$ & $1,242^{\dagger}$ & 1,255 & $34.7^{+}$ & $24.5+$ & $29.2^{+}$ & $50.0^{+}$ & 50.5 \\
\hline Buffalo-Cheektowaga-Niagara Falls, NY & 664 & 959 & 985 & 1,157 & 1,138 & 113.4 & 164.0 & 168.9 & 197.5 & 194.3 \\
\hline Charlotte-Concord-Gastonia, NC-SC & 1,962 & 2,419 & 2,296 & 2,554 & 2,632 & 160.2 & 193.8 & 180.1 & 196.4 & 202.4 \\
\hline Chicago-Naperville-Elgin, IL-IN-WI & 5,662 & 5,696 & 6,481 & 6,997 & 6,943 & 115.9 & 116.8 & 133.5 & 143.8 & 142.7 \\
\hline Cincinnati, OH-KY-IN & 1,913 & 2,020 & 2,183 & 2,481 & $2,576^{+}$ & 174.3 & 183.4 & 197.7 & 223.3 & $231.9^{+}$ \\
\hline Cleveland-Elyria, $\mathrm{OH}$ & 2,021 & 1,745 & 2,134 & 3,043 & 2,719 & 189.2 & 163.6 & 200.7 & 285.9 & 255.5 \\
\hline Columbus, $\mathrm{OH}$ & 1,473 & 1,638 & 2,105 & 2,165 & 2,311 & 145.3 & 159.5 & 203.0 & 205.1 & 218.9 \\
\hline Dallas-Fort Worth-Arlington, TX & 4,153 & 4,963 & 4,577 & 5,504 & 5,343 & 117.5 & 137.4 & 124.5 & 146.5 & 142.2 \\
\hline Denver-Aurora-Lakewood, CO & 780 & 1,096 & 1,403 & 2,083 & 1,920 & 56.4 & 77.7 & 98.1 & 144.2 & 132.9 \\
\hline Detroit-Warren-Dearborn, MI & 2,698 & 2,592 & 3,190 & 4,008 & 3,867 & 122.0 & 117.2 & 144.5 & 181.0 & 174.7 \\
\hline Hartford-West Hartford-East Hartford, CT & 425 & $310^{+}$ & $412^{+}$ & 796 & $776^{+}$ & 68.3 & $50.0^{+}$ & $66.7^{\dagger}$ & 128.5 & $125.3^{+}$ \\
\hline Houston-The Woodlands-Sugar Land, TX & 4,151 & 4,113 & 4,422 & 4,422 & 4,439 & 127.2 & 122.8 & 129.7 & 127.5 & 128.0 \\
\hline Indianapolis-Carmel-Anderson, IN & 1,828 & 1,791 & 2,264 & 2,533 & 2,345 & 181.3 & 176.1 & 220.9 & 244.3 & 226.1 \\
\hline Jacksonville, FL & 1,288 & 1,237 & 1,402 & 1,643 & 1,784 & 176.9 & 166.5 & 184.9 & 213.0 & 231.3 \\
\hline Kansas City, MO-KS & 1,361 & 1,409 & 1,875 & 2,448 & 2,479 & 128.9 & 132.6 & 174.9 & 225.9 & 228.8 \\
\hline Las Vegas-Henderson-Paradise, NV & 1,039 & 1,129 & 1,341 & 1,518 & 1,958 & 100.5 & 106.7 & 124.1 & 137.4 & 177.3 \\
\hline Los Angeles-Long Beach-Anaheim, CA & 5,029 & 5,778 & 7,083 & 8,808 & 9,187 & 74.8 & 85.4 & 104.9 & 130.1 & 135.7 \\
\hline Louisville-Jefferson County, KY-IN & 992 & 1,012 & 1,278 & 1,493 & $1,198^{\dagger}$ & 152.6 & 154.8 & 194.8 & 225.8 & $181.2^{\dagger}$ \\
\hline Memphis, TN-MS-AR & 1,371 & 1,469 & 1,818 & 2,304 & 2,552 & 196.0 & 209.8 & 259.7 & 327.8 & 363.1 \\
\hline Miami-Fort Lauderdale-West Palm Beach, FL & 2,123 & 2,252 & 2,606 & 2,699 & 2,816 & 69.5 & 72.7 & 83.4 & 85.2 & 88.9 \\
\hline Milwaukee-Waukesha-West Allis, WI & 1,298 & 1,850 & 2,208 & 2,346 & 2,195 & 160.8 & 228.8 & 274.0 & 290.5 & 271.8 \\
\hline Minneapolis-St. Paul-Bloomington, MN-WI & 1,388 & 1,229 & 1,676 & 2,256 & 2,412 & 78.6 & 69.0 & 93.5 & 124.3 & 132.8 \\
\hline Nashville-Davidson-Murfreesboro-Franklin, TN & 790 & 888 & 1,129 & 1,036 & 1,236 & 86.0 & 94.7 & 118.1 & 106.4 & 127.0 \\
\hline New Orleans-Metairie, LA & 1,339 & 1,423 & 1,527 & 1,512 & 1,414 & 207.2 & 218.1 & 233.1 & 229.1 & 214.3 \\
\hline New York-Newark-Jersey City, NY-NJ-PA & 6,544 & 7,349 & 7,134 & 8,210 & 8,565 & 63.1 & 70.6 & 68.6 & 78.3 & 81.7 \\
\hline Oklahoma City, OK & 1,310 & 1,267 & 1,558 & 1,771 & 1,757 & 193.2 & 183.8 & 223.8 & 252.6 & 250.6 \\
\hline Orlando-Kissimmee-Sanford, FL & 1,109 & 1,254 & 1,326 & 1,569 & 1,633 & 93.4 & 102.7 & 106.2 & 122.3 & 127.3 \\
\hline Philadelphia-Camden-Wilmington, PA-NJ-DE-MD & 4,461 & 4,277 & 4,640 & 4,544 & 4,413 & 142.7 & 136.4 & 148.0 & 144.3 & 140.2 \\
\hline Phoenix-Mesa-Scottsdale, AZ & 2,658 & 2,708 & 3,259 & 3,807 & 3,752 & 117.6 & 117.6 & 138.9 & 159.8 & 157.5 \\
\hline Pittsburgh, PA & 1,415 & 1,239 & 1,153 & 948 & 1,035 & 116.8 & 102.5 & 95.9 & 79.2 & 86.4 \\
\hline Portland-Vancouver-Hillsboro, OR-WA & 382 & 764 & 1,005 & 1,208 & 1,478 & 32.2 & 63.2 & 82.0 & 97.5 & 119.3 \\
\hline Providence-Warwick, RI-MA & $325^{+}$ & $260^{+}$ & $368^{+}$ & $559^{\dagger}$ & 687 & $39.2^{+}$ & $31.3^{+}$ & $44.3^{+}$ & $67.0^{\dagger}$ & 82.4 \\
\hline Raleigh, NC & 638 & 857 & 785 & 1,101 & 1,126 & 100.2 & 131.4 & 117.4 & 161.0 & 164.7 \\
\hline Richmond, VA & 1,194 & 1,141 & 1,473 & 1,432 & 1,319 & 183.4 & 173.6 & 222.2 & 214.0 & 197.1 \\
\hline Riverside-San Bernardino-Ontario, CA & 1,966 & 2,208 & 2,515 & 3,101 & 3,255 & 88.1 & 97.9 & 110.5 & 134.8 & 141.5 \\
\hline Sacramento-Roseville-Arden-Arcade, CA & 1,246 & 1,538 & 1,463 & 1,613 & 1,891 & 108.8 & 132.5 & 124.7 & 135.8 & 159.2 \\
\hline Salt Lake City, UT & 376 & 325 & 480 & 549 & 578 & 65.5 & 55.8 & 81.3 & 91.7 & 96.5 \\
\hline San Antonio-New Braunfels, TX & 1,445 & 1,785 & 1,981 & 2,228 & 1,804 & 122.4 & 147.8 & 161.1 & 178.1 & 144.2 \\
\hline San Diego-Carlsbad, CA & 1,038 & 1,018 & 1,479 & 1,593 & 2,091 & 63.9 & 62.1 & 89.7 & 96.0 & 126.1 \\
\hline San Francisco-Oakland-Hayward, CA & 1,836 & 2,101 & 2,183 & 2,646 & 2,719 & 78.9 & 89.1 & 92.2 & 110.7 & 113.8 \\
\hline San Jose-Sunnyvale-Santa Clara, CA & 557 & 642 & 614 & 772 & 663 & 57.4 & 65.4 & 62.6 & 78.1 & 67.0 \\
\hline Seattle-Tacoma-Bellevue, WA & 1,412 & 1,662 & 1,556 & 2,011 & 2,137 & 76.9 & 89.0 & 81.9 & 104.1 & 110.6 \\
\hline St. Louis, MO-IL & 2,087 & 2,436 & 2,911 & 2,969 & 3,173 & 144.3 & 168.1 & 201.4 & 205.4 & 219.5 \\
\hline Tampa-St. Petersburg-Clearwater, FL & 1,619 & 1,676 & 1,815 & 1,955 & 1,786 & 107.6 & 109.2 & 116.0 & 122.7 & 112.1 \\
\hline Virginia Beach-Norfolk-Newport News, VA-NC & 1,711 & 1,701 & 2,118 & 2,340 & 2,082 & 196.4 & 194.4 & 241.5 & 267.0 & 237.5 \\
\hline Washington-Arlington-Alexandria, DC-VA-MD-WV & 1,163 & 1,064 & 1,489 & 3,031 & 2,931 & 37.7 & 34.1 & 47.5 & 95.4 & 92.2 \\
\hline SELECTED MSAs TOTAL & 89,905 & 95,471 & 109,713 & 127,953 & 127,411 & 100.7 & 105.9 & 120.9 & 139.5 & 139.0 \\
\hline
\end{tabular}

* MSAs were selected on the basis of the largest population in the 2010 US Census.

${ }^{+}$The variable used to identify county, which is used to classify cases into MSAs, was complete for $\leq 95 \%$ of cases in a state contributing data to this MSA. See Section A1.4 in the Appendix for more information.

₹ 2016 county data for Alabama have been corrected and may not match previous reports.

NOTE: Cases reported with unknown sex are not included in this table. 
Table 19. Gonorrhea Among Males - Reported Cases and Rates of Reported Cases in Selected Metropolitan Statistical Areas (MSAs) in Alphabetical Order, United States, 2014-2018

\begin{tabular}{|c|c|c|c|c|c|c|c|c|c|c|}
\hline \multirow[b]{2}{*}{ MSAs } & \multicolumn{5}{|c|}{ Cases } & \multicolumn{5}{|c|}{ Rates per 100,000 Population } \\
\hline & 2014 & 2015 & 2016 & 2017 & 2018 & 2014 & 2015 & 2016 & 2017 & 2018 \\
\hline Atlanta-Sandy Springs-Roswell, GA & 4,177 & $3,885^{+}$ & 6,960 & 7,622 & $6,658^{\dagger}$ & 153.5 & $140.5^{\dagger}$ & 248.6 & 267.9 & $234.0^{+}$ \\
\hline Austin-Round Rock, TX & 1,635 & 2,144 & 2,499 & 2,882 & 2,994 & 168.2 & 214.2 & 242.9 & 271.7 & 282.3 \\
\hline Baltimore-Columbia-Towson, MD & 1,840 & 2,202 & 3,359 & 3,833 & 3,457 & 137.0 & 163.2 & 249.0 & 283.1 & 255.3 \\
\hline Birmingham-Hoover, AL & 979 & 1,075 & $1,068^{\ddagger}$ & 1,827 & 1,841 & 177.9 & 195.2 & $193.4^{\ddagger}$ & 330.4 & 333.0 \\
\hline Boston-Cambridge-Newton, MA-NH & $1,870^{+}$ & $1,881^{\dagger}$ & $2,274^{+}$ & $3,120^{+}$ & 4,075 & $81.4^{+}$ & $81.1^{\dagger}$ & $97.6^{+}$ & $132.7^{+}$ & 173.3 \\
\hline Buffalo-Cheektowaga-Niagara Falls, NY & 678 & 1,023 & 1,195 & 1,291 & 1,415 & 123.1 & 185.8 & 217.5 & 234.3 & 256.8 \\
\hline Charlotte-Concord-Gastonia, NC-SC & 1,683 & 2,251 & 2,451 & 2,856 & 3,127 & 145.6 & 191.0 & 204.4 & 233.2 & 255.3 \\
\hline Chicago-Naperville-Elgin, IL-IN-WI & 6,947 & 7,780 & 10,133 & 11,514 & 12,632 & 148.7 & 166.5 & 217.6 & 246.6 & 270.6 \\
\hline Cincinnati, OH-KY-IN & 1,431 & 1,693 & 1,913 & 2,233 & $2,409^{+}$ & 136.0 & 160.2 & 180.3 & 209.0 & $225.5^{+}$ \\
\hline Cleveland-Elyria, $\mathrm{OH}$ & 1,781 & 1,683 & 2,071 & 2,800 & 2,681 & 178.9 & 169.3 & 208.7 & 281.6 & 269.6 \\
\hline Columbus, $\mathrm{OH}$ & 1,787 & 2,038 & 2,716 & 3,032 & 3,346 & 182.2 & 204.9 & 270.3 & 296.4 & 327.1 \\
\hline Dallas-Fort Worth-Arlington, TX & 5,033 & 6,357 & 6,498 & 7,331 & 7,885 & 147.1 & 182.0 & 182.8 & 201.3 & 216.5 \\
\hline Denver-Aurora-Lakewood, CO & 1,236 & 1,742 & 2,445 & 3,325 & 3,350 & 90.1 & 124.1 & 171.8 & 230.3 & 232.0 \\
\hline Detroit-Warren-Dearborn, MI & 2,606 & 2,894 & 3,622 & 4,648 & 4,947 & 125.0 & 138.5 & 173.3 & 221.4 & 235.7 \\
\hline Hartford-West Hartford-East Hartford, CT & 466 & $416^{+}$ & $551^{+}$ & 1,033 & $1,203^{+}$ & 78.7 & $70.4^{+}$ & $93.5^{+}$ & 174.8 & $203.6^{+}$ \\
\hline Houston-The Woodlands-Sugar Land, TX & 4,146 & 5,167 & 5,930 & 6,343 & 6,370 & 128.5 & 156.2 & 176.3 & 185.3 & 186.1 \\
\hline Indianapolis-Carmel-Anderson, IN & 1,927 & 1,920 & 2,542 & 2,892 & 2,551 & 200.1 & 197.5 & 259.5 & 291.6 & 257.3 \\
\hline Jacksonville, FL & 1,316 & 1,501 & 1,764 & 2,076 & 2,270 & 190.5 & 212.5 & 245.0 & 283.0 & 309.4 \\
\hline Kansas City, MO-KS & 1,281 & 1,534 & 2,134 & 2,826 & 3,178 & 126.1 & 149.7 & 206.6 & 270.3 & 304.0 \\
\hline Las Vegas-Henderson-Paradise, NV & 1,612 & 1,837 & 2,306 & 2,905 & 3,330 & 155.6 & 173.9 & 214.4 & 264.2 & 302.8 \\
\hline Los Angeles-Long Beach-Anaheim, CA & 12,071 & 14,066 & 18,060 & 20,803 & 21,963 & 184.7 & 213.9 & 275.3 & 315.9 & 333.6 \\
\hline Louisville-Jefferson County, KY-IN & 961 & 1,170 & 1,670 & 1,910 & $1,444^{+}$ & 155.1 & 187.3 & 266.2 & 301.9 & $228.2^{\dagger}$ \\
\hline Memphis, TN-MS-AR & 1,254 & 1,674 & 1,926 & 2,347 & 2,905 & 194.8 & 260.0 & 299.6 & 363.7 & 450.1 \\
\hline Miami-Fort Lauderdale-West Palm Bea & 3,999 & 4,651 & 5,374 & 6,146 & 6,801 & 139.0 & 159.6 & 182.6 & 205.4 & 227.3 \\
\hline Milwaukee-Waukesha-West Allis, WI & 1,281 & 1,864 & 2,241 & 2,560 & 2,551 & 167.4 & 242.9 & 292.4 & 333.1 & 331.9 \\
\hline Minneapolis-St. Paul-Bloomington, MN-WI & 1,942 & 2,058 & 2,439 & 2,993 & 3,414 & 112.4 & 118.0 & 138.6 & 167.7 & 191.3 \\
\hline Nashville-Davidson-Murfreesboro-Franklin, TN & 1,130 & 1,312 & 1,566 & 1,670 & 1,866 & 129.3 & 147.0 & 172.3 & 179.6 & 200.7 \\
\hline New Orleans-Metairie, LA & 1,328 & 1,506 & 1,887 & 2,126 & 2,170 & 219.2 & 246.7 & 307.5 & 345.2 & 352.3 \\
\hline New York-Newark-Jersey City, NY-NJ-PA & 13,448 & 16,290 & 18,976 & 23,737 & 26,170 & 138.4 & 166.8 & 194.6 & 241.4 & 266.1 \\
\hline Oklahoma City, OK & 1,056 & 1,136 & 1,394 & 1,647 & 1,839 & 160.3 & 169.7 & 205.9 & 241.2 & 269.4 \\
\hline Orlando-Kissimmee-Sanford, FL & 1,461 & 1,818 & 2,067 & 2,720 & 2,674 & 128.8 & 155.9 & 173.2 & 221.7 & 217.9 \\
\hline Philadelphia-Camden-Wilmington, PA-NJ-DE-MD & 5,152 & 5,439 & 6,736 & 7,540 & 7,695 & 176.2 & 185.4 & 229.4 & 255.8 & 261.1 \\
\hline Phoenix-Mesa-Scottsdale, AZ & 3,286 & 3,780 & 4,824 & 5,602 & 5,669 & 147.4 & 166.4 & 208.3 & 237.8 & 240.7 \\
\hline Pittsburgh, PA & 1,187 & 1,182 & 1,448 & 1,422 & 1,592 & 103.7 & 103.3 & 127.0 & 125.2 & 140.1 \\
\hline Portland-Vancouver-Hillsborc & 1,116 & 1,621 & 2,172 & 2,236 & 2,754 & 96.2 & 137.3 & 181.1 & 184.2 & 226.9 \\
\hline Providence-Warwick, RI-MA & $587^{+}$ & $572^{+}$ & $740^{+}$ & $1,001^{\dagger}$ & 1,266 & $75.3^{+}$ & $73.2^{+}$ & $94.4^{+}$ & $127.2^{+}$ & 160.9 \\
\hline Raleigh, NC & 770 & 1,161 & 1,130 & 1,390 & 1,428 & 127.0 & 186.9 & 178.1 & 213.4 & 219.3 \\
\hline Richmond, VA & 978 & 1,058 & 1,712 & 1,743 & 1,648 & 160.6 & 172.3 & 276.7 & 278.8 & 263.7 \\
\hline Riverside-San Bernardino-Ontario, CA & 2,321 & 2,692 & 3,437 & 4,207 & 4,708 & 105.0 & 120.5 & 152.6 & 184.5 & 206.5 \\
\hline Sacramento-Roseville-Arden-Arcade, CA & 1,362 & 1,771 & 1,934 & 2,433 & 2,490 & 124.0 & 159.1 & 172.3 & 213.9 & 219.0 \\
\hline Salt Lake City, UT & 650 & 753 & 982 & 1,144 & 1,355 & 112.3 & 128.1 & 164.8 & 189.3 & 224.3 \\
\hline San Antonio-New Braunfels, TX & 1,710 & 2,375 & 2,798 & 2,663 & 2,494 & 149.0 & 201.9 & 233.2 & 217.7 & 203.9 \\
\hline San Diego-Carlsbad, CA & 2,354 & 2,668 & 3,498 & 4,365 & 4,079 & 143.5 & 160.8 & 209.7 & 260.0 & 242.9 \\
\hline San Francisco-Oakland-H & 5,261 & 7,201 & 8,441 & 9,482 & 9,840 & 232.2 & 313.3 & 365.3 & 405.7 & 421.0 \\
\hline San Jose-Sunnyvale-Santa Clara, CA & 995 & 1,212 & 1,361 & 1,756 & 1,565 & 101.3 & 121.8 & 136.3 & 173.9 & 155.0 \\
\hline Seattle-Tacoma-Bellevue, WA & 2,519 & 3,104 & 3,593 & 4,654 & 5,049 & 137.3 & 166.3 & 189.2 & 240.5 & 260.9 \\
\hline St. Louis, MO-IL & 2,256 & 2,802 & 3,646 & 3,726 & 4,085 & 166.0 & 205.6 & 267.8 & 273.6 & 299.9 \\
\hline Tampa-St. Petersburg-Clearwater, FL & 1,823 & 2,240 & 2,592 & 2,704 & 2,566 & 129.2 & 155.6 & 176.6 & 180.5 & 171.3 \\
\hline Virginia Beach-Norfolk-Newport News, VA-NC & 1,489 & 1,595 & 2,195 & 2,423 & 2,408 & 176.2 & 187.7 & 258.3 & 285.5 & 283.7 \\
\hline Washington-Arlington-Alexandria, DC-VA-MD-WV & 1,811 & 1,943 & 2,838 & 6,613 & 6,338 & 61.4 & 65.2 & 94.8 & 217.6 & 208.6 \\
\hline SELECTED MSAs TOTAL & 121,989 & 143,737 & 178,108 & 212,152 & 222,545 & 142.5 & 166.1 & 204.4 & 240.7 & 252.5 \\
\hline
\end{tabular}

* MSAs were selected on the basis of the largest population in the 2010 US Census.

${ }^{+}$The variable used to identify county, which is used to classify cases into MSAs, was complete for $\leq 95 \%$ of cases in a state contributing data to this MSA. See Section A1.4 in the Appendix for more information.

${ }^{\ddagger} 2016$ county data for Alabama have been corrected and may not match previous reports.

NOTE: Cases reported with unknown sex are not included in this table. 
Table 20. Gonorrhea - Reported Cases and Rates of Reported Cases in Counties and Independent Cities* Ranked by Number of Reported Cases, United States, 2018

\begin{tabular}{|c|c|c|c|c|}
\hline Rank* & County/Independent City & Cases & Rate per 100,000 Population & Cumulative Percentage \\
\hline 1 & Los Angeles County, CA & 27,266 & 268.3 & 5 \\
\hline 2 & Cook County, IL & 16,117 & 309.3 & 7 \\
\hline 3 & New York County, NY & 9,067 & 544.7 & 9 \\
\hline 4 & Maricopa County, AZ & 8,888 & 206.4 & 11 \\
\hline 5 & Harris County, TX & 8,730 & 187.6 & 12 \\
\hline 6 & Kings County, NY & 7,574 & 285.9 & 13 \\
\hline 7 & Dallas County, TX & 7,326 & 279.8 & 15 \\
\hline 8 & Philadelphia County, PA & 7,205 & 455.8 & 16 \\
\hline 9 & Wayne County, MI & 6,287 & 358.5 & 17 \\
\hline 10 & San Diego County, CA & 6,184 & 185.3 & 18 \\
\hline 11 & San Francisco County, CA & 5,887 & 665.7 & 19 \\
\hline 12 & Clark County, NV & 5,294 & 240.2 & 20 \\
\hline 13 & Bronx County, NY & 5,204 & 353.7 & 21 \\
\hline 14 & Franklin County, $\mathrm{OH}$ & 4,921 & 380.9 & 22 \\
\hline 15 & Cuyahoga County, $\mathrm{OH}$ & 4,552 & 364.6 & 22 \\
\hline 16 & Milwaukee County, WI & 4,547 & 477.6 & 23 \\
\hline 17 & King County, WA & 4,406 & 201.3 & 24 \\
\hline 18 & Shelby County, TN & 4,328 & 461.9 & 25 \\
\hline 19 & Miami-Dade County, FL & 4,309 & 156.6 & 25 \\
\hline 20 & Washington, D.C. & 4,240 & 611.0 & 26 \\
\hline 21 & Riverside County, CA & 4,084 & 168.5 & 27 \\
\hline 22 & Marion County, IN & 4,013 & 422.4 & 27 \\
\hline 23 & Orange County, CA & 3,940 & 123.5 & 28 \\
\hline 24 & San Bernardino County, CA & 3,893 & 180.4 & 29 \\
\hline 25 & Bexar County, TX & 3,884 & 198.3 & 30 \\
\hline 26 & Broward County, FL & 3,846 & 198.7 & 30 \\
\hline 27 & Sacramento County, CA & 3,806 & 248.7 & 31 \\
\hline 28 & Queens County, NY & 3,796 & 160.9 & 31 \\
\hline 29 & Alameda County, CA & 3,777 & 227.1 & 32 \\
\hline 30 & Baltimore (City), MD & 3,596 & 587.9 & 33 \\
\hline 31 & Hamilton County, $\mathrm{OH}$ & 3,577 & 439.5 & 33 \\
\hline 32 & Duval County, FL & 3,492 & 372.3 & 34 \\
\hline 33 & Jackson County, MO & 3,443 & 492.6 & 35 \\
\hline 34 & Travis County, TX & 3,409 & 277.9 & 35 \\
\hline 35 & Fulton County, GA & 3,368 & 323.4 & 36 \\
\hline 36 & Hennepin County, MN & 3,308 & 264.2 & 36 \\
\hline 37 & Tarrant County, TX & 3,212 & 156.3 & 37 \\
\hline 38 & Mecklenburg County, NC & 3,192 & 296.4 & 37 \\
\hline 39 & Orange County, FL & 2,937 & 217.7 & 38 \\
\hline 40 & Jefferson County, AL & 2,809 & 426.1 & 38 \\
\hline 41 & St. Louis County, MO & 2,791 & 280.0 & 39 \\
\hline 42 & Oklahoma County, OK & 2,752 & 349.3 & 39 \\
\hline 43 & Denver County, CO & 2,704 & 383.8 & 40 \\
\hline 44 & Multnomah County, OR & 2,477 & 306.7 & 40 \\
\hline 45 & Suffolk County, MA & 2,464 & 308.8 & 41 \\
\hline 46 & Kern County, CA & 2,311 & 258.8 & 41 \\
\hline 47 & Hillsborough County, FL & 2,292 & 162.7 & 41 \\
\hline 48 & Bernalillo County, NM & 2,287 & 337.9 & 42 \\
\hline 49 & St. Louis (City), MO & 2,280 & 738.8 & 42 \\
\hline 50 & Erie County, NY & 2,249 & 243.0 & 43 \\
\hline 51 & Fresno County, CA & 2,246 & 227.0 & 43 \\
\hline 52 & DeKalb County, GA & 2,188 & 290.5 & 43 \\
\hline 53 & Santa Clara County, CA & 2,179 & 112.4 & 44 \\
\hline 54 & Jefferson County, KY & 2,158 & 279.8 & 44 \\
\hline 55 & Wake County, NC & 2,143 & 199.9 & 44 \\
\hline 56 & Orleans Parish, LA & 2,114 & 537.5 & 45 \\
\hline 57 & Tulsa County, OK & 2,086 & 322.8 & 45 \\
\hline 58 & Allegheny County, PA & 2,060 & 168.4 & 46 \\
\hline 59 & Contra Costa County, CA & 2,057 & 179.3 & 46 \\
\hline 60 & Prince George's County, MD & 2,020 & 221.3 & 46 \\
\hline 61 & Essex County, NJ & 2,011 & 248.8 & 47 \\
\hline 62 & Guilford County, NC & 1,970 & 373.8 & 47 \\
\hline 63 & Pima County, AZ & 1,925 & 188.2 & 47 \\
\hline 64 & Pierce County, WA & 1,917 & 218.6 & 48 \\
\hline 65 & Salt Lake County, UT & 1,907 & 167.9 & 48 \\
\hline 66 & Davidson County, TN & 1,880 & 272.0 & 48 \\
\hline 67 & Monroe County, NY & 1,867 & 249.7 & 49 \\
\hline 68 & Richland County, SC & 1,834 & 445.6 & 49 \\
\hline 69 & Hartford County, CT & 1,806 & 201.7 & 49 \\
\hline 70 & Lucas County, OH & 1,799 & 417.5 & 49 \\
\hline
\end{tabular}

* The top 70 counties and independent cities ranked in descending order by number of cases reported in 2018 then by rate are displayed.

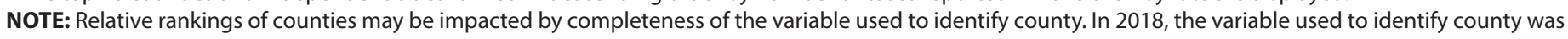
complete for $\leq 95 \%$ of cases in Connecticut, Georgia, Hawaii, Kentucky, and South Dakota. See Section A1.4 in the Appendix for more information. 
Table 21. Gonorrhea - Reported Cases and Rates of Reported Cases by Age Group and Sex, United States, 2014-2018

\begin{tabular}{|c|c|c|c|c|c|c|c|c|}
\hline & \multirow{2}{*}{$\begin{array}{l}\text { Age } \\
\text { Group }\end{array}$} & \multicolumn{4}{|c|}{ Cases } & \multicolumn{3}{|c|}{ Rates per 100,000 Population* } \\
\hline & & Total & Male & Female & Unknown Sex & Total & Male & Female \\
\hline \multirow{14}{*}{$\underset{\mathbb{D}}{\stackrel{0}{O}}$} & $0-4$ & 154 & 47 & 105 & 2 & 0.8 & 0.5 & 1.1 \\
\hline & $5-9$ & 53 & 7 & 46 & 0 & 0.3 & 0.1 & 0.5 \\
\hline & $10-14$ & 2,450 & 440 & 2,005 & 5 & 11.9 & 4.2 & 19.8 \\
\hline & $15-19$ & 68,468 & 23,981 & 44,399 & 88 & 325.0 & 222.4 & 431.7 \\
\hline & $20-24$ & 116,200 & 56,714 & 59,329 & 157 & 507.2 & 483.1 & 531.0 \\
\hline & $25-29$ & 69,587 & 40,602 & 28,899 & 86 & 316.5 & 363.8 & 266.9 \\
\hline & $30-34$ & 38,393 & 24,349 & 13,988 & 56 & 178.3 & 225.3 & 130.5 \\
\hline & $35-39$ & 20,803 & 14,129 & 6,654 & 20 & 104.4 & 142.1 & 66.7 \\
\hline & $40-44$ & 12,687 & 9,349 & 3,320 & 18 & 61.6 & 91.5 & 32.0 \\
\hline & $45-54$ & 15,322 & 12,388 & 2,917 & 17 & 35.3 & 57.8 & 13.2 \\
\hline & $55-64$ & 4,549 & 3,859 & 680 & 10 & 11.4 & 20.0 & 3.3 \\
\hline & $65+$ & 911 & 790 & 121 & 0 & 2.0 & 3.9 & 0.5 \\
\hline & Unknown Age & 485 & 288 & 145 & 52 & & & \\
\hline & TOTAL & 350,062 & 186,943 & 162,608 & 511 & 109.8 & 119.1 & 100.4 \\
\hline \multirow{14}{*}{$\underset{\mathrm{v}}{\mathrm{v}}$} & $0-4$ & 148 & 47 & 98 & 3 & 0.7 & 0.5 & 1.0 \\
\hline & $5-9$ & 78 & 11 & 66 & 1 & 0.4 & 0.1 & 0.7 \\
\hline & $10-14$ & 2,312 & 385 & 1,923 & 4 & 11.2 & 3.7 & 19.0 \\
\hline & $15-19$ & 72,001 & 26,401 & 45,477 & 123 & 341.1 & 244.5 & 441.1 \\
\hline & $20-24$ & 124,592 & 63,289 & 61,105 & 198 & 547.9 & 542.4 & 551.9 \\
\hline & $25-29$ & 82,867 & 50,089 & 32,662 & 116 & 368.9 & 439.0 & 295.5 \\
\hline & $30-34$ & 45,681 & 29,751 & 15,867 & 63 & 210.7 & 273.2 & 147.1 \\
\hline & $35-39$ & 26,137 & 18,198 & 7,897 & 42 & 128.3 & 178.9 & 77.4 \\
\hline & $40-44$ & 15,042 & 11,116 & 3,898 & 28 & 74.4 & 110.8 & 38.3 \\
\hline & $45-54$ & 18,779 & 15,379 & 3,375 & 25 & 43.5 & 72.2 & 15.4 \\
\hline & $55-64$ & 6,035 & 5,175 & 849 & 11 & 14.8 & 26.2 & 4.0 \\
\hline & $65+$ & 1,191 & 1,032 & 158 & 1 & 2.5 & 4.9 & 0.6 \\
\hline & Unknown Age & 353 & 197 & 139 & 17 & & & \\
\hline & TOTAL & 395,216 & 221,070 & 173,514 & 632 & 123.0 & 139.7 & 106.3 \\
\hline \multirow{14}{*}{$\frac{N}{a}$} & $0-4$ & 187 & 72 & 113 & 2 & 0.9 & 0.7 & 1.2 \\
\hline & $5-9$ & 98 & 16 & 81 & 1 & 0.5 & 0.2 & 0.8 \\
\hline & $10-14$ & 2,436 & 498 & 1,929 & 9 & 11.8 & 4.7 & 19.1 \\
\hline & $15-19$ & 80,172 & 30,316 & 49,710 & 146 & 379.4 & 280.7 & 481.3 \\
\hline & $20-24$ & 138,130 & 71,967 & 65,930 & 233 & 617.2 & 626.3 & 605.4 \\
\hline & $25-29$ & 101,283 & 62,189 & 38,881 & 213 & 442.5 & 534.7 & 345.3 \\
\hline & $30-34$ & 57,646 & 38,193 & 19,306 & 147 & 264.6 & 348.2 & 178.5 \\
\hline & $35-39$ & 34,058 & 23,744 & 10,230 & 84 & 163.9 & 228.8 & 98.4 \\
\hline & $40-44$ & 19,104 & 14,116 & 4,935 & 53 & 97.0 & 144.4 & 49.7 \\
\hline & $45-54$ & 24,142 & 19,762 & 4,330 & 50 & 56.4 & 93.6 & 20.0 \\
\hline & $55-64$ & 8,138 & 6,947 & 1,178 & 13 & 19.6 & 34.7 & 5.5 \\
\hline & $65+$ & 1,599 & 1,403 & 191 & 5 & 3.2 & 6.4 & 0.7 \\
\hline & Unknown Age & 1,521 & 810 & 685 & 26 & & & \\
\hline & TOTAL & 468,514 & 270,033 & 197,499 & 982 & 145.0 & 169.7 & 120.4 \\
\hline \multirow{14}{*}{$\underset{\mathbf{v}}{\vec{v}}$} & $0-4$ & 203 & 56 & 144 & 3 & 1.0 & 0.5 & 1.5 \\
\hline & $5-9$ & 110 & 19 & 90 & 1 & 0.5 & 0.2 & 0.9 \\
\hline & $10-14$ & 2,725 & 507 & 2,212 & 6 & 13.1 & 4.8 & 21.7 \\
\hline & $15-19$ & 92,608 & 34,918 & 57,573 & 117 & 438.2 & 323.3 & 557.3 \\
\hline & $20-24$ & 155,862 & 81,036 & 74,578 & 248 & 704.7 & 714.0 & 692.5 \\
\hline & $25-29$ & 121,880 & 75,123 & 46,577 & 180 & 521.5 & 631.2 & 406.1 \\
\hline & $30-34$ & 71,603 & 47,342 & 24,157 & 104 & 325.9 & 426.9 & 222.0 \\
\hline & $35-39$ & 43,792 & 30,277 & 13,448 & 67 & 206.3 & 285.2 & 126.7 \\
\hline & $40-44$ & 24,108 & 17,753 & 6,331 & 24 & 122.7 & 182.0 & 64.0 \\
\hline & $45-54$ & 29,428 & 23,803 & 5,580 & 45 & 69.4 & 113.9 & 26.0 \\
\hline & $55-64$ & 10,867 & 9,311 & 1,538 & 18 & 25.9 & 46.0 & 7.1 \\
\hline & $65+$ & 2,063 & 1,818 & 233 & 12 & 4.1 & 8.1 & 0.8 \\
\hline & Unknown Age & 359 & 206 & 126 & 27 & & & \\
\hline & TOTAL & 555,608 & 322,169 & 232,587 & 852 & 170.6 & 200.8 & 140.7 \\
\hline \multirow{14}{*}{$\underset{\infty}{\stackrel{0}{0}}$} & $0-4$ & 224 & 77 & 142 & 5 & 1.1 & 0.8 & 1.5 \\
\hline & $5-9$ & 111 & 13 & 98 & 0 & 0.5 & 0.1 & 1.0 \\
\hline & $10-14$ & 2,683 & 509 & 2,168 & 6 & 12.9 & 4.8 & 21.3 \\
\hline & $15-19$ & 91,373 & 34,614 & 56,628 & 131 & 432.4 & 320.5 & 548.1 \\
\hline & $20-24$ & 157,708 & 81,813 & 75,663 & 232 & 713.0 & 720.9 & 702.6 \\
\hline & $25-29$ & 129,385 & 80,216 & 48,991 & 178 & 553.6 & 674.0 & 427.2 \\
\hline & $30-34$ & 80,507 & 53,362 & 27,026 & 119 & 366.4 & 481.2 & 248.3 \\
\hline & $35-39$ & 48,399 & 33,556 & 14,769 & 74 & 228.0 & 316.1 & 139.1 \\
\hline & $40-44$ & 26,953 & 19,552 & 7,353 & 48 & 137.2 & 200.5 & 74.3 \\
\hline & $45-54$ & 31,270 & 25,092 & 6,132 & 46 & 73.8 & 120.0 & 28.6 \\
\hline & $55-64$ & 12,091 & 10,399 & 1,675 & 17 & 28.8 & 51.3 & 7.7 \\
\hline & $65+$ & 2,332 & 2,036 & 283 & 13 & 4.6 & 9.0 & 1.0 \\
\hline & Unknown Age & 369 & 162 & 146 & 61 & & & \\
\hline & TOTAL & 583,405 & 341,401 & 241,074 & 930 & 179.1 & 212.8 & 145.8 \\
\hline
\end{tabular}

* No population data are available for unknown sex and age; therefore, rates are not calculated. NOTE: Cases in the $0-4$ age group may include cases due to perinatal transmission. 
Table 22A. Gonorrhea - Reported Cases by Race/Hispanic Ethnicity, Age Group, and Sex, United States, 2018

\begin{tabular}{|c|c|c|c|c|c|c|c|c|c|c|c|c|}
\hline \multirow{2}{*}{$\begin{array}{l}\text { Age } \\
\text { Group }\end{array}$} & \multicolumn{3}{|c|}{$\begin{array}{l}\text { American Indians/ } \\
\text { Alaska Natives }\end{array}$} & \multicolumn{3}{|c|}{ Asians } & \multicolumn{3}{|c|}{ Blacks } & \multicolumn{3}{|c|}{$\begin{array}{c}\text { Native Hawaiians/ } \\
\text { Other Pacific Islanders }\end{array}$} \\
\hline & Total* & Male & Female & Total* & Male & Female & Total* & Male & Female & Total* & Male & Female \\
\hline $0-4$ & 3 & 1 & 2 & 0 & 0 & 0 & 65 & 18 & 47 & 0 & 0 & 0 \\
\hline $5-9$ & 3 & 0 & 3 & 0 & 0 & 0 & 43 & 3 & 40 & 0 & 0 & 0 \\
\hline $10-14$ & 28 & 2 & 26 & 14 & 3 & 11 & 1,336 & 297 & 1,038 & 3 & 1 & 2 \\
\hline $15-19$ & 1,002 & 279 & 721 & 515 & 217 & 298 & 43,237 & 17,561 & 25,660 & 128 & 44 & 84 \\
\hline $20-24$ & 1,872 & 649 & 1,222 & 1,446 & 957 & 485 & 68,405 & 36,106 & 32,254 & 272 & 124 & 148 \\
\hline $25-29$ & 1,900 & 764 & 1,135 & 1,612 & 1,277 & 334 & 50,009 & 31,538 & 18,436 & 272 & 170 & 101 \\
\hline $30-34$ & 1,415 & 587 & 827 & 1,111 & 894 & 214 & 26,045 & 18,205 & 7,823 & 155 & 91 & 64 \\
\hline $35-39$ & 854 & 372 & 481 & 696 & 578 & 118 & 13,978 & 10,467 & 3,506 & 109 & 62 & 47 \\
\hline $40-44$ & 403 & 175 & 228 & 441 & 370 & 71 & 7,410 & 5,895 & 1,508 & 52 & 37 & 15 \\
\hline $45-54$ & 323 & 161 & 162 & 461 & 398 & 63 & 8,378 & 7,190 & 1,183 & 46 & 39 & 6 \\
\hline $55-64$ & 89 & 58 & 30 & 127 & 95 & 32 & 3,599 & 3,229 & 367 & 7 & 6 & 1 \\
\hline $65+$ & 25 & 19 & 6 & 34 & 23 & 11 & 588 & 541 & 47 & 2 & 2 & 0 \\
\hline Unknown Age & 3 & 2 & 1 & 6 & 4 & 2 & 49 & 26 & 19 & 0 & 0 & 0 \\
\hline TOTAL & 7,920 & 3,069 & 4,844 & 6,463 & 4,816 & 1,639 & 223,142 & 131,076 & 91,928 & 1,046 & 576 & 468 \\
\hline
\end{tabular}

\begin{tabular}{|c|c|c|c|c|c|c|c|c|c|c|c|c|}
\hline \multirow{2}{*}{$\begin{array}{l}\text { Age } \\
\text { Group }\end{array}$} & \multicolumn{3}{|c|}{ Whites } & \multicolumn{3}{|c|}{ Multirace } & \multicolumn{3}{|c|}{ Hispanics } & \multicolumn{3}{|c|}{$\begin{array}{l}\text { Other/ } \\
\text { Unknown }\end{array}$} \\
\hline & Total* & Male & Female & Total* & Male & Female & Total* & Male & Female & Total* & Male & Female \\
\hline $0-4$ & 50 & 19 & 31 & 3 & 1 & 2 & 30 & 9 & 21 & 73 & 29 & 39 \\
\hline $5-9$ & 22 & 5 & 17 & 0 & 0 & 0 & 22 & 3 & 19 & 21 & 2 & 19 \\
\hline $10-14$ & 364 & 34 & 330 & 28 & 0 & 28 & 256 & 44 & 212 & 654 & 128 & 521 \\
\hline $15-19$ & 14,951 & 3,991 & 10,955 & 988 & 280 & 708 & 9,239 & 3,442 & 5,791 & 21,313 & 8,800 & 12,411 \\
\hline $20-24$ & 31,718 & 14,478 & 17,211 & 1,717 & 767 & 946 & 18,184 & 10,053 & 8,117 & 34,094 & 18,679 & 15,280 \\
\hline $25-29$ & 31,051 & 17,345 & 13,687 & 1,465 & 927 & 535 & 15,978 & 10,763 & 5,191 & 27,098 & 17,432 & 9,572 \\
\hline $30-34$ & 23,351 & 13,919 & 9,416 & 1,015 & 747 & 268 & 10,230 & 7,372 & 2,846 & 17,185 & 11,547 & 5,568 \\
\hline $35-39$ & 14,851 & 9,392 & 5,451 & 592 & 448 & 143 & 6,367 & 4,702 & 1,655 & 10,952 & 7,535 & 3,368 \\
\hline $40-44$ & 8,406 & 5,621 & 2,775 & 294 & 238 & 55 & 3,572 & 2,689 & 877 & 6,375 & 4,527 & 1,824 \\
\hline $45-54$ & 10,588 & 8,306 & 2,273 & 346 & 314 & 31 & 3,440 & 2,827 & 606 & 7,688 & 5,857 & 1,808 \\
\hline $55-64$ & 4,314 & 3,729 & 583 & 91 & 85 & 6 & 840 & 720 & 120 & 3,024 & 2,477 & 536 \\
\hline $65+$ & 856 & 771 & 84 & 12 & 12 & 0 & 121 & 105 & 16 & 694 & 563 & 119 \\
\hline Unknown Age & 62 & 33 & 24 & 1 & 1 & 0 & 47 & 27 & 20 & 201 & 69 & 80 \\
\hline TOTAL & 140,584 & 77,643 & 62,837 & 6,552 & 3,820 & 2,722 & 68,326 & 42,756 & 25,491 & 129,372 & 77,645 & 51,145 \\
\hline
\end{tabular}

* Total includes cases reported with unknown sex.

NOTE: These tables should be used only for race/Hispanic ethnicity comparisons. See Table 21 for age-specific cases and rates and Tables $14-16$ for total and sex-specific cases and rates. Cases in the $0-4$ age group may include cases due to perinatal transmission. 
Table 22B. Gonorrhea — Rates of Reported Cases* by Race/Hispanic Ethnicity, Age Group, and Sex, United States, 2018

\begin{tabular}{|c|c|c|c|c|c|c|c|c|c|c|c|c|}
\hline \multirow{2}{*}{$\begin{array}{l}\text { Age } \\
\text { Group }\end{array}$} & \multicolumn{3}{|c|}{$\begin{array}{l}\text { American Indians/ } \\
\text { Alaska Natives }\end{array}$} & \multicolumn{3}{|c|}{ Asians } & \multicolumn{3}{|c|}{ Blacks } & \multicolumn{3}{|c|}{$\begin{array}{l}\text { Native Hawaiians/ } \\
\text { Other Pacific Islanders }\end{array}$} \\
\hline & Total $^{\dagger}$ & Male & Female & Total $^{\dagger}$ & Male & Female & Total $^{\dagger}$ & Male & Female & Total $^{\dagger}$ & Male & Female \\
\hline $0-4$ & 1.8 & 1.2 & 2.5 & 0.0 & 0.0 & 0.0 & 2.4 & 1.3 & 3.5 & 0.0 & 0.0 & 0.0 \\
\hline $5-9$ & 1.7 & 0.0 & 3.5 & 0.0 & 0.0 & 0.0 & 1.5 & 0.2 & 2.9 & 0.0 & 0.0 & 0.0 \\
\hline $10-14$ & 15.8 & 2.2 & 29.8 & 1.3 & 0.6 & 2.1 & 47.4 & 20.8 & 74.6 & 7.3 & 4.8 & 9.8 \\
\hline $15-19$ & 554.9 & 303.8 & 812.5 & 47.8 & 40.1 & 55.6 & $1,457.7$ & $1,166.7$ & $1,756.4$ & 314.5 & 210.8 & 423.6 \\
\hline $20-24$ & 995.4 & 676.7 & 1,325.9 & 112.0 & 146.6 & 76.1 & $2,129.0$ & $2,212.1$ & $2,040.3$ & 618.7 & 553.0 & 687.1 \\
\hline $25-29$ & 965.7 & 759.8 & $1,179.8$ & 101.9 & 164.1 & 41.5 & $1,474.0$ & $1,860.7$ & $1,085.9$ & 524.7 & 632.3 & 404.8 \\
\hline $30-34$ & 844.6 & 698.9 & 989.8 & 69.7 & 117.0 & 25.8 & 913.9 & $1,315.3$ & 533.7 & 308.7 & 354.2 & 261.1 \\
\hline $35-39$ & 557.1 & 488.0 & 624.0 & 46.1 & 82.0 & 14.7 & 512.7 & 807.1 & 245.3 & 239.0 & 265.3 & 211.3 \\
\hline $40-44$ & 287.1 & 253.3 & 319.9 & 30.7 & 55.2 & 9.2 & 298.3 & 504.7 & 114.6 & 134.4 & 188.0 & 78.9 \\
\hline $45-54$ & 107.8 & 110.5 & 105.3 & 18.1 & 33.6 & 4.6 & 159.6 & 292.0 & 42.4 & 64.6 & 111.0 & 16.6 \\
\hline $55-64$ & 30.7 & 42.6 & 19.5 & 6.2 & 10.3 & 2.9 & 74.6 & 146.4 & 14.0 & 11.8 & 20.8 & 3.3 \\
\hline $65+$ & 9.2 & 15.5 & 4.0 & 1.5 & 2.4 & 0.9 & 12.8 & 29.2 & 1.7 & 3.8 & 8.1 & 0.0 \\
\hline \multicolumn{13}{|c|}{ Unknown Age } \\
\hline TOTAL & 329.5 & 259.3 & 397.1 & 35.1 & 55.0 & 17.0 & 548.9 & 674.4 & 433.3 & 181.4 & 198.6 & 163.2 \\
\hline
\end{tabular}

\begin{tabular}{|c|c|c|c|c|c|c|c|c|c|}
\hline \multirow{2}{*}{$\begin{array}{l}\text { Age } \\
\text { Group }\end{array}$} & \multicolumn{3}{|c|}{ Whites } & \multicolumn{3}{|c|}{ Multirace } & \multicolumn{3}{|c|}{ Hispanics } \\
\hline & Total $^{\dagger}$ & Male & Female & Total $^{+}$ & Male & Female & Total $^{\dagger}$ & Male & Female \\
\hline $0-4$ & 0.5 & 0.4 & 0.6 & 0.3 & 0.2 & 0.4 & 0.6 & 0.3 & 0.8 \\
\hline $5-9$ & 0.2 & 0.1 & 0.3 & 0.0 & 0.0 & 0.0 & 0.4 & 0.1 & 0.7 \\
\hline $10-14$ & 3.4 & 0.6 & 6.3 & 3.4 & 0.0 & 6.9 & 5.0 & 1.7 & 8.4 \\
\hline $15-19$ & 132.9 & 69.1 & 200.1 & 137.5 & 76.9 & 199.8 & 188.6 & 137.6 & 241.7 \\
\hline $20-24$ & 266.3 & 236.3 & 297.5 & 270.0 & 238.7 & 300.7 & 376.0 & 402.4 & 347.2 \\
\hline $25-29$ & 242.1 & 265.5 & 217.4 & 278.5 & 360.5 & 199.0 & 333.4 & 428.6 & 227.6 \\
\hline $30-34$ & 188.7 & 222.3 & 154.0 & 250.1 & 388.1 & 125.6 & 225.9 & 310.0 & 132.4 \\
\hline $35-39$ & 123.5 & 154.9 & 91.4 & 167.7 & 269.9 & 76.5 & 144.1 & 205.6 & 77.7 \\
\hline $40-44$ & 75.1 & 99.8 & 49.8 & 102.1 & 175.7 & 36.1 & 88.1 & 130.5 & 44.0 \\
\hline $45-54$ & 39.5 & 62.1 & 16.9 & 68.7 & 132.2 & 11.6 & 49.9 & 81.3 & 17.7 \\
\hline $55-64$ & 14.5 & 25.7 & 3.8 & 21.7 & 42.8 & 2.7 & 17.9 & 31.5 & 5.0 \\
\hline $65+$ & 2.2 & 4.4 & 0.4 & 3.1 & 7.0 & 0.0 & 2.9 & 5.8 & 0.7 \\
\hline \multicolumn{10}{|c|}{ Unknown Age } \\
\hline TOTAL & 71.1 & 79.6 & 62.7 & 94.4 & 111.6 & 77.4 & 115.9 & 143.6 & 87.4 \\
\hline
\end{tabular}

* Per 100,000.

† Total includes cases reported with unknown sex.

NOTE: These tables should be used only for race/Hispanic ethnicity comparisons. See Table 21 for age-specific cases and rates and Tables $14-16$ for total and sex-specific cases and rates. Cases in the 0-4 age group may include cases due to perinatal transmission. No population data exist for unknown sex, unknown age, or other/unknown race; therefore rates are not calculated. 
Table 23A. Gonorrhea - Reported Cases and Rates of Reported Cases Among Females Aged 15-24 Years by Age, United States, 2014-2018

\begin{tabular}{|c|c|c|c|c|c|c|c|c|c|c|}
\hline \multirow[b]{2}{*}{ Ages } & \multicolumn{5}{|c|}{ Cases } & \multicolumn{5}{|c|}{ Rates per 100,000 Females } \\
\hline & 2014 & 2015 & 2016 & 2017 & 2018 & 2014 & 2015 & 2016 & 2017 & 2018 \\
\hline 15 & 3,487 & 3,477 & 3,678 & 3,976 & 3,750 & 171.3 & 167.4 & 179.0 & 197.2 & 186.0 \\
\hline 16 & 6,188 & 6,090 & 6,573 & 7,600 & 6,921 & 303.9 & 297.8 & 315.4 & 367.7 & 334.9 \\
\hline 17 & 8,830 & 9,117 & 9,855 & 11,479 & 11,047 & 431.6 & 444.9 & 479.7 & 547.0 & 526.4 \\
\hline 18 & 12,196 & 12,769 & 14,304 & 16,429 & 16,679 & 591.9 & 619.3 & 694.1 & 793.0 & 805.1 \\
\hline 19 & 13,698 & 14,024 & 15,300 & 18,089 & 18,231 & 650.5 & 674.8 & 737.8 & 870.4 & 877.3 \\
\hline 20 & 13,801 & 13,835 & 14,657 & 16,950 & 17,477 & 642.1 & 651.7 & 701.7 & 811.3 & 836.5 \\
\hline 21 & 13,324 & 13,331 & 14,149 & 15,968 & 16,556 & 611.5 & 615.2 & 663.1 & 758.8 & 786.8 \\
\hline 22 & 12,031 & 12,597 & 13,540 & 14,933 & 14,912 & 536.0 & 573.2 & 621.4 & 694.4 & 693.4 \\
\hline 23 & 10,746 & 11,271 & 12,245 & 14,046 & 13,670 & 467.5 & 497.7 & 553.9 & 639.5 & 622.3 \\
\hline 24 & 9,427 & 10,071 & 11,339 & 12,681 & 13,048 & 409.6 & 434.2 & 497.8 & 569.0 & 585.5 \\
\hline Total & 103,728 & 106,582 & 115,640 & 132,151 & 132,291 & 483.4 & 498.5 & 545.0 & 626.3 & 627.0 \\
\hline
\end{tabular}

NOTE: Cases reported with unknown sex are not included in this table.

Table 23B. Gonorrhea - Reported Cases and Rates of Reported Cases Among Males Aged 15-24 Years by Age, United States, 2014-2018

\begin{tabular}{|c|c|c|c|c|c|c|c|c|c|c|}
\hline \multirow[b]{2}{*}{ Ages } & \multicolumn{5}{|c|}{ Cases } & \multicolumn{5}{|c|}{ Rates per 100,000 Males } \\
\hline & 2014 & 2015 & 2016 & 2017 & 2018 & 2014 & 2015 & 2016 & 2017 & 2018 \\
\hline 15 & 1,169 & 1,222 & 1,294 & 1,335 & 1,333 & 54.9 & 56.2 & 60.4 & 63.6 & 63.5 \\
\hline 16 & 2,427 & 2,645 & 3,078 & 3,374 & 3,189 & 113.9 & 123.6 & 141.1 & 156.5 & 147.9 \\
\hline 17 & 4,250 & 4,825 & 5,504 & 6,301 & 6,185 & 198.7 & 224.9 & 256.0 & 286.7 & 281.4 \\
\hline 18 & 7,001 & 7,778 & 8,945 & 10,482 & 10,356 & 323.4 & 360.8 & 414.4 & 483.2 & 477.4 \\
\hline 19 & 9,134 & 9,931 & 11,495 & 13,426 & 13,551 & 411.3 & 454.6 & 529.8 & 616.3 & 622.1 \\
\hline 20 & 10,684 & 11,394 & 12,949 & 15,085 & 15,280 & 470.7 & 508.6 & 589.3 & 689.6 & 698.5 \\
\hline 21 & 11,498 & 12,668 & 14,178 & 15,985 & 16,089 & 497.6 & 553.2 & 629.3 & 722.0 & 726.7 \\
\hline 22 & 11,707 & 13,171 & 14,754 & 16,484 & 16,421 & 494.4 & 564.9 & 640.9 & 726.1 & 723.3 \\
\hline 23 & 11,675 & 13,085 & 15,060 & 16,663 & 16,968 & 486.5 & 548.0 & 643.0 & 718.6 & 731.8 \\
\hline 24 & 11,150 & 12,971 & 15,026 & 16,819 & 17,055 & 466.3 & 536.4 & 627.0 & 713.0 & 723.0 \\
\hline Total & 80,695 & 89,690 & 102,283 & 115,954 & 116,427 & 358.3 & 399.2 & 458.8 & 523.5 & 525.6 \\
\hline
\end{tabular}

NOTE: Cases reported with unknown sex are not included in this table. 
Table 24. All Stages of Syphilis* - Reported Cases and Rates of Reported Cases by State/Territory and Region in Alphabetical Order, United States, 2014-2018

\begin{tabular}{|c|c|c|c|c|c|c|c|c|c|c|}
\hline \multirow[b]{2}{*}{ State/Territory } & \multicolumn{5}{|c|}{ Cases } & \multicolumn{5}{|c|}{ Rates per 100,000 Population } \\
\hline & 2014 & 2015 & 2016 & 2017 & 2018 & 2014 & 2015 & 2016 & 2017 & 2018 \\
\hline Alabama & 475 & 657 & 905 & 1,202 & 1,285 & 9.8 & 13.5 & 18.6 & 24.7 & 26.4 \\
\hline Alaska & 45 & 24 & 24 & 28 & 113 & 6.1 & 3.3 & 3.2 & 3.8 & 15.3 \\
\hline Arizona & 1,459 & 1,496 & 1,905 & 2,424 & 3,249 & 21.7 & 21.9 & 27.5 & 34.5 & 46.3 \\
\hline Arkansas & 390 & 500 & 567 & 726 & 964 & 13.1 & 16.8 & 19.0 & 24.2 & 32.1 \\
\hline California & 11,443 & 14,449 & 17,603 & 21,804 & 25,256 & 29.5 & 36.9 & 44.8 & 55.1 & 63.9 \\
\hline Colorado & 355 & 553 & 739 & 817 & 1,084 & 6.6 & 10.1 & 13.3 & 14.6 & 19.3 \\
\hline Connecticut & 169 & 220 & 217 & 283 & 264 & 4.7 & 6.1 & 6.1 & 7.9 & 7.4 \\
\hline Delaware & 110 & 110 & 149 & 194 & 129 & 11.8 & 11.6 & 15.7 & 20.2 & 13.4 \\
\hline District of Columbia & 281 & 322 & 568 & 845 & 763 & 42.6 & 47.9 & 83.4 & 121.8 & 109.9 \\
\hline Florida & 6,103 & 7,132 & 8,333 & 8,951 & 10,700 & 30.7 & 35.2 & 40.4 & 42.7 & 51.0 \\
\hline Georgia & 3,384 & 4,156 & 4,112 & 4,310 & 4,928 & 33.5 & 40.7 & 39.9 & 41.3 & 47.3 \\
\hline Hawaii & 106 & 163 & 215 & 165 & 210 & 7.5 & 11.4 & 15.1 & 11.6 & 14.7 \\
\hline Idaho & 46 & 102 & 127 & 151 & 134 & 2.8 & 6.2 & 7.5 & 8.8 & 7.8 \\
\hline Illinois & 2,796 & 3,290 & 4,039 & 3,838 & 4,471 & 21.7 & 25.6 & 31.6 & 30.0 & 34.9 \\
\hline Indiana & 475 & 699 & 778 & 788 & 985 & 7.2 & 10.6 & 11.7 & 11.8 & 14.8 \\
\hline lowa & 239 & 232 & 276 & 290 & 286 & 7.7 & 7.4 & 8.8 & 9.2 & 9.1 \\
\hline Kansas & 200 & 240 & 303 & 338 & 495 & 6.9 & 8.2 & 10.4 & 11.6 & 17.0 \\
\hline Kentucky & 447 & 433 & 572 & 722 & 881 & 10.1 & 9.8 & 12.9 & 16.2 & 19.8 \\
\hline Louisiana & 2,173 & 2,466 & 2,599 & 2,856 & 2,747 & 46.7 & 52.8 & 55.5 & 61.0 & 58.6 \\
\hline Maine & 23 & 38 & 64 & 132 & 147 & 1.7 & 2.9 & 4.8 & 9.9 & 11.0 \\
\hline Maryland & 1,475 & 1,870 & 1,842 & 2,059 & 2,536 & 24.7 & 31.1 & 30.6 & 34.0 & 41.9 \\
\hline Massachusetts & 813 & 1,263 & 1,446 & 1,474 & 1,305 & 12.1 & 18.6 & 21.2 & 21.5 & 19.0 \\
\hline Michigan & 1,095 & 1,089 & 1,092 & 1,267 & 1,691 & 11.0 & 11.0 & 11.0 & 12.7 & 17.0 \\
\hline Minnesota & 631 & 653 & 853 & 934 & 918 & 11.6 & 11.9 & 15.5 & 16.7 & 16.5 \\
\hline Mississippi & 642 & 760 & 925 & 937 & 1,454 & 21.4 & 25.4 & 30.9 & 31.4 & 48.7 \\
\hline Missouri & 771 & 778 & 955 & 1,337 & 1,913 & 12.7 & 12.8 & 15.7 & 21.9 & 31.3 \\
\hline Montana & 9 & 20 & 24 & 85 & 104 & 0.9 & 1.9 & 2.3 & 8.1 & 9.9 \\
\hline Nebraska & 96 & 81 & 121 & 118 & 219 & 5.1 & 4.3 & 6.3 & 6.1 & 11.4 \\
\hline Nevada & 893 & 915 & 1,313 & 1,684 & 2,000 & 31.5 & 31.7 & 44.7 & 56.2 & 66.7 \\
\hline New Hampshire & 79 & 84 & 100 & 109 & 137 & 6.0 & 6.3 & 7.5 & 8.1 & 10.2 \\
\hline New Jersey & 1,172 & 1,306 & 1,620 & 1,867 & 1,777 & 13.1 & 14.6 & 18.1 & 20.7 & 19.7 \\
\hline New Mexico & 283 & 332 & 470 & 510 & 812 & 13.6 & 15.9 & 22.6 & 24.4 & 38.9 \\
\hline New York & 7,129 & 7,795 & 9,456 & 9,877 & 10,183 & 36.1 & 39.4 & 47.9 & 49.8 & 51.3 \\
\hline North Carolina & 1,998 & 2,741 & 2,655 & 2,949 & 2,987 & 20.1 & 27.3 & 26.2 & 28.7 & 29.1 \\
\hline North Dakota & 51 & 42 & 61 & 78 & 84 & 6.9 & 5.5 & 8.0 & 10.3 & 11.1 \\
\hline Ohio & 1,229 & 1,348 & 1,600 & 1,900 & 1,908 & 10.6 & 11.6 & 13.8 & 16.3 & 16.4 \\
\hline Oklahoma & 414 & 521 & 696 & 953 & 1,138 & 10.7 & 13.3 & 17.7 & 24.2 & 29.0 \\
\hline Oregon & 582 & 783 & 810 & 848 & 1,032 & 14.7 & 19.4 & 19.8 & 20.5 & 24.9 \\
\hline Pennsylvania & 1,523 & 1,788 & 2,037 & 2,235 & 2,414 & 11.9 & 14.0 & 15.9 & 17.5 & 18.9 \\
\hline Rhode Island & 160 & 163 & 234 & 221 & 284 & 15.2 & 15.4 & 22.2 & 20.9 & 26.8 \\
\hline South Carolina & 750 & 834 & 974 & 1,096 & 1,152 & 15.5 & 17.0 & 19.6 & 21.8 & 22.9 \\
\hline South Dakota & 95 & 71 & 57 & 75 & 74 & 11.1 & 8.3 & 6.6 & 8.6 & 8.5 \\
\hline Tennessee & 977 & 1,241 & 1,448 & 1,453 & 1,725 & 14.9 & 18.8 & 21.8 & 21.6 & 25.7 \\
\hline Texas & 7,805 & 8,250 & 9,564 & 12,127 & 12,969 & 29.0 & 30.0 & 34.3 & 42.8 & 45.8 \\
\hline Utah & 149 & 169 & 259 & 299 & 423 & 5.1 & 5.6 & 8.5 & 9.6 & 13.6 \\
\hline Vermont & 12 & 15 & 37 & 26 & 29 & 1.9 & 2.4 & 5.9 & 4.2 & 4.6 \\
\hline Virginia & 702 & 1,023 & 1,304 & 1,758 & 2,038 & 8.4 & 12.2 & 15.5 & 20.8 & 24.1 \\
\hline Washington & 854 & 1,109 & 1,414 & 1,751 & 1,912 & 12.1 & 15.5 & 19.4 & 23.6 & 25.8 \\
\hline West Virginia & 55 & 109 & 151 & 123 & 185 & 3.0 & 5.9 & 8.2 & 6.8 & 10.2 \\
\hline Wisconsin & 285 & 262 & 423 & 551 & 509 & 5.0 & 4.5 & 7.3 & 9.5 & 8.8 \\
\hline Wyoming & 6 & 10 & 17 & 19 & 42 & 1.0 & 1.7 & 2.9 & 3.3 & 7.2 \\
\hline US TOTAL & 63,454 & 74,707 & 88,053 & 101,584 & 115,045 & 19.9 & 23.2 & 27.3 & 31.2 & 35.3 \\
\hline Northeast & 11,080 & 12,672 & 15,211 & 16,224 & 16,540 & 19.7 & 22.5 & 27.1 & 28.7 & 29.3 \\
\hline Midwest & 7,963 & 8,785 & 10,558 & 11,514 & 13,553 & 11.8 & 12.9 & 15.5 & 16.9 & 19.9 \\
\hline South & 28,181 & 33,125 & 37,364 & 43,261 & 48,581 & 23.5 & 27.3 & 30.5 & 35.0 & 39.3 \\
\hline West & 16,230 & 20,125 & 24,920 & 30,585 & 36,371 & 21.6 & 26.5 & 32.5 & 39.5 & 47.0 \\
\hline American Samoa & NR & NR & NR & NR & 0 & - & - & - & - & 0.0 \\
\hline Guam & 13 & 22 & 13 & 21 & 30 & 8.1 & 13.6 & 7.8 & 12.5 & 17.9 \\
\hline Northern Mariana Islands & NR & NR & NR & NR & 2 & - & - & - & - & 3.8 \\
\hline Puerto Rico & 960 & 1,267 & 1,185 & 1,055 & 1,088 & 27.1 & 36.5 & 34.7 & 31.5 & 32.5 \\
\hline Virgin Islands & 6 & 25 & 2 & 0 & NR & 5.8 & 24.3 & 1.9 & 0.0 & - \\
\hline TERRITORIES & 979 & 1,314 & 1,200 & 1,076 & 1,120 & 25.7 & 35.1 & 32.6 & 29.7 & 30.9 \\
\hline TOTAL & 64,433 & 76,021 & 89,253 & 102,660 & 116,165 & 20.0 & 23.4 & 27.3 & 31.2 & 35.3 \\
\hline
\end{tabular}

* See Section A1.9 in the Appendix for definition. See Section A1.11 in the Appendix for more information on interpreting case counts and rates in US territories. $\mathrm{NR}=$ No report. 
Table 25. All Stages of Syphilis* - Reported Cases and Rates of Reported Cases in Selected Metropolitan Statistical Areas (MSAs) ${ }^{\dagger}$ in Alphabetical Order, United States, 2014-2018

\begin{tabular}{|c|c|c|c|c|c|c|c|c|c|c|}
\hline \multirow[b]{2}{*}{ MSAs } & \multicolumn{5}{|c|}{ Cases } & \multicolumn{5}{|c|}{ Rates per 100,000 Population } \\
\hline & 2014 & 2015 & 2016 & 2017 & 2018 & 2014 & 2015 & 2016 & 2017 & 2018 \\
\hline Atlanta-Sandy Springs-Roswell, GA & 2,669 & 3,106 & 3,219 & 3,281 & 3,559 & 47.5 & 54.4 & 55.6 & 55.8 & 60.5 \\
\hline Austin-Round Rock, TX & 681 & 623 & 825 & 1,012 & 985 & 35.0 & 31.1 & 40.1 & 47.8 & 46.6 \\
\hline Baltimore-Columbia-Towson, MD & 816 & 1,015 & 1,031 & 1,084 & 1,392 & 29.3 & 36.3 & 36.8 & 38.6 & 49.6 \\
\hline Birmingham-Hoover, AL & 157 & 197 & $248^{5}$ & 314 & 344 & 13.7 & 17.2 & $21.6^{\S}$ & 27.3 & 29.9 \\
\hline Boston-Cambridge-Newton, MA-NH & 597 & $827^{\ddagger}$ & $1,000^{\ddagger}$ & 1,068 & 973 & 12.6 & $17.3^{\ddagger}$ & $20.9^{\ddagger}$ & 22.1 & 20.1 \\
\hline Buffalo-Cheektowaga-Niagara Falls, NY & 135 & 182 & 146 & 176 & 162 & 11.9 & 16.0 & 12.9 & 15.5 & 14.2 \\
\hline Charlotte-Concord-Gastonia, NC-SC & 533 & 732 & 851 & 876 & 843 & 22.4 & 30.2 & 34.4 & 34.7 & 33.4 \\
\hline Chicago-Naperville-Elgin, IL-IN-WI & 2,559 & 3,060 & 3,805 & 3,536 & 4,096 & 26.8 & 32.0 & 40.0 & 37.1 & 43.0 \\
\hline Cincinnati, OH-KY-IN & 381 & 319 & 269 & 326 & 430 & 17.7 & 14.8 & 12.4 & 15.0 & 19.7 \\
\hline Cleveland-Elyria, $\mathrm{OH}$ & 199 & 229 & 378 & 430 & 358 & 9.6 & 11.1 & 18.4 & 20.9 & 17.4 \\
\hline Columbus, $\mathrm{OH}$ & 441 & 518 & 578 & 700 & 564 & 22.1 & 25.6 & 28.3 & 33.7 & 27.1 \\
\hline Dallas-Fort Worth-Arlington, TX & 2,231 & 2,261 & 2,661 & 3,849 & 3,938 & 32.1 & 31.8 & 36.8 & 52.0 & 53.2 \\
\hline Denver-Aurora-Lakewood, CO & 298 & 426 & 552 & 618 & 793 & 10.8 & 15.1 & 19.3 & 21.4 & 27.5 \\
\hline Detroit-Warren-Dearborn, MI & 804 & 790 & 751 & 802 & 1,155 & 18.7 & 18.4 & 17.5 & 18.6 & 26.8 \\
\hline Hartford-West Hartford-East Hartford, CT & 52 & 84 & 55 & 66 & 77 & 4.3 & 6.9 & 4.6 & 5.5 & 6.4 \\
\hline Houston-The Woodlands-Sugar Land, TX & 2,316 & 2,568 & 2,817 & 3,244 & 3,644 & 35.7 & 38.6 & 41.6 & 47.1 & 52.9 \\
\hline Indianapolis-Carmel-Anderson, IN & 285 & 408 & 415 & 457 & 521 & 14.5 & 20.5 & 20.7 & 22.5 & 25.7 \\
\hline Jacksonville, FL & 270 & 435 & 445 & 644 & 686 & 19.0 & 30.0 & 30.1 & 42.8 & 45.6 \\
\hline Kansas City, MO-KS & 406 & 365 & 434 & 470 & 795 & 19.6 & 17.5 & 20.6 & 22.1 & 37.3 \\
\hline Las Vegas-Henderson-Paradise, NV & 830 & 826 & 1,194 & 1,504 & 1,700 & 40.1 & 39.1 & 55.4 & 68.2 & 77.1 \\
\hline Los Angeles-Long Beach-Anaheim, CA & 4,739 & 5,813 & 7,098 & 8,705 & 9,783 & 35.7 & 43.6 & 53.3 & 65.2 & 73.3 \\
\hline Louisville-Jefferson County, KY-IN & 239 & 270 & 381 & 421 & 508 & 18.8 & 21.1 & 29.7 & 32.5 & 39.3 \\
\hline Memphis, TN-MS-AR & 475 & 575 & 762 & 661 & 786 & 35.4 & 42.8 & 56.7 & 49.0 & 58.3 \\
\hline Miami-Fort Lauderdale-West Palm Beach, FL & 3,314 & 3,640 & 4,102 & 4,279 & 5,106 & 55.9 & 60.5 & 67.6 & 69.5 & 82.9 \\
\hline Milwaukee-Waukesha-West Allis, WI & 184 & 148 & 228 & 295 & 242 & 11.7 & 9.4 & 14.5 & 18.7 & 15.4 \\
\hline Minneapolis-St. Paul-Bloomington, MN-WI & 585 & 592 & 742 & 791 & 719 & 16.7 & 16.8 & 20.9 & 22.0 & 20.0 \\
\hline Nashville-Davidson-Murfreesboro-Franklin, TN & 305 & 359 & 383 & 363 & 577 & 17.0 & 19.6 & 20.5 & 19.1 & 30.3 \\
\hline New Orleans-Metairie, LA & 736 & 765 & 849 & 855 & 916 & 58.8 & 60.6 & 66.9 & 67.0 & 71.8 \\
\hline New York-Newark-Jersey City, NY-NJ-PA & 7,481 & 8,172 & 10,116 & 10,577 & 10,752 & 37.2 & 40.5 & 50.2 & 52.0 & 52.9 \\
\hline Oklahoma City, OK & 231 & 264 & 406 & 534 & 540 & 17.3 & 19.4 & 29.6 & 38.6 & 39.0 \\
\hline Orlando-Kissimmee-Sanford, FL & 784 & 916 & 1,183 & 1,196 & 1,391 & 33.8 & 38.4 & 48.5 & 47.7 & 55.4 \\
\hline Philadelphia-Camden-Wilmington, PA-NJ-DE-MD & 1,273 & 1,393 & 1,647 & 1,820 & 1,775 & 21.0 & 22.9 & 27.1 & 29.9 & 29.1 \\
\hline Phoenix-Mesa-Scottsdale, AZ & 1,065 & 1,126 & 1,501 & 1,882 & 2,504 & 23.7 & 24.6 & 32.2 & 39.7 & 52.9 \\
\hline Pittsburgh, PA & 154 & 273 & 236 & 192 & 244 & 6.5 & 11.6 & 10.1 & 8.2 & 10.5 \\
\hline Portland-Vancouver-Hillsboro, OR-WA & 473 & 604 & 665 & 654 & 794 & 20.1 & 25.3 & 27.4 & 26.7 & 32.4 \\
\hline Providence-Warwick, RI-MA & 205 & 233 & $288^{\ddagger}$ & $295^{\ddagger}$ & 341 & 12.7 & $14.4^{\ddagger}$ & $17.8^{\ddagger}$ & 18.2 & 21.0 \\
\hline Raleigh, NC & 317 & 410 & 384 & 438 & 450 & 25.5 & 32.2 & 29.5 & 32.8 & 33.7 \\
\hline Richmond, VA & 145 & 207 & 305 & 394 & 480 & 11.5 & 16.3 & 23.8 & 30.4 & 37.1 \\
\hline Riverside-San Bernardino-Ontario, CA & 950 & 1,165 & 1,554 & 1,960 & 2,587 & 21.4 & 26.0 & 34.3 & 42.8 & 56.5 \\
\hline Sacramento-Roseville-Arden-Arcade, CA & 371 & 609 & 611 & 807 & 1,092 & 16.5 & 26.8 & 26.6 & 34.7 & 47.0 \\
\hline Salt Lake City, UT & 109 & 122 & 188 & 203 & 270 & 9.5 & 10.4 & 15.8 & 16.9 & 22.4 \\
\hline San Antonio-New Braunfels, TX & 1,017 & 988 & 1,126 & 1,445 & 1,485 & 43.7 & 41.4 & 46.3 & 58.4 & 60.0 \\
\hline San Diego-Carlsbad, CA & 987 & 1,209 & 1,419 & 1,722 & 1,802 & 30.2 & 36.6 & 42.8 & 51.6 & 54.0 \\
\hline San Francisco-Oakland-Hayward, CA & 2,111 & 2,355 & 2,427 & 2,947 & 3,104 & 46.0 & 50.6 & 51.9 & 62.3 & 65.7 \\
\hline San Jose-Sunnyvale-Santa Clara, CA & 304 & 360 & 504 & 661 & 872 & 15.6 & 18.2 & 25.5 & 33.1 & 43.6 \\
\hline Seattle-Tacoma-Bellevue, WA & 590 & 759 & 968 & 1,185 & 1,238 & 16.1 & 20.3 & 25.5 & 30.6 & 32.0 \\
\hline St. Louis, MO-IL & 412 & 417 & 528 & 782 & 877 & 14.7 & 14.8 & 18.8 & 27.9 & 31.2 \\
\hline Tampa-St. Petersburg-Clearwater, FL & 807 & 958 & 1,129 & 1,061 & 1,258 & 27.7 & 32.2 & 37.2 & 34.3 & 40.7 \\
\hline Virginia Beach-Norfolk-Newport News, VA-NC & 220 & 375 & 516 & 566 & 658 & 12.8 & 21.7 & 29.9 & 32.8 & 38.1 \\
\hline Washington-Arlington-Alexandria, DC-VA-MD-WV & 811 & 1,058 & 1,107 & 2,295 & 2,437 & 13.4 & 17.4 & 18.1 & 36.9 & 39.2 \\
\hline SELECTED MSAs TOTAL & 48,054 & 55,106 & 65,027 & 74,443 & 82,603 & 27.5 & 31.2 & 36.5 & 41.4 & 45.9 \\
\hline
\end{tabular}

* See Section A1.9 in the Appendix for definition.

+ MSAs were selected on the basis of the largest population in the 2010 US Census.

₹ The variable used to identify county, which is used to classify cases into MSAs, was complete for $\leq 95 \%$ of cases in a state contributing data to this MSA. See Section A1.4 in the Appendix for more information.

§ 2016 county data for Alabama have been corrected and may not match previous reports.

NOTE: County was misclassified in the 2017 District of Columbia STD morbidity data resulting in inaccurate county-level case counts and rates. 
Table 26. Primary and Secondary Syphilis - Reported Cases and Rates of Reported Cases by State, Ranked by Rates, United States, 2018

\begin{tabular}{|c|c|c|c|}
\hline Rank* & State & Cases & Rate per 100,000 Population \\
\hline 1 & Nevada & 682 & 22.7 \\
\hline 2 & California & 7,607 & 19.2 \\
\hline 3 & Mississippi & 464 & 15.5 \\
\hline 4 & Georgia & 1,607 & 15.4 \\
\hline 5 & Arizona & 1,047 & 14.9 \\
\hline 6 & New Mexico & 304 & 14.6 \\
\hline 7 & Louisiana & 669 & 14.3 \\
\hline 8 & Florida & 2,880 & 13.7 \\
\hline 9 & Oklahoma & 531 & 13.5 \\
\hline 10 & New York & 2,654 & 13.4 \\
\hline 11 & Missouri & 806 & 13.2 \\
\hline 12 & Maryland & 737 & 12.2 \\
\hline 13 & Illinois & 1,408 & 11.0 \\
\hline \multirow[t]{2}{*}{14} & Washington & 802 & 10.8 \\
\hline & US TOTAL ${ }^{+}$ & 35,063 & 10.8 \\
\hline 15 & North Carolina & 1,098 & 10.7 \\
\hline 16 & Oregon & 424 & 10.2 \\
\hline 17 & Alabama & 477 & 9.8 \\
\hline 18 & Arkansas & 288 & 9.6 \\
\hline 19 & Rhode Island & 96 & 9.1 \\
\hline 20 & Texas & 2,538 & 9.0 \\
\hline 21 & Virginia & 702 & 8.3 \\
\hline 22 & Tennessee & 553 & 8.2 \\
\hline 23 & Kentucky & 366 & 8.2 \\
\hline 24 & Massachusetts & 552 & 8.0 \\
\hline 25 & South Carolina & 384 & 7.6 \\
\hline 26 & Alaska & 55 & 7.4 \\
\hline 27 & Michigan & 649 & 6.5 \\
\hline 28 & Hawaii & 92 & 6.4 \\
\hline 29 & Ohio & 740 & 6.3 \\
\hline 30 & New Jersey & 570 & 6.3 \\
\hline 31 & Pennsylvania & 797 & 6.2 \\
\hline 32 & Nebraska & 119 & 6.2 \\
\hline 33 & Colorado & 337 & 6.0 \\
\hline 34 & Maine & 74 & 5.5 \\
\hline 35 & Indiana & 367 & 5.5 \\
\hline 36 & Utah & 169 & 5.4 \\
\hline 37 & North Dakota & 41 & 5.4 \\
\hline 38 & Minnesota & 292 & 5.2 \\
\hline 39 & Kansas & 152 & 5.2 \\
\hline 40 & New Hampshire & 64 & 4.8 \\
\hline 41 & South Dakota & 41 & 4.7 \\
\hline 42 & Montana & 45 & 4.3 \\
\hline 43 & Wyoming & 23 & 4.0 \\
\hline 44 & West Virginia & 65 & 3.6 \\
\hline 45 & Delaware & 30 & 3.1 \\
\hline 46 & lowa & 86 & 2.7 \\
\hline 47 & Idaho & 46 & 2.7 \\
\hline 48 & Wisconsin & 152 & 2.6 \\
\hline 49 & Connecticut & 91 & 2.5 \\
\hline 50 & Vermont & 11 & 1.8 \\
\hline
\end{tabular}

* States were ranked by rate, then by case count, then in alphabetical order, with rates shown rounded to the nearest tenth.

${ }^{\dagger}$ Total includes cases reported by the District of Columbia with 279 cases and a rate of 40.2 cases per 100,000 population, but excludes territories. 
Table 27. Primary and Secondary Syphilis - Reported Cases and Rates of Reported Cases by State/Territory and Region in Alphabetical Order, United States, 2014-2018

\begin{tabular}{|c|c|c|c|c|c|c|c|c|c|c|}
\hline \multirow[b]{2}{*}{ State/Territory } & \multicolumn{5}{|c|}{ Cases } & \multicolumn{5}{|c|}{ Rates per 100,000 Population } \\
\hline & 2014 & 2015 & 2016 & 2017 & 2018 & 2014 & 2015 & 2016 & 2017 & 2018 \\
\hline Alabama & 161 & 280 & 376 & 424 & 477 & 3.3 & 5.8 & 7.7 & 8.7 & 9.8 \\
\hline Alaska & 15 & 8 & 8 & 13 & 55 & 2.0 & 1.1 & 1.1 & 1.8 & 7.4 \\
\hline Arizona & 577 & 589 & 721 & 943 & 1,047 & 8.6 & 8.6 & 10.4 & 13.4 & 14.9 \\
\hline Arkansas & 121 & 134 & 150 & 234 & 288 & 4.1 & 4.5 & 5.0 & 7.8 & 9.6 \\
\hline California & 3,835 & 4,908 & 5,891 & 6,708 & 7,607 & 9.9 & 12.5 & 15.0 & 17.0 & 19.2 \\
\hline Colorado & 186 & 245 & 250 & 292 & 337 & 3.5 & 4.5 & 4.5 & 5.2 & 6.0 \\
\hline Connecticut & 86 & 92 & 110 & 110 & 91 & 2.4 & 2.6 & 3.1 & 3.1 & 2.5 \\
\hline Delaware & 47 & 41 & 58 & 57 & 30 & 5.0 & 4.3 & 6.1 & 5.9 & 3.1 \\
\hline District of Columbia & 116 & 95 & 161 & 274 & 279 & 17.6 & 14.1 & 23.6 & 39.5 & 40.2 \\
\hline Florida & 1,740 & 2,083 & 2,406 & 2,390 & 2,880 & 8.7 & 10.3 & 11.7 & 11.4 & 13.7 \\
\hline Georgia & 1,234 & 1,413 & 1,350 & 1,489 & 1,607 & 12.2 & 13.8 & 13.1 & 14.3 & 15.4 \\
\hline Hawaii & 68 & 91 & 112 & 94 & 92 & 4.8 & 6.4 & 7.8 & 6.6 & 6.4 \\
\hline Idaho & 12 & 57 & 50 & 64 & 46 & 0.7 & 3.4 & 3.0 & 3.7 & 2.7 \\
\hline Illinois & 863 & 1,085 & 1,260 & 1,225 & 1,408 & 6.7 & 8.4 & 9.8 & 9.6 & 11.0 \\
\hline Indiana & 168 & 285 & 326 & 319 & 367 & 2.5 & 4.3 & 4.9 & 4.8 & 5.5 \\
\hline lowa & 72 & 75 & 89 & 101 & 86 & 2.3 & 2.4 & 2.8 & 3.2 & 2.7 \\
\hline Kansas & 60 & 87 & 124 & 133 & 152 & 2.1 & 3.0 & 4.3 & 4.6 & 5.2 \\
\hline Kentucky & 158 & 145 & 219 & 262 & 366 & 3.6 & 3.3 & 4.9 & 5.9 & 8.2 \\
\hline Louisiana & 575 & 696 & 750 & 679 & 669 & 12.4 & 14.9 & 16.0 & 14.5 & 14.3 \\
\hline Maine & 16 & 28 & 42 & 65 & 74 & 1.2 & 2.1 & 3.2 & 4.9 & 5.5 \\
\hline Maryland & 449 & 509 & 509 & 573 & 737 & 7.5 & 8.5 & 8.5 & 9.5 & 12.2 \\
\hline Massachusetts & 301 & 418 & 489 & 538 & 552 & 4.5 & 6.2 & 7.2 & 7.8 & 8.0 \\
\hline Michigan & 421 & 403 & 365 & 480 & 649 & 4.2 & 4.1 & 3.7 & 4.8 & 6.5 \\
\hline Minnesota & 257 & 246 & 306 & 292 & 292 & 4.7 & 4.5 & 5.5 & 5.2 & 5.2 \\
\hline Mississippi & 189 & 219 & 326 & 310 & 464 & 6.3 & 7.3 & 10.9 & 10.4 & 15.5 \\
\hline Missouri & 352 & 307 & 400 & 507 & 806 & 5.8 & 5.0 & 6.6 & 8.3 & 13.2 \\
\hline Montana & 8 & 13 & 14 & 48 & 45 & 0.8 & 1.3 & 1.3 & 4.6 & 4.3 \\
\hline Nebraska & 50 & 45 & 67 & 43 & 119 & 2.7 & 2.4 & 3.5 & 2.2 & 6.2 \\
\hline Nevada & 357 & 335 & 444 & 587 & 682 & 12.6 & 11.6 & 15.1 & 19.6 & 22.7 \\
\hline New Hampshire & 36 & 40 & 40 & 43 & 64 & 2.7 & 3.0 & 3.0 & 3.2 & 4.8 \\
\hline New Jersey & 297 & 372 & 472 & 499 & 570 & 3.3 & 4.2 & 5.3 & 5.5 & 6.3 \\
\hline New Mexico & 126 & 118 & 189 & 193 & 304 & 6.0 & 5.7 & 9.1 & 9.2 & 14.6 \\
\hline New York & 1,727 & 2,006 & 2,455 & 2,355 & 2,654 & 8.7 & 10.1 & 12.4 & 11.9 & 13.4 \\
\hline North Carolina & 733 & 1,196 & 1,082 & 1,138 & 1,098 & 7.4 & 11.9 & 10.7 & 11.1 & 10.7 \\
\hline North Dakota & 13 & 11 & 33 & 44 & 41 & 1.8 & 1.5 & 4.4 & 5.8 & 5.4 \\
\hline Ohio & 568 & 560 & 716 & 832 & 740 & 4.9 & 4.8 & 6.2 & 7.1 & 6.3 \\
\hline Oklahoma & 151 & 209 & 264 & 373 & 531 & 3.9 & 5.3 & 6.7 & 9.5 & 13.5 \\
\hline Oregon & 272 & 345 & 327 & 352 & 424 & 6.9 & 8.6 & 8.0 & 8.5 & 10.2 \\
\hline Pennsylvania & 532 & 655 & 755 & 793 & 797 & 4.2 & 5.1 & 5.9 & 6.2 & 6.2 \\
\hline Rhode Island & 71 & 77 & 90 & 71 & 96 & 6.7 & 7.3 & 8.5 & 6.7 & 9.1 \\
\hline South Carolina & 250 & 294 & 316 & 361 & 384 & 5.2 & 6.0 & 6.4 & 7.2 & 7.6 \\
\hline South Dakota & 53 & 39 & 26 & 33 & 41 & 6.2 & 4.5 & 3.0 & 3.8 & 4.7 \\
\hline Tennessee & 237 & 349 & 390 & 488 & 553 & 3.6 & 5.3 & 5.9 & 7.3 & 8.2 \\
\hline Texas & 1,636 & 1,680 & 1,955 & 2,233 & 2,538 & 6.1 & 6.1 & 7.0 & 7.9 & 9.0 \\
\hline Utah & 47 & 65 & 92 & 117 & 169 & 1.6 & 2.2 & 3.0 & 3.8 & 5.4 \\
\hline Vermont & 5 & 9 & 23 & 13 & 11 & 0.8 & 1.4 & 3.7 & 2.1 & 1.8 \\
\hline Virginia & 289 & 334 & 459 & 536 & 702 & 3.5 & 4.0 & 5.5 & 6.3 & 8.3 \\
\hline Washington & 344 & 445 & 565 & 677 & 802 & 4.9 & 6.2 & 7.8 & 9.1 & 10.8 \\
\hline West Virginia & 28 & 52 & 53 & 62 & 65 & 1.5 & 2.8 & 2.9 & 3.4 & 3.6 \\
\hline Wisconsin & 86 & 79 & 132 & 173 & 152 & 1.5 & 1.4 & 2.3 & 3.0 & 2.6 \\
\hline Wyoming & 4 & 5 & 7 & 4 & 23 & 0.7 & 0.9 & 1.2 & 0.7 & 4.0 \\
\hline US TOTAL & 19,999 & 23,872 & 27,814 & 30,644 & 35,063 & 6.3 & 7.4 & 8.6 & 9.4 & 10.8 \\
\hline Northeast & 3,071 & 3,697 & 4,476 & 4,487 & 4,909 & 5.5 & 6.6 & 8.0 & 7.9 & 8.7 \\
\hline Midwest & 2,963 & 3,222 & 3,844 & 4,182 & 4,853 & 4.4 & 4.7 & 5.7 & 6.1 & 7.1 \\
\hline South & 8,114 & 9,729 & 10,824 & 11,883 & 13,668 & 6.8 & 8.0 & 8.8 & 9.6 & 11.1 \\
\hline West & 5,851 & 7,224 & 8,670 & 10,092 & 11,633 & 7.8 & 9.5 & 11.3 & 13.0 & 15.0 \\
\hline American Samoa & NR & NR & NR & NR & 0 & - & - & - & - & 0.0 \\
\hline Guam & 7 & 2 & 2 & 13 & 18 & 4.3 & 1.2 & 1.2 & 7.8 & 10.8 \\
\hline Northern Mariana Islands & NR & NR & NR & NR & 1 & - & - & - & - & 1.9 \\
\hline Puerto Rico & 484 & 531 & 493 & 411 & 365 & 13.6 & 15.3 & 14.5 & 12.3 & 10.9 \\
\hline Virgin Islands & 2 & 8 & 0 & 0 & NR & 1.9 & 7.8 & 0.0 & 0.0 & - \\
\hline TERRITORIES & 493 & 541 & 495 & 424 & 384 & 12.9 & 14.5 & 13.4 & 11.7 & 10.6 \\
\hline TOTAL & 20,492 & 24,413 & 28,309 & 31,068 & 35,447 & 6.4 & 7.5 & 8.7 & 9.4 & 10.8 \\
\hline
\end{tabular}

$\mathrm{NR}=$ No report.

NOTE: See Section A1.11 in the Appendix for more information on interpreting case counts and rates in US territories.

Centers for Disease Control and Prevention: STD Surveillance 2018

Tables 
Table 28. Primary and Secondary Syphilis Among Females - Reported Cases and Rates of Reported Cases by State/Territory and Region in Alphabetical Order, United States, 2014-2018

\begin{tabular}{|c|c|c|c|c|c|c|c|c|c|c|}
\hline \multirow[b]{2}{*}{ State/Territory } & \multicolumn{5}{|c|}{ Cases } & \multicolumn{5}{|c|}{ Rates per 100,000 Population } \\
\hline & 2014 & 2015 & 2016 & 2017 & 2018 & 2014 & 2015 & 2016 & 2017 & 2018 \\
\hline Alabama & 17 & 37 & 80 & 70 & 88 & 0.7 & 1.5 & 3.2 & 2.8 & 3.5 \\
\hline Alaska & 1 & 1 & 0 & 1 & 4 & 0.3 & 0.3 & 0.0 & 0.3 & 1.1 \\
\hline Arizona & 50 & 47 & 80 & 158 & 177 & 1.5 & 1.4 & 2.3 & 4.5 & 5.0 \\
\hline Arkansas & 23 & 26 & 37 & 64 & 68 & 1.5 & 1.7 & 2.4 & 4.2 & 4.4 \\
\hline California & 318 & 476 & 744 & 902 & 1,255 & 1.6 & 2.4 & 3.8 & 4.5 & 6.3 \\
\hline Colorado & 6 & 6 & 16 & 18 & 25 & 0.2 & 0.2 & 0.6 & 0.6 & 0.9 \\
\hline Connecticut & 7 & 15 & 12 & 13 & 5 & 0.4 & 0.8 & 0.7 & 0.7 & 0.3 \\
\hline Delaware & 2 & 2 & 3 & 6 & 7 & 0.4 & 0.4 & 0.6 & 1.2 & 1.4 \\
\hline District of Columbia & 5 & 6 & 5 & 7 & 12 & 1.4 & 1.7 & 1.4 & 1.9 & 3.3 \\
\hline Florida & 137 & 210 & 266 & 321 & 356 & 1.3 & 2.0 & 2.5 & 3.0 & 3.3 \\
\hline Georgia & 96 & 94 & 113 & 139 & 184 & 1.9 & 1.8 & 2.1 & 2.6 & 3.4 \\
\hline Hawaii & 2 & 1 & 10 & 6 & 7 & 0.3 & 0.1 & 1.4 & 0.8 & 1.0 \\
\hline Idaho & 0 & 9 & 6 & 15 & 5 & 0.0 & 1.1 & 0.7 & 1.8 & 0.6 \\
\hline Illinois & 81 & 84 & 95 & 85 & 120 & 1.2 & 1.3 & 1.5 & 1.3 & 1.8 \\
\hline Indiana & 11 & 28 & 36 & 27 & 26 & 0.3 & 0.8 & 1.1 & 0.8 & 0.8 \\
\hline lowa & 6 & 5 & 6 & 9 & 11 & 0.4 & 0.3 & 0.4 & 0.6 & 0.7 \\
\hline Kansas & 14 & 17 & 12 & 16 & 24 & 1.0 & 1.2 & 0.8 & 1.1 & 1.6 \\
\hline Kentucky & 22 & 23 & 21 & 41 & 70 & 1.0 & 1.0 & 0.9 & 1.8 & 3.1 \\
\hline Louisiana & 132 & 189 & 192 & 186 & 166 & 5.6 & 7.9 & 8.0 & 7.8 & 6.9 \\
\hline Maine & 3 & 6 & 3 & 9 & 7 & 0.4 & 0.9 & 0.4 & 1.3 & 1.0 \\
\hline Maryland & 49 & 58 & 54 & 47 & 81 & 1.6 & 1.9 & 1.7 & 1.5 & 2.6 \\
\hline Massachusetts & 23 & 25 & 24 & 26 & 41 & 0.7 & 0.7 & 0.7 & 0.7 & 1.2 \\
\hline Michigan & 31 & 34 & 31 & 34 & 69 & 0.6 & 0.7 & 0.6 & 0.7 & 1.4 \\
\hline Minnesota & 21 & 39 & 37 & 38 & 44 & 0.8 & 1.4 & 1.3 & 1.4 & 1.6 \\
\hline Mississippi & 17 & 32 & 58 & 88 & 152 & 1.1 & 2.1 & 3.8 & 5.7 & 9.9 \\
\hline Missouri & 34 & 54 & 66 & 93 & 161 & 1.1 & 1.7 & 2.1 & 3.0 & 5.2 \\
\hline Montana & 2 & 1 & 1 & 9 & 10 & 0.4 & 0.2 & 0.2 & 1.7 & 1.9 \\
\hline Nebraska & 4 & 3 & 5 & 7 & 14 & 0.4 & 0.3 & 0.5 & 0.7 & 1.5 \\
\hline Nevada & 23 & 23 & 50 & 75 & 119 & 1.6 & 1.6 & 3.4 & 5.0 & 8.0 \\
\hline New Hampshire & 4 & 3 & 4 & 4 & 3 & 0.6 & 0.4 & 0.6 & 0.6 & 0.4 \\
\hline New Jersey & 16 & 26 & 24 & 34 & 58 & 0.3 & 0.6 & 0.5 & 0.7 & 1.3 \\
\hline New Mexico & 14 & 11 & 27 & 19 & 46 & 1.3 & 1.0 & 2.6 & 1.8 & 4.4 \\
\hline New York & 49 & 59 & 115 & 121 & 171 & 0.5 & 0.6 & 1.1 & 1.2 & 1.7 \\
\hline North Carolina & 68 & 112 & 109 & 127 & 160 & 1.3 & 2.2 & 2.1 & 2.4 & 3.0 \\
\hline North Dakota & 5 & 0 & 3 & 5 & 10 & 1.4 & 0.0 & 0.8 & 1.4 & 2.7 \\
\hline Ohio & 76 & 68 & 94 & 112 & 92 & 1.3 & 1.1 & 1.6 & 1.9 & 1.5 \\
\hline Oklahoma & 15 & 21 & 41 & 67 & 144 & 0.8 & 1.1 & 2.1 & 3.4 & 7.3 \\
\hline Oregon & 22 & 35 & 35 & 52 & 92 & 1.1 & 1.7 & 1.7 & 2.5 & 4.4 \\
\hline Pennsylvania & 47 & 52 & 62 & 76 & 99 & 0.7 & 0.8 & 1.0 & 1.2 & 1.5 \\
\hline Rhode Island & 5 & 4 & 3 & 7 & 12 & 0.9 & 0.7 & 0.6 & 1.3 & 2.2 \\
\hline South Carolina & 23 & 37 & 52 & 56 & 67 & 0.9 & 1.5 & 2.0 & 2.2 & 2.6 \\
\hline South Dakota & 34 & 7 & 6 & 4 & 11 & 8.0 & 1.6 & 1.4 & 0.9 & 2.6 \\
\hline Tennessee & 34 & 23 & 56 & 47 & 67 & 1.0 & 0.7 & 1.6 & 1.4 & 1.9 \\
\hline Texas & 242 & 230 & 230 & 313 & 424 & 1.8 & 1.7 & 1.6 & 2.2 & 3.0 \\
\hline Utah & 1 & 2 & 6 & 4 & 10 & 0.1 & 0.1 & 0.4 & 0.3 & 0.6 \\
\hline Vermont & 0 & 0 & 3 & 1 & 2 & 0.0 & 0.0 & 0.9 & 0.3 & 0.6 \\
\hline Virginia & 17 & 17 & 47 & 60 & 77 & 0.4 & 0.4 & 1.1 & 1.4 & 1.8 \\
\hline Washington & 18 & 30 & 51 & 70 & 100 & 0.5 & 0.8 & 1.4 & 1.9 & 2.7 \\
\hline West Virginia & 6 & 9 & 10 & 21 & 19 & 0.6 & 1.0 & 1.1 & 2.3 & 2.1 \\
\hline Wisconsin & 7 & 0 & 7 & 12 & 18 & 0.2 & 0.0 & 0.2 & 0.4 & 0.6 \\
\hline Wyoming & 0 & 1 & 1 & 0 & 5 & 0.0 & 0.3 & 0.3 & 0.0 & 1.8 \\
\hline US TOTAL & 1,840 & 2,298 & 3,049 & 3,722 & 4,995 & 1.1 & 1.4 & 1.9 & 2.3 & 3.0 \\
\hline Northeast & 154 & 190 & 250 & 291 & 398 & 0.5 & 0.7 & 0.9 & 1.0 & 1.4 \\
\hline Midwest & 324 & 339 & 398 & 442 & 600 & 0.9 & 1.0 & 1.2 & 1.3 & 1.7 \\
\hline South & 905 & 1,126 & 1,374 & 1,660 & 2,142 & 1.5 & 1.8 & 2.2 & 2.6 & 3.4 \\
\hline West & 457 & 643 & 1,027 & 1,329 & 1,855 & 1.2 & 1.7 & 2.7 & 3.4 & 4.8 \\
\hline American Samoa & NR & NR & NR & NR & 0 & - & - & - & - & 0.0 \\
\hline Guam & 2 & 0 & 0 & 2 & 0 & 2.5 & 0.0 & 0.0 & 2.5 & 0.0 \\
\hline Northern Mariana Islands & $N R$ & NR & NR & NR & 0 & - & - & - & - & 0.0 \\
\hline Puerto Rico & 30 & 70 & 86 & 66 & 52 & 1.6 & 3.9 & 4.8 & 3.8 & 3.0 \\
\hline Virgin Islands & 1 & 4 & 0 & 0 & NR & 1.8 & 7.3 & 0.0 & 0.0 & - \\
\hline TERRITORIES & 33 & 74 & 86 & 68 & 52 & 1.7 & 3.8 & 4.5 & 3.6 & 2.8 \\
\hline TOTAL & 1,873 & 2,372 & 3,135 & 3,790 & 5,047 & 1.1 & 1.4 & 1.9 & 2.3 & 3.0 \\
\hline
\end{tabular}

$\mathrm{NR}=$ No report.

NOTE: Cases reported with unknown sex are not included in this table. See Section A1.11 in the Appendix for more information on interpreting case counts and rates in US territories. 
Table 29. Primary and Secondary Syphilis Among Males - Reported Cases and Rates of Reported Cases by State/Territory and Region in Alphabetical Order, United States, 2014-2018

\begin{tabular}{|c|c|c|c|c|c|c|c|c|c|c|}
\hline \multirow[b]{2}{*}{ State/Territory } & \multicolumn{5}{|c|}{ Cases } & \multicolumn{5}{|c|}{ Rates per 100,000 Population } \\
\hline & 2014 & 2015 & 2016 & 2017 & 2018 & 2014 & 2015 & 2016 & 2017 & 2018 \\
\hline Alabama & 144 & 243 & 296 & 354 & $\overline{389}$ & 6.1 & 10.3 & 12.6 & 15.0 & 16.5 \\
\hline Alaska & 14 & 7 & 8 & 12 & 51 & 3.6 & 1.8 & 2.1 & 3.1 & 13.2 \\
\hline Arizona & 527 & 542 & 641 & 785 & 870 & 15.8 & 16.0 & 18.6 & 22.5 & 24.9 \\
\hline Arkansas & 98 & 108 & 113 & 170 & 220 & 6.7 & 7.4 & 7.7 & 11.5 & 14.9 \\
\hline California & 3,515 & 4,430 & 5,143 & 5,804 & 6,349 & 18.2 & 22.8 & 26.4 & 29.5 & 32.3 \\
\hline Colorado & 180 & 239 & 234 & 274 & 312 & 6.7 & 8.7 & 8.4 & 9.7 & 11.1 \\
\hline Connecticut & 79 & 77 & 98 & 97 & 84 & 4.5 & 4.4 & 5.6 & 5.5 & 4.8 \\
\hline Delaware & 45 & 39 & 55 & 51 & 23 & 9.9 & 8.5 & 11.9 & 11.0 & 4.9 \\
\hline District of Columbia & 106 & 83 & 152 & 264 & 264 & 33.9 & 26.1 & 47.0 & 80.2 & 80.2 \\
\hline Florida & 1,602 & 1,873 & 2,140 & 2,069 & 2,524 & 16.5 & 18.9 & 21.3 & 20.2 & 24.6 \\
\hline Georgia & 1,138 & 1,319 & 1,237 & 1,350 & 1,421 & 23.1 & 26.5 & 24.6 & 26.6 & 28.0 \\
\hline Hawaii & 66 & 90 & 102 & 88 & 85 & 9.2 & 12.4 & 14.2 & 12.3 & 11.9 \\
\hline Idaho & 12 & 48 & 44 & 49 & 41 & 1.5 & 5.8 & 5.2 & 5.7 & 4.8 \\
\hline Illinois & 782 & 1,001 & 1,165 & 1,140 & 1,287 & 12.4 & 15.9 & 18.5 & 18.1 & 20.5 \\
\hline Indiana & 157 & 257 & 290 & 292 & 341 & 4.8 & 7.9 & 8.9 & 8.9 & 10.4 \\
\hline lowa & 66 & 70 & 83 & 92 & 75 & 4.3 & 4.5 & 5.3 & 5.9 & 4.8 \\
\hline Kansas & 46 & 70 & 112 & 117 & 128 & 3.2 & 4.8 & 7.7 & 8.1 & 8.8 \\
\hline Kentucky & 136 & 122 & 198 & 221 & 296 & 6.3 & 5.6 & 9.1 & 10.1 & 13.5 \\
\hline Louisiana & 443 & 507 & 558 & 493 & 503 & 19.5 & 22.2 & 24.4 & 21.5 & 22.0 \\
\hline Maine & 13 & 22 & 39 & 54 & 67 & 2.0 & 3.4 & 6.0 & 8.3 & 10.2 \\
\hline Maryland & 400 & 451 & 455 & 526 & 656 & 13.8 & 15.5 & 15.6 & 17.9 & 22.4 \\
\hline Massachusetts & 277 & 391 & 464 & 512 & 499 & 8.5 & 11.9 & 14.0 & 15.4 & 15.0 \\
\hline Michigan & 390 & 369 & 334 & 446 & 580 & 8.0 & 7.6 & 6.8 & 9.1 & 11.8 \\
\hline Minnesota & 235 & 207 & 267 & 252 & 248 & 8.7 & 7.6 & 9.7 & 9.1 & 8.9 \\
\hline Mississippi & 172 & 187 & 268 & 222 & 312 & 11.8 & 12.9 & 18.5 & 15.4 & 21.6 \\
\hline Missouri & 318 & 253 & 334 & 414 & 645 & 10.7 & 8.5 & 11.2 & 13.8 & 21.5 \\
\hline Montana & 6 & 12 & 13 & 39 & 35 & 1.2 & 2.3 & 2.5 & 7.4 & 6.6 \\
\hline Nebraska & 46 & 42 & 62 & 36 & 105 & 4.9 & 4.4 & 6.5 & 3.8 & 11.0 \\
\hline Nevada & 334 & 312 & 394 & 512 & 563 & 23.4 & 21.5 & 26.7 & 34.0 & 37.4 \\
\hline New Hampshire & 32 & 37 & 36 & 39 & 61 & 4.9 & 5.6 & 5.4 & 5.9 & 9.2 \\
\hline New Jersey & 281 & 346 & 448 & 465 & 511 & 6.4 & 7.9 & 10.3 & 10.6 & 11.6 \\
\hline New Mexico & 112 & 107 & 162 & 174 & 258 & 10.8 & 10.4 & 15.7 & 16.8 & 24.9 \\
\hline New York & 1,675 & 1,933 & 2,319 & 2,214 & 2,483 & 17.5 & 20.1 & 24.2 & 23.0 & 25.8 \\
\hline North Carolina & 665 & 1,084 & 973 & 1,011 & 937 & 13.7 & 22.2 & 19.7 & 20.2 & 18.7 \\
\hline North Dakota & 8 & 11 & 30 & 39 & 31 & 2.1 & 2.8 & 7.7 & 10.1 & 8.0 \\
\hline Ohio & 492 & 492 & 622 & 720 & 648 & 8.7 & 8.7 & 10.9 & 12.6 & 11.3 \\
\hline Oklahoma & 136 & 188 & 223 & 306 & 387 & 7.1 & 9.7 & 11.5 & 15.7 & 19.9 \\
\hline Oregon & 250 & 310 & 292 & 298 & 332 & 12.7 & 15.6 & 14.4 & 14.5 & 16.2 \\
\hline Pennsylvania & 485 & 602 & 693 & 717 & 698 & 7.8 & 9.6 & 11.1 & 11.4 & 11.1 \\
\hline Rhode Island & 66 & 73 & 87 & 64 & 84 & 12.9 & 14.3 & 17.0 & 12.4 & 16.3 \\
\hline South Carolina & 227 & 257 & 264 & 305 & 317 & 9.7 & 10.8 & 11.0 & 12.5 & 13.0 \\
\hline South Dakota & 19 & 32 & 20 & 29 & 30 & 4.4 & 7.4 & 4.6 & 6.6 & 6.8 \\
\hline Tennessee & 203 & 326 & 334 & 441 & 486 & 6.4 & 10.1 & 10.3 & 13.5 & 14.8 \\
\hline Texas & 1,394 & 1,450 & 1,725 & 1,920 & 2,114 & 10.4 & 10.6 & 12.5 & 13.7 & 15.0 \\
\hline Utah & 46 & 63 & 86 & 113 & 159 & 3.1 & 4.2 & 5.6 & 7.2 & 10.2 \\
\hline Vermont & 5 & 9 & 20 & 12 & 9 & 1.6 & 2.9 & 6.5 & 3.9 & 2.9 \\
\hline Virginia & 272 & 316 & 403 & 471 & 617 & 6.6 & 7.7 & 9.7 & 11.3 & 14.8 \\
\hline Washington & 326 & 414 & 514 & 606 & 701 & 9.2 & 11.5 & 14.1 & 16.4 & 18.9 \\
\hline West Virginia & 22 & 43 & 43 & 41 & 46 & 2.4 & 4.7 & 4.7 & 4.6 & 5.1 \\
\hline Wisconsin & 79 & 79 & 125 & 161 & 134 & 2.8 & 2.8 & 4.4 & 5.6 & 4.6 \\
\hline Wyoming & 4 & 4 & 6 & 4 & 18 & 1.3 & 1.3 & 2.0 & 1.4 & 6.1 \\
\hline US TOTAL & 18,146 & 21,547 & 24,724 & 26,885 & 30,034 & 11.6 & 13.6 & 15.5 & 16.8 & 18.7 \\
\hline Northeast & 2,913 & 3,490 & 4,204 & 4,174 & 4,496 & 10.7 & 12.7 & 15.3 & 15.2 & 16.3 \\
\hline Midwest & 2,638 & 2,883 & 3,444 & 3,738 & 4,252 & 7.9 & 8.6 & 10.3 & 11.1 & 12.6 \\
\hline South & 7,203 & 8,596 & 9,437 & 10,215 & 11,512 & 12.3 & 14.5 & 15.7 & 16.9 & 19.0 \\
\hline West & 5,392 & 6,578 & 7,639 & 8,758 & 9,774 & 14.4 & 17.4 & 20.0 & 22.7 & 25.3 \\
\hline American Samoa & NR & NR & NR & NR & 0 & - & - & - & - & 0.0 \\
\hline Guam & 5 & 2 & 2 & 11 & 18 & 6.1 & 2.4 & 2.3 & 12.8 & 20.9 \\
\hline Northern Mariana Islands & NR & NR & NR & NR & 1 & - & - & - & - & 3.6 \\
\hline Puerto Rico & 454 & 461 & 407 & 345 & 311 & 26.6 & 27.8 & 25.1 & 21.7 & 19.5 \\
\hline Virgin Islands & 1 & 4 & 0 & 0 & NR & 2.1 & 8.4 & 0.0 & 0.0 & - \\
\hline TERRITORIES & 460 & 467 & 409 & 356 & 330 & 25.1 & 26.1 & 23.2 & 20.6 & 19.1 \\
\hline TOTAL & 18,606 & 22,014 & 25,133 & 27,241 & 30,364 & 11.7 & 13.8 & 15.6 & 16.8 & 18.7 \\
\hline
\end{tabular}

$\mathrm{NR}=$ No report.

NOTE: Cases reported with unknown sex are not included in this table. See Section A1.11 in the Appendix for more information on interpreting case counts and rates in US territories. 
Table 30. Primary and Secondary Syphilis - Reported Cases and Rates of Reported Cases in Selected Metropolitan Statistical Areas (MSAs)* in Alphabetical Order, United States, 2014-2018

\begin{tabular}{|c|c|c|c|c|c|c|c|c|c|c|}
\hline \multirow[b]{2}{*}{ MSAs } & \multicolumn{5}{|c|}{ Cases } & \multicolumn{5}{|c|}{ Rates per 100,000 Population } \\
\hline & 2014 & 2015 & 2016 & 2017 & 2018 & 2014 & 2015 & 2016 & 2017 & 2018 \\
\hline Atlanta-Sandy Springs-Roswell, GA & 996 & 1,097 & 1,018 & 1,095 & 1,094 & 17.7 & 19.2 & 17.6 & 18.6 & 18.6 \\
\hline Austin-Round Rock, TX & 227 & 203 & 317 & 359 & 294 & 11.7 & 10.1 & 15.4 & 17.0 & 13.9 \\
\hline Baltimore-Columbia-Towson, MD & 282 & 343 & 332 & 342 & 446 & 10.1 & 12.3 & 11.9 & 12.2 & 15.9 \\
\hline Birmingham-Hoover, AL & 58 & 74 & $109^{\ddagger}$ & 122 & 124 & 5.1 & 6.5 & $9.5^{\ddagger}$ & 10.6 & 10.8 \\
\hline Boston-Cambridge-Newton, MA-NH & 227 & $271^{+}$ & $320^{+}$ & 373 & 390 & 4.8 & $5.7^{\dagger}$ & $6.7^{+}$ & 7.7 & 8.1 \\
\hline Buffalo-Cheektowaga-Niagara Falls, NY & 49 & 92 & 63 & 60 & 53 & 4.3 & 8.1 & 5.6 & 5.3 & 4.7 \\
\hline Charlotte-Concord-Gastonia, NC-SC & 220 & 333 & 378 & 355 & 333 & 9.2 & 13.7 & 15.3 & 14.1 & 13.2 \\
\hline Chicago-Naperville-Elgin, IL-IN-WI & 811 & 1,047 & 1,202 & 1,140 & 1,280 & 8.5 & 11.0 & 12.6 & 12.0 & 13.4 \\
\hline Cincinnati, OH-KY-IN & 153 & 93 & 81 & 103 & 158 & 7.1 & 4.3 & 3.7 & 4.7 & 7.3 \\
\hline Cleveland-Elyria, $\mathrm{OH}$ & 80 & 72 & 156 & 180 & 116 & 3.9 & 3.5 & 7.6 & 8.7 & 5.6 \\
\hline Columbus, $\mathrm{OH}$ & 250 & 274 & 316 & 353 & 241 & 12.5 & 13.6 & 15.5 & 17.0 & 11.6 \\
\hline Dallas-Fort Worth-Arlington, TX & 508 & 476 & 542 & 699 & 739 & 7.3 & 6.7 & 7.5 & 9.4 & 10.0 \\
\hline Denver-Aurora-Lakewood, CO & 153 & 192 & 187 & 216 & 241 & 5.6 & 6.8 & 6.6 & 7.5 & 8.3 \\
\hline Detroit-Warren-Dearborn, MI & 317 & 284 & 253 & 291 & 466 & 7.4 & 6.6 & 5.9 & 6.7 & 10.8 \\
\hline Hartford-West Hartford-East Hartford, CT & 26 & 44 & 32 & 32 & 30 & 2.1 & 3.6 & 2.7 & 2.6 & 2.5 \\
\hline Houston-The Woodlands-Sugar Land, TX & 414 & 439 & 411 & 386 & 647 & 6.4 & 6.6 & 6.1 & 5.6 & 9.4 \\
\hline Indianapolis-Carmel-Anderson, IN & 109 & 172 & 158 & 186 & 173 & 5.5 & 8.6 & 7.9 & 9.2 & 8.5 \\
\hline Jacksonville, FL & 69 & 91 & 118 & 194 & 219 & 4.9 & 6.3 & 8.0 & 12.9 & 14.6 \\
\hline Kansas City, MO-KS & 220 & 191 & 201 & 214 & 355 & 10.6 & 9.1 & 9.6 & 10.1 & 16.7 \\
\hline Las Vegas-Henderson-Paradise, NV & 318 & 305 & 398 & 519 & 554 & 15.4 & 14.4 & 18.5 & 23.5 & 25.1 \\
\hline Los Angeles-Long Beach-Anaheim, CA & 1,407 & 1,832 & 2,123 & 2,365 & 2,800 & 10.6 & 13.7 & 15.9 & 17.7 & 21.0 \\
\hline Louisville-Jefferson County, KY-IN & 83 & 87 & 149 & 150 & 219 & 6.5 & 6.8 & 11.6 & 11.6 & 16.9 \\
\hline Memphis, TN-MS-AR & 94 & 121 & 173 & 153 & 201 & 7.0 & 9.0 & 12.9 & 11.3 & 14.9 \\
\hline Miami-Fort Lauderdale-West Palm Beach, FL & 821 & 884 & 925 & 919 & 1,079 & 13.8 & 14.7 & 15.2 & 14.9 & 17.5 \\
\hline Milwaukee-Waukesha-West Allis, WI & 52 & 39 & 50 & 79 & 64 & 3.3 & 2.5 & 3.2 & 5.0 & 4.1 \\
\hline Minneapolis-St. Paul-Bloomington, MN-WI & 243 & 228 & 261 & 248 & 238 & 7.0 & 6.5 & 7.3 & 6.9 & 6.6 \\
\hline Nashville-Davidson-Murfreesboro-Franklin, TN & 74 & 116 & 115 & 160 & 227 & 4.1 & 6.3 & 6.2 & 8.4 & 11.9 \\
\hline New Orleans-Metairie, LA & 221 & 218 & 235 & 210 & 236 & 17.7 & 17.3 & 18.5 & 16.5 & 18.5 \\
\hline New York-Newark-Jersey City, NY-NJ-PA & 1,721 & 2,037 & 2,551 & 2,461 & 2,693 & 8.6 & 10.1 & 12.7 & 12.1 & 13.3 \\
\hline Oklahoma City, OK & 91 & 113 & 162 & 219 & 252 & 6.8 & 8.3 & 11.8 & 15.8 & 18.2 \\
\hline Orlando-Kissimmee-Sanford, FL & 239 & 299 & 384 & 318 & 366 & 10.3 & 12.5 & 15.7 & 12.7 & 14.6 \\
\hline Philadelphia-Camden-Wilmington, PA-NJ-DE-MD & 446 & 458 & 639 & 636 & 594 & 7.4 & 7.5 & 10.5 & 10.4 & 9.7 \\
\hline Phoenix-Mesa-Scottsdale, AZ & 416 & 452 & 574 & 775 & 819 & 9.3 & 9.9 & 12.3 & 16.4 & 17.3 \\
\hline Pittsburgh, PA & 78 & 150 & 100 & 71 & 91 & 3.3 & 6.4 & 4.3 & 3.0 & 3.9 \\
\hline Portland-Vancouver-Hillsboro, OR-WA & 206 & 261 & 263 & 255 & 300 & 8.8 & 10.9 & 10.8 & 10.4 & 12.2 \\
\hline Providence-Warwick, RI-MA & 93 & $111^{+}$ & $115^{+}$ & 98 & 127 & 5.8 & $6.9^{\dagger}$ & $7.1^{+}$ & 6.0 & 7.8 \\
\hline Raleigh, NC & 129 & 168 & 137 & 136 & 173 & 10.4 & 13.2 & 10.5 & 10.2 & 13.0 \\
\hline Richmond, VA & 68 & 73 & 104 & 124 & 210 & 5.4 & 5.7 & 8.1 & 9.6 & 16.2 \\
\hline Riverside-San Bernardino-Ontario, CA & 288 & 341 & 445 & 495 & 622 & 6.5 & 7.6 & 9.8 & 10.8 & 13.6 \\
\hline Sacramento-Roseville-Arden-Arcade, CA & 162 & 265 & 272 & 343 & 474 & 7.2 & 11.7 & 11.8 & 14.8 & 20.4 \\
\hline Salt Lake City, UT & 39 & 49 & 69 & 87 & 119 & 3.4 & 4.2 & 5.8 & 7.2 & 9.9 \\
\hline San Antonio-New Braunfels, TX & 247 & 237 & 243 & 329 & 284 & 10.6 & 9.9 & 10.0 & 13.3 & 11.5 \\
\hline San Diego-Carlsbad, CA & 371 & 493 & 524 & 585 & 555 & 11.4 & 14.9 & 15.8 & 17.5 & 16.6 \\
\hline San Francisco-Oakland-Hayward, CA & 767 & 830 & 872 & 1,030 & 1,035 & 16.7 & 17.8 & 18.6 & 21.8 & 21.9 \\
\hline San Jose-Sunnyvale-Santa Clara, CA & 120 & 134 & 222 & 217 & 296 & 6.1 & 6.8 & 11.2 & 10.9 & 14.8 \\
\hline Seattle-Tacoma-Bellevue, WA & 235 & 311 & 397 & 440 & 501 & 6.4 & 8.3 & 10.5 & 11.4 & 13.0 \\
\hline St. Louis, MO-IL & 153 & 112 & 215 & 278 & 345 & 5.5 & 4.0 & 7.7 & 9.9 & 12.3 \\
\hline Tampa-St. Petersburg-Clearwater, FL & 320 & 393 & 436 & 364 & 457 & 11.0 & 13.2 & 14.4 & 11.8 & 14.8 \\
\hline Virginia Beach-Norfolk-Newport News, VA-NC & 85 & 117 & 206 & 170 & 209 & 5.0 & 6.8 & 11.9 & 9.9 & 12.1 \\
\hline Washington-Arlington-Alexandria, DC-VA-MD-WV & 226 & 230 & 273 & 640 & 720 & 3.7 & 3.8 & 4.5 & 10.3 & 11.6 \\
\hline SELECTED MSAs TOTAL & 14,982 & 17,252 & 19,826 & 21,574 & 24,259 & 8.6 & 9.8 & 11.1 & 12.0 & 13.5 \\
\hline
\end{tabular}

* MSAs were selected on the basis of the largest population in the 2010 US Census.

t The variable used to identify county, which is used to classify cases into MSAs, was complete for $\leq 95 \%$ of cases in a state contributing data to this MSA. See Section A1.4 in the Appendix for more information.

${ }^{\ddagger} 2016$ county data for Alabama have been corrected and may not match previous reports. 
Table 31. Primary and Secondary Syphilis Among Females - Reported Cases and Rates of Reported Cases in Selected Metropolitan Statistical Areas (MSAs)* in Alphabetical Order, United States, 2014-2018

\begin{tabular}{|c|c|c|c|c|c|c|c|c|c|c|}
\hline \multirow[b]{2}{*}{ MSAs } & \multicolumn{5}{|c|}{ Cases } & \multicolumn{5}{|c|}{ Rates per 100,000 Population } \\
\hline & 2014 & 2015 & 2016 & 2017 & 2018 & 2014 & 2015 & 2016 & 2017 & 2018 \\
\hline Atlanta-Sandy Springs-Roswell, GA & 58 & 61 & 61 & 78 & 97 & 2.0 & 2.1 & 2.0 & 2.6 & 3.2 \\
\hline Austin-Round Rock, TX & 14 & 16 & 17 & 29 & 29 & 1.4 & 1.6 & 1.7 & 2.7 & 2.7 \\
\hline Baltimore-Columbia-Towson, MD & 42 & 49 & 46 & 39 & 54 & 2.9 & 3.4 & 3.2 & 2.7 & 3.7 \\
\hline Birmingham-Hoover, AL & 5 & 8 & $25^{\ddagger}$ & 21 & 23 & 0.8 & 1.3 & $4.2^{\ddagger}$ & 3.5 & 3.9 \\
\hline Boston-Cambridge-Newton, MA-NH & 14 & $13^{+}$ & $9^{+}$ & 13 & 24 & 0.6 & $0.5^{+}$ & $0.4^{+}$ & 0.5 & 1.0 \\
\hline Buffalo-Cheektowaga-Niagara Falls, NY & 1 & 0 & 1 & 6 & 4 & 0.2 & 0.0 & 0.2 & 1.0 & 0.7 \\
\hline Charlotte-Concord-Gastonia, NC-SC & 6 & 16 & 25 & 31 & 43 & 0.5 & 1.3 & 2.0 & 2.4 & 3.3 \\
\hline Chicago-Naperville-Elgin, IL-IN-WI & 76 & 86 & 88 & 79 & 103 & 1.6 & 1.8 & 1.8 & 1.6 & 2.1 \\
\hline Cincinnati, OH-KY-IN & 33 & 21 & 11 & 11 & 18 & 3.0 & 1.9 & 1.0 & 1.0 & 1.6 \\
\hline Cleveland-Elyria, $\mathrm{OH}$ & 5 & 1 & 12 & 23 & 10 & 0.5 & 0.1 & 1.1 & 2.2 & 0.9 \\
\hline Columbus, $\mathrm{OH}$ & 34 & 27 & 48 & 45 & 33 & 3.4 & 2.6 & 4.6 & 4.3 & 3.1 \\
\hline Dallas-Fort Worth-Arlington, TX & 63 & 56 & 62 & 75 & 101 & 1.8 & 1.6 & 1.7 & 2.0 & 2.7 \\
\hline Denver-Aurora-Lakewood, CO & 4 & 6 & 11 & 15 & 19 & 0.3 & 0.4 & 0.8 & 1.0 & 1.3 \\
\hline Detroit-Warren-Dearborn, MI & 23 & 26 & 26 & 20 & 46 & 1.0 & 1.2 & 1.2 & 0.9 & 2.1 \\
\hline Hartford-West Hartford-East Hartford, CT & 2 & 9 & 2 & 8 & 2 & 0.3 & 1.5 & 0.3 & 1.3 & 0.3 \\
\hline Houston-The Woodlands-Sugar Land, TX & 64 & 58 & 49 & 63 & 97 & 2.0 & 1.7 & 1.4 & 1.8 & 2.8 \\
\hline Indianapolis-Carmel-Anderson, IN & 5 & 12 & 15 & 12 & 9 & 0.5 & 1.2 & 1.5 & 1.2 & 0.9 \\
\hline Jacksonville, FL & 9 & 8 & 26 & 50 & 41 & 1.2 & 1.1 & 3.4 & 6.5 & 5.3 \\
\hline Kansas City, MO-KS & 23 & 41 & 39 & 43 & 59 & 2.2 & 3.9 & 3.6 & 4.0 & 5.4 \\
\hline Las Vegas-Henderson-Paradise, NV & 17 & 17 & 40 & 55 & 93 & 1.6 & 1.6 & 3.7 & 5.0 & 8.4 \\
\hline Los Angeles-Long Beach-Anaheim, CA & 67 & 108 & 144 & 177 & 273 & 1.0 & 1.6 & 2.1 & 2.6 & 4.0 \\
\hline Louisville-Jefferson County, KY-IN & 14 & 10 & 12 & 16 & 21 & 2.2 & 1.5 & 1.8 & 2.4 & 3.2 \\
\hline Memphis, TN-MS-AR & 22 & 13 & 25 & 21 & 40 & 3.1 & 1.9 & 3.6 & 3.0 & 5.7 \\
\hline Miami-Fort Lauderdale-West Palm Beach, FL & 44 & 75 & 66 & 71 & 102 & 1.4 & 2.4 & 2.1 & 2.2 & 3.2 \\
\hline Milwaukee-Waukesha-West Allis, WI & 6 & 0 & 1 & 5 & 7 & 0.7 & 0.0 & 0.1 & 0.6 & 0.9 \\
\hline Minneapolis-St. Paul-Bloomington, MN-WI & 19 & 37 & 31 & 24 & 16 & 1.1 & 2.1 & 1.7 & 1.3 & 0.9 \\
\hline Nashville-Davidson-Murfreesboro-Franklin, TN & 5 & 4 & 12 & 10 & 21 & 0.5 & 0.4 & 1.3 & 1.0 & 2.2 \\
\hline New Orleans-Metairie, LA & 18 & 29 & 25 & 36 & 25 & 2.8 & 4.4 & 3.8 & 5.5 & 3.8 \\
\hline New York-Newark-Jersey City, NY-NJ-PA & 52 & 69 & 123 & 119 & 152 & 0.5 & 0.7 & 1.2 & 1.1 & 1.4 \\
\hline Oklahoma City, OK & 7 & 14 & 27 & 39 & 70 & 1.0 & 2.0 & 3.9 & 5.6 & 10.0 \\
\hline Orlando-Kissimmee-Sanford, FL & 8 & 14 & 21 & 24 & 17 & 0.7 & 1.1 & 1.7 & 1.9 & 1.3 \\
\hline Philadelphia-Camden-Wilmington, PA-NJ-DE-MD & 39 & 43 & 49 & 63 & 79 & 1.2 & 1.4 & 1.6 & 2.0 & 2.5 \\
\hline Phoenix-Mesa-Scottsdale, AZ & 42 & 39 & 56 & 128 & 131 & 1.9 & 1.7 & 2.4 & 5.4 & 5.5 \\
\hline Pittsburgh, PA & 6 & 11 & 11 & 6 & 4 & 0.5 & 0.9 & 0.9 & 0.5 & 0.3 \\
\hline Portland-Vancouver-Hillsboro, OR-WA & 9 & 19 & 20 & 31 & 37 & 0.8 & 1.6 & 1.6 & 2.5 & 3.0 \\
\hline Providence-Warwick, RI-MA & 8 & $8^{+}$ & $6^{+}$ & 8 & 14 & 1.0 & $1.0^{+}$ & $0.7^{+}$ & 1.0 & 1.7 \\
\hline Raleigh, NC & 6 & 13 & 10 & 17 & 29 & 0.9 & 2.0 & 1.5 & 2.5 & 4.2 \\
\hline Richmond, VA & 2 & 4 & 15 & 18 & 29 & 0.3 & 0.6 & 2.3 & 2.7 & 4.3 \\
\hline Riverside-San Bernardino-Ontario, CA & 15 & 17 & 47 & 67 & 118 & 0.7 & 0.8 & 2.1 & 2.9 & 5.1 \\
\hline Sacramento-Roseville-Arden-Arcade, CA & 11 & 26 & 34 & 43 & 127 & 1.0 & 2.2 & 2.9 & 3.6 & 10.7 \\
\hline Salt Lake City, UT & 1 & 1 & 5 & 0 & 3 & 0.2 & 0.2 & 0.8 & 0.0 & 0.5 \\
\hline San Antonio-New Braunfels, TX & 47 & 43 & 43 & 54 & 49 & 4.0 & 3.6 & 3.5 & 4.3 & 3.9 \\
\hline San Diego-Carlsbad, CA & 20 & 17 & 20 & 31 & 41 & 1.2 & 1.0 & 1.2 & 1.9 & 2.5 \\
\hline San Francisco-Oakland-Hayward, CA & 34 & 46 & 50 & 58 & 85 & 1.5 & 2.0 & 2.1 & 2.4 & 3.6 \\
\hline San Jose-Sunnyvale-Santa Clara, CA & 12 & 10 & 32 & 29 & 80 & 1.2 & 1.0 & 3.3 & 2.9 & 8.1 \\
\hline Seattle-Tacoma-Bellevue, WA & 10 & 6 & 15 & 5 & 25 & 0.5 & 0.3 & 0.8 & 0.3 & 1.3 \\
\hline St. Louis, MO-IL & 17 & 14 & 24 & 34 & 50 & 1.2 & 1.0 & 1.7 & 2.4 & 3.5 \\
\hline Tampa-St. Petersburg-Clearwater, FL & 41 & 44 & 48 & 42 & 37 & 2.7 & 2.9 & 3.1 & 2.6 & 2.3 \\
\hline Virginia Beach-Norfolk-Newport News, VA-NC & 8 & 5 & 22 & 24 & 18 & 0.9 & 0.6 & 2.5 & 2.7 & 2.1 \\
\hline Washington-Arlington-Alexandria, DC-VA-MD-WV & 7 & 10 & 8 & 19 & 41 & 0.2 & 0.3 & 0.3 & 0.6 & 1.3 \\
\hline SELECTED MSAs TOTAL & 1,095 & 1,280 & 1,615 & 1,935 & 2,646 & 1.2 & 1.4 & 1.8 & 2.1 & 2.9 \\
\hline
\end{tabular}

* MSAs were selected on the basis of the largest population in the 2010 US Census.

+ The variable used to identify county, which is used to classify cases into MSAs, was complete for $\leq 95 \%$ of cases in a state contributing data to this MSA. See Section A1.4 in the Appendix for more information.

₹ 2016 county data for Alabama have been corrected and may not match previous reports.

NOTE: Cases reported with unknown sex are not included in this table. 
Table 32. Primary and Secondary Syphilis Among Males - Reported Cases and Rates of Reported Cases in Selected Metropolitan Statistical Areas (MSAs)* in Alphabetical Order, United States, 2014-2018

\begin{tabular}{|c|c|c|c|c|c|c|c|c|c|c|}
\hline \multirow[b]{2}{*}{ MSAs } & \multicolumn{5}{|c|}{ Cases } & \multicolumn{5}{|c|}{ Rates per 100,000 Population } \\
\hline & 2014 & 2015 & 2016 & 2017 & 2018 & 2014 & 2015 & 2016 & 2017 & 2018 \\
\hline Atlanta-Sandy Springs-Roswell, GA & 938 & 1,036 & 957 & 1,017 & 995 & 34.5 & 37.5 & 34.2 & 35.7 & 35.0 \\
\hline Austin-Round Rock, TX & 213 & 187 & 300 & 330 & 265 & 21.9 & 18.7 & 29.2 & 31.1 & 25.0 \\
\hline Baltimore-Columbia-Towson, MD & 240 & 294 & 286 & 303 & 392 & 17.9 & 21.8 & 21.2 & 22.4 & 28.9 \\
\hline Birmingham-Hoover, AL & 53 & 66 & $84^{\ddagger}$ & 101 & 101 & 9.6 & 12.0 & $15.2^{\ddagger}$ & 18.3 & 18.3 \\
\hline Boston-Cambridge-Newton, MA-NH & 213 & $257^{+}$ & $311^{+}$ & 360 & 357 & 9.3 & $11.1^{\dagger}$ & $13.4^{+}$ & 15.3 & 15.2 \\
\hline Buffalo-Cheektowaga-Niagara Falls, NY & 48 & 92 & 62 & 54 & 49 & 8.7 & 16.7 & 11.3 & 9.8 & 8.9 \\
\hline Charlotte-Concord-Gastonia, NC-SC & 214 & 317 & 353 & 324 & 290 & 18.5 & 26.9 & 29.4 & 26.5 & 23.7 \\
\hline Chicago-Naperville-Elgin, IL-IN-WI & 735 & 961 & 1,114 & 1,061 & 1,176 & 15.7 & 20.6 & 23.9 & 22.7 & 25.2 \\
\hline Cincinnati, OH-KY-IN & 120 & 72 & 70 & 92 & 140 & 11.4 & 6.8 & 6.6 & 8.6 & 13.1 \\
\hline Cleveland-Elyria, $\mathrm{OH}$ & 75 & 71 & 144 & 157 & 106 & 7.5 & 7.1 & 14.5 & 15.8 & 10.7 \\
\hline Columbus, $\mathrm{OH}$ & 216 & 247 & 268 & 308 & 208 & 22.0 & 24.8 & 26.7 & 30.1 & 20.3 \\
\hline Dallas-Fort Worth-Arlington, TX & 445 & 420 & 480 & 624 & 638 & 13.0 & 12.0 & 13.5 & 17.1 & 17.5 \\
\hline Denver-Aurora-Lakewood, CO & 149 & 186 & 176 & 201 & 222 & 10.9 & 13.3 & 12.4 & 13.9 & 15.4 \\
\hline Detroit-Warren-Dearborn, MI & 294 & 258 & 227 & 271 & 420 & 14.1 & 12.3 & 10.9 & 12.9 & 20.0 \\
\hline Hartford-West Hartford-East Hartford, CT & 24 & 35 & 30 & 24 & 27 & 4.1 & 5.9 & 5.1 & 4.1 & 4.6 \\
\hline Houston-The Woodlands-Sugar Land, TX & 350 & 381 & 362 & 323 & 550 & 10.8 & 11.5 & 10.8 & 9.4 & 16.1 \\
\hline Indianapolis-Carmel-Anderson, IN & 104 & 160 & 143 & 174 & 164 & 10.8 & 16.5 & 14.6 & 17.5 & 16.5 \\
\hline Jacksonville, FL & 60 & 83 & 92 & 144 & 178 & 8.7 & 11.7 & 12.8 & 19.6 & 24.3 \\
\hline Kansas City, MO-KS & 197 & 150 & 162 & 171 & 296 & 19.4 & 14.6 & 15.7 & 16.4 & 28.3 \\
\hline Las Vegas-Henderson-Paradise, NV & 301 & 288 & 358 & 464 & 461 & 29.1 & 27.3 & 33.3 & 42.2 & 41.9 \\
\hline Los Angeles-Long Beach-Anaheim, CA & 1,340 & 1,724 & 1,978 & 2,188 & 2,527 & 20.5 & 26.2 & 30.2 & 33.2 & 38.4 \\
\hline Louisville-Jefferson County, KY-IN & 69 & 77 & 137 & 134 & 198 & 11.1 & 12.3 & 21.8 & 21.2 & 31.3 \\
\hline Memphis, TN-MS-AR & 72 & 108 & 148 & 132 & 161 & 11.2 & 16.8 & 23.0 & 20.5 & 24.9 \\
\hline Miami-Fort Lauderdale-West Palm Beach, FL & 777 & 809 & 859 & 848 & 977 & 27.0 & 27.8 & 29.2 & 28.3 & 32.6 \\
\hline Milwaukee-Waukesha-West Allis, WI & 46 & 39 & 49 & 74 & 57 & 6.0 & 5.1 & 6.4 & 9.6 & 7.4 \\
\hline Minneapolis-St. Paul-Bloomington, MN-WI & 223 & 191 & 228 & 222 & 222 & 12.9 & 10.9 & 13.0 & 12.4 & 12.4 \\
\hline Nashville-Davidson-Murfreesboro-Franklin, TN & 69 & 112 & 103 & 150 & 206 & 7.9 & 12.5 & 11.3 & 16.1 & 22.2 \\
\hline New Orleans-Metairie, LA & 203 & 189 & 210 & 174 & 211 & 33.5 & 31.0 & 34.2 & 28.3 & 34.3 \\
\hline New York-Newark-Jersey City, NY-NJ-PA & 1,666 & 1,954 & 2,407 & 2,322 & 2,540 & 17.1 & 20.0 & 24.7 & 23.6 & 25.8 \\
\hline Oklahoma City, OK & 84 & 99 & 135 & 180 & 182 & 12.8 & 14.8 & 19.9 & 26.4 & 26.7 \\
\hline Orlando-Kissimmee-Sanford, FL & 231 & 285 & 363 & 294 & 349 & 20.4 & 24.4 & 30.4 & 24.0 & 28.4 \\
\hline Philadelphia-Camden-Wilmington, PA-NJ-DE-MD & 407 & 415 & 590 & 573 & 515 & 13.9 & 14.1 & 20.1 & 19.4 & 17.5 \\
\hline Phoenix-Mesa-Scottsdale, AZ & 374 & 413 & 518 & 647 & 688 & 16.8 & 18.2 & 22.4 & 27.5 & 29.2 \\
\hline Pittsburgh, PA & 72 & 139 & 89 & 65 & 87 & 6.3 & 12.1 & 7.8 & 5.7 & 7.7 \\
\hline Portland-Vancouver-Hillsboro, OR-WA & 197 & 242 & 243 & 224 & 263 & 17.0 & 20.5 & 20.3 & 18.5 & 21.7 \\
\hline Providence-Warwick, RI-MA & 84 & $103^{+}$ & $109^{\dagger}$ & 90 & 113 & 10.8 & $13.2^{\dagger}$ & $13.9^{+}$ & 11.4 & 14.4 \\
\hline Raleigh, NC & 123 & 155 & 127 & 119 & 144 & 20.3 & 24.9 & 20.0 & 18.3 & 22.1 \\
\hline Richmond, VA & 66 & 69 & 87 & 103 & 176 & 10.8 & 11.2 & 14.1 & 16.5 & 28.2 \\
\hline Riverside-San Bernardino-Ontario, CA & 273 & 324 & 398 & 428 & 504 & 12.4 & 14.5 & 17.7 & 18.8 & 22.1 \\
\hline Sacramento-Roseville-Arden-Arcade, CA & 151 & 239 & 238 & 300 & 346 & 13.7 & 21.5 & 21.2 & 26.4 & 30.4 \\
\hline Salt Lake City, UT & 38 & 48 & 64 & 87 & 116 & 6.6 & 8.2 & 10.7 & 14.4 & 19.2 \\
\hline San Antonio-New Braunfels, TX & 200 & 194 & 200 & 275 & 235 & 17.4 & 16.5 & 16.7 & 22.5 & 19.2 \\
\hline San Diego-Carlsbad, CA & 351 & 476 & 504 & 554 & 514 & 21.4 & 28.7 & 30.2 & 33.0 & 30.6 \\
\hline San Francisco-Oakland-Hayward, CA & 731 & 783 & 820 & 970 & 948 & 32.3 & 34.1 & 35.5 & 41.5 & 40.6 \\
\hline San Jose-Sunnyvale-Santa Clara, CA & 108 & 124 & 190 & 188 & 216 & 11.0 & 12.5 & 19.0 & 18.6 & 21.4 \\
\hline Seattle-Tacoma-Bellevue, WA & 225 & 305 & 382 & 434 & 475 & 12.3 & 16.3 & 20.1 & 22.4 & 24.5 \\
\hline St. Louis, MO-IL & 136 & 98 & 191 & 244 & 295 & 10.0 & 7.2 & 14.0 & 17.9 & 21.7 \\
\hline Tampa-St. Petersburg-Clearwater, FL & 278 & 349 & 388 & 322 & 420 & 19.7 & 24.2 & 26.4 & 21.5 & 28.0 \\
\hline Virginia Beach-Norfolk-Newport News, VA-NC & 77 & 112 & 178 & 144 & 190 & 9.1 & 13.2 & 20.9 & 17.0 & 22.4 \\
\hline Washington-Arlington-Alexandria, DC-VA-MD-WV & 219 & 219 & 264 & 618 & 674 & 7.4 & 7.4 & 8.8 & 20.3 & 22.2 \\
\hline SELECTED MSAs TOTAL & 13,879 & 15,955 & 18,176 & 19,606 & 21,584 & 16.2 & 18.4 & 20.9 & 22.2 & 24.5 \\
\hline
\end{tabular}

* MSAs were selected on the basis of the largest population in the 2010 US Census.

t The variable used to identify county, which is used to classify cases into MSAs, was complete for $\leq 95 \%$ of cases in a state contributing data to this MSA. See Section A1.4 in the Appendix for more information.

${ }^{\ddagger} 2016$ county data for Alabama have been corrected and may not match previous reports.

NOTE: Cases reported with unknown sex are not included in this table. 
Table 33. Primary and Secondary Syphilis - Reported Cases and Rates of Reported Cases in Counties and Independent Cities* Ranked by Number of Reported Cases, United States, 2018

\begin{tabular}{|c|c|c|c|c|}
\hline Rank* & County/Independent City & Cases & Rate per 100,000 Population & Cumulative Percentage \\
\hline 1 & Los Angeles County, CA & 2,350 & 23.1 & 7 \\
\hline 2 & Cook County, IL & 1,090 & 20.9 & 10 \\
\hline 3 & Maricopa County, AZ & 784 & 18.2 & 12 \\
\hline 4 & New York County, NY & 686 & 41.2 & 14 \\
\hline 5 & Harris County, TX & 565 & 12.1 & 16 \\
\hline 6 & San Francisco County, CA & 561 & 63.4 & 17 \\
\hline 7 & San Diego County, CA & 555 & 16.6 & 19 \\
\hline 8 & Clark County, NV & 554 & 25.1 & 20 \\
\hline 9 & Miami-Dade County, FL & 544 & 19.8 & 22 \\
\hline 10 & Kings County, NY & 496 & 18.7 & 23 \\
\hline 11 & Fulton County, GA & 460 & 44.2 & 25 \\
\hline 12 & Bronx County, NY & 459 & 31.2 & 26 \\
\hline 13 & Orange County, CA & 450 & 14.1 & 27 \\
\hline 14 & Broward County, FL & 445 & 23.0 & 29 \\
\hline 15 & Sacramento County, CA & 417 & 27.2 & 30 \\
\hline 16 & Philadelphia County, PA & 408 & 25.8 & 31 \\
\hline 17 & King County, WA & 392 & 17.9 & 32 \\
\hline 18 & Dallas County, TX & 368 & 14.1 & 33 \\
\hline 19 & Queens County, NY & 356 & 15.1 & 34 \\
\hline 20 & San Bernardino County, CA & 316 & 14.6 & 35 \\
\hline 21 & Riverside County, CA & 306 & 12.6 & 36 \\
\hline 22 & Wayne County, MI & 299 & 17.1 & 37 \\
\hline 23 & Santa Clara County, CA & 291 & 15.0 & 38 \\
\hline 24 & Tarrant County, TX & 284 & 13.8 & 38 \\
\hline 25 & Kern County, CA & 280 & 31.4 & 39 \\
\hline 26 & Washington, D.C. & 279 & 40.2 & 40 \\
\hline 27 & Orange County, FL & 278 & 20.6 & 41 \\
\hline 28 & Baltimore (City), MD & 277 & 45.3 & 41 \\
\hline 29 & Bexar County, TX & 273 & 13.9 & 42 \\
\hline 30 & San Joaquin County, CA & 270 & 36.2 & 43 \\
\hline 31 & DeKalb County, GA & 249 & 33.1 & 44 \\
\hline 32 & Mecklenburg County, NC & 249 & 23.1 & 44 \\
\hline 33 & Travis County, TX & 239 & 19.5 & 45 \\
\hline 34 & Hillsborough County, FL & 239 & 17.0 & 46 \\
\hline 35 & Jackson County, MO & 233 & 33.3 & 46 \\
\hline 36 & Alameda County, CA & 222 & 13.3 & 47 \\
\hline 37 & Franklin County, $\mathrm{OH}$ & 213 & 16.5 & 48 \\
\hline 38 & Multnomah County, OR & 195 & 24.1 & 48 \\
\hline 39 & Fresno County, CA & 195 & 19.7 & 49 \\
\hline 40 & Oklahoma County, OK & 192 & 24.4 & 49 \\
\hline 41 & Duval County, FL & 191 & 20.4 & 50 \\
\hline 42 & Pinellas County, FL & 191 & 19.7 & 50 \\
\hline 43 & Jefferson County, KY & 180 & 23.3 & 51 \\
\hline 44 & Bernalillo County, NM & 169 & 25.0 & 51 \\
\hline 45 & Stanislaus County, CA & 167 & 30.5 & 52 \\
\hline 46 & Contra Costa County, CA & 162 & 14.1 & 52 \\
\hline 47 & Suffolk County, MA & 157 & 19.7 & 53 \\
\hline 48 & Orleans Parish, LA & 156 & 39.7 & 53 \\
\hline 49 & Shelby County, TN & 156 & 16.6 & 54 \\
\hline 50 & Wake County, NC & 156 & 14.5 & 54 \\
\hline 51 & Hennepin County, MN & 156 & 12.5 & 55 \\
\hline 52 & Tulsa County, OK & 154 & 23.8 & 55 \\
\hline 53 & Prince George's County, MD & 153 & 16.8 & 56 \\
\hline 54 & Sonoma County, CA & 142 & 28.2 & 56 \\
\hline 55 & Marion County, IN & 141 & 14.8 & 56 \\
\hline 56 & Davidson County, TN & 139 & 20.1 & 57 \\
\hline 57 & Monroe County, NY & 139 & 18.6 & 57 \\
\hline 58 & Denver County, CO & 132 & 18.7 & 57 \\
\hline 59 & St. Louis County, MO & 132 & 13.2 & 58 \\
\hline 60 & St. Louis (City), MO & 131 & 42.4 & 58 \\
\hline 61 & Pima County, AZ & 128 & 12.5 & 59 \\
\hline 62 & Middlesex County, MA & 124 & 7.7 & 59 \\
\hline 63 & Salt Lake County, UT & 119 & 10.5 & 59 \\
\hline 64 & Hamilton County, $\mathrm{OH}$ & 117 & 14.4 & 60 \\
\hline 65 & Jefferson County, AL & 112 & 17.0 & 60 \\
\hline 66 & Durham County, NC & 111 & 35.6 & 60 \\
\hline 67 & Gwinnett County, GA & 109 & 11.8 & 61 \\
\hline 68 & Spokane County, WA & 106 & 20.9 & 61 \\
\hline 69 & Nueces County, TX & 105 & 29.1 & 61 \\
\hline 70 & Baltimore County, MD & 103 & 12.4 & 61 \\
\hline
\end{tabular}

* The top 70 counties and independent cities ranked in descending order by number of cases reported in 2018 then by rate are displayed.

NOTE: Relative rankings of counties may be impacted by completeness of the variable used to identify county. See Section A1.4 in the Appendix for more information. 
Table 34. Primary and Secondary Syphilis - Reported Cases and Rates of Reported Cases by Age Group and Sex, United States, 2014-2018

\begin{tabular}{|c|c|c|c|c|c|c|c|c|}
\hline & \multirow{2}{*}{$\begin{array}{l}\text { Age } \\
\text { Group }\end{array}$} & \multicolumn{4}{|c|}{ Cases } & \multicolumn{3}{|c|}{ Rates per 100,000 Population* } \\
\hline & & Total & Male & Female & Unknown Sex & Total & Male & Female \\
\hline \multirow{14}{*}{$\underset{ }{\stackrel{0}{\circ}}$} & $0-4$ & 0 & 0 & 0 & 0 & 0.0 & 0.0 & 0.0 \\
\hline & $5-9$ & 0 & 0 & 0 & 0 & 0.0 & 0.0 & 0.0 \\
\hline & $10-14$ & 12 & 4 & 8 & 0 & 0.1 & 0.0 & 0.1 \\
\hline & $15-19$ & 1,023 & 761 & 262 & 0 & 4.9 & 7.1 & 2.5 \\
\hline & $20-24$ & 4,137 & 3,632 & 503 & 2 & 18.1 & 30.9 & 4.5 \\
\hline & $25-29$ & 4,092 & 3,727 & 361 & 4 & 18.6 & 33.4 & 3.3 \\
\hline & $30-34$ & 2,887 & 2,635 & 248 & 4 & 13.4 & 24.4 & 2.3 \\
\hline & $35-39$ & 2,045 & 1,868 & 177 & 0 & 10.3 & 18.8 & 1.8 \\
\hline & $40-44$ & 1,758 & 1,654 & 103 & 1 & 8.5 & 16.2 & 1.0 \\
\hline & $45-54$ & 2,966 & 2,830 & 135 & 1 & 6.8 & 13.2 & 0.6 \\
\hline & $55-64$ & 897 & 860 & 36 & 1 & 2.2 & 4.5 & 0.2 \\
\hline & $65+$ & 176 & 169 & 7 & 0 & 0.4 & 0.8 & 0.0 \\
\hline & Unknown Age & 6 & 6 & 0 & 0 & & & \\
\hline & TOTAL & 19,999 & 18,146 & 1,840 & 13 & 6.3 & 11.6 & 1.1 \\
\hline \multirow{14}{*}{ 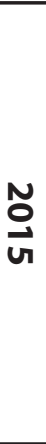 } & $0-4$ & 2 & 0 & 1 & 1 & 0.0 & 0.0 & 0.0 \\
\hline & $5-9$ & 1 & 0 & 1 & 0 & 0.0 & 0.0 & 0.0 \\
\hline & $10-14$ & 9 & 1 & 8 & 0 & 0.0 & 0.0 & 0.1 \\
\hline & $15-19$ & 1,148 & 865 & 283 & 0 & 5.4 & 8.0 & 2.7 \\
\hline & $20-24$ & 4,766 & 4,186 & 573 & 7 & 21.0 & 35.9 & 5.2 \\
\hline & $25-29$ & 5,168 & 4,671 & 491 & 6 & 23.0 & 40.9 & 4.4 \\
\hline & $30-34$ & 3,549 & 3,234 & 311 & 4 & 16.4 & 29.7 & 2.9 \\
\hline & $35-39$ & 2,482 & 2,249 & 229 & 4 & 12.2 & 22.1 & 2.2 \\
\hline & $40-44$ & 1,897 & 1,744 & 152 & 1 & 9.4 & 17.4 & 1.5 \\
\hline & $45-54$ & 3,488 & 3,294 & 190 & 4 & 8.1 & 15.5 & 0.9 \\
\hline & $55-64$ & 1,153 & 1,099 & 54 & 0 & 2.8 & 5.6 & 0.3 \\
\hline & $65+$ & 207 & 202 & 5 & 0 & 0.4 & 1.0 & 0.0 \\
\hline & Unknown Age & 2 & 2 & 0 & 0 & & & \\
\hline & TOTAL & 23,872 & 21,547 & 2,298 & 27 & 7.4 & 13.6 & 1.4 \\
\hline \multirow{14}{*}{ 음 } & $0-4$ & 2 & 0 & 2 & 0 & 0.0 & 0.0 & 0.0 \\
\hline & $5-9$ & 2 & 1 & 1 & 0 & 0.0 & 0.0 & 0.0 \\
\hline & $10-14$ & 15 & 6 & 9 & 0 & 0.1 & 0.1 & 0.1 \\
\hline & $15-19$ & 1,298 & 957 & 340 & 1 & 6.1 & 8.9 & 3.3 \\
\hline & $20-24$ & 5,172 & 4,418 & 744 & 10 & 23.1 & 38.4 & 6.8 \\
\hline & $25-29$ & 6,177 & 5,538 & 624 & 15 & 27.0 & 47.6 & 5.5 \\
\hline & $30-34$ & 4,278 & 3,806 & 464 & 8 & 19.6 & 34.7 & 4.3 \\
\hline & $35-39$ & 3,043 & 2,729 & 311 & 3 & 14.6 & 26.3 & 3.0 \\
\hline & $40-44$ & 2,140 & 1,944 & 193 & 3 & 10.9 & 19.9 & 1.9 \\
\hline & $45-54$ & 3,953 & 3,691 & 261 & 1 & 9.2 & 17.5 & 1.2 \\
\hline & $55-64$ & 1,418 & 1,338 & 80 & 0 & 3.4 & 6.7 & 0.4 \\
\hline & $65+$ & 279 & 269 & 10 & 0 & 0.6 & 1.2 & 0.0 \\
\hline & Unknown Age & 37 & 27 & 10 & 0 & & & \\
\hline & TOTAL & 27,814 & 24,724 & 3,049 & 41 & 8.6 & 15.5 & 1.9 \\
\hline \multirow{14}{*}{$\stackrel{\sim}{\stackrel{\circ}{\vec{v}}}$} & $0-4$ & 5 & 0 & 5 & 0 & 0.0 & 0.0 & 0.1 \\
\hline & $5-9$ & 1 & 0 & 1 & 0 & 0.0 & 0.0 & 0.0 \\
\hline & $10-14$ & 20 & 6 & 14 & 0 & 0.1 & 0.1 & 0.1 \\
\hline & $15-19$ & 1,421 & 1,092 & 327 & 2 & 6.7 & 10.1 & 3.2 \\
\hline & $20-24$ & 5,580 & 4,728 & 848 & 4 & 25.2 & 41.7 & 7.9 \\
\hline & $25-29$ & 6,838 & 6,033 & 795 & 10 & 29.3 & 50.7 & 6.9 \\
\hline & $30-34$ & 4,870 & 4,313 & 549 & 8 & 22.2 & 38.9 & 5.0 \\
\hline & $35-39$ & 3,580 & 3,145 & 431 & 4 & 16.9 & 29.6 & 4.1 \\
\hline & $40-44$ & 2,290 & 2,005 & 282 & 3 & 11.7 & 20.6 & 2.9 \\
\hline & $45-54$ & 4,091 & 3,753 & 334 & 4 & 9.7 & 18.0 & 1.6 \\
\hline & $55-64$ & 1,586 & 1,468 & 117 & 1 & 3.8 & 7.2 & 0.5 \\
\hline & $65+$ & 349 & 329 & 19 & 1 & 0.7 & 1.5 & 0.1 \\
\hline & Unknown Age & 13 & 13 & 0 & 0 & & & \\
\hline & TOTAL & 30,644 & 26,885 & 3,722 & 37 & 9.4 & 16.8 & 2.3 \\
\hline \multirow{14}{*}{$\underset{\infty}{\stackrel{0}{\circ}}$} & $0-4$ & 2 & 1 & 1 & 0 & 0.0 & 0.0 & 0.0 \\
\hline & $5-9$ & 0 & 0 & 0 & 0 & 0.0 & 0.0 & 0.0 \\
\hline & $10-14$ & 20 & 9 & 11 & 0 & 0.1 & 0.1 & 0.1 \\
\hline & $15-19$ & 1,618 & 1,175 & 442 & 1 & 7.7 & 10.9 & 4.3 \\
\hline & $20-24$ & 6,140 & 5,061 & 1,076 & 3 & 27.8 & 44.6 & 10.0 \\
\hline & $25-29$ & 7,712 & 6,625 & 1,077 & 10 & 33.0 & 55.7 & 9.4 \\
\hline & $30-34$ & 5,907 & 5,084 & 816 & 7 & 26.9 & 45.8 & 7.5 \\
\hline & $35-39$ & 4,200 & 3,574 & 621 & 5 & 19.8 & 33.7 & 5.8 \\
\hline & $40-44$ & 2,690 & 2,328 & 358 & 4 & 13.7 & 23.9 & 3.6 \\
\hline & $45-54$ & 4,398 & 3,973 & 423 & 2 & 10.4 & 19.0 & 2.0 \\
\hline & $55-64$ & 1,929 & 1,782 & 145 & 2 & 4.6 & 8.8 & 0.7 \\
\hline & $65+$ & 437 & 412 & 25 & 0 & 0.9 & 1.8 & 0.1 \\
\hline & Unknown Age & 10 & 10 & 0 & 0 & & & \\
\hline & TOTAL & 35,063 & 30,034 & 4,995 & 34 & 10.8 & 18.7 & 3.0 \\
\hline
\end{tabular}

* No population data are available for unknown sex and age; therefore, rates are not calculated. 
Table 35A. Primary and Secondary Syphilis - Reported Cases by Race/Hispanic Ethnicity, Age Group, and Sex, United States, 2018

\begin{tabular}{|c|c|c|c|c|c|c|c|c|c|c|c|c|}
\hline \multirow{2}{*}{$\begin{array}{l}\text { Age } \\
\text { Group }\end{array}$} & \multicolumn{3}{|c|}{$\begin{array}{c}\text { American Indians/ } \\
\text { Alaska Natives }\end{array}$} & \multicolumn{3}{|c|}{ Asians } & \multicolumn{3}{|c|}{ Blacks } & \multicolumn{3}{|c|}{$\begin{array}{c}\text { Native Hawaiians/ } \\
\text { Other Pacific Islanders }\end{array}$} \\
\hline & Total* & Male & Female & Total* & Male & Female & Total* & Male & Female & Total* & Male & Female \\
\hline $0-4$ & 0 & 0 & 0 & 0 & 0 & 0 & 2 & 1 & 1 & 0 & 0 & 0 \\
\hline $5-9$ & 0 & 0 & 0 & 0 & 0 & 0 & 0 & 0 & 0 & 0 & 0 & 0 \\
\hline $10-14$ & 0 & 0 & 0 & 0 & 0 & 0 & 11 & 4 & 7 & 0 & 0 & 0 \\
\hline 15-19 & 26 & 15 & 11 & 26 & 23 & 3 & 703 & 493 & 210 & 4 & 4 & 0 \\
\hline $20-24$ & 59 & 43 & 16 & 169 & 158 & 11 & 2,411 & 1,934 & 476 & 21 & 16 & 5 \\
\hline $25-29$ & 69 & 44 & 25 & 184 & 176 & 8 & 3,022 & 2,588 & 429 & 21 & 19 & 2 \\
\hline $30-34$ & 74 & 56 & 18 & 154 & 145 & 9 & 1,945 & 1,705 & 237 & 17 & 15 & 2 \\
\hline $35-39$ & 60 & 39 & 21 & 97 & 91 & 6 & 1,247 & 1,073 & 170 & 11 & 10 & 1 \\
\hline $40-44$ & 35 & 23 & 12 & 73 & 69 & 4 & 643 & 549 & 91 & 5 & 5 & 0 \\
\hline $45-54$ & 36 & 23 & 13 & 103 & 100 & 3 & 946 & 836 & 109 & 11 & 11 & 0 \\
\hline $55-64$ & 12 & 9 & 3 & 26 & 25 & 1 & 396 & 358 & 38 & 4 & 4 & 0 \\
\hline $65+$ & 1 & 1 & 0 & 13 & 12 & 1 & 91 & 78 & 13 & 0 & 0 & 0 \\
\hline Unknown Age & 0 & 0 & 0 & 1 & 1 & 0 & 2 & 2 & 0 & 0 & 0 & 0 \\
\hline TOTAL & 372 & 253 & 119 & 846 & 800 & 46 & 11,419 & 9,621 & 1,781 & 94 & 84 & 10 \\
\hline
\end{tabular}

\begin{tabular}{|c|c|c|c|c|c|c|c|c|c|c|c|c|}
\hline \multirow{2}{*}{$\begin{array}{l}\text { Age } \\
\text { Group }\end{array}$} & \multicolumn{3}{|c|}{ Whites } & \multicolumn{3}{|c|}{ Multirace } & \multicolumn{3}{|c|}{ Hispanics } & \multicolumn{3}{|c|}{$\begin{array}{l}\text { Other/ } \\
\text { Unknown }\end{array}$} \\
\hline & Total* & Male & Female & Total* & Male & Female & Total* & Male & Female & Total* & Male & Female \\
\hline $0-4$ & 0 & 0 & 0 & 0 & 0 & 0 & 0 & 0 & 0 & 0 & 0 & 0 \\
\hline $5-9$ & 0 & 0 & 0 & 0 & 0 & 0 & 0 & 0 & 0 & 0 & 0 & 0 \\
\hline $10-14$ & 3 & 2 & 1 & 0 & 0 & 0 & 4 & 3 & 1 & 2 & 0 & 2 \\
\hline $15-19$ & 353 & 248 & 105 & 26 & 20 & 6 & 393 & 307 & 85 & 87 & 65 & 22 \\
\hline $20-24$ & 1,468 & 1,177 & 290 & 126 & 100 & 26 & 1,496 & 1,295 & 200 & 390 & 338 & 52 \\
\hline $25-29$ & 2,079 & 1,708 & 370 & 132 & 120 & 12 & 1,753 & 1,564 & 185 & 452 & 406 & 46 \\
\hline $30-34$ & 1,909 & 1,560 & 349 & 118 & 109 & 9 & 1,343 & 1,196 & 144 & 347 & 298 & 48 \\
\hline $35-39$ & 1,468 & 1,227 & 241 & 93 & 75 & 18 & 960 & 844 & 116 & 264 & 215 & 48 \\
\hline $40-44$ & 1,085 & 929 & 155 & 52 & 49 & 3 & 627 & 558 & 69 & 170 & 146 & 24 \\
\hline $45-54$ & 2,154 & 1,958 & 195 & 72 & 69 & 3 & 807 & 736 & 71 & 269 & 240 & 29 \\
\hline $55-64$ & 1,102 & 1,032 & 68 & 25 & 25 & 0 & 229 & 205 & 24 & 135 & 124 & 11 \\
\hline $65+$ & 246 & 241 & 5 & 5 & 5 & 0 & 48 & 43 & 5 & 33 & 32 & 1 \\
\hline Unknown Age & 4 & 4 & 0 & 0 & 0 & 0 & 3 & 3 & 0 & 0 & 0 & 0 \\
\hline TOTAL & 11,871 & 10,086 & 1,779 & 649 & 572 & 77 & 7,663 & 6,754 & 900 & 2,149 & 1,864 & 283 \\
\hline
\end{tabular}

* Total includes cases reported with unknown sex.

NOTE: These tables should be used only for race/Hispanic ethnicity comparisons. See Table 34 for age-specific cases and rates and Tables $27-29$ for total and sex-specific cases and rates. Primary and secondary syphilis reported among children aged 0-4 may represent the misclassification of congenitally-acquired syphilis. Cases reported as congenitally-acquired syphilis (congenital syphilis) can be found in Table 40. 
Table 35B. Primary and Secondary Syphilis - Rates of Reported Cases* by Race/Hispanic Ethnicity, Age Group, and Sex, United States, 2018

\begin{tabular}{|c|c|c|c|c|c|c|c|c|c|c|c|c|}
\hline \multirow{2}{*}{$\begin{array}{l}\text { Age } \\
\text { Group }\end{array}$} & \multicolumn{3}{|c|}{$\begin{array}{l}\text { American Indians/ } \\
\text { Alaska Natives }\end{array}$} & \multicolumn{3}{|c|}{ Asians } & \multicolumn{3}{|c|}{ Blacks } & \multicolumn{3}{|c|}{$\begin{array}{c}\text { Native Hawaiians/ } \\
\text { Other Pacific Islanders }\end{array}$} \\
\hline & Total $^{+}$ & Male & Female & Total $^{+}$ & Male & Female & Total $^{\dagger}$ & Male & Female & Total $^{+}$ & Male & Female \\
\hline $0-4$ & 0.0 & 0.0 & 0.0 & 0.0 & 0.0 & 0.0 & 0.1 & 0.1 & 0.1 & 0.0 & 0.0 & 0.0 \\
\hline $5-9$ & 0.0 & 0.0 & 0.0 & 0.0 & 0.0 & 0.0 & 0.0 & 0.0 & 0.0 & 0.0 & 0.0 & 0.0 \\
\hline $10-14$ & 0.0 & 0.0 & 0.0 & 0.0 & 0.0 & 0.0 & 0.4 & 0.3 & 0.5 & 0.0 & 0.0 & 0.0 \\
\hline $15-19$ & 14.4 & 16.3 & 12.4 & 2.4 & 4.3 & 0.6 & 23.7 & 32.8 & 14.4 & 9.8 & 19.2 & 0.0 \\
\hline $20-24$ & 31.4 & 44.8 & 17.4 & 13.1 & 24.2 & 1.7 & 75.0 & 118.5 & 30.1 & 47.8 & 71.4 & 23.2 \\
\hline $25-29$ & 35.1 & 43.8 & 26.0 & 11.6 & 22.6 & 1.0 & 89.1 & 152.7 & 25.3 & 40.5 & 70.7 & 8.0 \\
\hline $30-34$ & 44.2 & 66.7 & 21.5 & 9.7 & 19.0 & 1.1 & 68.2 & 123.2 & 16.2 & 33.9 & 58.4 & 8.2 \\
\hline $35-39$ & 39.1 & 51.2 & 27.2 & 6.4 & 12.9 & 0.7 & 45.7 & 82.7 & 11.9 & 24.1 & 42.8 & 4.5 \\
\hline $40-44$ & 24.9 & 33.3 & 16.8 & 5.1 & 10.3 & 0.5 & 25.9 & 47.0 & 6.9 & 12.9 & 25.4 & 0.0 \\
\hline $45-54$ & 12.0 & 15.8 & 8.4 & 4.0 & 8.4 & 0.2 & 18.0 & 34.0 & 3.9 & 15.4 & 31.3 & 0.0 \\
\hline $55-64$ & 4.1 & 6.6 & 1.9 & 1.3 & 2.7 & 0.1 & 8.2 & 16.2 & 1.5 & 6.8 & 13.9 & 0.0 \\
\hline $65+$ & 0.4 & 0.8 & 0.0 & 0.6 & 1.2 & 0.1 & 2.0 & 4.2 & 0.5 & 0.0 & 0.0 & 0.0 \\
\hline \multicolumn{13}{|c|}{ Unknown Age } \\
\hline TOTAL & 15.5 & 21.4 & 9.8 & 4.6 & 9.1 & 0.5 & 28.1 & 49.5 & 8.4 & 16.3 & 29.0 & 3.5 \\
\hline
\end{tabular}

\begin{tabular}{|c|c|c|c|c|c|c|c|c|c|}
\hline \multirow{2}{*}{$\begin{array}{l}\text { Age } \\
\text { Group }\end{array}$} & \multicolumn{3}{|c|}{ Whites } & \multicolumn{3}{|c|}{ Multirace } & \multicolumn{3}{|c|}{ Hispanics } \\
\hline & Total $^{\dagger}$ & Male & Female & Total $^{+}$ & Male & Female & Total $^{+}$ & Male & Female \\
\hline $0-4$ & 0.0 & 0.0 & 0.0 & 0.0 & 0.0 & 0.0 & 0.0 & 0.0 & 0.0 \\
\hline $5-9$ & 0.0 & 0.0 & 0.0 & 0.0 & 0.0 & 0.0 & 0.0 & 0.0 & 0.0 \\
\hline $10-14$ & 0.0 & 0.0 & 0.0 & 0.0 & 0.0 & 0.0 & 0.1 & 0.1 & 0.0 \\
\hline 15-19 & 3.1 & 4.3 & 1.9 & 3.6 & 5.5 & 1.7 & 8.0 & 12.3 & 3.5 \\
\hline $20-24$ & 12.3 & 19.2 & 5.0 & 19.8 & 31.1 & 8.3 & 30.9 & 51.8 & 8.6 \\
\hline $25-29$ & 16.2 & 26.1 & 5.9 & 25.1 & 46.7 & 4.5 & 36.6 & 62.3 & 8.1 \\
\hline $30-34$ & 15.4 & 24.9 & 5.7 & 29.1 & 56.6 & 4.2 & 29.7 & 50.3 & 6.7 \\
\hline $35-39$ & 12.2 & 20.2 & 4.0 & 26.4 & 45.2 & 9.6 & 21.7 & 36.9 & 5.4 \\
\hline $40-44$ & 9.7 & 16.5 & 2.8 & 18.1 & 36.2 & 2.0 & 15.5 & 27.1 & 3.5 \\
\hline $45-54$ & 8.0 & 14.7 & 1.5 & 14.3 & 29.0 & 1.1 & 11.7 & 21.2 & 2.1 \\
\hline $55-64$ & 3.7 & 7.1 & 0.4 & 6.0 & 12.6 & 0.0 & 4.9 & 9.0 & 1.0 \\
\hline $65+$ & 0.6 & 1.4 & 0.0 & 1.3 & 2.9 & 0.0 & 1.1 & 2.4 & 0.2 \\
\hline \multicolumn{10}{|c|}{ Unknown Age } \\
\hline TOTAL & 6.0 & 10.3 & 1.8 & 9.4 & 16.7 & 2.2 & 13.0 & 22.7 & 3.1 \\
\hline
\end{tabular}

* Per 100,000.

† Total includes cases reported with unknown sex.

NOTE: These tables should be used only for race/Hispanic ethnicity comparisons. See Table 34 for age-specific cases and rates and Tables $27-29$ for total and sex-specific cases and rates. Primary and secondary syphilis reported among children aged 0-4 may represent the misclassification of congenitally-acquired syphilis. Cases reported as congenitally-acquired syphilis (congenital syphilis) can be found in Table 40. No population data exist for unknown sex, unknown age, or other/unknown race; therefore rates are not calculated. 
Table 36. Early Non-Primary Non-Secondary Syphilis - Reported Cases and Rates of Reported Cases by State/ Territory and Region in Alphabetical Order, United States, 2014-2018

\begin{tabular}{|c|c|c|c|c|c|c|c|c|c|c|}
\hline \multirow[b]{2}{*}{ State/Territory } & \multicolumn{5}{|c|}{ Cases } & \multicolumn{5}{|c|}{ Rates per 100,000 Population } \\
\hline & 2014 & 2015 & 2016 & 2017 & 2018 & 2014 & 2015 & 2016 & 2017 & 2018 \\
\hline Alabama & 144 & 177 & 293 & 425 & 447 & 3.0 & 3.6 & 6.0 & 8.7 & 9.2 \\
\hline Alaska & 25 & 13 & 13 & 9 & 41 & 3.4 & 1.8 & 1.8 & 1.2 & 5.5 \\
\hline Arizona & 311 & 361 & 488 & 620 & 898 & 4.6 & 5.3 & 7.0 & 8.8 & 12.8 \\
\hline Arkansas & 152 & 216 & 280 & 328 & 364 & 5.1 & 7.3 & 9.4 & 10.9 & 12.1 \\
\hline California & 3,396 & 4,435 & 5,289 & 7,028 & 7,708 & 8.8 & 11.3 & 13.5 & 17.8 & 19.5 \\
\hline Colorado & 164 & 212 & 274 & 281 & 362 & 3.1 & 3.9 & 4.9 & 5.0 & 6.5 \\
\hline Connecticut & 62 & 97 & 84 & 145 & 77 & 1.7 & 2.7 & 2.3 & 4.0 & 2.1 \\
\hline Delaware & 33 & 47 & 57 & 49 & 36 & 3.5 & 5.0 & 6.0 & 5.1 & 3.7 \\
\hline District of Columbia & 142 & 200 & 355 & 341 & 336 & 21.6 & 29.8 & 52.1 & 49.1 & 48.4 \\
\hline Florida & 1,886 & 2,288 & 2,634 & 3,033 & 3,939 & 9.5 & 11.3 & 12.8 & 14.5 & 18.8 \\
\hline Georgia & 1,078 & 1,477 & 1,263 & 1,218 & 1,517 & 10.7 & 14.5 & 12.2 & 11.7 & 14.5 \\
\hline Hawaii & 25 & 56 & 89 & 58 & 87 & 1.8 & 3.9 & 6.2 & 4.1 & 6.1 \\
\hline Idaho & 12 & 24 & 33 & 41 & 33 & 0.7 & 1.5 & 2.0 & 2.4 & 1.9 \\
\hline Illinois & 819 & 889 & 1,138 & 1,192 & 1,464 & 6.4 & 6.9 & 8.9 & 9.3 & 11.4 \\
\hline Indiana & 129 & 220 & 247 & 250 & 359 & 2.0 & 3.3 & 3.7 & 3.7 & 5.4 \\
\hline lowa & 82 & 69 & 59 & 91 & 85 & 2.6 & 2.2 & 1.9 & 2.9 & 2.7 \\
\hline Kansas & 92 & 153 & 178 & 202 & 291 & 3.2 & 5.3 & 6.1 & 6.9 & 10.0 \\
\hline Kentucky & 169 & 164 & 189 & 236 & 294 & 3.8 & 3.7 & 4.3 & 5.3 & 6.6 \\
\hline Louisiana & 372 & 439 & 568 & 623 & 576 & 8.0 & 9.4 & 12.1 & 13.3 & 12.3 \\
\hline Maine & 7 & 10 & 6 & 36 & 43 & 0.5 & 0.8 & 0.5 & 2.7 & 3.2 \\
\hline Maryland & 529 & 594 & 598 & 683 & 857 & 8.9 & 9.9 & 9.9 & 11.3 & 14.2 \\
\hline Massachusetts & 282 & 355 & 538 & 549 & 620 & 4.2 & 5.2 & 7.9 & 8.0 & 9.0 \\
\hline Michigan & 243 & 282 & 290 & 330 & 407 & 2.5 & 2.8 & 2.9 & 3.3 & 4.1 \\
\hline Minnesota & 159 & 185 & 251 & 313 & 286 & 2.9 & 3.4 & 4.5 & 5.6 & 5.1 \\
\hline Mississippi & 336 & 405 & 490 & 555 & 937 & 11.2 & 13.5 & 16.4 & 18.6 & 31.4 \\
\hline Missouri & 240 & 247 & 276 & 423 & 546 & 4.0 & 4.1 & 4.5 & 6.9 & 8.9 \\
\hline Montana & 1 & 5 & 6 & 23 & 25 & 0.1 & 0.5 & 0.6 & 2.2 & 2.4 \\
\hline Nebraska & 19 & 5 & 19 & 26 & 37 & 1.0 & 0.3 & 1.0 & 1.4 & 1.9 \\
\hline Nevada & 389 & 439 & 510 & 498 & 512 & 13.7 & 15.2 & 17.3 & 16.6 & 17.1 \\
\hline New Hampshire & 22 & 16 & 33 & 37 & 41 & 1.7 & 1.2 & 2.5 & 2.8 & 3.1 \\
\hline New Jersey & 612 & 714 & 755 & 865 & 788 & 6.8 & 8.0 & 8.4 & 9.6 & 8.8 \\
\hline New Mexico & 76 & 71 & 118 & 120 & 161 & 3.6 & 3.4 & 5.7 & 5.7 & 7.7 \\
\hline New York & 2,307 & 2,802 & 3,504 & 3,914 & 4,097 & 11.7 & 14.2 & 17.7 & 19.7 & 20.6 \\
\hline North Carolina & 468 & 753 & 799 & 771 & 797 & 4.7 & 7.5 & 7.9 & 7.5 & 7.8 \\
\hline North Dakota & 22 & 17 & 12 & 12 & 13 & 3.0 & 2.2 & 1.6 & 1.6 & 1.7 \\
\hline Ohio & 265 & 326 & 389 & 454 & 481 & 2.3 & 2.8 & 3.3 & 3.9 & 4.1 \\
\hline Oklahoma & 198 & 222 & 339 & 478 & 342 & 5.1 & 5.7 & 8.6 & 12.2 & 8.7 \\
\hline Oregon & 149 & 214 & 250 & 205 & 299 & 3.8 & 5.3 & 6.1 & 4.9 & 7.2 \\
\hline Pennsylvania & 641 & 770 & 982 & 1,100 & 1,192 & 5.0 & 6.0 & 7.7 & 8.6 & 9.3 \\
\hline Rhode Island & 49 & 38 & 63 & 71 & 71 & 4.6 & 3.6 & 6.0 & 6.7 & 6.7 \\
\hline South Carolina & 467 & 496 & 613 & 687 & 732 & 9.7 & 10.1 & 12.4 & 13.7 & 14.6 \\
\hline South Dakota & 23 & 11 & 14 & 19 & 9 & 2.7 & 1.3 & 1.6 & 2.2 & 1.0 \\
\hline Tennessee & 236 & 312 & 337 & 412 & 472 & 3.6 & 4.7 & 5.1 & 6.1 & 7.0 \\
\hline Texas & 1,984 & 2,471 & 2,872 & 3,680 & 4,245 & 7.4 & 9.0 & 10.3 & 13.0 & 15.0 \\
\hline Utah & 41 & 31 & 61 & 85 & 101 & 1.4 & 1.0 & 2.0 & 2.7 & 3.3 \\
\hline Vermont & 7 & 6 & 14 & 13 & 18 & 1.1 & 1.0 & 2.2 & 2.1 & 2.9 \\
\hline Virginia & 274 & 410 & 602 & 659 & 668 & 3.3 & 4.9 & 7.2 & 7.8 & 7.9 \\
\hline Washington & 198 & 293 & 446 & 588 & 599 & 2.8 & 4.1 & 6.1 & 7.9 & 8.1 \\
\hline West Virginia & 23 & 40 & 51 & 34 & 55 & 1.2 & 2.2 & 2.8 & 1.9 & 3.0 \\
\hline Wisconsin & 91 & 95 & 150 & 199 & 163 & 1.6 & 1.6 & 2.6 & 3.4 & 2.8 \\
\hline Wyoming & 1 & 1 & 5 & 4 & 11 & 0.2 & 0.2 & 0.9 & 0.7 & 1.9 \\
\hline US TOTAL & 19,452 & 24,173 & 28,924 & 34,013 & 38,539 & 6.1 & 7.5 & 9.0 & 10.4 & 11.8 \\
\hline Northeast & 3,989 & 4,808 & 5,979 & 6,730 & 6,947 & 7.1 & 8.5 & 10.6 & 11.9 & 12.3 \\
\hline Midwest & 2,184 & 2,499 & 3,023 & 3,511 & 4,141 & 3.2 & 3.7 & 4.4 & 5.1 & 6.1 \\
\hline South & 8,491 & 10,711 & 12,340 & 14,212 & 16,614 & 7.1 & 8.8 & 10.1 & 11.5 & 13.4 \\
\hline West & 4,788 & 6,155 & 7,582 & 9,560 & 10,837 & 6.4 & 8.1 & 9.9 & 12.3 & 14.0 \\
\hline American Samoa & NR & NR & NR & NR & 0 & - & - & - & - & 0.0 \\
\hline Guam & 1 & 2 & 1 & 3 & 3 & 0.6 & 1.2 & 0.6 & 1.8 & 1.8 \\
\hline Northern Mariana Islands & NR & NR & NR & NR & 0 & - & - & - & - & 0.0 \\
\hline Puerto Rico & 375 & 565 & 570 & 527 & 577 & 10.6 & 16.3 & 16.7 & 15.7 & 17.2 \\
\hline Virgin Islands & 0 & 7 & 2 & 0 & NR & 0.0 & 6.8 & 1.9 & 0.0 & - \\
\hline TERRITORIES & 376 & 574 & 573 & 530 & 580 & 9.9 & 15.4 & 15.5 & 14.6 & 16.0 \\
\hline TOTAL & 19,828 & 24,747 & 29,497 & 34,543 & 39,119 & 6.1 & 7.6 & 9.0 & 10.5 & 11.9 \\
\hline
\end{tabular}

$\mathrm{NR}=$ No report.

NOTE: See Section A1.11 in the Appendix for more information on interpreting case counts and rates in US territories. 
Table 37. Early Non-Primary Non-Secondary Syphilis - Reported Cases and Rates of Reported Cases in Selected Metropolitan Statistical Areas (MSAs)* in Alphabetical Order, United States, 2014-2018

\begin{tabular}{|c|c|c|c|c|c|c|c|c|c|c|}
\hline \multirow[b]{2}{*}{ MSAs } & \multicolumn{5}{|c|}{ Cases } & \multicolumn{5}{|c|}{ Rates per 100,000 Population } \\
\hline & 2014 & 2015 & 2016 & 2017 & 2018 & 2014 & 2015 & 2016 & 2017 & 2018 \\
\hline Atlanta-Sandy Springs-Roswell, GA & 863 & 1,067 & 1,053 & 1,027 & 1,197 & 15.4 & 18.7 & 18.2 & 17.5 & 20.3 \\
\hline Austin-Round Rock, TX & 207 & 242 & 291 & 424 & 388 & 10.7 & 12.1 & 14.2 & 20.0 & 18.3 \\
\hline Baltimore-Columbia-Towson, MD & 296 & 344 & 358 & 337 & 454 & 10.6 & 12.3 & 12.8 & 12.0 & 16.2 \\
\hline Birmingham-Hoover, AL & 46 & 60 & $79^{\ddagger}$ & 124 & 135 & 4.0 & 5.2 & $6.9^{\ddagger}$ & 10.8 & 11.7 \\
\hline Boston-Cambridge-Newton, MA-NH & 208 & $235^{+}$ & $408^{+}$ & 429 & 479 & 4.4 & $4.9^{+}$ & $8.5^{+}$ & 8.9 & 9.9 \\
\hline Buffalo-Cheektowaga-Niagara Falls, NY & 19 & 37 & 29 & 32 & 44 & 1.7 & 3.3 & 2.6 & 2.8 & 3.9 \\
\hline Charlotte-Concord-Gastonia, NC-SC & 129 & 206 & 264 & 259 & 258 & 5.4 & 8.5 & 10.7 & 10.3 & 10.2 \\
\hline Chicago-Naperville-Elgin, IL-IN-WI & 734 & 814 & 1,058 & 1,091 & 1,369 & 7.7 & 8.5 & 11.1 & 11.4 & 14.4 \\
\hline Cincinnati, OH-KY-IN & 98 & 92 & 74 & 83 & 117 & 4.6 & 4.3 & 3.4 & 3.8 & 5.4 \\
\hline Cleveland-Elyria, $\mathrm{OH}$ & 31 & 37 & 63 & 73 & 58 & 1.5 & 1.8 & 3.1 & 3.5 & 2.8 \\
\hline Columbus, $\mathrm{OH}$ & 82 & 130 & 149 & 183 & 167 & 4.1 & 6.4 & 7.3 & 8.8 & 8.0 \\
\hline Dallas-Fort Worth-Arlington, TX & 644 & 932 & 1,038 & 1,256 & 1,334 & 9.3 & 13.1 & 14.4 & 17.0 & 18.0 \\
\hline Denver-Aurora-Lakewood, CO & 145 & 175 & 212 & 213 & 283 & 5.3 & 6.2 & 7.4 & 7.4 & 9.8 \\
\hline Detroit-Warren-Dearborn, MI & 163 & 206 & 194 & 196 & 265 & 3.8 & 4.8 & 4.5 & 4.5 & 6.1 \\
\hline Hartford-West Hartford-East Hartford, CT & 16 & 31 & 20 & 31 & 20 & 1.3 & 2.6 & 1.7 & 2.6 & 1.7 \\
\hline Houston-The Woodlands-Sugar Land, TX & 444 & 522 & 585 & 740 & 1,026 & 6.8 & 7.8 & 8.6 & 10.7 & 14.9 \\
\hline Indianapolis-Carmel-Anderson, IN & 91 & 143 & 165 & 161 & 223 & 4.6 & 7.2 & 8.2 & 7.9 & 11.0 \\
\hline Jacksonville, FL & 69 & 162 & 137 & 191 & 202 & 4.9 & 11.2 & 9.3 & 12.7 & 13.4 \\
\hline Kansas City, MO-KS & 132 & 133 & 140 & 158 & 247 & 6.4 & 6.4 & 6.7 & 7.4 & 11.6 \\
\hline Las Vegas-Henderson-Paradise, NV & 375 & 413 & 470 & 451 & 447 & 18.1 & 19.5 & 21.8 & 20.5 & 20.3 \\
\hline Los Angeles-Long Beach-Anaheim, CA & 1,619 & 2,052 & 2,403 & 3,119 & 3,565 & 12.2 & 15.4 & 18.1 & 23.4 & 26.7 \\
\hline Louisville-Jefferson County, KY-IN & 82 & 90 & 118 & 143 & 166 & 6.5 & 7.0 & 9.2 & 11.1 & 12.8 \\
\hline Memphis, TN-MS-AR & 143 & 195 & 246 & 244 & 256 & 10.6 & 14.5 & 18.3 & 18.1 & 19.0 \\
\hline Miami-Fort Lauderdale-West Palm Beach, FL & 1,094 & 1,220 & 1,282 & 1,569 & 2,071 & 18.4 & 20.3 & 21.1 & 25.5 & 33.6 \\
\hline Milwaukee-Waukesha-West Allis, WI & 69 & 66 & 108 & 130 & 93 & 4.4 & 4.2 & 6.9 & 8.2 & 5.9 \\
\hline Minneapolis-St. Paul-Bloomington, MN-WI & 155 & 170 & 226 & 280 & 214 & 4.4 & 4.8 & 6.4 & 7.8 & 5.9 \\
\hline Nashville-Davidson-Murfreesboro-Franklin, TN & 83 & 82 & 72 & 102 & 179 & 4.6 & 4.5 & 3.9 & 5.4 & 9.4 \\
\hline New Orleans-Metairie, LA & 122 & 171 & 242 & 244 & 271 & 9.7 & 13.5 & 19.1 & 19.1 & 21.2 \\
\hline New York-Newark-Jersey City, NY-NJ-PA & 2,681 & 3,210 & 4,008 & 4,405 & 4,522 & 13.3 & 15.9 & 19.9 & 21.7 & 22.3 \\
\hline Oklahoma City, OK & 107 & 114 & 195 & 270 & 179 & 8.0 & 8.4 & 14.2 & 19.5 & 12.9 \\
\hline Orlando-Kissimmee-Sanford, FL & 180 & 266 & 377 & 359 & 490 & 7.8 & 11.1 & 15.4 & 14.3 & 19.5 \\
\hline Philadelphia-Camden-Wilmington, PA-NJ-DE-MD & 512 & 616 & 736 & 835 & 796 & 8.5 & 10.1 & 12.1 & 13.7 & 13.1 \\
\hline Phoenix-Mesa-Scottsdale, AZ & 240 & 268 & 381 & 440 & 697 & 5.3 & 5.9 & 8.2 & 9.3 & 14.7 \\
\hline Pittsburgh, PA & 63 & 111 & 125 & 109 & 129 & 2.7 & 4.7 & 5.3 & 4.7 & 5.5 \\
\hline Portland-Vancouver-Hillsboro, OR-WA & 124 & 170 & 226 & 187 & 266 & 5.3 & 7.1 & 9.3 & 7.6 & 10.8 \\
\hline Providence-Warwick, RI-MA & 64 & $48^{+}$ & $71^{+}$ & 92 & 91 & 4.0 & $3.0^{+}$ & $4.4^{\dagger}$ & 5.7 & 5.6 \\
\hline Raleigh, NC & 77 & 115 & 135 & 130 & 105 & 6.2 & 9.0 & 10.4 & 9.7 & 7.9 \\
\hline Richmond, VA & 68 & 98 & 162 & 157 & 162 & 5.4 & 7.7 & 12.6 & 12.1 & 12.5 \\
\hline Riverside-San Bernardino-Ontario, CA & 223 & 311 & 379 & 513 & 605 & 5.0 & 6.9 & 8.4 & 11.2 & 13.2 \\
\hline Sacramento-Roseville-Arden-Arcade, CA & 74 & 137 & 133 & 194 & 244 & 3.3 & 6.0 & 5.8 & 8.3 & 10.5 \\
\hline Salt Lake City, UT & 31 & 23 & 43 & 55 & 71 & 2.7 & 2.0 & 3.6 & 4.6 & 5.9 \\
\hline San Antonio-New Braunfels, TX & 308 & 258 & 339 & 485 & 553 & 13.2 & 10.8 & 14.0 & 19.6 & 22.4 \\
\hline San Diego-Carlsbad, CA & 299 & 343 & 461 & 550 & 535 & 9.2 & 10.4 & 13.9 & 16.5 & 16.0 \\
\hline San Francisco-Oakland-Hayward, CA & 839 & 964 & 919 & 1,218 & 1,264 & 18.3 & 20.7 & 19.6 & 25.8 & 26.7 \\
\hline San Jose-Sunnyvale-Santa Clara, CA & 58 & 96 & 135 & 200 & 233 & 3.0 & 4.9 & 6.8 & 10.0 & 11.7 \\
\hline Seattle-Tacoma-Bellevue, WA & 143 & 221 & 303 & 424 & 422 & 3.9 & 5.9 & 8.0 & 11.0 & 10.9 \\
\hline St. Louis, MO-IL & 139 & 138 & 151 & 245 & 233 & 5.0 & 4.9 & 5.4 & 8.7 & 8.3 \\
\hline Tampa-St. Petersburg-Clearwater, FL & 227 & 258 & 364 & 296 & 392 & 7.8 & 8.7 & 12.0 & 9.6 & 12.7 \\
\hline Virginia Beach-Norfolk-Newport News, VA-NC & 90 & 167 & 245 & 270 & 244 & 5.2 & 9.7 & 14.2 & 15.6 & 14.1 \\
\hline Washington-Arlington-Alexandria, DC-VA-MD-WV & 286 & 320 & 355 & 820 & 893 & 4.7 & 5.2 & 5.8 & 13.2 & 14.4 \\
\hline SELECTED MSAs TOTAL & 14,992 & 18,279 & 21,727 & 25,544 & 28,654 & 8.6 & 10.3 & 12.2 & 14.2 & 15.9 \\
\hline
\end{tabular}

* MSAs were selected on the basis of the largest population in the 2010 US Census.

${ }^{\dagger}$ The variable used to identify county, which is used to classify cases into MSAs, was complete for $\leq 95 \%$ of cases in a state contributing data to this MSA. See Section A1.4 in the Appendix for more information.

‡ 2016 county data for Alabama have been corrected and may not match previous reports. 
Table 38. Unknown Duration or Late Syphilis* - Reported Cases and Rates of Reported Cases by State/ Territory and Region in Alphabetical Order, United States, 2014-2018

\begin{tabular}{|c|c|c|c|c|c|c|c|c|c|c|}
\hline \multirow[b]{2}{*}{ State/Territory } & \multicolumn{5}{|c|}{ Cases } & \multicolumn{5}{|c|}{ Rates per 100,000 Population } \\
\hline & 2014 & 2015 & 2016 & 2017 & 2018 & 2014 & 2015 & 2016 & 2017 & 2018 \\
\hline Alabama & 167 & 197 & 232 & 347 & 354 & 3.4 & 4.1 & 4.8 & 7.1 & 7.3 \\
\hline Alaska & 5 & 3 & 3 & 6 & 16 & 0.7 & 0.4 & 0.4 & 0.8 & 2.2 \\
\hline Arizona & 558 & 532 & 680 & 829 & 1,243 & 8.3 & 7.8 & 9.8 & 11.8 & 17.7 \\
\hline Arkansas & 110 & 145 & 131 & 156 & 287 & 3.7 & 4.9 & 4.4 & 5.2 & 9.6 \\
\hline California & 4,110 & 4,966 & 6,216 & 7,787 & 9,609 & 10.6 & 12.7 & 15.8 & 19.7 & 24.3 \\
\hline Colorado & 5 & 96 & 211 & 240 & 378 & 0.1 & 1.8 & 3.8 & 4.3 & 6.7 \\
\hline Connecticut & 21 & 30 & 23 & 28 & 94 & 0.6 & 0.8 & 0.6 & 0.8 & 2.6 \\
\hline Delaware & 30 & 21 & 34 & 88 & 63 & 3.2 & 2.2 & 3.6 & 9.1 & 6.5 \\
\hline District of Columbia & 23 & 26 & 51 & 230 & 148 & 3.5 & 3.9 & 7.5 & 33.1 & 21.3 \\
\hline Florida & 2,429 & 2,723 & 3,233 & 3,435 & 3,773 & 12.2 & 13.4 & 15.7 & 16.4 & 18.0 \\
\hline Georgia & 1,055 & 1,245 & 1,478 & 1,580 & 1,773 & 10.4 & 12.2 & 14.3 & 15.1 & 17.0 \\
\hline Hawaii & 13 & 14 & 13 & 10 & 27 & 0.9 & 1.0 & 0.9 & 0.7 & 1.9 \\
\hline Idaho & 22 & 21 & 44 & 46 & 54 & 1.3 & 1.3 & 2.6 & 2.7 & 3.1 \\
\hline Illinois & 1,087 & 1,285 & 1,623 & 1,399 & 1,570 & 8.4 & 10.0 & 12.7 & 10.9 & 12.3 \\
\hline Indiana & 170 & 189 & 197 & 211 & 258 & 2.6 & 2.9 & 3.0 & 3.2 & 3.9 \\
\hline lowa & 84 & 88 & 127 & 96 & 112 & 2.7 & 2.8 & 4.1 & 3.1 & 3.6 \\
\hline Kansas & 48 & 0 & 0 & 3 & 44 & 1.7 & 0.0 & 0.0 & 0.1 & 1.5 \\
\hline Kentucky & 117 & 123 & 159 & 218 & 212 & 2.7 & 2.8 & 3.6 & 4.9 & 4.8 \\
\hline Louisiana & 1,180 & 1,277 & 1,233 & 1,495 & 1,456 & 25.4 & 27.3 & 26.3 & 31.9 & 31.1 \\
\hline Maine & 0 & 0 & 16 & 31 & 30 & 0.0 & 0.0 & 1.2 & 2.3 & 2.2 \\
\hline Maryland & 481 & 749 & 719 & 783 & 913 & 8.0 & 12.5 & 12.0 & 12.9 & 15.1 \\
\hline Massachusetts & 227 & 486 & 416 & 387 & 133 & 3.4 & 7.2 & 6.1 & 5.6 & 1.9 \\
\hline Michigan & 416 & 393 & 424 & 447 & 622 & 4.2 & 4.0 & 4.3 & 4.5 & 6.2 \\
\hline Minnesota & 215 & 220 & 289 & 327 & 330 & 3.9 & 4.0 & 5.2 & 5.9 & 5.9 \\
\hline Mississippi & 116 & 136 & 107 & 71 & 50 & 3.9 & 4.5 & 3.6 & 2.4 & 1.7 \\
\hline Missouri & 178 & 220 & 271 & 397 & 544 & 2.9 & 3.6 & 4.4 & 6.5 & 8.9 \\
\hline Montana & 0 & 2 & 4 & 13 & 34 & 0.0 & 0.2 & 0.4 & 1.2 & 3.2 \\
\hline Nebraska & 26 & 31 & 34 & 48 & 63 & 1.4 & 1.6 & 1.8 & 2.5 & 3.3 \\
\hline Nevada & 142 & 133 & 347 & 575 & 775 & 5.0 & 4.6 & 11.8 & 19.2 & 25.9 \\
\hline New Hampshire & 21 & 28 & 27 & 29 & 31 & 1.6 & 2.1 & 2.0 & 2.2 & 2.3 \\
\hline New Jersey & 263 & 220 & 381 & 489 & 406 & 2.9 & 2.5 & 4.3 & 5.4 & 4.5 \\
\hline New Mexico & 80 & 141 & 160 & 196 & 337 & 3.8 & 6.8 & 7.7 & 9.4 & 16.1 \\
\hline New York & 3,073 & 2,975 & 3,484 & 3,592 & 3,404 & 15.6 & 15.0 & 17.6 & 18.1 & 17.1 \\
\hline North Carolina & 791 & 783 & 756 & 1,015 & 1,075 & 8.0 & 7.8 & 7.5 & 9.9 & 10.5 \\
\hline North Dakota & 16 & 14 & 16 & 22 & 30 & 2.2 & 1.8 & 2.1 & 2.9 & 4.0 \\
\hline Ohio & 381 & 445 & 483 & 596 & 667 & 3.3 & 3.8 & 4.2 & 5.1 & 5.7 \\
\hline Oklahoma & 59 & 83 & 90 & 95 & 253 & 1.5 & 2.1 & 2.3 & 2.4 & 6.4 \\
\hline Oregon & 159 & 218 & 227 & 283 & 299 & 4.0 & 5.4 & 5.5 & 6.8 & 7.2 \\
\hline Pennsylvania & 346 & 356 & 295 & 335 & 416 & 2.7 & 2.8 & 2.3 & 2.6 & 3.2 \\
\hline Rhode Island & 40 & 48 & 81 & 79 & 117 & 3.8 & 4.5 & 7.7 & 7.5 & 11.0 \\
\hline South Carolina & 28 & 41 & 36 & 40 & 27 & 0.6 & 0.8 & 0.7 & 0.8 & 0.5 \\
\hline South Dakota & 16 & 21 & 15 & 20 & 23 & 1.9 & 2.4 & 1.7 & 2.3 & 2.6 \\
\hline Tennessee & 502 & 575 & 713 & 543 & 688 & 7.7 & 8.7 & 10.7 & 8.1 & 10.2 \\
\hline Texas & 4,110 & 4,047 & 4,666 & 6,035 & 5,819 & 15.2 & 14.7 & 16.7 & 21.3 & 20.6 \\
\hline Utah & 61 & 73 & 106 & 97 & 152 & 2.1 & 2.4 & 3.5 & 3.1 & 4.9 \\
\hline Vermont & 0 & 0 & 0 & 0 & 0 & 0.0 & 0.0 & 0.0 & 0.0 & 0.0 \\
\hline Virginia & 137 & 276 & 235 & 551 & 659 & 1.6 & 3.3 & 2.8 & 6.5 & 7.8 \\
\hline Washington & 310 & 366 & 400 & 480 & 504 & 4.4 & 5.1 & 5.5 & 6.5 & 6.8 \\
\hline West Virginia & 4 & 17 & 45 & 25 & 64 & 0.2 & 0.9 & 2.5 & 1.4 & 3.5 \\
\hline Wisconsin & 108 & 88 & 140 & 176 & 193 & 1.9 & 1.5 & 2.4 & 3.0 & 3.3 \\
\hline Wyoming & 1 & 4 & 5 & 11 & 8 & 0.2 & 0.7 & 0.9 & 1.9 & 1.4 \\
\hline US TOTAL & 23,541 & 26,170 & 30,676 & 35,992 & 40,137 & 7.4 & 8.1 & 9.5 & 11.1 & 12.3 \\
\hline Northeast & 3,991 & 4,143 & 4,723 & 4,970 & 4,631 & 7.1 & 7.4 & 8.4 & 8.8 & 8.2 \\
\hline Midwest & 2,745 & 2,994 & 3,619 & 3,742 & 4,456 & 4.1 & 4.4 & 5.3 & 5.5 & 6.5 \\
\hline South & 11,339 & 12,464 & 13,918 & 16,707 & 17,614 & 9.5 & 10.3 & 11.4 & 13.5 & 14.2 \\
\hline West & 5,466 & 6,569 & 8,416 & 10,573 & 13,436 & 7.3 & 8.6 & 11.0 & 13.7 & 17.4 \\
\hline American Samoa & NR & NR & NR & NR & 0 & - & - & - & - & 0.0 \\
\hline Guam & 5 & 16 & 10 & 5 & 9 & 3.1 & 9.9 & 6.0 & 3.0 & 5.4 \\
\hline Northern Mariana Islands & NR & NR & NR & NR & 1 & - & - & - & - & 1.9 \\
\hline Puerto Rico & 101 & 166 & 117 & 110 & 138 & 2.8 & 4.8 & 3.4 & 3.3 & 4.1 \\
\hline Virgin Islands & 4 & 10 & 0 & 0 & NR & 3.8 & 9.7 & 0.0 & 0.0 & - \\
\hline TERRITORIES & 110 & 192 & 127 & 115 & 148 & 2.9 & 5.1 & 3.4 & 3.2 & 4.1 \\
\hline TOTAL & 23,651 & 26,362 & 30,803 & 36,107 & 40,285 & 7.3 & 8.1 & 9.4 & 11.0 & 12.2 \\
\hline
\end{tabular}

* The case classification of 'Unknown duration or late syphilis' went into effect in January 2018. During 2014-2017, cases in this category include cases classified as late latent syphilis and late syphilis with clinical manifestations. See Appendix A1.9 for a detailed explanation of changes to the syphilis case definition. $\mathrm{NR}=$ No report.

NOTE: See Section A1.11 in the Appendix for more information on interpreting case counts and rates in US territories. 
Table 39. Unknown Duration or Late Syphilis* - Reported Cases and Rates of Reported Cases in Selected Metropolitan Statistical Areas (MSAs) ${ }^{\dagger}$ in Alphabetical Order, United States, 2014-2018

\begin{tabular}{|c|c|c|c|c|c|c|c|c|c|c|}
\hline \multirow[b]{2}{*}{ MSAs } & \multicolumn{5}{|c|}{ Cases } & \multicolumn{5}{|c|}{ Rates per 100,000 Population } \\
\hline & 2014 & 2015 & 2016 & 2017 & 2018 & 2014 & 2015 & 2016 & 2017 & 2018 \\
\hline Atlanta-Sandy Springs-Roswell, GA & 804 & $927^{\S}$ & 1,135 & 1,146 & 1,249 & 14.3 & $16.2^{\S}$ & 19.6 & 19.5 & 21.2 \\
\hline Austin-Round Rock, TX & 246 & 175 & 212 & 226 & 295 & 12.7 & 8.7 & 10.3 & 10.7 & 13.9 \\
\hline Baltimore-Columbia-Towson, MD & 226 & 316 & 327 & 389 & 470 & 8.1 & 11.3 & 11.7 & 13.9 & 16.7 \\
\hline Birmingham-Hoover, AL & 53 & 62 & $57^{\ddagger}$ & 68 & 85 & 4.6 & 5.4 & $5.0^{\ddagger}$ & 5.9 & 7.4 \\
\hline Boston-Cambridge-Newton, MA-NH & $159^{\S}$ & $319^{\S}$ & $271^{\S}$ & 266 & 104 & $3.4^{\S}$ & $6.7^{\S}$ & $5.7^{\S}$ & 5.5 & 2.2 \\
\hline Buffalo-Cheektowaga-Niagara Falls, NY & 67 & 53 & 54 & 83 & 65 & 5.9 & 4.7 & 4.8 & 7.3 & 5.7 \\
\hline Charlotte-Concord-Gastonia, NC-SC & 183 & 191 & 201 & 252 & 247 & 7.7 & 7.9 & 8.1 & 10.0 & 9.8 \\
\hline Chicago-Naperville-Elgin, IL-IN-WI & 988 & 1,169 & 1,529 & 1,288 & 1,424 & 10.3 & 12.2 & 16.1 & 13.5 & 14.9 \\
\hline Cincinnati, OH-KY-IN & 124 & 129 & 105 & 136 & 149 & 5.8 & 6.0 & 4.8 & 6.2 & 6.8 \\
\hline Cleveland-Elyria, $\mathrm{OH}$ & 88 & 120 & 159 & 176 & 181 & 4.3 & 5.8 & 7.7 & 8.5 & 8.8 \\
\hline Columbus, $\mathrm{OH}$ & 101 & 106 & 109 & 155 & 149 & 5.1 & 5.2 & 5.3 & 7.5 & 7.2 \\
\hline Dallas-Fort Worth-Arlington, TX & 1,065 & 837 & 1,069 & 1,854 & 1,773 & 15.3 & 11.8 & 14.8 & 25.1 & 24.0 \\
\hline Denver-Aurora-Lakewood, CO & 0 & 59 & 150 & 185 & 268 & 0.0 & 2.1 & 5.3 & 6.4 & 9.3 \\
\hline Detroit-Warren-Dearborn, MI & 312 & 291 & 295 & 312 & 412 & 7.3 & 6.8 & 6.9 & 7.2 & 9.6 \\
\hline Hartford-West Hartford-East Hartford, CT & 10 & 8 & 3 & 3 & 27 & 0.8 & 0.7 & 0.2 & 0.2 & 2.2 \\
\hline Houston-The Woodlands-Sugar Land, TX & 1,430 & 1,592 & 1,805 & 2,060 & 1,857 & 22.0 & 23.9 & 26.7 & 29.9 & 26.9 \\
\hline Indianapolis-Carmel-Anderson, IN & 83 & 93 & 87 & 106 & 125 & 4.2 & 4.7 & 4.3 & 5.2 & 6.2 \\
\hline Jacksonville, FL & 128 & 179 & 181 & 249 & 254 & 9.0 & 12.3 & 12.2 & 16.5 & 16.9 \\
\hline Kansas City, MO-KS & 54 & 40 & 87 & 92 & 183 & 2.6 & 1.9 & 4.1 & 4.3 & 8.6 \\
\hline Las Vegas-Henderson-Paradise, NV & 133 & 102 & 315 & 513 & 675 & 6.4 & 4.8 & 14.6 & 23.3 & 30.6 \\
\hline Los Angeles-Long Beach-Anaheim, CA & 1,679 & 1,902 & 2,532 & 3,164 & 3,338 & 12.7 & 14.3 & 19.0 & 23.7 & 25.0 \\
\hline Louisville-Jefferson County, KY-IN & 70 & 92 & 112 & 127 & 121 & 5.5 & 7.2 & 8.7 & 9.8 & 9.4 \\
\hline Memphis, TN-MS-AR & 236 & 256 & 336 & 257 & 322 & 17.6 & 19.0 & 25.0 & 19.1 & 23.9 \\
\hline Miami-Fort Lauderdale-West Palm Beach, FL & 1,371 & 1,524 & 1,868 & 1,745 & 1,920 & 23.1 & 25.3 & 30.8 & 28.3 & 31.2 \\
\hline Milwaukee-Waukesha-West Allis, WI & 63 & 43 & 70 & 84 & 85 & 4.0 & 2.7 & 4.5 & 5.3 & 5.4 \\
\hline Minneapolis-St. Paul-Bloomington, MN-WI & 187 & 192 & 251 & 261 & 265 & 5.4 & 5.4 & 7.1 & 7.2 & 7.4 \\
\hline Nashville-Davidson-Murfreesboro-Franklin, TN & 148 & 161 & 196 & 101 & 168 & 8.3 & 8.8 & 10.5 & 5.3 & 8.8 \\
\hline New Orleans-Metairie, LA & 383 & 370 & 363 & 391 & 394 & 30.6 & 29.3 & 28.6 & 30.6 & 30.9 \\
\hline New York-Newark-Jersey City, NY-NJ-PA & 3,057 & 2,915 & 3,534 & 3,687 & 3,506 & 15.2 & 14.4 & 17.5 & 18.1 & 17.3 \\
\hline Oklahoma City, OK & 31 & 32 & 47 & 42 & 104 & 2.3 & 2.4 & 3.4 & 3.0 & 7.5 \\
\hline Orlando-Kissimmee-Sanford, FL & 364 & 344 & 416 & 509 & 522 & 15.7 & 14.4 & 17.0 & 20.3 & 20.8 \\
\hline Philadelphia-Camden-Wilmington, PA-NJ-DE-MD & 311 & 314 & 266 & 342 & 380 & 5.1 & 5.2 & 4.4 & 5.6 & 6.2 \\
\hline Phoenix-Mesa-Scottsdale, AZ & 397 & 394 & 534 & 645 & 945 & 8.8 & 8.6 & 11.5 & 13.6 & 19.9 \\
\hline Pittsburgh, PA & 13 & 11 & 11 & 12 & 23 & 0.6 & 0.5 & 0.5 & 0.5 & 1.0 \\
\hline Portland-Vancouver-Hillsboro, OR-WA & 143 & 171 & 173 & 207 & 221 & 6.1 & 7.2 & 7.1 & 8.4 & 9.0 \\
\hline Providence-Warwick, RI-MA & $48^{\S}$ & $74^{\S}$ & $102^{\S}$ & $105^{\S}$ & 123 & $3.0^{\S}$ & $4.6^{\S}$ & $6.3^{\S}$ & $6.5^{5}$ & 7.6 \\
\hline Raleigh, NC & 111 & 125 & 109 & 169 & 170 & 8.9 & 9.8 & 8.4 & 12.7 & 12.7 \\
\hline Richmond, VA & 9 & 36 & 35 & 111 & 107 & 0.7 & 2.8 & 2.7 & 8.6 & 8.3 \\
\hline Riverside-San Bernardino-Ontario, CA & 432 & 508 & 707 & 911 & 1,319 & 9.7 & 11.3 & 15.6 & 19.9 & 28.8 \\
\hline Sacramento-Roseville-Arden-Arcade, CA & 134 & 205 & 199 & 264 & 361 & 6.0 & 9.0 & 8.7 & 11.4 & 15.5 \\
\hline Salt Lake City, UT & 39 & 50 & 76 & 61 & 80 & 3.4 & 4.3 & 6.4 & 5.1 & 6.6 \\
\hline San Antonio-New Braunfels, TX & 448 & 483 & 531 & 612 & 585 & 19.2 & 20.3 & 21.9 & 24.7 & 23.6 \\
\hline San Diego-Carlsbad, CA & 310 & 367 & 425 & 576 & 697 & 9.5 & 11.1 & 12.8 & 17.3 & 20.9 \\
\hline San Francisco-Oakland-Hayward, CA & 502 & 553 & 629 & 691 & 800 & 10.9 & 11.9 & 13.4 & 14.6 & 16.9 \\
\hline San Jose-Sunnyvale-Santa Clara, CA & 125 & 129 & 145 & 237 & 339 & 6.4 & 6.5 & 7.3 & 11.9 & 17.0 \\
\hline Seattle-Tacoma-Bellevue, WA & 211 & 224 & 268 & 321 & 314 & 5.7 & 6.0 & 7.1 & 8.3 & 8.1 \\
\hline St. Louis, MO-IL & 119 & 165 & 160 & 254 & 295 & 4.2 & 5.9 & 5.7 & 9.0 & 10.5 \\
\hline Tampa-St. Petersburg-Clearwater, FL & 255 & 299 & 326 & 392 & 393 & 8.7 & 10.0 & 10.8 & 12.7 & 12.7 \\
\hline Virginia Beach-Norfolk-Newport News, VA-NC & 45 & 90 & 65 & 122 & 201 & 2.6 & 5.2 & 3.8 & 7.1 & 11.7 \\
\hline Washington-Arlington-Alexandria, DC-VA-MD-WV & 295 & 501 & 474 & 827 & 816 & 4.9 & 8.2 & 7.7 & 13.3 & 13.1 \\
\hline SELECTED MSAs TOTAL & 17,790 & 19,298 & 23,111 & 26,786 & 28,906 & 10.2 & 10.9 & 13.0 & 14.9 & 16.1 \\
\hline
\end{tabular}

* The case classification of 'Unknown duration or late syphilis' went into effect in January 2018. During 2014-2017, cases in this category include cases classified as late latent syphilis and late syphilis with clinical manifestations. See Appendix A1.9 for a detailed explanation of changes to the syphilis case definition.

${ }^{+}$MSAs were selected on the basis of the largest population in the 2010 US Census.

₹ 2016 county data for Alabama have been corrected and may not match previous reports.

${ }^{5}$ The variable used to identify county, which is used to classify cases into MSAs, was complete for $\leq 95 \%$ of cases in a state contributing data to this MSA. See Section A1.4 in the Appendix for more information. 
Table 40. Congenital Syphilis - Reported Cases and Rates of Reported Cases by State, Ranked by Rates, United States, 2018

\begin{tabular}{|c|c|c|c|}
\hline Rank* & State $^{\dagger}$ & Cases & Rate per 100,000 Live Births \\
\hline 1 & Texas & 367 & 92.2 \\
\hline 2 & Nevada & 31 & 85.5 \\
\hline 3 & Louisiana & 46 & 72.8 \\
\hline 4 & Arizona & 61 & 72.2 \\
\hline 5 & California & 332 & 67.9 \\
\hline 6 & Arkansas & 25 & 65.3 \\
\hline 7 & Florida & 108 & 48.0 \\
\hline 8 & New Mexico & 10 & 40.5 \\
\hline \multirow[t]{2}{*}{9} & Maryland & 29 & 39.7 \\
\hline & US TOTAL ${ }^{\ddagger}$ & 1,306 & 33.1 \\
\hline 10 & Georgia & 31 & 23.8 \\
\hline 11 & Oklahoma & 12 & 22.8 \\
\hline 12 & Missouri & 17 & 22.8 \\
\hline 13 & Hawaii & 4 & 22.1 \\
\hline 14 & Oregon & 10 & 22.0 \\
\hline 15 & Kansas & 8 & 21.0 \\
\hline 16 & Illinois & 29 & 18.8 \\
\hline 17 & Kentucky & 9 & 16.2 \\
\hline 18 & South Carolina & 9 & 15.7 \\
\hline 19 & Tennessee & 12 & 14.9 \\
\hline 20 & Ohio & 20 & 14.5 \\
\hline 21 & Minnesota & 10 & 14.3 \\
\hline 22 & North Carolina & 17 & 14.1 \\
\hline 23 & New Jersey & 13 & 12.7 \\
\hline 24 & New York & 28 & 12.0 \\
\hline 25 & Alabama & 7 & 11.8 \\
\hline 26 & Michigan & 13 & 11.5 \\
\hline 27 & Colorado & 7 & 10.5 \\
\hline 28 & Alaska & 1 & 8.9 \\
\hline 29 & Virginia & 9 & 8.8 \\
\hline 30 & New Hampshire & 1 & 8.2 \\
\hline 31 & South Dakota & 1 & 8.1 \\
\hline 32 & Mississippi & 3 & 7.9 \\
\hline 33 & Washington & 7 & 7.7 \\
\hline 34 & lowa & 3 & 7.6 \\
\hline 35 & Pennsylvania & 9 & 6.5 \\
\hline 36 & Connecticut & 2 & 5.6 \\
\hline 37 & West Virginia & 1 & 5.2 \\
\hline 38 & Idaho & 1 & 4.4 \\
\hline 39 & Utah & 1 & 2.0 \\
\hline 40 & Wisconsin & 1 & 1.5 \\
\hline \multirow[t]{10}{*}{41} & Indiana & 1 & 1.2 \\
\hline & Delaware & 0 & 0.0 \\
\hline & Maine & 0 & 0.0 \\
\hline & Massachusetts & 0 & 0.0 \\
\hline & Montana & 0 & 0.0 \\
\hline & Nebraska & 0 & 0.0 \\
\hline & North Dakota & 0 & 0.0 \\
\hline & Rhode Island & 0 & 0.0 \\
\hline & Vermont & 0 & 0.0 \\
\hline & Wyoming & 0 & 0.0 \\
\hline
\end{tabular}

* States were ranked by rate, then by case count, then in alphabetical order, with rates shown rounded to the nearest tenth.

${ }^{\dagger}$ Mother's state of residence was used to assign case.

${ }^{\ddagger}$ Total includes cases reported by the District of Columbia with 0 cases and a rate of 0.0 cases per 100,000 live births, but excludes territories. 
Table 41. Congenital Syphilis - Reported Cases and Rates of Reported Cases by Year of Birth, State/Territory* and Region in Alphabetical Order, United States, 2014-2018

\begin{tabular}{|c|c|c|c|c|c|c|c|c|c|c|}
\hline \multirow[b]{2}{*}{ State/Territory } & \multicolumn{5}{|c|}{ Cases } & \multicolumn{5}{|c|}{ Rates per 100,000 Live Births } \\
\hline & 2014 & 2015 & 2016 & 2017 & 2018 & 2014 & 2015 & 2016 & 2017 & 2018 \\
\hline Alabama & 3 & 3 & 4 & 6 & 7 & 5.0 & 5.0 & 6.8 & 10.1 & 11.8 \\
\hline Alaska & 0 & 0 & 0 & 0 & 1 & 0.0 & 0.0 & 0.0 & 0.0 & 8.9 \\
\hline Arizona & 13 & 14 & 16 & 32 & 61 & 15.0 & 16.4 & 18.9 & 37.9 & 72.2 \\
\hline Arkansas & 7 & 5 & 6 & 8 & 25 & 18.2 & 12.9 & 15.7 & 20.9 & 65.3 \\
\hline California & 102 & 140 & 207 & 281 & 332 & 20.3 & 28.5 & 42.3 & 57.5 & 67.9 \\
\hline Colorado & 0 & 0 & 4 & 4 & 7 & 0.0 & 0.0 & 6.0 & 6.0 & 10.5 \\
\hline Connecticut & 0 & 1 & 0 & 0 & 2 & 0.0 & 2.8 & 0.0 & 0.0 & 5.6 \\
\hline Delaware & 0 & 1 & 0 & 0 & 0 & 0.0 & 9.0 & 0.0 & 0.0 & 0.0 \\
\hline District of Columbia & 0 & 1 & 1 & 0 & 0 & 0.0 & 10.4 & 10.1 & 0.0 & 0.0 \\
\hline Florida & 48 & 38 & 60 & 93 & 108 & 21.8 & 16.9 & 26.7 & 41.3 & 48.0 \\
\hline Georgia & 17 & 21 & 21 & 23 & 31 & 13.0 & 16.0 & 16.1 & 17.7 & 23.8 \\
\hline Hawaii & 0 & 2 & 1 & 3 & 4 & 0.0 & 10.9 & 5.5 & 16.6 & 22.1 \\
\hline Idaho & 0 & 0 & 0 & 0 & 1 & 0.0 & 0.0 & 0.0 & 0.0 & 4.4 \\
\hline Illinois & 27 & 31 & 18 & 22 & 29 & 17.0 & 19.6 & 11.7 & 14.2 & 18.8 \\
\hline Indiana & 8 & 5 & 8 & 8 & 1 & 9.5 & 5.9 & 9.6 & 9.6 & 1.2 \\
\hline lowa & 1 & 0 & 1 & 2 & 3 & 2.5 & 0.0 & 2.5 & 5.1 & 7.6 \\
\hline Kansas & 0 & 0 & 1 & 0 & 8 & 0.0 & 0.0 & 2.6 & 0.0 & 21.0 \\
\hline Kentucky & 3 & 1 & 5 & 6 & 9 & 5.3 & 1.8 & 9.0 & 10.8 & 16.2 \\
\hline Louisiana & 46 & 54 & 48 & 59 & 46 & 71.3 & 83.5 & 76.0 & 93.4 & 72.8 \\
\hline Maine & 0 & 0 & 0 & 0 & 0 & 0.0 & 0.0 & 0.0 & 0.0 & 0.0 \\
\hline Maryland & 16 & 18 & 16 & 20 & 29 & 21.6 & 24.5 & 21.9 & 27.3 & 39.7 \\
\hline Massachusetts & 3 & 4 & 3 & 0 & 0 & 4.2 & 5.6 & 4.2 & 0.0 & 0.0 \\
\hline Michigan & 15 & 11 & 13 & 10 & 13 & 13.1 & 9.7 & 11.5 & 8.8 & 11.5 \\
\hline Minnesota & 0 & 2 & 7 & 2 & 10 & 0.0 & 2.9 & 10.0 & 2.9 & 14.3 \\
\hline Mississippi & 1 & 0 & 2 & 1 & 3 & 2.6 & 0.0 & 5.3 & 2.6 & 7.9 \\
\hline Missouri & 1 & 4 & 8 & 10 & 17 & 1.3 & 5.3 & 10.7 & 13.4 & 22.8 \\
\hline Montana & 0 & 0 & 0 & 1 & 0 & 0.0 & 0.0 & 0.0 & 8.1 & 0.0 \\
\hline Nebraska & 1 & 0 & 1 & 1 & 0 & 3.7 & 0.0 & 3.8 & 3.8 & 0.0 \\
\hline Nevada & 5 & 8 & 12 & 24 & 31 & 13.9 & 22.0 & 33.1 & 66.2 & 85.5 \\
\hline New Hampshire & 0 & 0 & 0 & 0 & 1 & 0.0 & 0.0 & 0.0 & 0.0 & 8.2 \\
\hline New Jersey & 0 & 0 & 12 & 14 & 13 & 0.0 & 0.0 & 11.7 & 13.6 & 12.7 \\
\hline New Mexico & 1 & 2 & 3 & 1 & 10 & 3.8 & 7.7 & 12.1 & 4.0 & 40.5 \\
\hline New York & 22 & 12 & 13 & 16 & 28 & 9.2 & 5.1 & 5.5 & 6.8 & 12.0 \\
\hline North Carolina & 6 & 9 & 18 & 25 & 17 & 5.0 & 7.4 & 14.9 & 20.7 & 14.1 \\
\hline North Dakota & 0 & 0 & 0 & 0 & 0 & 0.0 & 0.0 & 0.0 & 0.0 & 0.0 \\
\hline Ohio & 15 & 17 & 12 & 18 & 20 & 10.8 & 12.2 & 8.7 & 13.0 & 14.5 \\
\hline Oklahoma & 6 & 7 & 3 & 7 & 12 & 11.2 & 13.2 & 5.7 & 13.3 & 22.8 \\
\hline Oregon & 2 & 6 & 6 & 8 & 10 & 4.4 & 13.1 & 13.2 & 17.6 & 22.0 \\
\hline Pennsylvania & 4 & 7 & 5 & 7 & 9 & 2.8 & 5.0 & 3.6 & 5.0 & 6.5 \\
\hline Rhode Island & 0 & 0 & 0 & 0 & 0 & 0.0 & 0.0 & 0.0 & 0.0 & 0.0 \\
\hline South Carolina & 5 & 3 & 9 & 8 & 9 & 8.7 & 5.2 & 15.7 & 14.0 & 15.7 \\
\hline South Dakota & 3 & 0 & 2 & 3 & 1 & 24.4 & 0.0 & 16.3 & 24.4 & 8.1 \\
\hline Tennessee & 2 & 5 & 8 & 10 & 12 & 2.5 & 6.1 & 9.9 & 12.4 & 14.9 \\
\hline Texas & 75 & 52 & 71 & 179 & 367 & 18.8 & 12.9 & 17.8 & 45.0 & 92.2 \\
\hline Utah & 0 & 0 & 0 & 0 & 1 & 0.0 & 0.0 & 0.0 & 0.0 & 2.0 \\
\hline Vermont & 0 & 0 & 0 & 0 & 0 & 0.0 & 0.0 & 0.0 & 0.0 & 0.0 \\
\hline Virginia & 2 & 3 & 8 & 12 & 9 & 1.9 & 2.9 & 7.8 & 11.7 & 8.8 \\
\hline Washington & 2 & 5 & 3 & 6 & 7 & 2.3 & 5.6 & 3.3 & 6.6 & 7.7 \\
\hline West Virginia & 0 & 0 & 2 & 2 & 1 & 0.0 & 0.0 & 10.5 & 10.5 & 5.2 \\
\hline Wisconsin & 0 & 0 & 1 & 3 & 1 & 0.0 & 0.0 & 1.5 & 4.5 & 1.5 \\
\hline Wyoming & 0 & 0 & 0 & 0 & 0 & 0.0 & 0.0 & 0.0 & 0.0 & 0.0 \\
\hline US TOTAL & 462 & 492 & 639 & 935 & 1,306 & 11.6 & 12.4 & 16.2 & 23.7 & 33.1 \\
\hline Northeast & 29 & 24 & 33 & 37 & 53 & 4.6 & 3.8 & 5.3 & 5.9 & 8.5 \\
\hline Midwest & 71 & 70 & 72 & 79 & 103 & 8.5 & 8.4 & 8.7 & 9.5 & 12.4 \\
\hline South & 237 & 221 & 282 & 459 & 685 & 15.4 & 14.3 & 18.4 & 29.9 & 44.7 \\
\hline West & 125 & 177 & 252 & 360 & 465 & 12.8 & 18.4 & 26.3 & 37.5 & 48.5 \\
\hline American Samoa & NR & NR & NR & NR & 0 & - & - & - & - & 0.0 \\
\hline Guam & 0 & 2 & 0 & 0 & 0 & 0.0 & 59.4 & 0.0 & 0.0 & 0.0 \\
\hline Northern Mariana Islands & NR & NR & NR & NR & 0 & - & - & - & - & 0.0 \\
\hline Puerto Rico & 0 & 5 & 5 & 7 & 8 & 0.0 & 16.0 & 17.7 & 24.8 & 28.3 \\
\hline Virgin Islands & 0 & 0 & 0 & 0 & NR & 0.0 & 0.0 & 0.0 & 0.0 & - \\
\hline TERRITORIES & 0 & 7 & 5 & 7 & 8 & 0.0 & 19.5 & 15.2 & 21.3 & 23.3 \\
\hline TOTAL & 462 & 499 & 644 & 942 & 1,314 & 11.5 & 12.4 & 16.2 & 23.7 & 33.0 \\
\hline
\end{tabular}

* Mother's state/territory of residence was used to assign case.

$\mathrm{NR}=$ No report.

NOTE: See Section A1.11 in the Appendix for more information on interpreting case counts and rates in US territories. 
Table 42. Congenital Syphilis - Reported Cases and Rates of Reported Cases* by Year of Birth and Race/ Hispanic Ethnicity of Mother, United States, 2014-2018

\begin{tabular}{|c|c|c|c|c|c|c|c|c|}
\hline \multirow[b]{2}{*}{ Year of Birth } & \multicolumn{2}{|c|}{$\begin{array}{c}\text { American Indians/Alaska } \\
\text { Natives }\end{array}$} & \multicolumn{2}{|c|}{ Asians/Pacific Islanders } & \multicolumn{2}{|c|}{ Blacks } & \multicolumn{2}{|c|}{ Whites } \\
\hline & Cases & Rates & Cases & Rates & Cases & Rates & Cases & Rates \\
\hline 2014 & 5 & 13.2 & 19 & 7.0 & 227 & 38.2 & 79 & 3.6 \\
\hline 2015 & 4 & 10.7 & 15 & 5.5 & 207 & 34.8 & 97 & 4.5 \\
\hline 2016 & 12 & 32.8 & 25 & 8.9 & 264 & 44.8 & 119 & 5.6 \\
\hline 2017 & 14 & 38.2 & 13 & 4.6 & 357 & 60.6 & 210 & 9.9 \\
\hline 2018 & 29 & 79.2 & 26 & 9.2 & 510 & 86.6 & 286 & 13.5 \\
\hline
\end{tabular}

\begin{tabular}{|c|c|c|c|c|c|c|c|c|}
\hline \multirow[b]{2}{*}{ Year of Birth } & \multicolumn{2}{|c|}{ Other } & \multicolumn{2}{|c|}{ Hispanics } & \multicolumn{2}{|c|}{ Unknown } & \multicolumn{2}{|c|}{ Total } \\
\hline & Cases & Rates & Cases & Rates & Cases & Rates & Cases & Rates \\
\hline 2014 & 9 & & 112 & 12.3 & 11 & & 462 & 11.6 \\
\hline 2015 & 7 & & 143 & 15.5 & 19 & & 492 & 12.4 \\
\hline 2016 & 8 & & 189 & 20.6 & 22 & & 639 & 16.2 \\
\hline 2017 & 12 & & 310 & 33.8 & 19 & & 935 & 23.7 \\
\hline 2018 & 13 & & 411 & 44.7 & 31 & & 1,306 & 33.1 \\
\hline
\end{tabular}

* Per 100,000 live births.

NOTE: No population data exist for other or unknown race; therefore, rates are not calculated. 
Table 43. Chancroid - Reported Cases and Rates of Reported Cases by State/Territory in Alphabetical Order, United States, 2014-2018

\begin{tabular}{|c|c|c|c|c|c|c|c|c|c|c|}
\hline \multirow[b]{2}{*}{ State/Territory } & \multicolumn{5}{|c|}{ Cases } & \multicolumn{5}{|c|}{ Rates per 100,000 Population } \\
\hline & 2014 & 2015 & 2016 & 2017 & 2018 & 2014 & 2015 & 2016 & 2017 & 2018 \\
\hline Alabama & 0 & 0 & 1 & 0 & 0 & 0.0 & 0.0 & 0.0 & 0.0 & 0.0 \\
\hline Alaska & 0 & 0 & 0 & 0 & 0 & 0.0 & 0.0 & 0.0 & 0.0 & 0.0 \\
\hline Arizona & 0 & 1 & 0 & 0 & 0 & 0.0 & 0.0 & 0.0 & 0.0 & 0.0 \\
\hline Arkansas & 0 & 0 & 0 & 0 & 0 & 0.0 & 0.0 & 0.0 & 0.0 & 0.0 \\
\hline California & 4 & 2 & 2 & 1 & 1 & 0.0 & 0.0 & 0.0 & 0.0 & 0.0 \\
\hline Colorado & 0 & 0 & 1 & 0 & 0 & 0.0 & 0.0 & 0.0 & 0.0 & 0.0 \\
\hline Connecticut & 0 & 0 & 0 & 0 & 0 & 0.0 & 0.0 & 0.0 & 0.0 & 0.0 \\
\hline Delaware & 0 & 0 & 0 & 0 & 0 & 0.0 & 0.0 & 0.0 & 0.0 & 0.0 \\
\hline District of Columbia & 0 & 0 & 0 & 0 & 0 & 0.0 & 0.0 & 0.0 & 0.0 & 0.0 \\
\hline Florida & 0 & 0 & 0 & 0 & 0 & 0.0 & 0.0 & 0.0 & 0.0 & 0.0 \\
\hline Georgia & 0 & 0 & 0 & 0 & 0 & 0.0 & 0.0 & 0.0 & 0.0 & 0.0 \\
\hline Hawaii & 0 & 0 & 0 & 0 & 0 & 0.0 & 0.0 & 0.0 & 0.0 & 0.0 \\
\hline Idaho & 0 & 0 & 0 & 0 & 0 & 0.0 & 0.0 & 0.0 & 0.0 & 0.0 \\
\hline Illinois & 0 & 0 & 0 & 0 & 0 & 0.0 & 0.0 & 0.0 & 0.0 & 0.0 \\
\hline Indiana & 0 & 1 & 0 & 0 & 0 & 0.0 & 0.0 & 0.0 & 0.0 & 0.0 \\
\hline lowa & 0 & 0 & 0 & 0 & 0 & 0.0 & 0.0 & 0.0 & 0.0 & 0.0 \\
\hline Kansas & 0 & 0 & 0 & 0 & 0 & 0.0 & 0.0 & 0.0 & 0.0 & 0.0 \\
\hline Kentucky & 0 & 0 & 0 & 0 & 0 & 0.0 & 0.0 & 0.0 & 0.0 & 0.0 \\
\hline Louisiana & 0 & 0 & 0 & 0 & 0 & 0.0 & 0.0 & 0.0 & 0.0 & 0.0 \\
\hline Maine & 0 & 0 & 0 & 0 & 0 & 0.0 & 0.0 & 0.0 & 0.0 & 0.0 \\
\hline Maryland & 0 & 0 & 0 & 0 & 0 & 0.0 & 0.0 & 0.0 & 0.0 & 0.0 \\
\hline Massachusetts & 1 & 3 & 1 & 2 & 0 & 0.0 & 0.0 & 0.0 & 0.0 & 0.0 \\
\hline Michigan & 0 & 0 & 0 & 1 & 0 & 0.0 & 0.0 & 0.0 & 0.0 & 0.0 \\
\hline Minnesota & 0 & 0 & 0 & 0 & 0 & 0.0 & 0.0 & 0.0 & 0.0 & 0.0 \\
\hline Mississippi & 0 & 0 & 0 & 0 & 0 & 0.0 & 0.0 & 0.0 & 0.0 & 0.0 \\
\hline Missouri & 0 & 0 & 0 & 0 & 0 & 0.0 & 0.0 & 0.0 & 0.0 & 0.0 \\
\hline Montana & 0 & 0 & 0 & 0 & 0 & 0.0 & 0.0 & 0.0 & 0.0 & 0.0 \\
\hline Nebraska & 0 & 0 & 0 & 0 & 0 & 0.0 & 0.0 & 0.0 & 0.0 & 0.0 \\
\hline Nevada & 0 & 0 & 0 & 0 & 0 & 0.0 & 0.0 & 0.0 & 0.0 & 0.0 \\
\hline New Hampshire & 0 & 0 & 0 & 0 & 0 & 0.0 & 0.0 & 0.0 & 0.0 & 0.0 \\
\hline New Jersey & 0 & 0 & 0 & 0 & 0 & 0.0 & 0.0 & 0.0 & 0.0 & 0.0 \\
\hline New Mexico & 0 & 0 & 0 & 0 & 0 & 0.0 & 0.0 & 0.0 & 0.0 & 0.0 \\
\hline New York & 0 & 0 & 0 & 0 & 0 & 0.0 & 0.0 & 0.0 & 0.0 & 0.0 \\
\hline North Carolina & 0 & 0 & 1 & 1 & 0 & 0.0 & 0.0 & 0.0 & 0.0 & 0.0 \\
\hline North Dakota & 0 & 0 & 0 & 0 & 0 & 0.0 & 0.0 & 0.0 & 0.0 & 0.0 \\
\hline Ohio & 0 & 0 & 0 & 0 & 0 & 0.0 & 0.0 & 0.0 & 0.0 & 0.0 \\
\hline Oklahoma & 0 & 0 & 0 & 0 & 0 & 0.0 & 0.0 & 0.0 & 0.0 & 0.0 \\
\hline Oregon & 0 & 0 & 0 & 0 & 0 & 0.0 & 0.0 & 0.0 & 0.0 & 0.0 \\
\hline Pennsylvania & 0 & 0 & 0 & 0 & 0 & 0.0 & 0.0 & 0.0 & 0.0 & 0.0 \\
\hline Rhode Island & 0 & 0 & 0 & 0 & 0 & 0.0 & 0.0 & 0.0 & 0.0 & 0.0 \\
\hline South Carolina & 0 & 0 & 1 & 0 & 1 & 0.0 & 0.0 & 0.0 & 0.0 & 0.0 \\
\hline South Dakota & 0 & 0 & 0 & 0 & 0 & 0.0 & 0.0 & 0.0 & 0.0 & 0.0 \\
\hline Tennessee & 0 & 0 & 0 & 0 & 0 & 0.0 & 0.0 & 0.0 & 0.0 & 0.0 \\
\hline Texas & 1 & 2 & 0 & 2 & 1 & 0.0 & 0.0 & 0.0 & 0.0 & 0.0 \\
\hline Utah & 0 & 0 & 0 & 0 & 0 & 0.0 & 0.0 & 0.0 & 0.0 & 0.0 \\
\hline Vermont & 0 & 0 & 0 & 0 & 0 & 0.0 & 0.0 & 0.0 & 0.0 & 0.0 \\
\hline Virginia & 0 & 0 & 0 & 0 & 0 & 0.0 & 0.0 & 0.0 & 0.0 & 0.0 \\
\hline Washington & 0 & 1 & 0 & 0 & 0 & 0.0 & 0.0 & 0.0 & 0.0 & 0.0 \\
\hline West Virginia & 0 & 0 & 0 & 0 & 0 & 0.0 & 0.0 & 0.0 & 0.0 & 0.0 \\
\hline Wisconsin & 0 & 0 & 0 & 0 & 0 & 0.0 & 0.0 & 0.0 & 0.0 & 0.0 \\
\hline Wyoming & 0 & 1 & 0 & 0 & 0 & 0.0 & 0.2 & 0.0 & 0.0 & 0.0 \\
\hline US TOTAL & 6 & 11 & 7 & 7 & 3 & 0.0 & 0.0 & 0.0 & 0.0 & 0.0 \\
\hline American Samoa & NR & NR & NR & NR & 0 & - & - & - & - & 0.0 \\
\hline Guam & 0 & 0 & 0 & 0 & 0 & 0.0 & 0.0 & 0.0 & 0.0 & 0.0 \\
\hline Northern Mariana Islands & NR & NR & NR & NR & 0 & - & - & - & - & 0.0 \\
\hline Puerto Rico & 0 & 0 & 0 & 0 & 0 & 0.0 & 0.0 & 0.0 & 0.0 & 0.0 \\
\hline Virgin Islands & 0 & 0 & 0 & 0 & NR & 0.0 & 0.0 & 0.0 & 0.0 & - \\
\hline TERRITORIES & 0 & 0 & 0 & 0 & 0 & 0.0 & 0.0 & 0.0 & 0.0 & 0.0 \\
\hline TOTAL & 6 & 11 & 7 & 7 & 3 & 0.0 & 0.0 & 0.0 & 0.0 & 0.0 \\
\hline
\end{tabular}

$\mathrm{NR}=$ No report.

NOTE: See Section A1.11 in the Appendix for more information on interpreting case counts and rates in US territories. 
Table 44. Selected STDs and Complications - Initial Visits to Physicians' Offices, National Disease and Therapeutic Index (NDTI), United States, 1966-2016

\begin{tabular}{|c|c|c|c|}
\hline Year & $\begin{array}{c}\text { Trichomonas vaginalis } \\
\text { Infections* }\end{array}$ & $\begin{array}{c}\text { Other } \\
\text { Vaginal } \\
\text { Infections* }\end{array}$ & $\begin{array}{c}\text { Pelvic } \\
\text { Inflammatory } \\
\text { Disease }^{\dagger} \\
\end{array}$ \\
\hline 1966 & 579,000 & $1,155,000$ & NA \\
\hline 1967 & 515,000 & $1,277,000$ & NA \\
\hline 1968 & 463,000 & $1,460,000$ & NA \\
\hline 1969 & 421,000 & $1,390,000$ & NA \\
\hline 1970 & 529,000 & $1,500,000$ & NA \\
\hline 1971 & 484,000 & $1,281,000$ & NA \\
\hline 1972 & 574,000 & $1,810,000$ & NA \\
\hline 1973 & 466,000 & $1,858,000$ & NA \\
\hline 1974 & 427,000 & $1,907,000$ & NA \\
\hline 1975 & 500,000 & $1,919,000$ & NA \\
\hline 1976 & 473,000 & $1,690,000$ & NA \\
\hline 1977 & 324,000 & $1,713,000$ & NA \\
\hline 1978 & 329,000 & $2,149,000$ & NA \\
\hline 1979 & 363,000 & $1,662,000$ & NA \\
\hline 1980 & 358,000 & $1,670,000$ & 423,000 \\
\hline 1981 & 369,000 & $1,742,000$ & 283,000 \\
\hline 1982 & 268,000 & $1,859,000$ & 374,000 \\
\hline 1983 & 424,000 & $1,932,000$ & 424,000 \\
\hline 1984 & 381,000 & $2,450,000$ & 381,000 \\
\hline 1985 & 291,000 & $2,728,000$ & 425,000 \\
\hline 1986 & 338,000 & $3,118,000$ & 457,000 \\
\hline 1987 & 293,000 & $3,087,000$ & 403,000 \\
\hline 1988 & 191,000 & $3,583,000$ & 431,000 \\
\hline 1989 & 165,000 & $3,374,000$ & 413,000 \\
\hline 1990 & 213,000 & $4,474,000$ & 358,000 \\
\hline 1991 & 198,000 & $3,822,000$ & 377,000 \\
\hline 1992 & 182,000 & $3,428,000$ & 335,000 \\
\hline 1993 & 207,000 & $3,755,000$ & 407,000 \\
\hline 1994 & 199,000 & $4,123,000$ & 332,000 \\
\hline 1995 & 141,000 & $3,927,000$ & 262,000 \\
\hline 1996 & 245,000 & $3,472,000$ & 286,000 \\
\hline 1997 & 176,000 & $3,100,000$ & 260,000 \\
\hline 1998 & 164,000 & $3,200,000$ & 233,000 \\
\hline 1999 & 171,000 & $3,077,000$ & 250,000 \\
\hline 2000 & 222,000 & $3,470,000$ & 254,000 \\
\hline 2001 & 210,000 & $3,365,000$ & 244,000 \\
\hline 2002 & 150,000 & $3,315,000$ & 197,000 \\
\hline 2003 & 179,000 & $3,516,000$ & 123,000 \\
\hline 2004 & 221,000 & $3,602,000$ & 132,000 \\
\hline 2005 & 165,000 & $4,071,000$ & 176,000 \\
\hline 2006 & 200,000 & $3,891,000$ & 106,000 \\
\hline 2007 & 205,000 & $3,723,000$ & 146,000 \\
\hline 2008 & 204,000 & $3,571,000$ & 104,000 \\
\hline 2009 & 216,000 & $3,063,000$ & 100,000 \\
\hline 2010 & 149,000 & $3,192,000$ & 113,000 \\
\hline 2011 & 168,000 & $3,102,000$ & 90,000 \\
\hline 2012 & 219,000 & $3,452,000$ & 106,000 \\
\hline 2013 & 225,000 & $3,278,000$ & 88,000 \\
\hline 2014 & 155,000 & $3,419,000$ & 51,000 \\
\hline 2015 & 139,000 & $3,215,000$ & 68,000 \\
\hline 2016 & 222,000 & $4,112,000$ & 90,000 \\
\hline
\end{tabular}

* Females only.

† Females aged 15-44 years only.

$\mathrm{NA}=$ Not available.

NOTE: Standard errors for estimates under 100,000 are not available. The relative standard errors for estimates 100,000-299,999 are from 23\% to 19\%; 300,000599,999 are from $19 \%$ to $16 \% ; 600,000-999,999$ are from $16 \%$ to $13 \%$; and $1,000,000-5,000,000$ are from $13 \%$ to $7 \%$.

SOURCE: National Disease and Therapeutic Index, IMS Health, Integrated Promotional Services. IMS Health report, 1966-2016. 


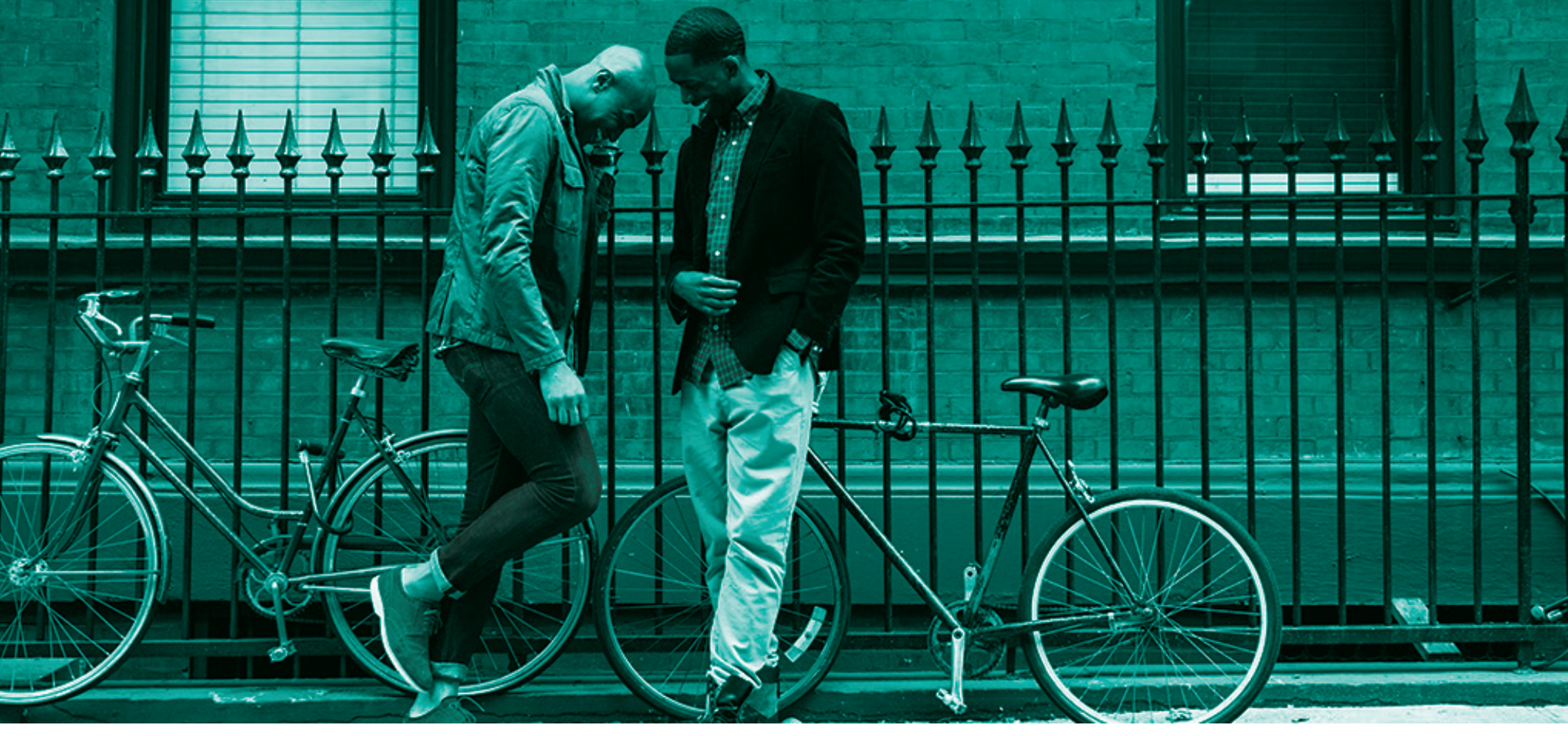

\section{Appendix}

\section{A. Interpreting STD Surveillance Data}

Sexually Transmitted Disease Surveillance 2018 presents surveillance information derived from the official statistics for the reported occurrence of nationally notifiable STDs in the United States, including data from sentinel surveillance and national surveys.

\section{A1. Nationally Notifiable STD Surveillance}

Nationally notifiable STD surveillance data are collected and compiled from reports sent by the STD control programs and health departments in all 50 states, the District of Columbia, selected cities, United States dependencies and possessions, and independent nations in free association with the United States to the Division of STD Prevention, National Center for HIV/AIDS, Viral Hepatitis, STD, and TB Prevention, Centers for Disease Control and Prevention (CDC). Included among the dependencies, possessions, and independent nations are Guam, Puerto Rico, Northern Mariana Islands, American Samoa, and the Virgin Islands. Selected tables and figures include data from these entities, identified as territories of the United States; however, the majority of national case counts and rates exclude data from these territories.

\section{A1.1 Reporting Formats}

STD morbidity data presented in this report are compiled from a combination of data reported on standardized hard copy reporting forms and electronic data received through the National Electronic Telecommunications System for Surveillance (NETSS) and via HL7 messaging using National Electronic Disease Surveillance System (NEDSS) standards. 


\section{Summary Report Forms}

The following hard copy forms were used to report national STD morbidity data:

1. FORM CDC 73.998: Monthly Surveillance Report of Early Syphilis. This monthly hard copy reporting form was used during 1984-2002 to report summary data for primary and secondary (P\&S) syphilis and early latent syphilis by county and state.

2. FORM CDC 73.688: Sexually Transmitted Disease Morbidity Report. This quarterly hard copy reporting form was used during 1963-2002 to report summary data for all stages of syphilis, congenital syphilis, gonorrhea, chancroid, chlamydia, and other STDs by sex and source of report (private versus public) for all 50 states, the District of Columbia, 64 selected cities (including San Juan, Puerto Rico), and territories of the United States.

Note: Chlamydial infection became a nationally notifiable condition in 1995 and the form was modified to support reporting of chlamydia that year. Congenital syphilis was dropped from this aggregate form in 1995 and replaced by the case-specific CDC 73.126 form, described later in this section.

3. FORM CDC 73.2638: Report of Civilian Cases of Primary \& Secondary Syphilis, Gonorrhea, and Chlamydia by Reporting Source, Sex, Race/Ethnicity, and Age Group. This annual hard copy form was used during 1981-2002 to report summary data for P\&S syphilis, gonorrhea, and chlamydia by age, race, sex, and source (public versus private) for all 50 states, seven large cities (Baltimore, Chicago, New York City, Los Angeles, Philadelphia, San Francisco, and the District of Columbia), and territories of the United States.

Note: Chlamydial infection became a nationally notifiable condition in 1995, and the form was modified to support reporting of chlamydia that year.

4. FORM CDC 73.126: Congenital Syphilis (CS) Case Investigation and Reporting. This case-specific hard copy form was first used in 1983 and continues to be used to report detailed case-specific data for congenital syphilis in some areas.

\section{National Electronic Telecommunications System for Surveillance}

As of December 31, 2003, all 50 states and the District of Columbia converted from summary hard copy reporting to electronic submission of line-listed (i.e., case-specific) data for chlamydia, gonorrhea, syphilis, and chancroid through NETSS. Though most of these areas report congenital syphilis and syphilitic stillbirths electronically, nine areas relied upon hard copy forms for reporting congenital syphilis and syphilitic stillbirths in 2018. Puerto Rico converted to electronic reporting in 2006 for all STDs, excluding congenital syphilis. American Samoa, Guam, Northern Mariana Islands, and the Virgin Islands continue to report STD data through summary hard copy forms.

Surveillance data sent to CDC through NETSS and on hard copy forms through June 19, 2019 are included in this report. The data presented in the figures and tables in this report supersede those in all earlier publications.

\section{National Electronic Disease Surveillance System}

In 2018, jurisdictions had the ability to transmit STD case notifications to CDC via HL7 messaging using NEDSS standards. Surveillance data sent to CDC via HL7 messaging from three jurisdictions (Connecticut, Idaho, and Oregon) are included in this report.

\section{A1.2 Population Denominators and Rate Calculations}

\section{0-2018 Rates and Population}

For those figures and tables presenting race using the 1997 Office of Management and Budget (OMB) standards, nonbridged-race data provided directly by the United States Census Bureau were used to calculate rates. The latest available year for population estimates at the time this report was written was 2017. Thus, 2017 population estimates were used to calculate 2018 rates. 
Once published, the 2018 population estimates will be used to calculate 2018 rates in Sexually Transmitted Disease Surveillance 2019.

Population estimates for Puerto Rico were obtained from the US Census Bureau Web site at: https://factfinder.census. gov

Population estimates for American Samoa, Guam, Northern Mariana Islands, and the Virgin Islands were obtained from the US Census Bureau International Programs Web site at: www.census.gov/programs-surveys/international-programs. $\underline{\mathrm{html}}$.

The 2018 rates by age and sex for American Samoa, Guam, Northern Mariana Islands, and the Virgin Islands were calculated using the latest population estimates available at: $\mathrm{https}$ ://factfinder.census.gov/faces/nav/jsf/pages/index. xhtml.

Because of the use of the updated population data, rates for 2000-2017 may be different from those presented in previous STD surveillance reports.

Several figures throughout this report depict state- or county-specific rates of reported cases of STDs. Rates were grouped and displayed by quintiles in Figures 3, 4, 16, 17, 37, A, C, I, J, K, L, M, N, O, P, Q, R, and AA. Rates were grouped and displayed in 4 categories_-zero cases and tertiles - in Figure 38.

\section{0-1999 Rates and Population}

The population counts for 1990 through 1999 incorporated the bridged single-race estimates of the April 1, 2000 US resident population. These files were prepared by the US Census Bureau with support from the National Cancer Institute.

\section{1-1989 Rates and Population}

Rates were calculated by using US Census Bureau population estimates for 1981 through 1989. ${ }^{1,2}$

\section{1-1980 Rates and Population}

Rates for 1941 through 1980 were based on population estimates from the US Census Bureau and are currently maintained by CDC's Division of STD Prevention.

\section{1-2018 Congenital Syphilis Rates and Live Births}

The congenital syphilis data in Table 1 of this report represent the number of congenital syphilis cases per 100,000 live births for all years during 1941-2018. Previous publications presented congenital syphilis rates per 100,000 population during 1941-1994 and rates for cases diagnosed at younger than 1 year of age per 100,000 live births during 19952005. To allow for trends in congenital syphilis rates to be compared for the period of 1941 through 2018, live births now are used as the denominator for congenital syphilis and case counts are no longer limited to those diagnosed within the first year of life. Congenital syphilis morbidity is assigned by year of birth. Rates of congenital syphilis for 1963 through 1988 were calculated by using published live birth data. ${ }^{3}$ Congenital syphilis rates for 1989 through 2018 were calculated by using live birth data based on information coded by the states and provided to the National Center for Health Statistics (NCHS) through the Vital Statistics Cooperative Program. Rates for 2018 were calculated by using live birth data for 2016.

\section{0-2018 Gay, Bisexual, and Other Men Who Have Sex with Men Rates and Population}

Figures 26 and AA show rates of reported cases of gonorrhea and P\&S syphilis among gay, bisexual, and other men who have sex with men (MSM). Population estimates of MSM are based on a method that combines published estimates of the prevalence of same-sex behavior among adult men with housing and population data from the American Community Survey 5-year summary file (2013-2017) ${ }^{4-7}$ County-specific estimates begin with MSM prevalence estimates that are determined by their urbanicity according to the NCHS urban-rural classification scheme for counties and their 
United States region. ${ }^{8}$ Estimates are then multiplied by a modified ratio of each county's percentage of male same-sex households to the total percentage of male same-sex households among all counties at the same level of urbanicity and within the same region. Thus, the final estimate for each county reflects what would be expected based on the county's geography, urban-rural classification, and observed concentration of households with a male head of household and a male partner. State-level estimates are then aggregated from the county-specific estimates.

\section{A1.3 Reporting Practices}

Although most state and local STD programs generally adhere to the national notifiable STD case definitions collaboratively developed by the Council of State and Territorial Epidemiologists (CSTE) and CDC, differences in policies and systems for collecting surveillance data may exist. Thus, comparisons of case numbers and rates between jurisdictions should be interpreted with caution. However, because case definitions and surveillance activities within a given area remain relatively stable over time, trends should be minimally affected by these differences.

Data collection for chlamydia began in 1984 and chlamydia was made nationally notifiable in 1995; however, chlamydia was not reportable in all 50 states and the District of Columbia until 2000. Data collection for gonorrhea, syphilis, and chancroid began in 1941; however, gonorrhea, syphilis, and chancroid became nationally notifiable in 1944. For more information on nationally notifiable conditions, please refer to the National Notifiable Disease Surveillance System (NNDSS) website: https://wwwn.cdc.gov/nndss/conditions/

\section{A1.4 Reporting of Surveillance Data by Geographic Areas}

\section{Metropolitan statistical area}

Sexually Transmitted Disease Surveillance 2018 continues the presentation of STD incidence data and rates for the 50 metropolitan statistical areas (MSA) with the largest populations according to 2010 United States census data. MSAs are defined by the OMB to provide nationally consistent definitions for collecting, tabulating, and publishing federal statistics for a set of geographic areas. ${ }^{9}$ An MSA is associated with at least one urbanized area that has a population of at least 50,000. The MSA comprises the central county or counties containing the central county, plus adjacent, outlying counties that have a high degree of social and economic integration with the central county as measured through commuting. The title of an MSA includes the name of the principal city with the largest 2010 census population. If there are multiple principal cities, the names of the second largest and third largest principal cities appear in the title in order of descending population size.

Reported cases are assigned to MSAs based on the reported county; cases reported with a missing a value for the county variable cannot be assigned to an MSA. Consequently, if a jurisdiction reports cases missing values for the county variable, reported rates for MSAs in their jurisdiction may be incomplete. Additionally, relative rankings of case counts by counties may be impacted by completeness of the variable used to identify county. Table A1 reports the percentage of cases reported with missing county information in each state for P\&S syphilis, chlamydia, and gonorrhea.

The MSA concept has been used as a statistical representation of the social and economic links between urban cores and outlying, integrated areas. However, MSAs do not equate to an urban-rural classification; all counties included in MSAs and many other counties contain both urban and rural territory and populations. STD programs that treat all parts of an MSA as if they were as urban as the densely settled core ignore the rural conditions that may exist in some parts of the area. In short, MSAs are not intended to be a general purpose geographic framework for nonstatistical activities or for use in program funding formulas.

For more information on the MSA definitions used in this report, go to: https://www.census.gov/programs-surveys/ metro-micro.html.

\section{County}

Figures 4, 17, and 38 show county-level maps with rates of reported cases of chlamydia, gonorrhea, and P\&S syphilis, respectively. Such county-level maps can be produced through NCHHSTP AtlasPlus, an interactive tool that allows users to create customized tables, maps, and charts using over 15 years of CDC's surveillance data on HIV, viral hepatitis, STD, and tuberculosis. Through the Map function, users can create custom maps to observe trends in the 
number of cases or rates of specific STDs by state or county. For more information on county-level rates, go to https:// www.cdc.gov/nchhstp/atlas.

\section{A1.5 Reporting of Data for Race/Hispanic Ethnicity}

In April 2008, the NETSS record layout was updated to conform to the OMB's current government-wide standard for race/Hispanic ethnicity data. The OMB standards were first issued in $1997 .{ }^{10}$ Beginning with the publication of Sexually Transmitted Disease Surveillance 2012, the race/Hispanic ethnicity data are presented according to the current OMB standard categories: American Indian or Alaska Native, Asian, Black or African American, Hispanic or Latino, Native Hawaiian or Other Pacific Islander, White, and Multirace. As of 2018, most reporting jurisdictions are locally compliant with current OMB standards and report in the current OMB standard race categories, including Multirace. However, a small number of jurisdictions reported race in pre-1997 single race categories, while other jurisdictions were using current OMB standards categories but were unable to report more than one race per person in 2018.

For this report, all race/Hispanic ethnicity data reported by jurisdictions are summarized in tables, charts, and interpretative text regardless of local compliance with the 1997 OMB standards. A small number of cases reported in the legacy 'Asian/Pacific Islander' category from non-compliant jurisdictions are re-coded to 'Unknown' because these cases cannot be properly re-coded into an appropriate current OMB standards category of 'Asian' or 'Native Hawaiian/ Other Pacific Islander.' No redistribution of cases is done; cases missing race and/or Hispanic ethnicity are not included in the calculation of rates by race/Hispanic ethnicity. As a consequence, rate data presented in this report underestimate actual case incidence in these population categories by a roughly similar proportion to the overall percentage of cases with missing/unknown race and Hispanic ethnicity.

Figures T, V, and X show rate ratios by race/Hispanic ethnicity and region. Rate ratios are calculated as the rate of reported gonorrhea cases per 100,000 for a given racial or ethnic minority population divided by the rate of reported gonorrhea cases per 100,000 population for Whites. Any population with a lower rate of reported cases of gonorrhea than the White population will have a rate ratio of less than 1:1.

All states and reporting jurisdictions are encouraged to continue efforts to upgrade local surveillance systems to be fully compliant with OMB standards for the collection of race and Hispanic ethnicity, to redouble efforts to ascertain complete information for all cases, and to implement CDC's HL7 case reporting guides at the earliest opportunity.

\section{A1.6 Management of Unknown, Missing, or Invalid Data for Age Group, Race/Hispanic Ethnicity, and Sex}

The percentage of unknown, missing, or invalid data for age group, race/Hispanic ethnicity, and sex varies from year to year, state to state, and by disease for reported STDs (Table A1).

Prior to the publication of Sexually Transmitted Disease Surveillance 2010, when the percentage of unknown, missing, or invalid values for age group, race/Hispanic ethnicity, and sex exceeded $50 \%$ for any state, the state's incidence and population data were excluded from the tables that presented data stratified by one or more of these variables. For the states for which $50 \%$ or more of their data were valid for age group, race/Hispanic ethnicity, and sex, the values for unknown, missing, or invalid data were redistributed on the basis of the state's distribution of known age group, race/ Hispanic ethnicity, and sex data. Beginning with the publication of Sexually Transmitted Disease Surveillance 2010, redistribution methodology is not applied to any of the data. The counts presented in this report are summations of all valid data reported in reporting year 2018.

As a result, rate data that are stratified by one or more of these variables reflect rates based on reported data only; caution should be used in interpreting specific rate data points as these may underestimate reported case incidence by race/Hispanic ethnicity due to the exclusion of cases missing these important demographic data.

\section{A1.7 Classification of STD Morbidity Reporting Sources}

Before 1996, states classified the source of case reports as either private source (including private physicians, hospitals, and institutions) or public source (primarily STD clinics). As states began reporting morbidity data electronically in 1996, the classification categories for source of case reports expanded to include the following data sources: STD 
clinics, HIV counseling and testing sites, drug treatment clinics, family planning clinics, prenatal/obstetrics clinics, tuberculosis clinics, private physicians/health maintenance organizations (HMOs), hospitals (inpatient), emergency rooms, correctional facilities, laboratories, blood banks, the National Job Training Program (NJTP), school-based clinics, mental health providers, the military, the Indian Health Service, and other unspecified sources. Figures 9, 10, 23, and 24 display trends in the proportion of cases reported in 2018 categorized by reporting source. Categories displayed vary across these figures and include the five most commonly reported sources for the population included in the figure, along with trends for all other reporting sources combined into the "All Other" category, and trends in the proportion of cases with unknown reporting source.

\section{A1.8 Interpreting Rates of Reported Cases of Chlamydia}

Trends in rates of reported cases of chlamydia are influenced by changes in incidence of infection, as well as changes in diagnostic, screening, and reporting practices. As chlamydial infections are usually asymptomatic, the number of infections identified and reported can increase as more people are screened even when incidence is flat or decreasing. During 2000-2011, the expanded use of more sensitive diagnostic tests (e.g., nucleic acid amplification tests [NAATs]) likely increased the number of infections identified and reported independently of increases in incidence. Also, although chlamydia has been a nationally notifiable condition since 1994, it was not until 2000 that all 50 states and the District of Columbia required reporting of chlamydia cases. National case rates prior to 2000 reflect incomplete reporting. The increased use of electronic laboratory reporting over the last decade or so also likely increased the proportion of diagnosed cases reported. Consequently, an increasing chlamydia case rate over time may reflect increases in incidence of infection, screening coverage, and use of more sensitive tests, as well as more complete reporting. Likewise, decreases in chlamydia case rates may suggest decreases in incidence of infection or screening coverage.

\section{A1.9 Syphilis Morbidity Reporting}

The surveillance case definition for syphilis has changed over time. Beginning in 2018, the category of "total syphilis" or "all stages of syphilis" includes: primary, secondary, early non-primary non-secondary, unknown duration or late, congenital syphilis, and syphilitic stillbirth. However, in previous years, "total syphilis" or "all stages of syphilis" have included different case classifications. For example, in the 1990 syphilis case definition, "total syphilis" or "all stages of syphilis" included: primary, secondary, latent, early latent, late latent, latent unknown duration, neurosyphilis, syphilitic stillbirth, and congenital syphilis. See Section C1.4 in the Appendix for information on current syphilis case definitions. More information on syphilis case definition changes over time can be found at: https://wwwn.cdc.gov/ nndss/conditions/syphilis/case-definition/2018/.

\section{A1.10 Congenital Syphilis Morbidity Reporting}

In 1988, the surveillance case definition for congenital syphilis was changed, resulting in a more sensitive definition. ${ }^{11}$ At the same time, many state and local STD programs began to greatly enhance active case finding for congenital syphilis. These surveillance changes, in addition to rising morbidity, led to a dramatic increase in the number of congenital syphilis cases reported during 1989-1991. By January 1, 1992, the new congenital syphilis case definition was fully implemented by all reporting areas. In addition to changing the case definition, CDC introduced a new congenital syphilis data collection form (CDC 73.126) in 1990; this was later revised in February 2013. Since 1995, congenital syphilis cases are reported by state and city of residence of the mother and by the reported race/Hispanic ethnicity of the mother.

Congenital syphilis reporting may be delayed as a result of case investigation and validation. Cases for previous years are added to CDC's surveillance databases throughout the year. Congenital syphilis data reported after publication of the current annual STD surveillance report will appear in subsequent reports and are assigned by the infant's year of birth.

\section{A1.11 Interpreting Surveillance Data from Territories}

There are a number of issues affecting the STD surveillance data reported to CDC from the US territories, including test kit stock-outs, resulting in an inability to test or screen for undetermined periods of time, as well as a variety of data collection, entry, and transmission issues. As such, the data likely underestimate the total STD burden in these areas and should be interpreted cautiously. 
In figures showing rates for US states and territories (Figures 3, 16, 37, A, C, I, J, K, L, M, and N), 2018 data from the Virgin Islands were not included as full data were not able to be obtained in time to include them in this report.

Data from American Samoa, Guam, and Northern Mariana Islands were not included in Figures O, P, Q, and R, as they do not participate in the NJTP.

In Figure AA, data from American Samoa, Guam, Northern Mariana Islands, Puerto Rico, and the Virgin Islands were not available.

\section{A2. Other Sources of Surveillance Data}

\section{A2.1 National Job Training Program}

Chlamydia and gonorrhea prevalence was calculated for males and females entering the NJTP. To increase the stability of the estimates, chlamydia or gonorrhea prevalence data are presented when valid test results for 100 or more students per year are available for the population subgroup and state. The majority of NJTP's chlamydia screening tests are conducted by a single national contract laboratory, which provides these data to CDC. Gonorrhea screening tests for male and female students in many training centers are conducted by local laboratories; these data are not available to CDC. Test results for students at centers that submit specimens to the national contract laboratory are included only if the number of gonorrhea tests submitted is greater than $90 \%$ of the number of chlamydia tests submitted from the same center for the same period. Prevalence data for state-specific figures were published with permission from the Department of Labor. Prevalence data are presented in Figures O, P, Q, and R.

\section{A2.2 STD Surveillance Network}

In 2005, CDC established the STD Surveillance Network (SSuN) as a collaborative network of state, county and/or city health departments following common protocols to conduct sentinel and enhanced STD surveillance activities. The purpose of SSuN is to improve the capacity of national, state and local STD programs to detect, monitor, and respond to trends in STDs through enhanced data collection, reporting, analysis, visualization, and interpretation of disease information.

Cycle 3 (2013-2018) of SSuN provided funding to 10 jurisdictions to conduct two core sentinel and enhanced STD surveillance activities. SSuN Cycle 3 sentinel surveillance activities included abstraction of clinical and demographic information on a full census of patients attending 30 STD clinics, and, through June 2018, among women 15-44 years of age presenting for care in facilities that provide family planning and reproductive health services. SSuN Cycle 3 enhanced surveillance activities included conducting health department registry matching, as well as provider and patient investigations on a probability sample of all persons diagnosed and reported with gonorrhea. Funded jurisdictions for core activities in SSuN Cycle 3 include Baltimore City (Maryland), California (excluding San Francisco County), Florida, Massachusetts, Minnesota, Multnomah County (Oregon), Philadelphia City (Pennsylvania), New York City (New York), San Francisco County (California), and Washington State.

In both components of SSuN Cycle 3, unique persons (diagnosed and reported with gonorrhea or seeking care in participating clinical facilities) were longitudinally followed using unique, coded IDs to provide information on repeat infections and/or care seeking behaviors. The primary unit of analysis for sentinel surveillance activities in clinical facilities is unique persons. These data are merged with multiple laboratory, diagnostic, and treatment observations to provide a comprehensive picture of services and diagnoses received for each individual patient. For enhanced, case-based surveillance activities in SSuN Cycle 3, the primary unit of analysis is a diagnosed and reported episode (case) of gonorrhea from any provider type or setting within the funded jurisdiction. Case data also included a unique person identifier, which allowed merging with multiple laboratory observations, matching with other health department disease registries, querying provider-based clinical information, and unique patient demographic and behavioral data obtained through direct patient interviews. For analysis in the population component, cases in the probability sample were weighted to reflect study design and to adjust for non-response by demographic category of the patient. Weighted analysis provides estimates of case-level and person-level characteristics representative of all reported cases in the funded jurisdictions. 
MSM are defined in all SSuN data collection activities as men who: a) reported having sex with another man in the preceding 2-3 months, and/or, b) those who reported that they considered themselves gay/homosexual or bisexual. Men who have sex with women (MSW) are defined as men who reported having sex with women exclusively, or who did not report the sex of their sex partners but reported that they considered themselves to be straight/heterosexual.

Data presented in figures in this report from the sentinel surveillance component of SSuN Cycle 3 include data from nine of the 10 participating jurisdictions (Baltimore [Maryland], Miami [Florida], Boston [Massachusetts], Minneapolis [Minnesota], Multnomah County [Oregon], New York City [New York], Philadelphia [Pennsylvania], San Francisco [California], and Seattle [Washington]), except for Figure GG which includes data from the seven jurisdictions which provided data on P\&S syphilis diagnoses (Baltimore [Maryland], Miami [Florida], Minneapolis [Minnesota], Multnomah County [Oregon], New York City [New York], San Francisco [California], and Seattle [Washington]).

Data presented in figures in this report from the population component of SSuN Cycle 3 for 2018 include gonorrhea cases sampled from all funded jurisdictions. Trend data across previous cycles of SSuN (Figure 26) include only those jurisdictions participating in both Cycles 2 and 3 (Baltimore, California [excluding San Francisco], Philadelphia, New York City, San Francisco and Washington State).

\section{A2.3 Gonococcal Isolate Surveillance Project}

Data on antimicrobial susceptibility in Neisseria gonorrhoeae were collected through the Gonococcal Isolate Surveillance Project (GISP), a sentinel system of selected STD clinics located at 25-30 GISP sentinel sites and regional laboratories in the United States. For more details on findings from GISP, go to: https://www.cdc.gov/std/GISP/.

For 2018, the antimicrobial agents tested by GISP were ceftriaxone, cefixime, azithromycin, ciprofloxacin, penicillin, tetracycline, and gentamicin.

The antimicrobial susceptibility criteria used in GISP for 2018 are as follows:

- Ceftriaxone, minimum inhibitory concentration (MIC) $\geq 0.5 \mu \mathrm{g} / \mathrm{ml}$ (decreased susceptibility)*

- Ceftriaxone, $\mathrm{MIC} \geq 0.125 \mu \mathrm{g} / \mathrm{ml}$ (elevated MIC)*

- Cefixime, $\mathrm{MIC} \geq 0.5 \mu \mathrm{g} / \mathrm{ml}$ (decreased susceptibility)*

- Cefixime, $\mathrm{MIC} \geq 0.25 \mu \mathrm{g} / \mathrm{ml}$ (elevated MIC)*

- Azithromycin, $\mathrm{MIC} \geq 2.0 \mu \mathrm{g} / \mathrm{ml}$ (elevated MIC)*

- Ciprofloxacin, MIC $\geq 1.0 \mu \mathrm{g} / \mathrm{ml}$ (resistance)

- Ciprofloxacin, MIC 0.125-0.5 $\mu \mathrm{g} / \mathrm{ml}$ (intermediate resistance)

- Penicillin, MIC $\geq 2.0 \mu \mathrm{g} / \mathrm{ml}$ (resistance)

- Tetracycline, $\mathrm{MIC} \geq 2.0 \mu \mathrm{g} / \mathrm{ml}$ (resistance)

- Gentamicin (MIC values correlated with susceptibility and resistance have not been established)*

The majority of these criteria are also recommended by the Clinical and Laboratory Standards Institute (CLSI). ${ }^{12}$

* As of December 2018, the CLSI criteria for resistance to ceftriaxone, cefixime, gentamicin, and azithromycin and for susceptibility to azithromycin and gentamicin have not been established for $N$. gonorrhoeae. 


\section{A2.4 National Health and Nutrition Examination Survey}

The National Health and Nutrition Examination Survey (NHANES) is a series of cross-sectional surveys designed to provide national statistics on the health and nutritional status of the general household population in the United States. Data are collected through household interviews, standardized physical examinations, and the collection of biological samples in special mobile examination centers. In 1999, NHANES became a continuous survey with data released every two years. The sampling plan of the survey is a stratified, multistage, probability cluster design that selects a sample representative of the United States civilian, non-institutionalized population. For more information, see: https://www. cdc.gov/nchs/nhanes.htm.

\section{A2.5 National Disease and Therapeutic Index}

The information on the number of initial visits to private physicians' offices for STDs was based on analysis of data from the National Disease and Therapeutic Index (NDTI) machine-readable files or summary statistics for 1966 through 2016. NDTI is a probability sample survey of private physicians' clinical management practices. For more information on this database, contact IMS Health, e-mail: ServiceCenter@us.imshealth.com; Telephone: (800) 523-5334.

\section{References}

1. US Census Bureau. United States population estimates by age, sex and race: 1980-1988. Washington, DC: US Government Printing Office;1990.

2. US Census Bureau. United States population estimates by age, sex and race: 1989. Washington, DC: US Government Printing Office; 1990.

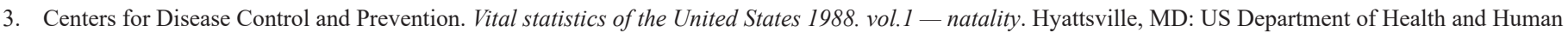
Services; 1990.

4. American Community Survey. 5-year summary file, 2013-2017. In: US Census Bureau; 2018.

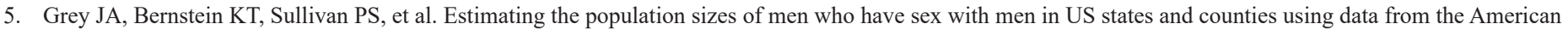
Community Survey. JMIR Public Health Surveill. 2016;2(1):e14.

6. Oster AM, Sternberg M, Lansky A, et al. Population size estimates for men who have sex with men and persons who inject drugs. $J$ Urban Health. 2015;92(4):733-743.

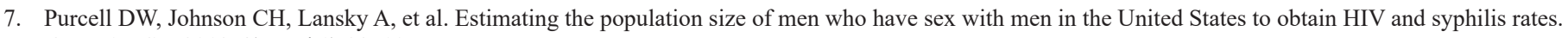
Open AIDS J. 2012;6(Suppl 1):98-107.

8. Ingram DD, Franco SJ. 2013 NCHS urban-rural classification scheme for counties. National Center for Health Statistics. Vital Health Stat 2(166). 2014.

9. Office of Management and Budget. Standards for defining metropolitan and micropolitan statistical areas. Federal Register. 2000;65(249):82228-82238.

10. Office of Management and Budget. Revisions to the Standards for Classification of Federal Data on Race and Ethnicity. October $30,1997$.

11. Kaufman RE, Jones OG, Blount JH, et al. Questionnaire survey of reported early congenital syphilis: Problems in diagnosis, prevention, and treatment. Sex Transm Dis. 1977;4(4):135-139.

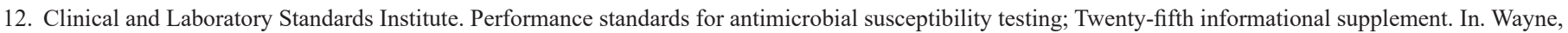
PA: Clinical and Laboratory Standards Institute; 2015. 
Table A1. Selected STDs - Percentage of Unknown, Missing, or Invalid Values for Selected Variables by State and by Nationally Notifiable STD, 2018

\begin{tabular}{|c|c|c|c|c|c|}
\hline \multirow[b]{2}{*}{ State } & \multicolumn{5}{|c|}{ Primary and Secondary Syphilis } \\
\hline & $\begin{array}{l}\text { Percentage } \\
\text { Unknown } \\
\text { Race/Hispanic } \\
\text { Ethnicity }\end{array}$ & $\begin{array}{c}\text { Percentage } \\
\text { Unknown } \\
\text { Age }\end{array}$ & $\begin{array}{c}\text { Percentage } \\
\text { Unknown } \\
\text { Sex }\end{array}$ & $\begin{array}{c}\text { Percentage } \\
\text { Unknown } \\
\text { Sex of Sex } \\
\text { Partners }\end{array}$ & $\begin{array}{c}\text { Percentage } \\
\text { Unknown } \\
\text { County }\end{array}$ \\
\hline Alabama & 0.2 & 0.2 & 0.0 & 39.4 & 0.0 \\
\hline Alaska & 0.0 & 0.0 & 0.0 & 3.6 & 0.0 \\
\hline Arizona & 1.1 & 0.0 & 0.0 & 9.1 & 0.0 \\
\hline Arkansas & 0.7 & 0.0 & 0.0 & 7.3 & 0.0 \\
\hline California & 7.9 & 0.1 & 0.0 & 13.6 & 0.0 \\
\hline Colorado & 2.7 & 0.0 & 0.0 & 15.4 & 0.3 \\
\hline Connecticut & 9.9 & 0.0 & 2.2 & 26.4 & 4.4 \\
\hline Delaware & 46.7 & 0.0 & 0.0 & 36.7 & 0.0 \\
\hline District of Columbia & 20.1 & 0.0 & 1.1 & 29.4 & 0.0 \\
\hline Florida & 6.7 & 0.0 & 0.0 & 14.1 & 0.0 \\
\hline Georgia & 3.2 & 0.0 & 0.1 & 28.1 & 0.2 \\
\hline Hawaii & 38.0 & 0.0 & 0.0 & 34.8 & 2.2 \\
\hline Idaho & 2.2 & 0.0 & 0.0 & 23.9 & 0.0 \\
\hline Illinois & 3.7 & 0.0 & 0.1 & 20.3 & 0.0 \\
\hline Indiana & 0.5 & 0.0 & 0.0 & 4.1 & 0.0 \\
\hline lowa & 0.0 & 0.0 & 0.0 & 4.7 & 0.0 \\
\hline Kansas & 0.0 & 0.0 & 0.0 & 9.2 & 0.7 \\
\hline Kentucky & 1.9 & 0.0 & 0.0 & 19.9 & 0.0 \\
\hline Louisiana & 0.0 & 0.0 & 0.0 & 2.8 & 0.0 \\
\hline Maine & 8.1 & 0.0 & 0.0 & 93.2 & 2.7 \\
\hline Maryland & 0.1 & 0.0 & 0.0 & 12.2 & 0.0 \\
\hline Massachusetts & 7.8 & 0.0 & 2.2 & 13.0 & 0.4 \\
\hline Michigan & 0.2 & 0.0 & 0.0 & 8.0 & 0.2 \\
\hline Minnesota & 2.4 & 0.0 & 0.0 & 8.9 & 0.0 \\
\hline Mississippi & 0.9 & 0.0 & 0.0 & 3.7 & 0.0 \\
\hline Missouri & 0.5 & 0.0 & 0.0 & 13.3 & 0.0 \\
\hline Montana & 2.2 & 0.0 & 0.0 & 20.0 & 0.0 \\
\hline Nebraska & 8.4 & 0.0 & 0.0 & 30.3 & 0.0 \\
\hline Nevada & 2.3 & 0.0 & 0.0 & 13.6 & 1.8 \\
\hline New Hampshire & 10.9 & 0.0 & 0.0 & 14.1 & 0.0 \\
\hline New Jersey & 5.1 & 0.0 & 0.2 & 12.8 & 0.0 \\
\hline New Mexico & 12.5 & 0.0 & 0.0 & 12.2 & 0.0 \\
\hline New York & 4.3 & 0.0 & 0.0 & 23.8 & 0.0 \\
\hline North Carolina & 0.1 & 0.0 & 0.1 & 10.3 & 0.0 \\
\hline North Dakota & 12.2 & 0.0 & 0.0 & 19.5 & 0.0 \\
\hline Ohio & 0.8 & 0.0 & 0.0 & 9.9 & 0.0 \\
\hline Oklahoma & 0.2 & 0.0 & 0.0 & 5.6 & 0.0 \\
\hline Oregon & 7.8 & 0.0 & 0.0 & 16.3 & 0.0 \\
\hline Pennsylvania & 7.0 & 0.0 & 0.0 & 8.2 & 0.0 \\
\hline Rhode Island & 15.6 & 0.0 & 0.0 & 16.7 & 0.0 \\
\hline South Carolina & 1.3 & 0.0 & 0.0 & 4.7 & 0.0 \\
\hline South Dakota & 0.0 & 0.0 & 0.0 & 7.3 & 0.0 \\
\hline Tennessee & 0.2 & 0.0 & 0.0 & 7.8 & 0.0 \\
\hline Texas & 2.7 & 0.1 & 0.0 & 29.4 & 0.4 \\
\hline Utah & 1.2 & 0.0 & 0.0 & 11.8 & 1.2 \\
\hline Vermont & 0.0 & 0.0 & 0.0 & 36.4 & 0.0 \\
\hline Virginia & 0.6 & 0.0 & 1.1 & 10.4 & 0.0 \\
\hline Washington & 5.7 & 0.0 & 0.1 & 4.0 & 0.0 \\
\hline West Virginia & 0.0 & 0.0 & 0.0 & 12.3 & 0.0 \\
\hline Wisconsin & 1.3 & 0.0 & 0.0 & 63.2 & 2.6 \\
\hline Wyoming & 8.7 & 0.0 & 0.0 & 26.1 & 0.0 \\
\hline U.S. TOTAL & 4.5 & 0.0 & 0.1 & 15.9 & 0.1 \\
\hline
\end{tabular}

Continued on next page. 
Table A1. Selected STDs - Percentage of Unknown, Missing, or Invalid Values for Selected Variables by State and by Nationally Notifiable STD, 2018 (continued)

\begin{tabular}{|c|c|c|c|c|c|c|c|c|}
\hline \multirow[b]{2}{*}{ State } & \multicolumn{4}{|c|}{ Gonorrhea } & \multicolumn{4}{|c|}{ Chlamydia } \\
\hline & $\begin{array}{c}\text { Percentage } \\
\text { Unknown } \\
\text { Race/Hispanic } \\
\text { Ethnicity }\end{array}$ & $\begin{array}{c}\text { Percentage } \\
\text { Unknown } \\
\text { Age }\end{array}$ & $\begin{array}{l}\text { Percentage } \\
\text { Unknown } \\
\text { Sex }\end{array}$ & $\begin{array}{c}\text { Percentage } \\
\text { Unknown } \\
\text { County }\end{array}$ & $\begin{array}{c}\text { Percentage } \\
\text { Unknown } \\
\text { Race/Hispanic } \\
\text { Ethnicity }\end{array}$ & $\begin{array}{c}\text { Percentage } \\
\text { Unknown } \\
\text { Age }\end{array}$ & $\begin{array}{l}\text { Percentage } \\
\text { Unknown } \\
\text { Sex }\end{array}$ & $\begin{array}{l}\text { Percentage } \\
\text { Unknown } \\
\text { County }\end{array}$ \\
\hline Alabama & 33.1 & 0.3 & 0.4 & 0.0 & 39.1 & 0.3 & 0.3 & 0.0 \\
\hline Alaska & 5.1 & 0.0 & 0.0 & 0.1 & 7.5 & 0.0 & 0.1 & 0.3 \\
\hline Arizona & 19.4 & 0.0 & 0.2 & 0.0 & 29.8 & 0.0 & 0.3 & 0.0 \\
\hline Arkansas & 10.1 & 0.0 & 0.0 & 0.0 & 11.0 & 0.0 & 0.0 & 0.0 \\
\hline California & 23.1 & 0.2 & 0.3 & 0.0 & 34.9 & 0.3 & 0.3 & 0.0 \\
\hline Colorado & 22.6 & 0.0 & 0.0 & 0.0 & 32.1 & 0.0 & 0.0 & 0.0 \\
\hline Connecticut & 57.2 & 0.1 & 0.4 & 8.6 & 76.4 & 0.2 & 0.9 & 5.4 \\
\hline Delaware & 20.2 & 0.0 & 0.3 & 0.1 & 32.6 & 0.1 & 0.2 & 0.0 \\
\hline District of Columbia & 92.0 & 0.2 & 0.9 & 0.0 & 94.4 & 0.7 & 0.7 & 0.0 \\
\hline Florida & 12.1 & 0.0 & 0.0 & 0.0 & 18.3 & 0.0 & 0.0 & 0.0 \\
\hline Georgia & 22.5 & 0.1 & 0.4 & 5.3 & 37.1 & 0.1 & 0.5 & 5.8 \\
\hline Hawaii & 50.9 & 0.4 & 0.3 & 11.4 & 58.0 & 0.1 & 0.2 & 7.5 \\
\hline Idaho & 25.2 & 0.0 & 0.3 & 0.0 & 31.8 & 0.0 & 0.3 & 0.0 \\
\hline Illinois & 12.2 & 0.0 & 0.0 & 0.0 & 16.4 & 0.0 & 0.0 & 0.0 \\
\hline Indiana & 7.9 & 0.0 & 0.1 & 0.2 & 12.8 & 0.0 & 0.1 & 0.2 \\
\hline lowa & 3.0 & 0.0 & 0.0 & 0.0 & 6.3 & 0.0 & 0.0 & 0.0 \\
\hline Kansas & 12.3 & 0.0 & 0.0 & 0.0 & 30.0 & 0.0 & 0.0 & 0.0 \\
\hline Kentucky & 44.5 & 0.3 & 0.9 & 5.0 & 50.5 & 0.2 & 0.7 & 4.9 \\
\hline Louisiana & 0.0 & 0.0 & 0.0 & 0.1 & 0.1 & 0.0 & 0.0 & 0.1 \\
\hline Maine & 4.5 & 0.4 & 0.1 & 0.3 & 28.6 & 20.0 & 0.0 & 0.0 \\
\hline Maryland & 17.4 & 0.0 & 0.0 & 0.0 & 35.3 & 0.0 & 0.1 & 0.0 \\
\hline Massachusetts & 33.1 & 0.4 & 0.8 & 1.9 & 59.0 & 0.2 & 0.4 & 4.0 \\
\hline Michigan & 15.2 & 0.0 & 0.0 & 0.2 & 20.4 & 0.0 & 0.1 & 0.1 \\
\hline Minnesota & 15.1 & 0.0 & 0.1 & 1.4 & 18.1 & 0.0 & 0.1 & 1.7 \\
\hline Mississippi & 23.5 & 0.0 & 0.2 & 0.0 & 31.5 & 0.0 & 0.2 & 0.0 \\
\hline Missouri & 9.0 & 0.0 & 0.0 & 0.0 & 13.0 & 0.0 & 0.0 & 0.0 \\
\hline Montana & 1.9 & 0.2 & 0.0 & 0.0 & 1.7 & 0.3 & 0.0 & 0.1 \\
\hline Nebraska & 10.7 & 0.0 & 0.0 & 0.0 & 19.3 & 0.0 & 0.0 & 0.0 \\
\hline Nevada & 36.1 & 0.0 & 0.2 & 0.6 & 45.1 & 0.0 & 0.4 & 0.4 \\
\hline New Hampshire & 10.6 & 0.0 & 0.0 & 0.0 & 25.4 & 0.0 & 0.0 & 0.0 \\
\hline New Jersey & 40.3 & 0.0 & 0.0 & 0.1 & 56.0 & 0.0 & 0.0 & 0.0 \\
\hline New Mexico & 30.4 & 0.0 & 0.0 & 0.0 & 38.4 & 0.0 & 0.0 & 0.0 \\
\hline New York & 25.0 & 0.0 & 0.1 & 0.0 & 40.4 & 0.1 & 0.0 & 0.0 \\
\hline North Carolina & 14.8 & 0.0 & 0.0 & 0.0 & 20.2 & 0.0 & 0.0 & 0.0 \\
\hline North Dakota & 7.1 & 0.0 & 0.0 & 0.0 & 10.4 & 0.0 & 0.0 & 0.0 \\
\hline Ohio & 14.9 & 0.0 & 0.0 & 1.5 & 21.2 & 0.0 & 0.0 & 1.7 \\
\hline Oklahoma & 6.2 & 0.0 & 0.0 & 0.0 & 8.7 & 0.0 & 0.0 & 0.0 \\
\hline Oregon & 10.6 & 0.0 & 0.1 & 0.0 & 25.1 & 0.0 & 0.1 & 0.0 \\
\hline Pennsylvania & 22.0 & 0.0 & 0.1 & 0.0 & 30.8 & 0.0 & 0.1 & 0.0 \\
\hline Rhode Island & 15.2 & 0.0 & 0.0 & 0.1 & 13.3 & 0.0 & 0.0 & 0.5 \\
\hline South Carolina & 32.6 & 0.0 & 0.3 & 0.1 & 38.7 & 0.0 & 0.3 & 0.1 \\
\hline South Dakota & 1.5 & 0.0 & 0.0 & 10.7 & 8.7 & 0.0 & 0.0 & 5.1 \\
\hline Tennessee & 1.6 & 0.0 & 0.0 & 0.0 & 2.1 & 0.0 & 0.0 & 0.0 \\
\hline Texas & 24.1 & 0.1 & 0.3 & 0.5 & 31.1 & 0.1 & 0.4 & 0.4 \\
\hline Utah & 4.7 & 0.0 & 0.0 & 0.1 & 6.2 & 0.0 & 0.0 & 0.0 \\
\hline Vermont & 19.0 & 0.0 & 0.7 & 0.0 & 23.7 & 0.1 & 0.8 & 0.0 \\
\hline Virginia & 15.7 & 0.0 & 0.4 & 0.0 & 27.6 & 0.1 & 0.4 & 0.0 \\
\hline Washington & 11.1 & 0.0 & 0.1 & 0.0 & 18.9 & 0.0 & 0.0 & 0.0 \\
\hline West Virginia & 15.0 & 0.0 & 0.0 & 0.0 & 18.7 & 0.0 & 0.0 & 0.0 \\
\hline Wisconsin & 10.9 & 0.0 & 0.1 & 0.0 & 10.8 & 0.0 & 0.1 & 0.0 \\
\hline Wyoming & 4.5 & 0.0 & 0.0 & 0.0 & 34.9 & 0.0 & 0.0 & 0.0 \\
\hline U.S. TOTAL & 19.6 & 0.1 & 0.2 & 0.6 & 28.5 & 0.1 & 0.2 & 0.6 \\
\hline
\end{tabular}

NOTE: For all categories, unknown included cases reported as unknown or missing. 
Table A2. Reported Cases of STDs by Reporting Source and Sex, United States, 2018

\begin{tabular}{|c|c|c|c|c|c|c|c|c|c|}
\hline \multirow[b]{2}{*}{ Disease } & \multicolumn{3}{|c|}{ Non-STD Clinic } & \multicolumn{3}{|c|}{ STD Clinic } & \multicolumn{3}{|c|}{ Total } \\
\hline & Male & Female & Total* & Male & Female & Total* & Male $^{\dagger}$ & Female $^{\dagger}$ & Total $^{\ddagger}$ \\
\hline Chlamydia & 467,861 & 939,649 & $1,409,760$ & 52,463 & 39,512 & 92,140 & 610,447 & $1,145,063$ & $1,758,668$ \\
\hline Gonorrhea & 258,366 & 194,513 & 453,535 & 35,142 & 13,871 & 49,077 & 341,401 & 241,074 & 583,405 \\
\hline Primary Syphilis & 7,742 & 1,130 & 8,875 & 2,017 & 156 & 2,173 & 11,280 & 1,498 & 12,793 \\
\hline Secondary Syphilis & 13,332 & 2,744 & 16,080 & 3,166 & 383 & 3,550 & 18,754 & 3,497 & 22,270 \\
\hline Early Non-Primary Non-Secondary Syphilis & 24,081 & 4,568 & 28,663 & 4,031 & 708 & 4,741 & 32,619 & 5,891 & 38,539 \\
\hline Syphilis, Unknown Duration or Late & 19,014 & 8,150 & 27,173 & 2,338 & 701 & 3,040 & 28,872 & 11,232 & 40,137 \\
\hline Chancroid & 2 & 0 & 2 & 0 & 0 & 0 & 3 & 0 & 3 \\
\hline
\end{tabular}

* Total includes cases reported with unknown sex.

${ }^{\dagger}$ Total includes cases reported with unknown reporting source.

₹ Total includes cases reported with unknown sex and reporting source. 


\section{B. National Objectives and Goals}

\section{B1. Healthy People 2020 Objectives}

For three decades, Healthy People has provided a comprehensive set of national 10-year health promotion and disease prevention objectives aimed at improving the health of all Americans. ${ }^{1}$ It is grounded in the principle that establishing objectives and providing benchmarks to track and monitor progress over time can motivate, guide, and focus action.

Healthy People 2020 (HP2020) continues in the tradition of its ambitious, yet achievable, 10-year agenda for improving the Nation's health. HP2020 is the result of a multiyear process that reflects input from a diverse group of individuals and organizations. HP2020 is organized into 42 topic areas, with more than 1,200 measures designed to drive action that will support its four overarching goals:

- Attain high-quality, longer lives free of preventable disease, disability, injury, and premature death.

- Achieve health equity, eliminate disparities, and improve the health of all groups.

- Create social and physical environments that promote good health for all.

- Promote quality of life, healthy development, and healthy behaviors across all life stages.

The topic area, Sexually Transmitted Diseases, contains objectives and measures related to STDs. Baselines, HP2020 targets, and annual progress toward the targets are reported in Table B1. The year 2020 targets for the diseases addressed in this report are as follows: primary and secondary (P\&S) syphilis (males), 6.7 cases per 100,000 males; P\&S syphilis (females), 1.3 cases per 100,000 females; congenital syphilis, 9.6 cases per 100,000 live births; gonorrhea (females aged 15-44 years), 251.9 cases per 100,000 females and gonorrhea (males aged 15-44 years), 194.8 cases per 100,000 males. The majority of the STD-related HP2020 targets were set using a standard percentage improvement with a standard default of a "10 percent improvement over the baseline."

\section{B2. Government Performance and Results Act of 1993}

The Government Performance and Results Act (GPRA) of 1993 was enacted by Congress to increase confidence in the capability of the federal government to increase the effectiveness and accountability of federal programs, to improve service delivery, to provide federal agencies a uniform tool for internal management, and to help Congress make decisions. GPRA requires each agency to have a performance plan with long-term outcomes and annual, measurable performance goals and to report on these plans annually, comparing results with annual goals.

STD has national level performance measures and CDC contextual indicators for a long term objective reported in CDC's Congressional Budget Justification, fulfilling this requirement. The long term objective is to reduce pelvic inflammatory disease (PID), which has specific measures of progress outlined in Table B2.

\section{References}

1. US Department of Health and Human Services. Healthy People 2020 (Healthy People 2020 Web site). https://www.healthypeople.gov/. Accessed August 16, 2019. 
Table B1. Healthy People 2020 (HP2020) Sexually Transmitted Diseases Objectives

\begin{tabular}{|c|c|c|c|c|c|c|c|}
\hline & HP2020 Objectives & $\begin{array}{l}\text { Baseline } \\
\text { Year }\end{array}$ & Baseline & 2016 & 2017 & 2018 & $\begin{array}{l}2020 \\
\text { Target }\end{array}$ \\
\hline \multirow[t]{4}{*}{1} & $\begin{array}{l}\text { Reduce the proportion of adolescents and young adults with } \\
\text { Chlamydia trachomatis infections }\end{array}$ & & & & & & \\
\hline & a. Among females aged 15 to 24 years attending family planning clinics & 2008 & $7.4 \%$ & $8.4 \%$ & $9.6 \%$ & $9.8 \% *$ & $6.7 \%$ \\
\hline & $\begin{array}{l}\text { b. Among females aged } 24 \text { years and under enrolled in a National Job } \\
\text { Training Program }\end{array}$ & 2008 & $12.8 \%$ & $11.4 \%$ & $11.8 \%$ & $12.5 \%$ & $11.5 \%$ \\
\hline & $\begin{array}{l}\text { c. Among males aged } 24 \text { years and under enrolled in a National Job } \\
\text { Training Program }\end{array}$ & 2008 & $7.0 \%$ & $7.1 \%$ & $6.6 \%$ & $6.6 \%$ & $6.3 \%$ \\
\hline \multirow[t]{3}{*}{2} & $\begin{array}{l}\text { Increase the proportion of sexually active females aged } 24 \text { years } \\
\text { and under enrolled in Medicaid plans who are screened for genital } \\
\text { Chlamydia infections during the measurement year }\end{array}$ & & & & & & \\
\hline & a. Females aged 16 to 20 years & 2008 & $52.7 \%$ & $56.4 \%$ & $54.2 \%$ & $\mathrm{~N} / \mathrm{A}$ & $70.9 \%$ \\
\hline & b. Females aged 21 to 24 years & 2008 & $59.4 \%$ & $64.9 \%$ & $63.7 \%$ & $\mathrm{~N} / \mathrm{A}$ & $80.0 \%$ \\
\hline \multirow[t]{3}{*}{3} & $\begin{array}{l}\text { Increase the proportion of sexually active females aged } 24 \text { years } \\
\text { and under enrolled in commercial health insurance plans who are } \\
\text { screened for genital Chlamydia infections during the measurement } \\
\text { year }\end{array}$ & & & & & & \\
\hline & a. Females aged 16 to 20 years & 2008 & $40.1 \%$ & $44.3 \%$ & $45.8 \%$ & $\mathrm{~N} / \mathrm{A}$ & $61.3 \%$ \\
\hline & b. Females aged 21 to 24 years & 2008 & $43.5 \%$ & $54.8 \%$ & $56.4 \%$ & N/A & $74.6 \%$ \\
\hline 4 & $\begin{array}{l}\text { Reduce the proportion of females aged } 15 \text { to } 44 \text { years who have ever } \\
\text { required treatment for pelvic inflammatory disease (PID) }\end{array}$ & $2006-2010$ & $4.2 \%$ & $\mathrm{~N} / \mathrm{A}$ & $3.2 \%^{\dagger}$ & $\mathrm{N} / \mathrm{A}$ & $3.8 \%$ \\
\hline \multirow[t]{3}{*}{5} & Reduce gonorrhea rates & & & & & & \\
\hline & a. Females aged 15 to 44 years & 2008 & 279.9 & 297.1 & 350.0 & 360.3 & 251.9 \\
\hline & b. Males aged 15 to 44 years & 2008 & 216.5 & 370.2 & 440.4 & 462.7 & 194.8 \\
\hline \multirow[t]{3}{*}{6} & $\begin{array}{l}\text { Reduce sustained domestic transmission of primary and secondary } \\
\text { syphilis }\end{array}$ & & & & & & \\
\hline & a. Among females & 2008 & 1.4 & 1.9 & 2.3 & 3.0 & 1.3 \\
\hline & b. Among males & 2008 & 7.4 & 15.6 & 16.9 & 18.7 & 6.7 \\
\hline 7 & Reduce congenital syphilis & 2008 & 10.7 & 15.7 & 23.3 & 33.1 & 9.6 \\
\hline 8 & $\begin{array}{l}\text { Reduce the proportion of young adults with genital herpes infection } \\
\text { due to herpes simplex type } 2\end{array}$ & $2005-2008$ & $10.5 \%$ & $7.6 \%^{\ddagger}$ & $\mathrm{N} / \mathrm{A}$ & N/A & $9.5 \%$ \\
\hline
\end{tabular}

HP2020

Objective

Data Source

\begin{tabular}{ll}
\hline $1 \mathrm{a}$ & STD Surveillance Network (SSuN), CDC \\
\hline $1 \mathrm{~b}, 1 \mathrm{c}$ & National Job Training Program (NJTP) \\
\hline $2 \mathrm{a}, 2 \mathrm{~b}, 3 \mathrm{a}, 3 \mathrm{~b}$ & Healthcare Effectiveness Data and Information Set (HEDIS), National Committee for Quality Assurance (NCQA) \\
\hline 4 & National Survey of Family Growth (NSFG), CDC \\
\hline $5 \mathrm{a}, 5 \mathrm{~b}, 6 \mathrm{a}, 6 \mathrm{~b}, 7$ & National Notifiable Disease Surveillance System (NNDSS), CDC \\
\hline 8 & National Health and Nutrition Examination Survey (NHANES), CDC \\
\hline
\end{tabular}

* Results based on data obtained from January through June 2018.

$+2015-2017$.

‡2015-2016.

NOTE: Data presented in this table reflect data reported to HP2020 in current and prior years. More information about HP2020 is available at: https://www.

healthypeople.gov/ 
Table B2. Congressional Budget Justification Sexually Transmitted Diseases Goals, Measures, and Target

\begin{tabular}{|c|c|c|c|c|}
\hline \multirow[b]{2}{*}{ Congressional Budget Justification Goals } & \multicolumn{3}{|c|}{ Actual } & \multirow{2}{*}{$\begin{array}{r}\text { Target } \\
2020\end{array}$} \\
\hline & 2016 & 2017 & 2018 & \\
\hline \multicolumn{5}{|l|}{ Contextual Indicators } \\
\hline $\begin{array}{l}\text { 2.7.6e. Proportion of sexually active females aged } 16-24 \text { years enrolled in Medicaid who are screened } \\
\text { for chlamydia infections }\end{array}$ & $60.0 \%$ & $60.7 \% *$ & N/A & $60.0 \%$ \\
\hline $\begin{array}{l}\text { 2.7.6f. Proportion of sexually active females aged } 16-24 \text { years enrolled in commercial health insurance } \\
\text { plans who are screened for chlamydia infections }\end{array}$ & $50.1 \%$ & $51.5 \% *$ & N/A & $50.1 \%$ \\
\hline 2.7.7. Reduce the rate ${ }^{\dagger}$ of symptomatic gonorrhea cases in men & 146.1 & 148.3 & 173.4 & 148.3 \\
\hline \multicolumn{5}{|l|}{ Performance Measures for Long Term Objective: Reduce pelvic inflammatory disease in the US } \\
\hline $\begin{array}{l}\text { 2.7.5. Increase the proportion of gonorrhea patients who are treated with a CDC-recommended } \\
\text { antibiotic regimen for gonorrhea }\end{array}$ & $81.3 \%$ & $82.4 \%$ & $85.8 \%$ & $87.6 \%$ \\
\hline 2.9.1. Reduce the incidence of $P \& S$ syphilis/100,000 population in women aged $15-44$ & 4.2 & 5.1 & 6.9 & 0.8 \\
\hline 2.9.2. Reduce the incidence of congenital syphilis $/ 100,000$ live births & 15.7 & 23.3 & 33.1 & 6.2 \\
\hline $\begin{array}{l}\text { 2.9.3. Increase the percentage of pregnant women that are screened for syphilis at least one month } \\
\text { before delivery }\end{array}$ & $89.5 \%$ & $89.9 \%$ & N/A & $87.2 \%$ \\
\hline 2.9.4. Increase the proportion of potential congenital syphilis cases averted & $75.0 \%$ & $72.0 \%$ & N/A & $75.0 \%$ \\
\hline 2.9.5. Reduce the rate of increase of $P \& S$ syphilis & $18.0 \%$ & $11.0 \%$ & $15.0 \%$ & $9.0 \%$ \\
\hline
\end{tabular}

Goals

2.7.6e, 2.7.6f

2.7.5, 2.7.7

2.9.1, 2.9.2, 2.9.4, 2.9.5

2.9 .3

Data Source

$\mathrm{P} \& \mathrm{~S}=$ primary and secondary.

* Proportion based on average screening coverage across plans, weighted by the number of sexually-active women in each plan.

${ }^{+}$Per $100,000$. 
This page intentionally left blank. 


\section{STD Surveillance Case Definitions}

\section{C1. Case Definitions for Nationally Notifiable Infectious Diseases}

The Council of State and Territorial Epidemiologists (CSTE) recommends that state health departments report cases of selected diseases to CDC's National Notifiable Diseases Surveillance System (NNDSS). Case definitions are periodically revised using CSTE's Position Statements and provide uniform criteria of nationally notifiable conditions for reporting purposes. The most current surveillance case definitions for nationally notifiable STDs are listed below. Please see the NNDSS website (https://wwwn.cdc.gov/nndss/case-definitions.html) for historical case definitions.

\section{C1.1 Chancroid (Revised 9/96)}

\section{Clinical description}

A sexually transmitted disease characterized by painful genital ulceration and inflammatory inguinal adenopathy. The disease is caused by infection with Haemophilus ducreyi.

\section{Laboratory criteria for diagnosis}

- Isolation of H. ducreyi from a clinical specimen.

\section{Case classification}

Probable: a clinically compatible case with both a) no evidence of Treponema pallidum infection by darkfield microscopic examination of ulcer exudate or by a serologic test for syphilis performed $\geq 7$ days after onset of ulcers, and b) either a clinical presentation of the ulcer(s) not typical of disease caused by herpes simplex virus (HSV) or a culture negative for HSV.

Confirmed: a clinically compatible case that is laboratory confirmed.

\section{C1.2 Chlamydia trachomatis Infection (Effective 1/10)}

\section{Clinical description}

Infection with Chlamydia trachomatis may result in urethritis, epididymitis, cervicitis, acute salpingitis, or other syndromes when sexually transmitted; however, the infection is often asymptomatic in women. Perinatal infections may result in inclusion conjunctivitis and pneumonia in newborns. Other syndromes caused by C. trachomatis include lymphogranuloma venereum (see Lymphogranuloma Venereum) and trachoma.

\section{Laboratory criteria for diagnosis}

- Isolation of C. trachomatis by culture, OR

- Demonstration of C. trachomatis in a clinical specimen by detection of antigen or nucleic acid.

\section{Case classification}

Confirmed: a case that is laboratory confirmed. 


\section{C1.3 Gonorrhea (Effective 1/14)}

\section{Clinical description}

A sexually transmitted infection commonly manifested by urethritis, cervicitis, proctitis, salpingitis, or pharyngitis. Infection may be asymptomatic.

\section{Laboratory criteria for diagnosis}

- Observation of gram-negative intracellular diplococci in a urethral smear obtained from a male or an endocervical smear obtained from a female, OR

- Isolation of typical gram-negative, oxidase-positive diplococci by culture (presumptive Neisseria gonorrhoeae) from a clinical specimen, OR

- Demonstration of N. gonorrhoeae in a clinical specimen by detection of antigen or nucleic acid.

\section{Case classification}

Probable: demonstration of gram-negative intracellular diplococci in a urethral smear obtained from a male or an endocervical smear obtained from a female.

Confirmed: a person with laboratory isolation of typical gram-negative, oxidase-positive diplococci by culture (presumptive $N$. gonorrhoeae) from a clinical specimen, or demonstration of $N$. gonorrhoeae in a clinical specimen by detection of antigen or detection of nucleic acid via nucleic acid amplification (e.g., polymerase chain reaction [PCR]) or hybridization with a nucleic acid probe.

\section{C1.4 Syphilis (Effective 1/18)}

Syphilis is a complex sexually transmitted disease that has a highly variable clinical course. Adherence to the surveillance case definitions will facilitate understanding the epidemiology of syphilis across the US.

\section{Syphilis, primary (Effective 1/18)}

\section{Clinical description}

A stage of infection with Treponema pallidum characterized by one or more ulcerative lesions (e.g., chancre), which might differ considerably in clinical appearance.

\section{Laboratory criteria for diagnosis}

\section{Confirmatory:}

- Demonstration of T. pallidum by darkfield microscopy in a clinical specimen that was not obtained from the oropharynx and is not potentially contaminated by stool, OR

- Demonstration of T. pallidum by polymerase chain reaction (PCR) or equivalent direct molecular methods in any clinical specimen.

Supportive:

- A reactive nontreponemal serologic test (Venereal Disease Research Laboratory [VDRL], rapid plasma reagin [RPR], or equivalent serologic methods), OR

- A reactive treponemal serologic test (T. pallidum particle agglutination [TP-PA], enzyme immunoassay [EIA], chemiluminescence immunoassay [CIA], or equivalent serologic methods).*

\footnotetext{
* These treponemal tests supersede older testing technologies, including microhemagglutination assay for antibody to T. pallidum [MHA-TP].
} 


\section{Case classification}

Probable: a case that meets the clinical description of primary syphilis and the supportive laboratory criteria.

Confirmed: a case that meets the clinical description of primary syphilis and the supportive confirmatory criteria.

\section{Syphilis, secondary (Effective 1/18)}

\section{Clinical description}

A stage of infection caused by T. pallidum characterized by localized or diffuse mucocutaneous lesions (e.g., rash - such as non-pruritic macular, maculopapular, papular, or pustular lesions), often with generalized lymphadenopathy. Other symptoms can include mucous patches, condyloma lata, and alopecia. The primary ulcerative lesion may still be present. Because of the wide array of symptoms and signs possibly indicating secondary syphilis, serologic tests for syphilis and a physical examination are crucial to determining if a case should be classified as secondary syphilis.

\section{Laboratory criteria for diagnosis}

Confirmatory:

- Demonstration of T. pallidum by darkfield microscopy in a clinical specimen that was not obtained from the oropharynx and is not potentially contaminated by stool, OR

- Demonstration of T. pallidum by polymerase chain reaction (PCR) or equivalent direct molecular methods in any clinical specimen.

Supportive:

- A reactive nontreponemal serologic test (VDRL, RPR, or equivalent serologic methods), AND

- A reactive treponemal serologic test (TP-PA, EIA, CIA, or equivalent serologic methods).

\section{Case classification}

Probable: a case that meets the clinical description of secondary syphilis and the supportive laboratory criteria.

Confirmed: a case that meets the clinical description of secondary syphilis and the confirmatory laboratory criteria.

\section{Syphilis, early non-primary non-secondary (Effective 1/18)}

\section{Clinical description}

A stage of infection caused by T. pallidum in which initial infection has occurred within the previous 12 months, but there are no signs or symptoms of primary or secondary syphilis.

\section{Laboratory criteria for diagnosis}

Supportive:

- A current nontreponemal test titer demonstrating fourfold or greater increase from the last nontreponemal test titer, unless there is evidence that this increase was not sustained for $>2$ weeks.

\section{Case classification}

Probable: a person with no clinical signs or symptoms of primary or secondary syphilis who has one of the following:

- No prior history of syphilis, AND a current reactive nontreponemal test (e.g., VDRL, RPR, or equivalent serologic methods), AND a current reactive treponemal test (e.g., TP-PA, EIA, CIA, or equivalent serologic methods), OR

- A prior history of syphilis and meets the supportive laboratory criteria. 
AND evidence of having acquired the infection within the previous 12 months based on one or more of the following criteria:

- Documented seroconversion or fourfold or greater increase in titer of a nontreponemal test during the previous 12 months, unless there is evidence that this increase was not sustained for $>2$ weeks

- Documented seroconversion of a treponemal test during the previous 12 months

- A history of symptoms consistent with primary or secondary syphilis during the previous 12 months

- Meets epidemiologic criteria.

Epidemiological criteria:

- A history of sexual exposure to a partner within the previous 12 months who had primary, secondary, or early non-primary non-secondary syphilis (documented independently as duration $<12$ months).

- Only sexual contact (sexual debut) was within the previous 12 months.

\section{Syphilis, unknown duration or late (Effective 1/18)}

\section{Clinical description}

A stage of infection caused by T. pallidum in which initial infection has occurred $>12$ months previously or in which there is insufficient evidence to conclude that infection was acquired during the previous 12 months.

\section{Case classification}

Probable: a person with no clinical signs or symptoms of primary or secondary syphilis who meets one of the following sets of criteria:

- No prior history of syphilis, and a current reactive nontreponemal test (e.g., VDRL, RPR, or equivalent serologic methods), and a current reactive treponemal test (e.g., TP-PA, EIA, CIA, or equivalent serologic methods), OR

- A prior history of syphilis, and a current nontreponemal test titer demonstrating fourfold or greater increase from the last nontreponemal test titer, unless there is evidence that this increase was not sustained for $>2$ weeks, OR

- Clinical signs or symptoms and laboratory results that meet the likely or verified criteria for neurologic, ocular, otic, or late clinical manifestations syphilis (see below)

- AND who has no evidence of having acquired the disease within the preceding 12 months (see Syphilis, early non-primary non-secondary).

Comments: Although cases of syphilis of unknown duration are grouped together with late syphilis for the purposes of surveillance, the conservative clinical and public health responses to these cases will differ when there is uncertainty about the duration of infection. When faced with uncertainty, clinicians should act conservatively and treat unknown duration syphilis as if it were late infection, with three doses of benzathine penicillin. In contrast, the most conservative approach for STD control programs would be to manage cases of syphilis of unknown duration as early non-primary non-secondary infections and search for partners who may have been recently infected. Because this would not be feasible for most STD control programs, programs should consider prioritizing cases of syphilis of unknown duration with higher nontreponemal titers (e.g., 1:32 or higher) for investigation and partner services. Although nontreponemal titers cannot reliably distinguish between early infection ( $<12$ months duration) and late infection $(>12$ months duration), nontreponemal titers usually are higher early in the course of syphilis infection. 


\section{Syphilis, Congenital (Effective 1/18)}

\section{Clinical description}

A condition caused by infection in utero with T. pallidum. A wide spectrum of severity exists, from inapparent infection to severe cases that are clinically apparent at birth. An infant or child (aged less than 2 years) may have signs such as hepatosplenomegaly, rash, condyloma lata, snuffles, jaundice (nonviral hepatitis), pseudoparalysis, anemia, or edema (nephrotic syndrome and/or malnutrition). An older child may have stigmata (e.g., interstitial keratitis, nerve deafness, anterior bowing of shins, frontal bossing, mulberry molars, Hutchinson teeth, saddle nose, rhagades, or Clutton joints).

\section{Laboratory criteria for diagnosis}

- Demonstration of $T$. pallidum by darkfield microscopy of lesions, body fluids, or neonatal nasal discharge, OR

- PCR or other equivalent direct molecular methods of lesions, neonatal nasal discharge, placenta, umbilical cord, or autopsy material, OR

- Immunohistochemistry (IHC), or special stains (e.g., silver staining) of specimens from lesions, placenta, umbilical cord, or autopsy material.

\section{Case classification}

Probable: a condition affecting an infant whose mother had untreated or inadequately treated* syphilis at delivery, regardless of signs in the infant, OR an infant or child who has a reactive non-treponemal test for syphilis (VDRL, RPR, or equivalent serologic methods) AND any one of the following:

- Any evidence of congenital syphilis on physical examination (see Clinical description).

- Any evidence of congenital syphilis on radiographs of long bones.

- A reactive CSF VDRL test.

- In a non-traumatic lumbar puncture, an elevated CSF leukocyte (white blood cell [WBC]) count or protein (without other cause):

- Suggested parameters for abnormal CSF WBC and protein values:

1. During the first 30 days of life, a CSF WBC count of $>15 \mathrm{WBC} / \mathrm{mm} 3$ or a CSF protein $>120 \mathrm{mg} / \mathrm{dL}$ is abnormal.

2. After the first 30 days of life, a CSF WBC count of $>5 \mathrm{WBC} \mathrm{mm} 3$ or a CSF protein $>40 \mathrm{mg} / \mathrm{dL}$, regardless of CSF serology.

The treating clinician should be consulted to interpret the CSF values for the specific patient.

* Adequate treatment is defined as completion of a penicillin-based regimen, in accordance with CDC treatment guidelines, appropriate for stage of infection, initiated 30 or more days before delivery.

Confirmed: a case that is laboratory confirmed.

Comments: Congenital and acquired syphilis may be difficult to distinguish when a child is seropositive after infancy. Signs of congenital syphilis may not be obvious, and stigmata may not yet have developed. Abnormal values for CSF VDRL, WBC count, and protein may be found in either congenital or acquired syphilis. Findings on radiographs of long bones may help because radiographic changes in the metaphysis and epiphysis are considered classic signs of congenitally acquired syphilis. While maternal antibodies can complicate interpretation of serologic tests in an infant, reactive tests past 18 months of age are considered to reflect the status of the child. The decision may ultimately be based on maternal history and clinical judgment. In a young child, the possibility of sexual abuse should be considered as a cause of acquired rather than congenital syphilis, depending on the clinical picture. For reporting purposes, congenital syphilis includes cases of congenitally acquired syphilis among infants and children as well as syphilitic stillbirths. 


\section{Syphilitic Stillbirth (Effective 1/18)}

\section{Clinical case definition}

A fetal death that occurs after a 20 -week gestation or in which the fetus weighs greater than $500 \mathrm{~g}$ and the mother had untreated or inadequately treated* syphilis at delivery.

\footnotetext{
* Adequate treatment is defined as completion of a penicillin-based regimen, in accordance with CDC treatment guidelines, appropriate for stage of infection, initiated 30 or more days before delivery.
}

Comments: For reporting purposes, congenital syphilis includes cases of congenitally acquired syphilis among infants and children as well as syphilitic stillbirths.

\section{Comments: Additional information to be collected on clinical manifestations of reported syphilis cases (Effective 1/18)}

Syphilis is a systemic infection that, if untreated, can cause a variety of clinical manifestations, including:

- Signs and symptoms of primary and secondary syphilis (see above case definitions).

- Latent infections (i.e., those lacking any signs or symptoms).

- Neurologic, ocular, or otic manifestations (neurosyphilis, ocular syphilis, or otosyphilis), which can occur at any stage of syphilis.

- Late clinical manifestations (tertiary syphilis), which generally occur after 15-30 years of untreated infection.

The following provides guidance for reporting neurologic, ocular, otic, and late clinical manifestations of syphilis. Cases should be reported according to stage of infection, as defined above (e.g., primary syphilis; secondary syphilis; early non-primary, non-secondary syphilis; or unknown duration or late syphilis) and the clinical manifestations should be reported in the case report data, as defined below.

\section{Neurologic manifestations:}

Neurologic manifestations (neurosyphilis) can occur at any stage of syphilis. If the patient has neurologic manifestations of syphilis, the case should be reported with the appropriate stage of infection (as if neurologic manifestations were not present) and neurologic manifestations should be noted in the case report data.

\section{Clinical description}

Infection of the central nervous system with T. pallidum, as evidenced by manifestations including syphilitic meningitis, meningovascular syphilis, general paresis, including dementia, and tabes dorsalis.

\section{Classification of neurologic manifestations (neurosyphilis)}

Possible: a person with a reactive nontreponemal test (e.g., VDRL, RPR, or equivalent serologic methods) and a reactive treponemal test (e.g., TP-PA, EIA, CIA or equivalent serologic methods) and clinical symptoms or signs that are consistent with neurosyphilis without other known causes for these clinical abnormalities.

Likely: a person with a reactive nontreponemal test (e.g., VDRL, RPR, or equivalent serologic methods) and a reactive treponemal test (e.g., TP-PA, EIA, CIA or equivalent serologic methods) with both of the following:

- Clinical symptoms or signs that are consistent with neurosyphilis without other known causes for these clinical abnormalities, AND

- Elevated CSF protein ( $>50 \mathrm{mg} / \mathrm{dL} 2)$ or leukocyte count $(>5 \mathrm{WBC} / \mathrm{mm} 3 \mathrm{CSF})$ in the absence of other known causes of these abnormalities. 
Verified: a person with a reactive nontreponemal test (e.g., VDRL, RPR, or equivalent serologic methods) and a reactive treponemal test (e.g., TP-PA, EIA, CIA or equivalent serologic methods) with both of the following:

- Clinical symptoms or signs that are consistent with neurosyphilis without other known causes for these clinical abnormalities, AND

- A reactive VDRL in CSF in the absence of grossly bloody contamination of the CSF.

\section{Ocular Manifestations:}

Ocular manifestations (ocular syphilis) can occur at any stage of syphilis. If the patient has ocular manifestations of syphilis, the case should be reported with the appropriate stage of infection (as if ocular manifestations were not present) and ocular manifestations should be noted in the case report data.

\section{Clinical description}

Infection of any eye structure with T. pallidum, as evidenced by manifestations including posterior uveitis, panuveitis, anterior uveitis, optic neuropathy, and retinal vasculitis. Ocular syphilis may lead to decreased visual acuity including permanent blindness.

\section{Classification of ocular manifestations (ocular syphilis)}

Possible: a person with a reactive nontreponemal test (e.g., VDRL, RPR, or equivalent serologic methods) and a reactive treponemal test (e.g., TP-PA, EIA, CIA or equivalent serologic methods) and clinical symptoms or signs consistent with ocular syphilis without other known causes for these clinical abnormalities.

Likely: a person with a reactive nontreponemal test (e.g., VDRL, RPR, or equivalent serologic methods) and a reactive treponemal test (e.g., TP-PA, EIA, CIA or equivalent serologic methods) and both of the following:

- Clinical symptoms or signs consistent with ocular syphilis without other known causes for these clinical abnormalities, AND

- Findings on exam by an ophthalmologist that are consistent with ocular syphilis in the absence of other known causes for these abnormalities.

Verified: a person with a reactive nontreponemal test (e.g., VDRL, RPR, or equivalent serologic methods) and a reactive treponemal test (e.g., TP-PA, EIA, CIA or equivalent serologic methods) and both of the following:

- Clinical symptoms or signs consistent with ocular syphilis without other known causes for these clinical abnormalities, AND

- Demonstration of T. pallidum in aqueous or vitreous fluid by darkfield microscopy, or by PCR or equivalent direct molecular methods.

\section{Otic Manifestations:}

Otic manifestations can occur at any stage of syphilis. If the patient has otic manifestations of syphilis, the case should be reported with the appropriate stage of infection (as if otic manifestations were not present) and otic manifestations should be noted in the case report data.

\section{Clinical description}

Infection of the cochleovestibular system with T. pallidum, as evidenced by manifestations including sensorineural hearing loss, tinnitus, and vertigo. 


\section{Classification of otic manifestations (otosyphilis)}

Possible: a person with a reactive nontreponemal test (e.g., VDRL, RPR, or equivalent serologic methods) and a reactive treponemal test (e.g., TP-PA, EIA, CIA or equivalent serologic methods) and clinical symptoms or signs consistent with otosyphilis without other known causes for these clinical abnormalities.

Likely: a person with a reactive nontreponemal test (e.g., VDRL, RPR, or equivalent serologic methods) and a reactive treponemal test (e.g., TP-PA, EIA, CIA or equivalent serologic methods) and both of the following:

- Clinical symptoms or signs consistent with otosyphilis without other known causes for these clinical abnormalities, AND

- Findings on exam by an otolaryngologist that are consistent with otosyphilis in the absence of other known causes for these abnormalities.

Verified: a person with a reactive nontreponemal test (e.g., VDRL, RPR, or equivalent serologic methods) and a reactive treponemal test (e.g., TP-PA, EIA, CIA or equivalent serologic methods) and both of the following:

- Clinical symptoms or signs consistent with otosyphilis without other known causes for these clinical abnormalities, AND

- Demonstration of T. pallidum in inner ear fluid by darkfield microscopy, or by PCR or equivalent direct molecular detection methods.

\section{Late Clinical Manifestations:}

Late clinical manifestations of syphilis usually develop only after a period of 15-30 years of untreated infection. Therefore, if the patient has late clinical manifestations of syphilis, the case should be reported with the appropriate stage of infection (for the vast majority of cases, unknown duration or late syphilis) and late clinical manifestations should be noted in the case report data.

\section{Clinical description}

Late clinical manifestations of syphilis (tertiary syphilis) may include inflammatory lesions of the cardiovascular system (e.g., aortitis, coronary vessel disease), skin (e.g., gummatous lesions), bone (e.g., osteitis), or other tissue. Rarely, other structures (e.g., the upper and lower respiratory tracts, mouth, eye, abdominal organs, reproductive organs, lymph nodes, and skeletal muscle) may be involved. In addition, certain neurologic manifestations (e.g., general paresis and tabes dorsalis) are also late clinical manifestations of syphilis.

\section{Classification of late clinical manifestations of syphilis (tertiary syphilis)}

Likely: a person with a reactive nontreponemal test (e.g., VDRL, RPR, or equivalent serologic methods) and a reactive treponemal test (e.g., TP-PA, EIA, CIA or equivalent serologic methods) with either of the following:

- Characteristic abnormalities or lesions of the cardiovascular system (e.g., aortitis, coronary vessel disease), skin (e.g., gummatous lesions), bone (e.g., osteitis), or other tissue, in the absence of other known causes of these abnormalities, OR

- Clinical signs and symptoms consistent with late neurologic manifestations of syphilis (e.g., general paresis, including dementia, or tabes dorsalis) in a case that meets the criteria for likely neurologic manifestations of syphilis (see above).

Verified: a person with a reactive nontreponemal test (e.g., VDRL, RPR, or equivalent serologic methods) and a reactive treponemal test (e.g., TP-PA, EIA, CIA or equivalent serologic methods) and either of the following:

- Characteristic abnormalities or lesions of the cardiovascular system (e.g., aortitis, coronary vessel disease), skin (e.g., gummatous lesions), bone (e.g., osteitis), or other tissue in the absence of other known causes of these abnormalities, in combination with either demonstration of T. pallidum in late lesions by special stains or equivalent methods, or by PCR or equivalent direct molecular methods, or demonstration of pathologic changes that are consistent with T. pallidum infection on histologic examination of late lesions, OR 
Clinical signs and symptoms consistent with late neurologic manifestations of syphilis (e.g., general paresis, including dementia, or tabes dorsalis) in a case that meets the criteria for verified neurologic manifestations of syphilis (see above).

\section{C2. Case Definitions For Non-Notifiable Infectious Diseases}

Although the conditions below are not currently nationally notifiable, they may be reportable in some jurisdictions. To provide uniform criteria for those jurisdictions, case definitions are provided by CSTE. Case definitions are periodically revised. The most current surveillance case definitions for non-notifiable STDs are listed below. Please see the NNDSS website (https://wwwn.cdc.gov/nndss/case-definitions.html) for historical case definitions.

\section{C2.1 Genital Herpes (Herpes Simplex Virus) (Revised 9/96)}

\section{Clinical description}

A condition characterized by visible, painful genital or anal lesions.

\section{Laboratory criteria for diagnosis}

- Isolation of herpes simplex virus from cervix, urethra, or anogenital lesion, OR

- Demonstration of virus by antigen detection technique in clinical specimens from cervix, urethra, or anogenital lesion, OR

- Demonstration of multinucleated giant cells on a Tzanck smear of scrapings from an anogenital lesion.

\section{Case classification}

Probable: a clinically compatible case (in which primary and secondary syphilis have been excluded by appropriate serologic tests and darkfield microscopy, when available) with either a diagnosis of genital herpes based on clinical presentation (without laboratory confirmation) or a history of one or more previous episodes of similar genital lesions.

Confirmed: a clinically compatible case that is laboratory confirmed.

\section{Comment}

Genital herpes should be reported only once per patient. The first diagnosis for a patient with no previous diagnosis should be reported.

\section{C2.2 Genital Warts (Revised 9/96)}

\section{Clinical description}

An infection characterized by the presence of visible, exophytic (raised) growths on the internal or external genitalia, perineum, or perianal region.

\section{Laboratory criteria for diagnosis}

- Histopathologic changes characteristic of human papillomavirus infection in specimens obtained by biopsy or exfoliative cytology, OR

- Demonstration of virus by antigen or nucleic acid detection in a lesion biopsy.

\section{Case classification}

Probable: a clinically compatible case without histopathologic diagnosis and without microscopic or serologic evidence that the growth is the result of secondary syphilis.

Confirmed: a clinically compatible case that is laboratory confirmed. 


\section{Comment}

Genital warts should be reported only once per patient. The first diagnosis for a patient with no previous diagnosis should be reported.

\section{C2.3 Granuloma Inguinale}

\section{Clinical description}

A slowly progressive ulcerative disease of the skin and lymphatics of the genital and perianal area caused by infection with Calymmatobacterium granulomatis. A clinically compatible case would have one or more painless or minimally painful granulomatous lesions in the anogenital area.

\section{Laboratory criteria for diagnosis}

- Demonstration of intracytoplasmic Donovan bodies in Wright or Giemsa-stained smears or biopsies of granulation tissue.

\section{Case classification}

Confirmed: a clinically compatible case that is laboratory confirmed.

\section{C2.4 Lymphogranuloma Venereum}

\section{Clinical description}

Infection with L1, L2, or, L3 serovars of $C$. trachomatis may result in a disease characterized by genital lesions, suppurative regional lymphadenopathy, or hemorrhagic proctitis. The infection is usually sexually transmitted.

\section{Laboratory criteria for diagnosis}

- Isolation of C. trachomatis, serotype L1, L2, or L3 from clinical specimen, OR

- Demonstration by immunofluorescence of inclusion bodies in leukocytes of an inguinal lymph node (bubo) aspirate, OR

- Positive microimmunofluorescent serologic test for a lymphogranuloma venereum strain of C. trachomatis.

\section{Case classification}

Probable: a clinically compatible case with one or more tender fluctuant inguinal lymph nodes or characteristic proctogenital lesions with supportive laboratory findings of a single C. trachomatis complement fixation titer of $>64$.

Confirmed: a clinically compatible case that is laboratory confirmed.

\section{C2.5 Mucopurulent Cervicitis (Revised 9/96)}

\section{Clinical description}

Cervical inflammation that is not the result of infection with $N$. gonorrhoeae or Trichomonas vaginalis. Cervical inflammation is defined by the presence of one of the following criteria:

- Mucopurulent secretion (from the endocervix) that is yellow or green when viewed on a white, cotton-tipped swab (positive swab test), OR

- Induced endocervical bleeding (bleeding when the first swab is placed in the endocervix).

\section{Laboratory criteria for diagnosis}

- No evidence of N. gonorrhoeae by culture, Gram stain, or antigen or nucleic acid detection, and no evidence of T. vaginalis on wet mount. 


\section{Case classification}

Confirmed: a clinically compatible case in a female who does not have either gonorrhea or trichomoniasis.

\section{Comment}

Mucopurulent cervicitis (MPC) is a clinical diagnosis of exclusion. The syndrome may result from infection with any of several agents (see C. trachomatis). If gonorrhea, trichomoniasis, and chlamydia are excluded, a clinically compatible illness should be classified as MPC. An illness in a female that meets the case definition of MPC and C. trachomatis infection should be classified as chlamydia.

\section{C2.6 Nongonococcal Urethritis (Revised 9/96)}

\section{Clinical description}

Urethral inflammation that is not the result of infection with $N$. gonorrhoeae. Urethral inflammation may be diagnosed by the presence of one of the following criteria:

- A visible abnormal urethral discharge, OR

- A positive leukocyte esterase test from a male aged $<60$ years who does not have a history of kidney disease or bladder infection, prostate enlargement, urogenital anatomic anomaly, or recent urinary tract instrumentation, OR

- Microscopic evidence of urethritis ( $\geq 5$ white blood cells per high-power field) on a Gram stain of a urethral smear.

\section{Laboratory criteria for diagnosis}

- No evidence of N. gonorrhoeae infection by culture, Gram stain, or antigen or nucleic acid detection.

\section{Case classification}

Confirmed: a clinically compatible case in a male in whom gonorrhea is not found, either by culture, Gram stain, or antigen or nucleic acid detection.

\section{Comment}

Nongonococcal urethritis (NGU) is a clinical diagnosis of exclusion. The syndrome may result from infection with any of several agents (see C. trachomatis). If gonorrhea and chlamydia are excluded, a clinically compatible illness should be classified as NGU. An illness in a male that meets the case definition of NGU and C. trachomatis infection should be classified as chlamydia.

\section{C2.7 Pelvic Inflammatory Disease (Revised 9/96)}

\section{Clinical case definition}

A clinical syndrome resulting from the ascending spread of microorganisms from the vagina and endocervix to the endometrium, fallopian tubes, and/or contiguous structures. In a female who has lower abdominal pain and who has not been diagnosed as having an established cause other than pelvic inflammatory disease (PID) (e.g., ectopic pregnancy, acute appendicitis, and functional pain), all the following clinical criteria must be present:

- Lower abdominal tenderness, AND

- Tenderness with motion of the cervix, AND

- Adnexal tenderness. 
In addition to the preceding criteria, at least one of the following findings must also be present:

- Meets the surveillance case definition of C. trachomatis infection or gonorrhea

- $\quad$ Temperature $>100.4 \mathrm{~F}(>38.0 \mathrm{C})$

- Leukocytosis $>10,000 \mathrm{WBC} / \mathrm{mm} 3$

- Purulent material in the peritoneal cavity obtained by culdocentesis or laparoscopy

- Pelvic abscess or inflammatory complex detected by bimanual examination or by sonography

- Patient is a sexual contact of a person known to have gonorrhea, chlamydia, or nongonococcal urethritis.

\section{Case classification}

Confirmed: a case that meets the clinical case definition.

\section{Comment}

For reporting purposes, a clinician's report of PID should be counted as a case. 


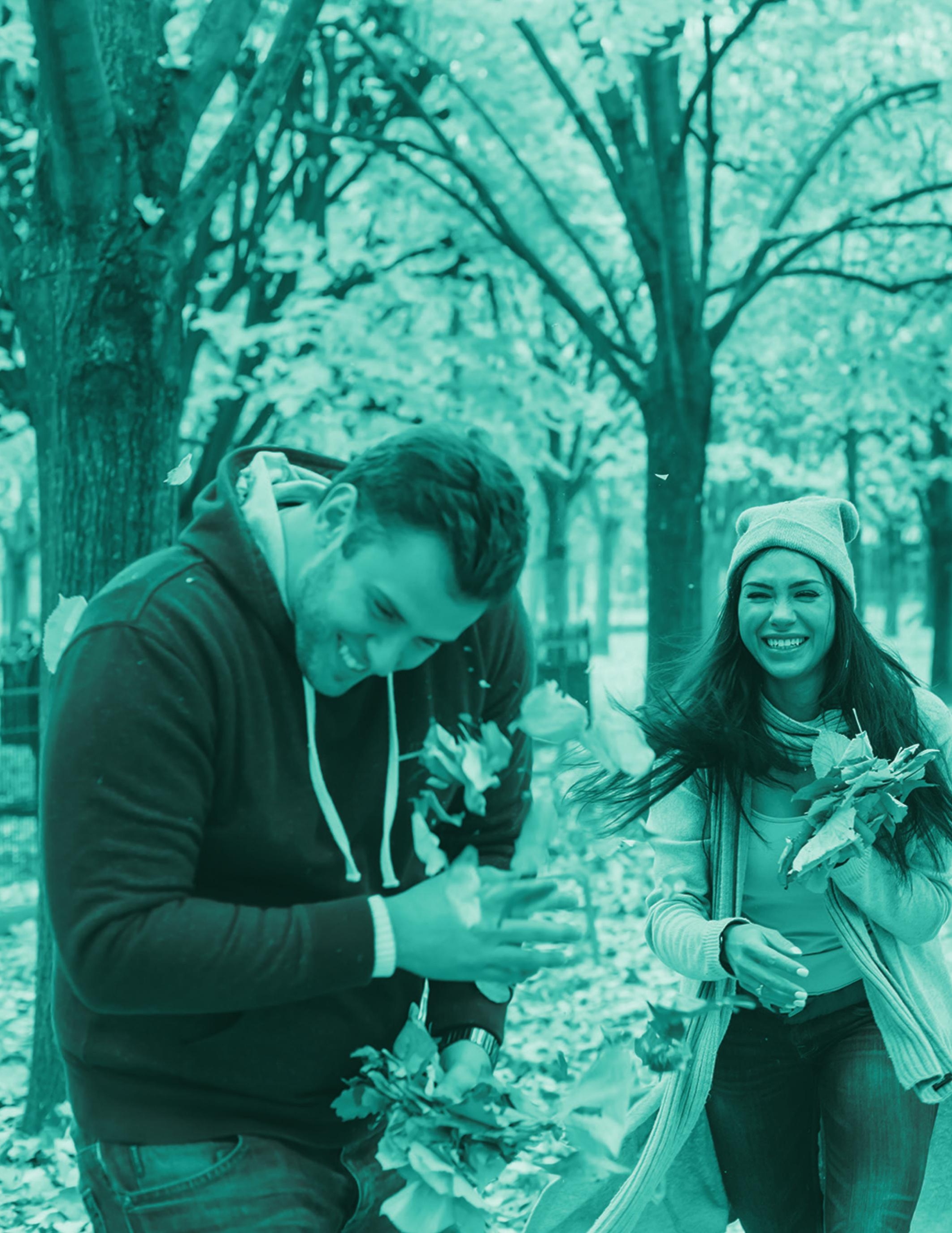




\section{Contributors}

We gratefully acknowledge the contributions of state STD project directors, STD program managers, state and territorial epidemiologists, and laboratory directors. The persons listed were in the positions shown as of August 23, 2019.

\begin{tabular}{|c|c|c|c|c|}
\hline State/City/Territory & STD Project Directors & STD Program Managers & State Epidemiologists & Laboratory Directors \\
\hline Alabama & Anthony Merriweather & Thomas Lee & Sherri L. Davidson & Sharon Massingale \\
\hline Alaska & Susan Jones & Tracy Smith & Joe McLaughlin & Bernard Jilly \\
\hline Arizona & Rebecca Scranton & Rebecca Scranton & Kenneth Komatsu & Victor Waddell \\
\hline Arkansas & Tiffany Vance & Brandi Roberts & Dirk Haselow & Glen Baker \\
\hline California & James Watt & James Watt & Gil F. Chavez & Katya Ledin \\
\hline Los Angeles & Mario Perez & Leo Moore & Gil F. Chavez & Nicole Green \\
\hline San Francisco & Susan Philip & Trang Nguyen & Gil F. Chavez & Godfred Masinde \\
\hline Colorado & Daniel Shodell & Yesenia Mendez & Rachel K. Herlihy & Scott Bookman \\
\hline Connecticut & Lynn Sosa & Linda Ferraro & Matthew Cartter & Jafar Razeq \\
\hline Delaware & Catherine Mosley & Catherine Mosley & Tabatha Offutt-Powell & Sergio Huerta \\
\hline District of Columbia & Michael Kharfen & Adam Visconti & John Davies-Cole & Anthony Tran \\
\hline Florida & Craig Wilson & Craig Wilson & Carina Blackmore & Carina Blackmore \\
\hline Georgia & LaTasha Terry & Mildred Banks & Cherie Drenzek & Elizabeth Franko \\
\hline Hawaii & Peter Whiticar & Gerald “Luke”" Hasty, Jr. & Sarah Park & Edward Desmond \\
\hline Idaho & Aimee Shipman & Kimberly Matulonis & Christine Hahn & Christopher Ball \\
\hline Illinois & Danny Brikshavana & Danny Brikshavana & Jennifer Layden & Matt Charles \\
\hline Chicago & Irina Tabidze & Tammy Rutledge & Jennifer Layden & Massimo Pacilli/Raj Rajendra \\
\hline Indiana & Caitlin Conrad & Caitlin Conrad & Pamela Pontones & Judith Lovchik \\
\hline lowa & Randy Mayer & George Walton & Caitlin Pedati & Michael Pentella \\
\hline Kansas & Jennifer VandeVelde & Jennifer VandeVelde & Farah Ahmed & N. Myron Gunsalus \\
\hline Kentucky & Robert Brawley & Chang Lee & Douglas Thoroughman & Jeremy Hart \\
\hline Louisiana & DeAnn Gruber & Chaquetta Johnson & Raoult Ratard & Richard Tulley \\
\hline Maine & Jayson Hunt & Emer Smith & Siiri Bennett & Kenneth Pote \\
\hline Maryland & Kenneth Ruby & Marcia Pearlowitz & David Blythe & Robert Myers \\
\hline Baltimore & Adena Greenbaum & LaNisha Childs & David Blythe & Irish Brown-Plowden \\
\hline Massachusetts & Kathleen Roosevelt & Betsy John/Lauren Molotnikov & Larry Madoff & Sandra Smole \\
\hline Michigan & Kathryn Macomber & Kristine Judd-Tuinier & Sarah Lyon-Callo & Sandip Shah \\
\hline Minnesota & Christine Jones & Peggy Darrett-Brewer & Ruth Lynfield & Joanne Bartkus \\
\hline Mississippi & Kendra Johnson & Christie Lewis & Paul Byers & Daphne Ware \\
\hline Missouri & Christine Smith & Craig Highfill & George Turabelidze & Bill Whitmar \\
\hline Montana & Cara Murolo & Cara Murolo & Laura Williamson & Ron Paul \\
\hline Nebraska & Jeri Weberg-Bryce & Jeri Weberg-Bryce & Tom Safranek & Peter Iwen \\
\hline Nevada & Kellie Ducker & Elizabeth Kessler & Melissa Peek-Bullock & Stephanie Van Hooser \\
\hline New Hampshire & Lindsay Pierce & Lorlette Moir/Hannah Bowen & Benjamin Chan & Christine Bean \\
\hline New Jersey & Greta Anschuetz & Greta Anschuetz & Christina Tan & Thomas J. Kirn \\
\hline New Mexico & Andrew Gans & Janine Waters & Michael Landen & Twila Kunde \\
\hline New York & Rachel Hart-Malloy & Margaret Carroll & Debra Blog & Jill Taylor \\
\hline New York City & Susan Blank & Kate Washburn & Debra Blog & Jennifer Rakeman \\
\hline North Carolina & Jacquelyn Clymore & Roger Follas & Zack Moore & Dee Pettit \\
\hline North Dakota & Lindsey VanderBusch & Lindsey VanderBusch & Tracy Miller & Christie Massen \\
\hline Ohio & Laurie Rickert & Karen Nicosia & Sietske de Fijter & Quanta Brown \\
\hline Oklahoma & Kristen Eberly & Sally Bouse & Laurence Burnsed & Samuel T. Dunn \\
\hline Oregon & Annick Benson-Scott & Joshua Ferrer & Thomas Jeanne & John Fontana \\
\hline Pennsylvania & Beth Butler & Kristine King & Sharon Watkins & Dongxiang Xia \\
\hline Philadelphia & Steven Alles & Cherie Walker-Baban & Sharon Watkins & Kerry Buchs \\
\hline
\end{tabular}




\begin{tabular}{|c|c|c|c|c|}
\hline State/City/Territory & STD Project Directors & STD Program Managers & State Epidemiologists & Laboratory Directors \\
\hline Rhode Island & Thomas Bertrand & Katharine Howe & Utpala Bandy & Ewa King \\
\hline South Carolina & Ali Mansaray & Bernard Gilliard & Linda Bell & Robert Brent Dixon \\
\hline South Dakota & Angela Jackley & Amanda Gill & Joshua Clayton & Timothy Southern \\
\hline Tennessee & Leo Parker & Parul Patel & Tim Jones & Richard Steece \\
\hline Texas & Shelley Lucas & Sydney Minnerly & Linda Gaul & Grace Kubin \\
\hline Utah & Scott White & Kassy Keen & Angela Dunn & Robyn Atkinson-Dunn \\
\hline Vermont & Daniel Daltry & Daniel Daltry & Patsy Kelso/Lori Cragin & Mary Celotti \\
\hline Virginia & Diana Prat & Oana Vasiliu & Lilian Peake & Denise Toney \\
\hline Washington & Elisabeth Crutsinger-Perry & Emalie Huriaux & $\begin{array}{l}\text { Scott Lindquist/Cathy } \\
\text { Wasserman }\end{array}$ & Romesh Gautom \\
\hline West Virginia & Loretta Haddy & Pamela Reynolds & Maria del Rosario & Sharon Lee Cibrik \\
\hline Wisconsin & Stephanie Smiley & Anthony Wade & Jon Meiman & James Schauer \\
\hline Wyoming & Debi Anderson & Vacant & Alexia Harrist & Shawna Dereemer \\
\hline American Samoa & Fara M. Utu & Ina Sagaga & Afili Tufa & June Vaifanua-Leo \\
\hline Federated States of Micronesia & Mayleen Ekiek & Mayleen Ekiek & Marcus Samo & $\begin{array}{l}\text { Maria Marfel, Kasian Otoko, } \\
\text { Maopa Raikabula, William Nena }\end{array}$ \\
\hline $\begin{array}{l}\text { Government of the Marshall } \\
\text { Islands }\end{array}$ & Mailynn K. Lang & Adela Sibok & Jill McGready & Paul Lalita \\
\hline Northern Mariana Islands & Vicenta Tenorio & Vicenta Tenorio & Paul White & Philip Dauterman \\
\hline Guam & Bernadette P. Schumann & Vince Aguon & Ann Pobustky & AnneMarie Santos \\
\hline Puerto Rico & Greduvel Duran Guzman & Javier Vazquez Melendez & Carmen Deseda & Gonzalo Gonzalez \\
\hline Republic of Palau & Sherilynn Madraisau & Connie B. Olikong & Tmong Cheryl Udui & Clarette Matlab, Francis Termeteet \\
\hline Virgin Islands & Jason Henry & Vacant & Esther Ellis & Brett Ellis \\
\hline
\end{tabular}


This page intentionally left blank. 



MEDIA MAIL
POSTAGE \& FEES PAID
CDC
Permit No. G-284

U.S. Department of Health \& Human Services Centers for Disease Control and Prevention, MS US12-2 Atlanta, Georgia 30329-4027

OFFICIAL BUSINESS

Penalty for Private Use $\$ 300$ 